\title{
Mechanische Relaxation in komplexen Fluiden
}

\author{
Dissertation
}

zur Erlangung des Doktorgrades der Mathematisch-Naturwissenschaftlichen Fakultäten der Georg-August-Universität zu Göttingen

\author{
Vorgelegt von \\ Peter Christian Rösner \\ aus Augsburg
}

Göttingen, 2004 
D 7

Referent: Prof. Dr. Konrad Samwer Korreferent: Prof. Dr. Helmar Teichler

Tag der mündlichen Prüfung: 01.03.2004 


\title{
Mechanische Relaxation in komplexen Fluiden
}

\author{
Peter Christian Rösner
}

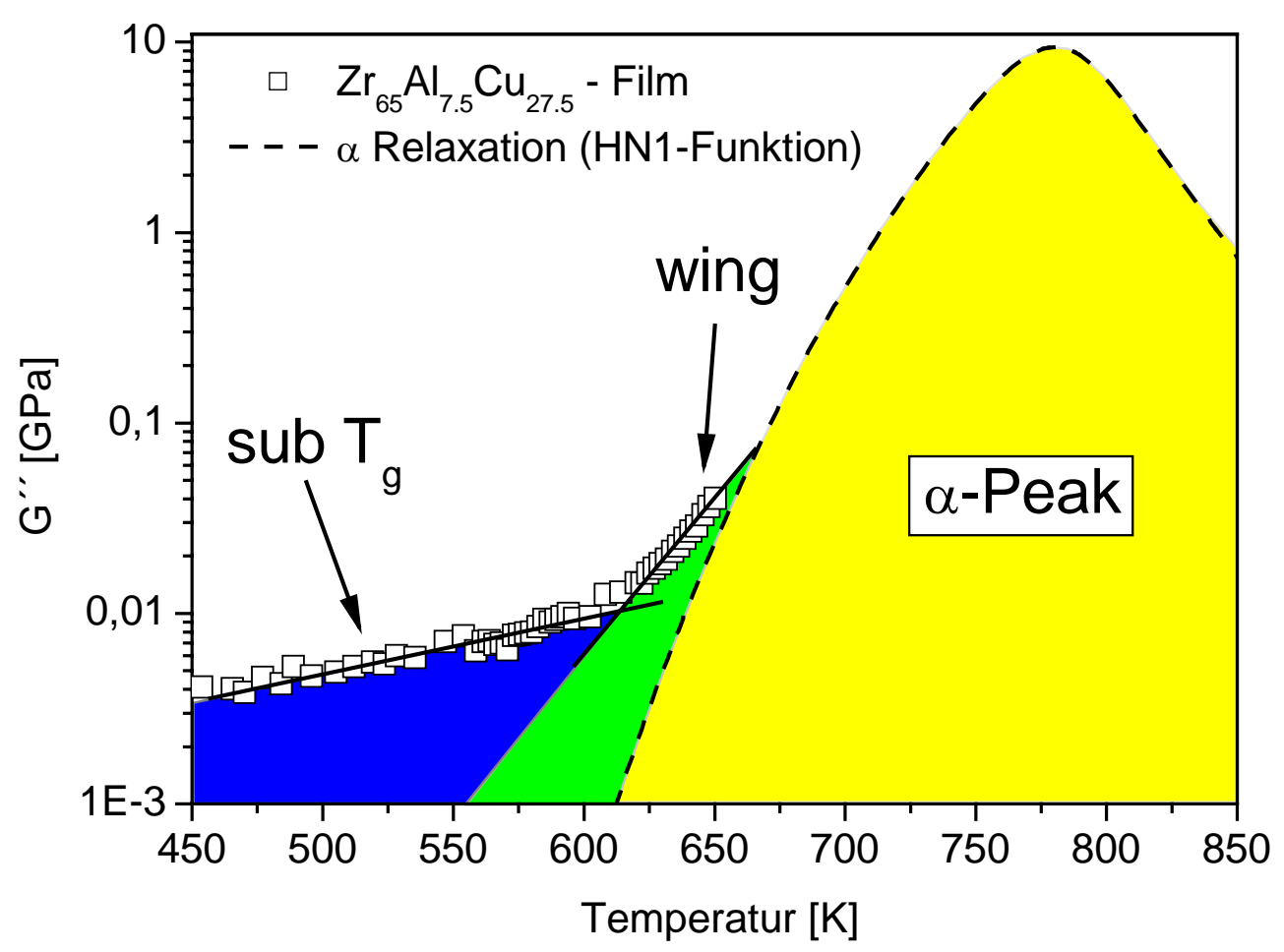

Titelbild:

Trennung der zusätzlichen dynamischen Beiträge zum primären $\alpha$-Relaxationsprozess (gelb) in einer $300 \mathrm{~nm}$ dicken amorphen $\mathrm{Zr}_{65} \mathrm{Al}_{7,5} \mathrm{Cu}_{27,5}$-Schicht bei tiefen Temperaturen in einen „excess-wing“ (grün) und sub- $\mathrm{T}_{\mathrm{g}}$-Beiträge (blau). 



\section{Inhaltsverzeichnis}

Inhaltsverzeichnis

1 Einleitung. 6

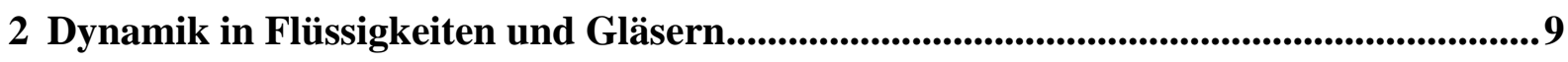

2.1 Phänomenologisches Frequenzspektrum im Überblick ............................................. 9

2.2 Glasübergang, viskoses Fließen und $\alpha$-Relaxation .............................................. 11

2.2.1 Glasübergang in strukturellen Gläsern ...................................................... 12

2.2.2 Viskosität und Fragilität von Gläsern .......................................................... 14

2.2.3 Relaxation im Zeitraum und Clustertheorie..................................................... 16

2.2.4 Temperaturabhängigkeit der Relaxationsdynamik ...................................... 18

2.2.4.1 Arrhenius-Gesetz ...................................................................... 18

2.2.4.2 Vogel-Fulcher-Tammann-Gleichung (VFT) ................................... 18

2.2.4.3 Erweiterte Freie-Volumen-Theorie (EFV) ...................................... 19

2.2.4.4 Frustration-Limited-Domain-Modell (FLD).................................... 19

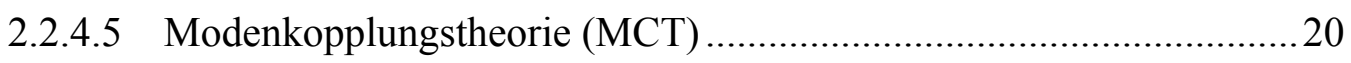

2.2.5 Übergang vom Zeit- in den Frequenzraum....................................................20

2.2.6 Temperaturabhängige Relaxationsfunktionen ..............................................23

2.2.6.1 Prozessführung bei konstanter Temperatur .................................... 23

2.2.6.2 Prozessführung bei konstanter Frequenz ........................................ 24

2.2.6.3 Zeit-Temperatur-Superposition ...................................................24

2.2.6.4 Beispiel für den Übergang Frequenz- in Temperaturraum ..................24

2.2.6.5 Schwierigkeiten beim Übergang in den Temperaturraum ..................27

2.3 Langsame $\beta$-Relaxation durch intramolekulare Dynamik .......................................27

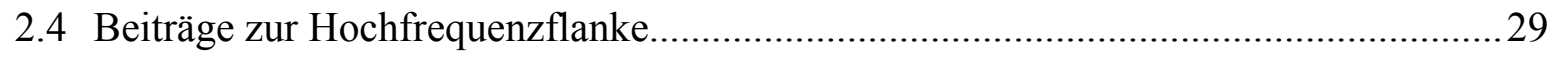

3 Experimentelles................................................................................................................................. 32

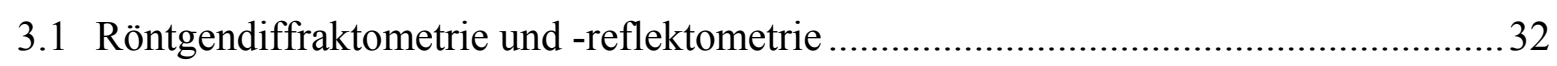

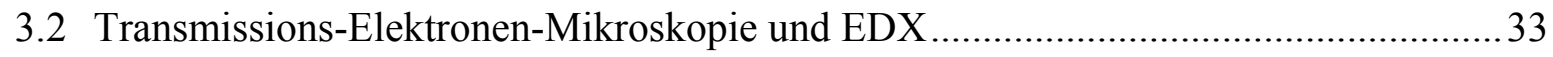

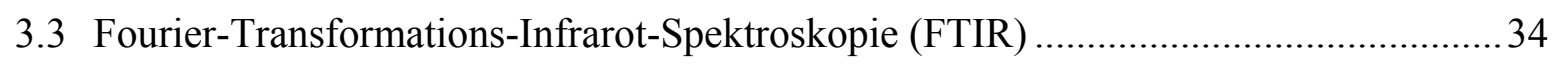

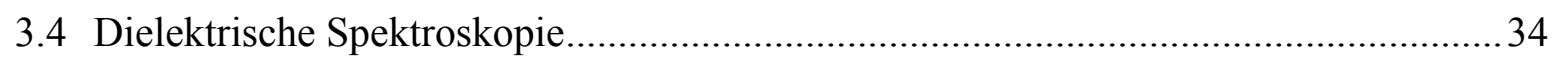

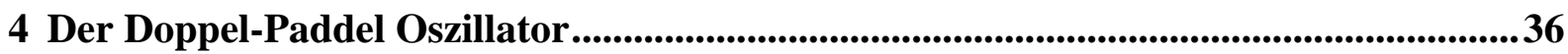

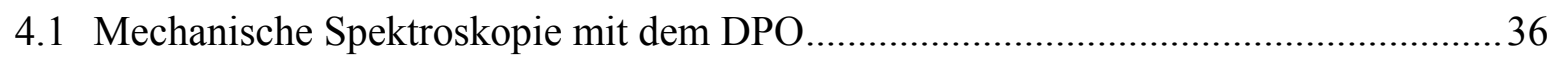

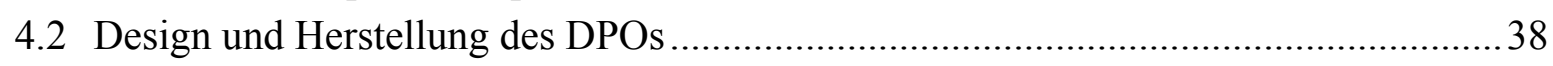

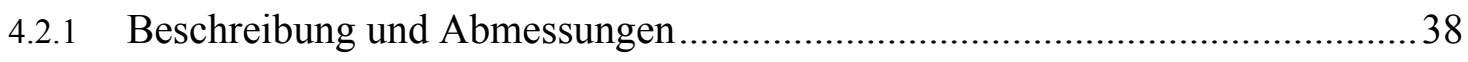


4.2.2 Herstellung durch Photolithografie (Cornell-Verfahren) ................................ 40

4.2.3 Herstellung durch Laserschneiden ........................................................... 43

4.2.4 Vergleich beider Methoden ........................................................................ 45

4.3 Konstruktion und Aufbau des UHV-Einsatzes....................................................... 48

4.3.1 Befestigung des Doppel-Paddel Oszillators ................................................ 48

4.3.2 Leitfähigkeit der Oszillator-Rückseite ........................................................ 48

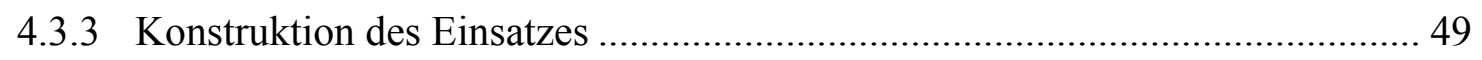

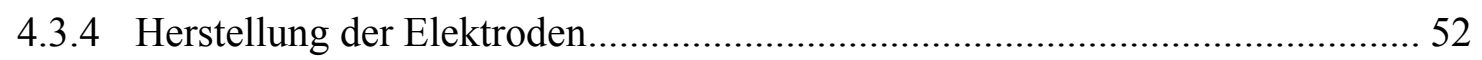

4.3.5 Temperaturmessung, -eichung und -regelung............................................ 53

4.4 Elektrischer Regelkreis, Steuerung und Datenerfassung ......................................... 59

4.4.1 Elektrostatische Anregung und Bias-Spannung ............................................ 59

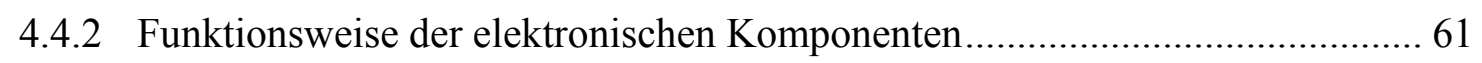

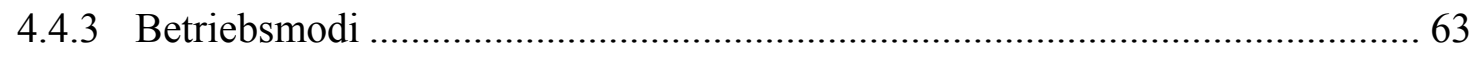

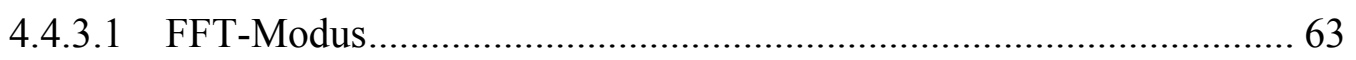

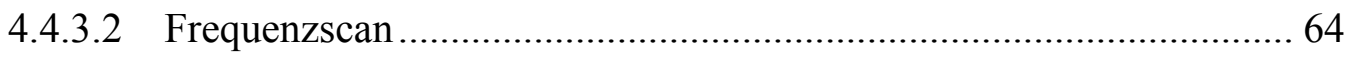

4.4.3.3 Phase Lock Loop (PLL) und Ring Down..................................... 64

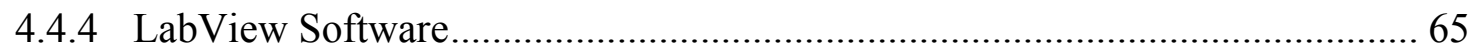

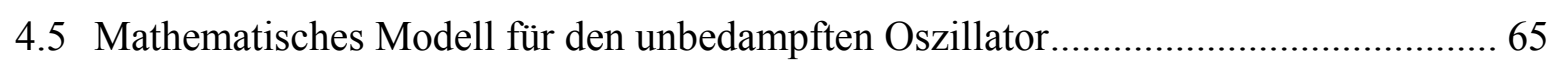

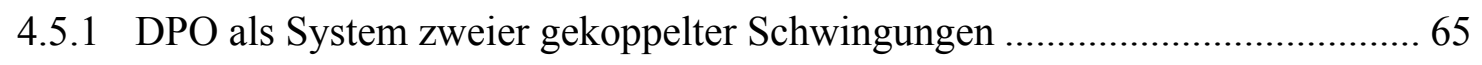

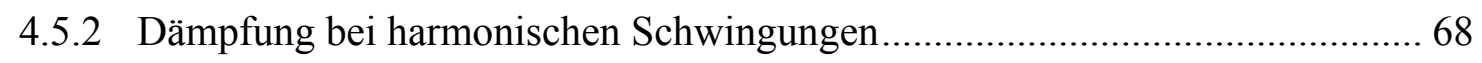

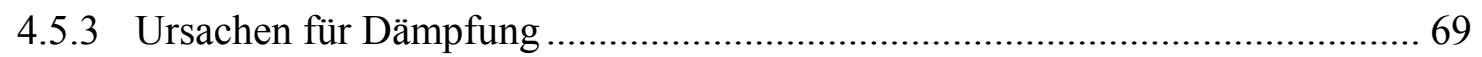

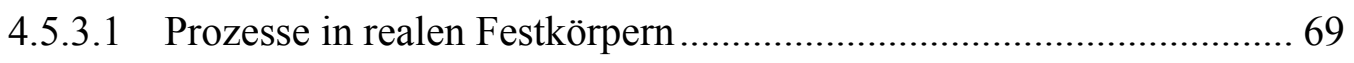

4.5.3.2 Verluste an der Oberfläche .......................................................... 70

4.5.3.3 Reibung in Gasen ..................................................................... 70

4.5.3.4 Verluste durch die Halterung …................................................... 70

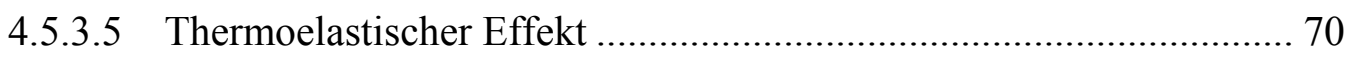

4.5.3.6 Phonon-Phonon-Wechselwirkungen ................................................. 71

4.5.3.7 Elektron-Phonon-Wechselwirkungen ............................................ 71

4.6 Schwingungsverhalten des leeren Paddels ................................................................ 71

4.6.1 Übersicht über die verschiedenen Eigenmoden ............................................ 71

4.6.2 Experimentelle Bestimmung der Dämpfung ................................................... 74

4.6.3 Einfluss von Phasenlage und Amplitude auf die Dämpfung ............................ 76

4.6.4 Temperaturabhängigkeit der Dämpfung .................................................... 78

4.7 Mathematisches Modell für das System Paddel - Schicht ....................................... 82

4.7.1 Beiträge zur Frequenzverschiebung .................................................... 82

4.7.2 Innere Reibung in dünnen Schichten........................................................... 85

4.7.3 Bestimmung des komplexen Schermoduls................................................ 87

4.7.4 Zusammenfassung der mathematischen Beschreibung ................................. 87

$5 \mathrm{Zr}_{65} \mathrm{Al}_{7,5} \mathrm{Cu}_{27,5}$ als metallisches Glas .................................................................................... 89

5.1 Herstellung und Charakterisierung der Schichten ............................................... 90 


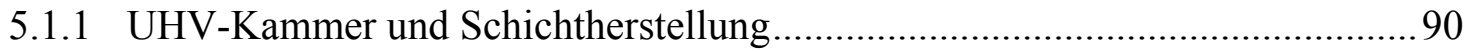

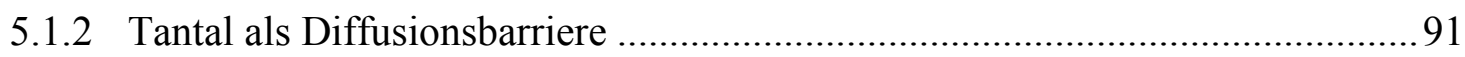

5.1.3 Zusammensetzung der Schichten in Wachstumsrichtung ............................... 91

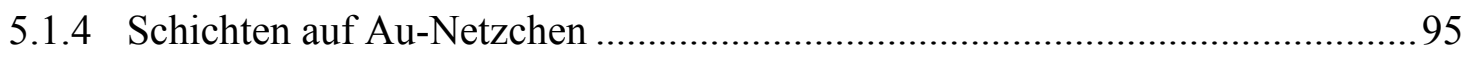

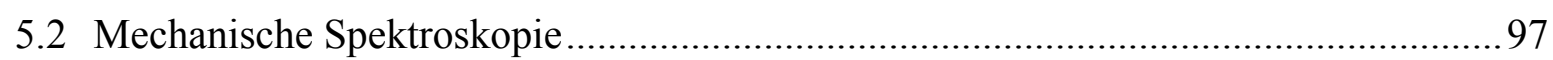

5.2.1 $\mathrm{Zr}_{65} \mathrm{Al}_{7,5} \mathrm{Cu}_{27,5}$ auf Silizium (leeres Paddel) .............................................. 97

5.2.1.1 Verschiebung der Resonanzfrequenz bei DPO 12 Schicht 1.............97

5.2.1.2 Dämpfungsfaktor bei DPO 12 Schicht 1 ........................................ 99

5.2.1.3 Isotherme Entwicklung bei DPO 12 Schicht 1 ................................ 100

5.2.1.4 Vergleich von DPO 12 Schicht 1 und DPO 21 Schicht 1 ................. 102

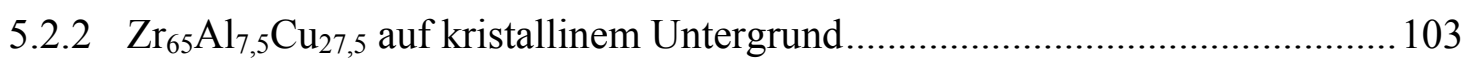

5.2.2.1 Verschiebung der Resonanzfrequenz bei DPO 12 Schicht 2........... 104

5.2.2.2 Skalierung der Frequenzverschiebung mit der Schichtdicke............ 104

5.2.2.3 Frequenzverschiebung bei mehrstufigem Temperaturprogramm..... 105

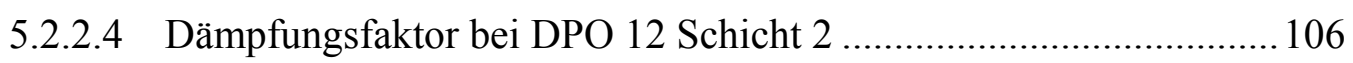

5.2.2.5 Skalierung des Dämpfungsfaktors mit der Schichtdicke .................. 107

5.2.2.6 Dämpfung bei mehrstufigem Temperaturprogramm ........................ 108

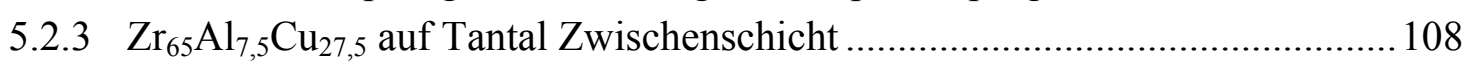

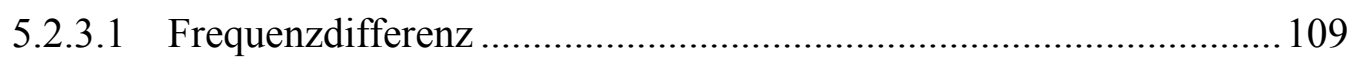

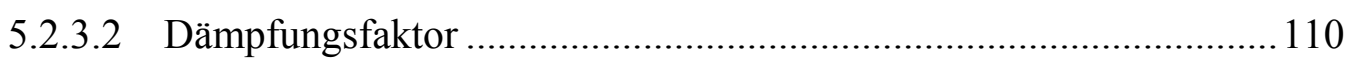

5.2.3.3 Isotherme Veränderung von Frequenz und Dämpfung..................... 112

5.2.4 Veränderung der ST-Resonanzfrequenz ................................................. 113

6 Polymethylmethacrylat als Polymerglas........................................................................ 115

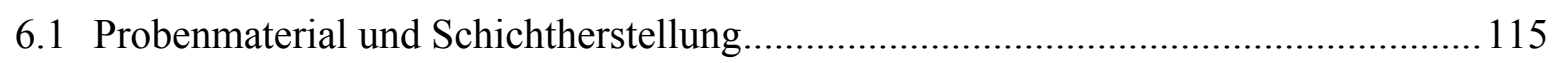

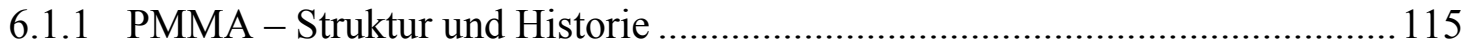

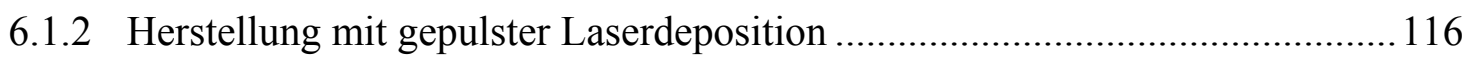

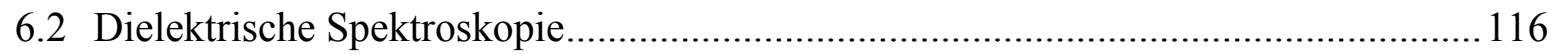

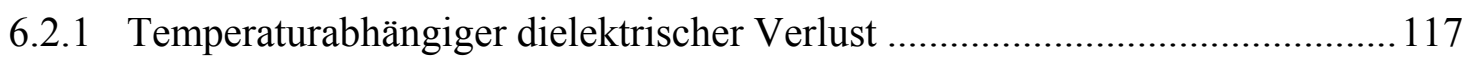

6.2.2 Vergleich Ausgangsmaterial - dünne Schicht ............................................... 118

6.2.3 Veränderungen im Spektrum mit steigender Endtemperatur ......................... 120

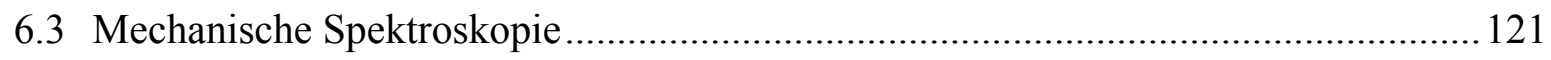

6.3.1 Temperaturabhängige Resonanzfrequenz.................................................. 122

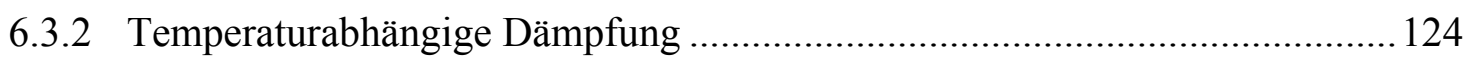

6.3.3 Isotherme Entwicklung von Frequenz und Dämpfung ............................... 127

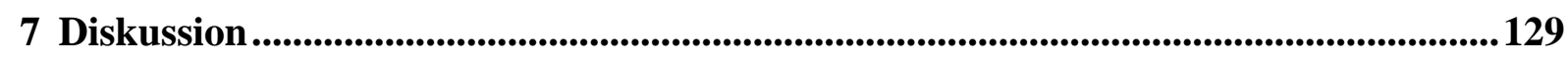

7.1 DPO als Substrat und Alternativen dazu......................................................... 129

7.1.1 Temperaturabhängigkeit der elastischen Konstanten von Si .......................... 129

7.1.2 Kristallografische Eigenschaften von Silizium........................................... 130 
7.1.3 Alternative Materialien für das DPO-Substrat ............................................. 131

7.1.4 Temperaturstabilität als empfindlichkeitsbegrenzender Faktor ..................... 131

7.1.5 Änderungen an der DPO-Geometrie ........................................................... 133

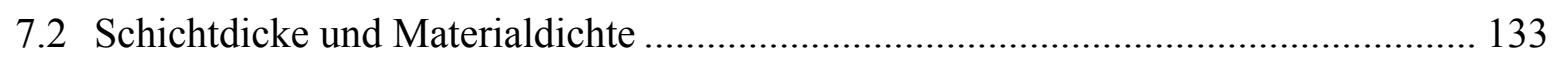

7.2.1 Beobachtungen bei $\mathrm{Zr}_{65} \mathrm{Al}_{7,5} \mathrm{Cu}_{27,5}$ - Frequenzverschiebungen ..................... 134

7.2.2 Beobachtung bei PMMA-Frequenzverschiebungen ................................... 135

7.2.3 Bewertung und Konsequenzen für die Interpretation.................................... 136

7.3 Abhängigkeit der $\mathrm{Zr}_{65} \mathrm{Al}_{7,5} \mathrm{Cu}_{27,5}$ Ergebnisse vom Substrat ................................... 137

7.3.1 Abhängigkeit der Frequenzverschiebung vom Substrat................................ 138

7.3.2 Rekonstruktion der Substratdämpfung bei DPO 19 ..................................... 140

7.3.3 Abhängigkeit der Dämpfung vom Substrat................................................. 141

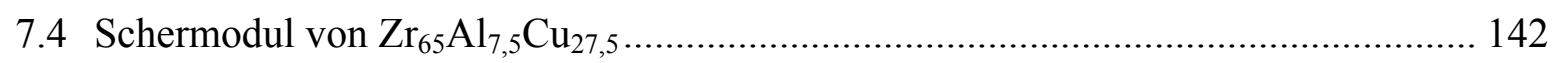

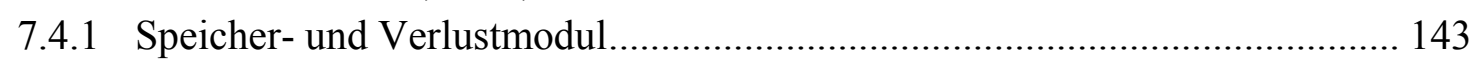

7.4.2 Vergleich des Verlustmoduls mit DMA-Resultaten ....................................... 144

7.4.3 Abschätzung der Poisson-Zahl v ................................................................... 146

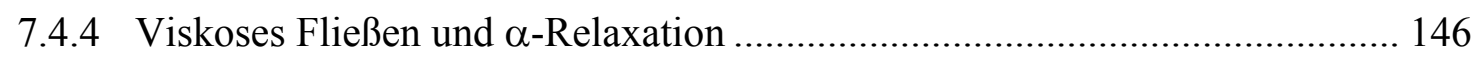

7.4.5 Zusätzliche Relaxationsbeiträge bei tiefen Temperaturen ............................. 149

7.4.5.1 „wing“ als Niedertemperaturflanke einer JG- $\beta$-Relaxation ............. 149

7.4.5.2 Überlagerung vieler unabhängiger Relaxationsprozesse ................ 150

7.4.5.3 Vergleich des JG- $\beta$ - und des „Cluster“-Ansatzes ........................... 151

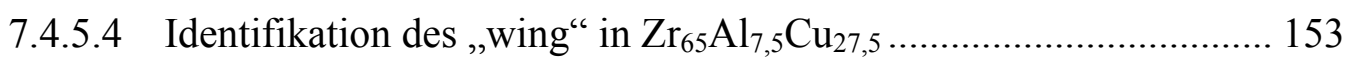

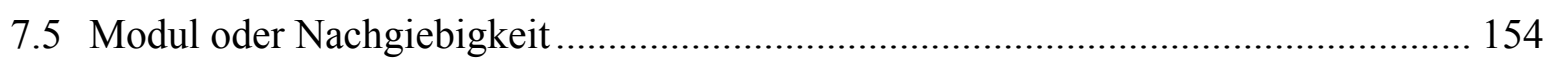

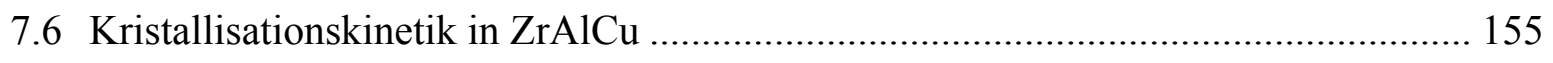

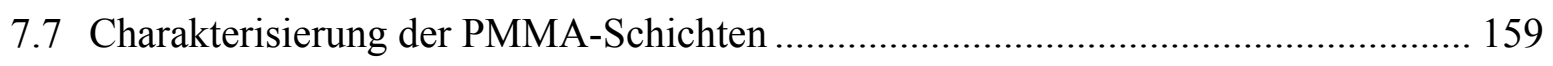

7.7.1 Strukturelle Eigenschaften von PMMA (FTIR) .......................................... 160

7.7.2 Abhängigkeit der Glastemperatur von der Kettenlänge ................................. 162

7.7.3 Thermische Eigenschaften von PMMA ..................................................... 164

7.7.4 Veränderungen in der Schicht bei hohen Temperaturen ................................ 164

7.8 Mechanische Eigenschaften von PMMA ................................................................ 165

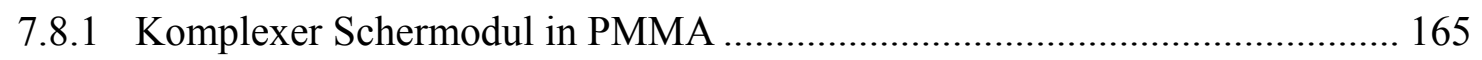

7.8.2 Vergleich des dielektrischen und des mechanischen Verlustes ...................... 167

7.8.3 Interpretation des mechanischen Verlustes ................................................. 169

8 Zusammenfassung und Ausblick ........................................................................................... 171

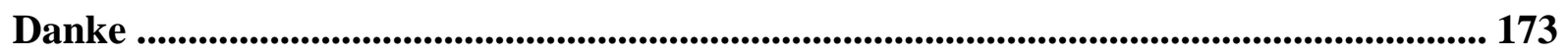

Anhang 1 - Dokumentation der LabView Software ................................................................. 175

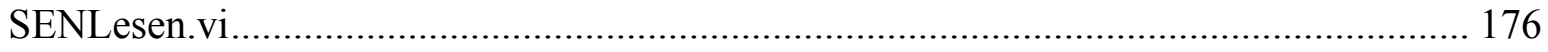

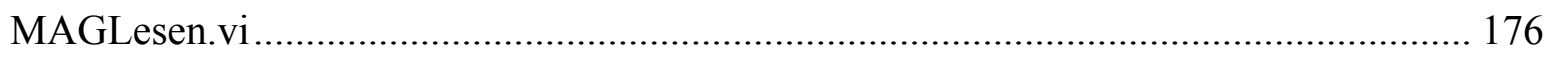




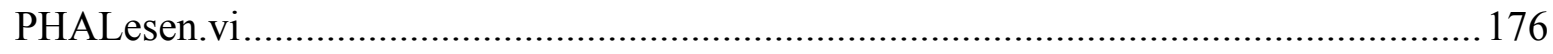

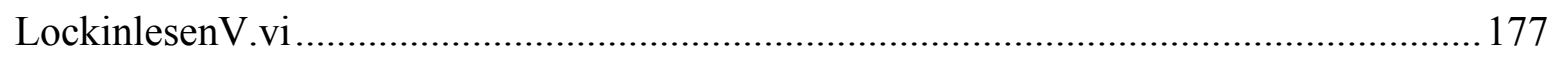

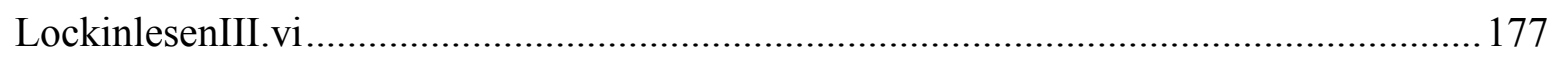

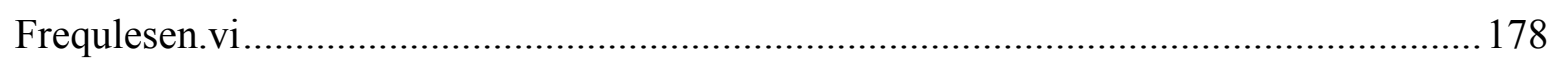

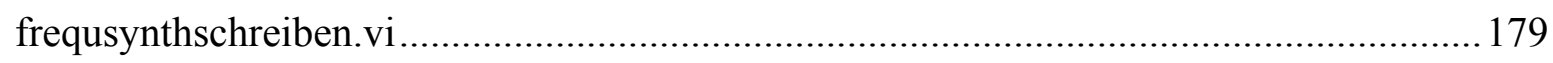

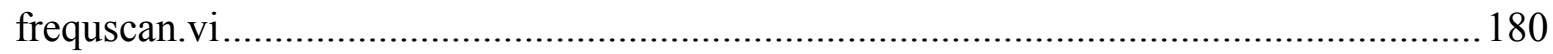

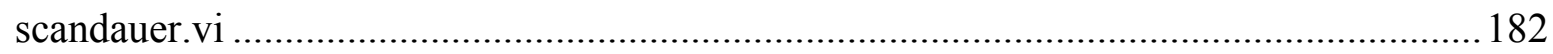

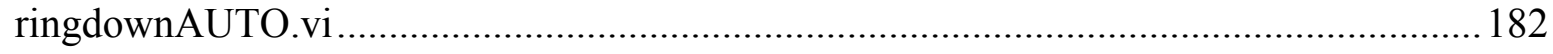

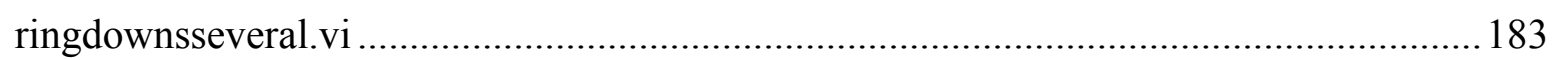

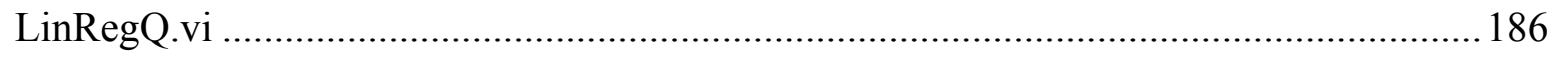

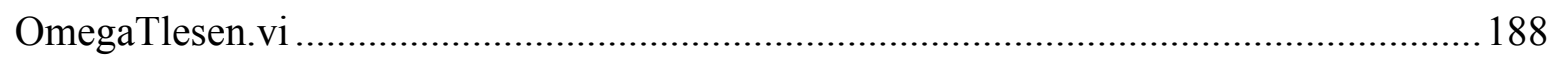

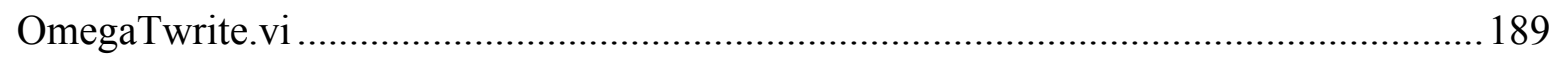

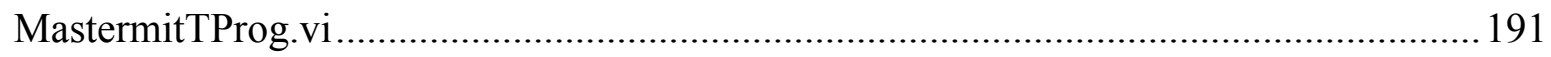

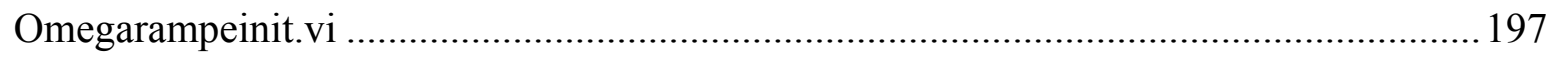

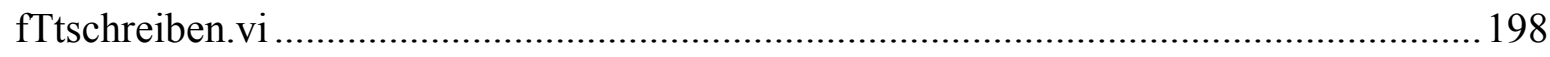

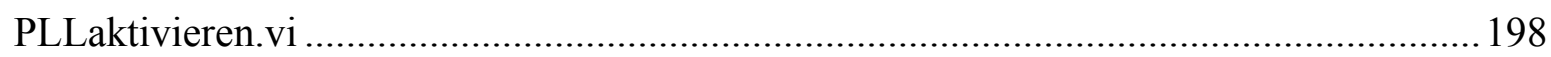

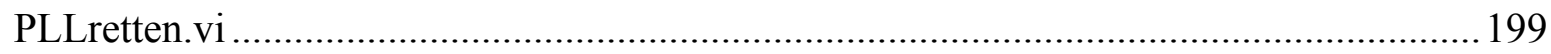

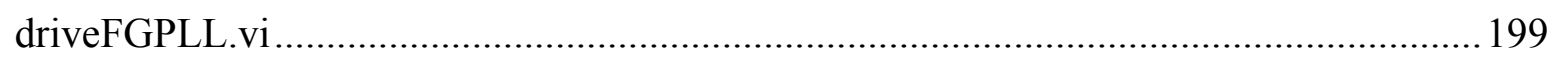

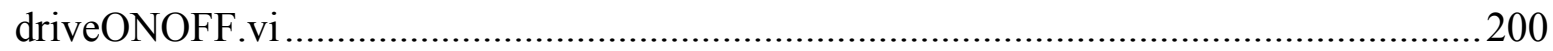

Anhang 2 - Real- und Imaginärteil der HN-Funktion ........................................................ 202

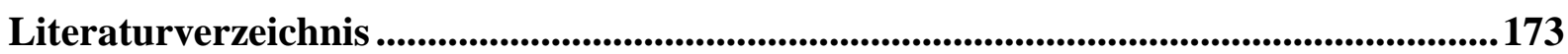




\section{Einleitung}

„The deepest and most interesting unsolved problem in solid state theory is probably the theory of the nature of glass and the glass transition."

Ph. W. Anderson in Science 267, 1615 (1995)

Gläser gehören zu den ältesten Werkstoffen der Menschheit. Lange vor unserer Zeit wurden aus dem vulkanischen Gestein Obsidian Messer und Pfeilspitzen hergestellt. Historische Funde im Nahen Osten belegen, dass bereits im 15. vorchristlichen Jahrhundert Vasen und Schüsseln aus Glas verwendet wurden. Es wird angenommen, dass sich die Kunst des Glasblasens etwa vor 2000 Jahren in Phönizien entwickelt hat. Neben den Gläsern auf Siliziumdioxid-Basis zählen auch Polymere und Keramiken zur Gruppe der amorphen Materialien. Sie sind nahezu allgegenwärtig in der heutigen Zeit und bilden die Grundlage für viele Produkte und technologische Anwendungen.

Die charakteristische Zeitskala, auf der sich die mikroskopischen Bausteine, aus denen das Glas aufgebaut ist, bewegen, verändert sich mit der Temperatur über viele Größenordnungen. Experimentelle Methoden erlauben es, diesen Übergang vom Glas als Festkörper hin zur Flüssigkeit, beispielsweise mit Messungen der Viskosität, genau zu studieren. Die Frage, wann eine amorphe Probe als fest und wann sie als flüssig zu betrachten ist, nimmt dabei philosophische Ausmaße an. Als dynamische Größe wird der Glasübergang oft bei der Temperatur festgelegt, bei der die charakteristische Relaxationszeit $\tau=200 \mathrm{~s}$ beträgt.

In den vergangenen Jahren sind - auch durch die Entwicklung von Computersimulationen bedeutende theoretische Fortschritte im Verständnis der mikroskopischen Vorgänge im Glaszustand, während des Erweichens am Glasübergang und in der unterkühlten Schmelze erzielt worden. Weitere Mosaiksteinchen liefern dazu die vielfältigen experimentellen Ergebnisse, die an unterschiedlichen Materialien mit verschiedensten Methoden gesammelt worden sind.

In Messungen des frequenzabhängigen dielektrischen Verlustes organischer Glasbildner bei verschiedenen Temperaturen konnte der primäre Relaxationsprozess durch viskoses Fließen zweifelsfrei nachgewiesen werden. Er zeigt sich in einem Maximum im Verlustspektrum, das sich mit zunehmender Temperatur zu größeren Frequenzen verschiebt. Dieser $\alpha$-Peak lässt sich mit der empirischen Havriliak-Negami-Funktion im Frequenzraum beschreiben. Die Re- 
laxationszeit $\tau$ gehorcht dabei einem Vogel-Fulcher-Tammann-Gesetz. Frequenz und Temperatur hängen über das Zeit-Temperatur-Superpositionsprinzip zusammen.

Haben die konstituierenden Bausteine des Glases zusätzliche innere Freiheitsgrade, was beispielsweise bei Polymermolekülen stets der Fall ist, so zeigt sich eine Anregung dieser Eigenmoden durch weitere Maxima im Verlustspektrum. Nun gibt es aber auch Gläser, bei denen sich sekundäre oder $\beta$-Relaxationsprozesse nicht durch einen klar ausgeprägten Peak oder eine Schulter zeigen. Vielmehr gibt es auf der Hochfrequenz- bzw. der Niedertemperaturflanke des $\alpha$-Peaks Abweichungen vom theoretisch erwarteten Potenzgesetz. Bis heute ist unklar, welche mikroskopischen Prozesse für diesen Zusatzbeitrag oder „excess-wing“ verantwortlich sind. Allerdings besteht Einigkeit darin, dass ein tieferes Verständnis des Glasübergangs nur möglich ist, wenn das „wing“-Phänomen aufgeklärt werden kann. Viele Theorien zum Glasübergang postulieren die Existenz von dynamischen Heterogenitäten und die korrelierte Bewegung von Atom- bzw. Molekülgruppen. In der Tat könnte der „,wing“ durch die überlagerte Relaxation solcher „Cluster“" zustande kommen.

Ziel dieser Arbeit ist es zu zeigen, dass es Zusatzbeiträge zum viskosen Fließen auch bei amorphen Metallen gibt und es sich somit um eine universelle Eigenschaft von Gläsern handeln könnte. Wenn der „wing“ in metallischen Gläsern existiert, ist das ein Indiz dafür, dass tatsächlich Heterogenitäten für dynamische Eigenschaften von Gläsern verantwortlich sind. Dies gilt umso mehr, da es in metallischen Systemen keine inneren Freiheitsgrade gibt und keine von diesen dominierte sekundäre Relaxationsmaxima auftreten.

Dynamische Prozesse in metallischen Gläsern zu messen ist allerdings ungleich komplizierter als in organischen Systemen. Metalle schirmen elektrische Felder ab und sind nicht polarisierbar, weshalb die Methode der dielektrischen Spektroskopie ausscheidet. Amorphe Metalle sind im Gegensatz zu Polymergläsern wegen der benötigten hohen Abkühlraten aus der Schmelze schwer herzustellen. Das thermisch stabile Intervall zwischen Glasübergang und Kristallisation ist klein.

In dieser Arbeit wird daher der mechanische Verlust an dünnen Schichten des metallischen Glases $\mathrm{Zr}_{65} \mathrm{Al}_{7,5} \mathrm{Cu}_{27,5}$ gemessen. Viele Eigenschaften dieses „Inoue-Glases“, darunter quasistatisches Kriechen und die Dynamik bei niedrigen Frequenzen wurden in Vorgängerarbeiten untersucht. Das thermisch stabile Intervall der unterkühlten Schmelze beträgt bis zu $80 \mathrm{~K}$. Als Substrate für die unter Ultrahochvakuumbedingungen kokondensierten Metallfilme werden Doppel-Paddel Oszillatoren (DPO) verwendet, deren große Güte ausreicht, um die erwartet 
geringen Verluste im komplexen Schermodul selbst in dünnen Schichten nachweisen zu können. Dabei wird in Kauf genommen, dass die Anregungsfrequenz durch die Geometrie des Oszillators mit 5,4 kHz fest vorgegeben ist und frequenzabhängige Messungen nicht möglich sind. Stattdessen wird das Zeit-Temperatur-Superpositionsprinzip genutzt und der Schermodul in Abhängigkeit von der Temperatur untersucht. Die Ergebnisse zeigen zweifelsfrei zusätzliche Beiträge zum Verlustmodul auf der Niedertemperaturflanke des $\alpha$-Peaks, was als erster Nachweis eines ,„wing“ in metallischen Gläsern gewertet werden kann.

Neben metallischen Schichten wurden auch Polymethylmethacrylat-(PMMA)-Filme untersucht, die mit gepulster Laserdeposition (PLD) auf die Oszillatoren aufgebracht wurden. Dynamische Eigenschaften von PMMA sind gut bekannt, die erwarteten Verlusteffekte sind groß und die Schichten kristallisieren nicht. PMMA erscheint daher als geeignetes Material, um die Funktionstüchtigkeit und die Empfindlichkeit der neu aufgebauten Apparatur zum Betrieb der Doppel-Paddel Oszillatoren zu testen. Zum Vergleich der unterschiedlichen Methoden wurde an unter gleichen Bedingungen hergestellten PMMA-Filmen an der Universität Augsburg dielektrische Spektroskopie durchgeführt. Dabei zeigen sich signifikante Unterschied im dielektrischen Verlust zwischen Ausgangsmaterial und Schicht, was die Frage aufwirft, ob und wie sich die Polymerstruktur vom Target auf die Schicht überträgt. Während elektrische Felder mit dem Dipolmoment der PMMA-Moleküle an deren Seitengruppen wechselwirken, führt die mechanische Auslenkung zu einer makroskopischen Torsion der Schichten. Gefundene Unterschiede im dielektrischen und im mechanischen Verlust werden so erklärt.

Diese Arbeit beginnt in Kapitel 2 mit der Beschreibung von dynamischen Prozessen in Flüssigkeiten und Gläsern. In Kapitel 3 werden experimentelle Methoden erläutert, bevor sich Kapitel 4 detailliert mit dem Aufbau und der Funktionsweise der Apparatur zum Betrieb des DPO beschäftigt. Die mathematische Modellbildung für die verschiedenen Eigenmoden wird ebenso erklärt, wie die Berechnung des komplexen Schermoduls aus den experimentellen Daten. Die Herstellung der Schichten und die Ergebnisse aus den DPO-Messungen werden für $\mathrm{Zr}_{65} \mathrm{Al}_{7,5} \mathrm{Cu}_{27,5}$ in Kapitel 5 und für PMMA in Kapitel 6 vorgestellt. Sie werden in Kapitel 7 ausführlich diskutiert und interpretiert, bevor die wesentlichen Resultate dieser Arbeit in Kapitel 8 zusammengefasst werden und ein Ausblick gegeben wird. 


\section{Dynamik in Flüssigkeiten und Gläsern}

In diesem Kapitel werden inter- und intramolekulare Prozesse beschrieben, die für dynamische Eigenschaften von Flüssigkeiten und Gläsern verantwortlich sind. Ausgehend von einem phänomenologischen Frequenzspektrum nach Lunkenheimer [1] mit seinen charakteristischen Merkmalen werden in den folgenden Abschnitten Theorien und Modelle vorgestellt, mit deren Hilfe sich die beobachteten Ausprägungen erklären lassen.

Kapitel 2.2 behandelt das viskose Fließen von unterkühlten Schmelzen bis zum Glasübergang. Gläser werden aufgrund von Eigenschaften wie Viskosität und Fragilität charakterisiert. Die Relaxation durch viskoses Fließen wird im Zeitraum beschrieben und verschiedene Modelle zur Temperaturabhängigkeit, darunter die Kohlrausch-Williams-Watts-Funktion, vorgestellt. Der Übergang vom Zeit- in den Frequenzraum wird in Abschnitt 2.2.5 vollzogen, in dem auch die für die Auswertung wichtige Havriliak-Negami-Funktion eingeführt wird. In Kapitel 2.2.6 schließlich wird die Relaxation bei konstanter Frequenz in Abhängigkeit von der Temperatur beschrieben. Das Zeit-Temperatur-Superpositionsprinzip stellt hierbei die entscheidende Verknüpfung zwischen den beiden äquivalenten Beschreibungen dar. Wegen seiner fundamentalen Bedeutung wird der Übergang vom Frequenz- in den Temperaturraum an einem Beispiel verdeutlicht.

Sekundäre Relaxationsprozesse die auf kürzeren Zeitskalen ablaufen und bei höheren Frequenzen beobachtet werden, sind in Abschnitt 2.3 beschrieben. Sie werden auf intramolekulare Vorgänge zurückgeführt. Unklar hingegen ist die mikroskopische Ursache für Beiträge zur Hochfrequenzflanke des viskosen Fließens, die in Kapitel 2.4 vorgestellt werden. Da der experimentell zugängliche Frequenzumfang für mechanische Spektroskopie nach oben beschränkt ist, wird auf höherfrequente Phänomene im Rahmen dieser Arbeit nicht genauer eingegangen.

\subsection{Phänomenologisches Frequenzspektrum im Überblick}

In Abbildung 2.1 ist das frequenzabhängige, dielektrische Verlustspektrum eines organischen Glasbildners schematisch dargestellt [1]. Es enthält verschiedene charakteristische Beiträge, die beginnend bei kleinen Frequenzen hier vorgestellt werden. 


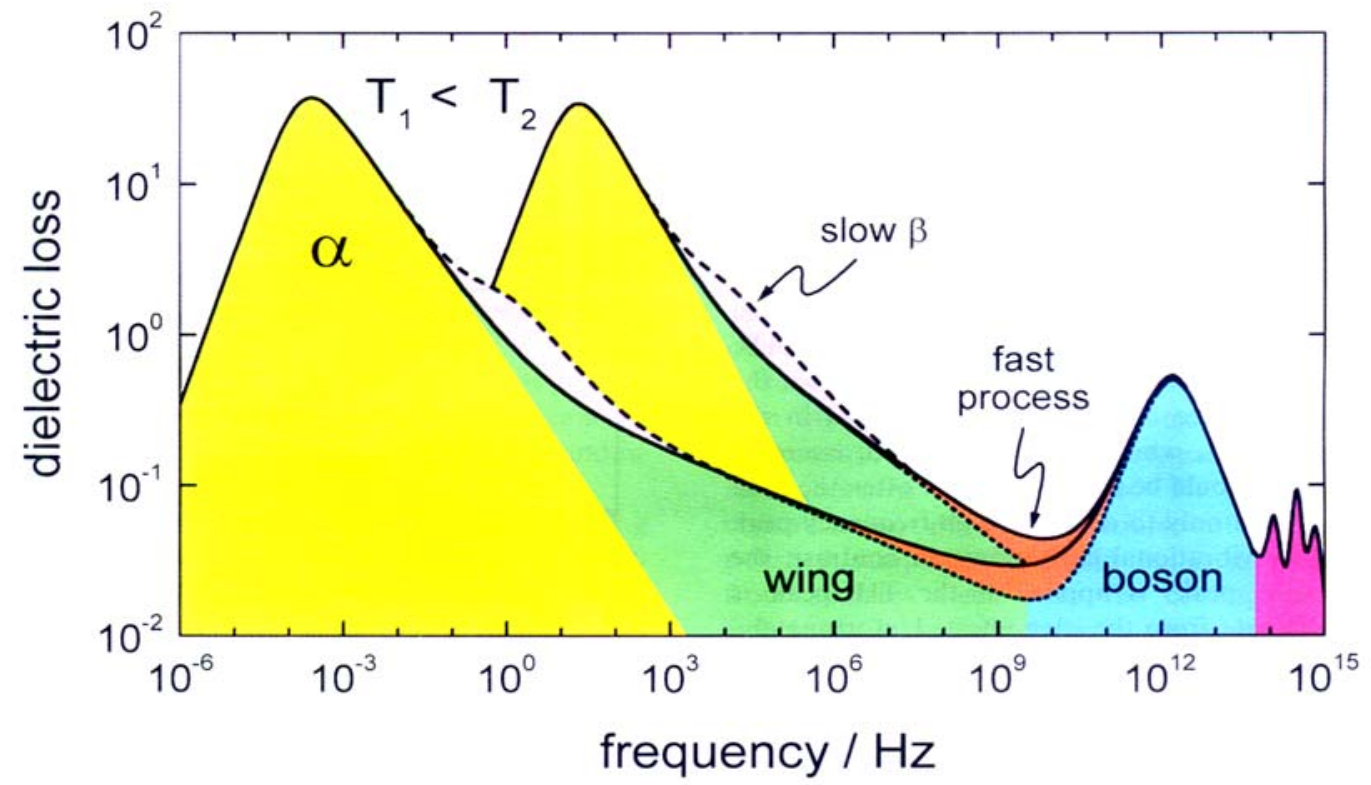

Abbildung 2.1: Schematischer Überblick des frequenzabhängigen dielektrischen Verlustes in organischen Glasbildnern bei zwei verschiedenen Temperaturen [1].

- Der $\alpha$-Prozess entspricht dem makroskopischen viskosen Fließen der Probe und führt zu einem ausgeprägten, in gelb dargestellten Maximum. Mit steigender Temperatur verschiebt sich die Peakposition, wegen der beschleunigten Dynamik, zu höheren Frequenzen.

- Vor allem in Materialien mit komplexer Molekülstruktur (z.B. Polymere), kann es Relaxationsprozesse geben, die als slow- $\beta,-\gamma,-\delta$, usw. bezeichnet werden und durch intramolekulare Bewegungen verursacht sind. Sie bilden zusätzliche Maxima auf der Hochfrequenzflanke des $\alpha$-Peaks aus. Ist die Relaxationsstärke dieser sekundären Prozesse klein, so beobachtet man statt eines Peaks nur eine schwächer ausgeprägte „Schulter“. Bei vielen Materialien ist überhaupt keine Änderung in der Krümmung der Hochfrequenzflanke der Primärrelaxation zu beobachten. Trotzdem zeigt sich ein verzögerten Abfall der Relaxationsfunktion. Dieses Phänomen wird als „excess-wing“ bezeichnet und ist in grün eingezeichnet.

- Die Modenkopplungstheorie sagt für das Minimum zwischen der abklingenden Hochfrequenzflanke und dem erneuten Anstieg zum Boson-Peak einen zusätzlichen Beitrag voraus, der als schneller $\beta$-Prozess bezeichnet wird (orange gezeichnet). 
- Bei Frequenzen von einigen THz stellt der Boson-Peak (blau) ein weiteres Charakteristikum im Frequenzspektrum von Gläsern dar. Dieses Maximum ist phononischen Ursprungs und bei vielen Substanzen mit Licht- und Neutronenstreuung gefunden worden. Schirmacher et al. [2] haben theoretisch gezeigt, dass ein ungeordnetes System klassischer harmonischer Oszillatoren mit einer kontinuierlichen Verteilung der Kopplungsparameter in diesem Frequenzbereich stets eine im Vergleich zum Debye-Modell erhöhte Zustandsdichte aufweist. Befindet sich das System in der Nähe einer Instabilität, so ist der BosonPeak besonders stark ausgeprägt.

- Im Infrarot-Bereich des Spektrums treten relativ scharfe Absorptionen auf, die mit intramolekularen Dreh- und Schwingungsresonanzen identifiziert werden können.

Da mit der mechanischen Spektroskopie im Rahmen dieser Arbeit Relaxationsprozesse bei einer Frequenz von etwa 5,4 kHz untersucht werden, sind nur die niederfrequenten Beiträge wie viskoses Fließen, intramolekulare Dynamik und „excess-wing“ von Bedeutung, die in den folgenden Kapiteln detailliert erläutert werden.

\subsection{Glasübergang, viskoses Fließen und $\alpha$-Relaxation}

Im glasverarbeitenden Gewerbe ist schon seit Jahrtausenden bekannt, dass die Viskosität von aufgeschmolzenem Quarzsand $\left(\mathrm{SiO}_{2}\right)$ mit einigen Zusätzen wie $\mathrm{Na}_{2} \mathrm{O}$ oder $\mathrm{CaO}$ über viele Größenordnungen hinweg mit der Temperatur reguliert werden kann. Kunstvoll geformte Gläser sind stumme Zeugen dieser Erkenntnis. Neben den oxidischen Gläsern lassen sich auch Salzschmelzen, Halbleiter, Polymere, Proteine, metallische Legierungen und magnetische Systeme bezüglich der Spinorientierung aus der ungeordneten Schmelze über die unterkühlte Flüssigkeit in einen glasartigen Zustand überführen. Durch ausreichend schnelles Abkühlen wird die ungeordnete, amorphe (,gestaltlose“) Struktur der Flüssigkeit „eingefroren“ und die Kristallisation unterdrückt. Die Atome oder Moleküle eines solchen strukturellen Glases besitzen zwar eine Nahordnung bezüglich ihrer Nachbarn, jedoch keine gitterperiodische Fernordnung. Zur Charakterisierung der Strukturverhältnisse im festen Glaszustand eignen sich Untersuchungsmethoden wie Röntgenbeugung oder Neutronenstreuung. 


\subsubsection{Glasübergang in strukturellen Gläsern}

Nicht immer wird in der Literatur zwischen den Begriffen ,amorpher Festkörper“ und „Glas“ unterschieden [3]. Allerdings gibt es eine allgemein anerkannte Abgrenzung [4]:

„Ein Glas ist ein amorpher Festkörper, der einen Glasübergang zeigt.“

Wird eine Flüssigkeit, von hohen Temperaturen kommend, rasch abgekühlt, so ändert sich am Glasübergang der Temperaturverlauf von extensiven thermodynamischen Größen wie dem Volumen V, der Entropie S oder der spezifischen Wärmekapazität $c_{p}$. In Abbildung 2.2 ist der Temperaturverlauf für die Entropie eines typischen Glases gezeigt [nach 3].

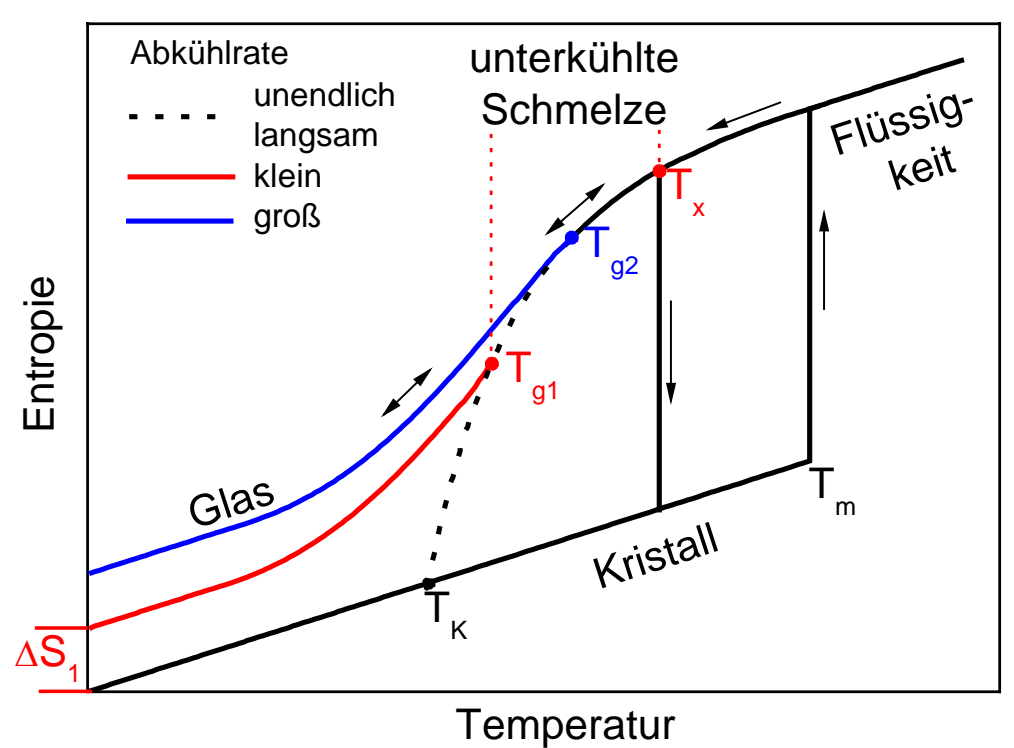

Abbildung 2.2: Schematische Temperaturabhängigkeit der Entropie eines strukturellen Glasbildners (nach [4]).

Rasches Abkühlen der Flüssigkeit unterdrückt die Kristallisation bei der Erstarrungstemperatur $\mathrm{T}_{\mathrm{m}}$. Die unterkühlte Schmelze geht bei der Glastemperatur $\mathrm{T}_{\mathrm{g}}$ in den Glaszustand über. Sowohl $\mathrm{T}_{\mathrm{g}}$ als auch die bei $\mathrm{T}=0 \mathrm{~K}$ verbleibende Restentropie $\Delta \mathrm{S}$ hängen dabei von der Kühlrate ab, wie aus Abbildung 2.2 hervorgeht.

Der rote Kurvenverlauf repräsentiert im Vergleich zur blauen Linie eine kleinere Kühlrate. Für die Glastemperaturen gilt damit: $\mathrm{T}_{\mathrm{g} 1}<\mathrm{T}_{\mathrm{g} 2}$. Die unterkühlte Flüssigkeit bleibt bei langsamerer Abkühlung länger im thermodynamischen Gleichgewicht. Bei unendlich kleiner Kühlrate würde bei der Kauzmann-Temperatur $\mathrm{T}_{\mathrm{K}}$ die Entropie der ungeordneten Flüssigkeit genauso groß wie die des geordneten Kristalls. Wegen der thermodynamischen Unmöglichkeit wird diese Überlegung in der Literatur als Kauzmann-Paradoxon bezeichnet [5]. Die in der 
Literatur in diesem Zusammenhang oft zu lesende Interpretation, dass $\mathrm{T}_{\mathrm{K}}$ eine untere Grenztemperatur für den Glasübergang darstellt, wird von Binder [6] abgelehnt. Er verweist auf theoretische Modelle, nach denen ein Glas beim Abkühlung zwei Übergänge durchläuft. Bei einer Temperatur $T_{c}>T_{g}$ gibt es zunächst den durch die Modenkopplungstheorie [7] beschriebenen dynamischen Übergang vom ergodischen in den nicht-ergodischen Zustand [8]. Die Lebensdauer des Zwischenzustandes der in zwei Schritten zerfallenden Selbstkorrelationsfunktion wird bei der Temperatur $T_{c}$ unendlich groß. Erst beim zweiten Übergang bei $T_{g}$ tritt diskontinuierlich ein statischer Ordnungsparameter auf. Mit diesem Übergang ist keine latente Wärme verbunden. Die Entropie in Abbildung 2.2 zeigt eine Stufe, keinen Sprung. Die Suszeptibilität des Glases bleibt endlich, weshalb Binder folgert [6], dass die durch Extrapolation der Entropie auf $\mathrm{S}=0$ definierte Temperatur $\mathrm{T}_{\mathrm{K}}$ keine physikalische Bedeutung hat.

Wird das Glas nun langsam wieder erwärmt, so stellt sich bei Temperaturen um $T_{g}$ ein metastabiles Gleichgewicht ein, falls nicht vorher Kristallisation einsetzt und das System den thermodynamisch stabilen Zustand erreicht. Diese Relaxation aus dem Nichtgleichgewichtszustand des abgeschreckten Glases in ein metastabiles Gleichgewicht der unterkühlten Schmelze findet oberhalb von $\mathrm{T}_{\mathrm{g}}$ statt, ist irreversibel und geht in der Regel mit einer Zunahme der Dichte und einer topologischen und chemischen Nahordnung einher [9].

Bei weiterer Temperaturerhöhung, geht das System bei $T_{x}$ schließlich in den thermodynamisch stabilen, kristallinen Zustand über. Wird das Glas mit konstanter Heizrate erwärmt, so ergibt sich ein Temperaturintervall zwischen $T_{g}$ und $T_{x}$, dessen Breite von der Heizrate abhängt. Die Kinetik der Kristallisation, die im Wesentlichen durch Keimbildung und -wachstum dominiert ist, wird in Zeit-Temperatur-Umwandlungs-Diagrammen veranschaulicht. $\mathrm{T}_{\mathrm{g}}$ und $T_{x}$ sind damit keine thermodynamischen Konstanten, sondern von der Prozessführung abhängig.

Gläser, die aus metallischen Legierungen hergestellt werden, kristallisieren relativ leicht. $\mathrm{Zu}$ ihrer Herstellung ist folglich eine große Abkühlrate nötig und der thermisch stabile Bereich der unterkühlten Schmelze ist klein. Metallische Gläser eignen sich wegen der Einfachheit ihrer atomaren Bausteine hervorragend als Modellsysteme zur experimentellen Überprüfung von theoretischen Vorhersagen im Bereich des Glasübergangs. Daher wurden große Anstrengungen unternommen, um Legierungssysteme zu finden, deren thermisch stabiles Intervall der unterkühlten Schmelze möglichst breit ist [10]. Wegen des relativ geringen experimentellen Aufwandes ist die mittels Differentialkalorimetrie gemessene spezifische Wärme die zur Charakterisierung am häufigsten verwendete thermodynamische Größe. 
Als Beispiele für die so bestimmte thermische Stabilität in metallischen Gläsern seien die „Inoue“-Gläser $\mathrm{Zr}_{65} \mathrm{Cu}_{27,5} \mathrm{Al}_{7,5}$ [11] und $\mathrm{Zr}_{65} \mathrm{Al}_{7,5} \mathrm{Cu}_{17,5} \mathrm{Ni}_{10}$ mit einem Stabilitätsbereich von etwa $90 \mathrm{~K}$ [12] (Heizrate: $40 \mathrm{~K} / \mathrm{min}$ ) und die „Johnson“-Gläser $\mathrm{Zr}_{41,2} \mathrm{Ti}_{13,8} \mathrm{Cu}_{12,5} \mathrm{Ni}_{10} \mathrm{Be}_{22,5}$ (Vit1) [13] und $\mathrm{Zr}_{46,8} \mathrm{Ti}_{8,2} \mathrm{Cu}_{7,5} \mathrm{Ni}_{10} \mathrm{Be}_{27,5}$ (Vit4) [14], thermisch stabil bis etwa $130 \mathrm{~K}$ oberhalb von $\mathrm{T}_{\mathrm{g}}$ [15] (Heizrate jeweils $20 \mathrm{~K} / \mathrm{min}$ ) genannt. Alternativ zu den Zr-basierten Gläsern wurden in der jüngeren Vergangenheit Systeme auf Pd-Basis diskutiert. Das $\mathrm{Pd}_{43} \mathrm{Cu}_{27} \mathrm{Ni}_{10} \mathrm{P}_{20}$-System ist bei einer Heizrate von $10 \mathrm{~K} / \mathrm{min}$ thermisch stabil bis etwa $100 \mathrm{~K}$ oberhalb von $\mathrm{T}_{\mathrm{g}}$. Bei einer Heizrate von $160 \mathrm{~K} / \mathrm{min}$ findet überhaupt keine Kristallisation statt und die Legierung geht vom Glas über die unterkühlte Schmelze direkt in die Flüssigkeit über [16].

Das Problem der thermischen Stabilität der unterkühlten Schmelze stellt sich bei Polymergläsern nicht. Die langkettigen und knäuelartigen Moleküle sind kaum in eine geordnete Struktur zu bringen. Die Glasbildung erfolgt daher auch bei kleinen Abkühlraten. Selbst nach langem Auslagern bei Temperaturen oberhalb von $\mathrm{T}_{\mathrm{g}}$ findet keine Kristallisation statt.

Dynamische Messgrößen wie die Viskosität $\eta$ und die mechanische oder dielektrische Relaxationszeit $\tau$ zeigen im Gegensatz zu den diskutierten extensiven Größen keine Anomalie in ihrer Temperaturabhängigkeit. Das Einfrieren der Flüssigkeit erfolgt kontinuierlich. Es liegt daher im Ermessen des Beobachters, ab welcher Viskosität ein Zustand als „fest“ bezeichnet wird. In der Literatur (beispielsweise [17] oder [18]) wird für den Glasübergang meist eine Viskosität von $\eta=10^{12}$ Pas festgelegt, was einer Relaxationszeit von 100 s entspricht.

\subsubsection{Viskosität und Fragilität von Gläsern}

Die physikalische Definition der dynamischen Viskosität $\eta$ beruht auf der Scherspannung (F/A), die bei der Bewegung einer Flüssigkeit durch innere Reibung auftritt. Mit dem räumlichen Geschwindigkeitsgradienten dv/dx gilt:

$$
\frac{F}{A}=\eta \cdot \frac{d v}{d x}
$$

Empirisch ist seit langem bekannt, dass die Viskosität einer Flüssigkeit bei abnehmender Temperatur stark ansteigt. Während sie bei typischen Flüssigkeiten am Schmelzpunkt bei etwa $10^{-3}$ Pas liegt, erreicht sie am Glasübergang den Wert von $10^{12}$ Pas.

Die Temperaturabhängigkeit der Viskosität am Glasübergang wird zur Charakterisierung glasbildender Systeme verwendet. Nach C. A. Angell [19] wird die Viskosität logarithmisch 
gegenüber $\mathrm{T}_{\mathrm{g}} / \mathrm{T}$ aufgetragen (Abbildung 2.3). Je nach Kurvenverlauf werden die Materialien in „starke“ und „fragile“ Gläser eingeteilt. Ein Maß hierfür ist der Fragilitätsindex m:

$$
m=\left.\frac{d \log \eta}{d\left(T_{g} / T\right)}\right|_{T_{g}=T}
$$

Die „Steilheit“ der Viskosität beim Glasübergang bedeutet beispielsweise für das „starke“ Glas $\mathrm{SiO}_{2}$ den Fragilitätsindex $\mathrm{m}=20$. Für die im Rahmen dieser Arbeit untersuchten Systeme liegen die Indizes für PMMA bei $\mathrm{m}=145$ [20] und für $\mathrm{Zr}_{65} \mathrm{Cu}_{27,5} \mathrm{Al}_{7,5}$ bei $\mathrm{m}=38,4$ [21].

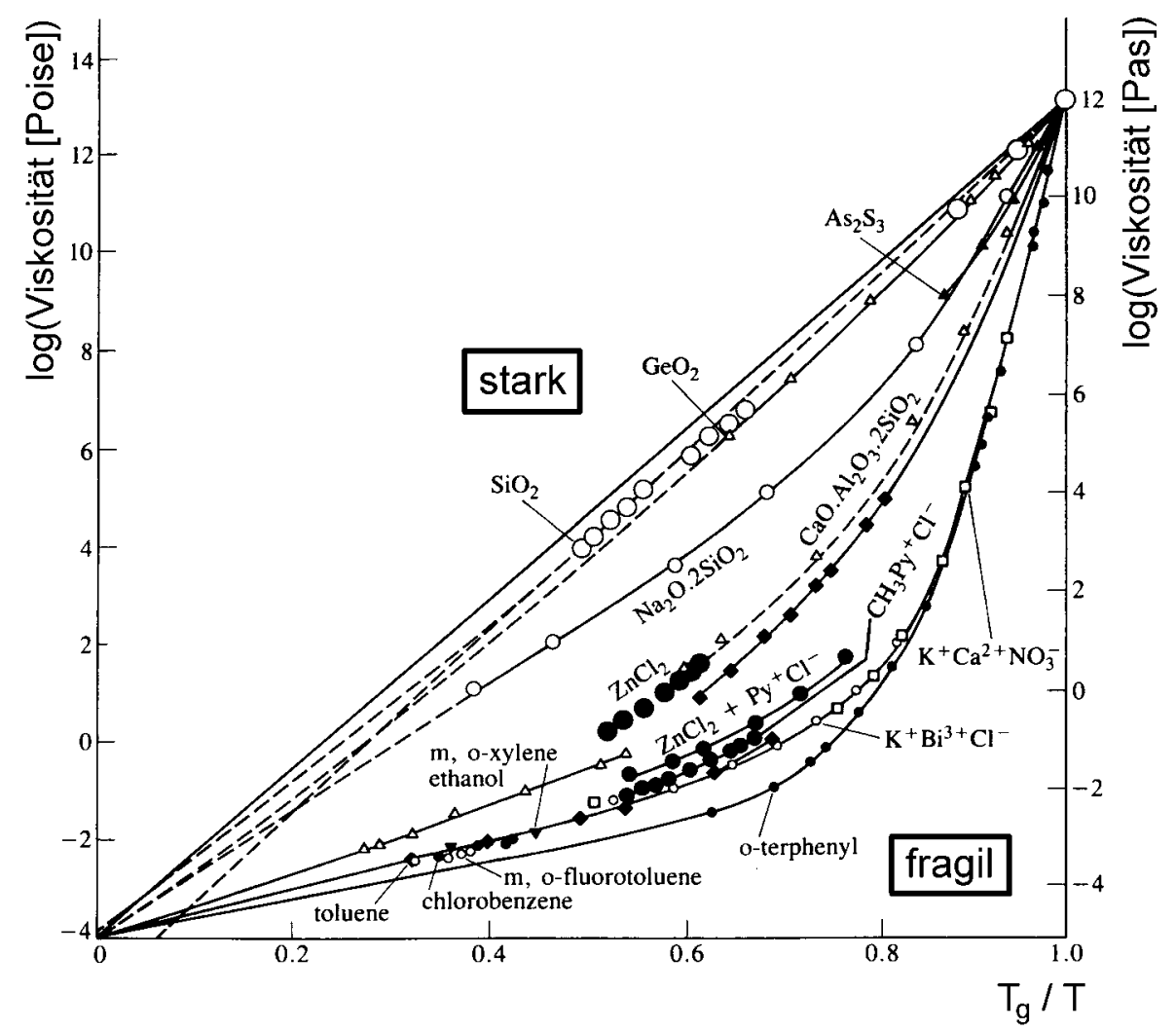

Abbildung 2.3: Angell-Plot zur Charakterisierung glasbildender Systeme nach ihrer Fragilität [19].

Mit der Viskosität ändert sich auch die intermolekulare Bewegungsdynamik um viele Größenordnungen. In der mechanischen oder dielektrischen Spektroskopie führt diese Änderung der Dynamik zu einem Maximum, dem sogenannten $\alpha$-Peak, im frequenzabhängigen Verlust (gelber Peak in Abbildung 2.1). 


\subsubsection{Relaxation im Zeitraum und Clustertheorie}

Neben der temperaturabhängigen Viskosität wird auch das Relaxationsverhalten zur Charakterisierung von Gläsern verwendet. Die relaxierende Größe $\phi(t)$, beispielsweise die anelastische Dehnung, die mechanische Spannung oder die dielektrische Polarisation wird dabei als Funktion der Zeit gemessen.

Nimmt man den einfachsten Fall der Relaxation nach einer stufenförmigen Änderung der äußeren Kraft oder des angelegten Feldes an und geht man weiter davon aus, dass die Atome nicht miteinander wechselwirken, so erhält man eine reine Debye-Antwortfunktion mit einer charakteristischen Relaxationszeit $\tau_{\mathrm{DB}}$ :

$$
\phi(t)_{D B}=\varphi_{0} \exp \left(-\frac{t}{\tau_{D B}}\right)
$$

Bei Gläsern zeigt sich, dass die Relaxation im Bereich des Glasübergangs zwar, wie im Debye-Fall auch, immer in Richtung des Gleichgewichts führt, allerdings von einer exakten Exponentialfunktion abweicht. Eine phänomenologische Beschreibung dieses Abweichens wurde schon Mitte des 19. Jahrhunderts von Kohlrausch [22] vorgeschlagen. Zusammen mit der Erweiterung auf dielektrische Relaxationen durch Williams und Watts [23] entstand die Kohlrausch-Williams-Watts-Funktion (KWW-Funktion), bei welcher die Debye-Stufenantwort um den KWW-Exponenten $\beta_{\mathrm{KWW}}\left(0<\beta_{\mathrm{KWW}} \leq 1\right)$ erweitert wird:

$$
\phi(t)_{K W W}=\varphi_{0} \exp \left(\left(-\frac{t}{\tau_{K W W}}\right)^{\beta_{K W W}}\right)
$$

Als Erklärung für die beobachtete gestreckte Exponentialfunktion wird angeführt, dass die Relaxation auf kooperativen Prozessen beruht, und dass es eine Verteilung verschiedener Relaxationszeiten gibt [24]. Viele prominente Theorien zum Glasübergang gehen davon aus, dass diese Verteilung durch dynamische Heterogenitäten, Dichtefluktuationen und von der Bewegung ganzer Atomgruppen („Cluster“) verursacht wird. Die Freie-Volumen-Theorie von Turnbull, Cohen und Grest [25, 26, 27] definiert festkörperähnliche „solid-like“ und flüssigkeitsähnliche „liquid-like“ Regionen. Die molekularkinetische Adam-Gibbs-Theorie [28] postuliert „,cooperative rearranging regions“ (CCR) und Chamberlin [29] geht in seinem Ansatz von einer heterogenen Verteilung dynamisch korrelierter Domänen aus. Je größer die Variation der Clustergrößen dabei ist, desto breiter ist das Spektrum der Relaxationszeiten und desto kleiner der KWW-Exponent. 
Eine andere Möglichkeit, die KWW-Gleichung abzuleiten, ist nach DiMarzio [30, 31], Potentialtöpfe im Phasenraum zu postulieren, in denen Teilchen gefangen sind. Die Wahrscheinlichkeit für die Existenz dieser Fallen klingt mit der Zeit exponentiell ab. Da die Potentialtöpfe unterschiedlich tief sind, ergeben sich verschiedene Abklingzeiten. In der Summe ergibt sich eine gestreckte Exponentialfunktion.

Nach einer Theorie von Moynihan und Schröder [32] berechnet sich das Volumen der kooperativen Bereiche aus der Breite des Relaxationszeitspektrums, die schließlich in $\beta_{\mathrm{KWw}}$ ausgedrückt ist. Als mittlere Clusterdimension ergibt sich am Glasübergang für $\mathrm{Zr}_{65} \mathrm{Cu}_{27,5} \mathrm{Al}_{7,5}$ ein Durchmesser von 2,1 nm [21,33]. Diese experimentelle Abschätzung wird durch Molekulardynamiksimulationen gestützt, in denen beispielsweise Teichler [34] kollektive Atombewegung als bestimmendes Element für die Dynamik identifiziert und die Größe der Cluster im Modellsystem $\mathrm{Zr}_{50} \mathrm{Ni}_{50}$ mit etwa $2 \mathrm{~nm}$ angibt.

Experimentell wurde am Glasübergang für PMMA ein $\beta_{\mathrm{Kww}}$ von 0,34 gefunden [20], für $\mathrm{Zr}_{65} \mathrm{Cu}_{27,5} \mathrm{Al}_{7,5}$ steigt der KWW-Exponent von 0,66 im Glaszustand auf 0,86 für die unterkühlte Schmelze knapp oberhalb der Glastemperatur an [21]. Weil die Dynamik durch kooperative Bewegung auf atomarer Skala bestimmt ist und die Größe dieser Bereiche von der Temperatur abhängt, kann auch die Breite der Verteilung an Relaxationszeiten und damit der KWWExponent $\beta_{\mathrm{KWW}}$ temperaturabhängig sein.

Die Gegenüberstellung experimenteller Daten für verschiedene Systeme zeigt eine klare Korrelation von $\beta_{\mathrm{KWw}}$ mit dem Fragilitätsindex m. Nach Böhmer [35] ist die Umrechnung beider Größen wie folgt möglich:

$$
m \approx(250 \pm 30)-320 \cdot \beta_{K W W}
$$

Die Modenkopplungstheorie (MCT) von Götze und Sjögren [7, 36] ist ein theoretischer Ansatz, die dynamischen Eigenschaften von „Harte-Kugel“-Gläsern selbstkonsistent zu erklären. Die MCT sagt eine Änderung des atomaren Transportmechanismus von lokalen Sprüngen (,hopping“) zu kollektiver Bewegung der Atome bei einer kritischen Temperatur $\mathrm{T}_{\mathrm{c}}$ voraus, die weit oberhalb von $T_{g}$ liegt. Beim Abkühlen friert die atomare Struktur bei $T_{c}$ ein, verursacht durch eine gleichzeitige Zunahme der Dichte. Atome können dann einem Käfig („,cage ") von Nachbaratomen nur durch Sprungprozesse entkommen, welche man sich als kollektive Prozesse vorstellt, an denen viele Atome beteiligt sind. 


\subsubsection{Temperaturabhängigkeit der Relaxationsdynamik}

Wie in den vorhergehenden Abschnitten bereits gezeigt, hängt die Viskosität von glasbildenden Flüssigkeiten stark von der Temperatur ab, was nichts anderes bedeutet, als dass die Dynamik und damit auch die charakteristische Zeitskala für Relaxationsprozesse temperaturabhängig ist.

\subsubsection{Arrhenius-Gesetz}

Bei der Suche nach einem Modell, um diese Abhängigkeit korrekt zu erfassen, wird im einfachsten Fall ein Doppelmuldenpotential mit einer Energiebarriere $E_{B}$ für das relaxierende System angenommen. Dies führt zu einem thermisch aktivierten Arrhenius-Gesetz [37].

$$
\tau_{\text {Arr }}=\tau_{0} \cdot \exp \left(\frac{E_{B}}{k_{B} T}\right)=\tau_{0} \cdot \exp \left(\frac{B}{T}\right)
$$

Der Vorfaktor $\tau_{0}$ wird als „Anklopfzeit“ bezeichnet und entspricht dem Zeitabstand zwischen zwei Versuchen der relaxierenden Teilchen, die Energiebarriere zu überwinden. Realistische „Anklopfzeiten“ für die strukturelle Relaxation liegen bei etwa $10^{-14} \mathrm{~s}$ [38].

Gleichung (2.6) beschreibt reale Flüssigkeiten gut. Allerdings weichen unterkühlte Flüssigkeiten und Gläser mit abnehmender Temperatur vom Arrhenius-Gesetz ab, was komplexere Modelle nötig macht.

\subsubsection{Vogel-Fulcher-Tammann-Gleichung (VFT)}

Zur empirischen Beschreibung der Temperaturabhängigkeit von Relaxationszeiten in realen Systemen entwickelten Vogel [39], Fulcher [40] und Tammann [41] die Vogel-Fulcher-Tammann-Gleichung (VFT):

$$
\tau_{V F T}=\tau_{0} \cdot \exp \left(\frac{B_{V F T}}{T-T_{V F T}}\right)=\tau_{0} \cdot \exp \left(\frac{D \cdot T_{V F T}}{T-T_{V F T}}\right)
$$

Die Relaxationszeit divergiert bei dem neu eingeführten Temperaturparameter $\mathrm{T}_{\mathrm{VFT}}$, der für viele Systeme in der Nähe der Kauzmann-Temperatur $T_{K}$ liegt [42] (Abschnitt 2.2.1). Die Anwendung der VFT-Gleichung ist wegen der Divergenz der Relaxationszeit nur für Temperaturen oberhalb von $T_{V F T}$ zulässig. Der Stärkeparameter D wurde von Angell [43] zur quantitati- 
ven Charakterisierung in „starke“ und „fragile“ Gläser vorgeschlagen. Nach Böhmer [44] sind der Stärkeparameter D und der Fragilitätsindex m (Abschnitt 2.2.2) jedoch gleichwertig:

$$
m=\frac{590}{D}+16
$$

Wird im Rahmen der Freie-Volumen-Theorie [25] das freie Volumen über die DoolittleBeziehung [45] mit der Viskosität verknüpft, so lässt sich die Williams-Landel-Ferry-Gleichung [46] ableiten, die wiederum äquivalent zur VFT-Beziehung ist.

Die Adam-Gibbs-Theorie [28] liefert eine Temperaturabhängigkeit der Relaxationszeit, die der VFT-Beziehung in Gleichung (2.7) nahezu identisch ist. Demnach vergrößern sich die CCR-Gebiete mit abnehmender Temperatur, bis das ganze System schließlich ein einziger großer Cluster und die Dynamik vollständig eingefroren ist. Nach der Theorie müsste

$$
T_{V F T}=\frac{3}{4} \cdot T_{g}
$$

gelten und dort ein Phasenübergang zweiter Ordnung stattfinden.

\subsubsection{Erweiterte Freie-Volumen-Theorie (EFV)}

In einer Erweiterung der Freien-Volumen-Theorie (EFV) beschreiben Cohen und Grest die Koexistenz der bereits in Abschnitt 2.2.3 erwähnten „liquid-like“ und „solid-like“ Bereiche [26, 27], im Rahmen eines Perkolationsübergangs. Für die Relaxationszeit gilt

$$
\tau_{E F V}=\tau_{0} \cdot \exp \left(\frac{B_{E F V}}{T-T_{E F V}+\sqrt{\left(T-T_{E F V}\right)^{2}+C T}}\right)
$$

mit den nun vier freien Parametern $\tau_{0}, \mathrm{~T}_{\mathrm{EFV}}, \mathrm{B}_{\mathrm{EFV}}$ und C. Gleichung (2.10) ähnelt formal Gleichung (2.7), die Parameter $\mathrm{B}_{\mathrm{EFV}}$ und $\mathrm{B}_{\mathrm{VFT}}$ sowie $\mathrm{T}_{\mathrm{VFT}}$ und $\mathrm{T}_{\mathrm{EFV}}$ entsprechen sich jeweils. Die Erweiterung gegenüber dem VFT-Ansatz steckt in der Wurzel im Nenner.

\subsubsection{Frustration-Limited-Domain-Modell (FLD)}

Liegt die kritische Temperatur bei den FVT- und EFV-Theorien unterhalb der Glastemperatur, so gibt es beim FLD-Modell eine kritische Temperatur T ${ }^{*}$, die oberhalb der Schmelztemperatur liegt. Nach Kivelson [47] und Tarjus [48] sind die Atome oder Moleküle unterhalb 
von $\mathrm{T}^{*}$ bestrebt, sich lokal in energetisch begünstigten Kristallstrukturen anzuordnen. Die Ausdehnung über das gesamte System ist aber nicht möglich, was schließlich zu folgender Temperaturabhängigkeit der Relaxationszeit führt:

$$
\tau_{F L D}= \begin{cases}\tau_{0} \cdot \exp \left(\frac{B_{F L D}}{T}\right) & \text { für } T \geq T^{*} \\ \tau_{0} \cdot \exp \left[\frac{B_{F L D}}{T}+\frac{F T^{*}}{T}\left(\frac{T^{*}-T}{T^{*}}\right)^{8 / 3}\right] & \text { für } T<T^{*}\end{cases}
$$

\subsubsection{Modenkopplungstheorie (MCT)}

Der Vollständigkeit halber sei auf die Vorhersage der (idealisierten) Modenkopplungstheorie für die Temperaturabhängigkeit der Relaxationszeit verwiesen [7, 36]:

$$
\tau_{M C T}=\frac{\tau_{c}}{\left(T-T_{c}\right)^{\gamma}} \quad \text { für } \quad T>T_{c}
$$

Da sie Vorhersagen für Temperaturen oberhalb der kritischen Temperatur $T_{c}$ macht, die wiederum deutlich oberhalb der Glastemperatur liegt, ist sie im Rahmen dieser Arbeit nicht beweisbar. Oberhalb von $\mathrm{T}_{\mathrm{c}}$ konnte die Modenkopplungstheorie, beispielsweise mittels Neutronenstreuung oder Diffusion radioaktiver Tracerisotope an nur schwach unterhalb die thermodynamische Erstarrungstemperatur unterkühlten metallischen Gläsern, experimentell bestätigt werden $[49,50,51]$.

\subsection{5 Übergang vom Zeit- in den Frequenzraum}

Bei der Betrachtung in Abschnitt 2.2.4 wurde die Stufenantwortfunktion der relaxierenden Größe in Abhängigkeit von der Zeit diskutiert. Mit dem mathematischen Instrument einer Fourier- bzw. Laplace-Transformation gelingt der Übergang vom Zeit- in den Frequenzraum. Für den Fall der dielektrischen Relaxation wird aus der zeitabhängigen dielektrischen Polarisation die frequenzabhängige Dielektrizitätskonstante und für den Fall der mechanischen Relaxation aus der zeitabhängigen Dehnung oder Torsion des Systems der frequenzabhängige Elastizitäts- oder Schermodul.

Für die Debye-Stufenantwortfunktion in Gleichung (2.3) im Zeitraum ergibt sich nach der Laplace-Transformation die Debye-Gleichung $(2.13)$ [52, 53], welche die komplexe Relaxa- 
tionsfunktion $\chi^{*}$ in Abhängigkeit von der anregenden Frequenz $\mathrm{f}$ für eine charakteristische Relaxationszeit $\tau_{\mathrm{DB}}$ beschreibt:

$$
\chi^{*}(f)_{D B}=\chi_{\infty}+\frac{\chi_{S}-\chi_{\infty}}{1+i \cdot 2 \pi f \tau_{D B}}
$$

Dabei sind $\chi_{S}$ und $\chi_{\infty}$ die asymptotischen reellen Größen für statische und für unendlich hochfrequente Anregung und $\Delta \chi=\chi_{\mathrm{S}}-\chi_{\infty}$ das Maß für die Relaxationsstärke. Der Real- und der Imaginärteil der komplexen Größe $\chi^{*}(\mathrm{f})$ ergibt sich nach Multiplikation mit ihrer konjugiert komplexen Zahl zu:

$$
\begin{aligned}
& \chi^{\prime}(f)_{D B}=\chi_{\infty}+\frac{\Delta \chi}{1+\left(2 \pi f \tau_{D B}\right)^{2}} \\
& \chi^{\prime \prime}(f)_{D B}=\frac{\Delta \chi \cdot 2 \pi f \tau_{D B}}{1+\left(2 \pi f \tau_{D B}\right)^{2}}
\end{aligned}
$$

Sowohl der Real-, als auch der Imaginärteil lassen sich aus der Antwortfunktion bestimmen und enthalten jeweils die komplette Information des Systems. Beide Größen stehen über die Kramers-Kronig-Relation miteinander in Beziehung [54, 55].

Debye geht von nicht-wechselwirkenden, relaxierenden Teilchen (Dipole, Atome, Moleküle) aus, bei denen es eine genau definierte charakteristische Relaxationszeit $\tau$ gibt. In unterkühlten Flüssigkeiten und Gläsern ist diese Annahme nicht aufrecht zu erhalten, wie in den Abschnitten 2.2.3 und 2.2.4 ausführlich diskutiert wurde. Der Realität näher kommt die Annahme einer Verteilung an Relaxationszeiten, wie sie in der gestreckt exponentiellen KWW-Relaxation im Zeitraum (Gleichung 2.4) verwirklicht ist. Allerdings lässt sich diese Funktion mittels Fourier-Transformation nicht analytisch in den Frequenzraum überführen.

In der Literatur werden die Ergebnisse verschiedenster Experimente, wie dielektrische Relaxation [56], mechanische Relaxation [57], Relaxation magnetischer Kernmomente [58] oder quasielastische Neutronenstreuung [59] mit einer von Havriliak und Negami [60] im Jahr 1967 empirisch eingeführten Relaxationsfunktion beschrieben:

$$
\chi^{*}(f)_{H N}=\chi_{\infty}+\frac{\chi_{S}-\chi_{\infty}}{\left[1+\left(i \cdot 2 \pi f \tau_{H N}\right)^{\alpha}\right]^{\gamma}}
$$


Der Real- und der Imaginärteil der Havriliak-Negami (HN-) Funktion sind:

$$
\begin{aligned}
& \chi^{\prime}=\chi_{\infty}+\left(\chi_{s}-\chi_{\infty}\right) \cdot \frac{\cos (\gamma \varphi)}{\left(1+2 \cdot\left(2 \pi f \tau_{H N}\right)^{\alpha} \cdot \cos \left(\alpha \frac{\pi}{2}\right)+\left(2 \pi f \tau_{H N}\right)^{2 \alpha}\right)^{\frac{\gamma}{2}}} \\
& \chi^{\prime \prime}=\left(\chi_{\infty}-\chi_{s}\right) \cdot \frac{\sin (\gamma \varphi)}{\left(1+2 \cdot\left(2 \pi f \tau_{H N}\right)^{\alpha} \cdot \cos \left(\alpha \frac{\pi}{2}\right)+\left(2 \pi f \tau_{H N}\right)^{2 \alpha}\right)^{\frac{\gamma}{2}}}
\end{aligned}
$$

Die Ableitung von Real- und Imaginärteil aus der komplexen Relaxationsfunktion ist nicht trivial und wird daher in Anhang 2 nachvollzogen.

Hinzugekommen sind, verglichen mit Gleichung (2.13), der Breiteparameter $\alpha$ und der Asymmetrieparameter $\gamma$, die jeweils Werte zwischen 0 und 1 annehmen können. Spezialfälle der HN-Gleichung sind die Cole-Davidson-Funktion (CD) [61, 62], bei der $\alpha=1$ gesetzt wird, und die symmetrische Cole-Cole-Funktion (CC) [63], bei der $\gamma=1$ ist.

Der Einfluss der beiden Parameter auf den Peak des Imaginärteils ist in Abbildung 2.4 gezeigt. Für alle Kurven wurde die Relaxationsstärke $\Delta \chi=1$ und die Relaxationszeit $\tau=1$ gewählt. $\chi^{\prime \prime}$ ist doppelt logarithmisch gegenüber der Frequenz $f$ aufgetragen. In beiden Graphen ist die Debye-Relaxation als schwarze Linie eingetragen.
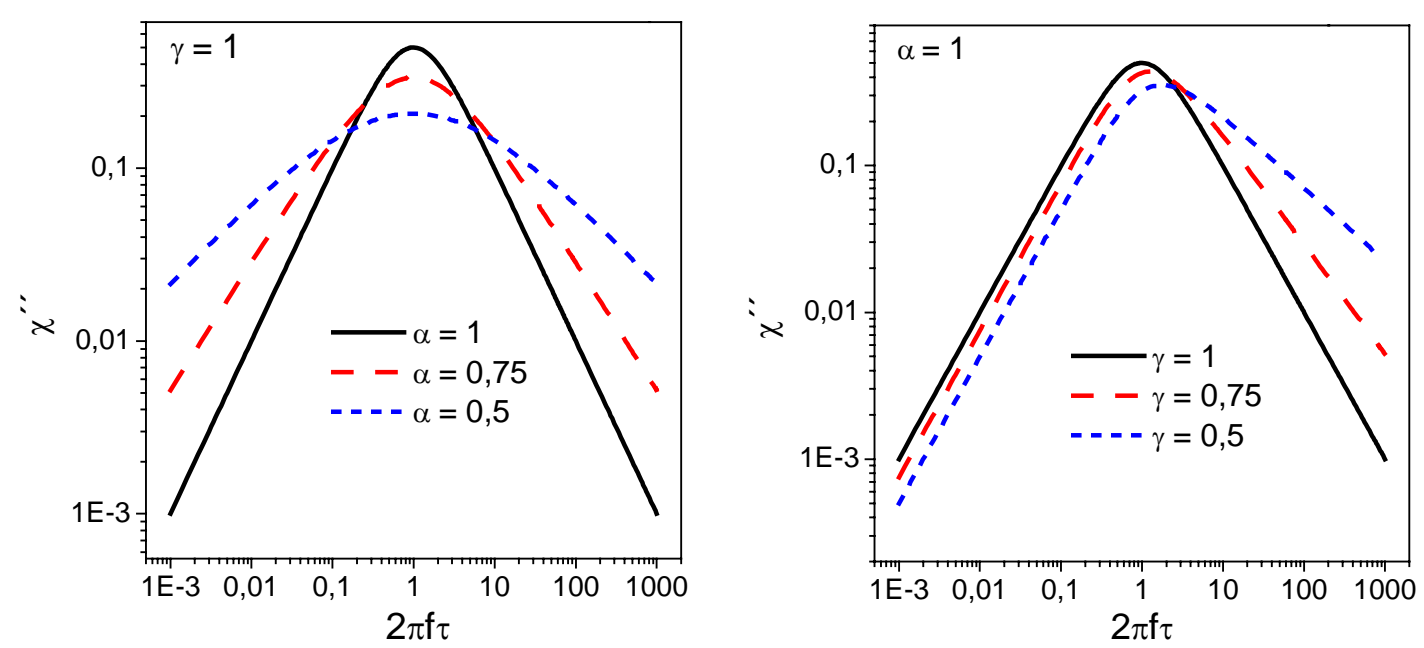

Abbildung 2.4: Einfluss der Parameter $\alpha$ (links) und $\gamma$ (rechts) auf den Imaginärteil der Havriliak-Negami- Relaxationsfunktion (2.16). Die schwarz eingezeichneten Linien mit $\alpha=1$ (links) bzw. $\gamma=1$ (rechts) entsprechen jeweils der Debye-Relaxation. 
Im linken Bild ist der Asymmetrieparameter $\gamma=1$ gewählt und die sich ergebende symmetrische Cole-Cole-Funktion für verschiedene Breiteparameter $\alpha$ gezeichnet. Während beim Debye-Peak die Höhe $\chi^{\prime \prime}{ }_{\max }=\Delta \chi / 2$ ist und die Halbwertsbreite 1,14 Dekaden beträgt, nimmt mit kleiner werdendem $\alpha$ das Maximum ab, während sich die Halbwertsbreite vergrößert.

Im rechten Graphen von Abbildung 2.4 ist der Breiteparameter $\alpha=1$ gesetzt. Mit kleiner werdendem $\gamma$ nimmt die Asymmetrie der Cole-Davidson-Funktion zu. Der niederfrequente Verlauf ändert sich dabei nicht, allerdings ist der Abfall von $\chi^{\prime \prime}$ auf der Hochfrequenzseite mit abnehmendem $\gamma$ weniger stark ausgeprägt.

Für den Fall einer Cole-Davidson Funktion entspricht der Parameter $\gamma$ gerade der negativen Steigung der Hochfrequenzflanke des Relaxationspeaks in der doppelt logarithmischen Auftragung von Abbildung 2.4. Für diesen Teilbereich der Funktion gilt folgendes Potenzgesetz:

$$
\chi^{\prime \prime}(f) \sim f^{-\gamma}
$$

Die gute experimentelle Übereinstimmung der KWW-Funktion im Zeit- und der HN-Funktion im Frequenzraum legt die Vermutung nahe, dass es einen Zusammenhang der freien Parameter beider Funktionen gibt. In der Tat gibt es mehrere Modelle, die einen solchen herstellen und es so ermöglichen, zwischen den KWW-Parametern $\beta_{\mathrm{KWw}}$ und $\tau_{\mathrm{KWw}}$ und den $\mathrm{HN}$ Parametern $\alpha, \gamma$ und $\tau_{\mathrm{HN}}$ hin- und her zu rechnen $[64,65,66]$.

\subsubsection{Temperaturabhängige Relaxationsfunktionen}

\subsubsection{Prozessführung bei konstanter Temperatur}

Bei der Havriliak-Negami-Gleichung wurde die relaxierende Größe $\chi^{*}(\mathrm{f})$ in Abhängigkeit von der Frequenz betrachtet. Mit der Wahl einer konstanten Temperatur stellt sich eine Verteilung an Relaxationszeiten ein, die je nach Modell (VFT, EVT, FLD) unterschiedlich, aber eindeutig definiert ist (Abschnitt 2.2.3). Wird das System nun von außen mit der Frequenz $\mathrm{f} z \mathrm{zu}$ Schwingungen angeregt, so beobachtet man die Relaxation genau für die charakteristische Zeit $\tau=1 /$ f. Mit variierender Frequenz werden bei isothermer Prozessführung verschiedene Relaxationszeiten abgetastet und der Real- oder Imaginäranteil von $\chi^{*}(\mathrm{f})$ gemessen. 


\subsubsection{Prozessführung bei konstanter Frequenz}

Mit höheren Temperaturen verschiebt sich die charakteristische Relaxationszeit $\tau$ des Systems zu kleineren Werten. Die dynamischen Prozesse nehmen an Geschwindigkeit zu. Bei Messung mit der konstanten Frequenz f lässt sich die Dynamik des Systems also dadurch abtasten, dass mit der Temperatur die Verteilungsfunktion der Relaxationszeiten verschoben wird.

\subsubsection{Zeit-Temperatur-Superposition}

Geht man davon aus, dass sich bei verschiedenen Temperaturen zwar die Position des Maximums der Verteilungsfunktion für die Relaxationszeiten verschiebt, dessen Form jedoch erhalten bleibt, so erhält man mit beiden Prozessführungen die exakt gleiche Information. Dass diese unterschiedliche Herangehensweise zu denselben Resultaten führt, drückt sich im ZeitTemperatur-Superpositionsprinzip aus, das auch in metallischen Gläsern mit Erfolg bestätigt wurde [67].

Als sehr erfolgreiche Anwendung dieses Prinzips wurde von Nagel und Mitarbeitern [68, 69, 70] eine Skalierungsmethode vorgeschlagen (Nagel-scaling), mit der frequenzabhängige Relaxationskurven temperaturunabhängig auf eine einzige Masterkurve im Frequenzraum abgebildet werden können. Eine Weiterentwicklung von Dendzik [71] bildet beliebige CD-Funktionen auf eine CD-Masterkurve ab, so dass Abweichungen, insbesondere der Hochfrequenzflanke, von einem idealen CD-Verhalten quantitativ erfasst werden können.

\subsubsection{Beispiel für den Übergang Frequenz- in Temperaturraum}

Zur Verdeutlichung und wegen der zentralen Bedeutung der Zeit-Temperatur-Äquivalenz für diese Arbeit, sei der Vorgang an einem fiktiven Beispiel noch einmal veranschaulicht.

Angenommen sei eine Relaxation nach Debye. Der Imaginärteil der Relaxationsfunktion $\chi^{\prime \prime}(\mathrm{f})$ berechnet sich nach Gleichung (2.15). Die Relaxationsstärke wird für alle Temperaturen mit $\Delta \chi=1$ festgelegt, die Relaxationszeit $\tau_{\mathrm{DB}}$ sei nach Gleichung (2.6) arrheniusabhängig, wobei die Parameter Anklopfzeit $\tau_{0}=10^{-14} \mathrm{~s}$ und $\mathrm{B}=1,2 \times 10^{4}$ gewählt werden (Abbildung 2.5). Je größer die Temperatur, desto kleiner wird die Relaxationszeit $\tau_{\mathrm{DB}}$ und desto schneller ist die Dynamik des untersuchten Prozesses. 


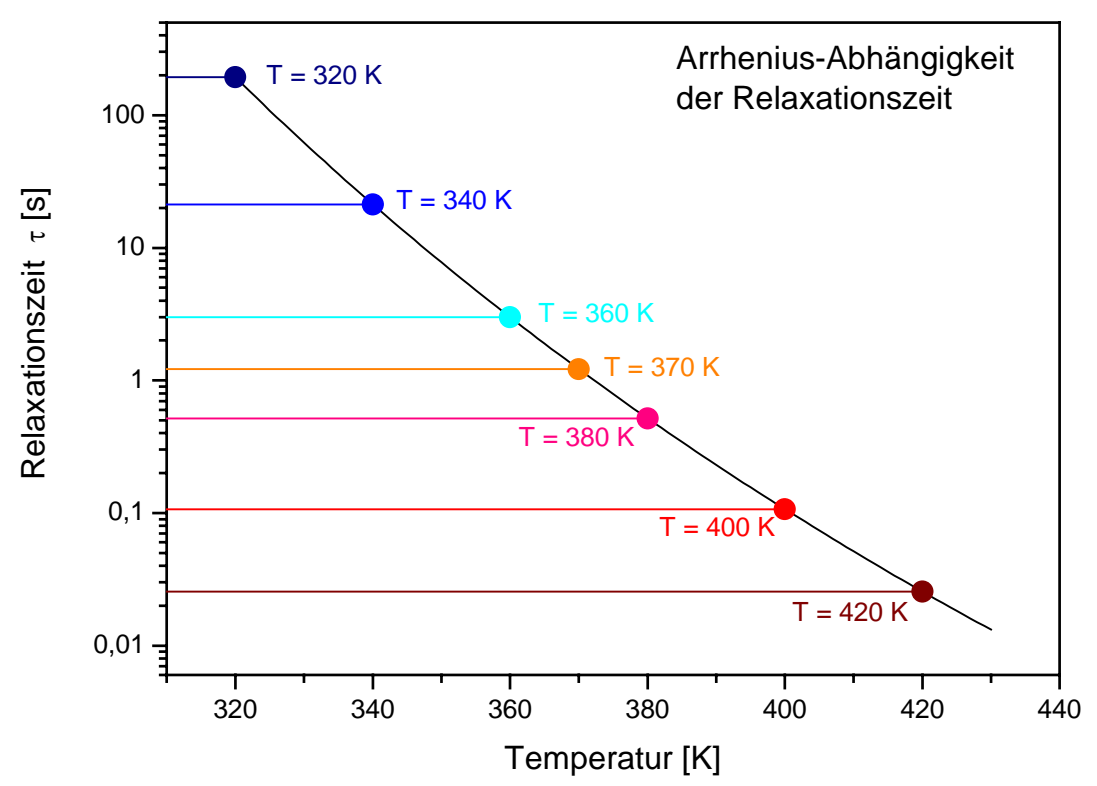

Abbildung 2.5: Abhängigkeit der Relaxationszeit von der Temperatur ( fiktives Beispiel).

Wird die Temperatur fest gewählt, so ergibt sich aus Abbildung 2.5 die Relaxationszeit, mit der, eingesetzt in Gleichung (2.15), der frequenzabhängige Imaginärteil $\chi^{\prime \prime}(\mathrm{f})$ der Relaxationsfunktion gegenüber der Frequenz für diese Temperatur berechnen werden kann. Abbildung 2.6 zeigt das Ergebnis in doppelt logarithmischer Auftragung für verschiedene Relaxationszeiten, die nach Arrhenius den angegebenen Temperaturen zuzuordnen sind.

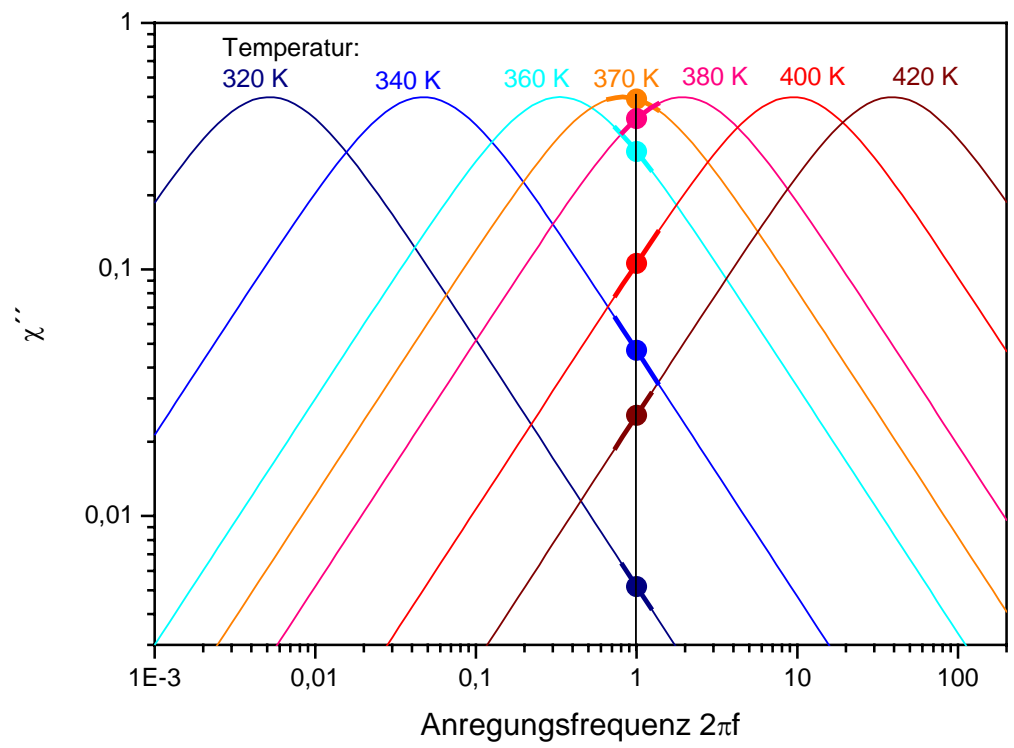

Abbildung 2.6: Frequenzabhängiger Imaginärteil der Relaxationsfunktion für verschiedene Temperaturen. 
Auch in Abbildung 2.6 ist ersichtlich, dass sich die Dynamik der Relaxation von langen Zeiten (kleinen Frequenzen) bei tiefen Temperaturen zu kurzen Zeiten (hohe Frequenzen) bei höheren Temperaturen verschiebt. Um vom Frequenz- in den Temperaturraum zu wechseln, muss bei einer konstanten Frequenz die Temperaturabhängigkeit der Funktion betrachtet werden. Im Beispiel wird die Frequenz $2 \pi \mathrm{f}=1$ gewählt (vertikale Linie in Abbildung 2.6).

Offensichtlich kann nun aus jeder Kurve der dargestellten Schar der Wert von $\chi^{\prime \prime}(2 \pi f=1)$ ermittelt werden (ausgefüllte Kreise). Durch die Verschiebung der Kurve mit steigender Temperatur nach rechts, werden niedrige Temperaturen auf der Hochfrequenzflanke der Relaxationsfunktion dargestellt. Mit steigender Temperatur wird das Maximum bei T $=372 \mathrm{~K}$ durchlaufen, bevor der Funktionswert auf der Niederfrequenzflanke wieder abfällt.

Werden die für verschiedene Temperaturen ermittelten Werte von $\chi^{\prime \prime}(2 \pi f=1)$ nun gegenüber der Temperatur aufgetragen, so ergibt sich der in Abbildung 2.7 gezeigte Verlauf.

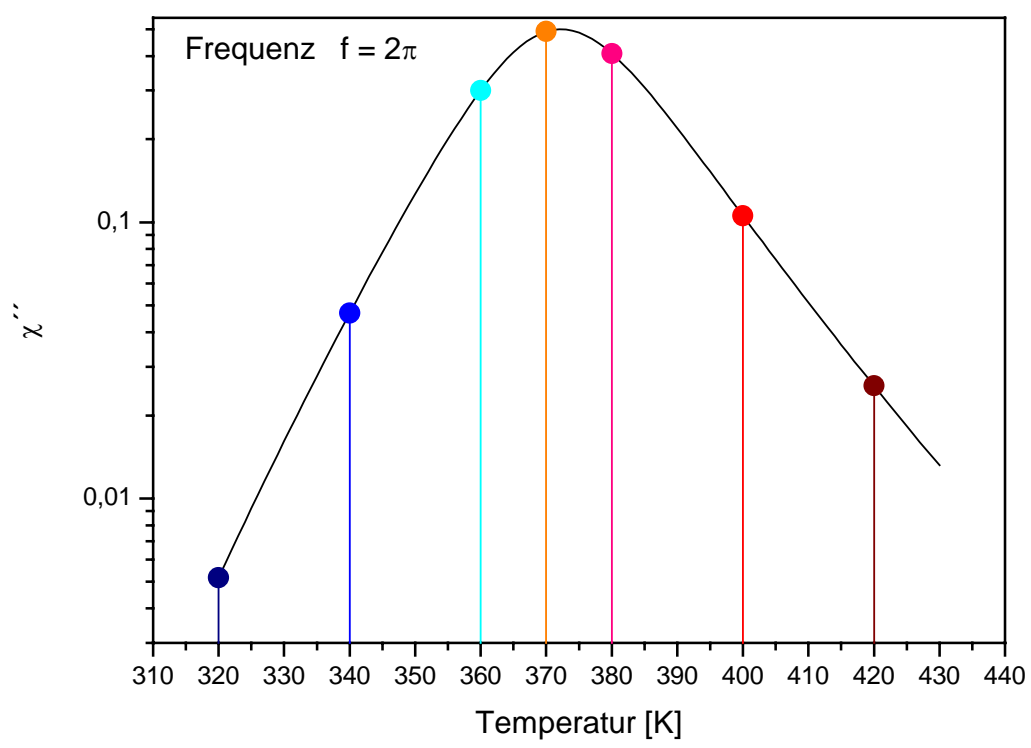

Abbildung 2.7: Relaxationsfunktion in Abhängigkeit von der Temperatur.

Die entscheidende Erkenntnis, die aus diesem Beispiel und der vergleichenden Betrachtung von Abbildung 2.6 und Abbildung 2.7 gewonnen werden kann, ist die Folgende:

Wird bei gewählter Frequenz die temperaturabhängige Relaxationsfunktion gemessen, beginnend bei kleinen Temperaturen, so entspricht diese Messung wegen des Zeit-Temperatur-Superpositionsprinzips einem Abtasten der Hochfrequenzflanke der frequenzabhängigen Messung bei konstanter Temperatur beginnend bei hohen Frequenzen. 


\subsubsection{Schwierigkeiten beim Übergang in den Temperaturraum}

Bei einem reellen glasbildenden System gestaltet sich der Übergang vom Frequenz- in den Temperaturraum, oder umgekehrt, ungleich komplexer. Dies hat mehrere Gründe:

- Die Temperaturabhängigkeit der charakteristischen Relaxationszeit (Abschnitt 2.2.4) ist vielfach nicht klar. Darüber hinaus divergieren die meisten Relaxationsfunktionen (beispielsweise die VFT-Funktion) bei einer bestimmten Temperatur und sind nur oberhalb dieser Singularität gültig. Eine geschlossene Darstellung der Dynamik vom tiefen Glaszustand in die unterkühlte Schmelze ist unmöglich.

- Aus dielektrischen Messungen, wie beispielsweise an PMMA, ist bekannt, dass die Relaxationsstärke $\Delta \chi$ temperaturabhängig ist [72]. Die Wechselwirkungen der umgebenden mit einem einzelnen Dipol bestimmen nach Onsager [73] die Relaxationsstärke. Bei realen Molekülen sind zusätzliche Wechselwirkungen, wie sterische Behinderungen oder Wasserstoffbrückenbindungen, zu berücksichtigen [74]. All das führt zur Kirkwood-Fröhlich-Gleichung [75, 76], mit der sich bei detailgenauer Kenntnis aller molekularer Wechselwirkungen die Temperaturabhängigkeit der Relaxationsstärke berechnen lässt.

- Wird für die Beschreibung von Messdaten die Havriliak-Negami-Funktion verwendet, so müssen im Gegensatz zur Debye-Relaxation die zusätzlichen Kurvenparameter $\alpha$ und $\gamma$ berücksichtigt werden. $\mathrm{Zu}$ deren Temperaturabhängigkeit gibt es bislang keine theoretischen Herleitungen, allerdings zeigt sich in frequenzabhängigen Experimenten an PMMA [72], dass sich beide Parameter mit der Temperatur ändern.

\subsection{Langsame $\beta$-Relaxation durch intramolekulare Dynamik}

Abweichungen vom reinen Debye- oder HN-Verhalten auf der Hochfrequenzflanke des dielektrischen Verlustes werden als „,slow- $\beta$ “ Prozesse bezeichnet und wurden erstmals in Polymeren unterhalb der Glastemperatur nachgewiesen. Sie werden der Bewegung von Seitengruppen zugeschrieben, die auch innerhalb der eingefrorenen Glasmatrix noch möglich ist [77]. Es gibt unterschiedliche mikroskopische Prozesse, die bei komplexen Molekülen, wie beispielsweise den Polymeren, zur Dynamik beitragen. Folgende Merkmale der $\beta$-Relaxationen stimmen in vielen Materialien überein: 
- Die Verteilung der Relaxationszeiten der $\beta$-Relaxation ist symmetrisch und, verglichen mit der $\alpha$-Relaxation, relativ breit [78, 79, 80, 81].

- Die Relaxationsstärke nimmt mit steigender Temperatur zu, während die Breite der Relaxation abnimmt $[82,96,83,80,84,85,86]$. Beide Parameter hängen oberhalb der Glastemperatur stärker von der Temperatur ab als unterhalb.

- Die Temperaturabhängigkeit der Relaxationszeit folgt einem Arrhenius-Gesetz, wie in Gleichung (2.6) beschrieben [87, 96].

Abbildung 2.8 verdeutlicht, warum der $\beta$-Prozess in vielen Systemen nur unterhalb der Glastemperatur aufgelöst werden kann. Seine charakteristische Relaxationszeit nähert sich oberhalb von $T_{g}$ der des $\alpha$-Prozesses so weit an, dass beide Prozesse nicht mehr getrennt beobachtet werden können. Dieses Phänomen wird in der Literatur als „merging“ kontrovers diskutiert $[88,89,90,91,92]$.

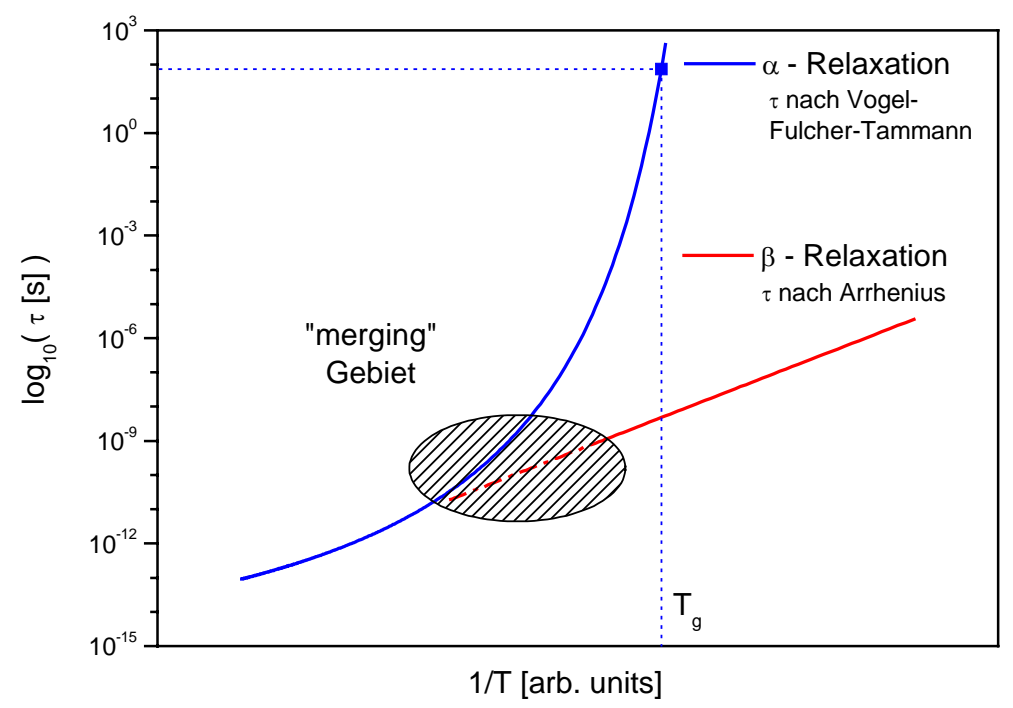

Abbildung 2.8: Abhängigkeit der charakteristischen Relaxationszeit von der Temperatur für den $\alpha$ - und den $\beta$ Prozess. Die Auftragung ist in der Arrhenius-Darstellung logarithmisch über 1/T.

Insbesondere die Frage, wie die Überlagerung beider Prozesse im „merging“ Gebiet mathematisch beschrieben werden sollte, ist dabei umstritten:

- Wird unterstellt, dass die mikroskopischen Ursachen beider Relaxationsprozesse voneinander unabhängig sind, so ergibt sich die beobachtete Relaxationsfunktion $\chi^{*}(\mathrm{f})$ aus der einfachen Addition beider Einzelbeiträge $\chi_{\alpha}^{*}(\mathrm{f})+\chi_{\beta}^{*}(\mathrm{f})$. 
- Wenn sich die Mechanismen beider Prozesse gegenseitig beeinflussen, so ist die Unabhängigkeitsannahme nicht mehr zulässig. Für diesen Fall wurde von Williams und Watts [93, 94] ein multiplikativer Ansatz vorgeschlagen, der mathematisch allerdings nur mit hohem Aufwand [80] zu verwirklichen ist. In diesem „Williams-Ansatz“ ist die gesamte, normalisierte Relaxationsfunktion im Zeitraum gegeben durch:

$$
\phi(t)=A \phi_{\alpha}(t)+(1-A) \phi_{\alpha}(t) \phi_{\beta}(t)
$$

Dabei sind $\phi_{\alpha}(\mathrm{t})$ und $\phi_{\beta}(\mathrm{t})$ und die normalisierten Relaxationsfunktionen für den $\alpha$ - und den $\beta$-Prozess, während A für den temperaturabhängigen Anteil der $\alpha$-Relaxation steht.

Trotz der prinzipiell unterschiedlichen Herangehensweise konnte gezeigt werden, dass sich beide Fälle auch im „merging“-Gebiet nur geringfügig unterscheiden [90, 91]. Sind die Relaxationszeiten beider Prozesse über mehrere Dekaden voneinander getrennt, so führen beide Ansätze zu identischen Ergebnissen.

\subsection{Beiträge zur Hochfrequenzflanke}

Neben den langsamen $\beta$-Prozessen, die sich durch die Betrachtung der intramolekularen Bewegung erklären lassen, wurden auch bei anderen glasbildenden Materialien $\beta$-Relaxationen gefunden [95, 96, 97]. Die Tatsache, dass es auf der Hochfrequenzflanke Beiträge in Form einer Schulter oder eines Peaks zum viskosen Fließen gibt, führt zu der Annahme, dass es weitere Prozesse geben könnte, die intrinsische Eigenschaften von unterkühlten Flüssigkeiten und Gläsern sind.

Die ersten detaillierten Untersuchungen zu einem neuen, fundamentalen Prozess jenseits des viskosen Fließens wurden von Johari und Goldstein [87, 98, 99] an Glasbildnern ohne dielektrisch aktiven Seitengruppen durchgeführt. In ihrem Modell [100] ist diese Johari-Goldstein$\beta$-Relaxation auf sogenannte, ,islands of mobility“ zurückzuführen. Es existieren lokale Bereiche, in denen einzelne Moleküle selbst unterhalb der Glastemperatur eine erhöhte Beweglichkeit aufweisen, was eine im Vergleich zur $\alpha$-Relaxation schnellere Dynamik zur Folge hat.

Demgegenüber geht Ngai davon aus, dass nicht nur einige, sondern alle Moleküle zu einer sekundären Relaxation beitragen. In seinem „coupling model“ [101] beschreibt er räumlich gleichförmige, zeitliche Dichteschwankungen, die für die Bewegung verantwortlich sind und zu der im statistischen Mittel alle Moleküle beitragen. Untersuchungen von Wagner und Ri- 
chert an Sorbit [102] und von Vogel und Rössler an Toluol [103] stützen dieses „,couplingmodel“.

Allerdings gibt es auch Gläser, bei denen sich weder ein Peak, noch eine Schulter auf der Hochfrequenzflanke der $\alpha$-Relaxation ausbilden [68, 104]. Stattdessen zeigt das Frequenzspektrum Zusatzbeiträge (,excess-wing“), ohne dass sich das Vorzeichen der Krümmung der Kurve ändert [105]. Dieser „wing“ geht aus dem Hochfrequenzlimit der Relaxationsfunktion hervor, das als Potenzgesetz $\chi^{\prime \prime}(\mathrm{f}) \sim \mathrm{f}^{\gamma}$ (Gleichung 2.19) etwa zwei bis drei Dekaden oberhalb der Peakfrequenz in ein zweites, schwächeres Potenzgesetz $\chi^{\prime \prime}(f) \sim \mathrm{f}^{\mathrm{g}}$ übergeht, wobei $\mathrm{g}<\gamma$ ist $[104,106]$.

Kaum ein Phänomen der Glasphysik wird in der Literatur so kontrovers diskutiert, wie die Frage, ob Johari-Goldstein $\beta$-Relaxation und der „excess-wing“ als völlig unterschiedlich voneinander anzusehen sind oder ob sie gar die gleiche physikalische Ursache haben. Bei der historisch älteren Auffassung, dass es sich um unterschiedliche Vorgänge handelt [68, 107, 108], hat Kudlik [107] folgende Klassifizierung glasbildender Systeme vorgeschlagen:

- Typ A Glasbildner zeigen einen ,excess-wing“ ohne ausgeprägte $\beta$-Relaxation.

- Typ B Glasbildner zeigen eine $\beta$-Relaxation, was bedeutet, dass sich ihr Krümmungsverhalten auf der Hochfrequenzflanke des $\alpha$-Peaks in Folge einer Schulter oder eines ausgeprägten Peaks verändert.

Allerdings gibt es auch Ansätze, eine Verbindung zwischen beiden Prozessen herzustellen. So hat Dixon [68] vorgeschlagen, den ,excess-wing“ als universelle Eigenschaft des $\alpha$-Prozesses in allen Glasbildnern zu akzeptieren. Die Tatsache, dass der „wing“ in Typ B Gläsern nicht gefunden wird liegt daran, dass er durch den starken $\beta$-Prozess überlagert wird. Noch weiter geht $\mathrm{Wu}$ [109], der fordert, auch bei Gläsern mit einem gut aufgelösten $\beta$-Peak den „excesswing“ bei der theoretischen Beschreibung der Kurven zu berücksichtigen. Das Modell dynamisch korrelierter Domänen von Chamberlin [110, 111] beschreibt den Zusammenhang beider Phänomene von einem theoretischen Standpunkt aus.

Die anschaulichste Interpretation für den „excess-wing“ ist es, die Existenz einer $\beta$-Relaxation zu postulieren, deren Peak allerdings durch den dominanten $\alpha$-Prozess vollständig überlagert ist. Diese Interpretation stützt die These einer universellen $\beta$-Relaxation und wurde in mehreren Publikationen erörtert [112, 113, 114, 115, 116, 117]. 
Dass in Materialien mit einem klar aufgelösten $\beta$-Peak die $\alpha$ - und $\beta$-Relaxationszeiten mit sinkender Temperatur unterschiedlich ansteigen (siehe auch Abbildung 2.8), lässt die Vermutung zu, dass der „excess-wing“ bei tiefen Temperaturen zu einer Schulter mutiert, wenn der $\alpha$-Peak zu kleineren Frequenzen verschoben ist und beide Prozesse weniger stark interferieren. Voraussetzung hierfür ist allerdings, dass die Wartezeit genügend groß ist, um bei den nötigen niedrigen Temperaturen unterhalb des Glasübergangs thermodynamisches Gleichgewicht zu gewährleisten. Solche Experimente wurden in jüngerer Vergangenheit durchgeführt mit dem Ergebnis, dass der excess-wing in der Tat die Hochfrequenzflanke eines unter dem $\alpha$-Peak versteckten $\beta$-Prozesses sein könnte $[92,118]$. 


\section{Experimentelles}

Die temperaturabhängigen dynamischen Eigenschaften der untersuchten dünnen Schichten wurden mit dem Doppel-Paddel Oszillator bestimmt. Weil die Apparatur hierzu im Rahmen dieser Arbeit aufgebaut wurde und wegen der Bedeutung für die Auswertung der Messergebnisse wird dieses Experiment und die zugehörigen mathematischen und physikalischen Grundlagen im zentralen Kapitel 4 behandelt.

An dieser Stelle werden die experimentellen Methoden vorgestellt, mit denen die Proben charakterisiert und analysiert wurden.

\subsection{Röntgendiffraktometrie und -reflektometrie}

Die strukturelle Analyse und die Bestimmung der Schichtdicke der Metallfilme wurde mit zwei kommerziellen Röntgendiffraktometern, einer D5000 der Firma Siemens und einer D8Advance der Firma Bruker AXS, durchgeführt. Als Röntgenquelle dient jeweils eine KupferAnode. Ein Einkristall-Monochromator im Fall der D5000 und ein Göbel-Spiegel bei der D8Advance selektiert die $\mathrm{Cu}-\mathrm{K}_{\alpha}-$ Strahlung mit einer Wellenlänge von $\lambda=0,15418 \mathrm{~nm}$. Die Messungen wurden in Bragg-Brentano-Geometrie durchgeführt.

Die Weitwinkel-Röntgendiffraktometrie ist eine Standardmethode für die strukturelle Untersuchung von Festkörpern. In Kristallen mit langreichweitiger Ordnung wird die eindringende Röntgenstrahlung an den Atomen der einzelnen Netzebenen gestreut. Die Überlagerung dieser Streuwellen liefert ein Beugungsbild, das der Fouriertransformierten der vorliegenden Kristallstruktur entspricht und somit Rückschlüsse auf die Anordnung der Atome erlaubt. Bei amorphen Materialien fehlt die langreichweitige Ordnung, weshalb keine scharf definierten Beugungsreflexe der Gitterebenen auftreten, sondern breite Intensitätsmaxima, die aus der statistischen radialen Verteilungsfunktion der Atome im Rahmen ihrer Nahordnung resultieren. Mit dieser Methode werden metallische Glasschichten auf Amorphizität oder Kristallinität untersucht.

Die Schichtdicke der metallischen Proben wurde mittels Kleinwinkel-Röntgenreflektometrie untersucht. Unter streifendem Einfall bei Winkeln kleiner als $3^{\circ}$ wird die Röntgenstrahlung sowohl an der Grenzfläche Schicht-Luft, als auch an der Grenzfläche Substrat-Schicht reflektiert. Die Interferenz beider Teilstrahlen führt zu einer Oszillation der Streuintensität, wobei 
die Position $\Theta_{\mathrm{i}}$ der Interferenzmaxima und -minima im Winkelraum $2 \Theta$ mit der Schichtdicke d wie folgt in Beziehung steht [119]:

$$
\Theta_{i}=2 \delta+\frac{\left(m_{i}+\Delta m\right)^{2} \lambda^{2}}{4 d^{2}}
$$

Dabei bezeichnet $\mathrm{m}_{\mathrm{i}}$ die Ordnung des Extremums, die durch Minimierung des Fehlerquadrates nach Hink und Petzold bestimmt wird [120]. Für Metallfilme auf Si-Substraten ist bei Mi$\operatorname{nima} \Delta \mathrm{m}=0$ und bei Maxima $\Delta \mathrm{m}=0,5$.

Dieses Verfahren ist für die Bestimmung der Schichtdicke bei dünnen, relativ glatten Schichten gut geeignet und wurde für „Tooling“--Messungen eingesetzt, um die Schichtdicke der einzelnen metallischen Aufdampfkomponenten am Ort des Paddels zu kalibrieren.

\subsection{Transmissions-Elektronen-Mikroskopie und EDX}

Zur Bestimmung von Struktur und Zusammensetzung der metallischen Glasschichten wurden Messungen von Michael Seibt und Yue-Lin Huang an einem Transmissions Elektronen Mikroskop (TEM) der Firma Philipps vom Typ CM-200-UT-FEG am IV. Physikalischen Institut der Universität Göttingen durchgeführt. Für die prinzipielle Funktionsweise und die theoretischen Grundlagen hierzu sei auf die Literatur verwiesen [121, 122].

Mittels energiedispersiver Röntgenanalyse (EDX) wird die Zusammensetzung der Proben untersucht. Hierzu gibt man für die erwarteten Elemente je einen festen Bereich im Energiespektrum so vor, dass in einem solchen Energieintervall nur charakteristische Röntgenstrahlung einer bestimmten Linie eines Elementes auftritt und es keinen Überlapp mit den charakteristischen Linien der anderen Elemente gibt. In einem Linescan über den zu messenden Probenbereich wird die Intensität dieser Linien ortsabhängig gemessen. Das Signal ist proportional zur Probendicke und zur atomaren Konzentration des jeweils betrachteten Elementes. Für die genauere quantitative Auswertung in Punktmessungen wird eine numerische Anpassung an das gemessene Hintergrundsignal durchgeführt, dieses vom gemessenen Signal subtrahiert und aus dem Verhältnis der Intensitäten der in den gewählten Fenstern auftreffenden Intensitäten die atomare Zusammensetzung der Probe berechnet.

Zwei der bedampften Oszillatoren, DPO 12 ohne und DPO 19 mit einer $50 \mathrm{~nm}$ dicken TantalDiffusionsbarriere wurden nach der Messung zerbrochen. Senkrecht zur Wachstumsrichtung der Schichten wurden Querschliffe des DPO-Halses präpariert. Um die Wirkung der 
Diffusionsbarriere zu überprüfen und die Elementverteilung an der Grenzfläche SubstratSchicht zu untersuchen, wurden EDX-Linescans und EDX-Punktmessungen senkrecht zur Paddeloberfläche durchgeführt. In diesen Messungen wird auch das homogene Wachstum der Schichten untersucht.

Zusätzlich zu den bedampften Oszillatoren wurden unabhängige Messungen an $50 \mathrm{~nm}$-dünnen Schichten durchgeführt, die in der UHV-Anlage auf Gold-Netzchen aufgedampft wurden. Diese Schichten wurden im TEM sowohl im Beugungs-, als auch im Hellfeldmodus betrachtet. Ziel dieser Kontrollmessungen war es, die metallischen Glasschichten auf ihre korrekte Zusammensetzung und ihre amorphe Struktur zu überprüfen.

\subsection{Fourier-Transformations-Infrarot-Spektroskopie (FTIR)}

Beim Durchstrahlen von Materie mit infrarotem Licht (IR) werden bei geeigneter Wellenlänge Eigenschwingungen der Molekülbindungen angeregt. Die Moleküle nehmen Energie auf und schwächen gleichzeitig die Intensität der Strahlung. Bestimmt man das Absorptionsverhalten in Abhängigkeit von der Wellenlänge der Strahlung, so gewinnt man Informationen über die chemische Struktur des Materials. Eine ausführliche Darstellung der Methode findet sich beispielsweise bei Günzler [123].

Bei einem Fourier-Transformations-Infrarot-Spektrometer (FTIR) wird die IR-Strahlung durch ein Michelson-Zweistrahl-Interferometer in spektrale Information umgewandelt. Das Verhältnis des Signals zum Rauschen wird dadurch bei hoher Messgeschwindigkeit optimiert. Mittels FTIR Spektroskopie wird geklärt, wie sich die molekulare Struktur des PMMA in der Schicht im Vergleich zum Ausgangsmaterial des Targets ändert und welche Auswirkungen die Temperaturzyklen bei der mechanischen Spektroskopie auf die Polymerstruktur haben.

Die Messungen im Rahmen dieser Arbeit wurden von E. Süske (Institut für Materialphysik der Universität Göttingen) an einem Bruker Tensor 37 FTIR-Spektrometer in Reflexion unter einem Winkel von $10^{\circ}$ durchgeführt.

\subsection{Dielektrische Spektroskopie}

Die Dynamik von strukturellen Glasbildnern umfasst einen Frequenzbereich von wenigen Mikrohertz bis hin zu mehreren Terahertz und hängt darüber hinaus stark von der Temperatur 
ab. Verschiedenste experimentelle Methoden wurden entwickelt, um die komplexe Dielektrizität zu bestimmen [38]. Der enorme Vorteil ist dabei, dass sowohl die Temperatur, als auch die Frequenz unabhängig voneinander mit relativ geringem experimentellem Aufwand variiert werden können. Beide Parameter, von denen die Dynamik wesentlich bestimmt wird, sind damit direkt zugänglich.

Um die Methoden der dielektrischen und der mechanischen Spektroskopie miteinander zu vergleichen, aber auch um eine Aussage zu Veränderungen durch den Ablationsprozess machen zu können, wurden PMMA-Proben von R. Wehn und P. Lunkenheimer (Lehrstuhl für Experimentalphysik V der Universität Augsburg) mit einem Novocontrol $\alpha$-Analyzer dielektrisch spektroskopiert [38].

Dazu wurde eine etwa $1 \mu \mathrm{m}$ dicke PMMA-Schicht auf ein elektrisch leitfähiges Kupferblech deponiert, bevor wiederum eine dünne Kupferschicht als Gegenelektrode auf die PMMAOberfläche aufgedampft wurde. Ein etwa $1 \mathrm{~mm}$ dicker Streifen des Ausgangsmaterials wurde aus dem Target geschnitten und auf beiden Seiten mit Kupfer bedampft. An beiden Proben wurden sowohl isotherme Frequenzscans durchgeführt, als auch bei verschiedenen Frequenzen temperaturabhängig gemessen. 


\section{Der Doppel-Paddel Oszillator}

Mit der Verwendung des Doppel-Paddel Oszillators (DPO) als Substrat können mechanische Eigenschaften von dünnen Schichten gemessen werden. Zu diesem Zweck wird der Oszillator im Rahmen dieser Arbeit erstmals bei Temperaturen größer als Raumtemperatur eingesetzt. Eine entsprechende Anlage wurde konstruiert und in Betrieb genommen. In diesem Kapitel werden im Detail die Grundlagen zum Verständnis der Experimente gelegt. Es gibt einen Überblick über den aktuellen Stand von Theorie und experimenteller Technik rund um den Doppel-Paddel Oszillator.

In Abschnitt 4.1 wird die grundsätzliche Idee erläutert, wie mit dieser Technik mechanische Spektroskopie betrieben werden kann. Die den Experimenten zu Grunde liegende physikalische Fragestellung wird beschrieben. Ausgehend von den verschiedenen Herstellungsverfahren für die Oszillatoren, die in Abschnitt 4.2 dargestellt sind, dokumentiert Abschnitt 4.3 die konstruktiven und experimentellen Erfordernisse für ihren Betrieb. Elektronik, Steuerung und Datenerfassung werden in Abschnitt 4.4 erklärt.

Ein gutes mathematisches Modell zur Beschreibung der Dynamik des Oszillators ist für die Auswertung der Daten unerlässlich. Die Abschnitte 4.5 und 4.6 sind zunächst dem leeren Oszillator gewidmet. Seine Schwingungseigenschaften werden modelliert und die erwarteten Resultate abgeschätzt. Die theoretischen Überlegungen werden mit experimentellen Ergebnissen verglichen. Schließlich wird in Abschnitt 4.7 gezeigt, wie eine dünne Schicht auf dem Oszillator dessen Eigenschaften verändert und wie man aus diesen Veränderungen auf Prozesse in der Schicht zurückschließen kann.

\subsection{Mechanische Spektroskopie mit dem DPO}

1985 wurde von Kleiman et al. [124, 125] ein neues Design für einen mechanischen Torsionsoszillator mit großer Güte vorgeschlagen. Dieser sogenannte Doppel-Paddel Oszillator (DPO) wurde in der Vergangenheit erfolgreich eingesetzt, um mechanische Eigenschaften dünner, auf dem Oszillator deponierter Schichten, bei tiefen Temperaturen zu untersuchen [126]. Beispiele hierfür sind Tunnelzustände in amorphen Filmen [127] und deren Verhalten in magnetischen Feldern [128] oder auch Messungen zur Schallgeschwindigkeit [129] und zu Alterungseigenschaften [130] von kristallinen Edelgasfilmen. Auch der DPO selbst war Objekt zahlreicher Arbeiten. Die Dynamik und Geometrie der verschiedenen Eigenmoden wurde 
mittels Finite Elemente Simulationen von Spiel et al. [131] berechnet und später von Liu et al. [132] mit Laser-Doppler-Vibrometrie gemessen.

Um mechanische Eigenschaften dünner Schichten zu untersuchen, wird der DPO resonant in der zweiten AntiSymmetrischen Eigenmode (AS2) betrieben. Die Resonanzfrequenz dieser speziellen Eigenschwingung liegt bei etwa 5,4 kHz. Wegen der speziellen Geometrie des Oszillators wird nur sehr wenig elastisch gespeicherte Energie durch Reibung dissipiert. Der Dämpfungsfaktor ist bei Temperaturen unterhalb von $10 \mathrm{~K}$ kleiner als $10^{-8}$. Houston et al. [133] haben gezeigt, dass sich das Temperaturverhalten der Güte dieser Eigenmode mit dem thermoelastischen Effekt quantitativ in einem Temperaturbereich von $50 \mathrm{~K}$ bis Raumtemperatur gut beschreiben lässt.

Bei den Experimenten im Rahmen dieser Arbeit werden die Oszillatoren bei Temperaturen bis zu $400{ }^{\circ} \mathrm{C}$ betrieben. Dabei hat sich herausgestellt, dass die Dämpfung der AS2-Mode auch bei hohen Temperaturen durch den thermoelastischen Effekt dominiert wird. Sie bleibt so niedrig, dass selbst dünne Schichten mit großer Empfindlichkeit untersucht werden können. Der DPO kann deshalb als Substrat verwendet werden, um Phänomene wie den Glasübergang, viskoses Fließen auf molekularer Ebene oder Kristallisation von amorphen Materialien zu untersuchen.

Wird eine dünne Schicht auf dem Oszillator deponiert, so verändern sich die Eigenschaften der AS2-Mode. Die zusätzliche Masse vergrößert die Trägheit des Systems. Gleichzeitig wird die Schicht Teil der tordierenden Bestandteile, was sich auf die rücktreibenden Drehmomente der Schwingung auswirkt. Beide Effekte verursachen eine Verschiebung der Resonanzfrequenz. Die Güte der Schwingung verschlechtert sich, da wegen innerer Reibung im Schichtmaterial zusätzlich Energie dissipiert wird. Durch den Vergleich der Dämpfung des beschichteten mit dem leeren Oszillator wird auf die mikroskopischen Prozesse in der Schicht zurückgeschlossen. Sowohl die Verschiebung der Resonanzfrequenz als auch die Änderung der Dämpfung werden temperaturabhängig gemessen und daraus der komplexe Schermodul des deponierten Materials berechnet.

Angeregt durch umfangreiche Arbeiten über den dielektrischen Verlust in organischen Glasbildnern [1] werden Schichten aus Polymethylmethacrylat (PMMA) untersucht, um die Methoden der dielektrischen und der mechanischen Spektroskopie zu vergleichen.

Ziel der Experimente am metallischen „Inoue“-Glas $\mathrm{Zr}_{65} \mathrm{Al}_{7.5} \mathrm{Cu}_{27.5}$ ist es, durch die Beobachtung des Relaxationsverhaltens und der inneren Reibung die Dynamik auf atomarer Skala bes- 
ser zu verstehen. Auf der Grundlage quasistatischer Experimente von M. Weiß [21] an diesem speziellen metallischen Glas wird der untersuchte Frequenzbereich erweitert. Die Torsionsschwingung und die Bestimmung des frequenzabhängigen Schermoduls ist dabei auch eine Alternative zu früheren Arbeiten mit Vibrating Reed Oszillatoren von Kempen et al. [134], mit denen der Elastizitätsmodul vermessen wurde.

\subsection{Design und Herstellung des DPOs}

\subsubsection{Beschreibung und Abmessungen}
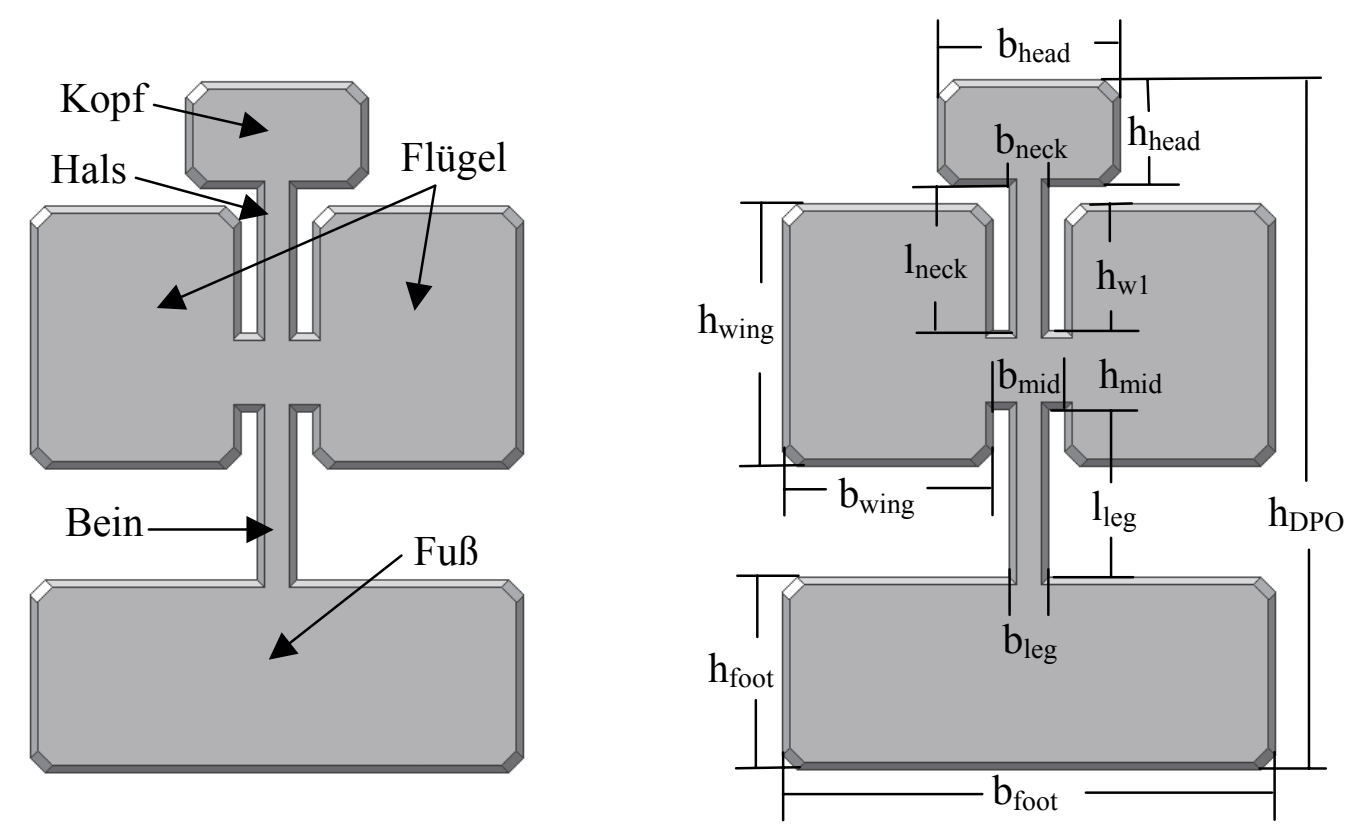

Abbildung 4.1: Doppel-Paddel Oszillator mit seinen charakteristischen Komponenten (links) und den physikalischen Dimensionsgrößen (rechts).

Der Doppel-Paddel Oszillator ist ein $300 \mu \mathrm{m}$ dickes, charakteristisch geformtes Gebilde mit etwa $2 \mathrm{~cm}$ Breite und $3 \mathrm{~cm}$ Höhe. Ausgangsmaterial für seine Herstellung sind (100)-orientierte Siliziumwafer hoher Reinheit. Die spezifische Leitfähigkeit bei Raumtemperatur ist gröBer als $10 \mathrm{k} \Omega \mathrm{cm}$. Bezugsquelle für die nach dem „Cornell-Verfahren“ (Abschnitt 4.2.2) hergestellten Wafer ist die Firma Wacker Chemie in Burghausen. Die Wafer für die lasergeschnittenen Paddel (Abschnitt 4.2.3) wurden von der Firma CrysTec in Berlin geliefert.

Abbildung 4.1 zeigt den Oszillator mit den in der Literatur üblichen Bezeichnung seiner Bestandteile. In Tabelle 4.1 sind seine Abmessungen angegeben. Die schrägen Kanten entstehen 
bei der Herstellung durch den Ätzprozess, der bei dem skizzierten Paddel von oben beginnt. Die Maße beziehen sich auf die längeren Kanten an der Rückseite. Bei der Laserherstellung entfällt diese Unterscheidung, weil durch die senkrechten Schnitte die Kantenlängen an Oberund Unterseite des Paddels gleich groß sind.

Die angegebenen Maße werden bei den Berechnungen in Abschnitt 4.5 eingesetzt und wurden auch von Spiel [131] für die Finite-Elemente-Simulationen benutzt. Bei den geätzten Paddeln werden dünne Schichten stets auf die Seite mit der größere Oberfläche aufgebracht, um zu gewährleisten, dass die Filmgeometrie die angegebenen Abmessungen hat.

\begin{tabular}{|c|c|c|}
\hline Größe & Beschreibung & Länge in $\mathrm{mm}$ \\
\hline $\mathrm{b}_{\text {head }}$ & Breite des Kopfes & 7,53 \\
\hline $\mathrm{h}_{\text {head }}$ & Höhe des Kopfes & 4,39 \\
\hline $\mathrm{b}_{\text {wing }}$ & Breite eines Flügels & 8,68 \\
\hline $\mathrm{h}_{\text {wing }}$ & Höhe eines Flügels & 10,87 \\
\hline $\mathrm{h}_{\mathrm{w} 1}$ & Überhöhung Flügel vom Mittelteil & 5,22 \\
\hline $\mathrm{b}_{\text {mid }}$ & Breite des Mittelteils & 3,01 \\
\hline $\mathrm{h}_{\text {mid }}$ & Höhe des Mittelteils & 3,27 \\
\hline $\mathrm{l}_{\text {neck }}$ & Länge des Halses & 5,97 \\
\hline $\mathrm{l}_{\text {leg }}$ & Länge des Beines & 6,98 \\
\hline $\mathrm{b}_{\text {neck }}$ & Breite des Halses & 1,61 \\
\hline $\mathrm{b}_{\text {leg }}$ & Breite des Beines & 1,0 \\
\hline $\mathrm{h}_{\text {foot }}$ & Höhe des Fußes & 7,97 \\
\hline $\mathrm{b}_{\text {foot }}$ & Breite des Fußes & 20,37 \\
\hline $\mathrm{h}_{\mathrm{DPO}}$ & Gesamthöhe des Oszillators & 28,58 \\
\hline $\mathrm{t}$ & Dicke des Oszillators & 0,3 \\
\hline
\end{tabular}

Tabelle 4.1: Abmessungen des Doppel-Paddel Oszillators. 


\subsubsection{Herstellung durch Photolithografie (Cornell-Verfahren)}

In Zusammenarbeit mit der Cornell Nanofabrication Facility (CNF) haben R.O. Pohl und Mitarbeiter ein Verfahren entwickelt, wie Doppel-Paddel Oszillatoren aus Siliziumwafern durch Photolithografie herstellt werden [136]. Die zwölf Einzelschritte dieses „Cornell-Verfahrens“ sind in Abbildung 4.2 skizziert und werden im Folgenden beschrieben:

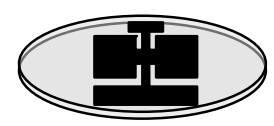

1. Maske

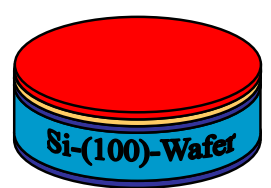

5. Photolack

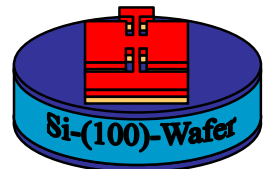

8. Entwickeln

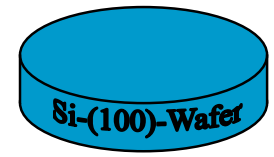

2. Si-Wafer

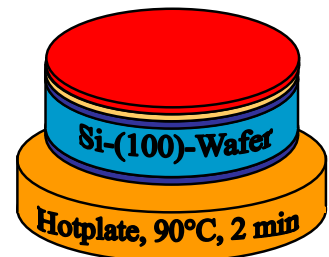

6. Hitzebehandlung

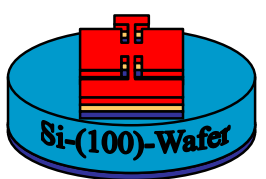

9. Plasmaätzen

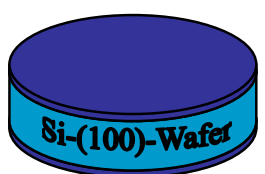

3. $\mathrm{Si}_{3} \mathrm{~N}_{4}-\mathrm{Schicht}$

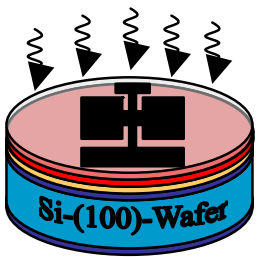

7. Belichten

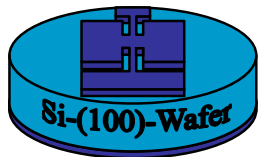

10. Aceton

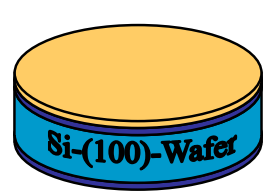

4. Primer

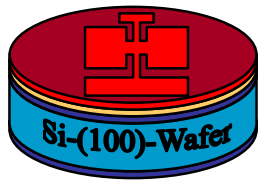

„Sonnenbrand“

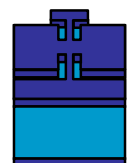

11. $\mathrm{KOH}$

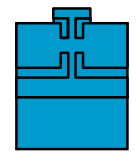

12. HF

Abbildung 4.2: Herstellung von Doppel-Paddel Oszillatoren nach dem „Cornell-Verfahren“.

\section{Maske}

Für die Strukturierung des Wafers durch Photolithografie wird eine Maske benötigt. Dazu werden die Paddel im Maßstab 1:1 mit einem Mann 3600 Muster-Generator auf eine Glasplatte übertragen. Anders als in der Schemaskizze angedeutet, passen mehrere Paddel auf einen Wafer. Die Belichtungsmaske ist ein Photopositiv. Die DPO-Form ist dunkel, wohingegen alle Bereiche, die in späteren Prozessen weggeätzt werden, durchlässig für die Strahlung sind und belichtet werden.

\section{Reinigen des Silizium-Wafers}

Ausgangsmaterial für die Oszillatoren sind $300 \mu \mathrm{m}$ dicke, (100)-orientierte Wafer aus einkristallinem Silizium, nach dem float-zone Verfahren hergestellt, beidseitig poliert und pTyp Bor dotiert mit einem spezifischen Widerstand $\mathrm{R}>10 \mathrm{k} \Omega \mathrm{cm}$. Auf einem mit 3000 
Umdrehungen pro Minute rotierendem Spincoater wird der Wafer mit Methylenchlorid behandelt, um eine eventuell vorhandene Oxydschicht zu entfernen. Anschließend wird der Wafer, ebenfalls während der Rotation, mit Isopropyl oder Methanol gereinigt.

\section{3. $\mathrm{Si}_{3} \mathbf{N}_{4}$ Schutzschicht}

Durch chemische Dampfabscheidung bei geringem Druck wird bei $800{ }^{\circ} \mathrm{C} \mathrm{Si}_{3} \mathrm{~N}_{4}$-Schicht von etwa $80 \mathrm{~nm}$ Dicke auf beide Seiten des Wafers aufgebracht. Das Siliziumnitrid dient als Schutzschicht beim späteren Ätzen mit Kalilauge.

\section{4. „Priming“ der Oberfläche}

Der Wafer wird bei einer Umdrehungsgeschwindigkeit von 3000 pro Minute auf dem Spinner zunächst mit Aceton gereinigt. Direkt anschließend, noch bevor das Aceton völlig abgetrocknet ist, wird er mit Isopropanol dehydratisiert. Nun wird der Spinner angehalten, und mit einer Pipette eine $20 \%$ Hexamethyldisilazan (HMDS) Lösung flächig auf den Wafer aufgetragen. Idealerweise ist das Lösungsmittel das gleiche, das später für den Photolack verwendet wird. Die Oberfläche muss blasenfrei und vollständig benetzt sein. Erst dann wird der Spinner wieder eingeschalten und der Wafer bei 3000 Umdrehungen pro Minute 60 Sekunden lang geschleudert. Die HDMS-Zwischenschicht, der sogenannte „Primer“, verbessert die Haftung des Photolacks durch Adhäsion und sorgt für eine gleichmäßigere Verteilung auf der Oberfläche. Die unbehandelte Siliziumnitrid-Schicht würde sehr schnell oxidieren und mit absorbiertem Wasser aus der Umgebung langreichweitige Wasserstoffbrücken bilden. Der Lack würde dann am Oberflächenwasser haften bleiben und sich sehr leicht wieder ablösen.

\section{Aufbringen des Photolacks}

Sobald der Spinner nach dem „Priming“ wieder zur Ruhe gekommen ist, wird der Photolack (Shipley 1400-23) mit einer sauberen Pipette so aufgetragen, dass die Oberfläche vollständig bedeckt ist. Für weitere 60 Sekunden wird nun der Spinner bei 3000 Umdrehungen pro Minute betrieben. Gleich zu Beginn der Rotation spritzt viel von dem aufgetragenen Photolack ab. Seine Farbe auf dem Wafer ändert sich mit der Dicke der Lackschicht. Nach dem Schleudern hat der Lack etwa eine Dicke von $2 \mu \mathrm{m}$.

\section{Ausbacken des Lösungsmittels}

Auf einer Heizplatte mit Vakuum-Ansaugmöglichkeit, um einen guten Wärmekontakt zu gewährleisten, wird der Wafer für 2 Minuten bei $90^{\circ} \mathrm{C}$ gebacken um dem Photolack das 
Lösungsmittel auszutreiben. Diese Parameter sind relativ kritisch, weil zu kurzes Backen zu ungenügenden mechanischen Eigenschaften des Photolacks führt, während zu langes Backen die photoempfindlichen Komponenten im Lack zerstört.

\section{Belichten}

Durch die vorher hergestellten Maske (Glasplatte) wird der Wafer in einem HTG contact printer mit intensiver UV-Strahlung belichtet. Dominante Emissionslinien der Hochdruck Quecksilber Lichtbogenlampe sind bei 365 nm (i-Linie), 405 nm (h-Linie) und 436 nm (gLinie). Entscheidend für die Qualität der Oszillatoren ist die Ausrichtung der Maske relativ zum Wafer. Die Oszillator-Symmetrieachse muss genau in $<110>$ Richtung des Wafers orientiert sein. Nach dem Belichten sind die Paddel auf dem Wafer schon zu erkennen („Sonnenbrand“-Effekt). Überbelichtung schadet dem Photolack nicht, weshalb die Belichtungszeit relativ unkritisch ist.

\section{Entwickeln}

In ein breites Becherglas wird unverdünnter Entwickler (Shipley MF-321) etwa $1 \mathrm{~cm}$ hoch eingefüllt. Der belichtete Wafer wird für 1 min eingetaucht. Einfache Entwickler basieren

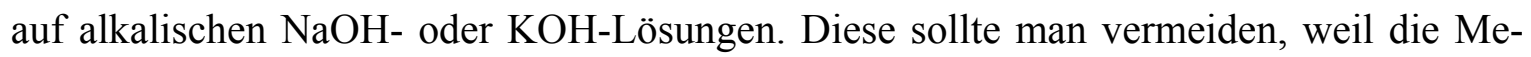
tallionen die Oberfläche kontaminieren könnten. Der Shipley MF-321 Entwickler ist speziell auf den Shipley 1400-23 Photolack abgestimmt. Während des Entwicklungsvorgangs färbt sich der Entwickler rötlich. Ist der Prozess abgeschlossen, wird der Wafer unter fließendem Wasser gut abgespült und mit Stickstoff trocken geblasen. Auf dem mit $\mathrm{Si}_{3} \mathrm{~N}_{4}$ bedeckten Wafer befindet sich nun Photolack in Oszillatorform.

\section{Plasmaätzen}

In einem Plasma, das aus $\mathrm{CHF}_{3}$ und $\mathrm{O}_{2}$ besteht, wird das freiliegende $\mathrm{Si}_{3} \mathrm{~N}_{4}$ in etwa drei Minuten trocken weggeätzt. Mit diesem Prozessschritt wird nur die belichtete Vorderseite des Wafers behandelt, die Rückseite bleibt mit der Siliziumnitridschicht vollständig bedeckt. Der Photolack dient als Schutzschicht für das Nitrid.

\section{Ablösen des Photolacks und des Primers}

In Aceton wird der in Oszillatorform verbliebene Photolack zusammen mit dem darunter liegenden Primer abgelöst. 


\section{KOH-Ätzen}

In heißer Kalilauge wird nun alles Silizium aufgelöst, das nicht auf beiden Seiten von der Siliziumnitrid-Schicht bedeckt ist. Um einen Wafer zu ätzen, werden $200 \mathrm{~g}$ festes $\mathrm{KOH}$ (Plätzchen) nach und nach in $800 \mathrm{~g}$ destilliertem Wasser aufgelöst. Die Konzentration der Lauge beträgt somit 20 gew\%. Die Temperatur wird vorsichtig auf idealerweise $99{ }^{\circ} \mathrm{C}$ erwärmt, allerdings darf die Lösung nicht kochen. Heiße Kalilauge ist ein stark anisotropes Ätzmittel, das die $<100>$ und die $<110>$ Richtungen stärker angreift, als die $<111>$ Richtungen. Die verbliebene $\mathrm{Si}_{3} \mathrm{~N}_{4}$ Oberfläche wird nur schwach angegriffen und schützt die von ihr bedeckten Flächen des Wafers. Nach etwa zwei Stunden sind die Doppel-Paddel Oszillatoren, die noch mit $\mathrm{Si}_{3} \mathrm{~N}_{4}$ bedeckt sind, freigeätzt und „schwimmen“ in der Lauge. Ein Teflonkäfig als Unterlage verhindert, dass sich die Oszillatoren ineinander verhaken und durch die Konvektion in der Lauge abbrechen.

Während des Ätzens wird das Becherglas abgedeckt, um zu schnelles Abdampfen der Lauge zu vermeiden. Die Paddel werden nun einzeln vorsichtig in destilliertes Wasser getaucht, um anhaftendes Ätzmittel zu entfernen.

Die verbliebene Siliziumnitrid-Schicht hat eine gute Schutzwirkung für die darunter liegende Siliziumoberfläche, weshalb es geschickt ist, diese Schutzschicht erst unmittelbar vor dem Einsatz des Paddels abzulösen.

\section{Ablösen des $\mathrm{Si}_{3} \mathrm{~N}_{4}$ in $\mathrm{HF}$}

Rund $50 \mathrm{ml}$ einer 25 \%igen Flusssäure wird in ein kleines Plastik-Becherglas gegeben. Die Paddel werden zum Ätzen möglichst aufrecht hineingestellt (der Ätzprozess muss an der Vorder- und an der Rückseite angreifen können. Nach etwa 60 Minuten ist der Vorgang abgeschlossen. Die Paddel werden vorsichtig in destilliertem Wasser gereinigt. Wenn keine Benetzung der Paddeloberfläche durch das Wasser mehr stattfindet, ist die Siliziumnitrid-Schicht vollständig abgelöst. Bleiben kleine Tröpfchen auf der Oberfläche haften, ist das ein Indiz dafür, dass der Ätzvorgang noch nicht vollständig abgeschlossen ist.

\subsubsection{Herstellung durch Laserschneiden}

Eine grundsätzlich andere Möglichkeit, Strukturen aus Silizium herzustellen ist, sie mit einem Laserstrahl aus dem Wafer herauszuschneiden. Doppel-Paddel Oszillatoren werden mit dieser 
Methode in Zusammenarbeit mit dem Fraunhofer Institut für Lasertechnik in Aachen hergestellt. Dabei wird ein gepulster ND:YAG-Laser vom Typ LASAG KLS246-040 FC (finecut) verwendet, der zum Schneiden, Bohren und Schweißen für industrielle Anwendungen konzipiert ist (Abbildung 4.3).
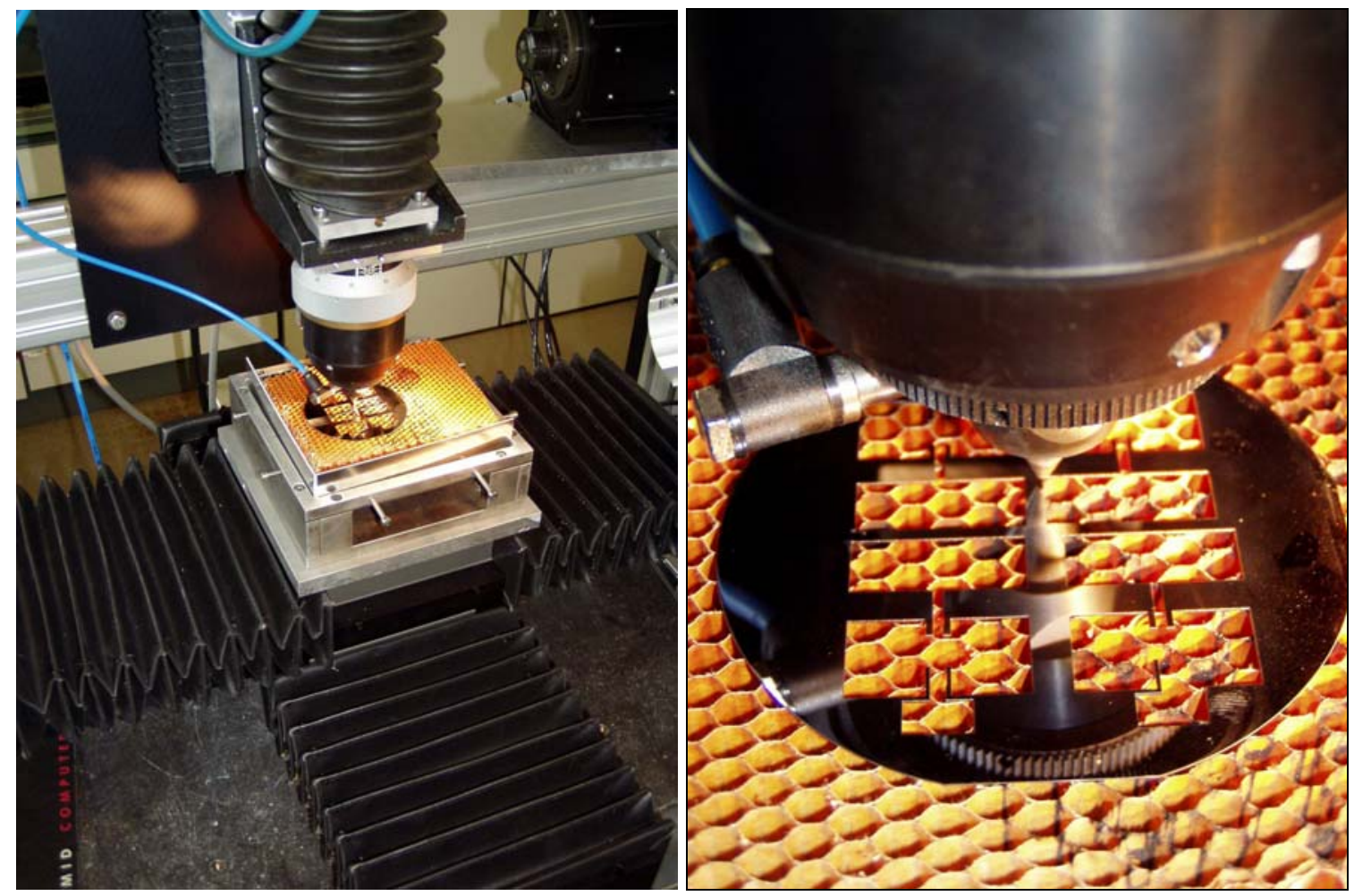

Abbildung 4.3: Anordnung zum Schneiden von Doppel-Paddel Oszillatoren mit einem Laserstrahl (links). In der Vergrößerung (rechts) sieht man den Wafer, aus dem die Paddel schon entnommen sind.

Mittels Feinsteuerung wird der Probentisch mit dem darauf liegenden Wafer bewegt. Der Laser wird bei einer Pulsfrequenz von $1 \mathrm{kHz}$, einer Pulsdauer von $30 \mu \mathrm{s}$, und einer Pulsenergie von $10 \mathrm{~mJ}$ betrieben. Der Durchmesser des Laserstrahls wird mit einer Blende auf 2,5 mm festgelegt. Nach Strahlaufweitung um den Faktor drei bis vier und Durchlaufen einer Fokussieroptik mit der Brennweite $50 \mathrm{~mm}$ beträgt der Strahldurchmesser auf dem Wafer noch etwa 20 bis $25 \mu \mathrm{m}$. Als Prozessgas wird Stickstoff mit 2 bis 3 bar Druck verwendet und aus einer Düse mit Öffnungsdurchmesser 0,5 mm geblasen. Die Vorschubgeschwindigkeit ist durch die Achsdynamik begrenzt und beträgt $300 \mathrm{~mm} / \mathrm{min}$. 


\subsubsection{Vergleich beider Methoden}

Das Entfernen von Silizium mit heißer Kalilauge ist ein stark anisotropes Ätzverfahren, das hauptsächlich senkrecht zur Waferebene wirkt. Trotzdem kann nicht verhindert werden, dass im Verlauf des Prozesses die schon freigelegten Strukturen des Oszillators auch von der Seite angegriffen werden. Die Verhältnisse der Ätzraten für die (111)-, (110)- und (100)-Ebenen betragen etwa 1:10:17 [135].

Die Anisotropie zeigt sich in schrägen Kanten, die eine geringe Rauhigkeit aufweisen und in abgerundeten Ecken. Die Querschnitte von Hals und Bein sind trapezförmig. Aufnahmen eines geätzten Paddels mit dem Raster-Elektronen-Mikroskop sind in Abbildung 4.4 gezeigt. Den Winkel der Kante zur Horizontalen hat Metcalf [136] zu 54,7 bestimmt.

Wird die Symmetrieachse des Paddels beim Belichten nicht exakt in [110]-Orientierung ausgerichtet, so führt dieser Fehler zu einer treppenartigen Struktur der Kanten. Das Ätzverfahren bewirkt insgesamt, dass die Abmessungen des Oszillators, wie durch die Maske vorgegeben, nur an der zuletzt von der Kalilauge erreichten Unterseite realisiert sind. Es wird allgemein angenommen [137], dass die Fehlorientierung und die daraus resultierende Treppenstruktur der Kanten einen Einfluss auf die beobachtete Dämpfung des Oszillators hat, obwohl diese Fragestellung bis heute nicht detailliert untersucht worden ist.

Die prinzipiell vollkommen unterschiedliche Herstellungsmethode des Laserschneidens führt zu Schnittkanten, die senkrecht auf der Paddel-Ebene stehen. Damit haben beide Seiten des Paddels gleiche Abmessungen und die Querschnitte von Hals und Bein sind rechteckig. Bei entsprechender Vergrößerung ist in Abbildung 4.5 jedoch zu sehen, dass die lasergeschnittenen Kanten extrem aufgeraut und zerklüftet sind. Darüber hinaus wird durch den herstellungsbedingten Gasstoß, der mit dem Laserpuls verbunden ist, aufgeschmolzenes Material von der Eintritts- zur Austrittsebene transportiert, bleibt zum Teil an deren Rand hängen oder lagert sich auf der Oberfläche ab. Diese Ablagerungen lassen sich mit einem dünnen Glasfaserpinsel größtenteils wieder entfernen.

Experimentell zeigt sich, dass die Resonanzfrequenzen von der Herstellungsmethode abhängen (Tabelle 4.2 in Abschnitt 4.6.1). Das ist nicht verwunderlich, weil sowohl die Trägheitsmomente, als auch die Torsionskonstanten durch die Massenverhältnisse und die exakten geometrischen Abmessungen bestimmt werden. 


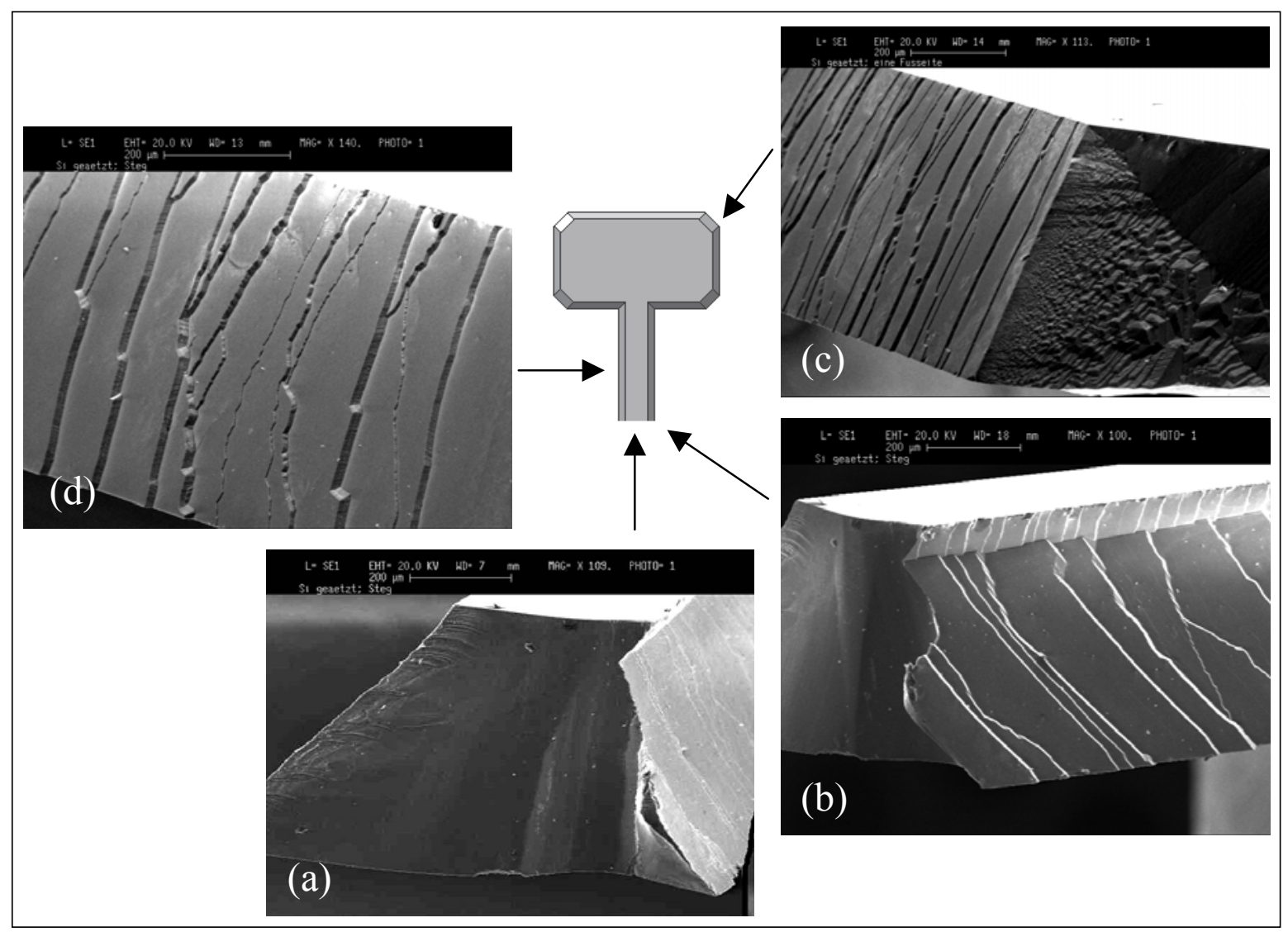

Abbildung 4.4: Untersuchung der Kanten mit dem Raster-Elektronen-Mikroskop. Durch den anisotropen Ätzprozess entsteht ein trapezförmiger Querschnitt des Halses (a), schräge Kanten (b), sowie Ecken (c), die an einen Geröllhang erinnern. Die treppenförmigen Kanten (b) und (d) entstehen durch Fehlorientierung beim Belichten.

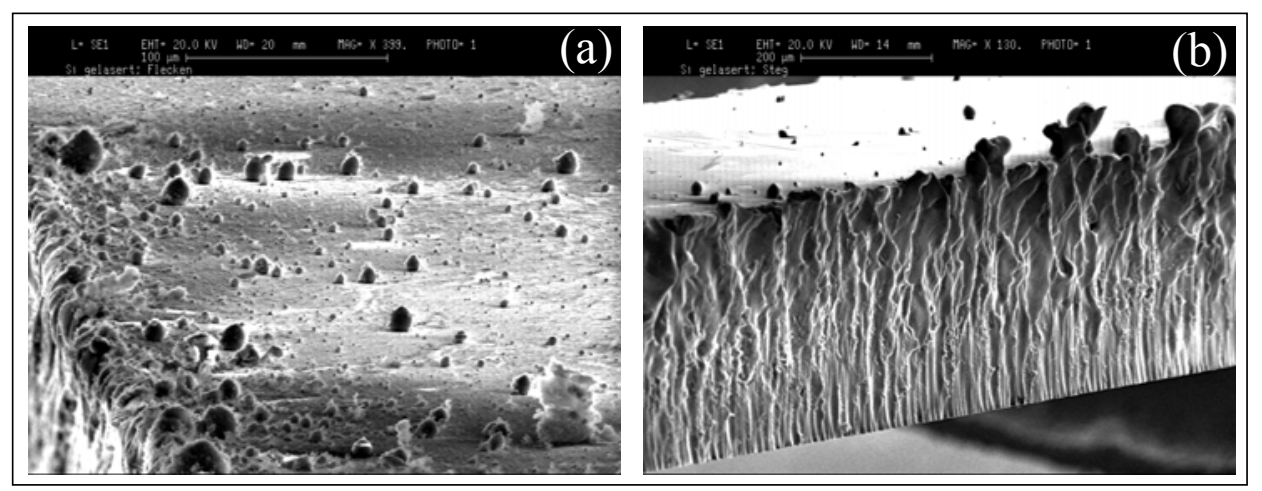

Abbildung 4.5: Materialauswurf beim Laserschneiden führt zu Ablagerungen auf der Oberfläche (a). Die Schnittkante (b) steht senkrecht auf der Paddelebene und ist sehr rau und zerklüftet. 
Die Dämpfung der AS2-Mode ist in Abbildung 4.6 für alle im Rahmen dieser Arbeit untersuchten Paddel vergleichend dargestellt. Bei den lasergeschnittenen Oszillatoren ist die Dämpfung im gesamten untersuchten Temperaturintervall deutlich größer als bei den geätzten. Diesen Umstand deutet die gepunktete Trennlinie qualitativ an. Die gezeigten Messungen an geätzten Paddeln liegen ausnahmslos unterhalb dieser Trennlinie, während sie für lasergeschnittene Oszillatoren darüber liegen. Eine mögliche Erklärung für die größere Dämpfung bei lasergeschnittenen Oszillatoren ist, dass beim Laserschneiden die Ränder des Paddels lokal aufgeschmolzen werden, wodurch die einkristalline Struktur zerstört wird. In diesen Bereichen kommt es bei Auslenkungen während der Schwingung zu inelastischer Verformung, wodurch Energie dissipiert wird und die Dämpfung ansteigt.

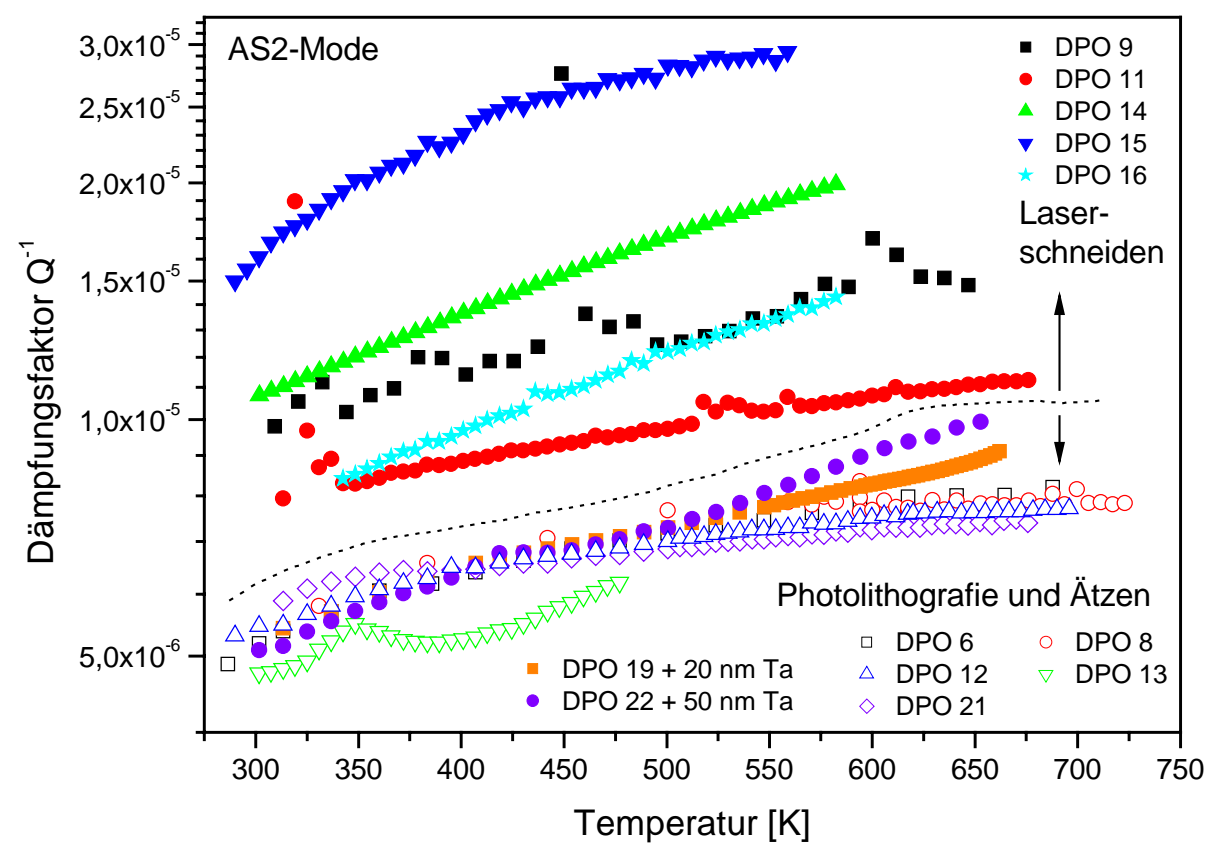

Abbildung 4.6: Dämpfungsfaktor der AS2-Mode in Abhängigkeit von der Temperatur für alle im Rahmen dieser Arbeit untersuchten Oszillatoren. Die für DPO 14 gezeigten Daten sind mathematisch rekonstruiert. DPO 19 und DPO 22 tragen bereits eine $20 \mathrm{bzw} .50 \mathrm{~nm}$ Ta-Schicht, alle anderen Oszillatoren sind unbedampft.

Insbesondere im Temperaturintervall zwischen $450 \mathrm{~K}$ und $600 \mathrm{~K}$ gibt es bei lasergeschnittenen Paddeln Abweichungen von der vermutlich glatten Kurve, die nicht reproduzierbar sind. Die Dämpfung springt bei einigen Messpunkten nach oben, um kurz darauf wieder im erwarteten Bereich zu liegen. Diese Ausreißer wurden nur bei lasergeschnittenen Oszillatoren beobachtet, weshalb prinzipielle Fehler der Messmethode oder der Elektronik ausgeschlossen werden. Die Ursachen für dieses Phänomen sind bislang nicht geklärt. 


\subsection{Konstruktion und Aufbau des UHV-Einsatzes}

\subsubsection{Befestigung des Doppel-Paddel Oszillators}

Voraussetzung für eine geringe Dämpfung ist, dass möglichst wenig elastische Energie während der Schwingung dissipiert wird. Da der Oszillator im Vakuum betrieben wird, kann Energie nur über seine Einspannung an die Umgebung abgegeben werden. Die Befestigung des Doppel-Paddel Oszillators am Fuß und seine Verbindung zur gesamten Apparatur hat daher eine große Bedeutung.

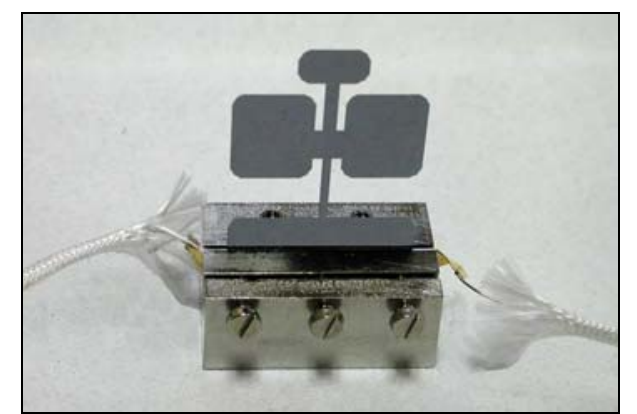

Abbildung 4.7: Der Doppel-Paddel Oszillator wird in einen Mini-Schraubstock eingespannt. Der zusätzlich mit eingeklemmtem Goldstreifen hat die Funktion eines Thermoelementes).

Bei der hier konstruierten Anlage wird die untere Hälfte des Paddelfußes fest zwischen zwei Backen eines kleinen Schraubstocks aus Edelstahl eingespannt. Beim Anziehen der drei Schrauben ist darauf zu achten, dass die Einspannung möglichst gleichmäßig erfolgt und der Fuß nicht verkantet. Beim Heizen könnte der Oszillator sonst Schaden nehmen oder sogar zerstört werden. Das Paddel bleibt nun für alle weiteren Untersuchungen in diesem Sockel eingespannt, was nicht nur den Transport des äußerst fragilen Oszillators erleichtert, sondern auch garantiert, dass der Energietransfer durch die Einspannung bei allen Messungen reproduzierbar in gleichem Umfang stattfindet.

\subsubsection{Leitfähigkeit der Oszillator-Rückseite}

Die Schwingung des Doppel-Paddel Oszillators wird kapazitiv durch elektrostatische Kräfte angeregt und ausgelesen. Dazu bilden die Rückseiten der beiden Flügel zusammen mit je einer in geringem Abstand darüber positionierten Elektrode zwei Kondensatoren. Wegen der 
geringen Leitfähigkeit des Siliziums ist die elektrostatische Kraft zwischen den Flügeln und den Elektroden zu klein. Deshalb wird die Rückseite des Paddels mit einer leitfähigen Schicht bedampft. In einer Edwards 306-Turbo Aufdampfanlage mit zwei thermischen Verdampfern wird zunächst eine rund $10 \mathrm{~nm}$ dicke Chrom-Zwischenschicht, und darauf einer $90 \mathrm{~nm}$ dicke Gold-Schicht deponiert. Beim Bedampfen wird der Mini-Schraubstock um einem Winkel von $45^{\circ}$ zur Horizontalen verkippt in die Aufdampfanlage eingesetzt. Dadurch wird insbesondere die Kante metallisiert, an der sich Oszillator-Fuß und Mini-Schraubstock berühren, was eine durchgängige elektrische Leitfähigkeit von den Flügeln bis zum Mini-Schraubstock, damit zur gesamten Anlage und dem elektrischen Null-Potential der Erde garantiert.

\subsubsection{Konstruktion des Einsatzes}

Der Mini-Schraubstock mit dem festgeklemmten Doppel-Paddel Oszillator mit leitfähiger Rückseite wird nun auf den Elektrodenhalter aufgeschraubt (Abbildung 4.8) und mit diesem in den im Rahmen dieser Arbeit konstruierten UHV-Einsatz eingebaut.

Die beiden Elektroden werden vorsichtig der Rückseite der beiden Oszillatorflügel angenähert und nach Erreichen ihrer endgültigen Position mit den seitlichen Schrauben fixiert. Der Abstand zwischen Elektrodenfläche und Flügel darf dabei höchstens 0,5 mm betragen. Wird diese Distanz überschritten, so wird das elektrische Übersprechen von Anregungs- und Detektionssignal der beiden Elektroden sehr groß. Zudem wird die elektrostatische Kraft so klein, dass große Anregungsspannungen nötig sind, was wieder das Übersprechen verstärkt. In der Praxis werden die Elektroden vorsichtig so nahe an die Flügel angenähert, solange man mit dem bloßen Auge gerade noch durch den entstehenden Schlitz hindurch sehen kann. Die Elektroden sind horizontal so positioniert, dass sich ihre radiale Symmetrieachse gerade über der oberen Hälfte der Flügel befindet, wie in Abbildung 4.8 angedeutet ist.

Soll eine Schwingungsmode angeregt oder ausgemessen werden, so setzt die kapazitive Methode voraus, dass sich der Abstand zwischen Flügel und Elektrode ändert, sich die Elektrode also an einem „Schwingungsbauch“ befindet. In einem früheren Versuch wurden die Elektroden genau über der Flügelmitte positioniert, wodurch nur die Biege- nicht jedoch die interessanteren Torsionsschwingungen angeregt werden konnten. 

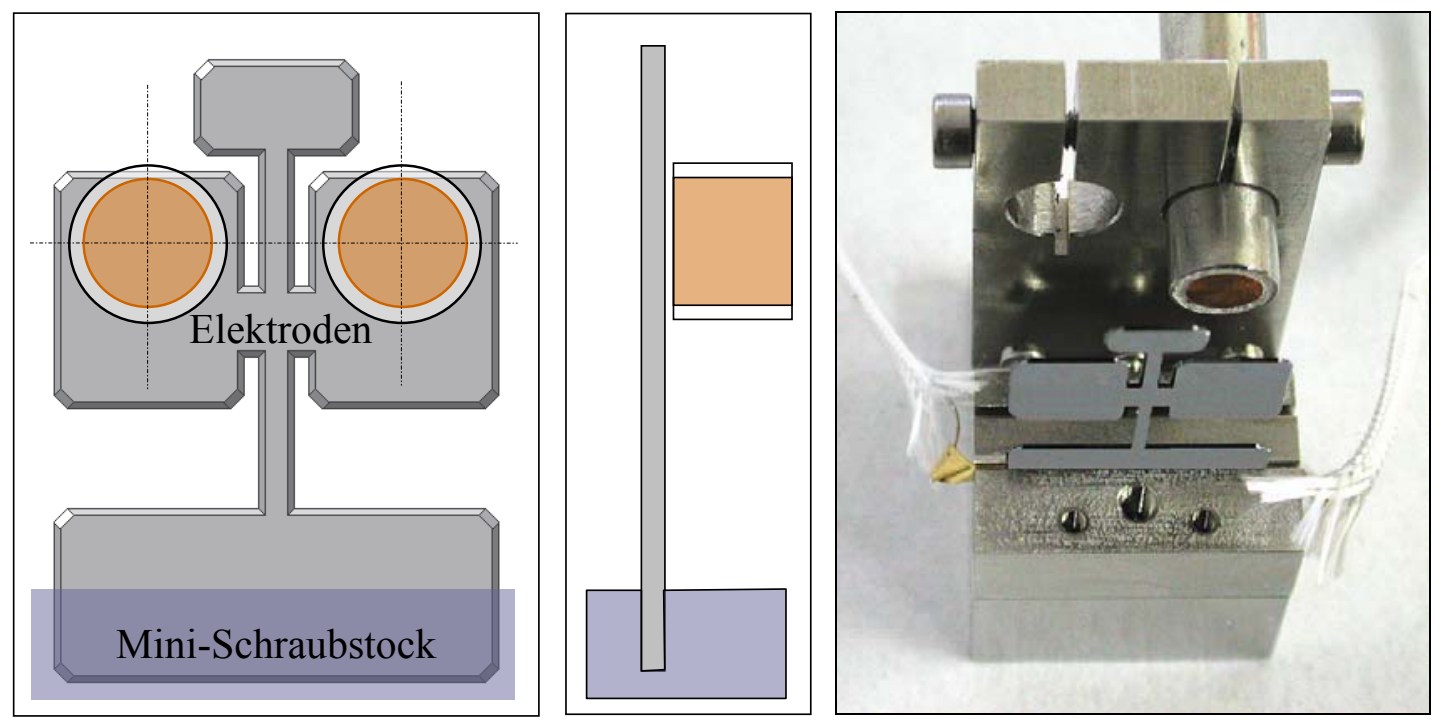

Abbildung 4.8: Mini-Schraubstock mit Oszillator und Elektrodenhalter. Die Elektrode im rechten Bild ist noch nicht in ihrer endgültigen Position.

Der Oszillator wird durch abgegebene Wärmestrahlung zweier Projektorbirnen der Firma Os$\operatorname{ram}(220$ V / 900 W) geheizt. Der Glaskörper der Birnen wird vorsichtig von den Glühwendeln abgelöst. Die Wendel sind in der Anlage parallel geschaltet und werden mittels einer Stromdurchführung extern angesteuert. Abbildung 4.9 zeigt die symmetrische Anordnung auf beiden Seiten des Oszillators. Abschirmbleche, die an der Unterseite dafür sorgen, dass die Heizstrahler nicht bedampft werden und an den übrigen Seiten des Paddels als Wärmeschild dienen, komplettieren den Einsatz (Abbildung 4.10).
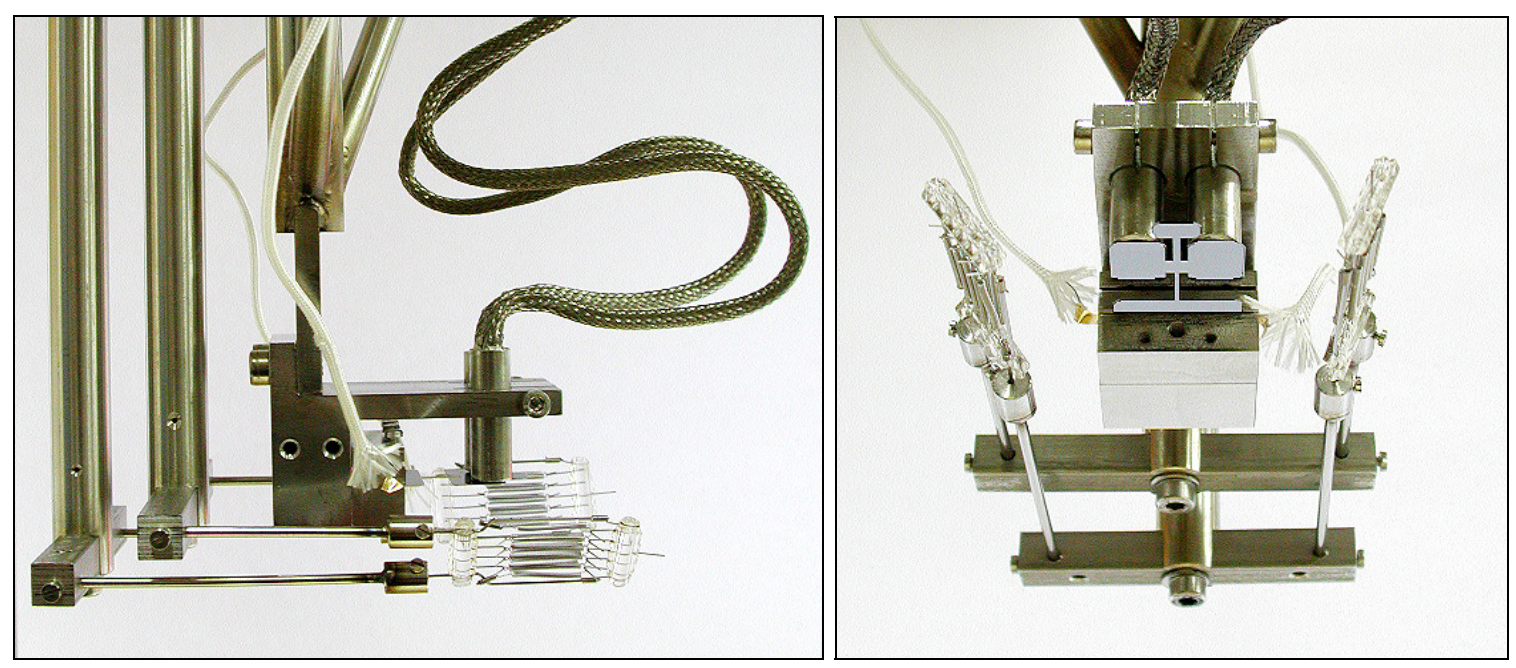

Abbildung 4.9: Elektrodenhalter eingebaut im UHV-Einsatz mit angenäherten Elektroden. 

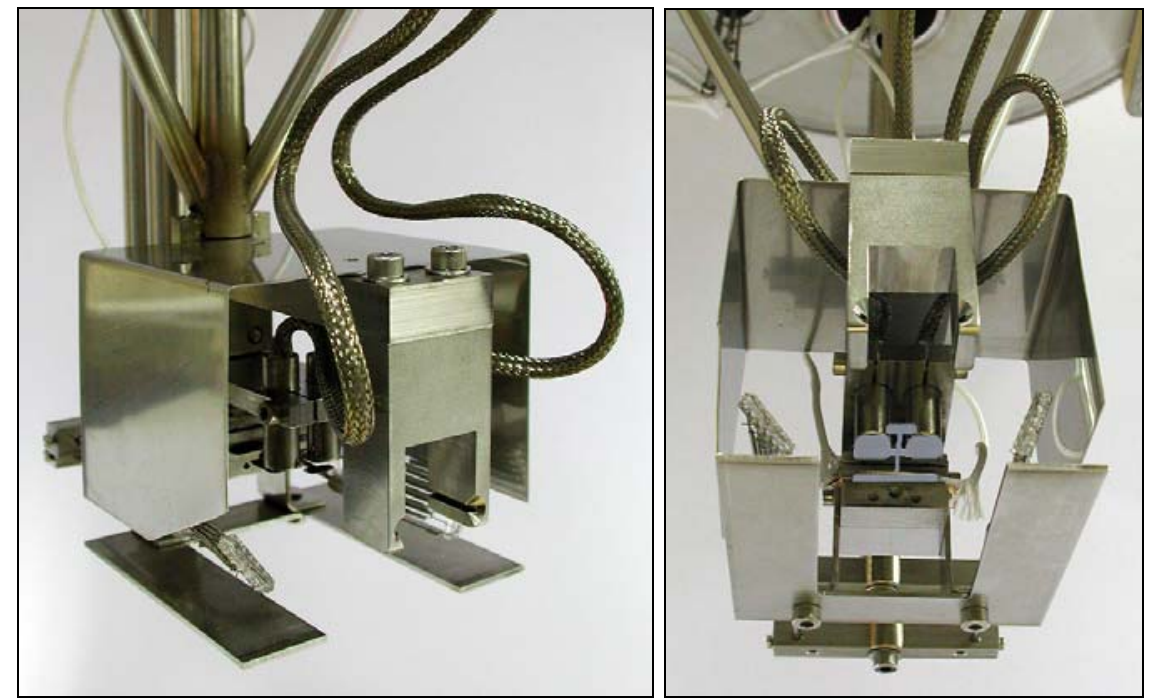

Abbildung 4.10: Kompletter UHV-Einsatz mit eingebautem Paddel und Abschirmblechen.

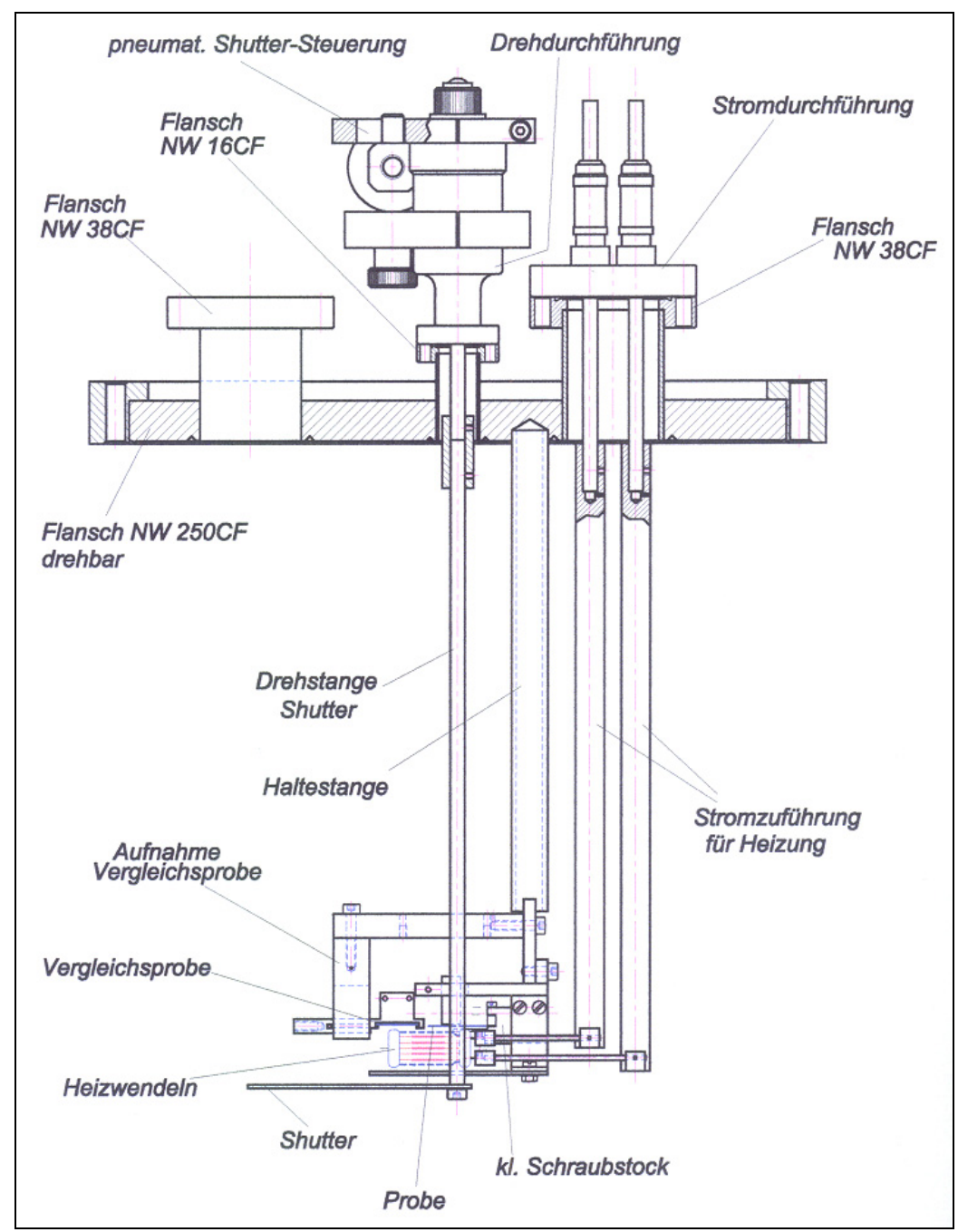

Abbildung 4.11: Schematische Skizze des UHV-Einsatzes mit seinen wichtigsten Komponenten. 
Mit dem hier beschriebenen Aufbau ist es unmöglich, die in der UHV-Kammer auf das Paddel aufgebrachte Schicht in situ durch weitere Methoden zu untersuchen oder zu charakterisieren. Deshalb bietet eine Haltevorrichtung die Möglichkeit, ein Vergleichssubstrat in direkte Nähe zum Paddelkopf zu bringen. Dieses Vergleichssubstrat kann mit bedampft werden und zu externen Untersuchungen aus der UHV-Kammer ausgeschleust werden, ohne das Vakuum zu brechen oder das Paddel ausbauen zu müssen.

Der gesamte Messkopf ist über ein vertikales Gestänge mit einem NW 250 CF Flansch verschweißt. An diesem befindet sich zusätzlich ein Sichtfenster, das zur Bedienung nötig ist, wenn Vergleichssubstrate ein- oder ausgeschleust werden, die Drehdurchführung für den Shutter, die elektrischen Durchführungen für zwei Thermoelemente und die Heizung, sowie die abgeschirmten Durchführungen für die Anregungs- und Signalelektrode. Der gesamte, so konstruierte UHV-Einsatz ist in Abbildung 4.11 gezeichnet.

\subsubsection{Herstellung der Elektroden}

Da die zu detektierenden elektrischen Signale sehr klein sind, kommt der Qualität der Elektroden, insbesondere ihrer elektrischen Abschirmung bis unmittelbar zu den DPO-Flügeln eine entscheidende Bedeutung zu.

Die Kernelektrode aus massivem Edelstahl hat einen Durchmesser von 5,5 mm und ist mit einer Isolationskeramik in einem äußeren Mantel fixiert. Dieser Mantel ist ein etwa $25 \mathrm{~mm}$ langes Edelstahlrohr mit einem Außendurchmesser von $8 \mathrm{~mm}$ und 0,2 mm Wandstärke. Zur Herstellung der Keramikmasse wird Durapot 809 Pulver im Verhältnis 100:13 mit demineralisiertem Wasser vermischt um eine gipsähnliche Konsistenz zu erreichen. Die Kernelektrode wird im Mantel zentriert und der verbleibende Hohlraum mit der Keramik gefüllt. Dabei sind Lufteinschlüsse zu vermeiden. Nach etwa zwei Tagen ist die Keramik bei Raumtemperatur eingetrocknet und die Vorderseite der Elektrode wird sorgfältig abgedreht. Überstehende Keramikkörner werden so entfernt. Um den Abstand zwischen der entstehenden Elektrodenebene und dem Oszillator später zu minimieren, ist darauf zu achten, dass die Ebene exakt plan wird und genau senkrecht zur Rotationsachse der Elektrode liegt. Die fertige Elektrode wird schließlich 24 Stunden lang im Vakuum bei einem Druck kleiner als $10^{-5}$ mbar bei $800{ }^{\circ} \mathrm{C}$ ausgebacken. 
Für die leitende Verbindung zwischen Elektrode und UHV-Durchführung sorgt ein Nickeldraht, der an der oszillatorabgewandten Seite der Elektrode festgeklemmt wird. Zur Isolation der Drähte werden CB 045 Alsinth Perlen von der Firma Caburn MDC verwendet. Der umperlte Draht wird mit einem Caburn-Abschirmmantel überzogen und an der UHV-Durchführung ebenfalls festgeklemmt.

\subsubsection{Temperaturmessung, -eichung und -regelung}

Die Temperaturregelung hat sich als der limitierende Faktor für die Empfindlichkeit der gesamten Apparatur erwiesen. Die Schwierigkeit besteht vor allem darin, dass die Temperaturen von Paddelkopf und -hals die Resonanzfrequenz zwar wesentlich beeinflussen, an diesen Stellen aber nicht direkt durch thermischen Kontakt gemessen werden können. Daher stellt sich zunächst das Problem der Temperaturmessung.

Bei den ersten Experimenten wurde beim Einspannen des Fußes in den Mini-Schraubstock ein dünner Gold-Streifen mit eingespannt. Durch seine gute Wärmeleitfähigkeit sollte realisiert werden, dass die an den beiden Streifenenden festgeschweißten Ni- bzw. CrNi-Thermodrähte die gleiche Temperatur haben, wie der Paddelfuß. Die ganze Anordnung arbeitet daher als Thermoelement. Allerdings hat sich gezeigt, dass Silizium bei hohen Temperaturen mit Gold legiert, weshalb sich der Streifen vom Paddelfuß nicht mehr ablösen lässt. Für jedes Paddel würde ein neues Thermoelement benötigt, dessen individuelle Eichung nicht möglich ist. Außerdem verringert sich durch das Legieren beider Elemente die Gesamtdicke in der Einspannung, weshalb der Oszillator nach einigen Temperaturzyklen nur noch locker im Mini-Schraubstock sitzt.

Die nächste Idee war, die Temperatur des Paddelkopfes indirekt durch thermische Strahlung zu detektieren. Versuche mit einem entsprechenden Pyrometer scheiterten, da der dünne Silizium-Oszillator für Infrarotstrahlung größtenteils durchlässig ist. Trotz Fokus auf den Paddelkopf wird nicht nur dessen Temperatur gemessen, sondern auch die aller Komponenten in räumlicher Anordnung hinter dem Kopf.

Die in dieser Arbeit veröffentlichten Temperaturen sind mit einem Ni/CrNi-Thermoelement gemessen, das mit einem Blech und zwei Schrauben an der Querseite des Mini-Schraubstockes befestigt ist. Daraus ergeben sich zwar gravierende, aber lösbare Probleme für die Temperaturmessung und -regelung: 
- Durch der Anordnung der Heizstrahler wird der gesamte Einsatz über das Paddel mitgeheizt. Der Mini-Schraubstock und alle anderen Komponenten der Anlage werden durch den thermischen Kontakt zwischen Bein, Fuß und Schraubstock erwärmt. Wegen der geringen Wärmekapazität des Paddels und des geringen Wärmeflusses durch das Bein mit seiner kleinen Querschnittsfläche, bildet sich bei großen Heizraten sehr schnell einen großer Temperaturgradient vom Paddelkopf bis zum Fuß und dem Thermoelement aus. Insbesondere wenn das Paddel mit dem Phase-Lock-Loop in Resonanz betrieben wird (Abschnitt 4.4.3), führen schnelle Temperaturänderungen dazu, dass sich die Frequenz so schnell verschiebt, dass die Schwingung zusammenbricht. Darüber hinaus sind starke Temperaturüberhöhungen für eine amorphe Metallschicht im Bereich der unterkühlten Schmelze wegen Kristallisationsgefahr unbedingt zu vermeiden. Die Heizrate darf deshalb nicht zu groß gewählt werden.

- Wegen der Temperaturüberhöhung dauert es eine gewisse Zeit, bis sich nach Einstellen einer neuen Temperatur thermisches Gleichgewicht zwischen Kopf und Fuß des Paddels eingestellt hat. Bis dahin wird die Temperatur am Kopf, wegen des Wärmeflusses durch das Bein, kontinuierlich abnehmen, was die Resonanzfrequenz verändert. Eine Messung von Frequenz und Dämpfung ist daher erst möglich, wenn sich thermisches Gleichgewicht eingestellt hat, was entsprechendes Warten nötig macht.

- Die reale Temperatur von Paddelkopf und -hals ist auch im thermischen Gleichgewicht größer als die am Schraubstock gemessene. Diese Differenz ist reproduzierbar, wenn keine baulichen Veränderungen vorgenommen werden. Mit einer Eichung, bei der die Temperatur am Schraubstock mit der des Paddelkopfes verglichen wird, lässt sich die Differenz bei den eigentlichen Experimenten korrigieren.

- Ist eine Temperatur eingestellt und thermisches Gleichgewicht erreicht, so erfordert die ausgleichende Regelung relativ große Schwankungen der Heizleistung und damit der Kopf- und Halstemperatur, um kleine Abweichungen der gemessenen Schraubstock-Temperatur vom Soll-Wert auszugleichen. Je höher die Absoluttemperatur ist, desto größer werden diese Schwankungen. Da die Resonanzfrequenz empfindlich von der Temperatur abhängt, übertragen sich die Schwankungen und die Frequenz kann nur innerhalb eines Bandes gemessen werden. 
Für die Temperatureichung wurde ein Thermoelement mit einem Tropfen Leitsilber am Kopf eines eingebauten Oszillators befestigt. Die an dieser Position gemessene Temperatur kann nun mit der des am Schraubstock festgeklemmten verglichen werden.

In Abbildung 4.12 ist die am Kopf gemessene Temperatur für die Heizraten $1{ }^{\circ} \mathrm{C} / \mathrm{min}$ (rote Kurve) und $2{ }^{\circ} \mathrm{C} / \mathrm{min}$ (schwarze Kurve) gezeigt, wobei die Temperatur am Mini-Schraubstock von $250^{\circ} \mathrm{C}$ auf $260{ }^{\circ} \mathrm{C}$ erhöht wurde. Die Rate wird dabei durch Temperaturerhöhungen um $1{ }^{\circ} \mathrm{C}$ im Abstand von $1 \mathrm{~min}$ und 0,5 min realisiert. Bei einer Rate von $1{ }^{\circ} \mathrm{C} / \mathrm{min}$ zeigt sich eine Temperaturüberhöhung von $18{ }^{\circ} \mathrm{C}$, bei $2{ }^{\circ} \mathrm{C} /$ min sogar um $30^{\circ} \mathrm{C}$. Allerdings ist die Gleichgewichtstemperatur, die sich am Kopf nach einer gewissen Zeit einstellt, offensichtlich unabhängig von der Heizrate. In für die Kristallisation kritischen Temperaturbereichen muss die Heizrate entsprechend klein gewählt werden.

Die Absolutwerte in Abbildung 4.12 zeigen, dass die Temperatur am Kopf nach Erreichen des thermischen Gleichgewichtes bei etwa $283{ }^{\circ} \mathrm{C}$ liegt, wenn am Sockel $250{ }^{\circ} \mathrm{C}$ geregelt wird. Wird die Soll-Temperatur am Schraubstock bei $260{ }^{\circ} \mathrm{C}$ eingestellt, beträgt die Kopftemperatur etwa $294{ }^{\circ} \mathrm{C}$.

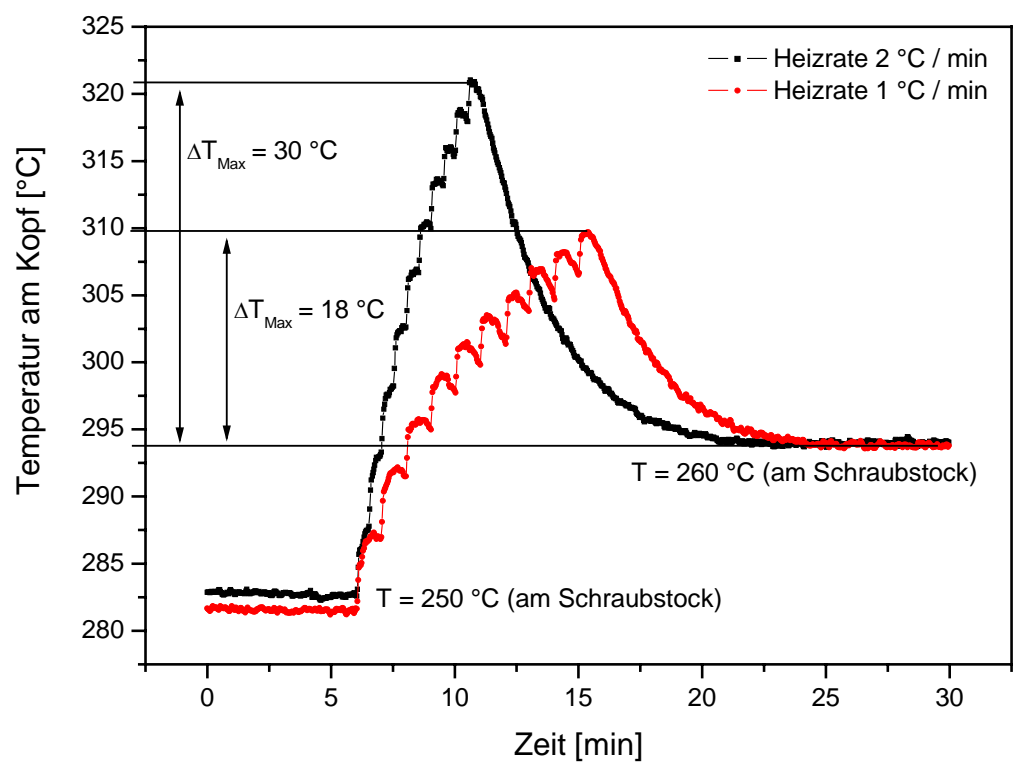

Abbildung 4.12: Heizratenabhängige Temperaturüberhöhung am Paddelkopf.

Nun wird die Temperatur am Sockel sukzessive erhöht und nach Erreichen des thermischen Gleichgewichtes mit der am Kopf gemessenen verglichen. Abbildung 4.13 zeigt diesen Vergleich. 
Der ermittelte lineare Zusammenhang gemessen in $\left[{ }^{\circ} \mathrm{C}\right]$ drückt sich in der folgenden Gradengleichung aus:

$$
T_{\text {Kopf }}=1,17 \times T_{\text {Schraubstock }}-6,59^{\circ} \mathrm{C}
$$

Temperaturangaben in dieser Arbeit beziehen sich im Folgenden stets auf die mit dieser Eichung berechnete Temperatur am Paddelkopf.

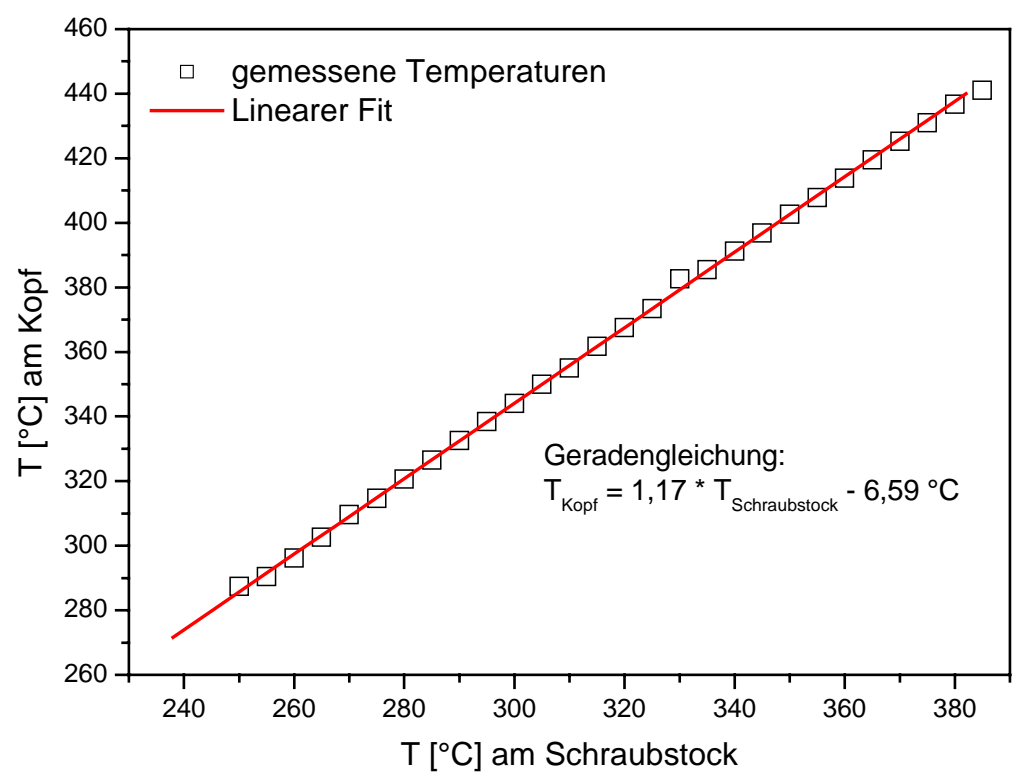

Abbildung 4.13: Vergleich der Temperaturen gemessen am Schraubstock (Abszisse) und am Paddelkopf (Ordinate). Der lineare Zusammenhang ergibt die Eichkurve für künftige Temperaturmessungen.

Die Resonanzfrequenz des Doppel-Paddel Oszillators hängt empfindlich von seiner Temperatur ab. Die Absoluttemperatur des Paddelkopfes ist im Vergleich zu der gemessenen am MiniSchraubstock durch die Eichmessung bekannt. Abbildung 4.14 zeigt den Zusammenhang zwischen der Temperatur des Paddelkopfes und der AS2-Resonanzfrequenz exemplarisch für einen Oszillator (DPO 11). Für diese Messung wird der Oszillator in Resonanz betrieben, mit dem Regler eine Temperatur am Schraubstock fest eingestellt und diese mit der Eichung in die Temperatur des Kopfes umgerechnet. Ist thermisches Gleichgewicht erreicht, dann bleibt die Resonanzfrequenz konstant und wird gemessen.

Offensichtlich hängt die Resonanzfrequenz reproduzierbar von der Temperatur ab und ist unabhängig von der Prozessführung. Die beim Heizen ermittelten Werte (rote Kurve) und die beim Kühlen gemessenen Frequenzen (blaue Kurve) stimmen überein. 
Für das Tuning des Temperaturreglers und das Abschätzen der Zeiten, bis sich thermisches Gleichgewicht eingestellt hat, dient die Resonanzfrequenz des Oszillators daher als Sonde für die tatsächliche Temperatur am Paddelkopf.

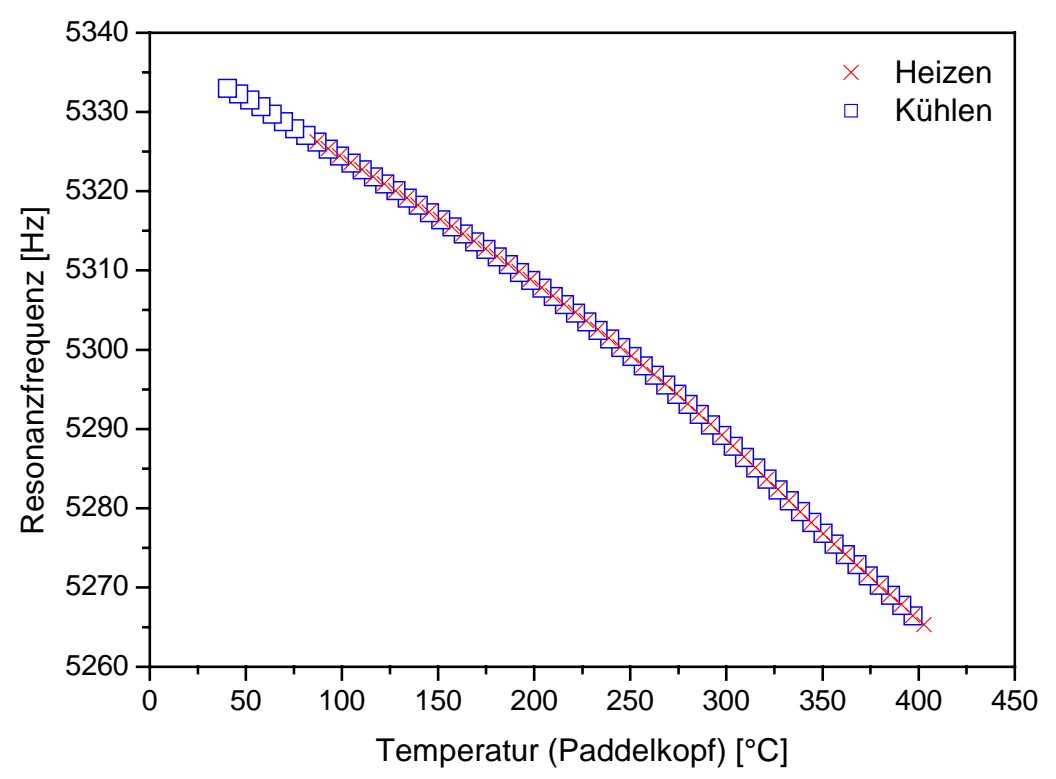

Abbildung 4.14: Abhängigkeit der AS2-Resonanzfrequenz von der Temperatur bei DPO 11. Die Frequenz ist reproduzierbar und nicht von der Prozessführung abhängig. Die Werte beim Heizen und beim anschließenden Kühlen sind gleich.

Der Temperaturregler ist ein Proportional-Integral-Differential-Regler (PID). Die Wahl der Regelparameter hat entscheidenden Einfluss auf kritisches Verhalten wie Überschwingen, Trägheit der Regelung oder Dauer, bis die Temperatur den Ist-Wert erreicht hat. In umfangreichen vergleichenden Messungen wurden die Parameter manuell optimiert und folgende Einstellungen gewählt:

- Proportionalband pb1 = 15. Je größer pb1 gewählt wird, desto langsamer reagiert der Regler auf Abweichungen vom Sollwert.

- Integrationszeit int $=800 \mathrm{~s}$. Je größer die Integrationszeit gewählt wird, desto träger ist der Regler und desto kleiner werden Überschwinger. Allerdings steigt damit auch die Zeit bis thermisches Gleichgewicht erreicht ist.

- Differentialteil $\mathrm{dEr}=3$. Je größer $\mathrm{dEr}$ gewählt wird, desto kleiner werden die Überschwinger, allerdings ergeben sich dann wieder große Schwankungen im output-Level für die Heizleistung. 
Mit diesen Einstellungen wurden nun verschiedene Temperaturen eingestellt und dabei die zeitliche Veränderung der Resonanzfrequenz verfolgt. In Abbildung 4.15 sind die Ergebnisse für vier verschiedene Temperaturen gezeigt. Sie wurden jeweils mit einer kontinuierlichen Heizrate von $0,5{ }^{\circ} \mathrm{C} / \mathrm{min}$ eingestellt, weshalb die Resonanzfrequenz in dieser Phase relativ linear abfällt. Ist der Soll-Wert der Temperatur erreicht, so zeigt sich, dass die Resonanzfrequenz zunächst wieder ansteigt. Die Überhitzung des Paddelkopfes wird abgebaut. Hat sich thermisches Gleichgewicht eingestellt, nimmt die Resonanzfrequenz einen konstanten Wert an.

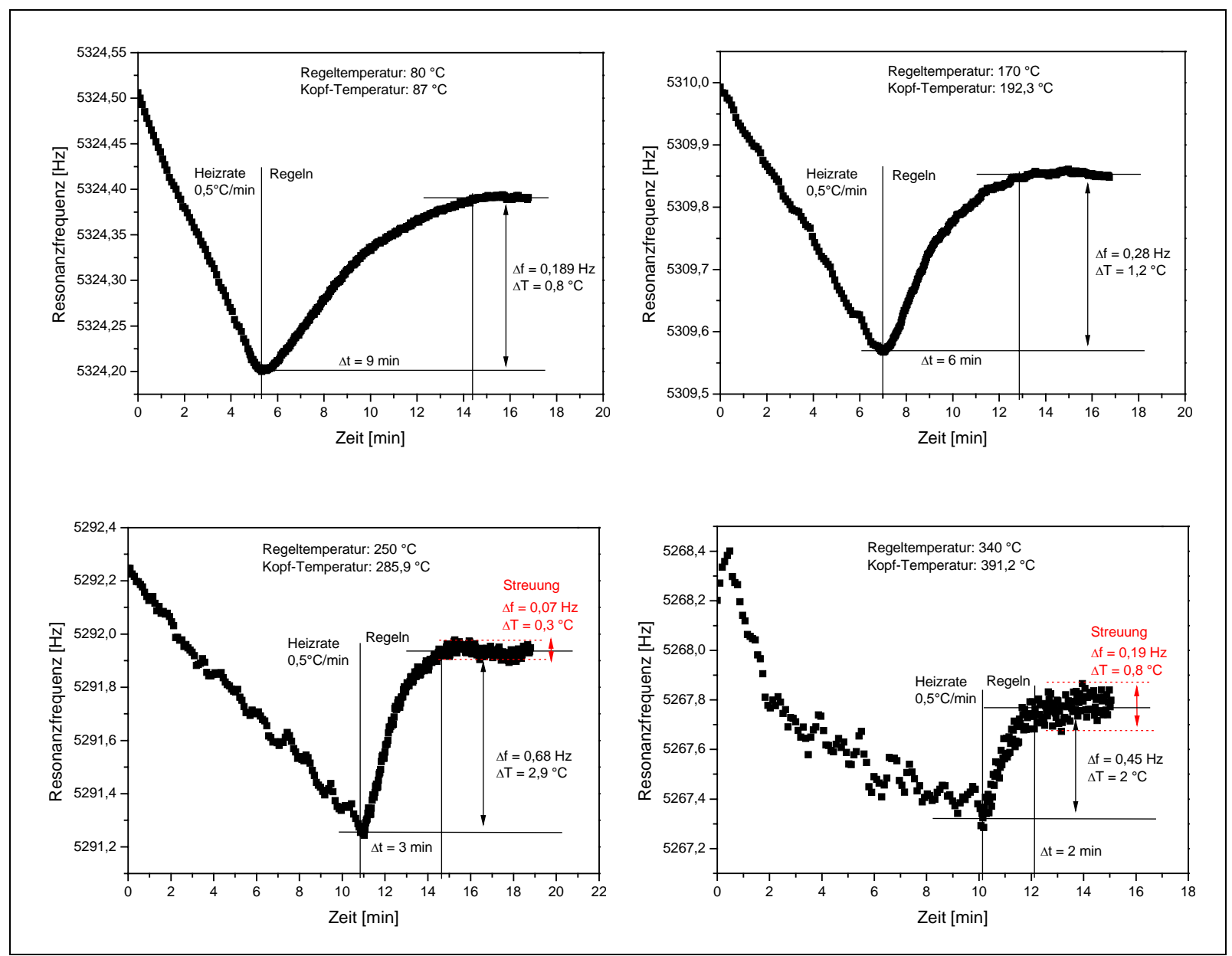

Abbildung 4.15: Wenn sich die Resonanzfrequenz nicht mehr ändert, ist die Temperatur am Paddelkopf konstant und thermisches Gleichgewicht erreicht. Die Zei $\Delta t$ wird vom Erreichen der Soll-Temperatur am Regler bis zur Einstellung des Gleichgewichtes gerechnet. Der Unterschied zwischen der kleinsten gemessenen Frequenz im Moment des Erreichens der Soll-Temperatur und der Gleichgewichts-Resonanzfrequenz bezeichnet das Überschießen der Frequenz $\Delta f$, was einer Temperaturüberhöhung $\Delta T$ am Kopf entspricht. Die Streuung der Resonanzfrequenz im thermischen Gleichgewicht ist bei $250^{\circ} \mathrm{C}$ und bei $340{ }^{\circ} \mathrm{C}$ rot eingetragen. 
Mit zunehmender Temperatur verkürzt sich die Zeitspanne, bis thermisches Gleichgewicht erreicht ist. Dauert es bei einer Soll-Temperatur von $80{ }^{\circ} \mathrm{C}$ noch $9 \mathrm{~min}$, so hat sich diese Zeit bei $340{ }^{\circ} \mathrm{C}$ auf 2 min reduziert. Mit zunehmender Absoluttemperatur vergrößert sich die Streuung der Resonanzfrequenz beträchtlich. Bei $340{ }^{\circ} \mathrm{C}$ schwanken die gemessenen Werte in einem Intervall von 0,19 Hz. Aus Abbildung 4.15 ist ersichtlich, dass die Temperatur am Paddelkopf dann etwa um $0,8{ }^{\circ} \mathrm{C}$ schwankt. Die Streuung der Resonanzfrequenz wird daher bei hohen Temperaturen relativ groß.

Sollen später kleine Änderungen im Schermodul einer dünnen Schicht durch die Verschiebung der Resonanzfrequenz ermittelt werden, so ist die dargestellte Regelgenauigkeit der limitierende Faktor bei der Messung. Eine detaillierte Abschätzung der Empfindlichkeit findet sich im Anschluss an die mathematische Modellbildung in Abschnitt 4.7.

\subsection{Elektrischer Regelkreis, Steuerung und Datenerfassung}

\subsubsection{Elektrostatische Anregung und Bias-Spannung}

Die mit einer $\mathrm{Cr} / \mathrm{Au}$-Schicht bedampfte, leitfähige Rückseite des Doppel-Paddel Oszillators und die Kupferelektrode bilden einen Plattenkondensator. Wird eine Spannung U angelegt, so herrscht zwischen den beiden Platten das elektrische Feld

$$
E=\frac{U}{d}
$$

Dieses Feld stammt von der auf die Leiterplatten verschobenen Ladung

$$
\pm Q= \pm C U= \pm \frac{\varepsilon_{0} A}{d} U
$$

Dabei ist C die Kapazität des Plattenkondensators, A die Plattenfläche, d der Abstand zwischen den Kondensatorplatten und $\varepsilon_{0}$ die Vakuum-Dielektrizitätskonstante. Soll die Kraftwirkung zwischen beiden Platten berechnet werden, so ist zu berücksichtigen, dass das Feld durch die Ladungen im Metall geschwächt wird, je mehr es in die Platten eindringt. Dicht außerhalb der Platten hat es den Wert E, im Innern des Metalls den Wert null. Im Mittel wirkt auf die Ladungen nur das halbe Feld E/2. Eine solche Anordnung wird in der Literatur als „Kirchhoff-Waage“ bezeichnet [138]. 
Die Kraft F, mit der beide Platten sich anziehen ist dann

$$
F=\frac{1}{2} Q E=\frac{1}{2} \frac{\varepsilon_{0} A}{d^{2}} U^{2}
$$

Die beim Doppel-Paddel Oszillators wirksame Elektrodenfläche ist etwa $0,2 \mathrm{~cm}^{2}$. Der Abstand zwischen Elektrode und Paddel wird mit 0,2 mm angenommen. Die angelegte Wechselspannung

$$
U=U_{0} \cos \left(\omega_{R} t\right)
$$

hat die Amplitude $\mathrm{U}_{0}=0,2 \mathrm{~V}$. Die Frequenz der Spannung, $\omega_{\mathrm{R}}$, ist die Resonanzfrequenz der anzuregenden Eigenmode. Unter Anwendung eines Additionstheorems beim Quadrieren der Cosinus-Funktion ergibt sich für die elektrostatische Kraft zwischen den Platten:

$$
F=\frac{1}{4} \frac{\varepsilon_{0} A}{d^{2}} U_{0}^{2} \cdot\left(1+\cos \left(2 \omega_{R} t\right)\right)
$$

Die Kraft oszilliert also mit der doppelten Anregungsfrequenz. Soll das Paddel in Resonanz betrieben werden, müsste die Anregungsfrequenz daher gerade halb so groß gewählt werden, wie die Eigenfrequenz. Mit den gegebenen Zahlwerten berechnet sich die Amplitude der Kraft zu $9 \times 10^{-11} \mathrm{~N}$.

Um die Kraft zwischen den Kondensatorplatten zu erhöhen und damit die Anregung effektiver zu gestalten, wird der Wechselspannung auf beiden Elektroden eine Gleichspannung $U_{\text {bias }}$ von $200 \mathrm{~V}$ überlagert. Im Schaltbild in Abbildung 4.16 ist die technische Ausführung skizziert. Die Gleichspannung wird über die Widerstände $\mathrm{R}=10 \mathrm{k} \Omega$ eingekoppelt. Die BlockKondensatoren $\mathrm{C}_{1}=1 \mu \mathrm{F}$ sorgen für eine Trennung der Vorspannung von der übrigen Elektronik, während die Kondensatoren $\mathrm{C}_{2}=1 \mu \mathrm{F}$ als Sperre für externe Störungen wirken.

Die insgesamt anliegende äußere Spannung ist nun

$$
U_{\text {ges }}=U_{\text {bias }}+U_{0} \cos \left(\omega_{R} t\right)
$$

Wegen der Proportionalität der Kraft zum Quadrat der Spannung gilt jetzt:

$$
\begin{aligned}
F & =\frac{1}{2} \frac{\varepsilon_{0} A}{d^{2}}\left[U_{\text {bias }}+U_{0} \cos \left(\omega_{R} t\right)\right]^{2}= \\
& =\frac{1}{2} \frac{\varepsilon_{0} A}{d^{2}}\left[U_{\text {bias }}{ }^{2}+2 U_{\text {bias }} U_{0} \cos \left(\omega_{R} t\right)+\left(U_{0} \cos \left(2 \omega_{R} t\right)\right)^{2}\right]
\end{aligned}
$$




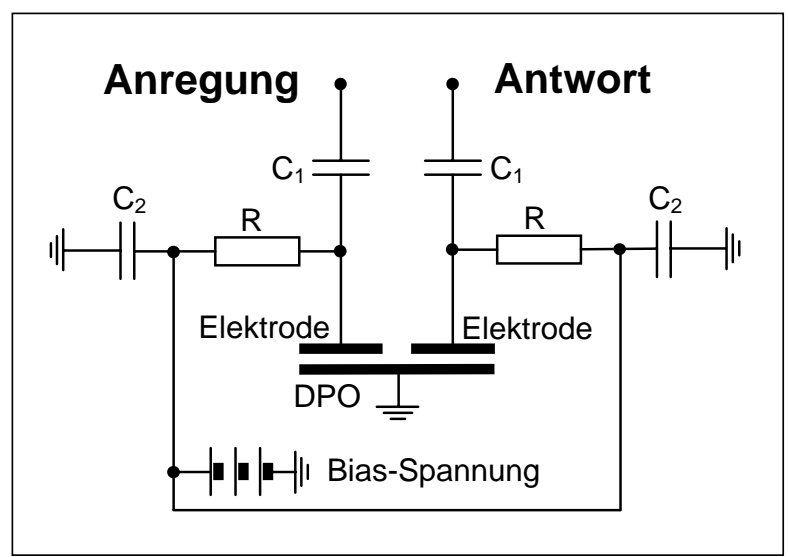

Abbildung 4.16: Schaltbild für die Realisierung einer Bias-Gleichspannung auf beiden Elektroden.

Der Gleichspannungs-Offset, proportional zu $\mathrm{U}_{\text {bias }}^{2}$, ist zeitunabhängig und spielt für die resonante Anregung keine Rolle. Da $U_{\text {bias }} U_{0} \gg U_{0}^{2}$ ist, wird $\left(U_{0} \cos \left(2 \omega_{R} t\right)\right)^{2}$ vernachlässigt und die treibende Kraft ist proportional zu $\mathrm{U}_{\text {bias }} \mathrm{U}_{0}$ :

$$
F=\frac{\varepsilon_{0} A}{d^{2}} U_{\text {bias }} U_{0} \cos \left(\omega_{R} t\right)
$$

Mit der angelegten Vorspannung ist die Amplitude der Kraft um den Faktor 2000 größer als ohne. Außerdem hat die oszillierende Kraft nun die gleiche Frequenz wie die anregende Wechselspannung. Die Amplitude der Kraft beträgt $1,8 \times 10^{-7} \mathrm{~N}$.

\subsubsection{Funktionsweise der elektronischen Komponenten}

Abbildung 4.17 zeigt das schematische Schaltbild der elektronischen Komponenten, die zum Betrieb des Doppel-Paddel Oszillators benötigt werden. Diese sind:

- Generator: Der HP 3325B Frequenzgenerator liefert eine sinusförmige Wechselspannung. Damit lässt sich der Oszillator extern mit beliebigen Frequenzen anregen.

- Zähler: Mit dem Fluke-Frequenzzähler PM 6669 wird die aktuelle Oszillator-Frequenz gemessen. Je nach Stellung der Schalter $S_{1}$ und $S_{2}$ ist dies die extern vorgegebene Frequenz oder die selbstangeregte Resonanzfrequenz.

- Vorverstärker: Auf der Antwort-Seite wird das Elektrodensignal mit einem DLPCA-200 Current-Amplifier der Firma Femto Messtechnik um den Faktor $10^{8}$ V/A verstärkt. 


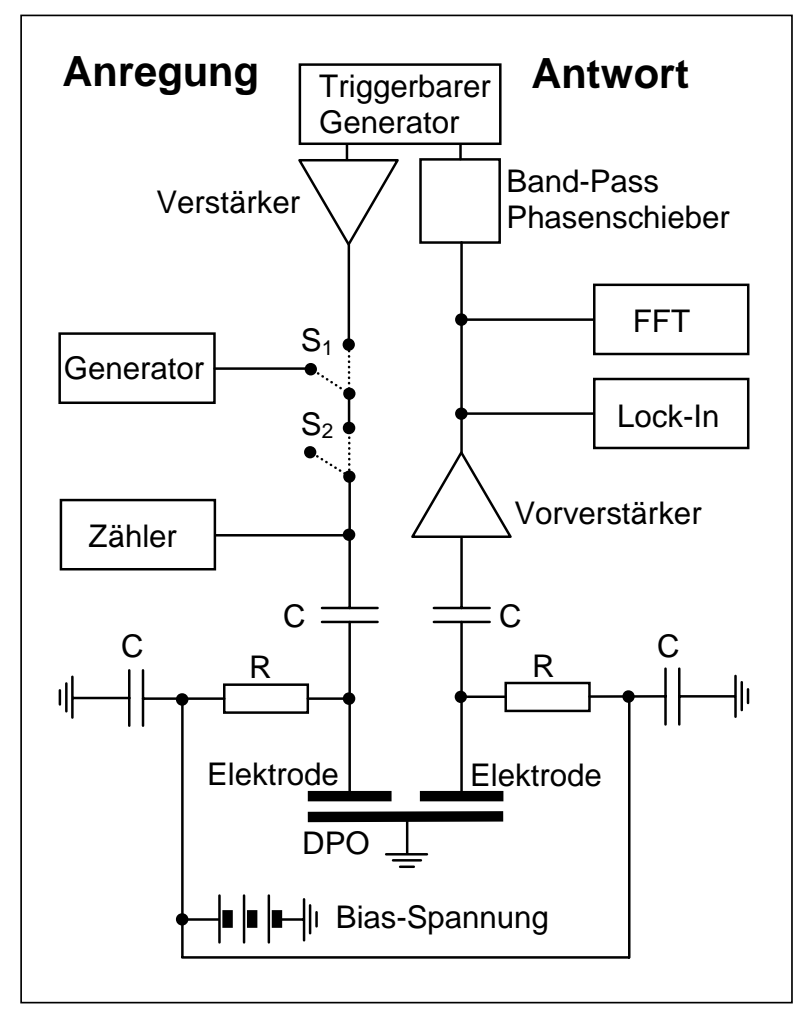

Abbildung 4.17: Elektronische Komponenten und ihre Schaltung zum Betrieb des DPOs.

- Lock-In: Die Amplitude des verstärkten Elektrodensignal und die Phasenlage zu dem als Referenz verwendeten Antriebssignal wird mit einem EG\&G 5210 Lock-In Amplifier gemessen.

- FFT: Um die Resonanzfrequenzen schnell identifizieren zu können steht ein Fast Fourier Transform Analyser „Phazer“ der Firma Gould Nicolet zur Verfügung.

- Band-Pass Phasenschieber, Triggerbarer Generator, Verstärker: Soll der Oszillator in Resonanz betrieben werden, so wird das detektierte Signal mithilfe eines PAR 116 Vorverstärkers noch einmal um den Faktor 100 verstärkt. Dieses Signal dient als Referenz für einen triggerbaren Generator, der in den PAR 124 Lock-In Verstärker integriert ist. Über einen ebenfalls in den PAR 124 Lock-In Verstärker integrierten Band-Pass-Filter wird ein um $90^{\circ}$ phasenverschobenes Signal erzeugt. Mit der Verstärker-Endstufe kann die Amplitude dieses Signals geregelt werden, mit dem dann der Oszillator wiederum angeregt wird. 


\subsubsection{Betriebsmodi}

Je nach gewünschter Anwendung kann der Doppel-Paddel Oszillator in drei verschiedenen Modi betrieben werden:

\subsubsection{FFT-Modus}

Sollen Resonanzfrequenzen schnell gefunden werden so ist der FFT-Analysator ein nützliches Instrument. Auch die Güte der Eigenmoden lässt sich automatisiert bestimmen. Die Schalter auf der Anregungsseite werden so eingestellt, dass das Signal des Frequenzgenerators auf der Anregungs-Elektrode liegt (Abbildung 4.18). Das Signal der Antwort-Elektrode wird auf den Eingang des FFT-Analysators gegeben.

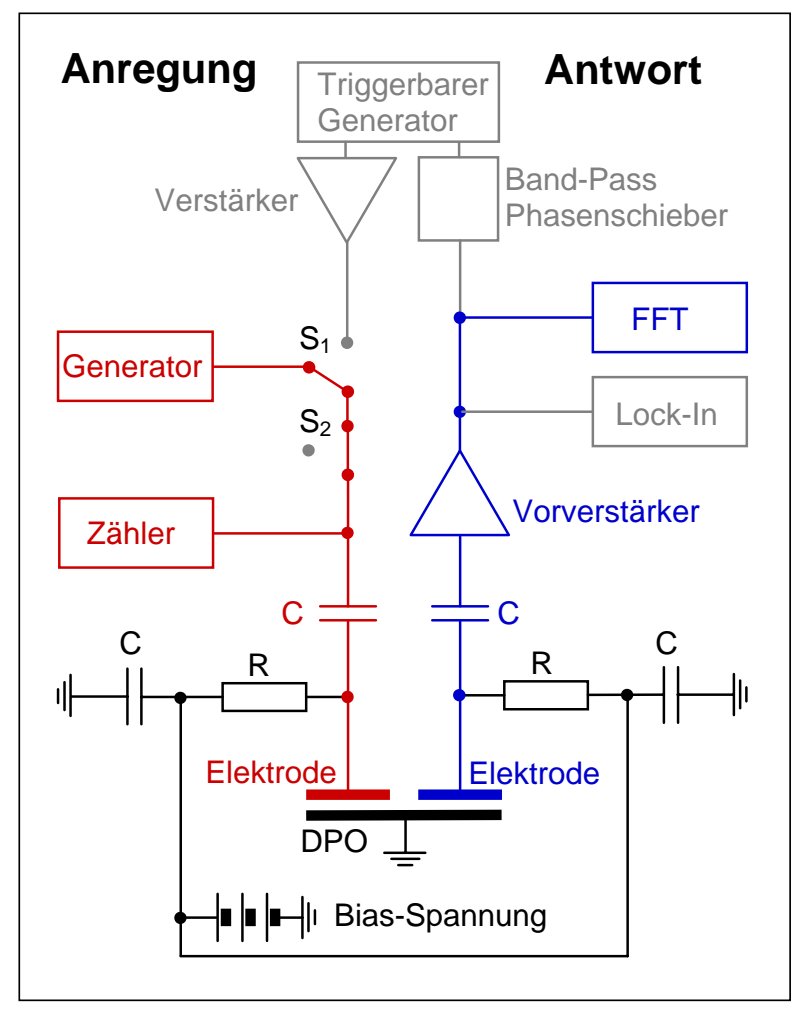

Abbildung 4.18: Schaltskizze für den FFT-Modus.

Mit der „Prism“-Software des FFT-Analysators kann ein Intervall um eine zentrale Frequenz festgelegt werden, in dem das Spektrum ausgewertet werden soll. Aus diesen Einstellungen, sowie aus der Anzahl der die Auflösung bestimmenden Linien, ergibt sich die benötigte Integrationszeit für die Messung. Zur Anregung wird die Sweep-Funktion des HP-Frequenzgene- 
rators verwendet. Nach Eingabe von Start- und Endfrequenz sowie der Sweep-Dauer werden kontinuierlich alle Frequenzen im angegebenen Intervall generiert. Zweckmäßigerweise wird die Sweep-Dauer gerade so groß gewählt wie die Integrationszeit des FFT-Analysators. Die Antwort des Paddels im Zeitraum wird mittels FFT direkt in den Frequenzraum übertragen.

\subsubsection{Frequenzscan}

In diesem Betriebsmodus wird die anregende Frequenz am HP-Generator manuell eingestellt und die Amplitude der Schwingung, sowie ihre Phasenlage zur Anregung mit dem EG\&G 5210 Lock-In Verstärker gemessen. Das Bode-Diagramm der einzelnen Eigenmoden kann durch schrittweise Veränderung der anregenden Frequenz mit nahezu beliebiger Genauigkeit ausgemessen werden.

\subsubsection{Phase Lock Loop (PLL) und Ring Down}
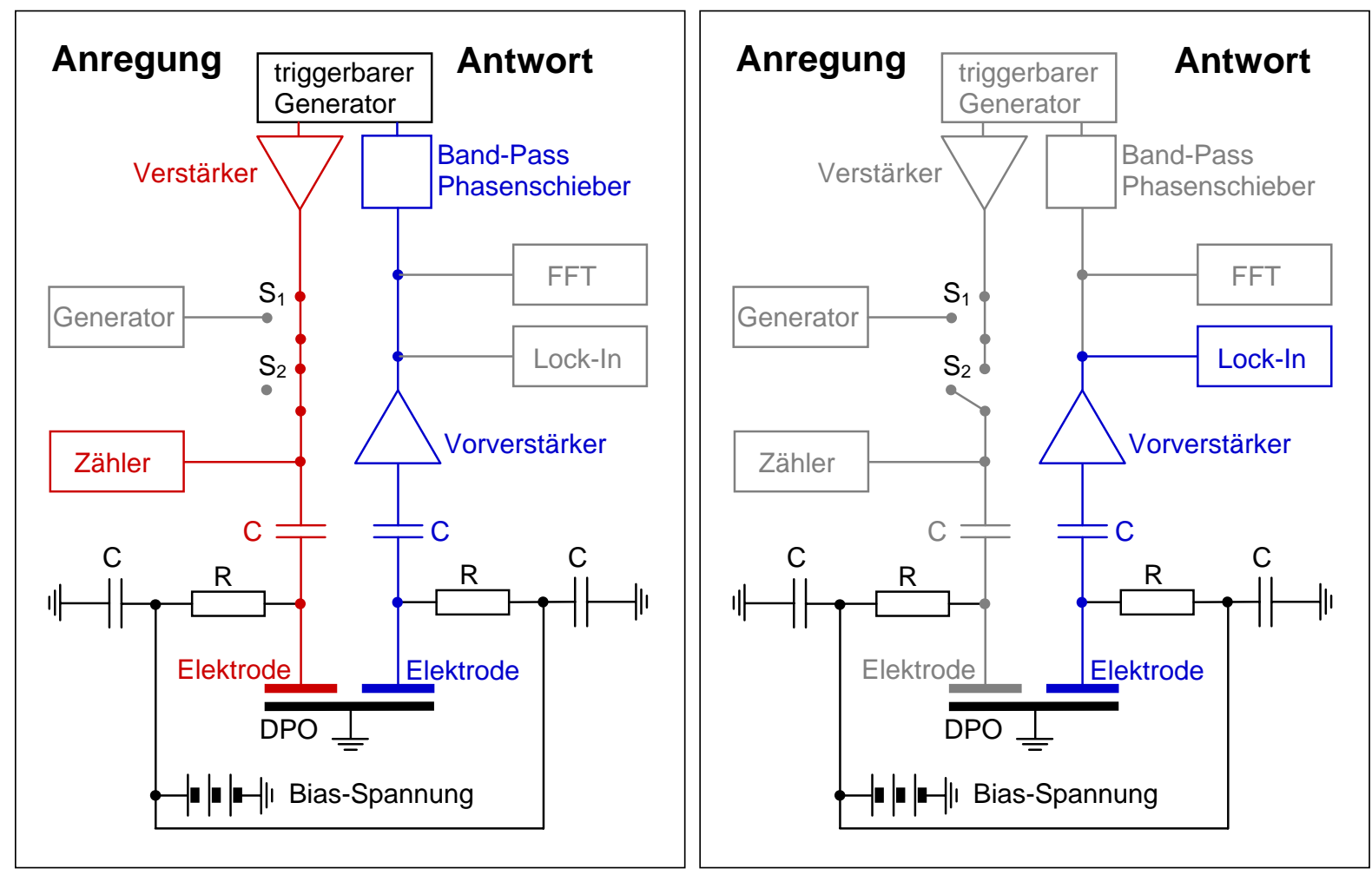

Abbildung 4.19: Schaltskizze für den Betrieb im PLL-Modus (links). Zum Start eines Ring-Down Experiments wird mit Schalter S2 die Anregung komplett abgeschaltet und die exponentiell abfallende Amplitude mit dem Lock-In Verstärker aufgezeichnet (rechts). 
Nach kurzzeitiger, externer Anregung durch den Frequenzgenerator wird mit dem elektrischen Relais $\mathrm{S}_{1}$ auf den Phase-Lock-Loop (PLL) Modus umgeschaltet. In diesem Fall (Abbildung 4.19 links) wird das Antwortsignal des Oszillators um 90 phasenverschoben, verstärkt, und so direkt als Anregung wieder auf den DPO gegeben. Dadurch wird das Paddel auf seiner Eigenmode ,eingelockt“ und schwingt mit konstanter Amplitude in Resonanz Wird nun die Anregung abgeschaltet, so lässt sich mit dem EG\&G-Lock-In-Verstärker die exponentiell abklingende Amplitude bestimmen (Abbildung 4.19 rechts). Aus dem logarithmischen Dekrement errechnen Dämpfungsfaktor und Güte der Schwingung. Diese Methode zur Bestimmung der Güte nimmt zwar deutlich mehr Zeit in Anspruch, als der FFT-Modus, allerdings ist die Genauigkeit und die Reproduzierbarkeit der Messung größer und die Streuung der Messdaten kleiner, wie in Abschnitt 4.6.4 gezeigt wird.

\subsubsection{LabView Software}

Zur Steuerung der elektronischen Komponenten und zur Datenerfassung wurde im Rahmen dieser Arbeit eine umfangreiche Software Bibliothek mit Hilfe von LabView 5.0 erstellt. Der komplette Quellcode, sowie eine ausführliche Dokumentation der einzelnen Programme sind im Anhang 1 wiedergegeben.

\subsection{Mathematisches Modell für den unbedampften Oszillator}

\subsubsection{DPO als System zweier gekoppelter Schwingungen}

In diesem Abschnitt wird ein mathematisches Modell für die Dynamik der beiden Torsionseigenmoden beschrieben. Nach einem Vorschlag von Van Cleve [139] besteht der Doppel-Paddel Oszillator aus zwei gekoppelten Drehschwingsystemen. Das eine System besteht aus dem um den Hals tordierenden Kopf, das andere aus den um das Bein tordierenden Flügeln. Für die Herleitung der Resonanzfrequenzen der gekoppelten Schwingung werden Energieverluste durch Dämpfung vernachlässigt.

Mit den Auslenkungen der Flügel $\phi_{\text {wing }}$ und des Kopfes $\phi_{\text {head }}$ aus der Ruhelage und den Winkelrichtgrößen $\kappa_{\text {leg }}$ des Beines und $\kappa_{\text {neck }}$ des Halses ergeben sich folgende rücktreibende Drehmomente auf Flügel und Kopf: 


$$
\begin{gathered}
M_{\text {wing }}=-\kappa_{\text {leg }} \varphi_{\text {wing }}-\kappa_{\text {neck }}\left(\varphi_{\text {wing }}-\varphi_{\text {head }}\right) \\
M_{\text {head }}=-\kappa_{\text {neck }}\left(\varphi_{\text {head }}-\varphi_{\text {wing }}\right)
\end{gathered}
$$

Mit Newtons Grundgesetz der Mechanik ergeben sich daraus die Bewegungsgleichungen:

$$
\begin{gathered}
I_{\text {wing }} \ddot{\varphi}_{\text {wing }}+k_{\text {leg }} \varphi_{\text {wing }}+k_{\text {neck }}\left(\varphi_{\text {wing }}-\varphi_{\text {head }}\right)=0 \\
I_{\text {head }} \ddot{\varphi}_{\text {head }}+k_{\text {neck }}\left(\varphi_{\text {head }}-\varphi_{\text {wing }}\right)=0
\end{gathered}
$$

$I_{\text {wing }}$ und $I_{\text {head }}$ sind dabei die Trägheitsmomente der Flügel und des Kopfes. Der Lösungsansatz für dieses System zweier gekoppelter Differentialgleichungen ist:

$$
\varphi_{\text {wing }}=A_{\text {wing }} e^{i \omega t} \text { und } \varphi_{\text {head }}=A_{\text {head }} e^{i \omega t} .
$$

Die beiden Lösungen für die Eigenfrequenzen $\omega_{ \pm}$des gekoppelten Systems sind:

$$
{\omega_{ \pm}}^{2}=\frac{1}{2 I_{\text {wing }} I_{\text {head }}}\left[\begin{array}{l}
I_{\text {head }}\left(\kappa_{\text {leg }}+\kappa_{\text {neck }}\right)+I_{\text {wing }} \kappa_{\text {neck }} \\
\pm \sqrt{\left(I_{\text {head }}\left(\kappa_{\text {leg }}+\kappa_{\text {neck }}\right)+I_{\text {wing }} \kappa_{\text {neck }}\right)^{2}-4 I_{\text {wing }} I_{\text {head }} \kappa_{\text {leg }} \kappa_{\text {neck }}}
\end{array}\right]
$$

Die negative Lösung beschreibt dabei die gemeinsame Torsion von Kopf und Flügeln in Phase um das Bein, wobei der Hals als starre Achse nicht verdreht wird. Bei der Lösung mit positivem Vorzeichen bleibt das Bein in Ruhe, während Kopf und Flügel gegeneinander mit einer Phasenverschiebung von $180^{\circ}$ um den Hals tordieren.

Experimente mit Laser-Doppler-Vibrometrie (Abschnitt 4.6.1) zur Aufklärung der tatsächlich Schwingungsformen bestätigen diese Interpretation. Die negative Lösung entspricht der symmetrischen ST-Torsionsmode, die positive Lösung der antisymmetrischen AS2-Torsionsmode. Die Frage, warum sich gerade die zweite Torsionsschwingung ergibt und nicht die AS1Mode beantwortet sich beim Betrachten der Bewegungsform. Die AS1-Mode beinhaltet gleichzeitig zur Torsion ein starkes Flattern der Flügel. Dieser Freiheitsgrad der Bewegung wird bei der AS2-Mode nicht oder nur sehr schwach angeregt. Da er in der Herleitung nicht berücksichtigt wird, ergibt sich konsequenterweise die AS2-Mode für die positive Lösung. Zur Abschätzung der Zahlwerte für die so hergeleiteten Torsions-Eigenfrequenzen, werden Kopf und Flügel durch rechteckige Plättchen beschrieben, für Hals und Bein werden „lange“ Stangen mit rechteckiger Querschnittsfläche angenommen (Abbildung 4.20). 

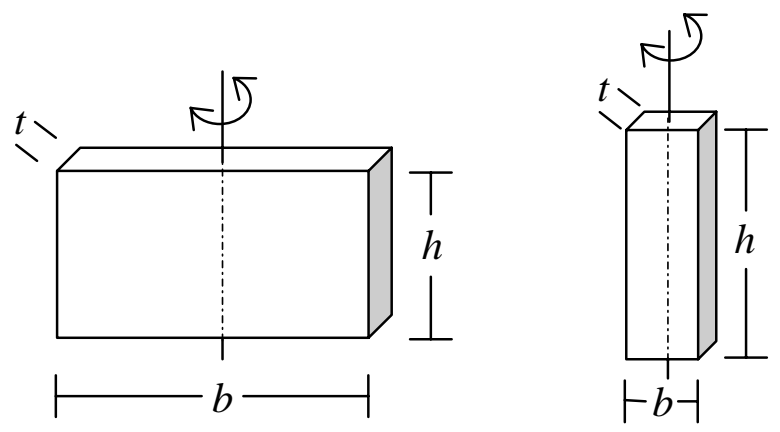

Abbildung 4.20: Zur Berechnung des Trägheitsmomentes eines dünnen Plättchens (links) und der Torsionskonstante einer langen Stange mit rechteckigem Querschnitt (rechts).

Das Trägheitsmoment eines rechteckigen Plättchens mit Breite $b$, Höhe $h$, Dicke $t$ und Massendichte $\rho$ ist [140]:

$$
I=\frac{1}{12} \rho t h b^{3}
$$

Für den Kopf lässt sich Gleichung 4.16 mit den in Tabelle 4.1 angegebenen Abmessungen direkt anwenden. Etwas komplizierter verhält es sich mit der Flügelpartie. Um ihr Trägheitsmoment zu bestimmen wird sie gedanklich in die beiden Einzelflügel und das Mittelelement zerlegt. Für das Mittelelement gilt wieder Gleichung 4.16. Bei den einzelnen Flügeln ist die Rotationsachse nun nicht mehr die Symmetrieachse des Plättchens, weshalb der Satz von Steiner [140] zur Anwendung kommt. Mit der Massendichte von Silizium $\rho=2332 \mathrm{~kg} / \mathrm{m}^{3}$ [147] errechnen sich die Trägheitsmomente von Kopf und Flügelpartie zu:

$$
\begin{aligned}
& I_{\text {head }}=1,22 \cdot 10^{-10} \mathrm{kgm}^{2} \\
& I_{\text {wing }}=5,93 \cdot 10^{-9} \mathrm{kgm}^{2}
\end{aligned}
$$

Der Torsionsmodul $\kappa$ einer „langen“ Stange mit der Länge h, der rechteckigen Querschnittsfläche $b \times t$, wobei $b$ die breitere und $t$ die schmalere Kante ist, und dem Schermodul in axialer Richtung G lässt sich analytisch berechnen [141]:

$$
\kappa=\frac{\beta b t^{3} G}{h}
$$

Dabei ist $\beta$ eine Funktion, die vom Verhältnis der Kantenlängen der Querschnittsfläche abhängt: 


$$
\beta=\frac{1}{3}\left(1-\frac{192}{\pi^{5}} \frac{t}{b} \sum_{n=1,3,5, \ldots}^{\infty} \frac{1}{n^{5}} \tanh \left(\frac{n \pi b}{2 t}\right)\right)
$$

Mit den in Tabelle 4.1 angegebenen Abmessungen und dem Schermodul für Silizium in $<110>$-Richtung $\mathrm{G}=66,2 \mathrm{GPa}$ [147] ergeben sich für die Winkelrichtgrößen von Hals und Bein:

$$
\begin{aligned}
& \kappa_{\text {neck }}=8,66 \cdot 10^{-2} \mathrm{Nm} \\
& \kappa_{\text {leg }}=3,45 \cdot 10^{-2} \mathrm{Nm}
\end{aligned}
$$

Werden die Winkelrichtgrößen aus (4.20) und die Trägheitsmomente aus (4.17) in Gleichung (4.15) eingesetzt, ergeben sich die beiden Kreisfrequenzen $\omega \mathrm{zu}$ :

$$
\omega_{-}=2,39 \cdot 10^{3} \frac{1}{\mathrm{~s}} \text { und } \omega_{+}=2,62 \cdot 10^{4} \frac{1}{\mathrm{~s}}
$$

und entsprechend die Frequenzen $\mathrm{f} z \mathrm{zu}$

$$
f_{-}=380 \mathrm{~Hz} \text { und } f_{+}=4170 \mathrm{~Hz} \text {. }
$$

Trotz der Vielzahl an Näherungen, die in dieses einfache mathematische Modell eingehen, angefangen von der vereinfachten Geometrie, bis hin zur völligen Vernachlässigung von Biegeverformungen während der Schwingung, liefert die Theorie der gekoppelten Torsionsschwingungen Ergebnisse, die in ihrer Größenordnung mit den tatsächlich gemessenen gut übereinstimmen (siehe Tabelle 4.2).

\subsubsection{Dämpfung bei harmonischen Schwingungen}

Es gibt zwei Möglichkeiten, die Dämpfung einer Eigenschwingung des Doppel-Paddel Oszillators experimentell zu bestimmen:

- Wird der Oszillator im PLL-Modus in Resonanz betrieben und die Anregung abgeschaltet, so ergibt sich eine abfallende Exponentialfunktion, wenn man die Amplitude A(t) der freien Schwingung gegenüber der Zeit t aufträgt:

$$
A(t)=A_{0} \exp \left(-f_{0} \pi Q^{-1} \cdot t\right)
$$

Dabei ist $\mathrm{f}_{0}$ die Resonanzfrequenz. Der Dämpfungskoeffizient $\mathrm{Q}^{-1}$ ist als Kehrbruch der Güte G definiert. Mit dem logarithmischen Dekrement $\Lambda=f_{0} \pi Q^{-1}$ ist die Güte: 


$$
Q=\frac{f_{0} \pi}{\Lambda}
$$

- Wird die Amplitude $A$ in Abhängigkeit von der anregenden äußeren Frequenz $f_{E}$ bestimmt, so errechnet sich die Güte aus der Breite $\Delta \mathrm{f}$ der Amplitudenresonanz bei $A_{\max } / \sqrt{2}$. Da die Energie der Schwingung quadratisch von ihrer Amplitude abhängt und die Güte als Verlust an Energie pro Schwingungsperiode bezogen auf die Gesamtenergie definiert ist, handelt es sich gerade um die Halbwertsbreite (FWHM) der Energie. Mit $\mathrm{f}_{0}$ als Position, bei der die Amplitude maximal ist, berechnet sich die Güte zu:

$$
Q=\frac{f_{0}}{\Delta f}
$$

\subsubsection{Ursachen für Dämpfung}

Dämpfung ist ein Phänomen, das bei allen realen Schwingungen auftritt. Beim ideal ungedämpften Oszillator wird von reversiblen Prozessen und rein elastischen Verformungen ausgegangen. In der Realität gibt es jedoch eine Vielzahl von Vorgängen, bei denen Energie aus der Schwingung thermodynamisch irreversibel dissipiert wird.

Im Folgenden wird ein Überblick über die Prozesse gegeben, die für die Dämpfung der AS2Mode des Doppel-Paddel Oszillators eine Rolle spielen [142]. Dabei geht es in den Abschnitten 4.5.3.1 bis 4.5.3.4 um Vorgänge, die durch entsprechend geschicktes Experimentieren wenn nicht völlig ausgeschlossen, so doch minimiert werden können. Dem gegenüber sind die Prozesse in 4.5.3.5 bis 4.5.3.7 prinzipiell nicht reduzierbar.

\subsubsection{Prozesse in realen Festkörpern}

In einem ideal elastischen Festkörper gibt es keine Dämpfung durch Defekte. Obwohl der Doppel-Paddel Oszillator aus hochreinem, einkristallinem Silizium hergestellt wird, ist er weit von einem idealen Festkörper entfernt. Leerstellen im thermischen Gleichgewicht, Fremdatome und andere Gitterbaufehler werden bei der Oszillation mitbewegt. Diese Bewegung ist irreversibel, mit Reibung verbunden und kostet Energie, die der Schwingung dann nicht mehr zur Verfügung steht. 


\subsubsection{Verluste an der Oberfläche}

Sowohl bei der Herstellung durch Laserschneiden, als auch beim Ätzen werden die Kanten und Ecken des Paddels so stark angegriffen und verändert, dass sie nicht mehr als einkristallin bezeichnet werden können. Die Gitterstruktur ist so stark gestört, dass Verformungen in diesen Bereichen irreversibel und nicht elastisch sind. Nicht zu vernachlässigen sind auch Verluste auf den Flächen des DPOs. Herstellungsbedingte Defekte durch Schneiden des Einkristalls und die sich anschließende Politur führen genauso zu Energieverlusten, wie chemische Rückstände (Kohlenstoff, Wasserstoff, Wasser).

\subsubsection{Reibung in Gasen}

Im UHV können die verbleibenden Moleküle als ideales Gas genähert werden. Trifft ein Gasmolekül den schwingenden Oszillator, so gibt es einen Impuls- und Energieübertrag auf das Teilchen, was für den Oszillator Dämpfung bedeutet.

\subsubsection{Verluste durch die Halterung}

Zwar ist bei der AS2-Mode durch die spezielle Form der Schwingung die Bewegung in Bein und Fuß auf ein Minimum reduziert, trotzdem kann Energie in Form von Phononen durch die Einspannung dissipiert werden. Festes Klemmen des Fußes in den Mini-Schraubstock und stabile Streben im UHV-Einsatz sorgen dafür, dass der DPO am Fuß nahezu keine Bewegungsmöglichkeiten mehr hat.

\subsubsection{Thermoelastischer Effekt}

Wird ein Einkristall gebogen, so entsteht ein Temperaturgradient zwischen den lokal gestauchten und den lokal gedehnten Bereichen im Gitter. Der resultierende Wärmefluss ist mit einem Entropieanstieg verbunden, wodurch Schwingungsenergie in thermische Energie umgewandelt wird. Wegen seiner Bedeutung zum Verständnis der temperaturabhängigen Dämpfung der AS2-Mode wird dieser Effekt in Abschnitt 4.6.4 ausführlich behandelt. 


\subsubsection{Phonon-Phonon-Wechselwirkungen}

Die Anharmonizität im Atompotential eines Kristallgitters führt zu Wechselwirkungen zwischen Schallwellen und thermischen Phononen. Durch die Vibrationen des Oszillators wird das Phononenspektrum verschoben. Der Prozess, der das thermische Gleichgewicht des Phononengases wieder einstellt, ist mit der Dissipation von Schallwellen und damit Energie verbunden.

\subsubsection{Elektron-Phonon-Wechselwirkungen}

Insbesondere wenn freie Elektronen zur Verfügung stehen, führen Oszillationen zu einer Änderung der lokalen elektrischen Felder der Atomrümpfe. Das Elektronengas folgt den Feldänderungen und dissipiert Energie. Für das Elektronengas wird dazu, analog einer Flüssigkeit, eine Viskosität definiert.

\subsection{Schwingungsverhalten des leeren Paddels}

\subsection{1 Übersicht über die verschiedenen Eigenmoden}

Wird die Amplitude der Paddelschwingung in Abhängigkeit von der Frequenz gemessen, so ergeben sich scharfe Resonanzmaxima wie in Abbildung 4.21 gezeigt. Die Bewegung und Dynamik der einzelnen Eigenmoden zu verstehen, wurde in den vergangenen Jahren als Schlüssel für folgende drei Forschungsziele betrachtet:

- Grundsätzliches Verständnis von Dämpfungsvorgängen in Festkörpern.

- Verbesserung des DPO-Designs, um die Güte noch weiter zu erhöhen.

- Weiterentwicklung des Oszillators um neue Anwendungen im Bereich der Mikro-Elektromechanischen Systeme (MEMS) zu erschließen.

Von X. Liu [143] wurden Laser-Doppler-Vibrometrie Experimente durchgeführt und die zunächst von C. Spiel [144] begonnenen Finite-Elemente-Rechnungen weiter verbessert. Bei der Laser-Doppler-Vibrometrie (Abbildung 4.22) wird der DPO in Resonanz betrieben und die Geschwindigkeit seiner Oberfläche senkrecht zur Oszillatorebene gemessen. 


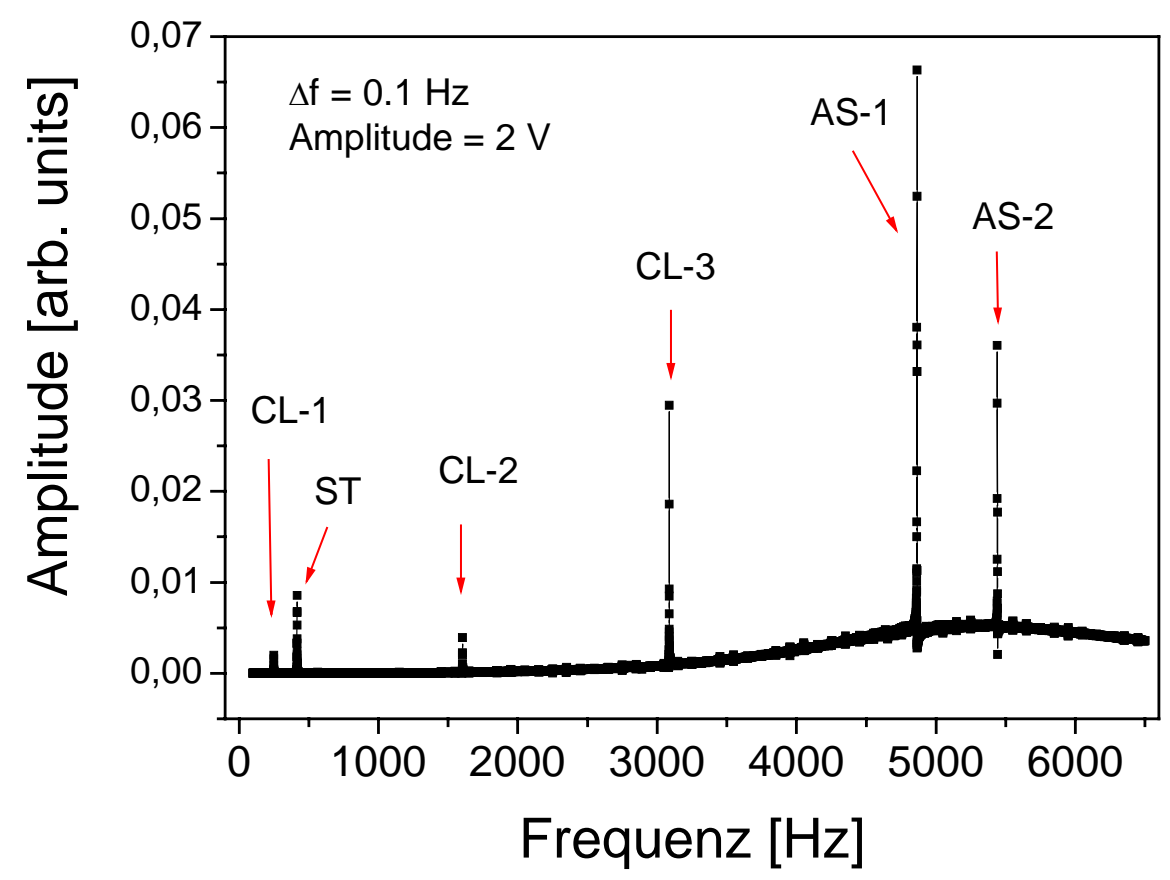

Abbildung 4.21: Frequenzspektrum des Doppel-Paddel Oszillators (DPO 11) mit seinen Eigenfrequenzen. Die Amplituden der Resonanzen erlauben wegen der großen Frequenzschritte keine Aussage über die tatsächliche Auslenkung.

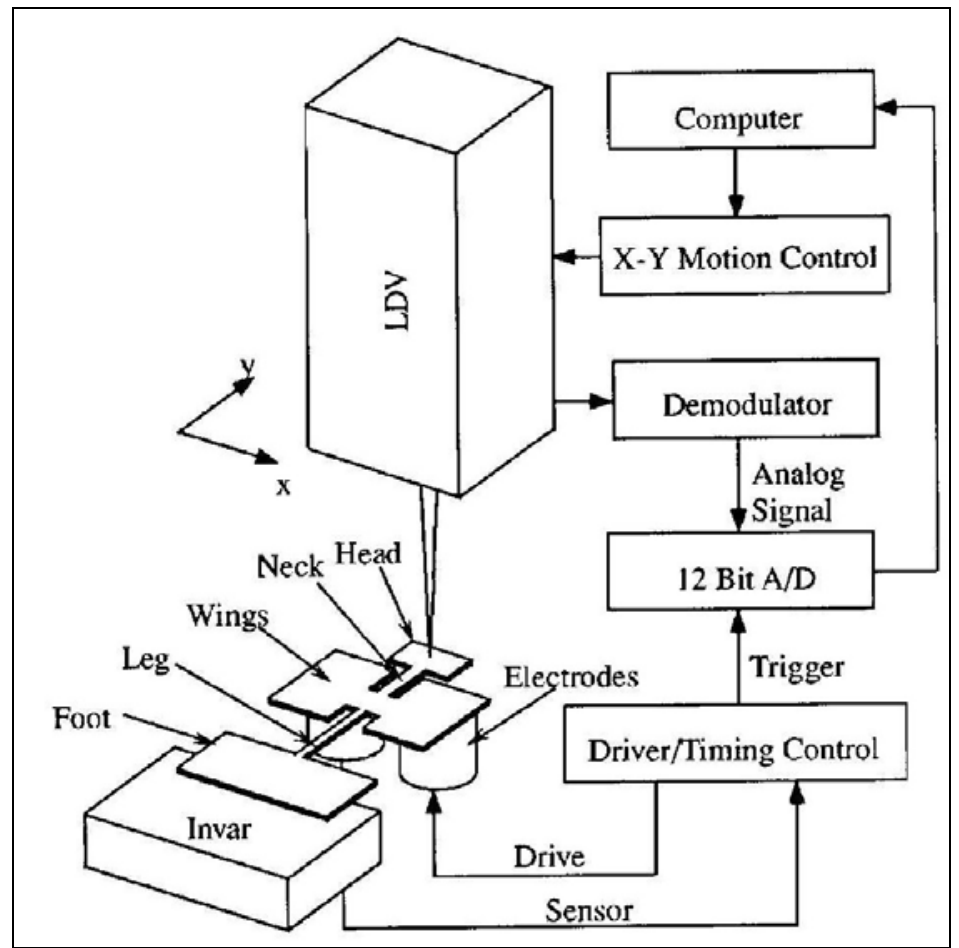

Abbildung 4.22: Experimenteller Aufbau für Laser-Doppler-Vibrometrie Messungen an einem DPO [143]. 

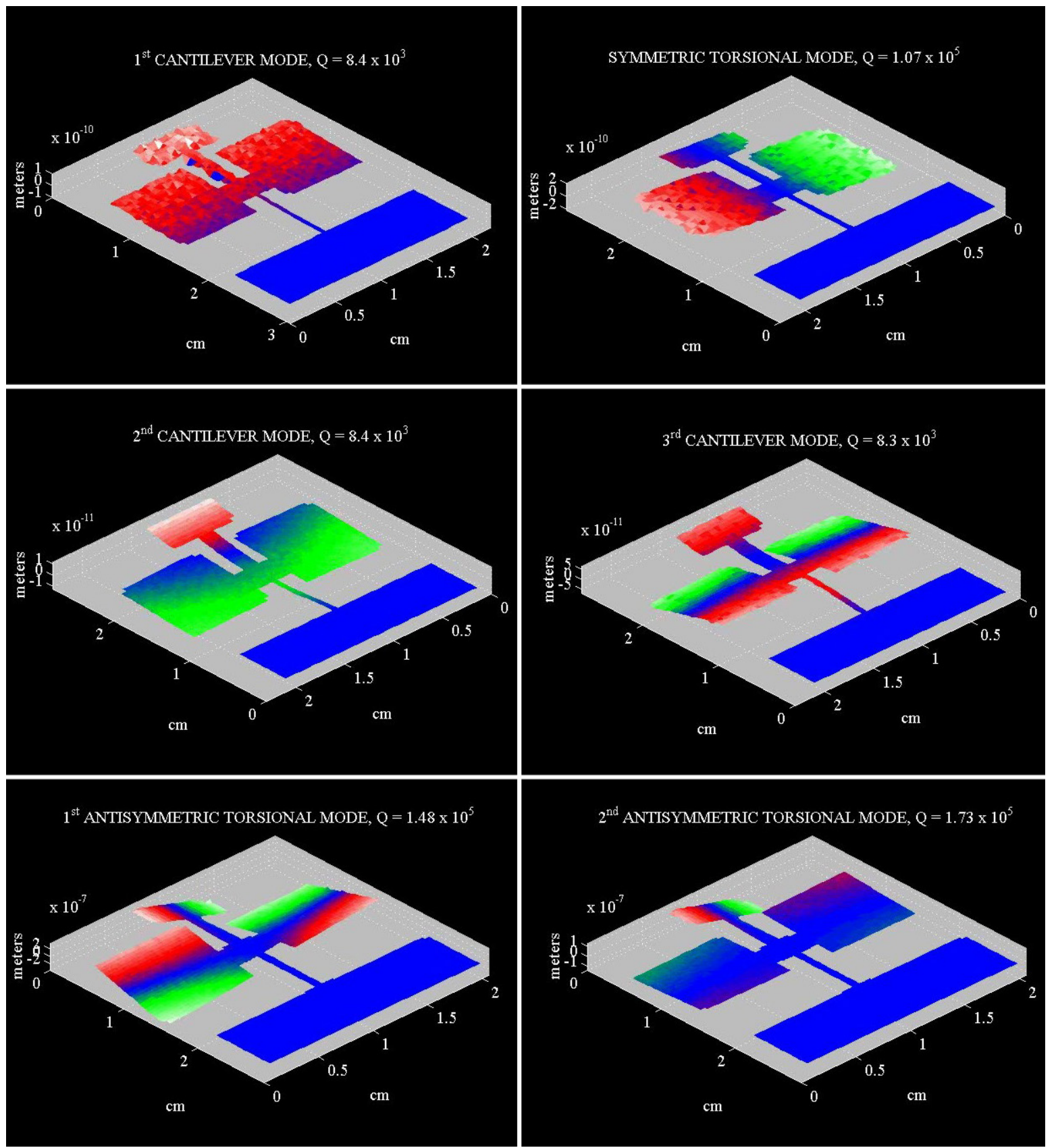

Abbildung 4.23: Darstellung der Auslenkung für die sechs Eigenmoden mit der niedrigsten Frequenz. Die Farbskala kennzeichnet die maximale Auslenkung aus der Ebene des Oszillators (blau). Rote Bereiche sind nach oben, grüne Bereiche nach unten ausgelenkt [143].

Um ein Bild der Geschwindigkeitsverteilung auf der Oberfläche zu erhalten, wird der DPO mit dem auf 0,45 mm Durchmesser fokussierten Laserstrahl komplett abgerastert. Aus der Dopplerverschiebung des reflektierten Strahls durch die Geschwindigkeit des sich auf die Laserquelle zu- oder von der Quelle wegbewegenden Oszillators errechnet sich die Auslenkung 
der verschiedenen Bereiche des Oszillators. In Abbildung 4.23 ist die Dynamik für die sechs Moden mit der niedrigsten Eigenfrequenz dargestellt.

Für die Finite-Elemente-Rechnungen wurde der Oszillator in 634 dreidimensionale Elemente mit 5041 Verknüpfungspunkten zerlegt, wobei die Dicke der Einzelelemente gerade der Oszillatordicke entspricht. Als Materialparameter wurden die elastischen Konstanten von Silizium bei Raumtemperatur $\mathrm{c}_{11}=165,8 \mathrm{GPa}, \mathrm{c}_{12}=63,94 \mathrm{GPa}, \mathrm{c}_{44}=79,62 \mathrm{GPa}$ [145] und die Silizium-Massendichte $\rho=2332 \mathrm{~kg} / \mathrm{m}^{3}$ verwendet. Die Ergebnisse der Rechnungen sind in Tabelle 4.2, zusammen mit gemessenen eigenen Werten mehrerer Doppel-Paddel Oszillatoren zusammengestellt. Dabei zeigt sich, dass die mathematische Modellierung die Realität relativ gut trifft. Es gibt charakteristische Unterschiede zwischen Paddeln, die auf unterschiedliche Weise hergestellt sind. Sie lassen sich verstehen, wenn die herstellungsbedingten Unterschiede ihrer Abmessungen betrachtet werden.

\begin{tabular}{|c|c|c|c|c|c|}
\cline { 2 - 6 } \multicolumn{1}{c|}{} & Simulation & \multicolumn{2}{c|}{ Laserschneiden } & \multicolumn{2}{c|}{ Photolithografie } \\
\hline Eigenmode & $\mathbf{f}_{\mathrm{FE}}$ & DPO 11 & DPO 20 & DPO 12 & DPO 22 \\
\hline CL1 1. cantilever & 253,01 & 267,4 & 271,3 & 248,4 & 245,65 \\
\hline ST symmetric torsion & 430,10 & 457,1 & 460,9 & 416,2 & 414,4 \\
\hline CL2 2. cantilever & 1629,9 & 1634,3 & 1644,9 & 1600,9 & 1598,9 \\
\hline CL3 3. cantilever & 3133,7 & 3231,2 & 3245,6 & 3085,2 & 3083,7 \\
\hline AS1 1. antisymm. torsion & 4817,5 & 4664,5 & 4673,8 & 4873,3 & 4888,1 \\
\hline AS2 2. antisymm. torsion & 5298,3 & 5332,2 & 5346,6 & 5441,0 & 5581,2 \\
\hline
\end{tabular}

Tabelle 4.2: Vergleich der aus Finite-Elemente-Rechnungen bestimmten Resonanzfrequenzen ( $\left.f_{F E}\right)$ mit gemessenen eigenen Werten. DPO 11 und DPO 20 wurden mit Laserschneiden, DPO 12 und DPO 22 mit Photolithografie und anschließendem Ätzen hergestellt. Die Frequenzen sind in Hz angegeben.

\subsubsection{Experimentelle Bestimmung der Dämpfung}

In Kapitel 4.5.2 wurden zwei unterschiedliche Methoden vorgestellt, mit denen die Dämpfung experimentell bestimmt werden kann. Um die Äquivalenz dieser Messmethoden zu beweisen, wurde der unbedampfte DPO 22 bei Raumtemperatur untersucht. 


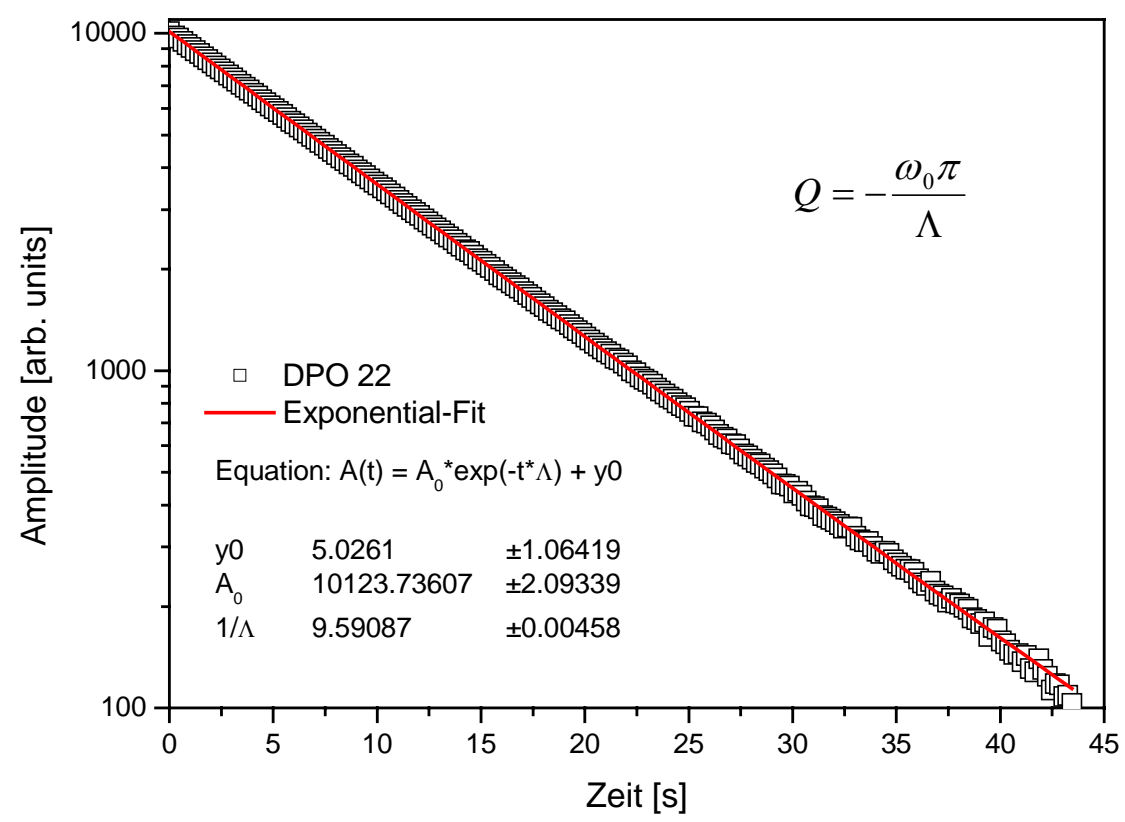

Abbildung 4.24: Logarithmische Darstellung der Abklingamplitude nach der Ring-Down-Methode.

Die abklingenden Ring-Down-Amplitude ist in Abbildung 4.24 logarithmisch dargestellt. Aus der Steigung der Geraden berechnet sich $Q=168055$ Aus der Amplitudenresonanzfunktion in Abbildung 4.25 ergibt sich $\mathrm{Q}=169959$. Beide Werte weichen um etwa 1\% voneinander ab, was im Rahmen des Fehlers die Gleichwertigkeit der beiden Methoden zeigt.

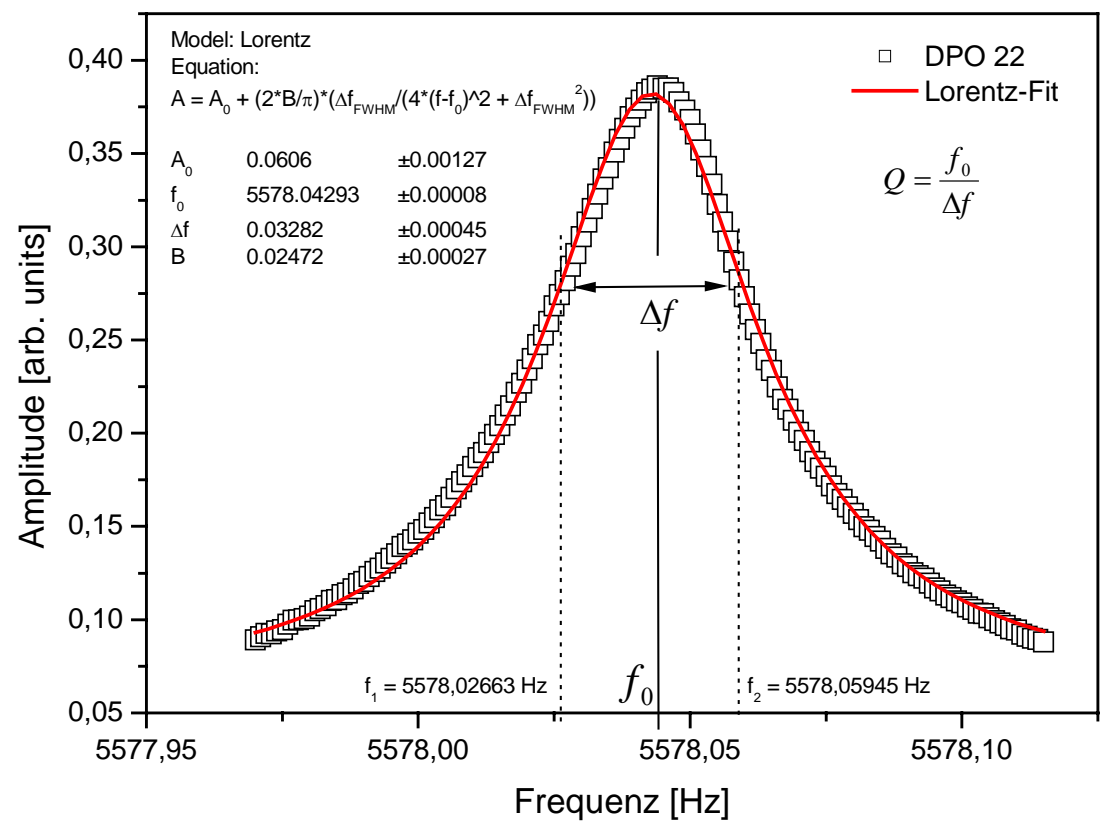

Abbildung 4.25: Experimentelle Bestimmung der Dämpfung mit der Amplitudenresonanzfunktion. 


\subsubsection{Einfluss von Phasenlage und Amplitude auf die Dämpfung}

Bevor ein Ring-Down-Experiment durchgeführt werden kann, muss der DPO im selbstangeregten Modus (PLL) betrieben werden. Die Parameter Anregungsamplitude und Phasenlage zwischen Anregung und Signal können dabei innerhalb von gewissen Grenzen frei gewählt werden. Dabei stellt sich die Frage, ob der im späteren Ring-Down-Experiment bestimmte Gütefaktor von diesen Parametern abhängt.

Die Phasenverschiebung zwischen Anregung und Signal wird mit dem Band-Pass Filter am PAR 124 Lock-In Verstärker festgelegt. Die Niederfrequenzflanke des Band-Pass Filters wird dabei als Phasenschieber benutzt. Je steiler die Flanke ist, desto definierter lässt sich die Phasenlage einstellen, weshalb in allen Experimenten eine Filtergüte von 100 gewählt wurde. Bei einer kleineren Güte ist der PLL nur schwer aufrecht zu erhalten. Die Filterfrequenz muss auf jeden Fall größer gewählt werden als die tatsächliche Resonanzfrequenz, weil man sich sonst nicht mehr auf der niederfrequenten Seite der Flanke befinden würde. Allerdings darf die Frequenz auch nicht zu groß gewählt werden, da zu größeren Frequenzen hin die Flanke immer flacher wird, bis der Filter schließlich sperrt.

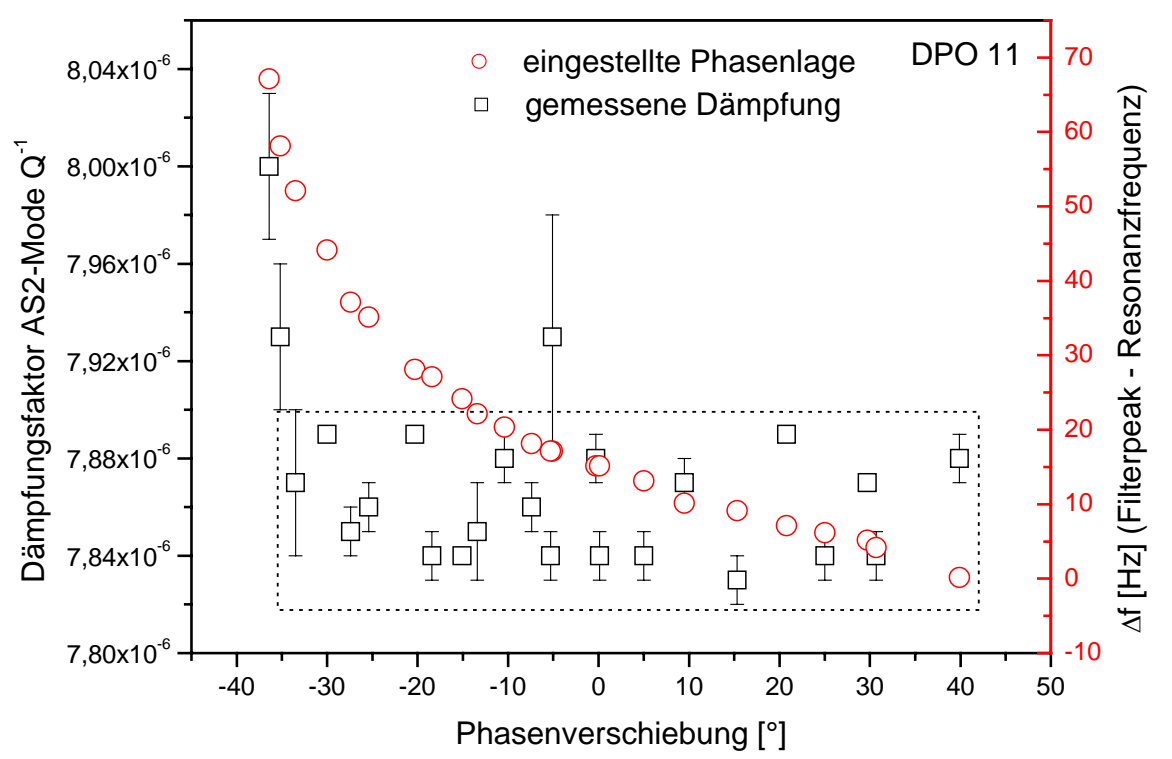

Abbildung 4.26: Abhängigkeit des Dämpfungsfaktors von der eingestellten Phasenlage zwischen Anregung und Signal bei DPO 11.

Abbildung 4.26 zeigt die Differenz der eingestellten Filterfrequenz zur Resonanzfrequenz (rechte Ordinate), die daraus resultierende Phasenverschiebung zwischen Anregung und Sig- 
nal (Abszisse) und den Dämpfungsfaktor (linke Ordinate) für den Oszillator DPO 11. Die Fehlerbalken des Dämpfungsfaktors ergeben sich als statistische Standardabweichung aus je fünf Ring-Down-Experimenten pro eingestellter Phasenlage. Offensichtlich gibt es im Rahmen des Fehlers, der kleiner ist als $10^{-7}$, keine Abhängigkeit der Dämpfung von der Phasenlage in den Grenzen von $-30^{\circ}$ bis $+40^{\circ}$. In der Praxis bedeutet das, dass Temperaturintervalle durchlaufen werden können, die eine Frequenzverschiebung von bis zu $70 \mathrm{~Hz}$ bedeuten, ohne den Band-Pass Filter nachregeln zu müssen.

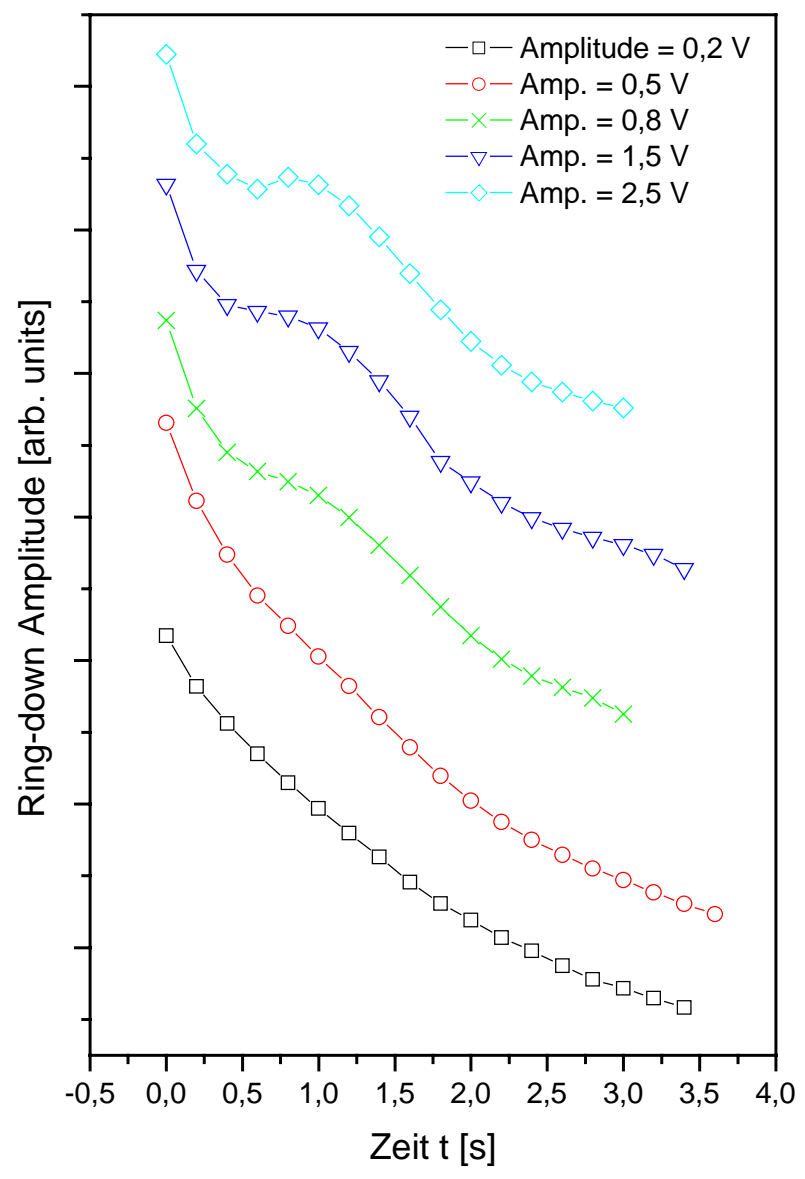

Abbildung 4.27: Einfluss der treibenden Kraft auf das Ring-Down-Verhalten von DPO 11. Die Kurven sind wegen der besseren Übersichtlichkeit vertikal verschoben.

Wesentlich gravierender ist der Einfluss der treibenden Kraft auf die Ring-Down-Experimente. In Abbildung 4.27 ist zu sehen, dass bei groß gewählten Spannungen am PAR-outputlevel Effekte auftreten, die an Interferenzen oder Schwebungen erinnern. Die resultierenden Ring-Down-Kurven weichen stark von der idealen abfallenden Exponentialfunktion ab. In allen Experimenten wurde daher sichergestellt, dass die treibende Kraft klein genug ist, um solche Effekte auszuschließen. 


\subsubsection{Temperaturabhängigkeit der Dämpfung}

Die Kenntnis der Temperaturabhängigkeit der Dämpfung des reinen Silizium Oszillators ist von fundamentaler Bedeutung, wenn kleine Änderungen in den mechanischen Eigenschaften von dünnen Schichten detektiert werden sollen. Deshalb wird die Dämpfung aller sechs Eigenmoden im Temperaturbereich von $300 \mathrm{~K}$ bis $650 \mathrm{~K}$ mit dem Gould Nicolet FFT-Analyser („Phazer“) detailliert untersucht. Bei den Messungen wird die maximale Zoom-Funktion der „Prism“-Software ausgenutzt, um eine bestmögliche Genauigkeit zu erzielen. Innerhalb des für die Integration zur Verfügung stehenden Zeitintervalls wird der Doppel-Paddel Oszillator mit Hilfe der Sweep-Funktion des Frequenzgenerators mit allen Frequenzen innerhalb des Zoom-Bereiches kontinuierlich angeregt. Das vom Phazer aufgenommene Zeitsignal wird mittels Fast-Fourier-Transformation in ein Frequenzsignal umgewandelt, das beispielhaft in Abbildung 4.28 gezeigt ist. Für geringe Gütefaktoren liefert diese Methode eine zufriedenstellende Genauigkeit, allerdings genügt die Zoom-Funktion, die maximal 3200 Linien auf ein Frequenzintervall von höchstens $15 \mathrm{~Hz}$ konzentriert, bei hohen Güten nicht, um eine gute Genauigkeit zu erzielen.

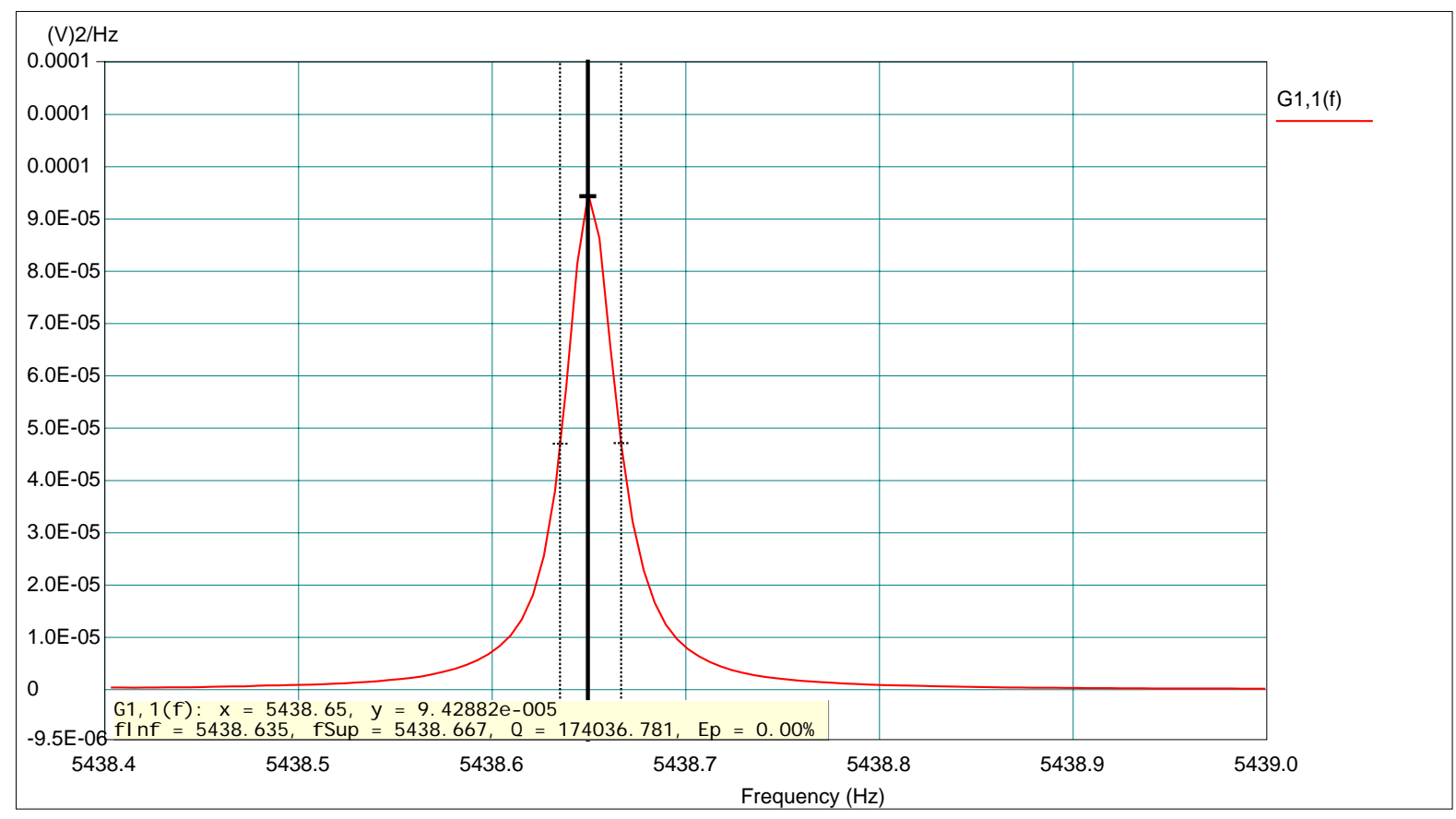

Abbildung 4.28: Exemplarisches Ergebnis der FFT-Phazer Software Prism für die AS2-Mode bei Raumtemperatur mit folgenden Einstellungen: Spectral Lines: 3200, Time Capture Points: 8192, Frequency Span: 15 Hz, Average Type: Peak Hold, Average Domain: Frequency, Average Number: 5, Frame Number: 5, Window Type: Hanning. 
Die so bestimmte temperaturabhängige Dämpfung aller sechs Eigenmoden, ist in Abbildung 4.29 dargestellt. Die Dämpfung hat für die AS1- und die AS2-Mode den geringsten Wert im untersuchten Temperaturintervall von $300 \mathrm{~K}$ bis $650 \mathrm{~K}$. Die Streuung liegt bei dieser Eigenschwingung im Rahmen des statistischen Fehlers des FFT-Phazers. Bei den anderen Eigenmoden ist die Streuung so groß, dass sie sich nicht mehr mit schlechter Statistik erklären lässt. Die Gründe für die Irreproduzierbarkeit der Dämpfung bei diesen Moden sind nicht klar, allerdings deckt sich die Beobachtung mit Messungen von C. Spiel [144] bei tiefen Temperaturen.

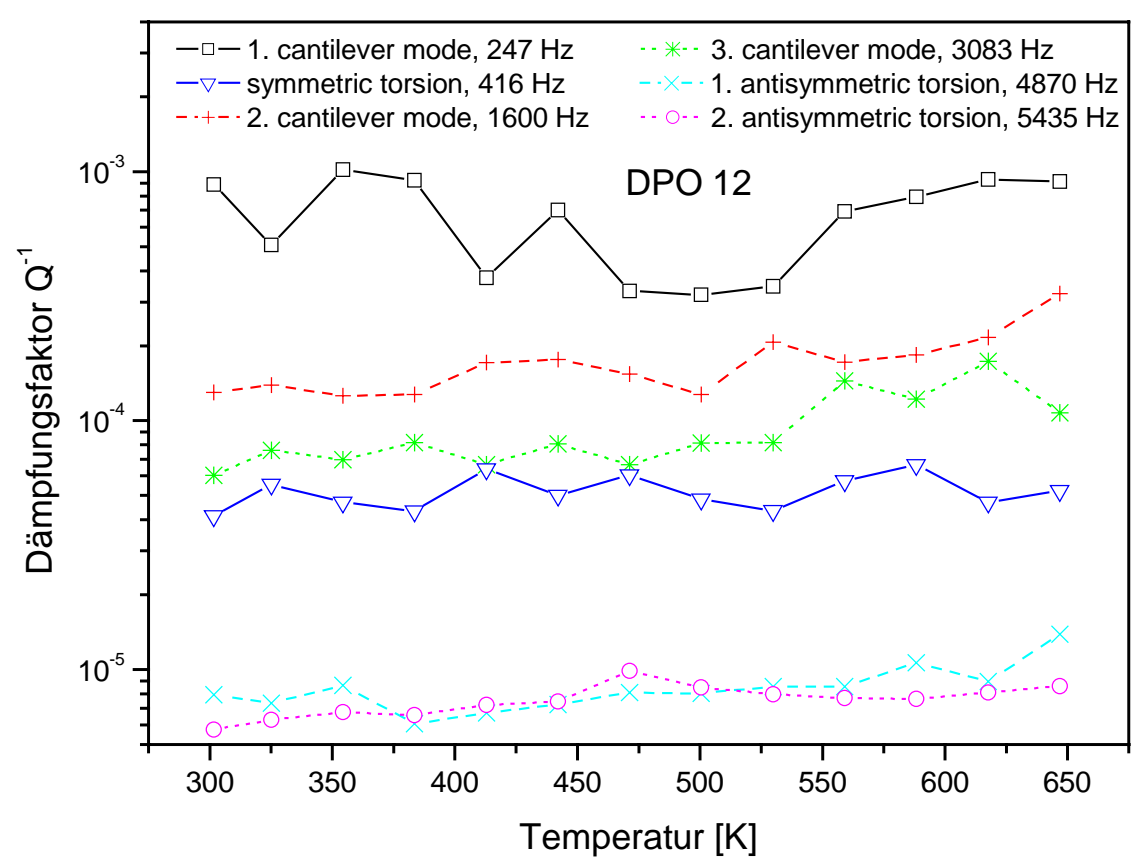

Abbildung 4.29: Temperaturabhängigkeit des Dämpfungsfaktors der sechs niedrigsten Eigenmoden. Die in der Legende angegebene Frequenz wurde bei Raumtemperatur gemessen.

Wegen der Bedeutung der AS2-Mode für die Experimente im Rahmen dieser Arbeit, wurde die Temperaturabhängigkeit dieser speziellen Mode mit Ring-Down-Experimenten noch einmal genauer untersucht. Abbildung 4.31 zeigt, dass die Dämpfung $\mathrm{Q}^{-1}$ von $5,3 \times 10^{-6}$ bei $300 \mathrm{~K}$ auf $\mathrm{Q}^{-1}=7,7 \times 10^{-6}$ bei $650 \mathrm{~K}$ ansteigt (schwarze Quadrate). Ebenfalls eingezeichnet sind Ergebnisse für die Dämpfung bei tiefen Temperaturen (Dreiecke), die in früheren Experimenten [133] bestimmt wurden. Erfreulich ist die gute Übereinstimmung der Daten bei Raumtemperatur, was belegt, dass es keine Abweichungen in den Ergebnissen aufgrund von Unterschieden im experimentellen Aufbau gibt. 
Die Frage ist nun, ob sich der beobachtete Anstieg der Dämpfung mit zunehmender Temperatur grundsätzlich verstehen lässt. Houston et al. [133] haben gezeigt, dass sich der Kurvenverlauf durch ein relativ einfaches Modell der thermoelastischen Energiedissipation beschreiben lässt. Grundlage dafür ist der thermoelastische Effekt bei reinen Biegeschwingungen. Zener [146] postuliert einen Wärmefluss und einen damit verbundenen Energietransport, der durch oszillierendes Komprimieren und Dehnen von elastischen Medien erzeugt wird. Die Ursache für den Energiefluss ist ein Temperaturgradient zwischen lokal komprimierten und lokal aufgeweiteten Bereichen des Gitters, wie in Abbildung 4.30 skizziert. Inelastische Streuung der thermisch erzeugten Phononen führt zu Energiedissipation. Diese Energie wird der zur Verfügung stehenden Schwingungsenergie entzogen, was zu einem Abklingen der Amplitude, also zu Dämpfung führt.

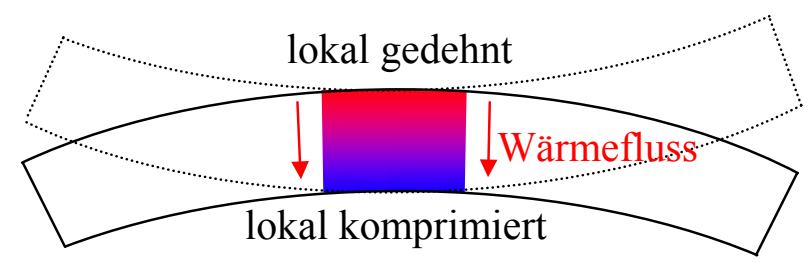

Abbildung 4.30: Schematische Darstellung des thermoelastischen Effektes bei einer reinen Biegeschwingung. Während durch die Verformung eine Seite des Festkörpers lokal gedehnt wird, ist die gegenüberliegende lokal komprimiert.

Da bei der AS2 Mode keine reine Torsion vorliegt, sondern immer auch eine leichte Biegung der Flügel (siehe auch Abbildung 4.23 rechts unten), ist die Voraussetzung für den thermoelastischen Effekt gegeben. Zur Berechnung wird die einfache Beschreibung von Zener um einen Faktor ergänzt, der für kompliziertere Schwingungsformen, wie bei der AS2-Mode einer Kombination aus Torsion und Biegeschwingung, nötig ist. Der Beitrag des thermoelastischen Effektes zur Dämpfung des Paddels ist:

$$
Q_{\text {th.e. }}^{-1}=p_{\text {th.e. }} \frac{E \alpha^{2} T}{c} \frac{\omega \tau}{1+\omega^{2} \tau^{2}}
$$

p $_{\text {th.e. }}$ ist der modenspezifische Faktor, der für die AS2 Mode mit Finite Elemente Rechnungen zu 0,0755 bestimmt wurde [133], E der Elastizitätsmodul (da die Torsionsachse des Oszillators in (110)-Richtung orientiert ist, gilt $\mathrm{E}=169 \mathrm{GPa}$ ), $\alpha$ der thermische Ausdehnungskoeffizient, c die spezifische Wärme pro Volumen, $\mathrm{T}$ die Temperatur, $\omega$ die Kreisfrequenz der Schwingung und $\tau$ die thermische Relaxationszeit, für die gilt: 


$$
\tau=\frac{t_{s}^{2} c}{\pi^{2} \kappa}
$$

Darin ist $t_{\mathrm{s}}$ die Dicke des Paddels und $\kappa$ die thermische Leitfähigkeit. Die temperaturabhängigen Materialeigenschaften von Silizium wurden für die Rechnung aus „EMIS Datareview Properties of Silicon“ [147] entnommen. Ergebnisse von Houston [133] für tiefe Temperaturen, die den Verlauf der Dämpfung in Abhängigkeit von der Temperatur gut beschreiben, wurden im Rahmen dieser Arbeit bis $650 \mathrm{~K}$ fortgesetzt (rote Linie in Abbildung 4.31) [148]. Der vergrößerte Bildausschnitt zeigt, dass auch bei Temperaturen oberhalb von Raumtemperatur Größenordnung und Verlauf der Dämpfung durch das Modell des thermoelastischen Effektes gut beschrieben werden. Dass die experimentellen Absolutwerte rund 20\% größer sind, hat seine Ursache darin, dass der thermoelastische Effekt nicht der einzige Beitrag zur Dämpfung des Oszillators ist, wie in Abschnitt 4.5.3 ausgeführt wurde. Allerdings zeigen die Ergebnisse, dass er die Temperaturabhängigkeit dominiert.

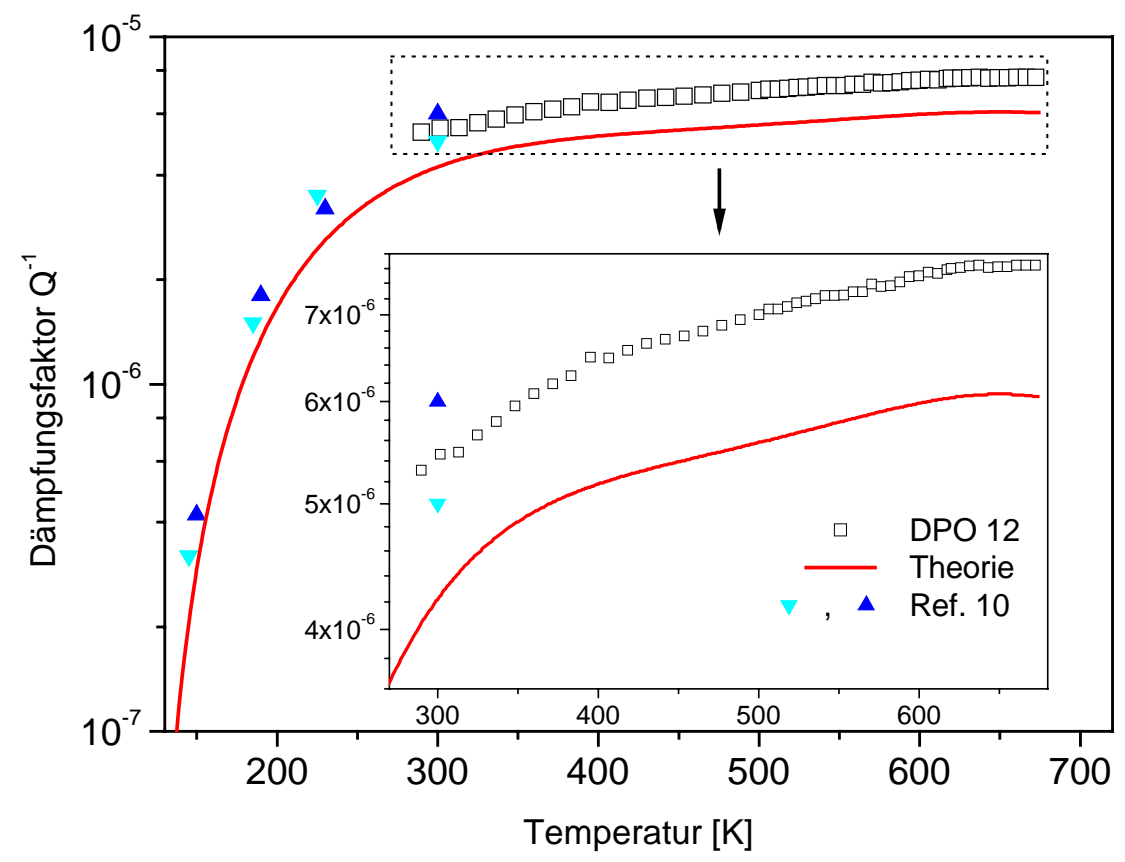

Abbildung 4.31: Temperaturabhängiger Dämpfungsfaktor für die AS2-Mode ermittelt nach der Ring-Down Methode (schwarze Quadrate. Die durchgezogene rote Linie symbolisiert den Beitrag durch den thermoelastischen Effekt, die eingezeichneten Dreiecke sind Literaturwerte [133]. 


\subsection{Mathematisches Modell für das System Paddel - Schicht}

\subsubsection{Beiträge zur Frequenzverschiebung}

Beginnend mit den Eigenfrequenzen der beiden unabhängigen Torsionsschwingungen (Abschnitt 4.5.1) wird nun der Einfluss einer zusätzlichen dünnen Schicht diskutiert.

Für die Torsion des Kopfes um den Hals gilt für den unbedampften Oszillator:

$$
\omega_{\text {hn }}=\sqrt{\frac{\kappa_{\text {neck }}}{I_{\text {head }}}}
$$

und für die Torsion der Flügel um das Bein:

$$
\omega_{w l}=\sqrt{\frac{\kappa_{\text {leg }}}{I_{\text {wing }}}}
$$

Die Kopplung beider schwingenden Systeme ergibt eine symmetrische und eine antisymmetrische Eigenschwingung. Deren Resonanzfrequenzen können alternativ zu Gleichung (4.15) für die ST-Mode auch durch

$$
\omega_{S T}=\omega_{w l}(1-\mu)
$$

und für die AS2-Mode durch

$$
\omega_{A S 2}=\omega_{h n}(1+\mu)
$$

beschrieben werden. Dabei wurde substituiert:

$$
\mu=I_{\text {head }} / I_{\text {wing }}
$$

Befindet sich auf dem gesamten Oszillator nun eine dünne Schicht, so wird sich sowohl das Trägheitsmoment, wegen der insgesamt größeren Masse, als auch die Torsionskonstante, wegen zusätzlicher elastischer Beiträge durch die Verformung der Schicht, verändern. Im Folgenden werden diese Einflüsse für die AS2-Mode diskutiert.

Die Kreisfrequenz der Torsion des Kopfes um den Hals ändert sich gemäß

$$
\omega_{h n}+\Delta \omega_{h n}=\sqrt{\frac{\kappa_{\text {neck }}+\Delta \kappa_{\text {neck }}}{I_{\text {head }}+\Delta I_{\text {head }}}}
$$


Sind die Änderungen $\Delta \mathrm{I}_{\text {head }}$ und $\Delta \kappa_{\text {neck }}$ des Trägheitsmomentes $\mathrm{I}_{\text {head }}$ und der Winkelrichtgröße $\kappa_{\text {neck }}$ durch die zusätzliche dünnen Schicht klein, so gilt in erster Näherung nach Taylor:

$$
\begin{aligned}
& 1+\frac{\Delta \omega_{\text {hn }}}{\omega_{\text {hn }}}=\sqrt{\frac{\kappa_{\text {neck }}+\Delta \kappa_{\text {neck }}}{I_{\text {head }}+\Delta I_{\text {head }}}} \cdot \sqrt{\frac{I_{\text {head }}}{\kappa_{\text {neck }}}}=\left(1+\frac{\Delta \kappa_{\text {neck }}}{\kappa_{\text {neck }}}\right)^{\frac{1}{2}}\left(1+\frac{\Delta I_{\text {head }}}{I_{\text {head }}}\right)^{-\frac{1}{2}} \\
& \approx\left(1+\frac{1}{2} \frac{\Delta \kappa_{\text {neck }}}{\kappa_{\text {neck }}}\right)\left(1-\frac{1}{2} \frac{\Delta I_{\text {head }}}{I_{\text {head }}}\right) \approx 1+\frac{1}{2} \frac{\Delta \kappa_{\text {neck }}}{\kappa_{\text {neck }}}-\frac{1}{2} \frac{\Delta I_{\text {head }}}{I_{\text {head }}}
\end{aligned}
$$

Aus der Frequenzverschiebung $\Delta \omega_{\mathrm{hn}}$ folgt eine Verschiebung für die AS2-Resonanz:

$$
\omega_{A S 2}+\Delta \omega_{A S 2}=\left(\omega_{h n}+\Delta \omega_{h n}\right)(1+\mu)
$$

Die eigentliche Messgröße ist allerdings nicht die Kreisfrequenz $\omega_{\mathrm{AS} 2}$, sondern die Frequenz $\mathrm{f}_{\mathrm{AS} 2}$. Für deren Verschiebung gilt:

$$
\frac{\Delta f_{A S 2}}{f_{A S 2}}=\frac{\Delta \omega_{A S 2}}{\omega_{A S 2}}=\frac{\Delta \omega_{h n}}{\omega_{h n}} \approx \frac{1}{2} \frac{\Delta \kappa_{\text {neck }}}{\kappa_{\text {neck }}}-\frac{1}{2} \frac{\Delta I_{\text {head }}}{I_{\text {head }}}
$$

Soll $\Delta \mathrm{f}_{\mathrm{AS} 2}$ nun tatsächlich berechnet werden, so werden die Beiträge $\Delta \mathrm{I}_{\text {head }}$ und $\Delta \kappa_{\text {neck }}$ benötigt, die durch den Film auf dem DPO verursacht sind. Es erscheint vernünftig anzunehmen, dass diese zusätzlichen Beiträge von der Schichtdicke abhängen, weshalb Klitsner [149] vorgeschlagen hat, ihre totalen Differentiale nach der Schichtdicke $\Delta \mathrm{t}$ zu berechnen:

$$
\Delta I_{\text {head }}=\frac{d I_{\text {head }}}{d t} \Delta t \text { und } \Delta \kappa_{\text {neck }}=\frac{d \kappa_{\text {neck }}}{d t} \Delta t
$$

Für die Ableitung der Torsionskonstante und des Trägheitsmomentes nach der Schichtdicke werden die gleichen geometrischen Näherungen gemacht, wie in Abschnitt 4.5.1 zur Herleitung der Resonanzfrequenzen. Aus den Gleichungen 4.15 und 4.17 folgt:

$$
\frac{d I_{\text {head }}}{d t}=\frac{1}{12} \rho h_{\text {head }} b_{\text {head }}^{3} \text { und } \frac{d \kappa_{\text {neck }}}{d t}=\frac{3 \beta b_{\text {neck }} t^{2} G}{h_{\text {neck }}}
$$

Mit den Größen $G_{\text {film }}$ für den Schermodul, $t_{\text {film }}$ für die Dicke und $\rho_{\text {film }}$ für die Massendichte der Schicht, ergibt sich für die dickenabhängigen Beiträge zu Trägheitsmoment und Torsionskonstante:

$$
\Delta I_{\text {head }}=\frac{1}{12} \rho_{\text {film }} h_{\text {head }} b_{\text {head }}^{3} t_{\text {film }} \text { und } \Delta \kappa_{\text {neck }}=\frac{3 \beta b_{\text {neck }} t^{2} G_{\text {film }} t_{\text {film }}}{h_{\text {neck }}}
$$


Da die geometrischen Abmessungen für das Substrat, wie auch die dünne Schicht mit Ausnahme ihrer unterschiedlichen Dicke gleich sind, ergeben sich folgende Verhältnisse:

$$
\frac{\Delta I_{\text {head }}}{I_{\text {head }}}=\frac{\rho_{\text {film }} t_{\text {film }}}{\rho_{\text {sub }} t_{\text {sub }}} \text { und } \frac{\Delta \kappa_{\text {neck }}}{\kappa_{\text {neck }}}=\frac{3 G_{\text {film }} t_{\text {film }}}{G_{\text {sub }} t_{\text {sub }}}
$$

Eingesetzt in Gleichung (4.36) ergibt sich für die Verschiebung der AS2-Resonanzfrequenz in Abhängigkeit von der Dicke der Schicht und ihren Materialeigenschaften:

$$
\frac{\Delta f_{\text {AS2 }}}{f_{\text {AS2 }}}=\frac{3 G_{\text {film }} t_{\text {film }}}{2 G_{\text {sub }} t_{\text {sub }}}-\frac{\rho_{\text {film }} t_{\text {film }}}{2 \rho_{\text {sub }} t_{\text {sub }}}
$$

Offensichtlich gibt es zwei konkurrierende Beiträge zur Frequenzverschiebung, die wegen des unterschiedlichen Vorzeichens die Resonanzfrequenz in unterschiedliche Richtung verschieben:

$$
\begin{aligned}
& \left.\frac{\Delta f_{\text {AS2 }}}{f_{\text {AS2 }}}\right|_{\text {elastisch }}=\frac{3 G_{\text {film }} t_{\text {film }}}{2 G_{\text {sub }} t_{\text {sub }}} \\
& \left.\frac{\Delta f_{\text {AS2 }}}{f_{\text {AS2 } 2}}\right|_{\text {Masse }}=-\frac{\rho_{\text {film }} t_{\text {film }}}{2 \rho_{\text {sub }} t_{\text {sub }}}
\end{aligned}
$$

Der elastische Beitrag in Gleichung (4.42) hängt wesentlich vom Schermodul der dünnen Schicht ab. Elastische Energie wird nun nicht mehr ausschließlich im tordierenden Silizium gespeichert, sondern zusätzlich in der Schicht. Der Hals als Torsionsfeder erscheint insgesamt „härter“, weshalb der elastische Beitrag die Resonanzfrequenz zu größeren Werten hin verschiebt. Der Beitrag in Gleichung (4.43) ist durch die zusätzliche Masse bedingt, was sich formal in seiner Abhängigkeit von der Dichte des Films ausdrückt. Je dicker die Schicht ist, desto träger wird das System. Die Resonanzfrequenz verschiebt sich zu kleinern Werten.

Eine sehr ähnliche Herleitung für die ST-Mode führt zu entsprechenden Resultaten.

Wird allerdings beim Bedampfen das Bein des Paddels abgeschattet, so gibt es keinen zusätzlichen elastischen Beitrag zur Torsionskonstante um den Fuß. Es gilt also:

$$
\left.\frac{\Delta f_{S T}}{f_{S T}}\right|_{\text {elastisch }}=0
$$

Da jedoch nur das Bein abgeschattet wird, die Flügel jedoch bedampft werden, gibt es sehr wohl einen Masseanteil zur ST-Frequenzverschiebung. Dieser berechnet sich analog Gleichung (4.43): 


$$
\left.\frac{\Delta f_{S T}}{f_{S T}}\right|_{\text {Masse }}=-\frac{\rho_{\text {film }} t_{\text {film }}}{2 \rho_{\text {sub }} t_{\text {sub }}}
$$

Die isolierte Betrachtung der Frequenzverschiebung der ST-Mode genügt schon, um das Produkt aus Dicke und Dichte der aufgebrachten Schicht bestimmen zu können.

$$
\rho_{\text {film }} t_{\text {film }}=-\left.2 \rho_{\text {sub }} t_{\text {sub }} \frac{\Delta f_{S T}}{f_{S T}}\right|_{\text {Masse }}
$$

Gleichung (4.46) in Gleichung (4.41) eingesetzt fasst also die unterschiedlichen Verschiebungen der ST- und der AS2-Resonanzfrequenzen zusammen. Daraus errechnet sich das Produkt aus Schermodul und Dicke der Schicht:

$$
G_{\text {film }} t_{\text {film }}=\frac{2}{3} G_{\text {sub }} t_{\text {sub }}\left(\frac{\Delta f_{A S 2}}{f_{A S 2}}-\frac{\Delta f_{S T}}{f_{S T}}\right)
$$

Ist durch eine unabhängige Messung die Dicke der Schicht $t_{\text {film }}$ bekannt, so lässt sich aus Gleichung (4.46) sofort die Massendichte $\rho_{\text {film }}$ und aus Gleichung (4.47) der Schermodul $G_{\text {film }}$ berechnen.

\subsubsection{Innere Reibung in dünnen Schichten}

Der Anteil $Q_{\text {film }}^{-1}$ an der gemessenen Gesamtdämpfung $Q_{D P O}^{-1}$ kann mittels eines Formalismus berechnet werden, der erstmals von White [150] vorgestellt wurde. Der Dämpfungsfaktor $Q^{-1}$ ist definiert als Verlust an elastischer Energie pro Schwingungsperiode $\Delta \mathrm{E}$ bezogen auf die gesamte im Oszillator gespeicherte elastische Energie E:

$$
Q^{-1}=\frac{\Delta E}{2 \pi E}
$$

Befindet sich eine dünne Schicht auf dem Oszillator, so ist Schwingungsenergie sowohl im Substrat, als auch in der Schicht gespeichert. Genauso wird elastische Energie in beiden Komponenten durch Reibung dissipiert.

$$
2 \pi Q_{D P O}^{-1}=\frac{\Delta E_{f i l m}+\Delta E_{\text {sub }}}{E_{\text {film }}+E_{\text {sub }}}
$$

Da die Dicke des Oszillators mit $\mathrm{t}_{\text {sub }}=300 \mu \mathrm{m}$ deutlich größer ist, als die der untersuchten dünnen Schichten, gilt $\mathrm{E}_{\text {sub }}>>\mathrm{E}_{\text {film }}$ und damit: 


$$
2 \pi Q_{D P O}^{-1} \approx \frac{\Delta E_{\text {film }}+\Delta E_{\text {sub }}}{E_{\text {sub }}}=Q_{\text {sub }}^{-1}+\frac{\Delta E_{\text {film }}}{E_{\text {sub }}}
$$

Mit der Umformung

$$
\frac{\Delta E_{f i l m}}{E_{\text {sub }}}=\frac{\Delta E_{\text {film }} \cdot E_{\text {film }}}{E_{\text {sub }} \cdot E_{\text {film }}}=2 \pi Q_{f l m}^{-1} \frac{E_{\text {film }}}{E_{\text {sub }}}
$$

ergibt sich:

$$
Q_{D P O}^{-1}-Q_{\text {sub }}^{-1}=\frac{E_{\text {film }}}{E_{\text {sub }}} Q_{\text {film }}^{-1}
$$

Die Bestimmung von $Q_{\text {film }}^{-1}$ ist damit auf die Dämpfung des leeren Paddels $Q_{\text {sub }}^{-1}$, des bedampften Paddels $Q_{D P O}^{-1}$ und auf das Verhältnis der in Schicht und Substrat gespeicherten elastischen Gesamtenergie der Schwingung reduziert. Mit dem einfachen Bild einer Torsionsschwingung berechnet sich diese Energie zu

$$
E=\frac{1}{2} \kappa \varphi^{2}
$$

wobei $\kappa$ die Torsionskonstante und $\varphi$ die Amplitude des Auslenkwinkels ist. Damit gilt zusammen mit Gleichung (4.40):

$$
\frac{E_{\text {film }}}{E_{\text {sub }}}=\frac{\kappa_{\text {film }}}{\kappa_{\text {sub }}}=\frac{\Delta \kappa}{\kappa}=\frac{3 G_{\text {film }} t_{\text {film }}}{G_{\text {sub }} t_{\text {sub }}}
$$

Für den Dämpfungsfaktor der Schicht aus Gleichung (4.52) gilt damit:

$$
Q_{\text {film }}^{-1}=\frac{G_{\text {sub }} t_{\text {sub }}}{3 G_{\text {film }} t_{\text {film }}}\left(Q_{D P O}^{-1}-Q_{\text {sub }}^{-1}\right)
$$

Zur Überprüfung dieser Gleichung wurden ebenfalls Finite Elemente Rechnungen durchgeführt. Erste Ergebnisse der Simulationen zeigen, dass sich $Q_{\text {film }}^{-1}$ mit Gleichung (4.55) gut beschreiben lässt [151]. 


\subsubsection{Bestimmung des komplexen Schermoduls}

Der Schermodul G eines amorphen Festkörpers ist eine komplexe Größe, die aus dem Speichermodul (Realteil $G^{\prime}$ ) und dem Verlustmodul (Imaginärteil $G^{\prime \prime}$ ) zusammensetzt ist. In der komplexen Schreibweise gilt damit:

$$
G=G^{\prime}+i G^{\prime \prime}
$$

In Gleichung 4.47 wurde mit $\mathrm{G}_{\text {film }}$ der Betrag $|\mathrm{G}|$ und in Gleichung 4.54 mit $Q_{\text {film }}^{-1}$ der Tangens des Verlustwinkels $\delta$ zwischen Real- und Imaginärteil in der Eulerschen Ebene berechnet. Es gelten folgende Zusammenhänge:

$$
\begin{gathered}
Q_{\text {film }}^{-1}=\tan (\delta)=\frac{G^{\prime \prime}}{G^{\prime}} \\
G_{\text {film }}=|G|=\sqrt{G^{\prime 2}+G^{\prime \prime 2}}
\end{gathered}
$$

Aus der Lösung dieses Gleichungssystems ergibt sich der Speicher- und der Verlustmodul:

$$
\begin{aligned}
& G^{\prime}=\frac{G_{\text {film }}}{\sqrt{1+\left(Q_{\text {film }}^{-1}\right)^{2}}} \\
& G^{\prime \prime}=\frac{Q_{\text {film }}^{-1} \cdot G_{\text {film }}}{\sqrt{1+\left(Q_{\text {film }}^{-1}\right)^{2}}}
\end{aligned}
$$

\subsubsection{Zusammenfassung der mathematischen Beschreibung}

Da die mathematische Beschreibung des Doppel-Paddel Oszillators zwar nicht besonders schwer, aber doch aufwändig ist, werden hier noch einmal die wichtigsten Schritte zusammengefasst. Alle gemessenen dünnen Schichten werden auf diese Weise ausgewertet.

1. Am unbedampften Oszillator wird die ST-Resonanzfrequenz bei Raumtemperatur, sowie die AS2-Resonanzfrequenz und die Dämpfung der AS2-Mode im interessierenden Temperaturintervall gemessen.

2. Die dünne Schicht wird auf Kopf, Hals und Flügel aufgebracht, während Bein und Fuß abgeschattet sind und nicht bedampft werden.

Aus der Frequenzverschiebung der ST-Mode bei Raumtemperatur ergibt sich das Produkt aus Dichte und Dicke der Schicht. Sind diese Größen schon vorher bekannt, so ist die Be- 
trachtung der ST-Mode unnötig. Insbesondere bei den Polymerschichten wurden Dichte und Dicke als gegeben vorausgesetzt, weil eine Abschattung des Beines bei der Schichtdeposition experimentell nicht möglich war.

3. Die temperaturabhängige Verschiebung der AS2-Resonanzfrequenz führt zum Betrag des Schermoduls der dünnen Schicht.

4. Die Messung der Dämpfung des Oszillators mit Schicht ergibt den temperaturabhängigen Dämpfungskoeffizienten der Schicht.

5. Real- und Imaginärteil des komplexen Schermoduls werden berechnet.

An dieser Stelle ist eine Bemerkung darüber angebracht, ob die vielen Näherungen, die zu diesem mathematischen Konzept führen, überhaupt brauchbare Resultate liefern können.

Es ist bekannt, dass die Form der Schwingung deutlich komplizierter ist, als durch die antisymmetrische Eigenschwingung der beiden gekoppelten Torsionspendel in Gleichung (4.15) modelliert. Aber für die reale AS2-Mode gibt es kein einfaches Masse-Feder-Modell, mit dem eine korrekte Beschreibung möglich wäre. Der Vergleich der berechneten mit den gemessenen Werten für die Eigenfrequenzen (Gleichung (4.22) und Tabelle 4.2) zeigt die zu erwartende Schwäche in den Ergebnissen auf. Unabhängig davon, ob die Absolutwerte exakt zu berechnen sind, erscheint die Annahme gerechtfertigt, dass sich Frequenzverschiebungen näherungsweise berechnen lassen, wenn die Schicht genügend dünn ist. Diese Annahme trifft für alle im Rahmen dieser Arbeit untersuchten Schichten zu. Die größte untersuchte Schichtdicke beträgt $1 \mu \mathrm{m}$, was im Vergleich zur DPO-Dicke von $300 \mu \mathrm{m}$ als genügend klein erachtet wird. Würde bei der realen und sehr komplizierten Schwingungsform jedes Detail berücksichtigt und ein exaktes mathematisches Modell entwickelt, dann müssten die Verhältnisse der Schermoduli und der Dichten von Schicht und Substrat in das Modell eingehen. Natürlich wäre weiter zu erwarten, dass die Trägheitsmomente von Kopf und Flügel, sowie die Torsionskonstanten von Hals und Kopf für die Eigenfrequenzen relevant sind. Dass diese proportional zur Wurzel aus dem Bruch von Torsionskonstante und Trägheitsmoment sind, rechtfertigt die Taylor-Entwicklung der Wurzel. Trotz aller Näherungen sind daher die wesentlichen Abhängigkeiten in Gleichung (4.39) durchaus glaubwürdig. Finite-Elemente-Simulationen zur Modellierung des Problems wurden begonnen [151], erste Ergebnisse stehen allerdings noch aus. 


\section{$5 \mathrm{Zr}_{65} \mathrm{Al}_{7,5} \mathrm{Cu}_{27,5}$ als metallisches Glas}

Für die mechanischen Untersuchungen der dynamischen Eigenschaften metallischer Gläser wurde als Modellsystem das „Inoue“-Glas $\mathrm{Zr}_{65} \mathrm{Al}_{7,5} \mathrm{Cu}_{27,5}[11,12]$ ausgewählt. Das thermisch stabile Intervall $\Delta \mathrm{T}$ der unterkühlten Schmelze zwischen der Glastemperatur $\mathrm{T}_{\mathrm{g}}$ und der Kristallisationstemperatur $T_{x}$ beträgt für diese speziellen Legierung bei einer Heizrate von $20 \mathrm{~K} / \mathrm{min}$ etwa $80 \mathrm{~K}$. Im ternären Phasendiagramm der drei Ausgangsmetalle in Abbildung 5.1 ist $\Delta \mathrm{T}$ in Abhängigkeit von der chemischen Zusammensetzung dargestellt.

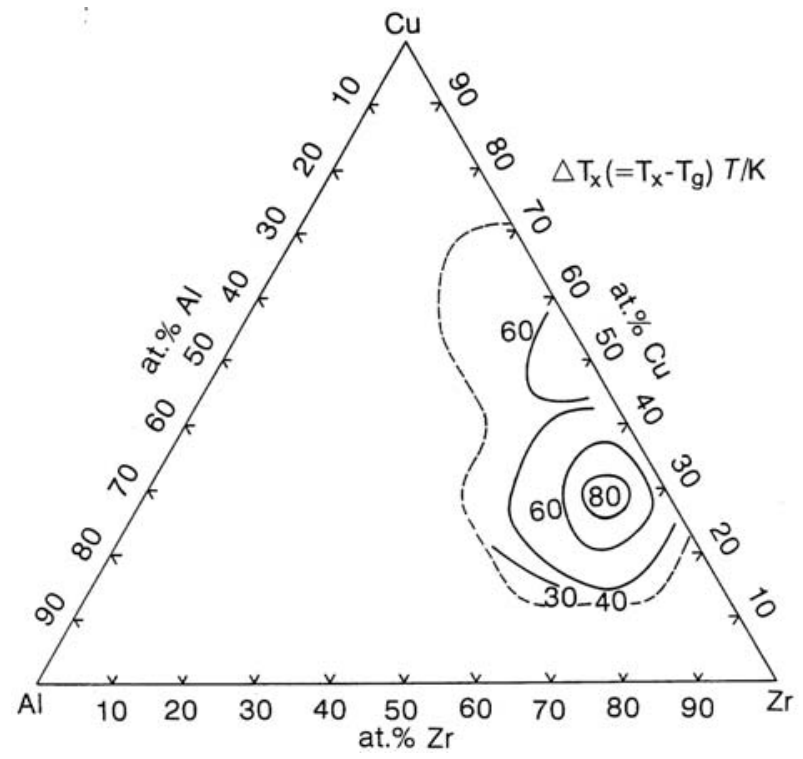

Abbildung 5.1: Ternäres Diagramm der Elemente Zr, Al und Cu zur Veranschaulichung der thermischen Stabilität der unterkühlten Schmelze der Legierung (aus [12]).

Eigenschaften wie die spezifische Wärme und die Stabilität der unterkühlten Schmelze [152], elektrische Transporteigenschaften, [153] Mechanismen zur Änderung der Nahordnung am Glasübergang [9] sowie strukturelle Relaxation und Viskosität [154] wurden am System $\mathrm{Zr}_{65} \mathrm{Al}_{7,5} \mathrm{Cu}_{27,5}$ detailliert untersucht. Die anelastische Relaxation wurde in quasistatischen Messungen ermittelt [21] und dynamische Prozesse mit der Vibrating Reed Technik betrachtet [134]. Neben diesen thermischen, elektrischen und mechanischen Eigenschaften war auch der Wachstumsprozess von dünnen $\mathrm{Zr}_{65} \mathrm{Al}_{7,5} \mathrm{Cu}_{27,5}$-Schichten selbst [155] und die Entwicklung ihrer Oberflächenrauhigkeit [156] Gegenstand intensiver Forschung. 


\subsection{Herstellung und Charakterisierung der Schichten}

\subsubsection{UHV-Kammer und Schichtherstellung}

Die Herstellung der Schichten und die dynamisch mechanischen Messungen daran, erfolgen in situ in einer Balzers UHV-Aufdampfanlage, die beispielsweise in [157] detailliert beschrieben ist. Die Kammer enthält drei Elektronenstrahlverdampfer mit jeweils einer Schwingquarzwaage, die in $120^{\circ}$-Symmetrie angeordnet sind. Mit einer Turbomolekularpumpe und einer Titan-Sublimationspumpe wird nach Ausheizen der Anlage ein Druck von $\mathrm{p}<10^{-9}$ mbar erreicht.

Die Schichten werden durch Verdampfen der drei Reinstmaterialien in separaten Elektronenstrahlverdampfern und Kokondensation der Legierung aus der Gasphase präpariert. Die Kenndaten der Reinstmaterialien und der Typ des jeweils verwendeten Elektronenstrahlverdampfers sind in Tabelle 5.1 angegeben.

\begin{tabular}{|l|l|l|l|}
\hline Element & Reinheit & Hersteller, Bezeichnung & Verdampfer \\
\hline Zirkon & 99,999 & Wah Chang, Albany, USA, $\mathrm{O}_{2}$ arm & ESV3, Leybold Heraeus \\
\hline Aluminium & 99,999 & Heraeus, Ingot, Nr. 009006 & EV1-8, AP\&T \\
\hline Kupfer & 99,999 & Alfa, Rod (13992), $\mathrm{HNO}_{3}$ geätzt & ESV4, Leybold-Heraeus \\
\hline
\end{tabular}

Tabelle 5.1: Für die Schichtherstellung verwendete Reinstmaterialien.

Vor der ersten Schichtdeposition wird das Material in den Verdampfern unter UHV-Bedingungen einmal vollständig auf- und zusammengeschmolzen, um eine homogene Pille herzustellen. Für gute Vakuumbedingungen wird die UHV-Kammer nach jedem Öffnen der Anlage bei mindestens $150{ }^{\circ} \mathrm{C}$ über mehrere Tage hindurch ausgeheizt.

Die gewünschte Schichtzusammensetzung der ternären Legierung wird durch die geeignete Wahl der Verhältnisse der Aufdampfraten der einzelnen Elemente eingestellt. Da sich der zu bedampfende Doppel-Paddel Oszillator etwa in der Mitte zwischen den Verdampfern befindet, die Schwingquarzwaagen jedoch direkt oberhalb der einzelnen Pillen angeordnet sind, muss die Aufdampfrate für jedes Element mit Hilfe eines Geometriefaktors, des „Tooling“Faktors, bestimmt werden. Er ergibt sich aus dem Verhältnis zwischen der mit KleinwinkelRöntgenreflektometrie (siehe auch Abschnitt 3.1) gemessenen Dicke der auf dem Substrat deponierten Schicht und dem vom Schwingquarz angezeigten Wert. Mit Hilfe der so bestimm- 
ten „Tooling“-Faktoren, der Molmasse und der Massendichte der einzelnen Elemente lassen sich dann die für die gewünschte Schichtzusammensetzung notwendigen Aufdampfraten berechnen. Diese Kalibrierung findet jedes Mal statt, wenn die Anlage geöffnet wird und Veränderungen an den Pillen oder dem Substrat vorgenommen werden.

Während des Aufdampfvorgangs wird die Rate durch die Leistung der einzelnen Verdampfer und die Elektronik der Schwingquarzwaagen geregelt und möglichst konstant gehalten. So wird gewährleistet, dass die Zusammensetzung der Schicht in Wachstumsrichtung homogen ist.

\subsubsection{Tantal als Diffusionsbarriere}

Aus der Halbleiterindustrie ist bekannt, dass insbesondere Kupfer bereits bei $200{ }^{\circ} \mathrm{C}$ mit Silizium reagiert [171]. Um das zu verhindern und eine eindeutige Grenzfläche zwischen dem Substrat aus Silizium und der amorphen $\mathrm{Zr}_{65} \mathrm{Al}_{7,5} \mathrm{Cu}_{27,5}$ Schicht zu erhalten, wurde jeweils eine etwa $50 \mathrm{~nm}$ dicke Tantal-Schicht auf die leeren Oszillatoren DPO 19 und DPO 22 aufgesputtert. Diese Schichten wurden von Yuansu Luo ex-situ in einer kommerziellen DC-Magnetronsputteranlage der Firma Electrotech Equipment GmbH vom Typ PLASMAFAB MS5200 hergestellt, die in der Dissertation von A. Käufler detailliert beschrieben ist [158]. Es ist bekannt, dass Tantal bis zu Temperaturen von $650{ }^{\circ} \mathrm{C}$ als Diffusionsbarriere zwischen Kupfer und Silizium wirkt $[171,172]$.

\subsubsection{Zusammensetzung der Schichten in Wachstumsrichtung}

Um die Wirkung dieser Tantal-Diffusionsbarrieren zu überprüfen, wurde die chemische $\mathrm{Zu}$ sammensetzung an DPO 12 (ohne Ta) und DPO 19 (mit Ta) in Wachstumsrichtung der Schichten ermittelt. Dazu wurden die bedampften Oszillatoren nach Abschluss aller mechanischen Messungen ausgebaut, ein Querschnitt des DPO-Halses mittels Ionenätzen gedünnt und im Transmissions Elektronen Mikroskop (TEM) untersucht. Diese Messungen wurden in Zusammenarbeit mit Yue-Lin Huang und Michael Seibt (IV. Physikalisches Institut) durchgeführt. In den Hellfeld-Aufnahmen in Abbildung 5.2 ist die Schicht als senkrechter heller Streifen zu erkennen. Die aus dem Vergrößerungsfaktor des Mikroskops ermittelte Gesamtdicke der deponierten $\mathrm{Zr}_{65} \mathrm{Al}_{7,5} \mathrm{Cu}_{27,5}$ Schichten ist angegeben. Sie ist deutlich geringer, als erwartet, was in Abschnitt 7.2.1 diskutiert wird. Ein Trend für die Konzentration der unterschiedlichen 
Elemente in der Probe wurde mit einem EDX-Linescan entlang der eingezeichneten Linie ermittelt. An den mit „,+“ markierten Stellen wurde mit genauen Punktmessungen die chemische Zusammensetzung bestimmt.
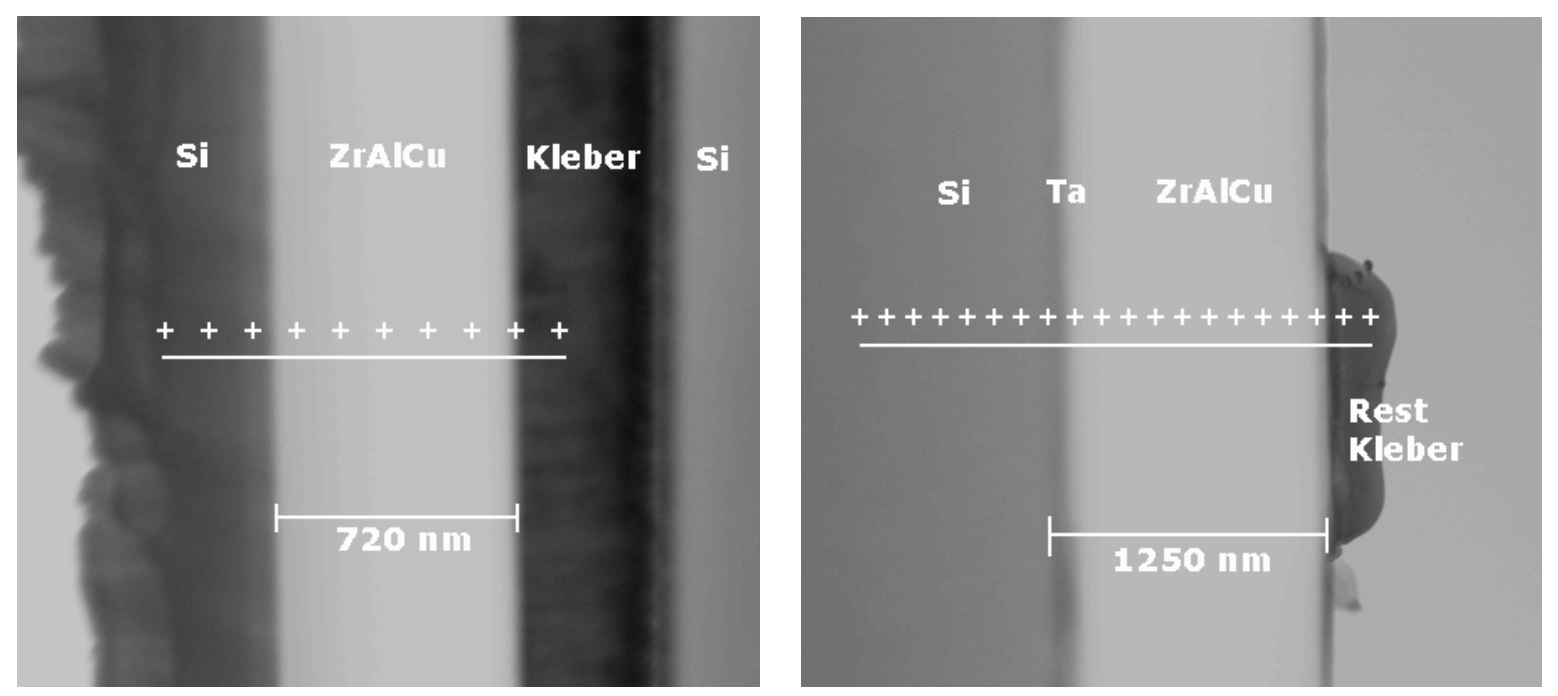

Abbildung 5.2: Hellfeldaufnahme von DPO 12 (links) und DPO 19 (rechts). Die $\mathrm{Zr}_{65} \mathrm{Al}_{7,5} \mathrm{Cu}_{27,5}$ Schicht erscheint als senkrechter, heller Streifen. Die chemische Zusammensetzung wurde entlang der waagrechten Linie und den mit „, “ gekennzeichneten Positionen bestimmt.

Das Energiespektrum in Abbildung 5.3 wird mit einem Elektronenstrahl aufgenommen, der nur so weit fokussiert ist, dass die gesamte Schicht noch vollständig überdeckt ist. Um die einzelnen Elemente zu identifizieren wird die Intensität der in rot gekennzeichnet Energiebereiche der einzelnen Linien ausgewertet.

Die Schwierigkeit der quantitativen Konzentrationsbestimmung besteht darin, dass für Silizium nur die $\mathrm{K}_{\alpha}$-und die $\mathrm{K}_{\beta}$-Linie, die bei nahezu identischer Energie auftreten, im zugänglichen Energiebereich des Detektors zwischen 0 und $20 \mathrm{keV}$ liegen. An dieser Stelle befindet sich aber auch die Tantal-M-Linie. Wenn die Intensität in diesem Bereich nun ansteigt, ist nicht zu identifizieren, ob Tantal oder Silizium die Ursache dafür ist.

Unsicherheiten gibt es auch bei der Aluminium K-Linie, deren Position in der Flanke der Silizium-K- und der Zirkon-L-Linie liegt, die beide große Intensitäten haben. Zusammen mit durch die keilförmige Schichtdicke verursachten Fluoreszenzeffekten führt dies Überlagerung zu einer systematischen Unterschätzung der Aluminium-Konzentration in der Schicht. 


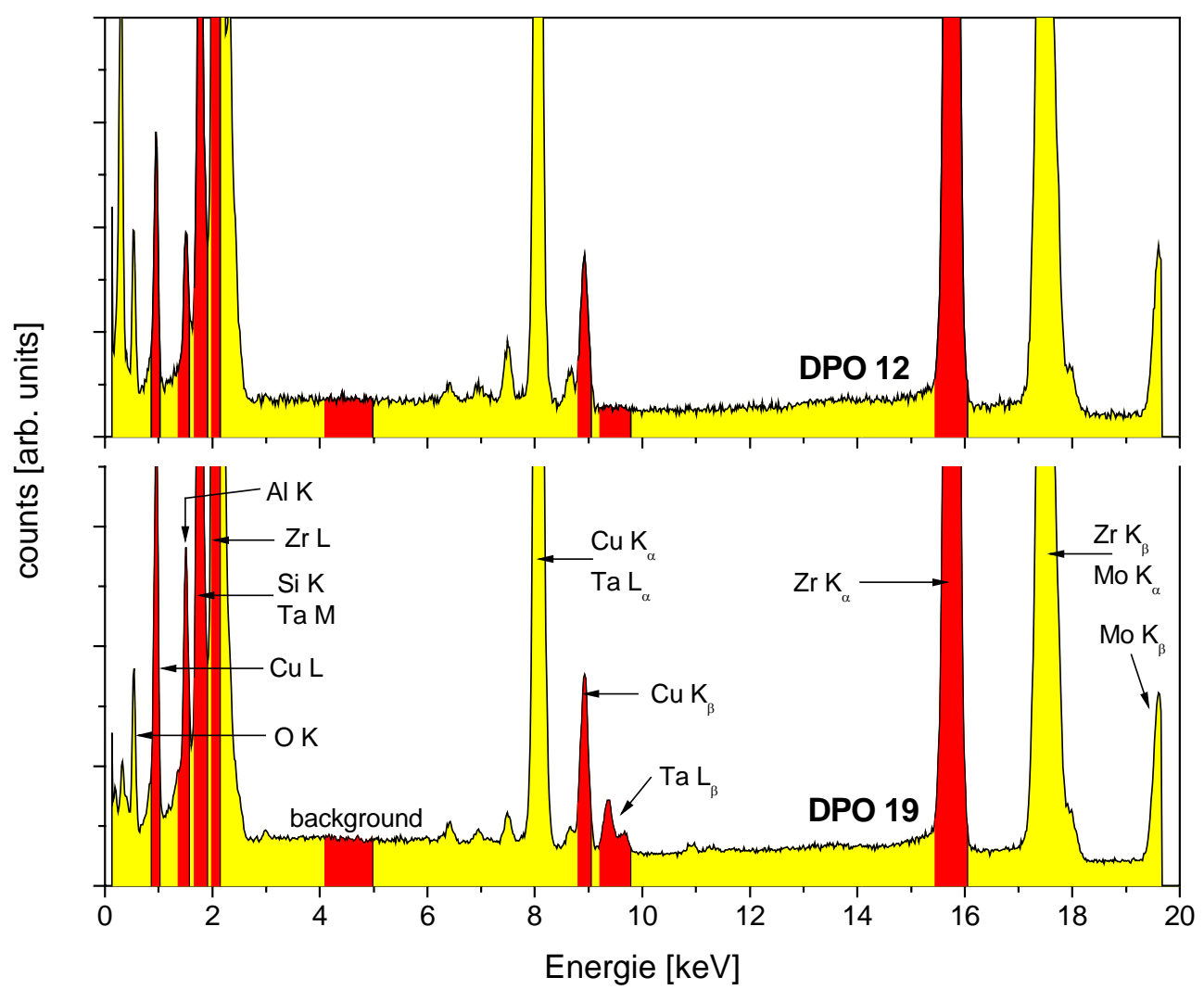

Abbildung 5.3: Energiespektrum von DPO 12 (oben) und DPO 19 (unten) gemittelt über den in Abbildung 5.2 gezeigten Querschliff der Schicht. Die rot markierten Linien dienen zur Konzentrationsberechnung.

In Abbildung 5.4 ist die Intensität der oben gekennzeichneten Energiebereiche von beiden untersuchten Oszillatoren in Wachstumsrichtung der Schicht gezeigt. Der vermeintlich starke Anstieg der Silizium-Intensität zwischen $-300 \mathrm{~nm}$ und -100 nm (links) ist mit der zunehmenden Dicke der Probe in diesem Bereich korreliert.
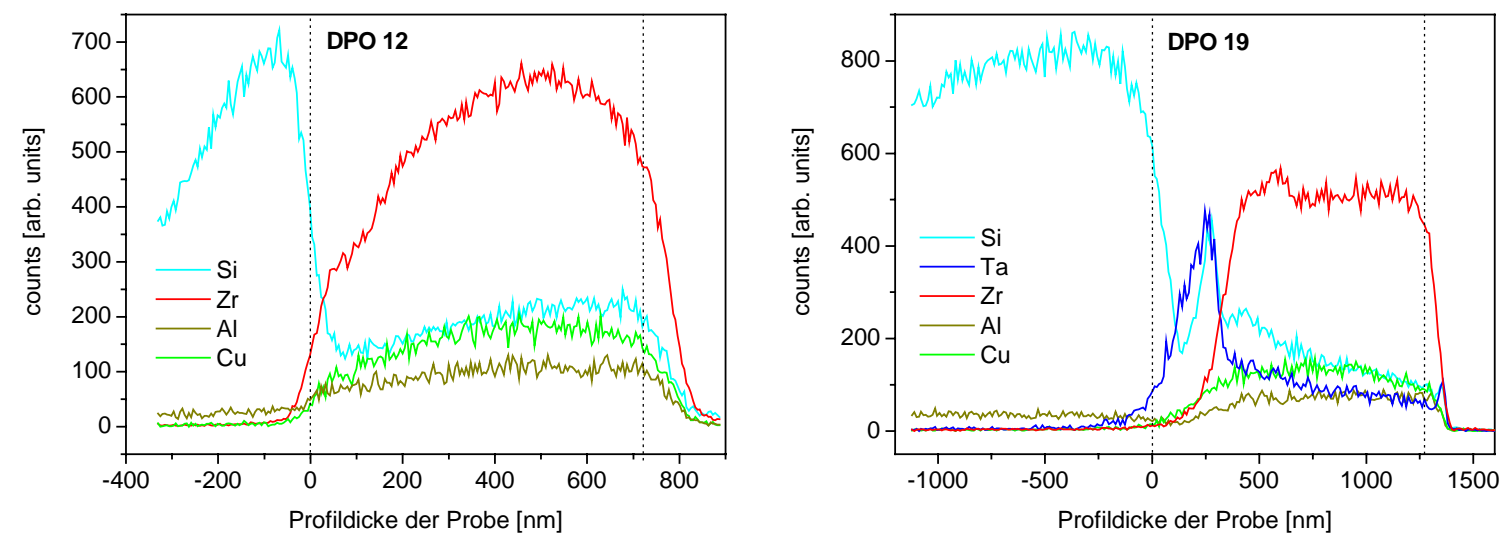

Abbildung 5.4: Intensitäten der in Abbildung 5.3 rot gekennzeichneten Energielinien aus einem EDX-Linescan entlang der in Abbildung 5.2 eingezeichneten Linien bei DPO 12 (links) und DPO 19 (rechts. 
In beiden Bildern ist klar erkennbar, dass die Silizium-Intensität an der Grenze SubstratSchicht deutlich abfällt, während gleichzeitig die Intensitäten für Zirkon, Kupfer und Aluminium ansteigen. Dass die Silizium-Intensität in der Schicht nicht, wie erwartet, auf null zurückgeht, kann an Verunreinigungen während des Ionenätzens bei der Präparation liegen, oder durch Fluoreszenzeffekte wegen der Nähe der Zr-L- und der Si-K-Linie erklärt werden.

Die Tantal-Zwischenschicht ist bei DPO 19 im rechten Bild durch die dunkelblaue Linie zu erkennen. Der korreliert auftretende Silizium-Peak (hellblau) tritt wegen der oben beschriebenen Überlagerung der Energiebereiche von Tantal und Silizium auf und ist nicht originär auf Silizium an dieser Schichtposition zurückzuführen.

Bei den Punktmessungen wird vom Gesamtsignal zunächst der Untergrund subtrahiert. Aus den Intensitätsverhältnissen der dann gemessenen elementspezifischen Energiebereiche kann dann die chemische Zusammensetzung an dieser Position berechnet werden. Beschränkt sich die Betrachtung auf die Komponenten Zirkon, Aluminium und Kupfer, so ergibt sich die Schichtkonzentration in Wachstumsrichtung. In Abbildung 5.5 ist gezeigt, dass die Schichten auf beiden Oszillatoren homogen aufgewachsen sind. Die Konzentration liegt für Zirkon etwas oberhalb der erwarteten 65 at\% und für Kupfer etwa bei dem eingestellten Wert von 27,5 at\%. Die Aluminium-Konzentration scheint sehr niedrig zu sein, was, wie oben beschrieben, durch die Position des für die Auswertung verwendeten Energiebereichs der AluminiumK-Linie verursacht sein könnte.
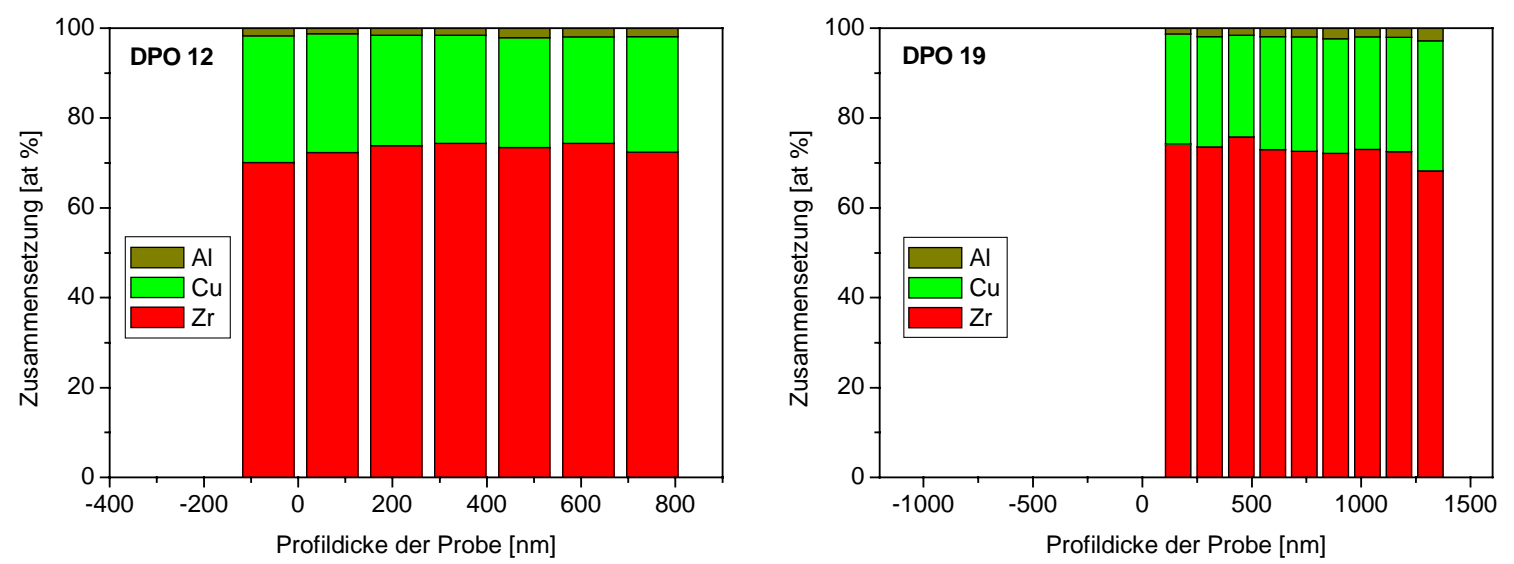

Abbildung 5.5: Chemische Zusammensetzung der Schichten auf DPO 12 (links) und DPO 19 (rechts) in Wachstumsrichtung, ermittelt durch EDX-Analyse an den in Abbildung 5.2 mit „, “ markierten Positionen. 


\subsubsection{Schichten auf Au-Netzchen}

Zur weiteren Überprüfung der korrekten chemischen Zusammensetzung wurden zwei $50 \mathrm{~nm}$ dicke $\mathrm{Zr}_{65} \mathrm{Al}_{7,5} \mathrm{Cu}_{27,5}$ Schichten untersucht, die unabhängig voneinander unter gleichen Bedingungen auf Gold-Netzchen aufgedampft wurden. Ihre Position in der UHV-Kammer entspricht genau der des Halses, falls ein DPO bedampft würde. Die bei der Auswertung störenden Silizium-Peaks im Energiespektrum sind nun ebenso ausgeschlossen, wie Verunreinigungen, die während des Ionenätzens auf der Probe abgelagert werden. Die Zusammensetzung der Schichten auf den Netzchen soll darüber Aufschluss geben, ob der in Abschnitt 5.1.3 ermittelte Aluminiumgehalt tatsächlich systematisch zu niedrig eingestellt wurde, oder ob es sich um einen Fehler handelt, der durch die Nähe des Aluminium- zum Silizium-Peak im Energiespektrum und durch Fluoreszenzeffekte wegen der keilförmigen Schichtdicke verursacht wird. Aus Tabelle 5.2 ist ersichtlich, dass die Ergebnisse dieser Kontrollmessungen gut mit der gewünschten Zusammensetzung von 7,5 at\% für den Aluminium-Anteil in den Schichten übereinstimmen.

\begin{tabular}{|l|c|c|c|}
\hline & Zr [at\%] & Al [at \%] & Cu [at \%] \\
\hline Au-Netzchen 1 & 65,38 & 6,78 & 27,84 \\
\hline Au-Netzchen 2 & 66,23 & 7,18 & 26,23 \\
\hline
\end{tabular}

Tabelle 5.2: Ergebnisse der Kontrollmessungen zur Zusammensetzung der Schichten auf Au-Netzchen

Das amorphe Wachstum der Schichten wurde ebenfalls an den bedampften Au-Netzchen überprüft. Abbildung 5.6 zeigt ein typisches Beugungsbild. Der helle Ring entspricht dem breiten Beugungsmaximum der nächsten Nachbaratome. Anhand der radial gemittelten Intensitätsverteilung kann, angedeutet durch die senkrechte weiße Linie, auch noch das zweite Maximum der übernächsten Nachbarn identifiziert werden. Jedenfalls sind keine kristallinen Reflexe in Form scharf ausgeprägter dünner Ringe zu erkennen was das amorphe Wachstum der Schichten beweist.

In Abbildung 5.7 ist die hochauflösende Hellfeldaufnahme einer solchen Schicht gezeigt. Auch hier sind keine kristallinen Bereiche zu erkennen. Die scheinbare Vorzugsrichtung könnte mit der Richtung der feinen Au-Drähte korreliert sein, aus denen das Au-Netzchen aufgebaut ist. 


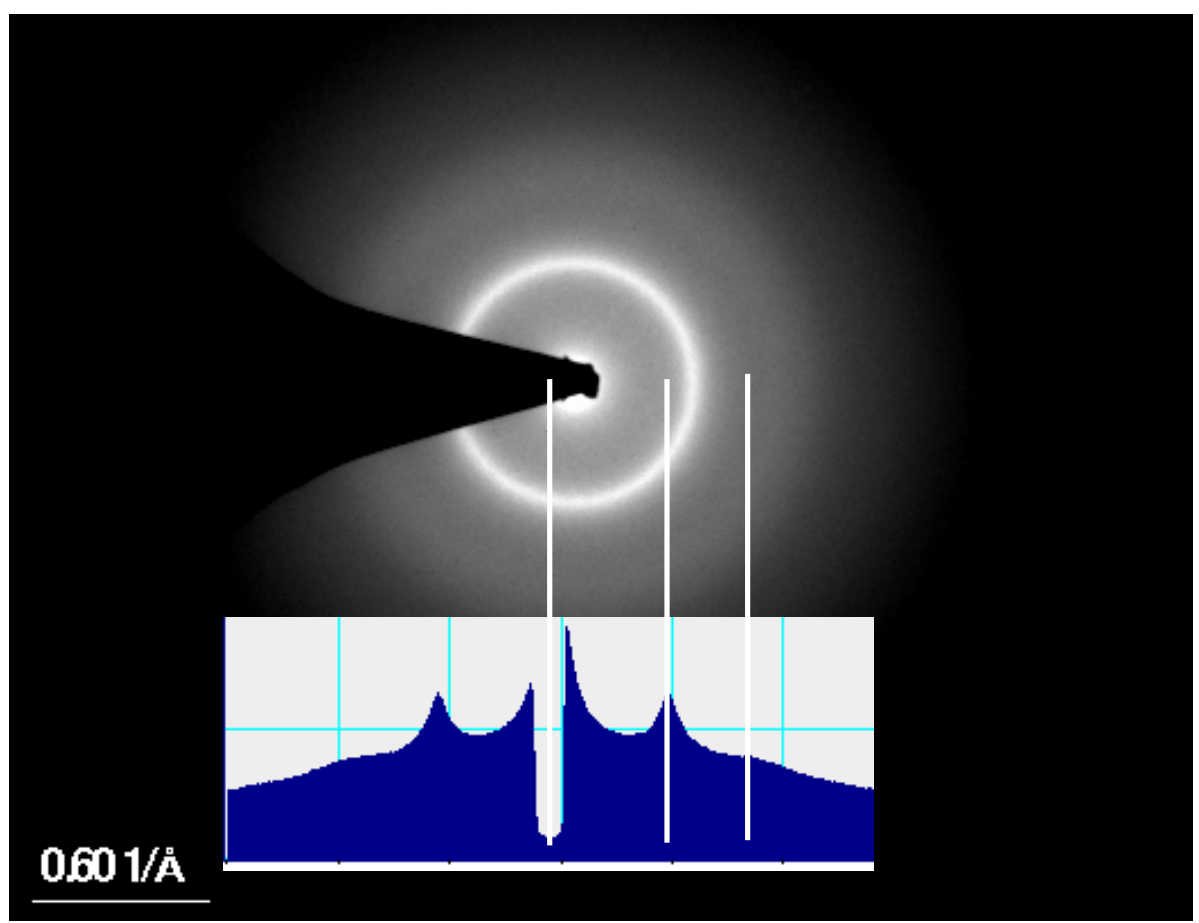

Abbildung 5.6: Beugungsbild einer $\mathrm{Zr}_{65} \mathrm{Al}_{7,5} \mathrm{Cu}_{27,5}$-Schicht auf einem Goldnetzchen. Das kleine Bild zeigt die radial gemittelte Intensitätsverteilung.

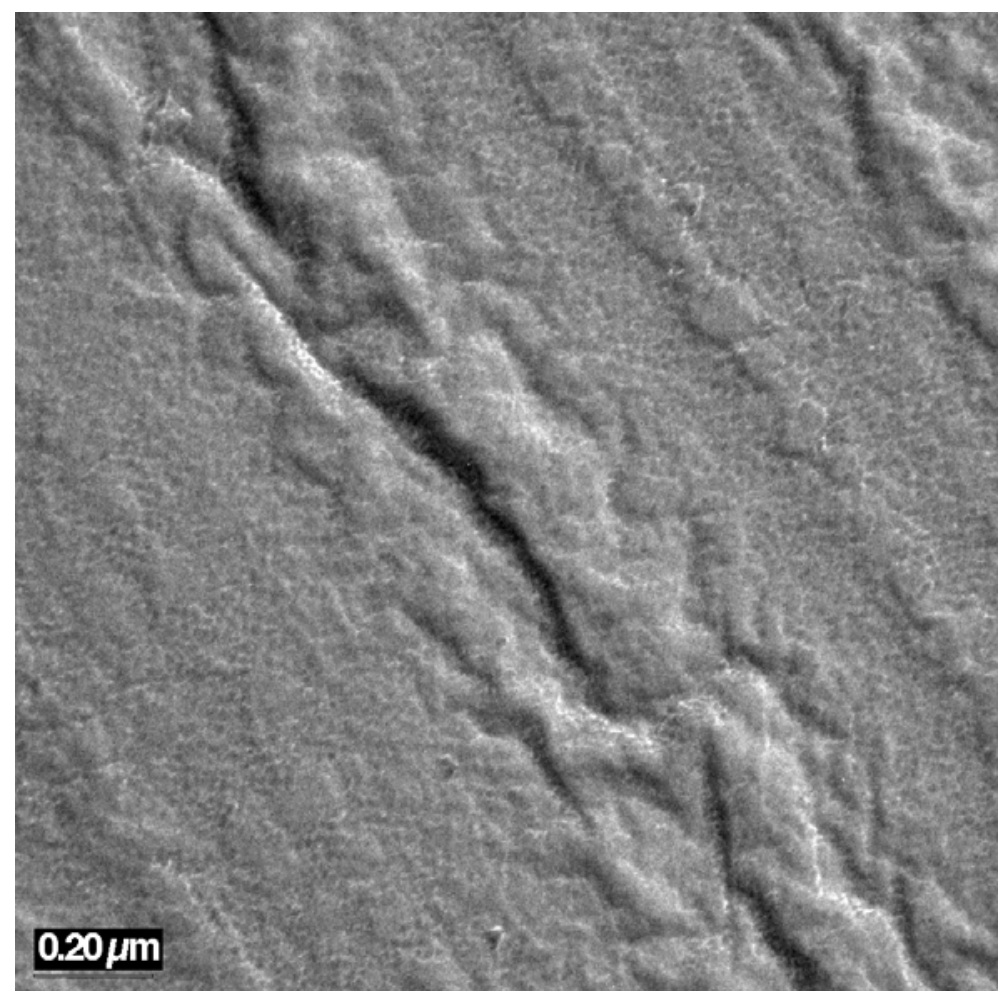

Abbildung 5.7: TEM-Hellfeldaufnahme einer auf ein Au-Netzchen aufgedampften $\mathrm{Zr}_{65} \mathrm{Al}_{7,5} \mathrm{Cu}_{27,5}$-Schicht. Die erkennbare Vorzugsrichtung von links oben nach rechts unten könnte mit der Struktur des Au-Netzchens korreliert sein. 


\subsection{Mechanische Spektroskopie}

In diesem Abschnitt werden exemplarisch die Ergebnisse aus dynamischen Messungen dünner Schichten des metallische Glases $\mathrm{Zr}_{65} \mathrm{Al}_{7,5} \mathrm{Cu}_{27,5}$ an vier verschiedenen Oszillatoren vorgestellt. Die hierfür verwendeten Paddel wurden nach dem in Abschnitt 4.2.2 erläuterten Cornell-Verfahren hergestellt und auf der Rückseite mit $\mathrm{Cr} / \mathrm{Au}$ bedampft (Abschnitt 4.3.2). Tabelle 5.3 zeigt im Überblick alle im Rahmen dieser Arbeit vorgestellten Schichten. Es hat sich herausgestellt, dass die mechanischen Eigenschaften der Schichten wesentlich von der Unterlage abhängen, auf die sie deponiert werden. Die im Folgenden präsentierten Ergebnisse sind deshalb nach Substratmaterialien geordnet. In Abschnitt 5.2.1 werden Schichten gezeigt, die direkt auf leere Silizium-Oszillatoren aufgedampft wurden. Bei den in Abschnitt 5.2.2 gezeigten Schichten dient eine kristallisierte $\mathrm{Zr}_{65} \mathrm{Al}_{7,5} \mathrm{Cu}_{27,5}$ Schicht als Untergrund, während die Schichten in Abschnitt 5.2.3 auf einer Tantal-Zwischenschicht deponiert sind.

\begin{tabular}{|c|c|c|c|}
\hline Oszillator & Bezeichnung & Untergrund & Schichtdicke \\
\hline DPO 12 & Schicht 1 & Si (leeres Paddel) & $300 \mathrm{~nm}$ \\
\hline & Schicht 2 & ZrAlCu - kristallin & $300 \mathrm{~nm}$ \\
\hline DPO 19 & Schicht 1 & $50 \mathrm{~nm}$ Ta & $300 \mathrm{~nm}$ \\
\hline & Schicht 2 & ZrAlCu - kristallin & $300 \mathrm{~nm}$ \\
\hline & Schicht 3 & ZrAlCu - kristallin & $600 \mathrm{~nm}$ \\
\hline DPO 21 & Schicht 1 & Si (leeres Paddel) & $300 \mathrm{~nm}$ \\
\hline DPO 22 & Schicht 1 & $50 \mathrm{~nm} \mathrm{Ta}$ & $356 \mathrm{~nm}$ \\
\hline
\end{tabular}

Tabelle 5.3: Überblick über die gemessenen Schichten auf verschiedenen Unterlagen und Oszillatoren

\subsection{1 $\mathrm{Zr}_{65} \mathrm{Al}_{7,5} \mathrm{Cu}_{27,5}$ auf Silizium (leeres Paddel)}

\subsubsection{Verschiebung der Resonanzfrequenz bei DPO 12 Schicht 1}

Zunächst wird in Abbildung 5.8 die Verschiebung der AS2-Resonanzfrequenz betrachtet, nachdem auf DPO 12 eine $300 \mathrm{~nm}$ dicke $\mathrm{Zr}_{65} \mathrm{Al}_{7,5} \mathrm{Cu}_{27,5}$-Schicht aufgedampft wurde. Der AS2-Frequenzunterschied zwischen bedampftem und leerem Oszillator beträgt bei Raumtemperatur etwa $-4 \mathrm{~Hz}$. Er verändert sich mit steigender Temperatur zu kleineren Werten (rote Quadrate), bevor er nach einem Minimum von etwa $-12 \mathrm{~Hz}$ bei $550 \mathrm{~K}$ wieder leicht ansteigt. 
Aus Gleichung (4.41) ist ersichtlich, dass es zwei verschiedene Beiträge mit entgegengesetztem Vorzeichen zur Frequenzverschiebung gibt. Die AS2-Resonanzfrequenz wird durch das zusätzliche Trägheitsmoment, bedingt durch die Masse der Schicht, zu kleineren Werten verschoben. Die elastischen Beiträge der Schicht bewirken stattdessen eine Verschiebung zu größeren Frequenzen.

Mit der Silizium-Dichte $\rho_{\text {sub }}=2332 \mathrm{~kg} / \mathrm{m}^{3}$ und der $\mathrm{Zr}_{65} \mathrm{Al}_{7,5} \mathrm{Cu}_{27,5}$ Dichte $\rho_{\text {film }}=6892 \mathrm{~kg} / \mathrm{m}^{3}$, der Paddeldicke $t_{\text {sub }}=300 \mu \mathrm{m}$ und der Schichtdicke $t_{\text {film }}=300 \mathrm{~nm}$ ergäbe sich eine massebedingte Verschiebung der gemessenen Resonanzfrequenz von $\mathrm{f}_{\mathrm{AS} 2}=5400 \mathrm{~Hz}$, gemäß Gleichung (4.43), um $\left.\Delta \mathrm{f}_{\mathrm{AS} 2}\right|_{\text {Masse }}=-7,98 \mathrm{~Hz}$.

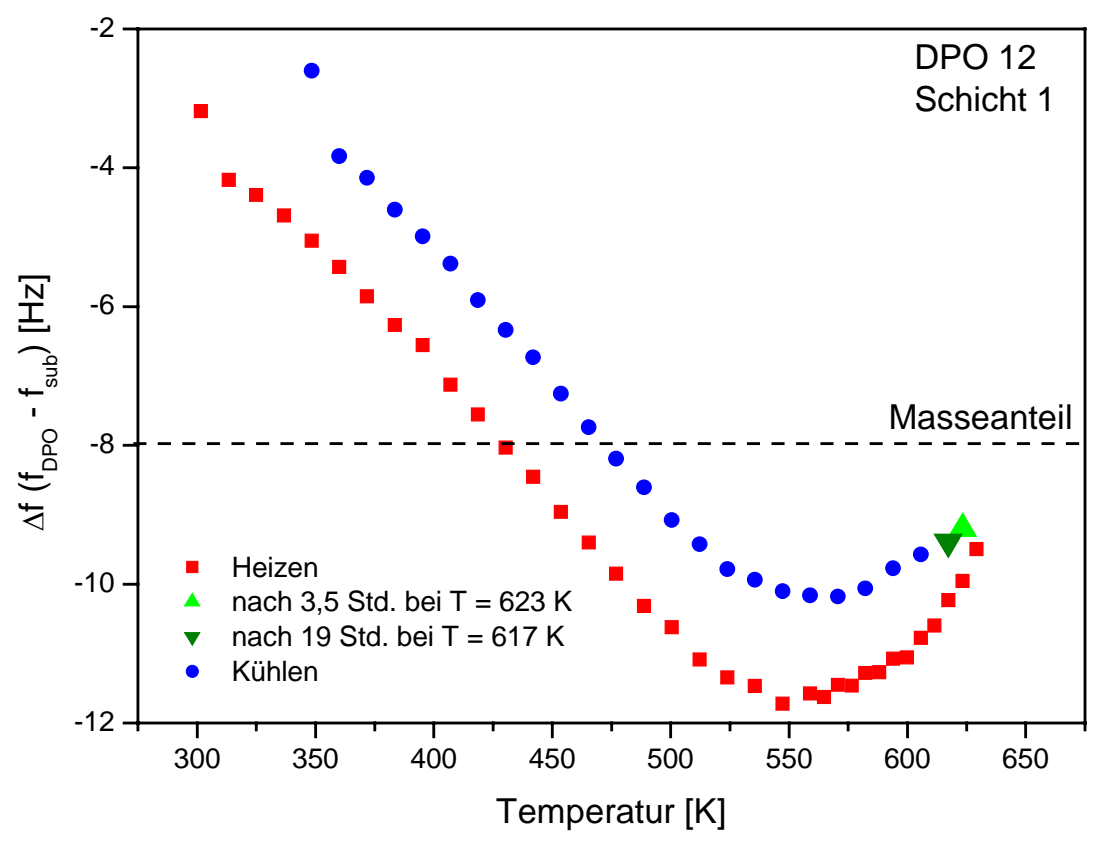

Abbildung 5.8: Temperaturverlauf der Differenz der AS2-Resonanzfrequenz der ersten Schicht auf DPO 12 beim Heizen, nach isothermer Auslagerung und beim Kühlen.

Da der in Gleichung (4.42) berechnete elastische Beitrag ein positives Vorzeichen hat, sind die genannten $-7,98 \mathrm{~Hz}$ eine untere Grenze für die Frequenzverschiebung (angedeutet durch die gestrichelte Linie in Abbildung 5.8). Erklärungsansätze, warum die Frequenzdifferenz bei höheren Temperaturen trotzdem deutlich unterhalb diese Grenze fällt, werden in Abschnitt 7.2 diskutiert.

Nach dem Aufheizen auf $630 \mathrm{~K}$ wurde die Schicht zunächst auf $623 \mathrm{~K}$ abgekühlt und isotherm für 3,5 Stunden ausgelagert. Anschließend wurde die Schicht auf $617 \mathrm{~K}$ abgekühlt und 
19 Stunden ausgelagert. Bei beiden Temperaturen wurde sowohl die Änderung der Resonanzfrequenz, als auch die Dämpfung fortwährend aufgezeichnet. Schließlich wurde die Probe wieder auf Raumtemperatur abgekühlt (blaue Kreise).

Abbildung 5.8 zeigt, dass die Resonanzverschiebung zwischen bedampftem und leerem Paddel während des Heiz- und Abkühlvorgangs nicht reversibel ist. Die negative Frequenzdifferenz ist beim Kühlen über nahezu den gesamten untersuchten Temperaturbereich um 1,5 Hz geringer. Da eine Veränderung der Masse ausgeschlossen wird, bedeutet das, dass sich die elastischen Anteile (Gleichung 4.42) entsprechend vergrößert haben.

\subsubsection{Dämpfungsfaktor bei DPO 12 Schicht 1}

Betrachtet man den Dämpfungsfaktor, so zeigt sich in Abbildung 5.9, dass die Dämpfung des bedampften Oszillators über die gesamte Messzeit und für das gesamte untersuchte Temperaturintervall größer ist als für das leere Paddel (durchgezogene schwarze Linie). Um die beobachteten Effekte deutlicher darzustellen, wird in Abbildung 5.10 die Differenz der Dämpfungsfaktoren zwischen bedampftem und leerem DPO 12 aufgetragen.

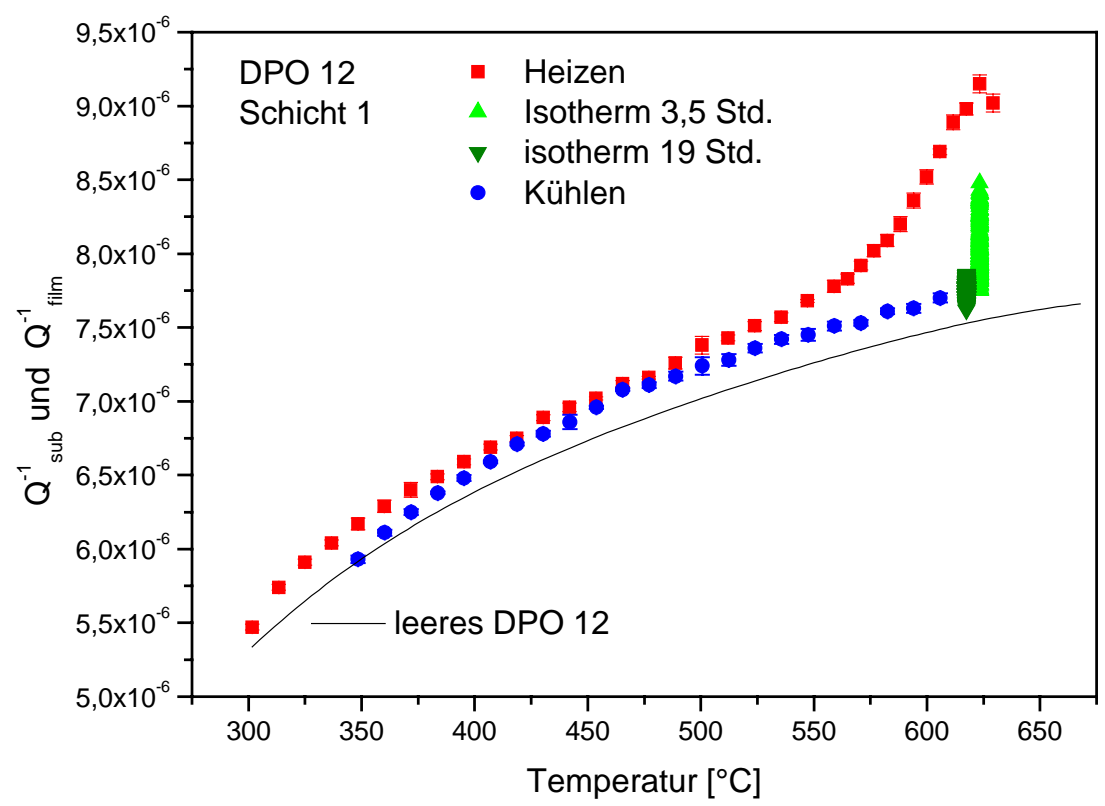

Abbildung 5.9: AS2-Dämpfungsfaktor des leeren (schwarze Linie) DPO 12 und des mit Schicht 1 bedampften Oszillator DPO 12 während des Heizens, isothermen Auslagerns und Kühlens. 


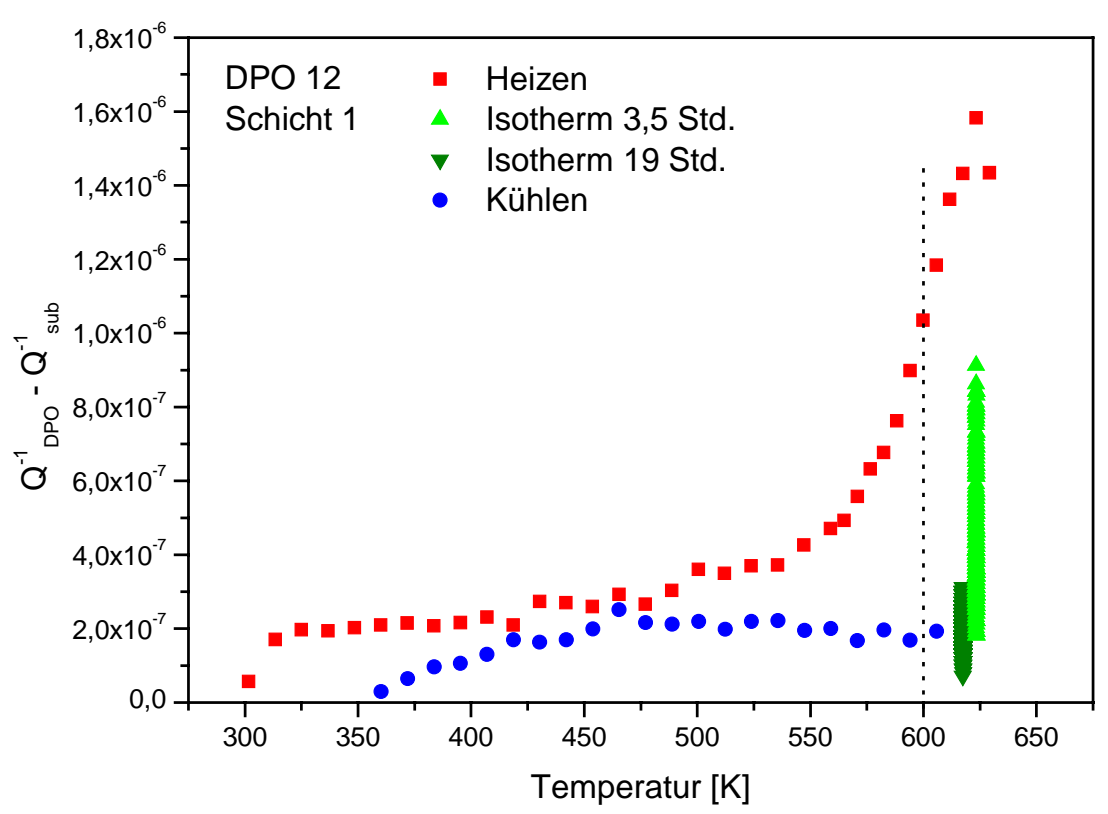

Abbildung 5.10: Differenz der Dämpfungsfaktoren zwischen leerem und mit Schicht 1 bedampften DPO 12.

Die Differenz der Dämpfungsfaktoren steigt beim Heizen von etwa $2 \times 10^{-7}$ bei Raumtemperatur auf etwa $4 \times 10^{-7}$ bei $550 \mathrm{~K}$ relativ gleichmäßig an. Dann nimmt der Anstieg dramatisch zu und erreicht bei $623 \mathrm{~K}$ sein Maximum von $1,6 \times 10^{-6}$, bevor sich bei noch höheren Temperaturen ein ebenso dramatischer Abfall andeutet.

Während der isothermen Messung bei $623 \mathrm{~K}$ fällt die Dämpfungsdifferenz mit der Zeit auf $2 \times 10^{-7} \mathrm{ab}$. In den 19 Stunden isothermen Auslagerns bei $617 \mathrm{~K}$ bleibt dieser Wert im Rahmen des Fehlers nahezu konstant. Auch während des nun folgenden Abkühlens auf Raumtemperatur bleibt die Dämpfungsdifferenz nahezu gleich. In weiteren Heiz- und Kühlzyklen wird die in Abbildung 5.10 gezeigte blaue Kurve nahezu reversibel durchlaufen.

\subsubsection{Isotherme Entwicklung bei DPO 12 Schicht 1}

Die isotherme Entwicklung während des Auslagerns bei $623 \mathrm{~K}$ ist in Abbildung 5.11 für die Differenz des Dämpfungsfaktors und in Abbildung 5.12 für die Frequenz dargestellt.

Aus dem zeitlichen Verlauf lässt sich ein guter Eindruck über die Unsicherheit und den statistischen Fehler der Messwerte gewinnen. Die Schwankungsbreite beträgt für die Dämpfungsdifferenz etwa $1 \times 10^{-7}$ und für die Frequenzdifferenz rund $0,15 \mathrm{~Hz}$. Diese Werte gelten 
bei hohen Temperaturen analog für alle anderen Darstellungen in diesem Kapitel, wenn nicht explizit etwas anderes vermerkt ist.

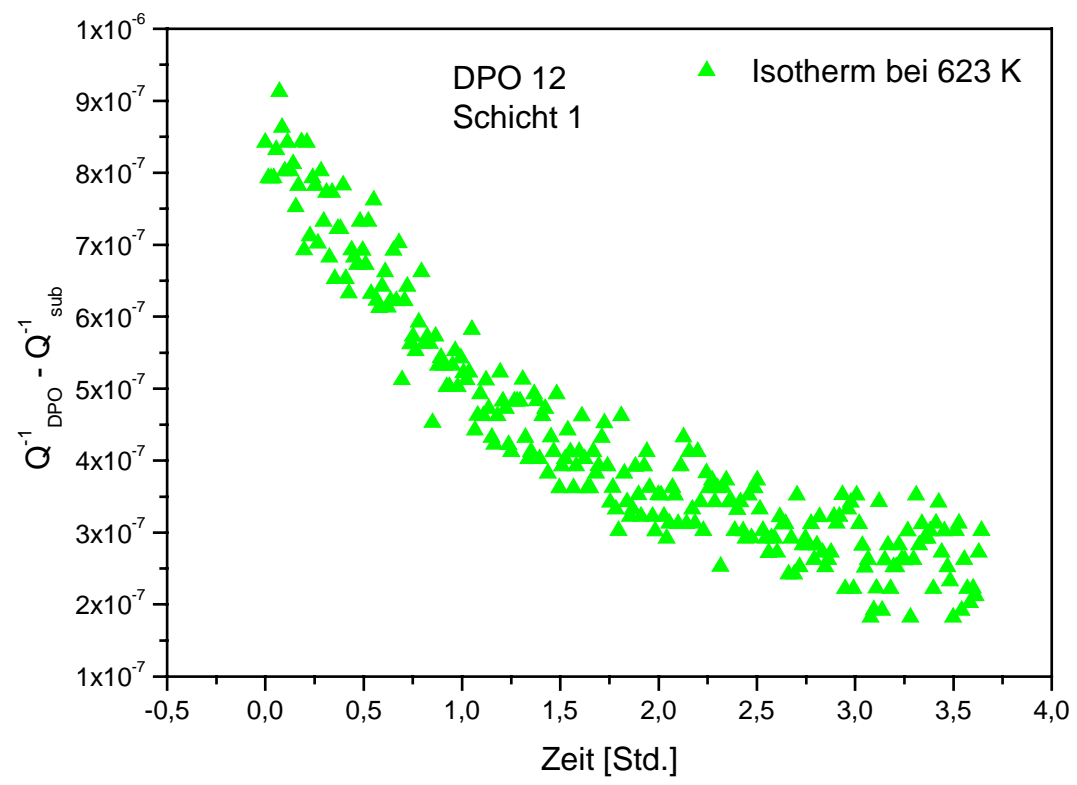

Abbildung 5.11: Isotherme Entwicklung der Dämpfungsdifferenz zwischen Schicht 1 auf DPO 12 und Substrat bei $623 \mathrm{~K}$

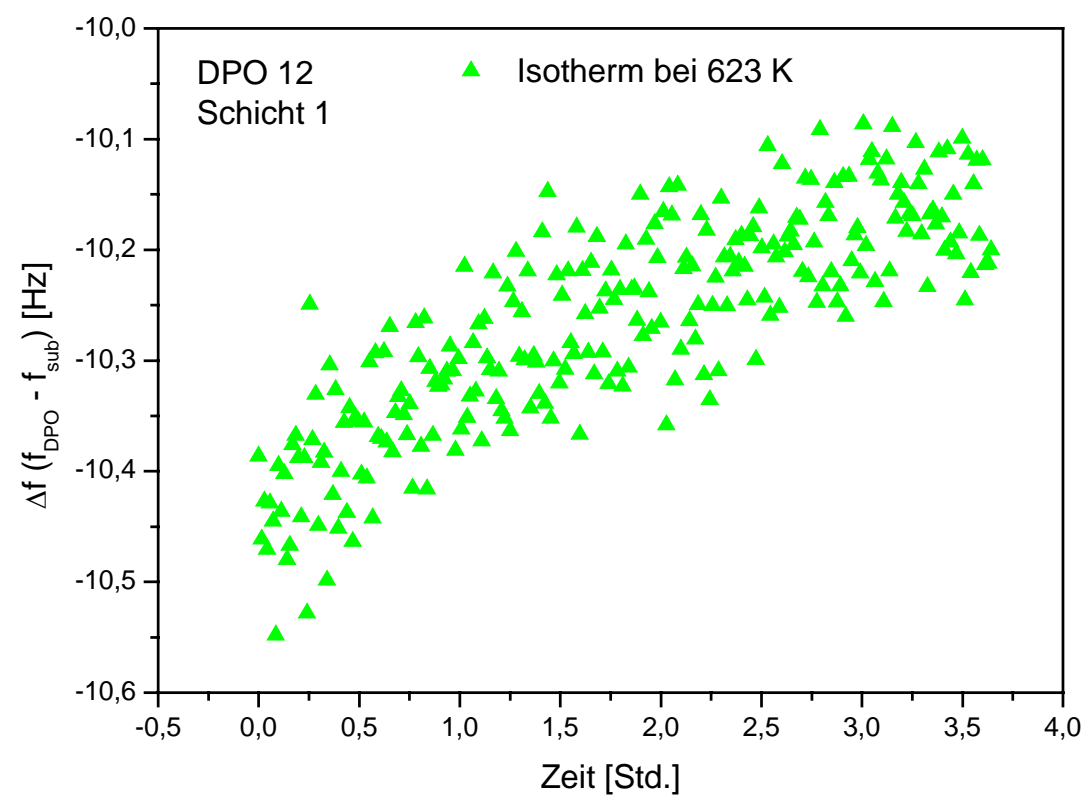

Abbildung 5.12: Isotherme Entwicklung der AS2-Frequenzdifferenz zwischen Schicht 1 auf DPO 12 und Substrat bei $623 \mathrm{~K}$. 


\subsubsection{Vergleich von DPO 12 Schicht 1 und DPO 21 Schicht 1}

Zur Verifizierung der Ergebnisse, insbesondere des überraschenden Frequenzverlaufs, wurde auch bei DPO 21 eine $300 \mathrm{~nm}$ dicke $\mathrm{Zr}_{65} \mathrm{Al}_{7,5} \mathrm{Cu}_{27,5}$-Schicht direkt auf den leeren Siliziumoszillator aufgedampft und untersucht. In Abbildung 5.13 ist die Frequenzdifferenz in Abhängigkeit von der Temperatur während des ersten Heizzyklus bei DPO 12 und bei DPO 21 vergleichend aufgetragen. Bei DPO 12 wurden die Daten ab $500 \mathrm{~K}$ in $7 \mathrm{~K}-\mathrm{Sch}$ ritten gemessen, wohingegen die Daten bei DPO 21 in $1 \mathrm{~K}-$ Schritten aufgenommen wurden. Für die Heizrate ergibt sich daraus 0,26 K/min für DPO 12 und 0,09 K/min für DPO 21.

Es zeigt sich, dass der Kurvenverlauf bei beiden Schichten ähnlich ist. Allerdings fällt die Kurve bei DPO 12 steiler ab und das Minimum ist mit etwa $-12 \mathrm{~Hz}$ deutlicher ausgeprägt als das Minimum von etwa $-10 \mathrm{~Hz}$ bei DPO 21. Bei beiden Messungen wird das durch den Massebeitrag erwartete negative Limit von $-7,98 \mathrm{~Hz}$ (Abschnitt 5.2.1.1) deutlich unterschritten.

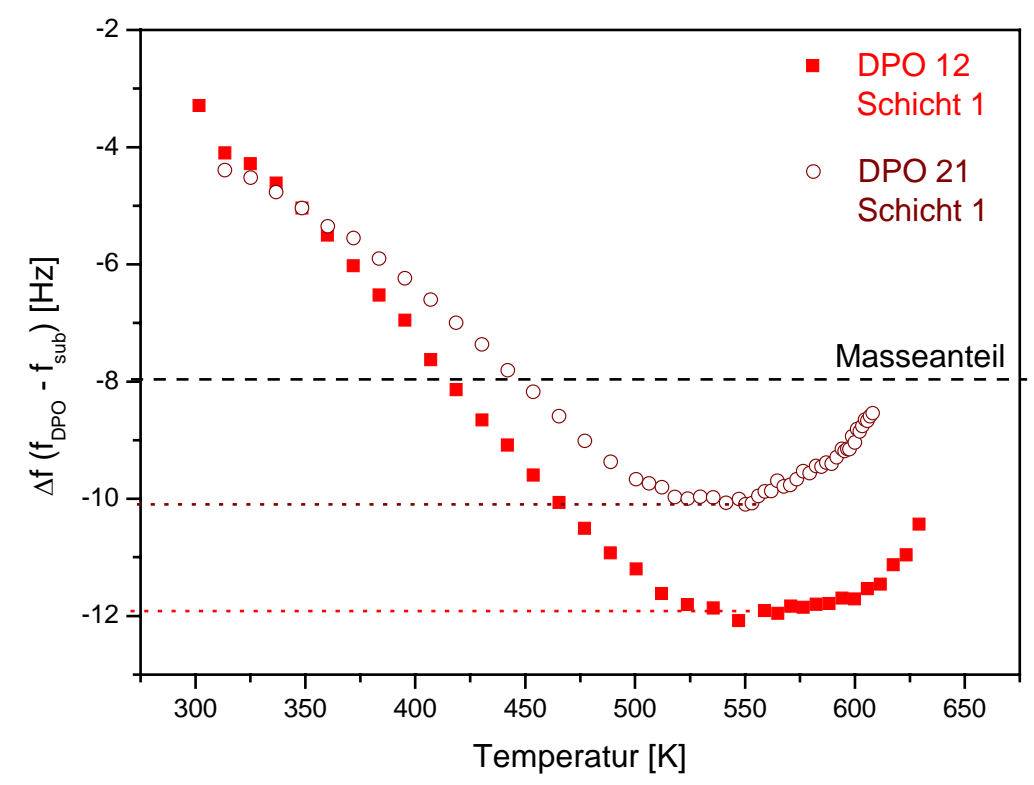

Abbildung 5.13: Frequenzdifferenz zwischen bedampften und leerem Paddel während des ersten Aufheizens der Schicht bei DPO 12 (ausgefüllte Quadrate) und DPO 21 (offene Kreise).

Beim Vergleich der Dämpfungsdifferenz in Abbildung 5.14 zeigt sich, dass die Werte von DPO $21 \mathrm{im}$ Temperaturbereich zwischen $350 \mathrm{~K}$ und $550 \mathrm{~K}$ höher liegen als die entsprechenden von DPO 12. Der Anstieg bis $550 \mathrm{~K}$ verläuft bei DPO 21 flacher, so dass die Werte für beide Messungen bei etwa $560 \mathrm{~K}$ gleich groß sind. Eine weitere Temperaturerhöhung führt in 
beiden Schichten zu einer starken Zunahme der Dämpfung, wiewohl sie bei DPO 21 nicht so dramatisch ausfällt wie bei DPO 12.

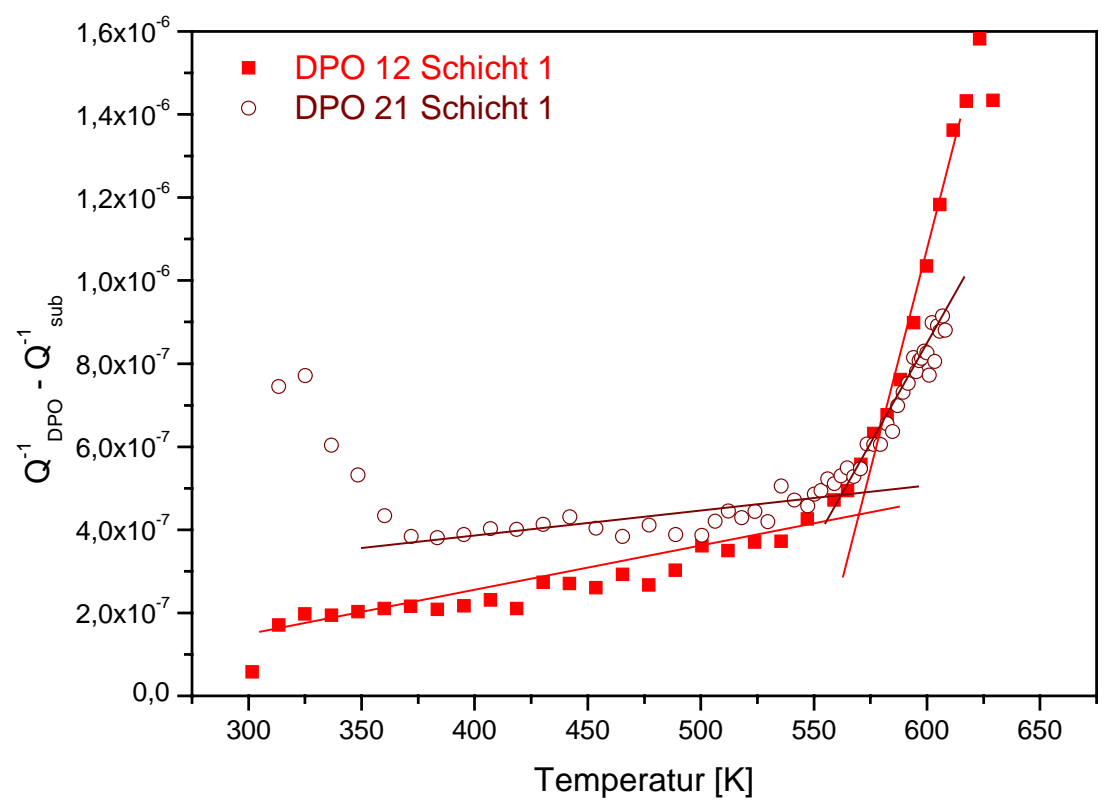

Abbildung 5.14: Dämpfungsdifferenz zwischen bedampftem und leerem Paddel während des ersten Aufheizens der Schicht bei DPO 12 (ausgefüllte Quadrate) und DPO 21 (offene Kreise) mit eingezeichneten Trendlinien.

\subsection{2 $\mathrm{Zr}_{65} \mathrm{Al}_{7,5} \mathrm{Cu}_{27,5}$ auf kristallinem Untergrund}

Um den hohen Aufwand zu umgehen, für jede Schicht einen neuen Oszillator einbauen zu müssen, wird folgende Strategie verfolgt: Wenn die Messungen an der ersten Schicht abgeschlossen sind, wird der Oszillator auf $700 \mathrm{~K}$ geheizt. Die Schicht ist nun vollständig kristallisiert. $\mathrm{Da}$ es in kristallinen $\mathrm{Zr}_{65} \mathrm{Al}_{7,5} \mathrm{Cu}_{27,5}$-Schichten unterhalb von $700 \mathrm{~K}$ keine strukturellen Änderungen gibt, wird erwartet, dass sowohl die Resonanzfrequenz, als auch die Dämpfung des Gesamtsystems nur von der Temperatur abhängen und reversibel durchlaufen werden. Dass dem so ist, wurde in mehreren Experimenten überprüft. Sowohl die Frequenz, als auch die Dämpfung hängen reproduzierbar von der Temperatur ab. Das Gesamtsystem des Oszillators mit der kristallinen Schicht wird nun als Substrat für eine neu aufzubringende Schicht genutzt. Während des Abkühlens von $700 \mathrm{~K}$ auf Raumtemperatur werden daher die Frequenz und die Dämpfung gemessen. Diese Referenzdaten werden zur Berechnung der Frequenzund der Dämpfungsdifferenz der folgenden Schicht subtrahiert. 


\subsubsection{Verschiebung der Resonanzfrequenz bei DPO 12 Schicht 2}

In Abbildung 5.15 ist die Verschiebung der AS 2-Resonanzfrequenz in Bezug zur neu definierten Referenz durch die $300 \mathrm{~nm}$ dicke Schicht 2 auf DPO 12 gezeigt [148]. Verglichen mit Schicht 1 auf DPO 12 (Abbildung 5.8) zeigt sich ein völlig anderer Verlauf. Nach dem Aufdampfen der Schicht beträgt der Frequenzunterschied etwa -4,5 Hz. Die Verschiebung zu negativen Werten verringert sich mit steigender Temperatur. Bei etwa $600 \mathrm{~K}$ ändert sich die Frequenzdifferenz dramatisch, bevor sie sich bei etwa $-2,5 \mathrm{~Hz}$ stabilisiert. Dieser Wert bleibt auch beim Abkühlen, sowie bei weiteren Temperaturzyklen konstant.

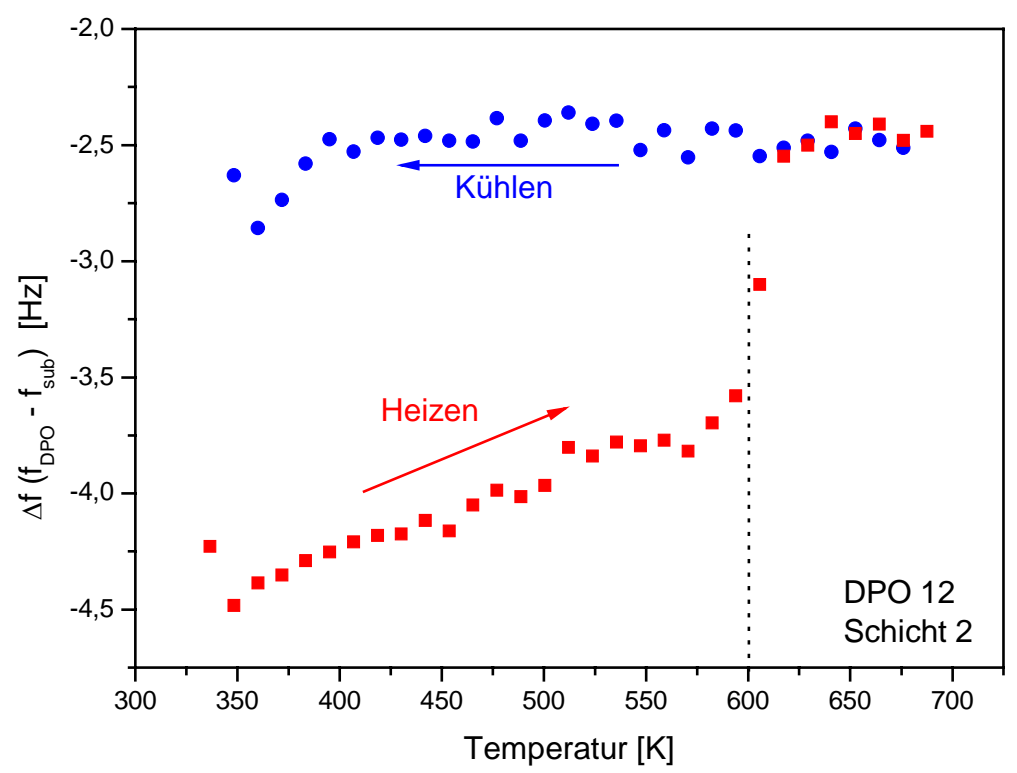

Abbildung 5.15: AS2-Frequenzverschiebung durch Schicht 2 auf DPO 12. Referenz ist DPO 12 mit vollständig kristallisierter Schicht 1.

\subsubsection{Skalierung der Frequenzverschiebung mit der Schichtdicke}

Um die Skalierung der Frequenzverschiebung mit der Schichtdicke zu analysieren, wurde bei DPO 19 auf kristallinem Untergrund die 600 nm dicke Schicht 3 aufgedampft. Die Frequenzverschiebung ist in Abbildung 5.16 gezeigt. Offensichtlich ist der qualitative Kurvenverlauf unabhängig von der Schichtdicke. Der Frequenzunterschied bei der $600 \mathrm{~nm}$ dicken Schicht beträgt bei Raumtemperatur etwa $-7,25$, im Vergleich $\mathrm{zu}-4,5 \mathrm{~Hz}$ bei der $300 \mathrm{~nm}$ dicken Schicht. Mit dem in Abschnitt 4.7.1 vorgestellten Formalismus würde man erwarten, dass die Frequenzverschiebung mit der Schichtdicke skaliert. Bei doppelter Schichtdicke müsste sie doppelt so groß ausfallen. Mögliche Ursachen, warum der Skalierungsfaktor hier nicht bei 2, 
sondern nur bei 1,6 liegt, werden in Abschnitt 7.2.3 diskutiert. Der dramatische Anstieg zu kleineren negativen Werten wird auch bei der dicken Schicht beobachtet. Er setzt bei etwa $620 \mathrm{~K}$ ein und damit etwas später als bei der dünneren Schicht.

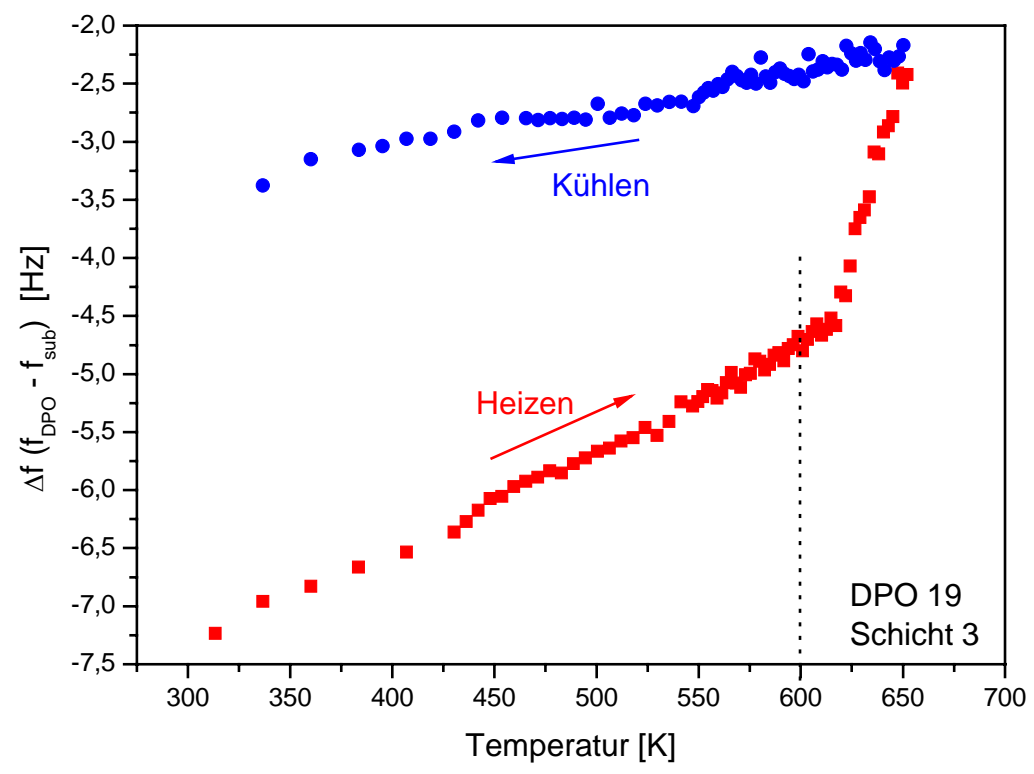

Abbildung 5.16: Frequenzverschiebung DPO 19 Schicht 3 (600 nm). Referenz ist DPO 19 mit $50 \mathrm{~nm}$ Tantal und vollständig kristallisierten Schichten 1 und 2.

\subsubsection{Frequenzverschiebung bei mehrstufigem Temperaturprogramm}

Schicht 2 auf DPO 19 wird mit einem mehrstufigen Temperaturprogramm analysiert. Wie in Abbildung 5.17 gezeigt, erfolgt der primäre Heizvorgang nur bis $590 \mathrm{~K}$ (rote Quadrate) und endet damit klar vor dem Beginn des starken Anstiegs im Frequenzunterschied. Der Kurvenverlauf beim anschließenden Abkühlen (blaue Kreise) weicht von der Heizkurve ab, was für irreversible Veränderungen bereits bei niedrigen Temperaturen spricht. Wird ein zweites Mal geheizt (orange Quadrate), so entspricht der Kurvenverlauf für den Frequenzunterschied reproduzierbar dem des vorhergehenden Abkühlens. Bei etwa $600 \mathrm{~K}$ ist wieder ein starker Anstieg zu erkennen. Ist die Endtemperatur des zweiten Heizvorgangs von $625 \mathrm{~K}$ erreicht, wird bis $460 \mathrm{~K}$ wieder abgekühlt (violette Kreise). Der folgende dritte Temperaturzyklus, nun bis 650 K Maximaltemperatur, zeigt keine wesentlichen Veränderungen im Kurvenverlauf mehr. Er reproduziert die Messwerte des zweiten Zyklus im Rahmen des Messfehlers. 
Anzumerken ist, dass die Frequenzverschiebung bei Schicht 2 auf DPO 19 nach der Herstellung bei Raumtemperatur $-3,25 \mathrm{~Hz}$ beträgt, was trotz gleicher Schichtdicke nicht mit den gemessenen $-4,5 \mathrm{~Hz}$ bei Schicht 2 auf DPO 12 übereinstimmt.

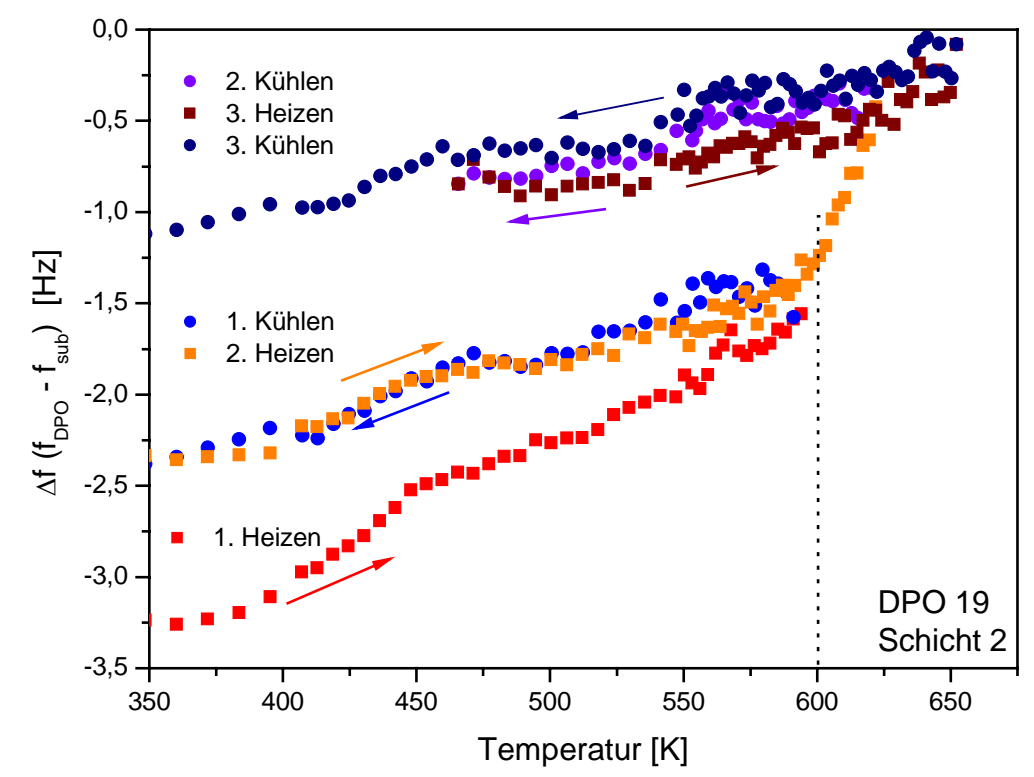

Abbildung 5.17: Frequenzverschiebung DPO 19 Schicht 2 (300 nm). Referenz ist DPO 19 mit 50 nm Tantal und vollständig kristallisierter Schicht 1. Dargestellt sind drei aufeinanderfolgende Temperaturzyklen.

\subsubsection{Dämpfungsfaktor bei DPO 12 Schicht 2}

In Abbildung 5.18 ist der Verlauf der Differenz der Dämpfungsfaktoren zwischen Substrat und Schicht 2 auf DPO 12 dargestellt. Verglichen mit Abbildung 5.10 macht es bei der Dämpfung kaum einen Unterschied, ob die Schicht auf das leere Siliziumsubstrat oder auf eine bereits kristallisierte $\mathrm{Zr}_{65} \mathrm{Al}_{7,5} \mathrm{Cu}_{27,5}$ Unterlage aufgedampft wird.

Wie in Abbildung 5.10 für Schicht 1 gezeigt, beträgt die Differenz der Dämpfungsfaktoren bei Raumtemperatur auch für Schicht 2 etwa $2 \times 10^{-7}$. Der Dämpfungsunterschied steigt mit der Temperatur allmählich an, bevor bei etwa $550 \mathrm{~K}$ ein dramatischer Anstieg bis zum Maximum von $1,6 \times 10^{-6}$ bei etwa $620 \mathrm{~K}$ einsetzt. Dann folgt ein scheinbar sprunghafter Abfall auf den Wert von $2 \times 10^{-7}$ zurück, der sich beim Abkühlen und auch bei weiteren Temperaturzyklen kaum mehr ändert [148]. 


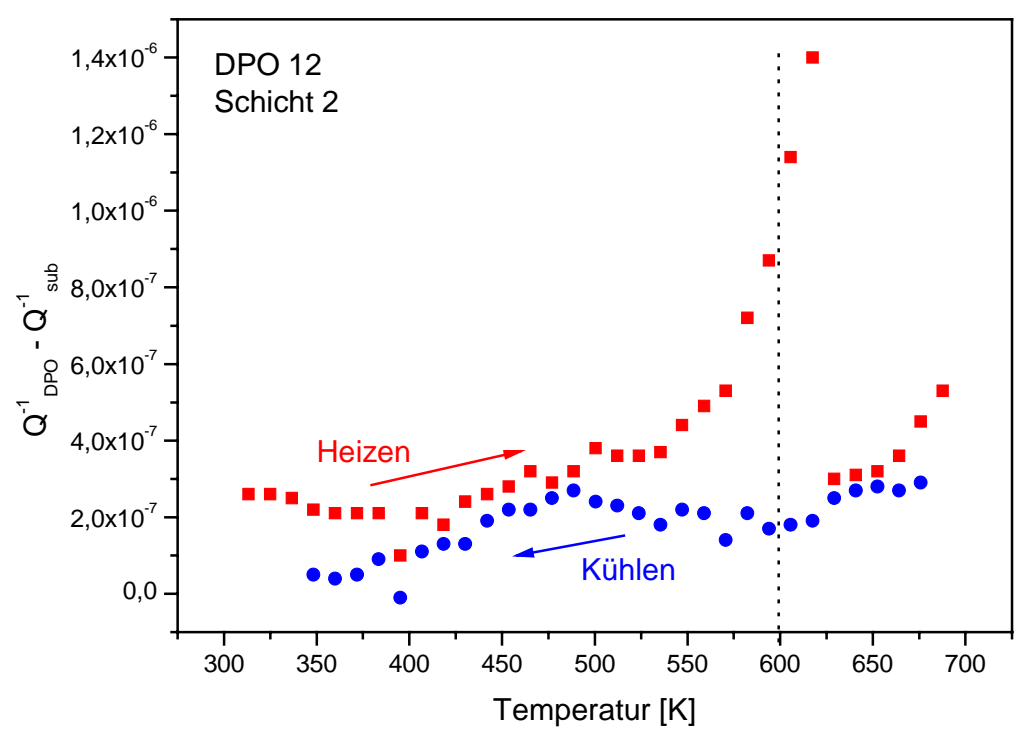

Abbildung 5.18: Differenz der Dämpfungsfaktoren zwischen Schicht 2 und Substrat (Si-Paddel mit $300 \mathrm{~nm}$ kristallisierte Schicht) bei DPO 12

\subsubsection{Skalierung des Dämpfungsfaktors mit der Schichtdicke}

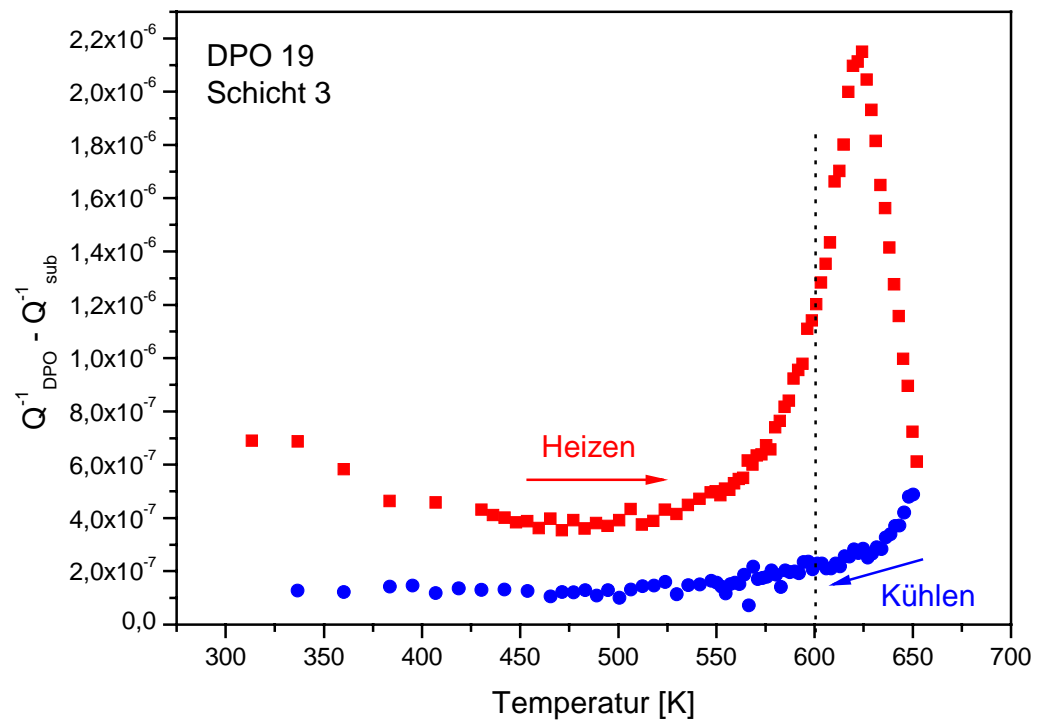

Abbildung 5.19: Differenz der Dämpfungsfaktoren zwischen DPO 19 Schicht 3 (600 nm) und Substrat.

Der Beitrag der $600 \mathrm{~nm}$ dicken Schicht 3 auf DPO 19 zur Dämpfung ist in Abbildung 5.19 dargestellt. Der Dämpfungsunterschied beträgt bis etwa $500 \mathrm{~K}$ im Rahmen des Fehlers $4 \times 10^{-7}$ und ist damit doppelt so groß wie bei den untersuchten $300 \mathrm{~nm}$ dicken Schichten. Auch hier 
beginnt die Dämpfung bei etwa $550 \mathrm{~K}$ dramatisch anzusteigen. Das Maximum liegt bei $625 \mathrm{~K}$ und ist mit $2,2 \times 10^{-6}$ deutlich höher als bei den dünneren Schichten.

\subsubsection{Dämpfung bei mehrstufigem Temperaturprogramm}

Wie für die Frequenz in Abschnitt 5.2.2.3 beschrieben, so wird in Abbildung 5.20 die Entwicklung des Dämpfungsunterschieds bei DPO 19 Schicht 2 in den drei aufeinanderfolgenden Temperaturzyklen gezeigt. Auffällig ist, dass der Dämpfungsfaktor schon beim ersten Abkühlen (blaue Kreise) Werte annimmt, die sehr klein sind und sogar negativ werden. Diese Abkühlkurve wird beim zweiten Heizen (orange Quadrate) reversibel durchlaufen, bevor bei etwa $600 \mathrm{~K}$ die maximale Dämpfung erreicht ist und die Werte mit zunehmender Temperatur wieder sinken. Im dritten Zyklus ändert sich der Kurvenverlauf nicht mehr. Diese Beobachtung deckt sich mit den in Abschnitt 5.2.2.3 beschriebenen für die Frequenz.

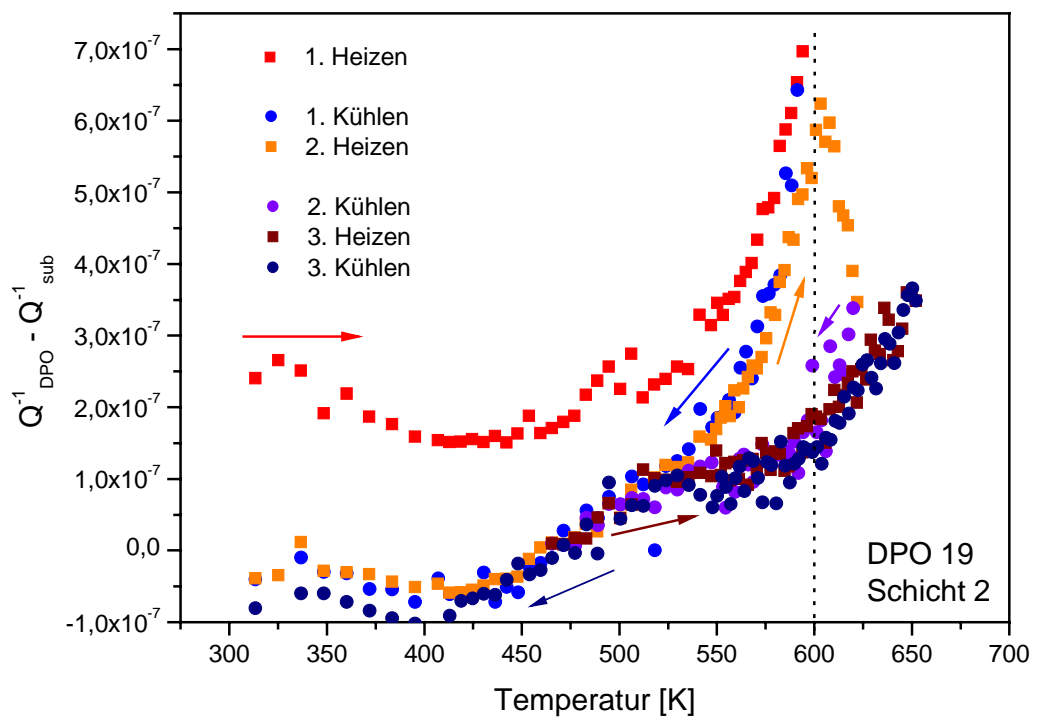

Abbildung 5.20: Differenz der Dämpfungsfaktoren zwischen Schicht 2 auf DPO 19 und Substrat.

\subsection{3 $\mathrm{Zr}_{65} \mathrm{Al}_{7,5} \mathrm{Cu}_{27,5}$ auf Tantal Zwischenschicht}

In diesem Kapitel wird eine dritte Strategie bezüglich der Schichtunterlage vorgestellt. Wie in Abschnitt 5.1.2 erläutert, wurde vor Beginn der Messungen auf DPO 19 und auf DPO 22 je eine $50 \mathrm{~nm}$ dicke Tantal-Schicht auf den Siliziumoszillator gesputtert. Die Eigenfrequenz und die Dämpfung der so vorpräparierten Oszillatoren wird gemessen und als Referenz für die $\mathrm{Zr}_{65} \mathrm{Al}_{7,5} \mathrm{Cu}_{27,5}$-Schichten betrachtet, die nun auf die Ta-Unterlage aufgedampft werden. 


\subsubsection{Frequenzdifferenz}

Abbildung 5.21 zeigt die Frequenzdifferenz für die $300 \mathrm{~nm}$ dicke Schicht 1 auf DPO 19 und Abbildung 5.22 für die 356 nm dicke Schicht 1 auf DPO 22. Im ersten Fall beträgt die Verschiebung nach der Herstellung bei Raumtemperatur -3,5 Hz, im zweiten Fall -5,2 Hz. Beiden Temperaturverläufen gleich ist die Zunahme der Differenz zu negativeren Werten mit steigender Temperatur.

In Abbildung 5.21 ist zu erkennen, dass es durch den primären Heizvorgang zu irreversiblen Veränderungen in der Schicht kommt. Die Frequenzdifferenz der roten Kurve liegt deutlich tiefer als für alle anderen Temperaturzyklen. Dann allerdings ist die Frequenzdifferenz vom ersten Abkühlen bis zum vierten Aufheizen mit der Temperatur relativ gut reproduzierbar. Erst ein isothermes Auslagern bei $654 \mathrm{~K}$ für 9 Stunden bewirkt weitere irreversible Änderungen. Der Kurvenverlauf beim 6. Abkühlen (schwarz) ähnelt qualitativ den vorhergehenden, liegt aber etwa 1,5 Hz darüber.

Ähnlich ist bei Schicht 1 auf DPO 22 in Abbildung $5.22 \mathrm{zu}$ erkennen, dass die Frequenzverschiebung beim ersten Abkühlen (blaue Kreise) zwar deutlich vom primären Heizverlauf abweicht, mit dem zweiten Heizvorgang (orange Dreiecke) aber relativ gut reproduziert wird. Wegen der sichtbar großen Schwankungen in den Daten wurden an dieser Schicht keine weiteren Messungen durchgeführt.

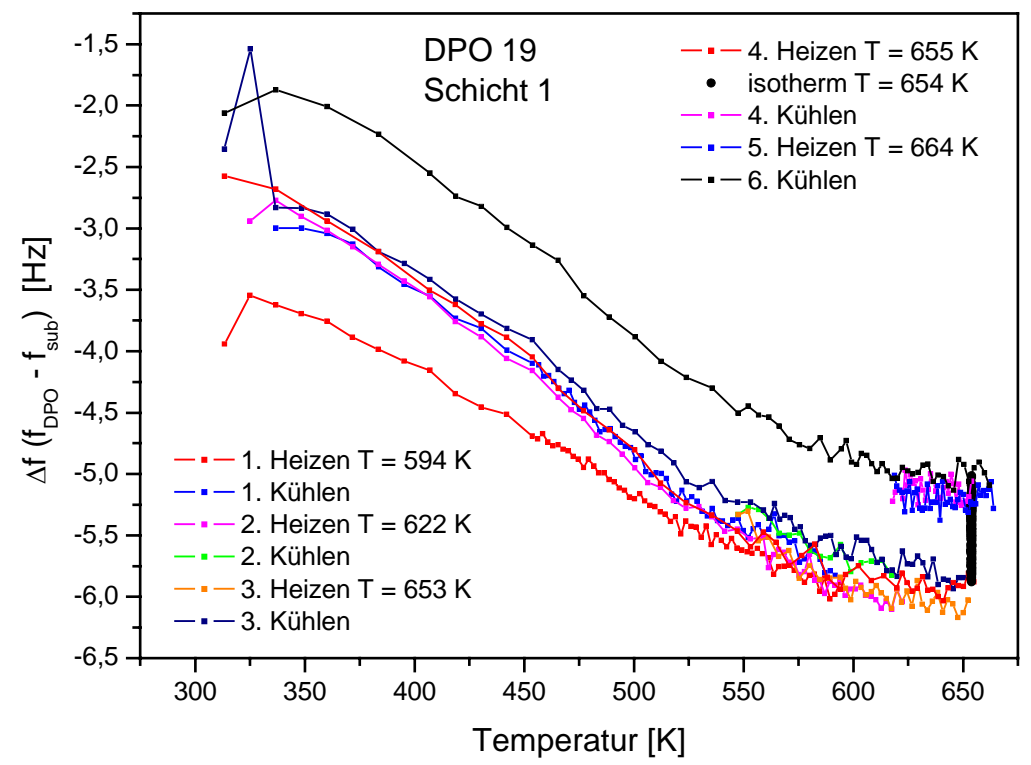

Abbildung 5.21: Frequenzverschiebung zwischen DPO 19 Schicht 1 und Substrat. Dargestellt sind aufeinanderfolgende Temperaturzyklen, sowie eine isotherme Messung bei 654 K über 9 Stunden. 


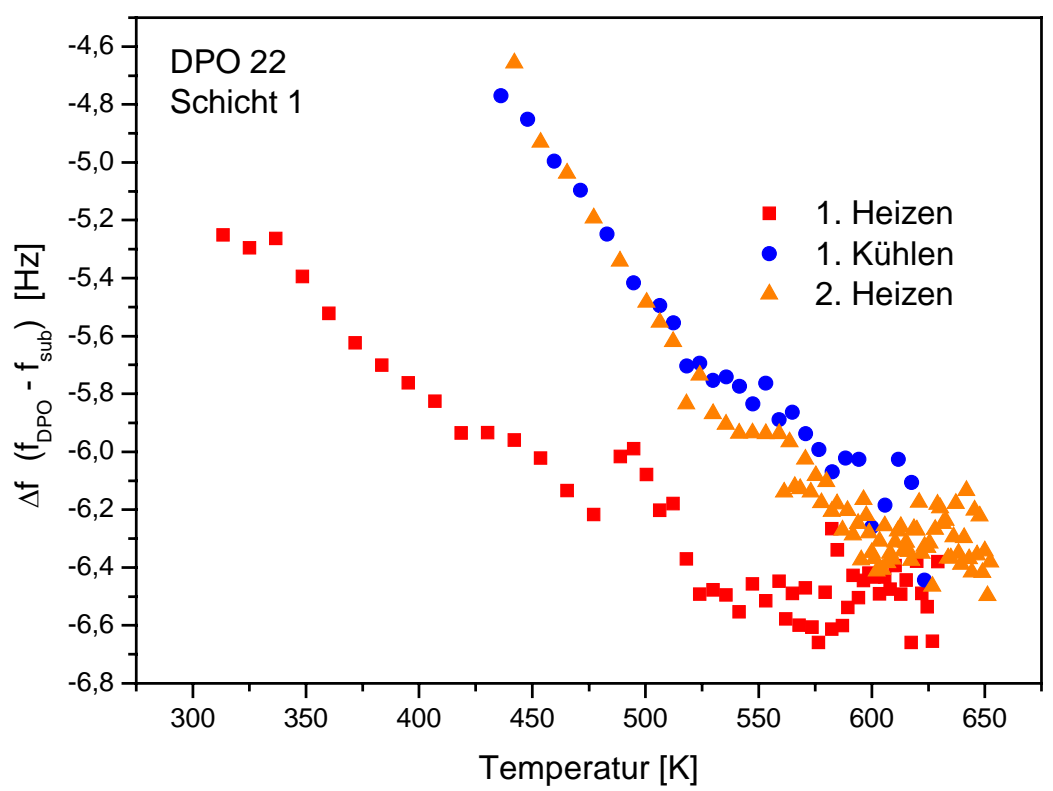

Abbildung 5.22: Frequenzverschiebung zwischen DPO 22 Schicht 1 und Substrat für aufeinanderfolgende Temperaturzyklen.

\subsubsection{Dämpfungsfaktor}

In Abbildung 5.23 und in Abbildung 5.24 sind die absolut gemessenen Dämpfungsfaktoren für beide auf Tantal aufgedampften Schichten gezeigt.

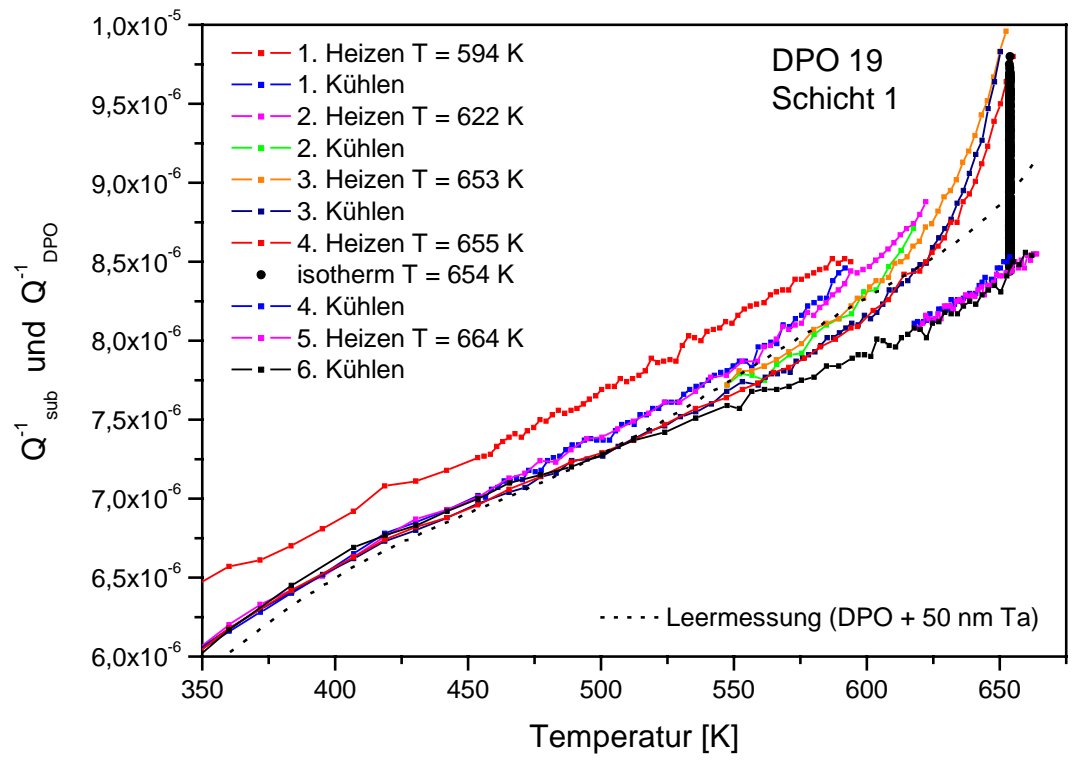

Abbildung 5.23: Dämpfungsfaktor für das Substrat (gestrichelte Linie) und für den mit Schicht 1 bedampften DPO 19 bei aufeinanderfolgenden Temperaturzyklen und einer isothermen Messung bei $654 \mathrm{~K}$. 


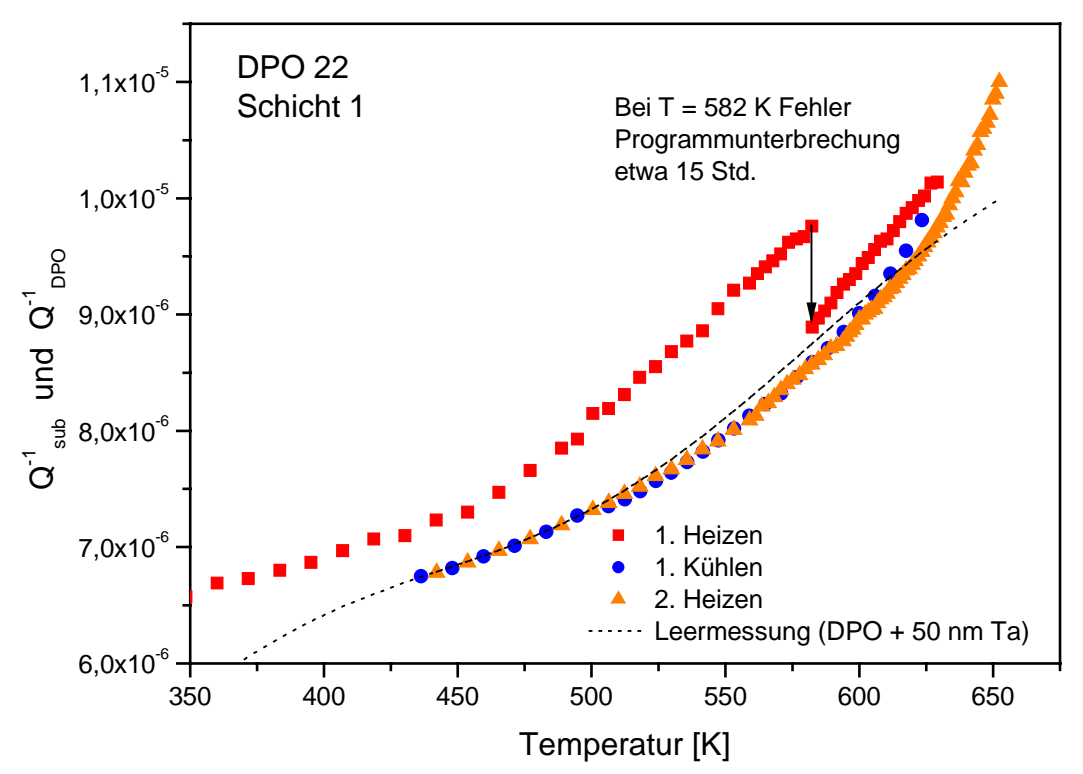

Abbildung 5.24: Dämpfungsfaktor für das Substrat (gestrichelte Linie) und für den mit Schicht 1 bedampften DPO 22 bei aufeinanderfolgenden Temperaturzyklen.

Zusätzlich eingezeichnet ist jeweils die Kurve für die nur mit der Ta-Schicht belegten Oszillatoren. Diese gestrichelte Linie sollte als Bezug für die Differenzbildung dienen. Bei beiden Schichten fällt allerdings auf, dass die Gesamtdämpfung des Systems unter diese Linie abfällt. Für die mit der metallischen Glasschicht bedampften Oszillatoren sind aber wegen der zusätzlichen Reibungsprozesse in dieser Schicht unbedingt größere Werte zu erwarten als für das Substrat. Im Temperaturbereich zwischen $500 \mathrm{~K}$ und $620 \mathrm{~K}$ ist die Differenzbildung um den Beitrag der Schicht zur Dämpfung zu ermitteln, so nicht sinnvoll. Es scheint so zu sein, dass während der Temperaturzyklen und im Laufe der Zeit Prozesse ablaufen, die die ursprüngliche Referenzkurve des Substrats zu kleineren Werten verschieben. Ursachen hierfür und Konsequenzen für die weitere Auswertung der Daten werden in Abschnitt 7.3 besprochen.

Auch bei den auf Tantal aufgedampften Schichten nimmt die Dämpfung ab etwa $550 \mathrm{~K}$ deutlich zu. Während sie jedoch bei Silizium und der kristallisierten Schicht als Unterlage bereits bei $625 \mathrm{~K}$ ihr Maximum erreicht und danach stark abfällt, bildet sich mit der Tantal-Zwischenschicht bis zu Temperaturen von $655 \mathrm{~K}$ kein Maximum aus. Betrachtet man in Abbildung 5.23 den dritten (orange) und den vierten Heizvorgang (rot), so stimmen die Werte für beide Kurven nahezu überein, was dafür spricht, dass der Kristallisationsprozess bis zu dieser Temperatur weitgehend unterdrückt wurde. 


\subsubsection{Isotherme Veränderung von Frequenz und Dämpfung}

Erst ein neunstündiger, isothermer Auslagerungsvorgang von Schicht 1 auf DPO 19 bei $654 \mathrm{~K}$ verursacht irreversible Veränderungen in Frequenz und Dämpfung.

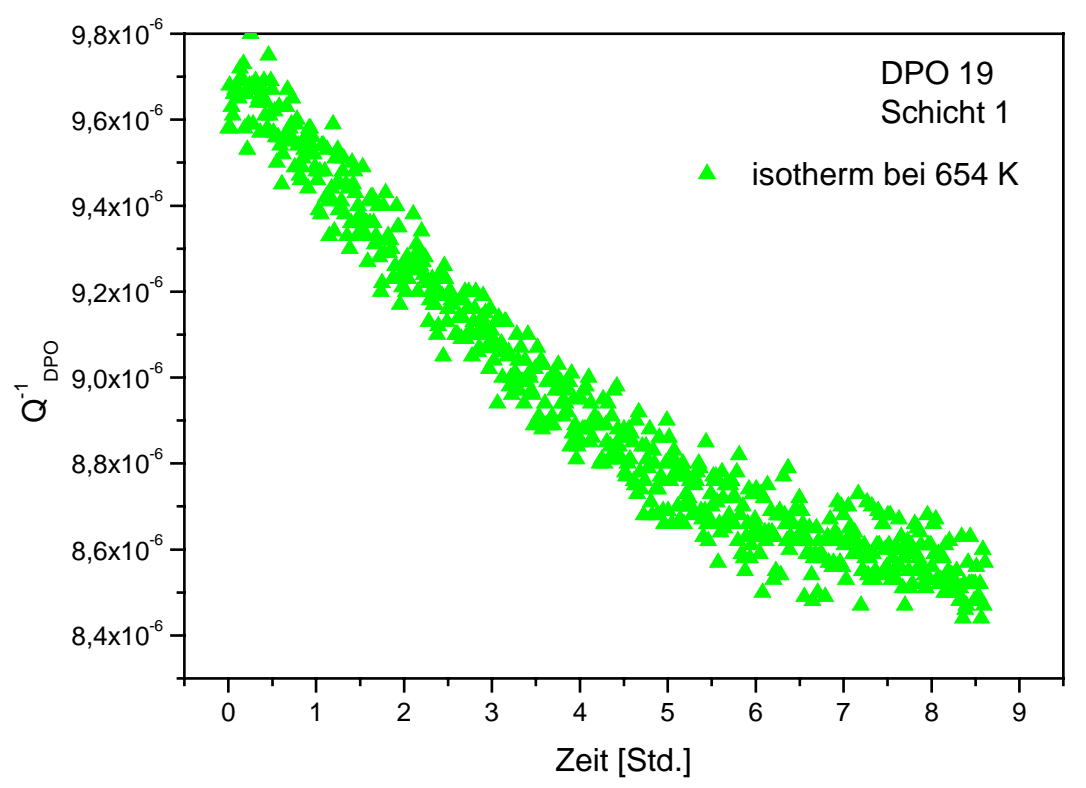

Abbildung 5.25: Isotherme Entwicklung des Dämpfungskoeffizienten von Schicht 1 auf DPO 19 bei 654 K.

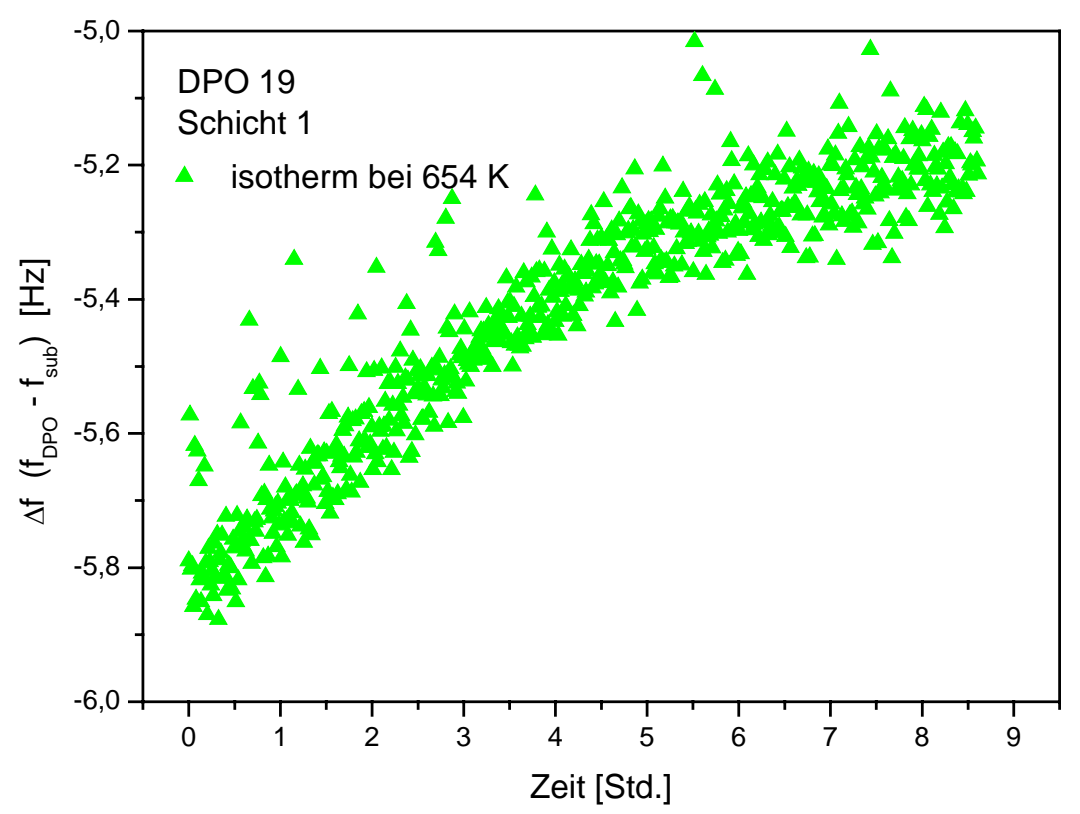

Abbildung 5.26: Isotherme Entwicklung der AS2-Frequenzdifferenz zwischen Schicht 1 auf DPO 19 und Substrat bei $654 \mathrm{~K}$. 
Der zeitliche Verlauf der Dämpfung ist in Abbildung 5.25 wiedergegeben. Auch die AS2-Resonanzfrequenz ändert sich zeitabhängig, wie in Abbildung 5.26 gezeigt ist. Beide Kurvenverläufe ähneln, abgesehen von der höheren Temperatur, bei der sie gemessen wurden, den in Abschnitt 5.2.1 vorgestellten isothermen Untersuchungen an Schicht 1 auf DPO 12.

\subsubsection{Veränderung der ST-Resonanzfrequenz}

Für das in Abschnitt 4.7.3 erläuterten Vorgehen zur Berechnung des komplexen Schermoduls dünner Schichten sind die bisher gezeigten Messergebnisse nur dann ausreichend, wenn Dichte und Dicke der untersuchten Schicht bekannt sind. Ist dies nicht der Fall, so kann das in die Berechnungen eingehende Produkt aus Dichte und Dicke experimentell aus der Verschiebung der ST-Resonanzfrequenz berechnet werden.

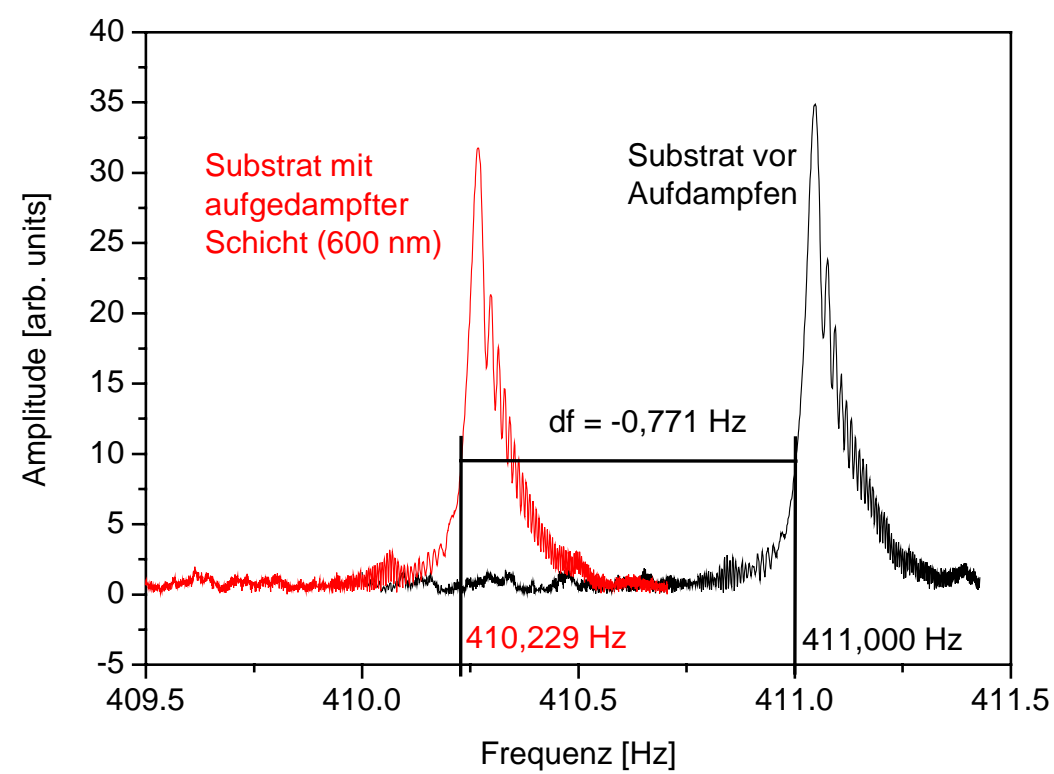

Abbildung 5.27: Verschiebung der ST-Mode durch die zusätzliche Masse einer aufgedampften Schicht. Frequenzscan zur Messung von DPO 19 Schicht 3 (600 nm).

Mit einem Frequenzscan (Abschnitt 4.4.3) mit einer Schrittweite von $1 \mathrm{mHz}$ wird zunächst die ST-Resonanzfrequenz bei einer Temperatur von exakt $40{ }^{\circ} \mathrm{C}$ ermittelt. Nun wird die zu untersuchende Schicht bei Raumtemperatur aufgedampft. Der Oszillator wird erneut auf exakt $40{ }^{\circ} \mathrm{C}$ erhitzt, und die ST-Resonanzfrequenz wieder gemessen. Da die Eigenfrequenzen stark temperaturabhängig sind ist es unerlässlich, die Resonanzfrequenz vor und nach dem Aufdampfen der Schicht bei exakt der gleichen Temperatur zu bestimmen. Für die AS2-Mode ist 
die Temperaturabhängigkeit in Abbildung 4.14 gezeigt. Sie gilt analog für die ST-Mode. In Abbildung 5.27 sind zwei typische Frequenzscans gezeigt, die vor dem Aufdampfen von Schicht 3 auf DPO 19 (schwarz) und danach (rot) aufgenommen wurden. Die Frequenzverschiebung beträgt in diesen Fall $-0,77 \mathrm{~Hz}$.

Tabelle 5.4 zeigt zusammenfassend die Verschiebung der ST-Resonanzfrequenz für alle untersuchten Schichten. Die gemessenen Ist-Werte werden mit den nach Gleichung (4.45) berechneten Soll-Daten verglichen. Übereinstimmend zeigt sich für alle Schichten, dass die tatsächliche Frequenzverschiebung nur etwa 2/3 des vorhergesagten Wertes beträgt. In Abschnitt 7.2 wird auf die Problematik der Schichtdickenbestimmung ausführlich eingegangen.

\begin{tabular}{|c|c|c|c|c|c|}
\hline \multirow[b]{2}{*}{ Oszillator } & \multirow[b]{2}{*}{ Bezeichnung } & \multirow[b]{2}{*}{ Dicke } & \multicolumn{3}{|c|}{ Verschiebung der ST-Resonanzfrequenz } \\
\hline & & & Ist & Soll & Ist zu Soll \\
\hline \multirow[t]{2}{*}{ DPO 12} & Schicht 1 & $300 \mathrm{~nm}$ & $-0,391 \mathrm{~Hz}$ & $-0,613 \mathrm{~Hz}$ & $64 \%$ \\
\hline & Schicht 2 & $300 \mathrm{~nm}$ & $-0,386 \mathrm{~Hz}$ & $-0,613 \mathrm{~Hz}$ & $63 \%$ \\
\hline \multirow[t]{3}{*}{ DPO 19} & Schicht 1 & $300 \mathrm{~nm}$ & $-0,387 \mathrm{~Hz}$ & $-0,609 \mathrm{~Hz}$ & $63 \%$ \\
\hline & Schicht 2 & $300 \mathrm{~nm}$ & $-0,369 \mathrm{~Hz}$ & $-0,609 \mathrm{~Hz}$ & $61 \%$ \\
\hline & Schicht 3 & $600 \mathrm{~nm}$ & $-0,771 \mathrm{~Hz}$ & $-1,218 \mathrm{~Hz}$ & $63 \%$ \\
\hline DPO 21 & Schicht 1 & $300 \mathrm{~nm}$ & $-0,405 \mathrm{~Hz}$ & $-0,611 \mathrm{~Hz}$ & $66 \%$ \\
\hline DPO 22 & Schicht 1 & $356 \mathrm{~nm}$ & $-0,450 \mathrm{~Hz}$ & $-0,725 \mathrm{~Hz}$ & $62 \%$ \\
\hline
\end{tabular}

Tabelle 5.4: ST-Resonanzverschiebung aller in dieser Arbeit beschriebenen Schichten. 


\section{Polymethylmethacrylat als Polymerglas}

\subsection{Probenmaterial und Schichtherstellung}

\subsubsection{PMMA - Struktur und Historie}

Im Jahre 1901 veröffentlichte Otto Röhm seine Dissertation „Polymerisationsprodukte der Akrylsäure“. Die entstandenen Polymere hatten Eigenschaften, die zwischen einem zähen, biegsamen Glas und einem steifen Kunststoff lagen.

1933 wurde eine Methode gefunden, Methylmethacrylat zwischen zwei Glasplatten zu gießen und darin zu polymerisieren. Der dabei entstandene glasklare, harte und bruchfeste Kunststoff Polymethylmethacrylat (PMMA) erhielt den Markennamen „Plexiglas“. Plexiglas schrumpft nicht, verzieht sich nicht und bricht splitterfrei, weshalb sich das Produkt ein breites Anwendungsfeld erobert hat. Dazu gehören flache und gewölbte Fensterscheiben, Abdeckgläser, Duschwände, Oberflächenbeschichtungen, Rundbanden für Eishockeystadien und Aquarien.

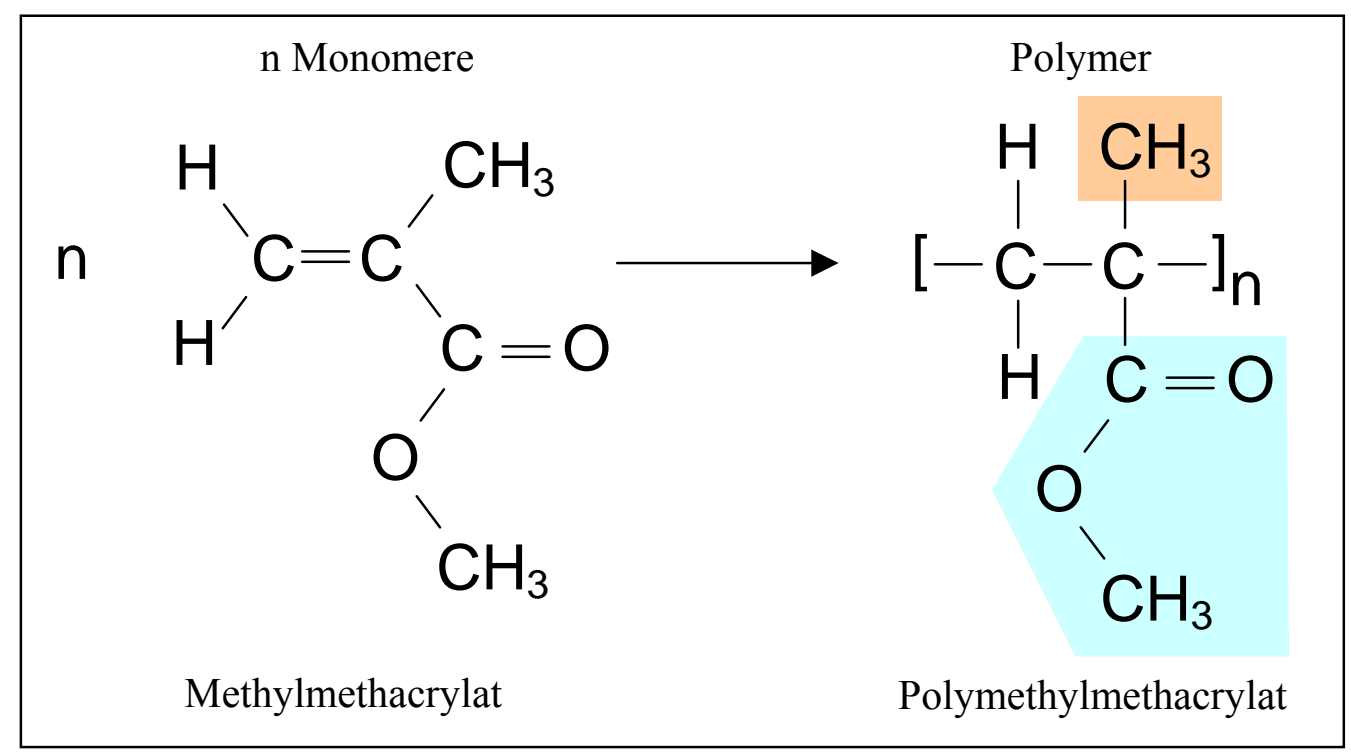

Abbildung 6.1: Herstellung von PMMA durch radikalische Polymerisation. Der Polymer-Backbone hat zwei Seitenketten, orange und blau unterlegt.

PMMA ist wie alle Polymere aus langen, kettenförmigen Molekülen aufgebaut, deren Zusammenhalt durch Van-der-Waals-Bindungen gewährleistet ist. Der Vernetzungsgrad ist gering, weshalb PMMA zur Klasse der Thermoplaste zählt. Jede einzelne Kette besteht aus einer vielfachen Wiederholung ihrer konstitutiven Einheit, dem Monomer und wird durch radikalische 
Polymerisation hergestellt (Abbildung 6.1). Die entstehenden Makromoleküle können aus bis zu 10.000 Monomer bestehen, wobei die Kettenlänge einstellbar ist. Wegen ihrer Größe und der freien Rotation um die kovalenten Einfachbindungen des Kohlenstoffs im Backbone der Kette, hat PMMA im festen Zustand eine ungeordnete, amorphe Struktur mit relativ hohem Anteil an freiem Volumen.

\subsubsection{Herstellung mit gepulster Laserdeposition}

Die in dieser Arbeit untersuchten dünnen PMMA-Schichten wurden mit gepulster Laserdeposition (PLD) in Zusammenarbeit mit E. Süske und H.-U. Krebs (Institut für Materialphysik) hergestellt $[159,160]$.

Ein Excimer-Laser (Lambda Physik LPX 110i) wird mit Krypton und Fluor betrieben und erzeugt für etwa $30 \mathrm{~ns}$ einen kohärenten Strahlungspuls der Wellenlänge $248 \mathrm{~nm}$. Der Laserstrahl wird fokussiert und trifft mit einer Energiedichte von $1,8 \mathrm{~J} / \mathrm{cm}^{2}$ unter einem Winkel von $45^{\circ}$ auf ein ataktisches PMMA-Target, das sich in einer UHV-Kammer bei einem Druck von $10^{-8}$ mbar befindet. Als Ausgangsmaterial werden PMMA-Folien der Firma Goodfellow verwendet, deren molare Masse mit $2 \times 10^{-6}$ bis $4 \times 10^{-6} \mathrm{~g} / \mathrm{mol}$ angegeben wird. Der zu beschichtende Doppel-Paddel Oszillator befindet sich, eingespannt in einen Mini-Schraubstock, im Abstand von etwa $40 \mathrm{~mm}$ parallel oberhalb des Targets. Ob und wie sich Struktur und Eigenschaften der PMMA-Schichten durch den Ablationsprozess verglichen mit dem Ausgangsmaterial verändern, wird in Abschnitt 7.7 diskutiert.

\subsection{Dielektrische Spektroskopie}

Wegen der industriellen und wirtschaftlichen Bedeutung von PMMA wurde die frequenzabhängige Dynamik mittels dielektrischer Spektroskopie in der Vergangenheit erfolgreich untersucht [z.B. 88, 161, 162]. Die an der Universität Augsburg durchgeführten (siehe Abschnitt 3.4) und in dieser Arbeit vorgestellten Messungen an mit PLD hergestellten Schichten sollen insbesondere die folgende drei fundamentalen Fragen beantworten helfen:

- Lässt sich der Verlust in einer dünnen Schicht, die mittels gepulster Laserdeposition hergestellt wurde, mit dem Verlust im kompakten Ausgangsmaterial vergleichen? 
- Wie verschiebt sich die Lage der Verlustmaxima bei temperaturabhängigen Messungen, wenn die anregende Frequenz konstant bleibt? Insbesondere bei der AS2-Resonanzfrequenz von 5,4 kHz sollen dielektrische und mechanische Messungen verglichen werden.

- Wie verändert sich das temperaturabhängige Spektrum bei mehreren Messdurchläufen, wobei die Endtemperatur jeweils erhöht wird?

\subsubsection{Temperaturabhängiger dielektrischer Verlust}

In Abbildung 6.2 ist der temperaturabhängige dielektrische Verlust im PMMA-Ausgangsmaterial für verschiedene Anregungsfrequenzen aufgetragen.

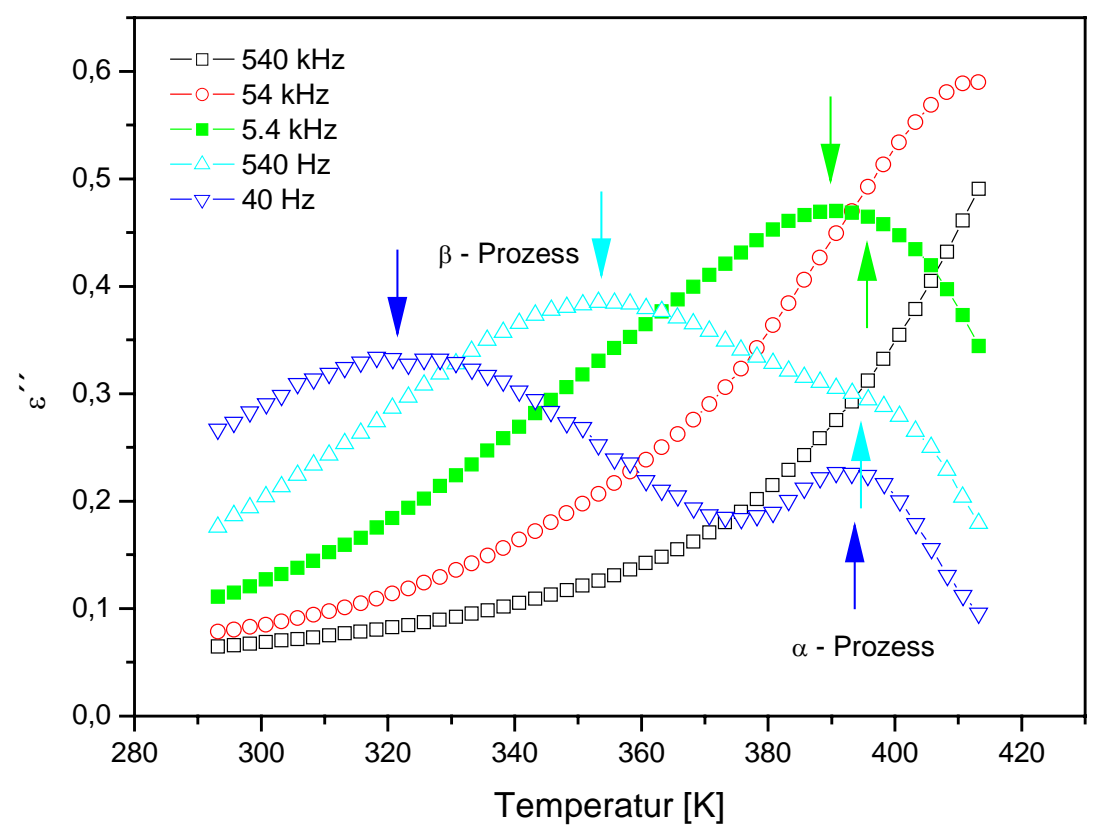

Abbildung 6.2: Dielektrischer Verlust im PMMA-Ausgangsmaterial in Abhängigkeit von der Temperatur für verschiedene Frequenzen. Die Pfeile indizieren die Positionen von $\alpha$ - und $\beta$-Peak.

Bei der kleinsten untersuchten Frequenz von $40 \mathrm{~Hz}$ (dunkelblau) lassen sich im Spektrum zwei Maxima klar voneinander unterscheiden. Der Peak bei etwa $320 \mathrm{~K}$ entsteht durch die Rotation der $-\mathrm{COOCH}_{3}$ Seitengruppen um den Polymer-Backbone, was in der Literatur [88] als slow- $\beta$-Prozess bezeichnet wird (Abschnitt 2.3). Das viskose Fließen der einzelnen Polymerketten gegeneinander ist der primäre $\alpha$-Relaxationsprozess und führt zu einem zweiten Peak bei etwa $395 \mathrm{~K}$. 
Da die Carboxylgruppen in den Seitenketten den Dipolmoment für das gesamte Polymermolekül tragen, relaxiert die elektrische Polarisation hauptsächlich im $\beta$-Prozess und nur zu einem geringen Anteil in Verschiebungen des Backbones, die im $\alpha$-Prozess sichtbar sind, weshalb das $\beta$-Maximum im dielektrischen Verlust in PMMA stark ausgeprägt ist.

Wird die Messfrequenz um rund eine Größenordnung erhöht, so lassen sich noch immer zwei getrennte Maxima im Verlustspektrum klar identifizieren. Die Position des $\alpha$-Peaks verändert sich kaum, wohingegen der $\beta$-Peak um $35 \mathrm{~K}$ verschoben ist und nun bei $355 \mathrm{~K}$ liegt. Diese Verschiebung ist nicht überraschend und lässt sich anschaulich erklären. Die charakteristische Zeitskala, mit der die Seitengruppen rotieren, ändert sich mit der Temperatur, wobei meist eine Arrheniusabhängigkeit angenommen wird (Abbildung 2.8). Je größer die Temperatur ist, desto schneller bewegen sich die Seitengruppen. Bei einer Frequenz von $40 \mathrm{~Hz}$ werden Prozesse auf einer charakteristische Zeitskala von $25 \mathrm{~ms}$ angeregt. Offensichtlich ist diese Relaxationszeit für die Seitengruppenrotation gerade bei $320 \mathrm{~K}$ erreicht. Bei $540 \mathrm{~Hz}$ ist die Anregungsdauer deutlich kürzer und liegt nur noch bei 1,8 ms. Um die Dynamik so zu beschleunigen muss die Temperatur erhöht werden, wodurch sich die Verschiebung der Peakposition hin zu $355 \mathrm{~K}$ erklärt.

Wählt man die Anregungsfrequenz mit 5,4 kHz noch eine Größenordnung höher, so werden Prozesse angeregt, deren Relaxationszeiten etwa $0,18 \mathrm{~ms}$ betragen. Für den $\beta$-Prozess bedeutet das, dass die Temperatur auf etwa $390 \mathrm{~K}$ erhöht werden muss, um die benötigte Rotationsgeschwindigkeit für die Seitengruppen zu erreichen. Bei dieser Temperatur hat aber gerade auch der Verlust durch das viskose Fließen sein Maximum. Die bei kleinen Anregungsfrequenzen noch getrennt auflösbaren Peaks der Einzelprozesse verschmieren nun zu einem einzigen Maximum und zeigen das in Abschnitt 2.3 beschriebene „merging“-Phänomen [162].

\subsubsection{Vergleich Ausgangsmaterial - dünne Schicht}

Eine entscheidende Frage zur Beurteilung der Ergebnisse an dünnen Schichten ist, ob und wie das Polymer bei der Schichtherstellung verändert wird. Materialparameter wie die Kettenlänge der Moleküle, die Massendichte oder der Vernetzungsgrad durch radikalisierte Seitengruppen beeinflussen die Eigenschaften des PMMA (siehe auch Abschnitt 7.7).

Aufschluss darüber kann auch der direkte Vergleich des temperaturabhängigen dielektrischen Verlustes im PMMA-Ausgangsmaterial (bulk) und in einer mit PLD hergestellten $1 \mu \mathrm{m}$ 
dicken Schicht geben, der in Abbildung 6.3 für zwei verschiedene Frequenzen zu sehen ist. Die Stärke des Antwortsignals ist dabei für die Schicht (rechte Skala) deutlich kleiner als für das massivere Ausgangsmaterial (linke Skala), was sich durch das unterschiedliche Probenvolumen erklärt.

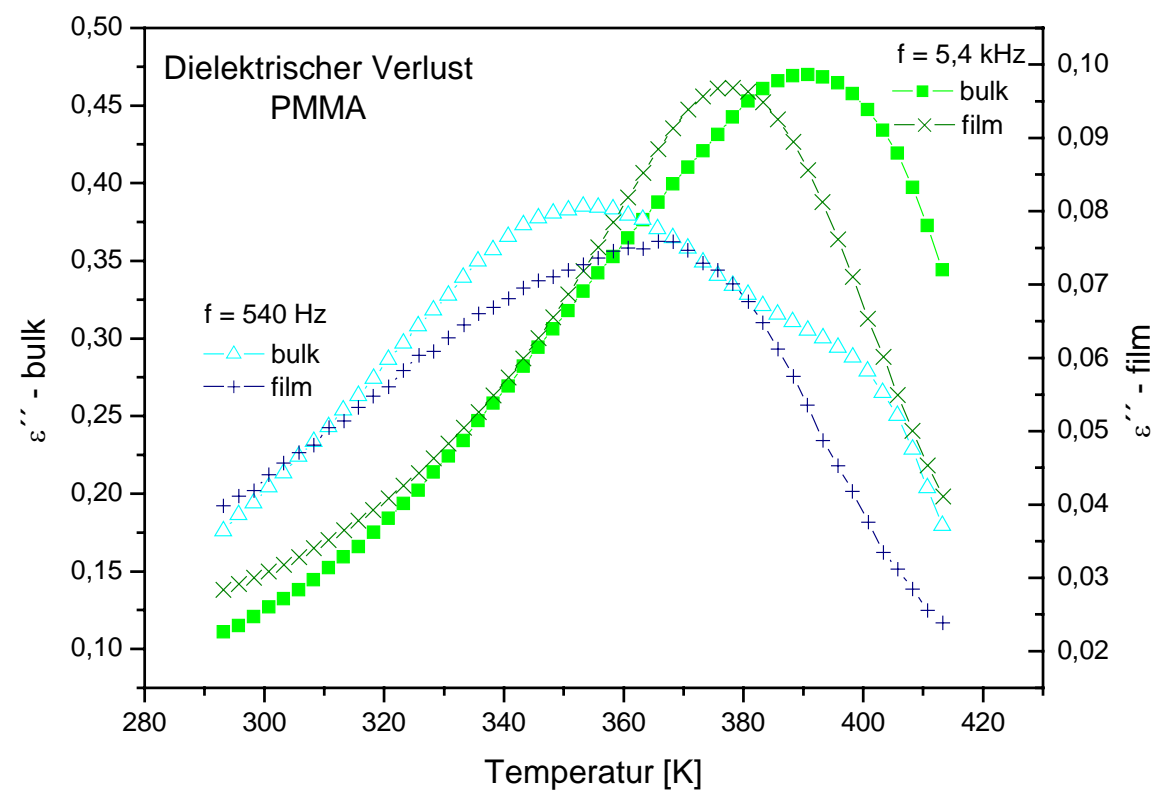

Abbildung 6.3: Vergleich des dielektrischen Verlustes in bulk-Material und in einer mit PLD hergestellten Schicht für zwei Frequenzen.

Die Kurven unterscheiden sich sowohl in ihrer Form, als auch in der Position der Maxima. So ist der bei $540 \mathrm{~Hz}$ noch deutlich erkennbare $\alpha$-Peak im bulk-Material (hellblau) im Vergleich zur Schicht (dunkelblau) deutlich zu kleineren Temperaturen hin verschoben. Das trifft auch bei der Anregung mit 5,4 kHz zu. Lag das Maximum im Ausgangsmaterial noch bei $395 \mathrm{~K}$, so befindet es sich in der Schicht bei etwa $375 \mathrm{~K}$. Dass sich der $\alpha$-Relaxationspeak um $20 \mathrm{~K}$ verschiebt, könnte auf eine veränderte Kettenlänge hinweisen (Abschnitt 7.7.2). Eine schlechtere thermische Ankopplung der dünnen Schicht als Ursache für die Verschiebung des Peaks schließen die Experimentatoren aus [163].

Die Frage, ob und wie sich auch die Position und Relaxationsstärke des $\beta$-Prozesses verändern bleibt mit den Ergebnissen aus Abbildung 6.3 unbeantwortet. Jedenfalls unterscheiden sich sowohl bei $540 \mathrm{~Hz}$, als auch bei $5,4 \mathrm{kHz}$ die Kurven für beide Proben in ihrer Niedertemperaturflanke. 


\subsubsection{Veränderungen im Spektrum mit steigender Endtemperatur}

Der temperaturabhängige dielektrische Verlust einer $1 \mu \mathrm{m}$ dicken PMMA-Schichten wird nun mehrmals hintereinander gemessen, wobei die Maximaltemperatur mit jedem Durchlauf erhöht wird. Die Ergebnisse für die Anregungsfrequenz von $5,4 \mathrm{kHz}$, sind in Abbildung 6.4 gezeigt.

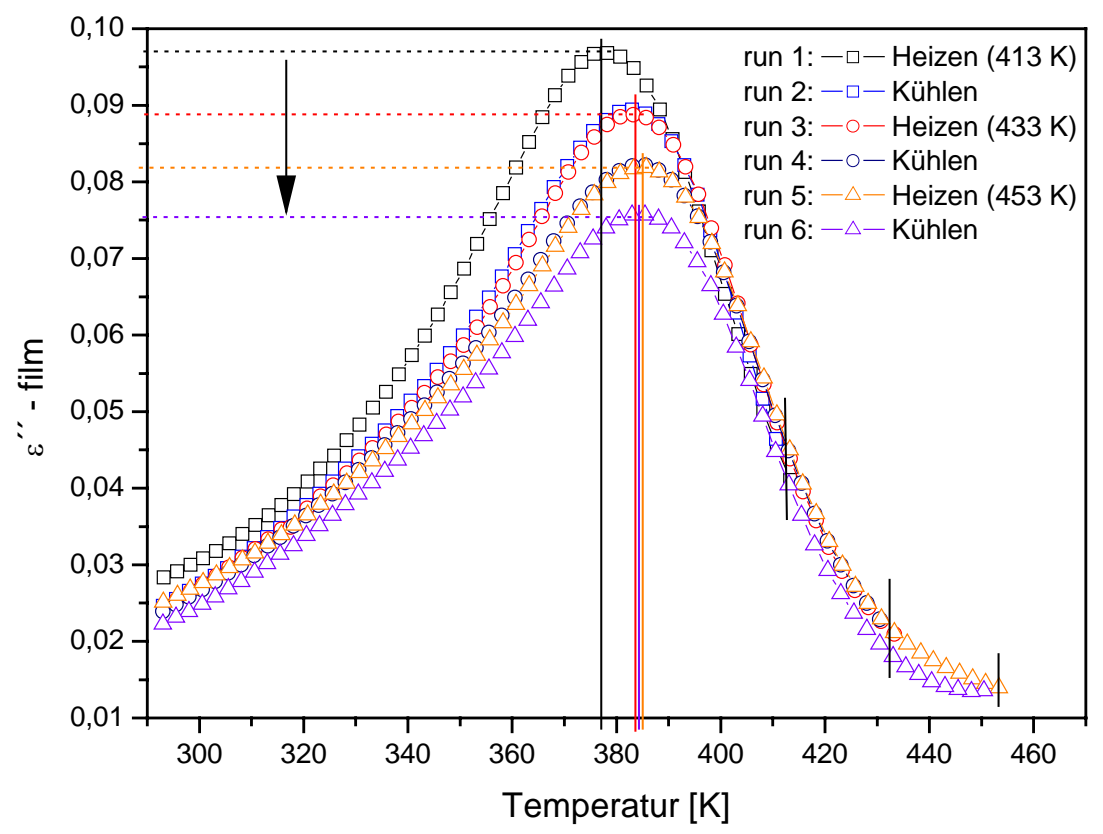

Abbildung 6.4: Dielektrischer Verlust von aufeinanderfolgenden Temperaturzyklen in einer $1 \mu$ m dicken PMMASchicht bei 5,4 kHz Anregungsfrequenz..

Beim ersten Heizen der Schicht (run 1, schwarze Quadrate) befindet sich das Maximum bei 376 K. Nach Erreichen der Endtemperatur für diesen Zyklus von 413 K wurde beim Abkühlen erneut gemessen (run 2, blaue Quadrate). Das Maximum liegt nun bei $383 \mathrm{~K}$ und die Relaxationsstärke hat abgenommen. Heizt man erneut (run 3, rote Kreise), so liegen die Messwerte relativ exakt auf der vorhergehenden Abkühlkurve (run 2). Dieses Mal wurde der Heizvorgang bis zur Endtemperatur von $433 \mathrm{~K}$ fortgesetzt, bevor wieder beim Abkühlen gemessen wurde (run 4, dunkelblaue Kreise). Die Position des Peaks verändert sich dabei nicht, allerdings ist die absolut gemessene Relaxationsstärke noch einmal verkleinert. Die gleichen Beobachtungen macht man auch für den dritten Zyklus (run 5 und run 6), wobei jetzt bis zur Endtemperatur von $453 \mathrm{~K}$ geheizt wurde. 


\subsection{Mechanische Spektroskopie}

Zwei Polymerschichten wurden mechanisch untersucht. Die Schicht auf DPO 14 hat eine Dicke von $1 \mu \mathrm{m}$, die Dicke auf DPO 16 ist mit $500 \mathrm{~nm}$ gerade halb so groß gewählt. DPO 14 und DPO 16 sind durch Laserschneiden hergestellt (Abschnitt 4.2.3). Bei DPO 16 wurden Frequenz und Dämpfung des leeren Oszillators gemessen, während dies für DPO 14 nicht der Fall war. Die entsprechenden Daten werden mathematisch rekonstruiert.

Beide Schichten wurden mit einem umfangreichen Temperaturprogramm untersucht, das in Tabelle 6.1 für DPO 14 und in Tabelle 6.2 für DPO 16 gezeigt ist. Die Abkürzung „run“ steht dabei für temperaturabhängige Messungen von Frequenz und Dämpfung, die Bezeichnung „iso“ für eine zeitabhängige, isotherme Untersuchung dieser Eigenschaften.

\begin{tabular}{|l|l|}
\hline run 1 & $302 \mathrm{~K} \rightarrow 419 \mathrm{~K}$ \\
\hline run 2 & $419 \mathrm{~K} \rightarrow 307 \mathrm{~K}$ \\
\hline run 3 & $307 \mathrm{~K} \rightarrow 430 \mathrm{~K}$ \\
\hline run 4 & $430 \mathrm{~K} \rightarrow 307 \mathrm{~K}$ \\
\hline run 5 & $307 \mathrm{~K} \rightarrow 442 \mathrm{~K}$ \\
\hline run 6 & $442 \mathrm{~K} \rightarrow 307 \mathrm{~K}$ \\
\hline run 7 & $307 \mathrm{~K} \rightarrow 454 \mathrm{~K}$ \\
\hline run 8 & $454 \mathrm{~K} \rightarrow 307 \mathrm{~K}$ \\
\hline
\end{tabular}

\begin{tabular}{|l|l|}
\hline run 9 & $307 \mathrm{~K} \rightarrow 465 \mathrm{~K}$ \\
\hline run 10 & $465 \mathrm{~K} \rightarrow 307 \mathrm{~K}$ \\
\hline run 11 & $307 \mathrm{~K} \rightarrow 501 \mathrm{~K}$ \\
\hline run 12 & $501 \mathrm{~K} \rightarrow 313 \mathrm{~K}$ \\
\hline run 13 & $313 \mathrm{~K} \rightarrow 524 \mathrm{~K}$ \\
\hline run 14 & $524 \mathrm{~K} \rightarrow 313 \mathrm{~K}$ \\
\hline run 15 & $313 \mathrm{~K} \rightarrow 524 \mathrm{~K}$ \\
\hline run 16 & $524 \mathrm{~K} \rightarrow 313 \mathrm{~K}$ \\
\hline
\end{tabular}

\begin{tabular}{|l|l|}
\hline run 17 & $313 \mathrm{~K} \rightarrow 524 \mathrm{~K}$ \\
\hline run 18 & $524 \mathrm{~K} \rightarrow 313 \mathrm{~K}$ \\
\hline run 19 & $313 \mathrm{~K} \rightarrow 536 \mathrm{~K}$ \\
\hline run 20 & $536 \mathrm{~K} \rightarrow 313 \mathrm{~K}$ \\
\hline $\operatorname{run} 21$ & $313 \mathrm{~K} \rightarrow 547 \mathrm{~K}$ \\
\hline run 22 & $547 \mathrm{~K} \rightarrow 313 \mathrm{~K}$ \\
\hline run 23 & $313 \mathrm{~K} \rightarrow 571 \mathrm{~K}$ \\
\hline run 24 & $571 \mathrm{~K} \rightarrow 313 \mathrm{~K}$ \\
\hline
\end{tabular}

Tabelle 6.1: Versuchsgang und Temperaturprogramm für DPO 14 mit 1000 nm PMMA.

\begin{tabular}{|l|l|}
\hline \multicolumn{2}{|c|}{ Temperatur bis $419 \mathrm{~K}$} \\
\hline run 1 & $296 \mathrm{~K} \rightarrow 419 \mathrm{~K}$ \\
\hline run 2 & $419 \mathrm{~K} \rightarrow 302 \mathrm{~K}$ \\
\hline run 3 & $302 \mathrm{~K} \rightarrow 419 \mathrm{~K}$ \\
\hline iso 1 & $419 \mathrm{~K}, \mathrm{t}=1 \mathrm{~h}$ \\
\hline run 4 & $419 \mathrm{~K} \rightarrow 307 \mathrm{~K}$ \\
\hline run 5 & $307 \mathrm{~K} \rightarrow 419 \mathrm{~K}$ \\
\hline iso 2 & $419 \mathrm{~K}, \mathrm{t}=8 \mathrm{~h}$ \\
\hline run 6 & $419 \mathrm{~K} \rightarrow 307 \mathrm{~K}$ \\
\hline run 7 & $307 \mathrm{~K} \rightarrow 419 \mathrm{~K}$ \\
\hline iso 3 & $419 \mathrm{~K}, \mathrm{t}=23 \mathrm{~h}$ \\
\hline run 8 & $419 \mathrm{~K} \rightarrow 307 \mathrm{~K}$ \\
\hline
\end{tabular}

\begin{tabular}{|l|l|}
\hline \multicolumn{2}{|l|}{ Temperatur bis $442 \mathrm{~K}$} \\
\hline run 9 & $307 \mathrm{~K} \rightarrow 430 \mathrm{~K}$ \\
\hline iso 4 & $430 \mathrm{~K}, \mathrm{t}=1,7 \mathrm{~h}$ \\
\hline run 10 & $430 \mathrm{~K} \rightarrow 307 \mathrm{~K}$ \\
\hline run 11 & $307 \mathrm{~K} \rightarrow 430 \mathrm{~K}$ \\
\hline iso 5 & $430 \mathrm{~K}, \mathrm{t}=5 \mathrm{~h}$ \\
\hline run 12 & $430 \mathrm{~K} \rightarrow 307 \mathrm{~K}$ \\
\hline run 13 & $307 \mathrm{~K} \rightarrow 430 \mathrm{~K}$ \\
\hline iso 6 & $430 \mathrm{~K}, \mathrm{t}=34 \mathrm{~h}$ \\
\hline run 14 & $430 \mathrm{~K} \rightarrow 307 \mathrm{~K}$ \\
\hline run 15 & $307 \mathrm{~K} \rightarrow 442 \mathrm{~K}$ \\
\hline iso 7 & $442 \mathrm{~K}, \mathrm{t}=17 \mathrm{~h}$ \\
\hline run 16 & $442 \mathrm{~K} \rightarrow 307 \mathrm{~K}$ \\
\hline
\end{tabular}

\begin{tabular}{|l|l|}
\hline \multicolumn{2}{|l|}{ Temperatur bis $512 \mathrm{~K}$} \\
\hline run 17 & $307 \mathrm{~K} \rightarrow 454 \mathrm{~K}$ \\
\hline run 18 & $454 \mathrm{~K} \rightarrow 307 \mathrm{~K}$ \\
\hline run 19 & $307 \mathrm{~K} \rightarrow 477 \mathrm{~K}$ \\
\hline run 20 & $477 \mathrm{~K} \rightarrow 307 \mathrm{~K}$ \\
\hline run 21 & $307 \mathrm{~K} \rightarrow 477 \mathrm{~K}$ \\
\hline Iso 8 & $477 \mathrm{~K}, \mathrm{t}=30 \mathrm{~h}$ \\
\hline run 22 & $477 \mathrm{~K} \rightarrow 307 \mathrm{~K}$ \\
\hline run 23 & $307 \mathrm{~K} \rightarrow 512 \mathrm{~K}$ \\
\hline run 24 & $512 \mathrm{~K} \rightarrow 307 \mathrm{~K}$ \\
\hline
\end{tabular}

Tabelle 6.2: Versuchsgang und Temperaturprogramm für DPO 16 mit 500 nm PMMA 


\subsubsection{Temperaturabhängige Resonanzfrequenz}

Als Konsequenz der zusätzlichen Masse auf Kopf und Flügel des Paddels und der elastischen Beiträge des PMMA bei der Torsion um den Hals ändert sich die AS2-Resonanzfrequenz. In Abbildung 6.5 ist diese Frequenzänderung für DPO 14 (oben) und für DPO 16 (unten) für jeweils das gesamte Temperaturprogramm gezeigt.
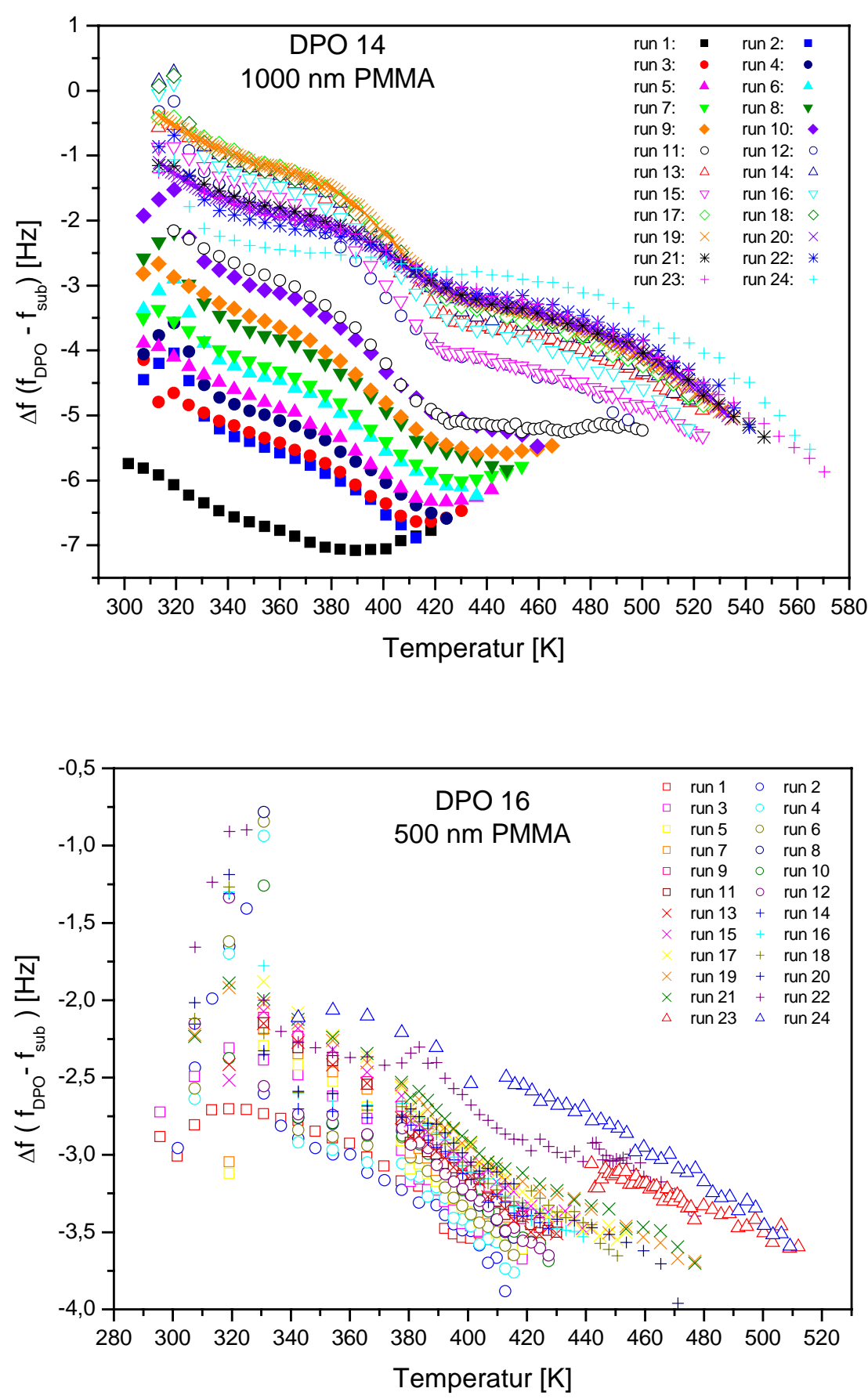

Abbildung 6.5: Frequenzverschiebung durch die PMMA-Schicht auf DPO 14 (oben) und DPO 16 (unten). Die Referenzdaten zur Differenzbildung wurden für DPO 14 mathematisch rekonstruiert. 
Innerhalb der einzelnen runs verändert sich die Frequenzdifferenz mit steigender Temperatur zu größeren negativen Werten. Betrachtet man in Abbildung 6.5 die ersten Durchläufe bei DPO 14, so bildet sich bei etwa $420 \mathrm{~K}$ ein Plateau relativ konstanter Frequenzverschiebung aus (bei run 11 bis $500 \mathrm{~K}$ ). Allerdings wird dieses Plateau nicht reversibel durchlaufen, sondern die Kurven verschieben sich mit steigender Endtemperatur zu kleineren negativen Werten. Diese Verschiebung ist auch bei DPO 16 zu beobachten, allerdings bildet sich kein Plateau wie bei DPO 14 aus.
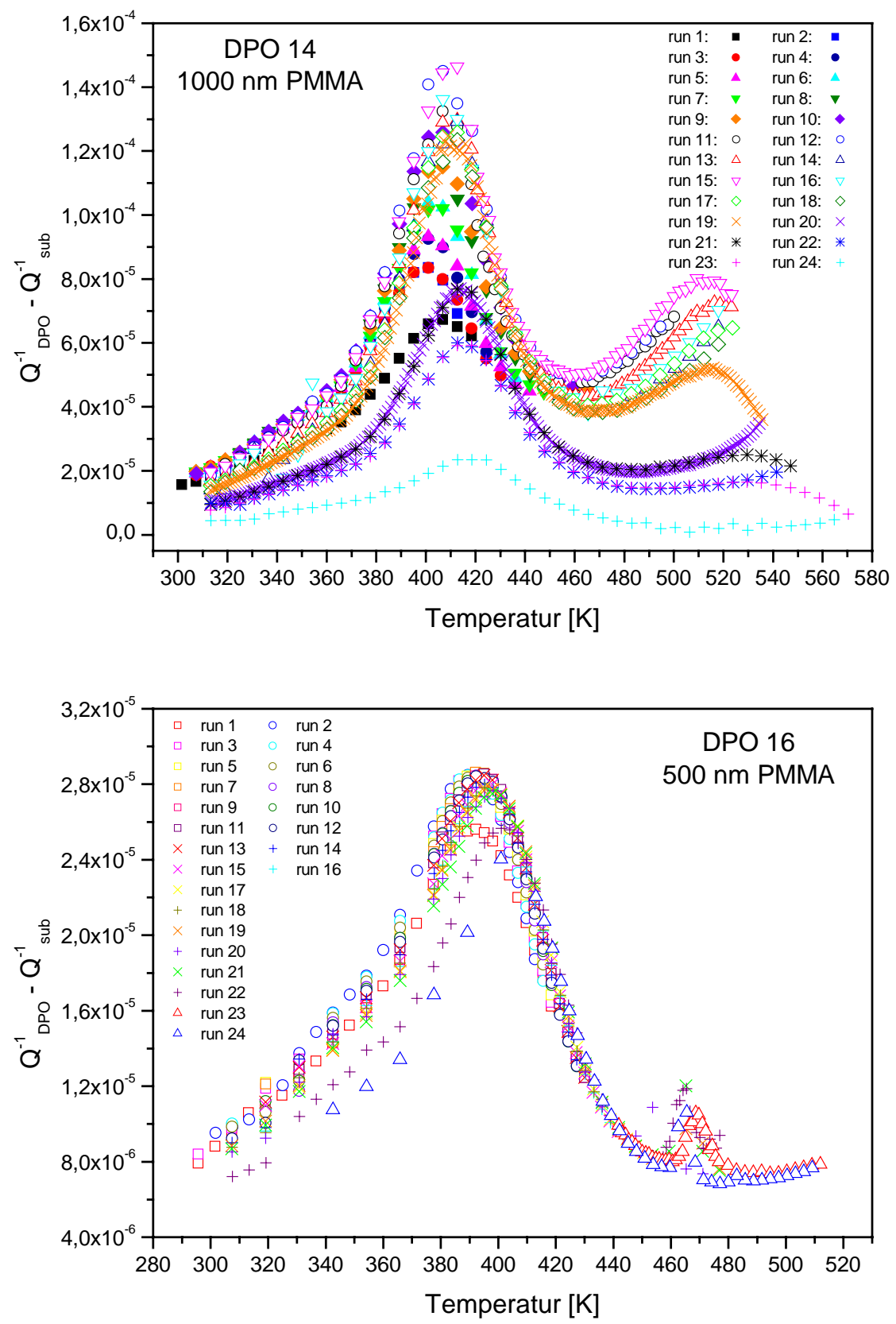

Abbildung 6.6: Dämpfungsdifferenz durch die PMMA-Schicht auf DPO 14 (oben) und DPO 16 (unten). Die Referenzdaten für die Differenzbildung bei DPO 14 wurden mathematisch rekonstruiert. 


\subsubsection{Temperaturabhängige Dämpfung}

Abbildung 6.6 zeigt für DPO 14 (1000 nm PMMA) und für DPO 16 (500 nm PMMA) die Dämpfungsdifferenz zwischen beschichtetem und leerem Oszillator. Herausstechendes Merkmal ist in beiden Bildern das ausgeprägte Maximum zwischen $380 \mathrm{~K}$ und $420 \mathrm{~K}$. Bei DPO 14 bildet sich ein zweites Maximum zwischen $500 \mathrm{~K}$ und $520 \mathrm{~K}$ aus, dessen Entstehung sich auch bei DPO 16 andeutet. Der hier zusätzlich reproduzierbar durchlaufene kleine Peak bei $470 \mathrm{~K}$ ist bei DPO 14 nicht zu erkennen.

Ein Effekt, der nur bei DPO 14 auftritt, ist in Abbildung 6.7 gezeigt. Der Maximalwert der Dämpfungsdifferenz steigt von $6,8 \times 10^{-5}$ für den ersten Durchlauf kontinuierlich in dem Maß auf $1,33 \times 10^{-4}$ an, in dem die erreichte Endtemperatur des vorhergehenden runs erhöht wurde. Im rechten Bild ist dieser Zusammenhang durch eine Trendlinie angedeutet.
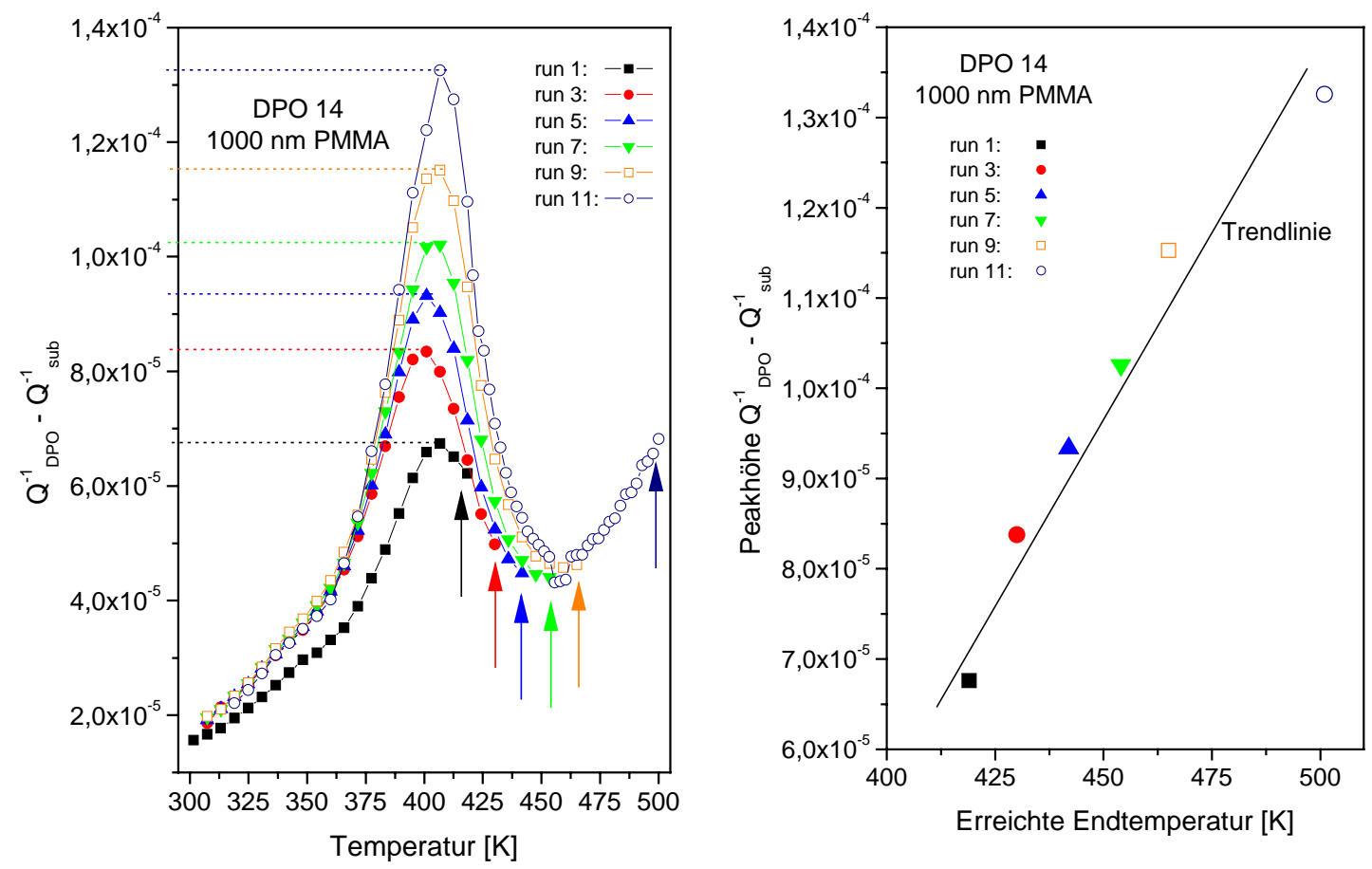

Abbildung 6.7: Im linken Bild ist die Entwicklung der Dämpfungsdifferenz bei DPO 14 mit zunehmender Endtemperatur (durch die Pfeile angedeutet) gezeigt. Das Dämpfungsmaximum ist im rechten Bild gegenüber der erreichten Endtemperatur aufgetragen.

Die Annahme, dass die Endtemperatur des vorhergehenden runs ausschlaggebend für die Veränderungen des Peaks ist, wird von der Beobachtung gestützt, dass die Heizkurven exakt auf den vorhergehenden Kühlkurven liegen. Diese Reversibilität ist in Abbildung 6.8 für einige 
Temperaturzyklen gezeigt. Im Gegensatz hierzu ergibt sich nach Erreichen einer jeweils neuen Maximaltemperatur ein veränderter Kurvenverlauf.

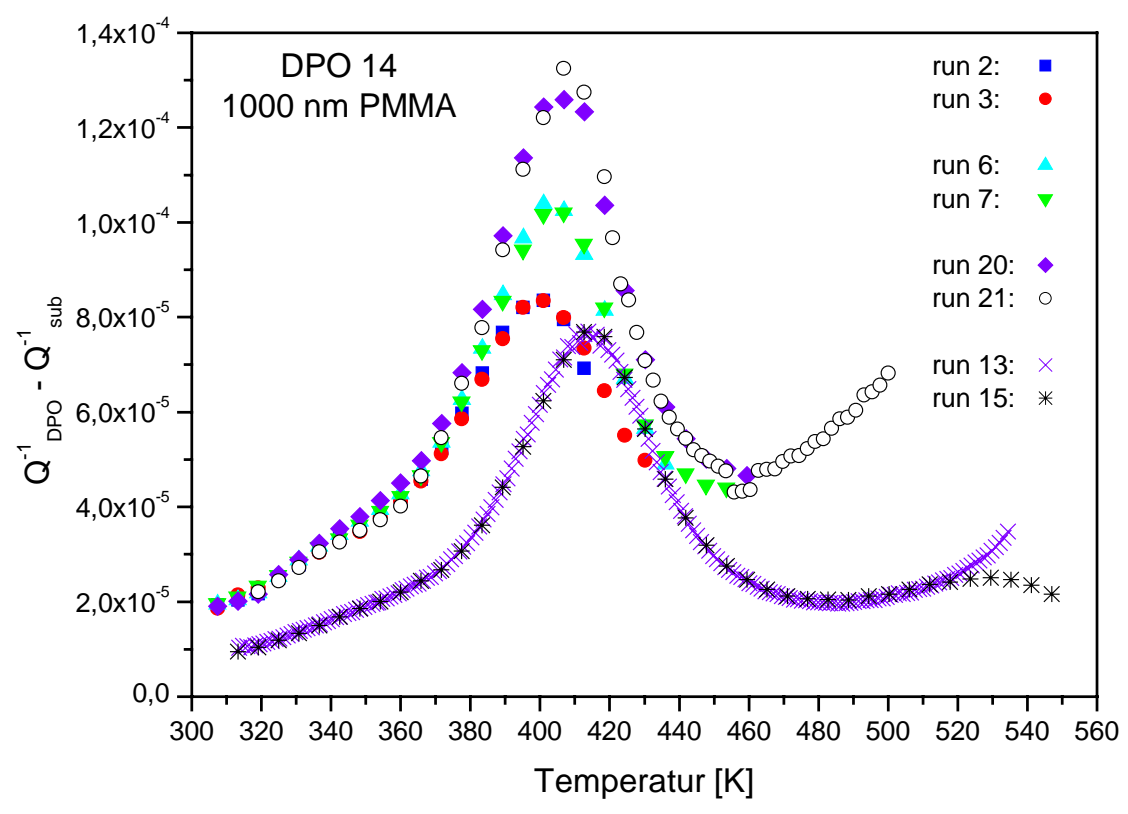

Abbildung 6.8: Reversibilität der Dämpfung für einige ausgewählte runs. Der Verlauf der Dämpfung beim Heizen deckt sich beinahe exakt mit dem Verlauf beim vorhergehenden Kühlen.

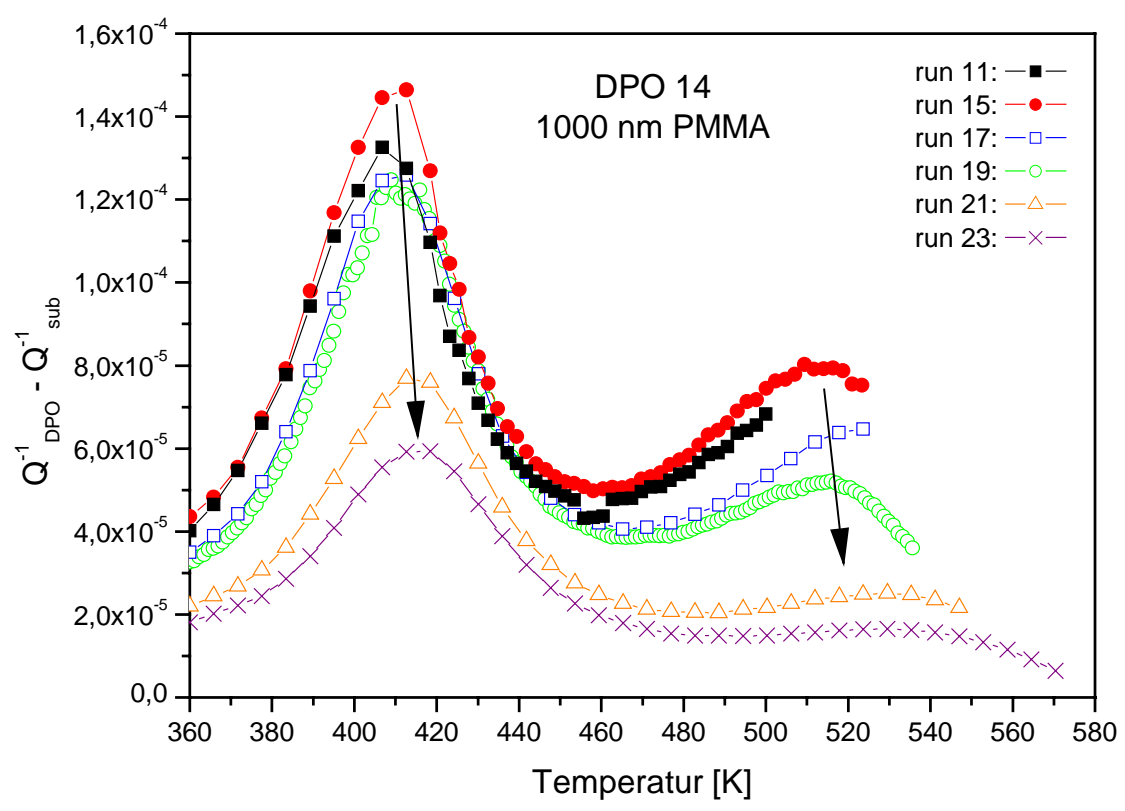

Abbildung 6.9: Abnahme der Höhe beider Peaks mit zunehmender Endtemperatur.

Wird die Maximaltemperatur auf über $500 \mathrm{~K}$ erhöht, so entsteht bei etwa $515 \mathrm{~K}$ ein zweites Maximum der Dämpfungsdifferenz (Abbildung 6.9). Allerdings bildet sich dieses Maximum 
sehr schnell zurück. Es ist bei run 21 kaum mehr zu erkennen. Die hohe Temperaturbeanspruchung des PMMA führt nun auch dazu, dass der Relaxationspeak bei etwa $410 \mathrm{~K}$ deutlich an Stärke verliert.
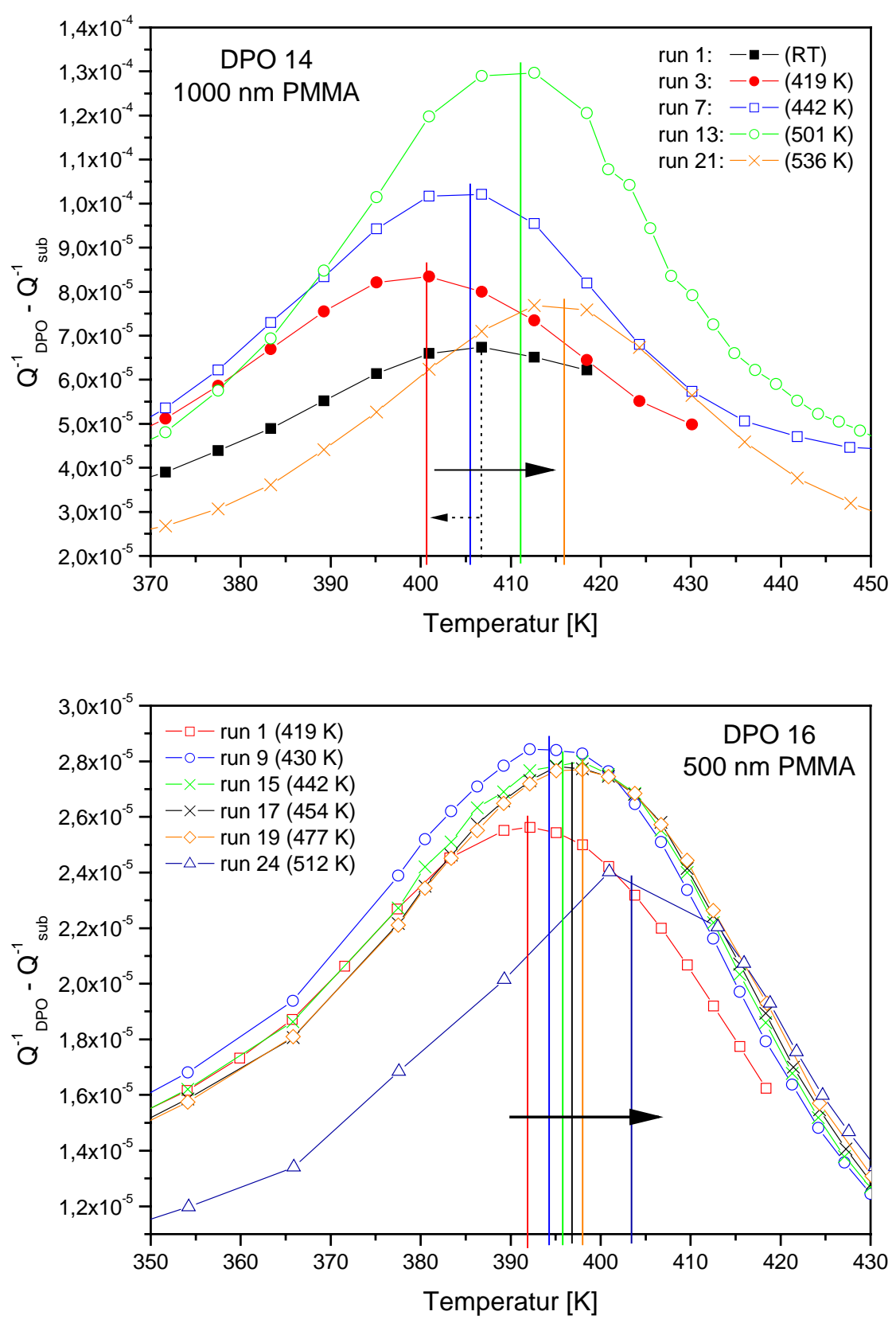

Abbildung 6.10: Veränderung der Peakposition bei PMMA auf DPO 14 (oben) und DPO 16 (unten) durch sukzessives Erhöhen der Maximaltemperatur. Der Pfeil signalisiert, dass sich das Maximum des Peaks im Verlauf der Versuchsdurchführung zu höheren Temperaturen hin verschiebt. Dargestellt sind ausgewählte runs. In Klammern ist die höchste bis zu diesem Zeitpunkt erreichte Temperatur angegeben. 
Sowohl bei DPO 14, als auch bei DPO 16 verschiebt sich die Position des Hauptmaximums im Laufe der Messreihen zu höheren Temperaturen. Diese Beobachtung ist in Abbildung 6.10 dargestellt. Im Verlauf des kompletten Temperaturprogramms beträgt die Verschiebung immerhin bis zu $15 \mathrm{~K}$. Bemerkenswert ist allerdings auch, dass die Position des Maximums für die PMMA-Schicht auf DPO 16 etwa $10 \mathrm{~K}$ niedriger liegt, als für die Schicht auf DPO 14.

\subsubsection{Isotherme Entwicklung von Frequenz und Dämpfung}

Bei DPO 16 wurde zusätzlich die isotherme Entwicklung der Resonanzfrequenz und der Dämpfung untersucht. Bei 419 K, 430 K und 477 K wurden diese Daten über mehrere Stunden aufgenommen. Dazwischen wurde in mehreren runs abgekühlt und wieder geheizt. In Abbildung 6.11 ist die zeitliche Entwicklung bei $419 \mathrm{~K}$ aufgetragen. Die Differenz der Resonanzfrequenz verschiebt sich im Lauf der Zeit zu kleineren negativen Werten, während die Dämpfungsdifferenz zunimmt. Beide Kurven streben einer Sättigung entgegen.
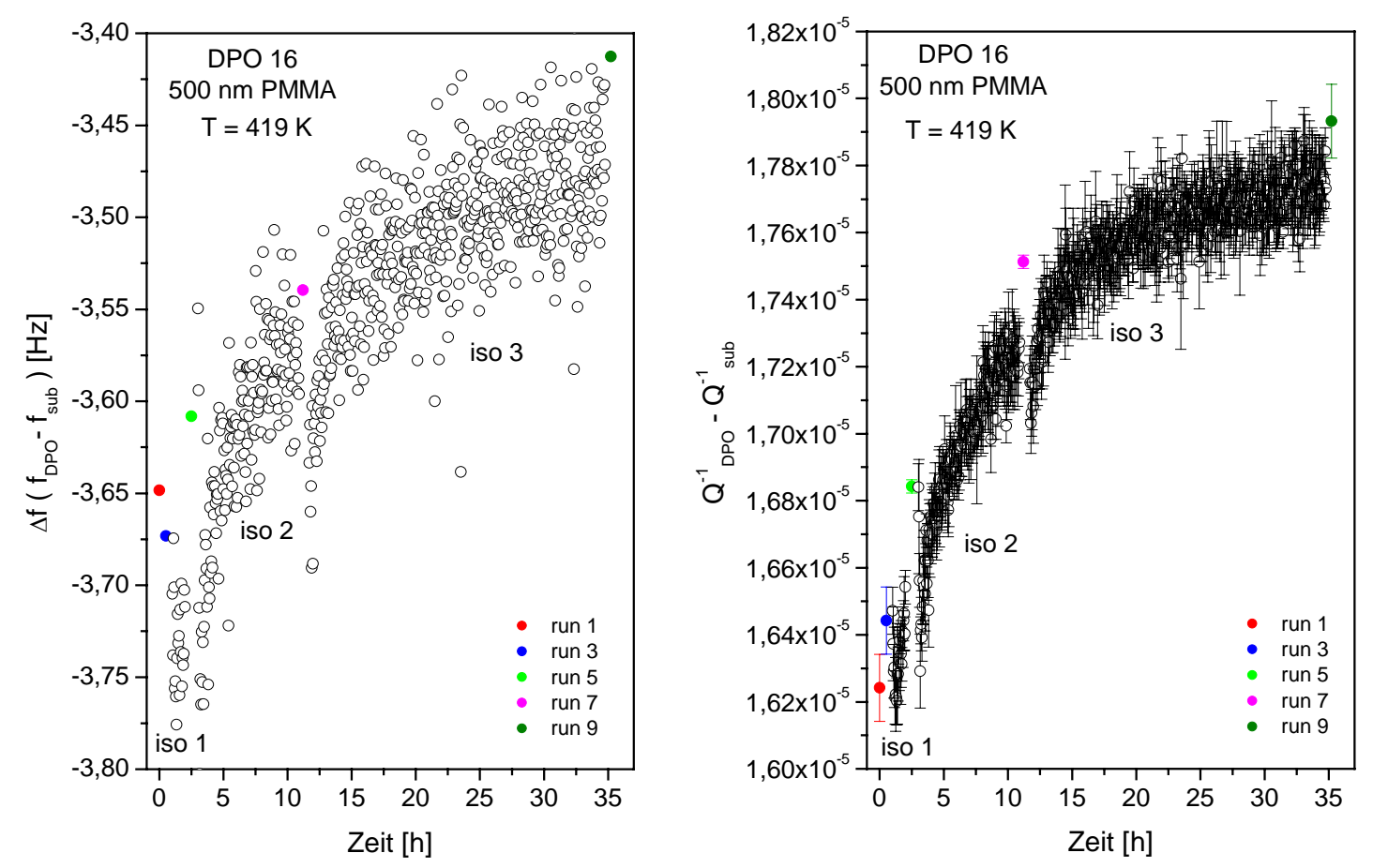

Abbildung 6.11: Frequenzdifferenz (links) und der Dämpfungsdifferenz (rechts) isotherm bei 419 K gemessen.

Bei $430 \mathrm{~K}$ (Abbildung 6.12) und bei $477 \mathrm{~K}$ (Abbildung 6.13) ergeben sich prinzipiell ähnliche zeitliche Verläufe, wobei der Anstieg in der Dämpfungsdifferenz bei $430 \mathrm{~K}$ deutlich geringer ausgeprägt ist, als bei 419 K. Die Sprünge in der Dämpfung bei $477 \mathrm{~K}$ sind im Rahmen dieser 
Betrachtung nicht zu erklären und werden auf Probleme mit der Einspannung des DPO-Fußes zurückgeführt. Rückschlüsse aus den gezeigten zeitlichen Verläufen der Resonanzfrequenz und der Dämpfung auf mikroskopische Prozesse werden in Abschnitt 7.7.4 besprochen.
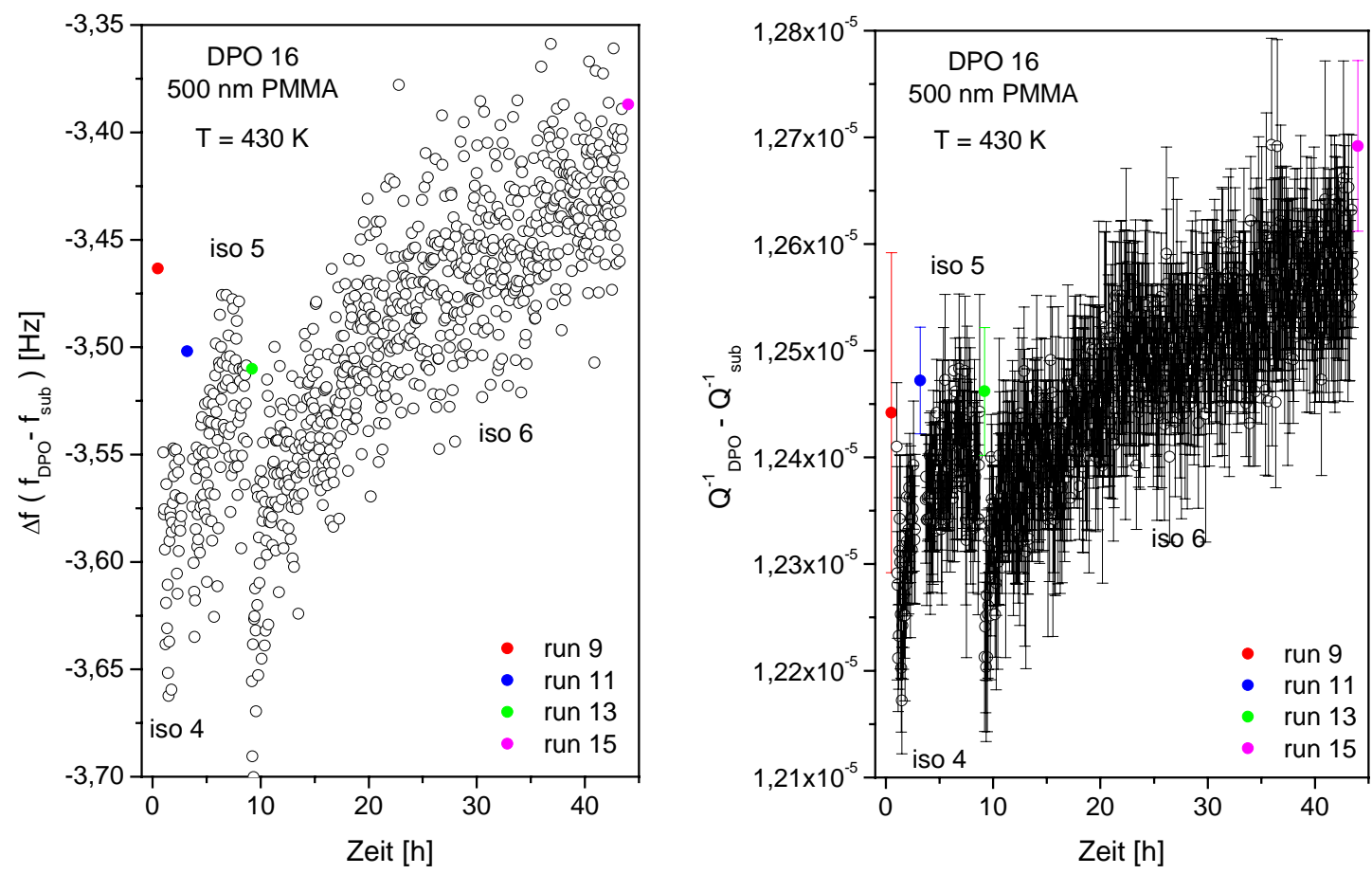

Abbildung 6.12: Frequenzdifferenz (links) und der Dämpfungsdifferenz (rechts) isotherm bei 430 K gemessen.
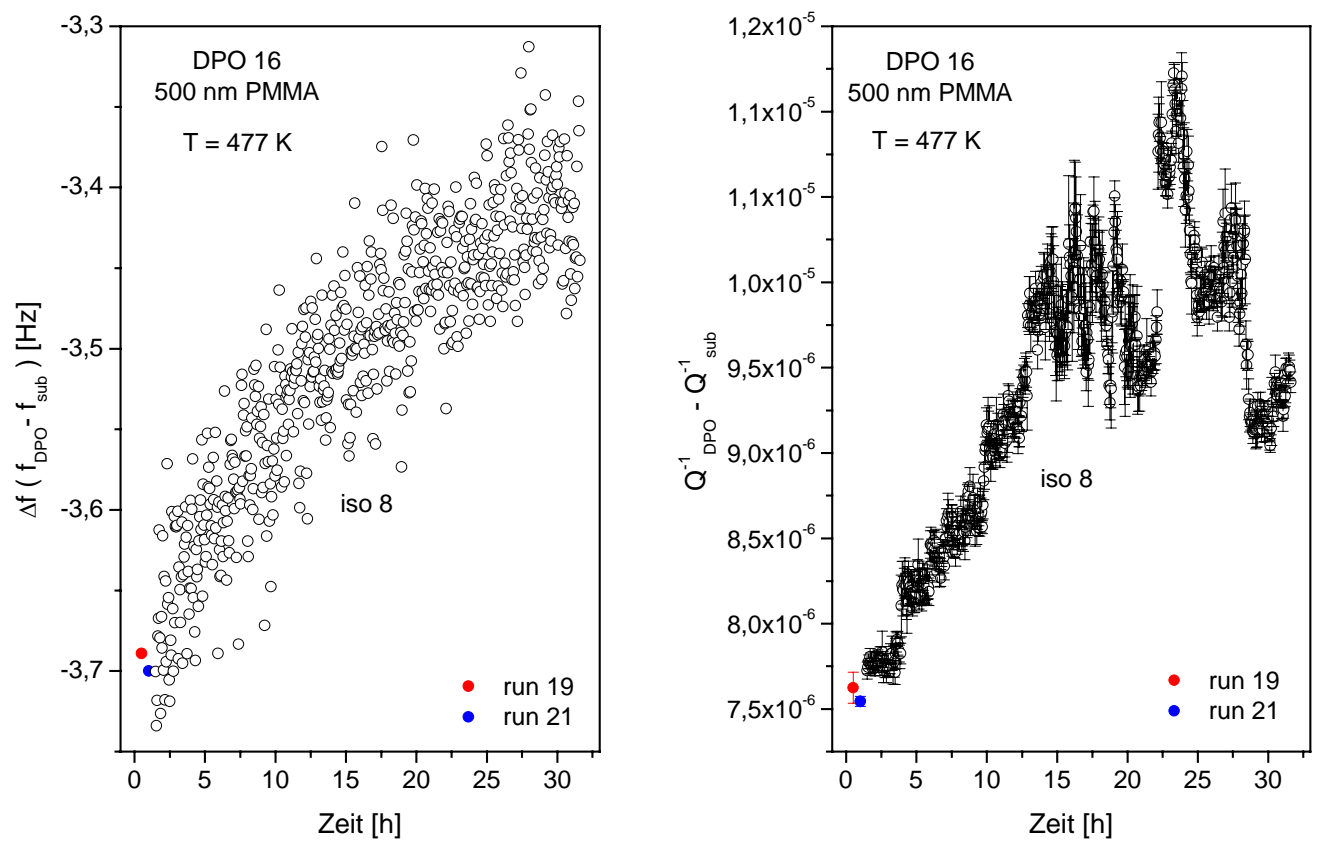

Abbildung 6.13: Frequenzdifferenz (links) und der Dämpfungsdifferenz (rechts) isotherm bei $477 \mathrm{~K}$ gemessen. 


\section{Diskussion}

\subsection{DPO als Substrat und Alternativen dazu}

Bei allen mechanischen Messungen wurde als Substrat für die dünnen Schichten ein DoppelPaddel Oszillator aus einkristallinem Silizium verwendet. In diesem Abschnitt werden die Materialeigenschaften von Silizium vorgestellt, die einen fundamentalen Einfluss auf das Verhalten des Oszillators haben. Alternative Geometrien und andere Materialien werden diskutiert.

\subsubsection{Temperaturabhängigkeit der elastischen Konstanten von Si}

In Abbildung 4.14 ist die Abhängigkeit der AS2-Resonanzfrequenz von der Temperatur gezeigt. Je größer die Temperatur wird, desto kleiner wird die Resonanzfrequenz. Aus diesen Daten kann der temperaturabhängige Schermodul von Silizium abgeleitet werden. Setzt man dazu Gleichung (4.28) und Gleichung (4.32) in Gleichung (4.31) ein, so ergibt sich folgende Beziehung für die AS2-Frequenz:

$$
\omega_{\text {AS2 }}=\sqrt{\frac{\kappa_{\text {neck }}}{I_{\text {head }}}} \cdot\left(1+\frac{I_{\text {head }}}{I_{\text {wing }}}\right)
$$

Die Trägheitsmomente sind konstant. Die Torsionskonstante $\kappa_{\text {neck }}$ in Gleichung (4.18) ist direkt proportional zum Schermodul G von Silizium. Wird die Wurzel in erster Näherung nach Taylor entwickelt, so erhält man für die den Zusammenhang zwischen den Änderungen von Resonanzfrequenz $\Delta \mathrm{f}$ und Schermodul $\Delta \mathrm{G}$ mit der Temperatur:

$$
\frac{\Delta f}{f}(T) \approx \frac{1}{2} \frac{\Delta G}{G}(T)
$$

Mit den Werten bei Raumtemperatur von $\mathrm{f}=5333 \mathrm{~Hz}$ und $\mathrm{G}=66,2 \mathrm{GPa}$ erhält man die in Abbildung 7.1 gezeigte Temperaturabhängigkeit des Schermoduls von Silizium. 


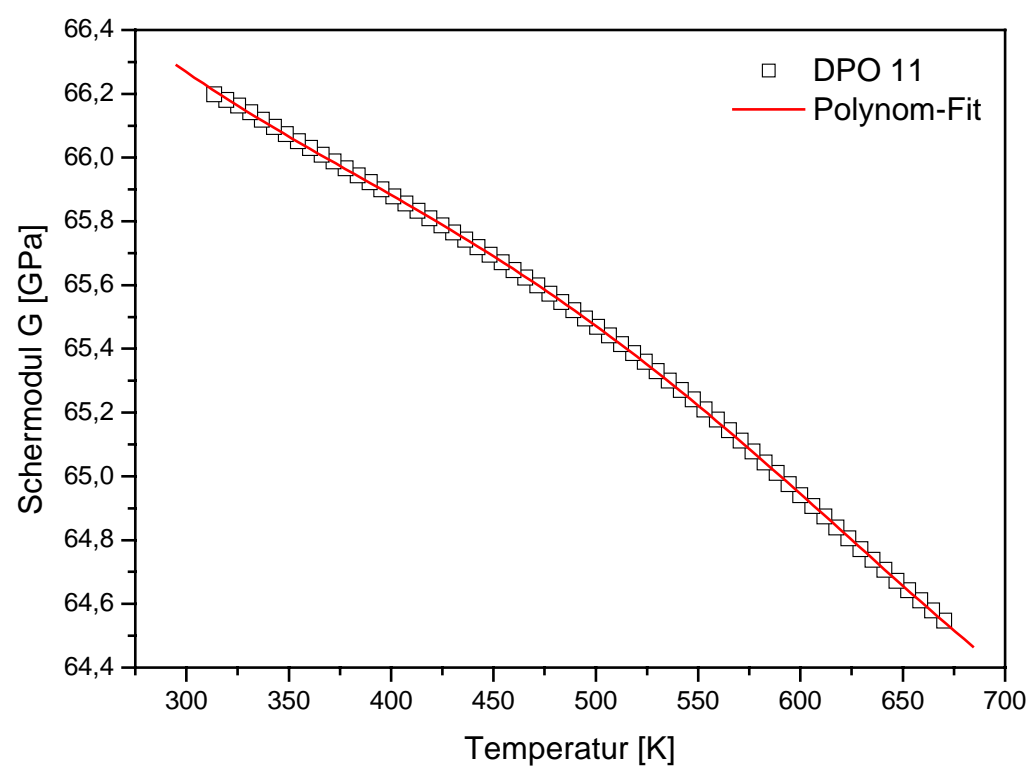

Abbildung 7.1: Veränderung des Schermoduls von Silizium in Abhängigkeit von der Temperatur. Messungen an DPO 11 (schwarze Quadrate) und Kurvenanpassung mit Polynom 4. Ordnung (rote Linie).

Dabei ist zu beachten, dass der Schermodul im untersuchten Temperaturintervall von Raumtemperatur bis etwa $670 \mathrm{~K}$ um rund 2,5\% abnimmt. Für die Kurvenanpassung ergibt sich bei Fit mit einem Polynom 4. Ordnung folgende Funktion:

$$
G(T)=70,79-0,035 \cdot T+1,032 \times 10^{-4} \cdot T^{2}-1,456 \times 10^{-7} \cdot T^{3}+7,248 \times 10^{-11} \cdot T^{4}
$$

Mit der temperaturunabhängigen Poisson-Zahl $v$ überträgt sich die Abhängigkeit des Schermoduls $\mathrm{G}$ von der Temperatur über der Beziehung

$$
G=\frac{E}{2(1+v)}
$$

auch auf den Elastizitätsmodul E. Die Werte für die $<110>$-Richtung von Silizium betragen bei Raumtemperatur $\mathrm{E}=169 \mathrm{GPa}, \mathrm{G}=66,2 \mathrm{GPa}$ und $v=0,276$ [147].

\subsubsection{Kristallografische Eigenschaften von Silizium}

Um die mechanischen Eigenschaften eines Festkörpers zu beschreiben, betrachtet man in der Elastizitätstheorie die Spannungs- und Dehnungskomponenten eines infinitesimal kleinen Volumenelementes in Form eines Parallelepipeds [164]. Die Spannungs- und Dehnungskomponenten, die dabei an jeder Fläche angreifen und in die drei Raumrichtungen wirken, bilden je 
einen Tensor zweiter Stufe mit neun Elementen. Das verallgemeinerte Hook-Gesetz für den nicht-isotropen Festkörper verknüpft beide Tensoren über die 81-elementige Matrix der elastischen Moduln oder äquivalent der elastischen Nachgiebigkeit. Diese große Anzahl an unabhängigen Matrixelementen reduziert sich, falls Kristallsymmetrien existieren [165]

Wegen der Bedeutung von Silizium für die Halbleiterindustrie sind die elastischen Konstanten der unterschiedlichen kristallografischen Raumrichtungen gut bekannt [166] und auch experimentell gemessen. So gibt Yi [167] den Young-Modulus E in Richtung der Hauptachsen eines Einkristalls mit $\mathrm{E}_{<100>}=115,3 \mathrm{GPa}, \mathrm{E}_{<110>}=169,2 \mathrm{GPa}$ und $\mathrm{E}_{<111>}=191,1 \mathrm{GPa}$ an. Durch das anisotrope Cornell-Ätzverfahren muss die Symmetrieachse der Doppel-Paddel Oszillatoren stets in $<110>$-Richtung orientiert sein. Diese herstellungsbedingte Vorgabe ist beim Laser-Cut-Verfahren nicht mehr notwendig. Würde der Siliziumwafer beim Schneiden um $45^{\circ}$ gedreht in die Apparatur eingesetzt, so wäre die Oszillatorachse in $<111>$-Richtung orientiert. Mit Ausgangswafern, die eine andere Kristallorientierung haben, sind auch andere Richtungen für die Oszillatorachse realisierbar.

Der dann veränderte Young-Modul entlang der Torsionsachse wirkt sich sowohl auf die Eigenfrequenzen, als auch auf die Dämpfung des leeren Oszillators aus. In welcher Größenordnung dies geschieht wurde in dieser Arbeit nicht untersucht, wäre jedoch für weitergehende Experimente eine interessante Fragestellung.

\subsubsection{Alternative Materialien für das DPO-Substrat}

Alternativ zu Silizium wurde ein Paddel mit dem Laser-Cut-Verfahren aus einem $200 \mu \mathrm{m}$ dicken Saphir-Substrat geschnitten und getestet. Da Saphir eine große Härte besitzt besteht die Vermutung, dass die Dämpfung bei diesem Material ebenso klein, oder gar noch geringer sein könnte als bei Oszillatoren aus Silizium. Die AS2-Resonanzfrequenz wurde bei etwa $5000 \mathrm{~Hz}$ identifiziert, allerdings war es nicht möglich, den Phase-Lock-Loop anzuregen, weshalb diese Experimente abgebrochen wurden.

\subsubsection{Temperaturstabilität als empfindlichkeitsbegrenzender Faktor}

Die große Genauigkeit, mit der Eigenfrequenzen bestimmt werden können und die geringe Dämpfung des unbedampften Doppel-Paddel Oszillators schaffen ideale Voraussetzungen um 
die mechanischen Eigenschaften selbst von sehr dünnen Schichten zu bestimmen. Allerdings ist die Temperaturstabilität ein begrenzendes Kriterium für die Empfindlichkeit.

Mit der in Abschnitt 4.3.5 vorgestellten Regelung lassen sich niedrige Temperaturen, bis etwa $350 \mathrm{~K}$, mit einer Genauigkeit besser als $0,05 \mathrm{~K}$ reproduzierbar einstellen und innerhalb eines solchen Intervalls auch regeln. Nach Gleichung (7.2) lässt sich die daraus resultierende Schwankung des Schermoduls für Silizium berechnen. Für die AS2-Resonanzfrequenz von etwa $5400 \mathrm{~Hz}$ bedeutet das eine Unsicherheit kleiner 0,01 Hz. Übertragen auf die Resonanzfrequenz der ST-Mode von etwa 415 Hz ergibt sich mit diesen Überlegungen ein durch Temperaturschwankungen verursachtes Intervall von $0,077 \mathrm{~Hz}$.

Soll nun eine dünne Schicht zweifelsfrei durch die Änderung der ST-Resonanzfrequenz detektiert werden, so muss die zusätzliche Masse auf dem Oszillator mindestens eine Verschiebung um ein solchen Intervall bewirken. Nach Gleichung (4.45) können, bei einer ähnlichen Dichte wie Silizium, Schichten genau dann noch detektiert werden, wenn ihre Dicke größer als $2,2 \mathrm{~nm}$ ist.

Diese große Empfindlichkeit verringert sich bei hohen Temperaturen dramatisch, wie in Abschnitt 4.3.5 gezeigt ist. So schwankt die Temperatur bei $660 \mathrm{~K}$ innerhalb eines Intervalls von $0,8 \mathrm{~K}$, was letztlich bedeutet, dass die Schicht mindestes $40 \mathrm{~nm}$ dick sein muss, um durch die Frequenzverschiebung eindeutig wahrnehmbar zu sein.

Statistische Abweichungen bei der Dämpfung des Oszillators liegen innerhalb eines Intervalls von $2 \times 10^{-7}$. Diese Schwankungsbreite ist relativ unabhängig von der Temperatur. Aus Gleichung (4.55) errechnet sich dann die Unsicherheit für das Produkt aus Schermodul und Schichtdicke. Ist der Schermodul einer Schicht in etwa so groß wie der von Silizium, und wird der Dämpfungsfaktor eines Materials zu $\mathrm{Q}^{-1}=0,005$ angenommen, dann können $4 \mathrm{~nm}$ dicke Filme gemessen werden.

Da aber der Schermodul der Schicht über die Verschiebung der Resonanzfrequenzen in Gleichung (4.47) berechnet wird, überträgt sich die Unsicherheit der Frequenzbestimmung durch Temperaturschwankungen auch auf die Dämpfung. Die schlechte Temperaturstabilität ist damit der limitierende Faktor für die Genauigkeit. Trotzdem können mit der hier vorgestellten Apparatur Schichten ab 40 nm Dicke untersucht werden. 


\subsection{5 Änderungen an der DPO-Geometrie}

Für die Interpretation der Daten aus der mechanischen Spektroskopie wären weitergehende Messungen bei verschiedenen Frequenzen wünschenswert. Dann könnten direkt, ohne die Temperaturabhängigkeit der Relaxationszeiten voraussetzen zu müssen, verschiedene charakteristische Zeitskalen abgefragt werden. Aus diesem Grund gibt es Überlegungen, die Abmessungen des Doppel-Paddel Oszillators so zu verändern, dass sich die AS2-Resonanzfrequenz signifikant verschiebt. In Abschnitt 4.5 wurde erläutert, wie die Eigenfrequenzen von den geometrischen Abmessungen abhängen.

In der Praxis würde eine Veränderung der Paddelgeometrie die Neukonstruktion der gesamten Apparatur bedingen. Relativ einfach zu testen sind allerdings Variationen der Oszillatordicke. So wurden Paddel nach dem Laser-Cut-Verfahren aus $200 \mu \mathrm{m}, 375 \mu \mathrm{m}$ und $500 \mu \mathrm{m}$ dicken Silizium-Wafern hergestellt und getestet. Für den $500 \mu \mathrm{m}$ dicken Oszillator liegt die AS2-Frequenz etwa bei 7000 Hz. Für das $200 \mu \mathrm{m}$ dicke Paddel beträgt sie etwa $3000 \mathrm{~Hz}$, allerdings konnte bei diesem Oszillator wegen der großen Grunddämpfung die AS2-Mode im PhaseLock-Loop nicht angeregt werden. Da frequenzabhängige Messungen mit einer um den Faktor 10 oder gar 100 variierenden Frequenz interessant wären, wurden diese Versuche nicht weiter verfolgt.

\subsection{Schichtdicke und Materialdichte}

Die Schichtdicke in situ auf einem eingebauten und bedampften DPO zu messen, ist mit der hier konstruierten Apparatur nicht möglich. Allerdings wurden sowohl an PMMA- als auch an $\mathrm{Zr}_{65} \mathrm{Al}_{7,5} \mathrm{Cu}_{27,5}$-Schichten, die unter genau gleichen Bedingungen hergestellt wurden, wie später die Schichten auf die Oszillatoren, unabhängige Dickenbestimmungen durchgeführt. Bei PMMA wurde die Dicke der Schichten mittels Kleinwinkel-Röntgenstreuung untersucht, bei $\mathrm{Zr}_{65} \mathrm{Al}_{7,5} \mathrm{Cu}_{27,5}$ durch Tooling-Messungen für die einzelnen Komponenten auf SiliziumSubstraten vor der eigentlichen Schichtherstellung. Eigentlich könnte die Dicke der Schichten nun als bekannt vorausgesetzt werden. Aber es gibt deutliche Hinweise, die Zweifel an dieser Aussage aufkommen lassen. Sie werden in diesem Abschnitt diskutiert.

Auch die Abschätzung der Massendichte für das Schichtmaterial ist problembehaftet. Bei $\mathrm{Zr}_{65} \mathrm{Al}_{7,5} \mathrm{Cu}_{27,5}$ wird sie durch ein gewichtetes Mittel aus den Einzeldichten berechnet: 


$$
\rho\left(Z_{65} A l_{7,5} C u_{27,5}\right)=\frac{65}{100} \rho(Z r)+\frac{7,5}{100} \rho(A l)+\frac{27,5}{100} \rho(C u) \approx 6892 \frac{\mathrm{kg}}{\mathrm{m}^{3}}
$$

Für die Dichten der Komponenten wird dabei die der Kompaktmaterialien eingesetzt. Weil aber während der Kokondensation aus der Gasphase, bedingt durch die amorphe Struktur der entstehenden Schichten, freies Volumen mit eingeschreckt wird, ist die tatsächliche Massendichte kleiner, als die in Gleichung (7.5) berechnete. Für PMMA-Schichten wird für die Dichte die des Ausgangsmaterials angenommen, die vom Hersteller mit $1200 \mathrm{~kg} / \mathrm{m}^{3}$ angegeben wird.

\subsubsection{Beobachtungen bei $\mathrm{Zr}_{65} \mathrm{Al}_{7,5} \mathrm{Cu}_{27,5}$ - Frequenzverschiebungen}

In Gleichung (4.46) wird beschrieben, dass die Verschiebung der Resonanzfrequenz der STMode ein direktes Maß für das Produkt aus Dichte und Dicke der dünnen Schichten ist. Die ST-Resonanzfrequenz muss daher vor und nach dem Aufbringen der Schicht bestimmt werden. Die Vorgehensweise ist in Abschnitt 5.2.4 beschrieben.

Für die untersuchten Schichten kann die theoretisch erwartete ST-Frequenzdifferenz mit der vorgegebenen Schichtdicke und der in Gleichung (7.5) berechneten Dichte ermittelt werden. Die Werte sind in Tabelle 5.4 dargestellt. Sie werden mit den experimentell gemessenen Frequenzverschiebungen verglichen. Es zeigt sich, dass bei allen Schichten die gemessene Frequenzdifferenz nur etwa $63 \%$ dessen beträgt, was nach dem mathematischen Modell erwartet würde. Für dieses Resultat gibt es drei plausible Erklärungen:

- Die mathematischen Näherungen in Abschnitt 4.7.1 sind so groß, dass Gleichung (4.45) den Prozess nicht ausreichend exakt wiedergibt. Würde man beispielsweise den Faktor 2 in Gleichung (4.46) durch den Faktor 3 ersetzen, würde die theoretische Beschreibung dem Experiment mit großer Genauigkeit entsprechen.

- Falls die Gleichungen (4.45) und (4.46) korrekt sind, würde das bedeuten, dass das Produkt aus Schichtdicke und-dichte nur $63 \%$ des erwarteten Wertes beträgt. Entweder ist also die Schicht nicht so dick wie angenommen, oder die Dichte ist deutlich kleiner, als durch das gewichtete Mittel abgeschätzt.

- Beim Bedampfen ist die Durchstrahl-Öffnung zum Oszillator für die Metallatome in der Gasphase durch verschiedene Abschattungen geometrisch relativ eng begrenzt. Bei den Tooling-Messungen wurde stets ein kleiner Bereich, exakt in der Mitte dieser Öffnung be- 
trachtet, wo sich beim Oszillator der Hals befindet. Es könnte aber sein, dass die äußeren Bereiche der Flügel auch wegen der $\cos ^{2}$-förmigen Gaskeulen aus den Verdampfern mit weniger Material belegt sind, als die zentrale Mittelposition von Kopf und Hals. Der senkrechte Abstand des Materials von der Torsionsachse wirkt sich quadratisch auf den zusätzlichen Trägheitsmoment durch die Schicht aus. Schon eine geringe Abnahme der Schichtdicke an den Flügelrändern würde den Masseeffekt zur Resonanzverschiebung überproportional klein werden lassen, wie ja tatsächlich beobachtet.

Die TEM-Bilder in Abbildung 5.2 sind mit einer Dickenskala versehen. Demnach sind die drei auf DPO 12 aufgedampften, je $300 \mathrm{~nm}$ dicken Schichten insgesamt nicht $900 \mathrm{~nm}$ wie angenommen, sondern nur $720 \mathrm{~nm}$ dick, was 80\% des erwarteten Wertes entspricht. Ähnlich verhält es sich bei DPO 19. Die im TEM bestimmte Dicke beträgt $1250 \mathrm{~nm}$ und beträgt damit nur 79\% der erwarteten Gesamtdicke auf diesem Oszillator von $1590 \mathrm{~nm}$.

\subsubsection{Beobachtung bei PMMA-Frequenzverschiebungen}

Weitere Hinweise ergeben sich aus der Betrachtung der PMMA-Resultate. Zwar wurde die ST-Resonanzfrequenz vor der externen Schichtherstellung nicht gemessen, allerdings sind aus der Verschiebung der AS2-Frequenz ähnliche Schlussfolgerungen möglich.

Wie aus Gleichung (4.41) ersichtlich ist, gibt es zwei gegenläufig orientierte Beiträge zur AS2-Frequenzverschiebung. Der Masseanteil führt zu kleineren Resonanzfrequenzen, weshalb das Vorzeichen in Gleichung (4.43) negativ ist. Elastische Beiträge der Schicht führen, je nach Größe ihres Schermoduls, zu größeren Frequenzen, wie durch das positive Vorzeichen in Gleichung (4.42) ausgedrückt.

In Abbildung 6.5 ist die Verschiebung der AS2-Frequenz durch eine $500 \mathrm{~nm}$ dicke PMMASchicht gezeigt. Offensichtlich wird selbst bei hohen Temperaturen die Grenze von $-4 \mathrm{~Hz}$ nicht unterschritten. Unterstellt man, dass bei hohen Temperaturen, in der unterkühlten Schmelze der Schicht, ihr Schermodul sehr klein wird, so kann der elastische Beitrag in Gleichung (4.42) näherungsweise gleich null gesetzt werden. Die gesamte beobachtete Resonanzverschiebung wird nun durch die zusätzliche Masse verursacht. Mit den gegebenen Daten für Dicke und Dichte dieser PMMA-Schicht errechnet sich nach Gleichung (4.43) eine Frequenzverschiebung von $-2,286 \mathrm{~Hz}$. Dieser betragsmäßig kleine Wert würde in der Konsequenz 
einen negativen Schermodul für die Schicht bedeuten, was physikalisch natürlich nicht sinnvoll ist. Prinzipiell gibt es zwei Erklärungen:

- Die beiden Gleichungen (4.41) und (4.43) beschreiben die Wirkung der zusätzlichen Masse der Schicht auf dem Oszillator nicht genau genug. Allerdings müsste der Faktor 2 in Gleichung (4.43) nicht wie bei den metallischen Glasschichten größer, sondern kleiner sein. Für die gezeigte Schicht müsste er bei etwa 1,14 liegen.

- Schichtdicke, -dichte, oder beide, sind nicht korrekt ermittelt. Das tatsächliche Produkt aus Dicke und Dichte wäre dann 1,75 mal so groß sein, wie mit den gegebenen Werten berechnet. In der Praxis bedeutet das, dass die Schichtdicke in Wirklichkeit größer ist, als angenommen, oder dass die Dichte der Schicht durch den Herstellungsprozess deutlich größer ist, als im PMMA-Ausgangsmaterial.

\subsubsection{Bewertung und Konsequenzen für die Interpretation}

Dass die Abweichungen von den Gleichungen aus Abschnitt 4.7.1 bei PMMA und bei $\mathrm{Zr}_{65} \mathrm{Al}_{7,5} \mathrm{Cu}_{27,5}$ zwar um etwa den gleichen Betrag aber in unterschiedliche Richtungen erfolgen, deutet darauf hin, dass in der mathematischen Ableitung keine prinzipiellen Fehler stecken. Die gegebenen Gleichungen werden daher als korrekt angenommen.

Die „Tooling“-Messungen der Zr-, Al- und Cu-Schichten wurden mit großer Akribie mehrmals durchgeführt. Trotzdem beträgt die in den TEM-Bildern gemessene Dicke nur etwa 80\% der eingestellten Soll-Dicke.

Die Dichte von $\mathrm{Zr}_{65} \mathrm{Al}_{7,5} \mathrm{Cu}_{27,5}$-Schichten wurde in Vorgängerarbeiten untersucht. Sie weicht nicht wesentlich von dem in Gleichung (7.5) berechneten gewichteten Mittel ab. Nun liegt die Vermutung nahe, dass die gemessene, zu kleine Frequenzverschiebung ihre Ursache in einer Kombination aus ungenauem Tooling, Skalierungsfehlern beim TEM und der mit zunehmendem Abstand von der Torsionsachse abnehmenden Schichtdicke auf den Oszillatorflügeln, hat.

Es stellt sich die Frage, ob sich eine ungleichmäßige Schichtdicke auf die ST- und die AS2Resonanzverschiebung gleichermaßen auswirken. Bei einer $300 \mathrm{~nm}$ dicken Schicht der Dichte von $6892 \mathrm{~kg} / \mathrm{m}^{3}$ beträgt die massebedingte AS2-Resonanzverschiebung $-8,02 \mathrm{~Hz}$. Überträgt man die beobachtete Abweichung bei den ST-Messungen, so dürfte die Verschiebung davon nur $63 \%$, also $-5,05 \mathrm{~Hz}$, betragen. In Abbildung 5.21 beobachtet man für eine solche Schicht 
bei hohen Temperaturen eine AS2-Verschiebung von $-6,3 \mathrm{~Hz}$. Wird diese Verschiebung ausschließlich dem Masseanteil zugeschrieben, so beträgt die gemessene Verschiebung der AS2-Resonanzfrequenz immerhin wären das $79 \%$ des theoretisch erwarteten Wertes. Berücksichtigt man nun noch, dass der Schermodul in der unterkühlten, zähen Flüssigkeit zwar klein, aber nicht null ist, so muss in jedem Fall zusätzlich ein elastischer Beitrag mit positivem Vorzeichen berücksichtigt werden.

Die widersprüchlichen Beobachtungen lassen sich im Rahmen dieser Arbeit nicht auflösen. Um weitere Auswertungen vornehmen zu können wird die massebedingte Verschiebung der AS2-Resonanzfrequenz künftig mit $90 \%$ des theoretisch erwarteten Wertes angesetzt. Diese Festlegung ist zwar willkürlich, erscheint nach Abwägung aller betrachteten Ergebnisse aber als nicht unrealistisch.

Die Schichtdicke von PMMA-Filmen mittels Kleinwinkel-Röntgenstreuung an Vergleichsschichten zu bestimmen ist zwar möglich, wegen der großen Rauhigkeit der Filme und der geringen Reflexion an der Grenzfläche Schicht-Luft, aber stark fehlerbehaftet. Beobachtungen von R. Wehn bei den dielektrischen Messungen deuten in der Tat auf eine größere Schichtdicke hin, als ursprünglich vermutet.

Bei Messungen des Elastizitätsmoduls und der Vickershärte an Schichten und am Ausgangsmaterial wurde von E. Süske für die Schichten ein größerer E-Modul und auch eine größere Härte festgestellt [196]. Kombiniert mit der verkürzten Kettenlänge der PMMA-Moleküle in der Schicht weist dies zusätzlich auf eine größere Gesamtdichte der Schicht im Vergleich zum Ausgangsmaterial hin.

Berücksichtigt man auch hier, dass der Schermodul der PMMA-Schmelze nicht kleiner als null werden kann, so wird der Massebeitrag zur AS2-Frequenzverschiebung bei PMMA künftig mit dem 1,8-fachen des theoretisch berechneten Wertes festgesetzt. Dabei wird davon ausgegangen, dass dieser Faktor im Wesentlichen durch eine größere Schichtdicke verursacht ist, weshalb auch bei späteren Auswertungen die Schichtdicke selbst mit dem Faktor 1,8 skaliert wird.

\subsection{Abhängigkeit der $\mathrm{Zr}_{65} \mathrm{Al}_{7,5} \mathrm{Cu}_{27,5}$ Ergebnisse vom Substrat}

In Kapitel 5.2 sind die Ergebnisse aus der mechanischen Spektroskopie an $\mathrm{Zr}_{65} \mathrm{Al}_{7,5} \mathrm{Cu}_{27,5}$ dargestellt. Offensichtlich ergeben sich signifikante Unterschiede, je nachdem, auf welcher Un- 
terlage die Schicht aufgedampft ist. Auf diese Unterschiede und mögliche Ursachen wird in nun detailliert eingegangen.

\subsubsection{Abhängigkeit der Frequenzverschiebung vom Substrat}

Abbildung 7.2 zeigt die Gegenüberstellung der AS2-Frequenzverschiebung dreier, jeweils $300 \mathrm{~nm}$ dicker, Schichten. Gemeinsam ist allen drei Kurvenverläufen, dass die gemessene Frequenzverschiebung beim Aufheizen gegenüber der beim Abkühlen zu größeren negativen Werten verschoben ist. Alle drei gezeigten Schichten sind bei hohen Temperaturen kristallisiert. Da elastische Beiträge zur Frequenzverschiebung durch die Schicht stets positives Vorzeichen haben (Gleichung 4.42), bedeutet dies, dass der Speichermodul für kristalline Schichten größer ist, als für amorphe.

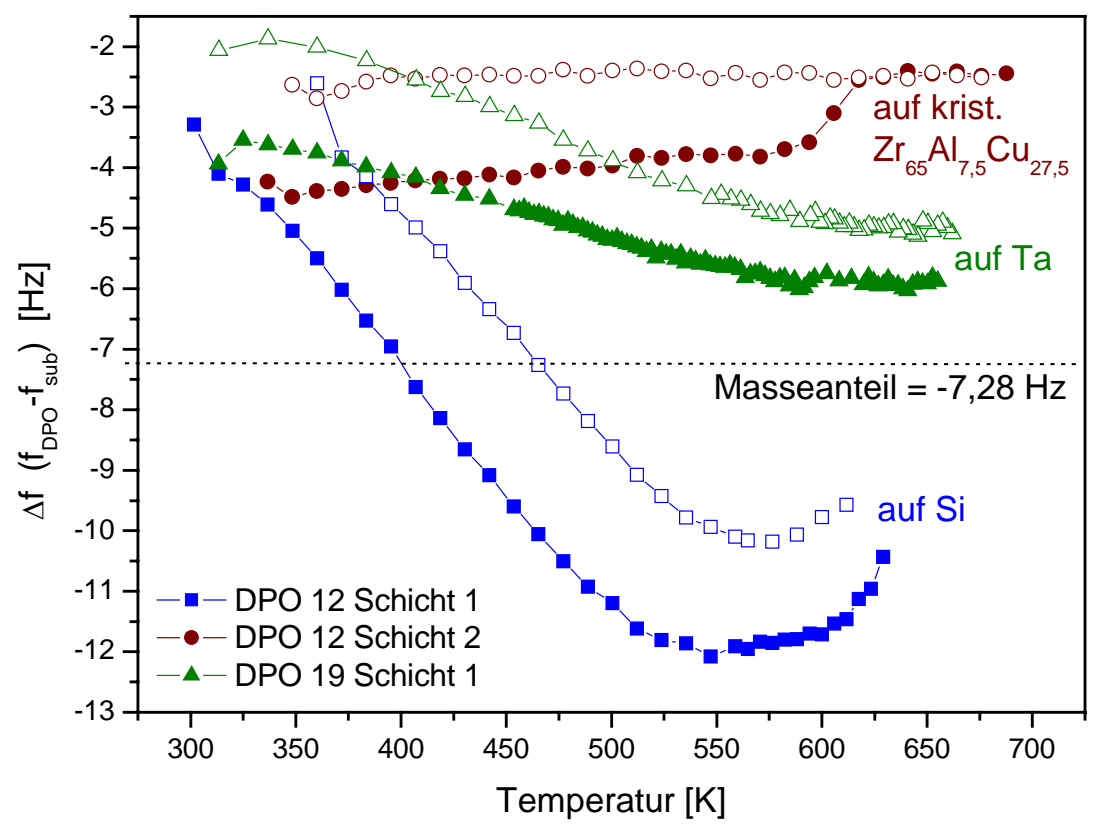

Abbildung 7.2: Verschiebung der AS2-Resonanzfrequenz durch eine $300 \mathrm{~nm}$ dicke Schicht in Abhängigkeit von der Temperatur. Bei DPO 12 Schicht 1 (blau) besteht die Unterlage aus Silizium, bei DPO 12 Schicht 2 (rot) aus einer kristallisierten $\mathrm{Zr}_{65} \mathrm{Al}_{7,5} \mathrm{Cu}_{27,5}$-Schicht und bei DPO 19 Schicht 1 (grün) aus einer Tantal-Zwischenschicht. Ausgefüllte Symbole zeigen den ersten Heizvorgang, offene Symbole das Abkühlen der Schicht.

Die gestrichelte, waagerechte Linie deutet die Verschiebung der Frequenz zu kleineren Werten durch den Beitrag der zusätzliche Masse auf dem Oszillator an. Werden die elastischen Beiträge, die stets entgegengesetztes Vorzeichen haben, berücksichtigt, so folgt, dass die be- 
obachtete gesamte Frequenzverschiebung für alle Temperaturen oberhalb der gestrichelten Linie liegen muss. Für DPO 12 Schicht 2, die auf die kristallisierte Vorgängerschicht aufgedampft wurde, und für DPO 19 Schicht 1 auf einer $50 \mathrm{~nm}$ dicken Tantal-Zwischenschicht, ist diese Bedingung erfüllt.

Für die direkt auf den Oszillator aufgedampfte Schicht 1 auf DPO 12 (blau) gilt sie nicht. Die Tatsache, dass die Frequenzverschiebung bis $\mathrm{zu}-12 \mathrm{~Hz}$ beträgt, lässt sich durch den Formalismus in Kapitel 4.7.1 nicht erklären. Eine denkbare Ursache ist, dass durch den Einfluss der Schicht der Schermodul des Siliziumssubstrats kleiner wird. Ein solcher Effekt könnte durch Diffusion von Metallatomen aus der Schicht ins Substrat verursacht sein. In der Tat ist aus der Literatur bekannt, dass insbesondere Kupfer bevorzugt in Silizium eindiffundiert. So können sich schon bei Raumtemperatur $\mathrm{Cu}_{3} \mathrm{Si}$-Phasen bilden [168], ab etwa $200{ }^{\circ} \mathrm{C}$ formiert sich eine Kupfersilizid-Schicht an der Grenzfläche $[169,170]$. Der Beweis, dass diese Effekte auch für die hier beobachteten Ergebnisse verantwortlich sind, konnte trotz der EDX-Untersuchungen am Elektronenmikroskop im Rahmen dieser Arbeit nicht zweifelsfrei erbracht werden (Abschnitt 5.1.3).

Wird die erste kristallisierte Schicht nun als Substrat für DPO 12 Schicht 2 verwendet, so zeigt sich (rot) ein völlig anderer Kurvenverlauf. Die Frequenzverschiebung bleibt für alle Temperaturen deutlich über dem gestrichelten Massebeitrag und steigt während des Heizens sogar leicht an. Dies bedeutet eine Zunahme der elastischen Beiträge und damit des Speichermoduls in der Schicht bei höheren Temperaturen und steht im Widerspruch zu der Annahme, dass die Fähigkeit eines amorphen Festkörpers, elastische Energie zu speichern, mit steigender Temperatur abnehmen sollte. Die in Abschnitt 5.2.2 gezeigten Ergebnisse deuten zweifelsfrei darauf hin, dass die kristallisierte Schicht als Unterlage ein makroskopisch großer Kristallisationskeim für die darauf gedampfte amorphe Schicht ist. Schon bei niedrigen Temperaturen verschiebt sich die vorliegende Grenzfläche zwischen kristalliner Unterlage und amorphem $\mathrm{Zr}_{65} \mathrm{Al}_{7,5} \mathrm{Cu}_{27,5}$ in Richtung der amorphen Schicht. In dem Maß, in dem sich die Grenzfläche verschiebt und die kristalline Phase wächst, steigt nun die Fähigkeit der Schicht, elastische Energie zu speichern, was den Frequenzverlauf erklärt.

Der dritte, noch einmal völlig unterschiedliche Kurvenverlauf (grün) zeigt sich, wenn zwischen Silizium-Substrat und amorpher Metallschicht Tantal als Diffusionsbarriere verwendet wurde. Tantal verhindert die Reaktion von Kupfer mit Silizium bis zu $650^{\circ} \mathrm{C}$ [171]. Erst dann bildet sich $\mathrm{TaSi}_{2}$, was keine Diffusionsbarriere für Kupfer mehr darstellt. Wiewohl es keine Tantal-Kupfer-Phasen gibt, wurde jedoch beobachtet, dass Tantal in Richtung einer Kupfer- 
Schicht diffundieren kann, was seine Barriereneigenschaft natürlich einschränkt [172]. Diese Diffusion von Tantal in die Schicht könnte im rechten Bild von Abbildung 5.4 zu erkennen sein. Möglicherweise ist die Intensitätslinie aber auch Fluoreszenzeffekten und Verunreinigungen bei der Präparation für das TEM zuzuschreiben. Trotz dieser Schwächen von Tantal als Diffusionsbarriere entspricht die grüne Kurve in Abbildung 7.2 dem erwarteten Verlauf für ein metallisches Glas am Besten. Der Speichermodul der Schicht nimmt mit zunehmender Temperatur ab. Offensichtlich verhindert die Tantal-Zwischenschicht sowohl die Reaktion des metallischen Glases mit dem Siliziumsubstrat, als auch die Kristallisation während des Aufheizens weitgehend.

\subsubsection{Rekonstruktion der Substratdämpfung bei DPO 19}

In Abbildung 5.23 ist phänomenologisch beschrieben, dass die Dämpfung des Systems DPOTantal- $\mathrm{Zr}_{65} \mathrm{Al}_{7,5} \mathrm{Cu}_{27,5}$ bei späteren Temperaturzyklen kleiner ist, als die Dämpfung des Systems DPO-Tantal, die als Referenz für die Differenzbildung dienen sollte. Dies steht in klarem Widerspruch zu der Tatsache, dass jede zusätzliche Schicht zur Dämpfung beiträgt. Es gibt folglich Prozesse, die die Referenzdämpfung des DPO-Tantal-Systems im Verlauf der Temperaturbehandlung zu kleineren Werten verschiebt. Mögliche Erklärungen sind Relaxationsprozesse in der gesputterten Tantal Schicht, Grenzflächenreaktionen zwischen Tantal und Silizium oder zwischen Tantal und der metallischen Glasschicht. Für die Auswertung muss die Substratdämpfung bekannt sein. Nur so lässt sich aus der Differenz zur Dämpfung des bedampften Substrats der Beitrag der zu untersuchenden Schicht bestimmen. Die Substratdämpfung wird daher mathematisch rekonstruiert.

$\mathrm{Zu}$ diesem Zweck wird angenommen, dass die Dämpfung des Gesamtsystems bei niedrigen Temperaturen etwa $10^{-7}$ größer ist, als die Substratdämpfung. Diese Annahme erscheint durch viele Messungen, unabhängig vom Untergrund, gerechtfertigt. Die Substratdämpfung wird auf diese Weise aus der gemessenen Gesamtdämpfung bis zu einer Temperatur von $500 \mathrm{~K}$ berechnet und mit einer Polynomfunktion gefittet. Der langsame, lineare Anstieg der so rekonstruierten Substratdämpfung wird nun zu höheren Temperaturen extrapoliert und stellt die Substratdämpfung für die Messung dar. In Abbildung 7.3 ist die aus den Messwerten rekonstruierte Substratdämpfung gezeigt. 


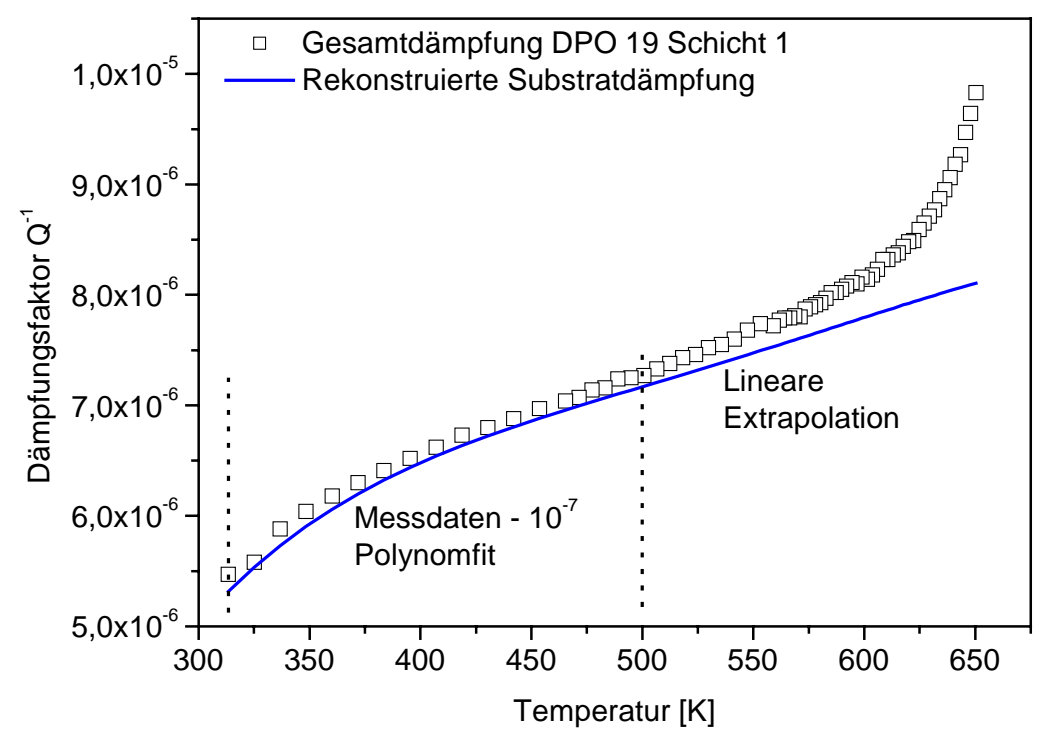

Abbildung 7.3: Rekonstruktion der Substratdämpfung für DPO 19 Schicht 1 aus den Messwerten.

\subsubsection{Abhängigkeit der Dämpfung vom Substrat}

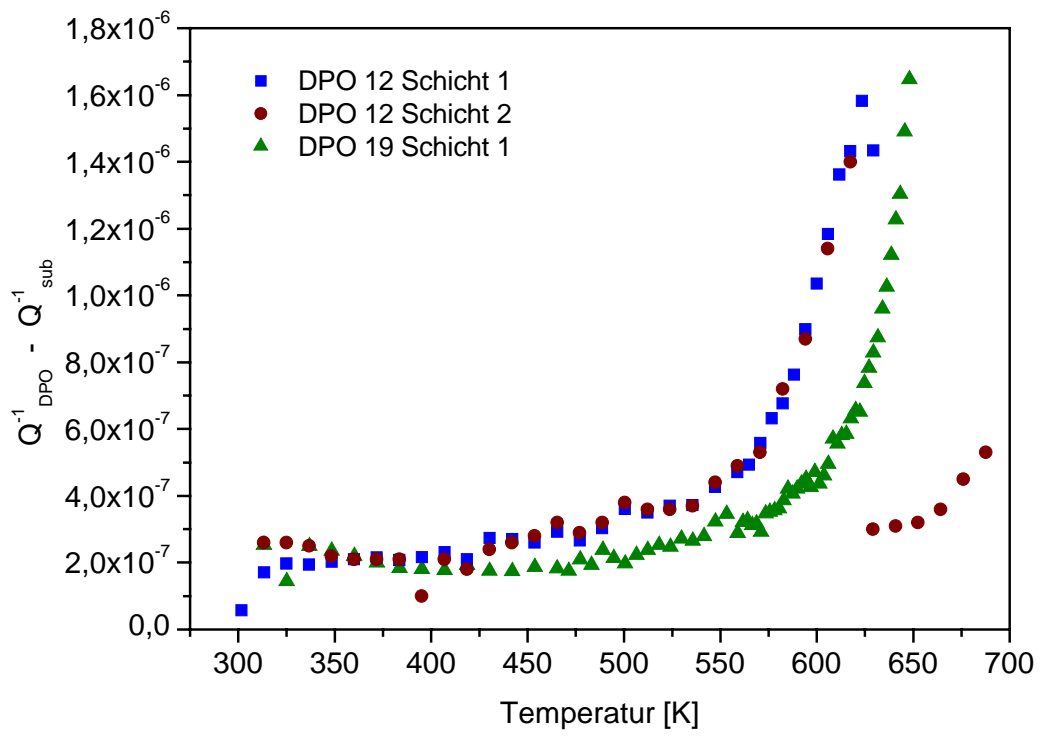

Abbildung 7.4: Differenz der Dämpfung zwischen bedampftem Oszillator und Substrat für verschiedene Unterlagen. Schicht 1 auf DPO 12 (blau) ist auf Silizium, Schicht 2 auf DPO 12 (rot) auf eine kristallisierte $\mathrm{Zr}_{65} \mathrm{Al}_{7,5} \mathrm{Cu}_{27,5}$ Schicht und Schicht 1 auf DPO 19 (grün) auf eine Tantal-Zwischenschicht aufgedampft.

Mit der so rekonstruierten Substratdämpfung lässt sich nun auch für die unterschiedliche Dämpfung von $\mathrm{Zr}_{65} \mathrm{Al}_{7,5} \mathrm{Cu}_{27,5}$-Schichten auf verschiedenen Unterlagen ein Vergleich ziehen. In Abbildung 7.4 ist die Dämpfungsdifferenz zum Substrat gezeigt. 
Offensichtlich spielt es für die Dämpfungsdifferenz keine Rolle, ob Silizium oder eine kristalline Schicht als Unterlage dient. In beiden Fällen beginnt bei etwa $550 \mathrm{~K}$ ein steiler Anstieg, bevor die Werte bei etwa $625 \mathrm{~K}$ rapide zu fallen beginnen. Dieser Abfall ist auf die Kristallisation der Schicht zurückzuführen. Ein ähnlich starker Anstieg ist auch für die auf Tantal aufgedampfte Schicht zu beobachten. Allerdings setzt er später ein und ist selbst bei $650 \mathrm{~K}$ noch nicht abgeschlossen.

\subsection{Schermodul von $\mathrm{Zr}_{65} \mathrm{Al}_{7,5} \mathrm{Cu}_{27,5}$}

Bei Raumtemperatur wird die gesamte Frequenzverschiebung für die amorphe Schicht, unabhängig vom Untergrund, zu etwa $-4,5 \mathrm{~Hz}$ angenommen (siehe Abbildung 7.2). Mit der massebedingten Verschiebung um $-7,25 \mathrm{~Hz}$, bedeutet das nach Gleichung (4.41) einen elastischen Beitrag von $+2,78 \mathrm{~Hz}$. Bei den kristallisierten Schichten findet man etwa $-2,5 \mathrm{~Hz}$ gesamte Frequenzverschiebung, weshalb der elastische Beitrag dazu bei $+4,78 \mathrm{~Hz}$ liegt. Der Schermodul der Schicht berechnet sich nun nach Gleichung (4.42) zu 22,7 GPa für die amorphe und zu 36,8 GPa für die kristalline Schicht. Dabei wurde die Resonanzfrequenz mit $5400 \mathrm{~Hz}$, der Schermodul von Silizium mit 66,2 GPa, die Substratdicke mit $300 \mu \mathrm{m}$ und die Schichtdicke mit $300 \mathrm{~nm}$ angenommen.

Mit Gleichung (4.42) lässt sich aus der gemessenen Frequenzverschiebung direkt der Betrag des komplexen Schermoduls der Schicht berechnen. Da es sich bei allen eingehenden Größen um konstante Faktoren handelt, folgt der Kurvenverlauf des Schermoduls mit der Temperatur exakt dem Verlauf der Frequenzverschiebung. In Abbildung 7.5 ist diese Analogie am Beispiel von DPO 19 Schicht 1 für das erste Aufheizen gezeigt. Der Schermodul der amorphen Schicht fällt mit steigender Temperatur von etwa $30 \mathrm{GPa}$ bei $320 \mathrm{~K}$ bis zu $10 \mathrm{GPa}$ bei $650 \mathrm{~K}$ ab. Bemerkenswert ist, dass dieses „Weichwerden“ der Schicht nicht etwa erst in der Nähe des Glasübergangs beginnt, sondern über das betrachtete Temperaturintervall kontinuierlich erfolgt. Ab etwa $600 \mathrm{~K}$ ändert sich der Schermodul kaum mehr und beträgt rund $12 \mathrm{GPa}$. 


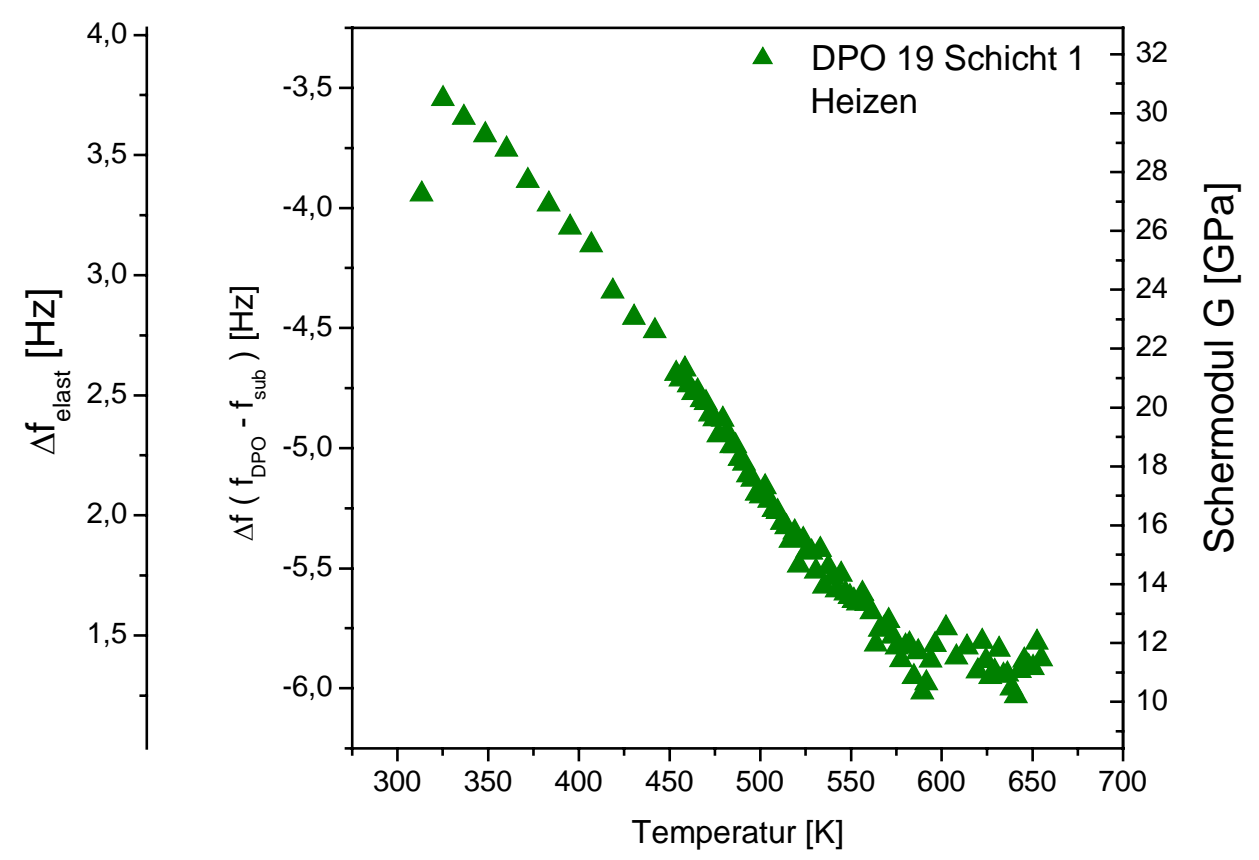

Abbildung 7.5: Analogie zwischen gemessener AS2-Frequenzverschiebung (linke Skala), dem elastischen Beitrag zur Frequenzverschiebung (Skala ganz links) und dem Betrag des Schermoduls (rechte Skala).

\subsubsection{Speicher- und Verlustmodul}

Aus der in Abbildung 7.4 gezeichneten Dämpfungsdifferenz zwischen bedampftem Oszillator und Substrat und dem in Abbildung 7.5 dargestellten Betrag des Schermoduls lassen sich mit dem in Abschnitt 4.7 ausführlich beschriebenen Formalismus der Real- und der Imaginärteil des komplexen Schermoduls berechnen. Dazu wird mit Gleichung (4.55) der Beitrag der Schicht zur Dämpfung und schließlich mit Gleichung (4.58) G' und G' ermittelt. Das Ergebnis ist für die $300 \mathrm{~nm}$ dicke $\mathrm{Zr}_{65} \mathrm{Al}_{7,5} \mathrm{Cu}_{27,5}$-Schicht 1 auf DPO 19 in Abbildung 7.6 gezeigt. Der Speichermodul nimmt kontinuierlich mit steigender Temperatur ab. Der Verlustmodul bleibt bis $500 \mathrm{~K}$ nahezu konstant, bevor er zunächst langsam und ab etwa $625 \mathrm{~K}$ sehr rasch ansteigt. 


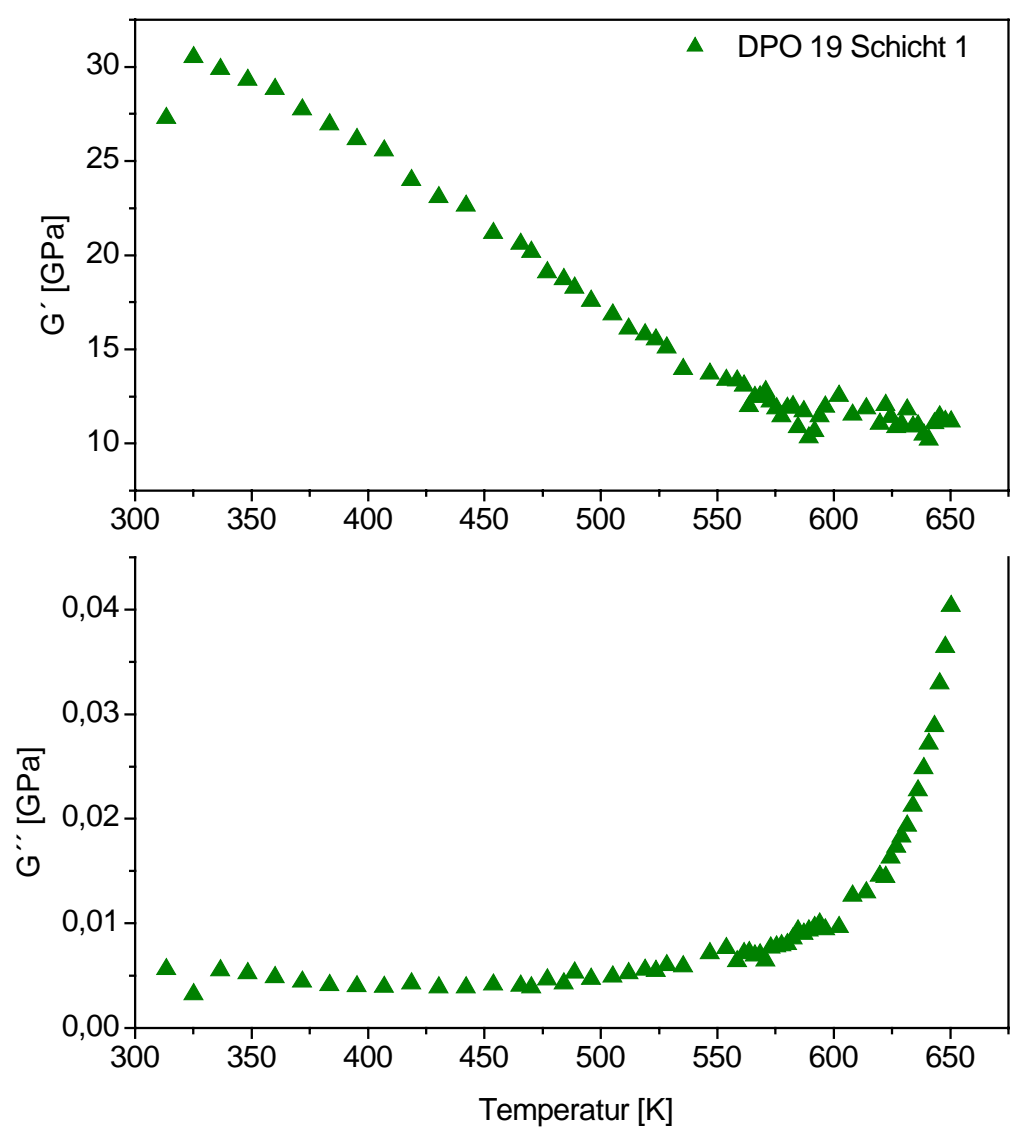

Abbildung 7.6: Realteil (oben) und Imaginärteil (unten) des komplexen Schermoduls einer $300 \mathrm{~nm}$ dicken $\mathrm{Zr}_{65} \mathrm{Al}_{7,5} \mathrm{Cu}_{27,5}$-Schicht in Abhängigkeit von der Temperatur.

\subsubsection{Vergleich des Verlustmoduls mit DMA-Resultaten}

In dynamisch mechanischen Messungen an $\mathrm{Zr}_{65} \mathrm{Al}_{7,5} \mathrm{Cu}_{27,5}$ Splat-Proben hat Rambousky [173] den komplexen Elastizitätsmodul ermittelt. Dabei gilt für den Zusammenhang zwischen dem Tangens des Verlustwinkels tan( $(\delta)$, dem Realteil E' und dem Imaginärteil E"' der analoge Zusammenhang wie in Gleichung (4.57) für den Schermodul G dargestellt.

Die kleine Grafik in Abbildung 7.7 zeigt die entsprechenden Ergebnisse von Rambousky. Im großen Bild wurden die Daten für E"' im Temperaturintervall zwischen $650 \mathrm{~K}$ und $670 \mathrm{~K}$ direkt aus den Originaldaten übernommen, für Temperaturen zwischen $610 \mathrm{~K}$ und $650 \mathrm{~K}$ wurden sie aus den gegebenen $\tan (\delta)$ und E' berechnet. Sie sind als schwarze Quadrate dargestellt. In das gleiche Bild wurden die Ergebnisse des imaginären Schermoduls von Schicht 1 auf DPO 19 als grüne Dreiecke eingetragen. Es zeigt sich eine gute Übereinstimmung im Kurvenverlauf, allerdings ist zu beachten, dass die beiden Temperaturskalen der oberen (für 
$\mathrm{G}^{\prime \prime}$ ) und der unteren Achse (für $\mathrm{E}^{\prime \prime}$ ) um $20 \mathrm{~K}$ gegeneinander verschoben sind. Der Anstieg im Verlustmodul des Schermoduls der dünnen Schicht beginnt etwa 20 K später wie der Anstieg im Verlustmodul E". Eine mögliche Erklärung für diese Temperaturverschiebung liegt in der Prozessführung begründet. So beträgt die Heizrate in den Rambousky-Experimenten $2 \mathrm{~K} / \mathrm{min}$, während sie beim Doppel-Paddel Oszillator etwa einen Faktor 10 kleiner ist. Bei kleineren Heizraten verschieben sich charakteristische Temperaturen wie der Glasübergang oder die Kristallisation zu niedrigeren Werten, was mit der Beobachtung in Abbildung 7.7 übereinstimmt.

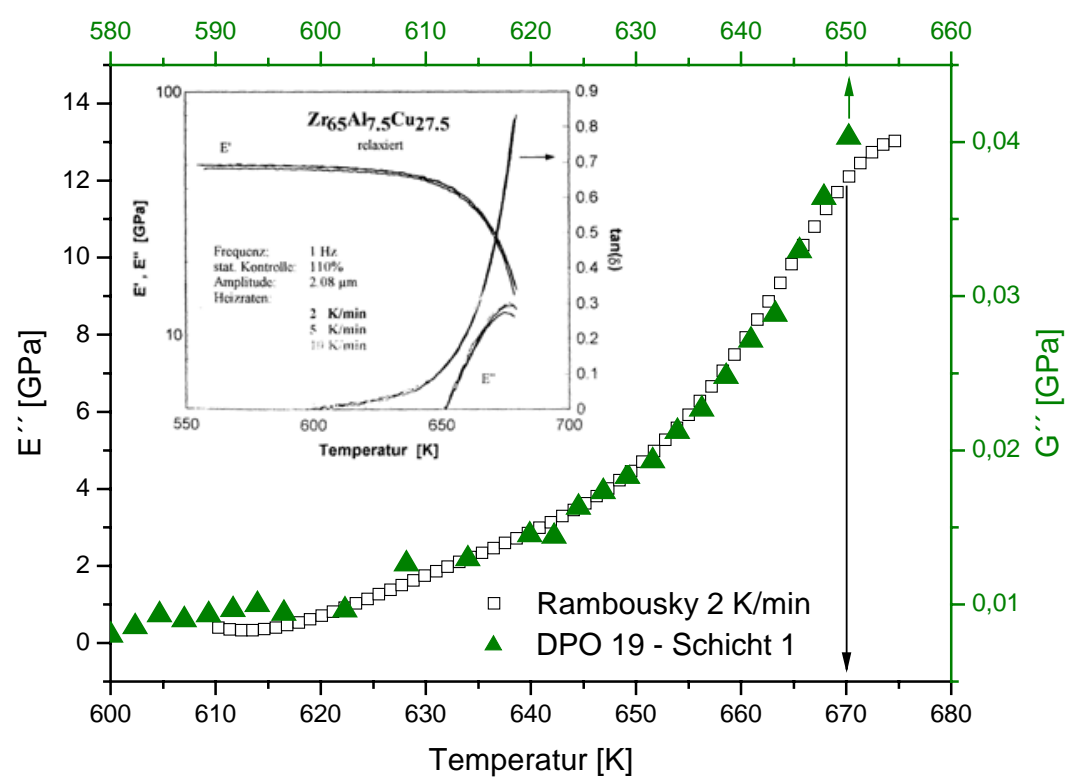

Abbildung 7.7: Vergleich des imaginären Schermoduls von DPO 19 Schicht 1 (Skala rechts und oben, grüne Dreiecke) mit dem dynamisch mechanischen gemessenen imaginären Elastizitätsmodul nach Rambousky [173] (schwarze Quadrate, Skala links und unten).

Ein weiteres Argument für die Temperaturverschiebung sind die verschiedenen Zeitskalen, die bei beiden Experimenten durch die unterschiedliche Anregungsfrequenz untersucht werden. So hat Rambousky dynamisch bei der Frequenz von $1 \mathrm{~Hz}$ gemessen, während die DPOUntersuchungen bei $5440 \mathrm{~Hz}$ durchgeführt wurden. Der Lunkenheimer-Plot in Abbildung 2.1 zeigt, dass die unterschiedlichen Frequenz und damit Relaxationszeiten gleichwertig zu einer Temperaturverschiebung des Kurvenverlaufs sind. Wegen des durch den Übergang vom Frequenz- in den Temperaturraum gespiegelten Graphen entspricht die höhere Frequenz einer Temperaturverschiebung zu niedrigeren Werten. 


\subsubsection{Abschätzung der Poisson-Zahl v}

Der Speichermodul E' für die relaxierte Splat-Probe kann aus dem kleinen Bild in Abbildung 7.7 direkt zu $50 \mathrm{GPa}$ abgelesen werden. In Vibrating Reed Messungen an $\mathrm{Zr}_{65} \mathrm{Al}_{7,5} \mathrm{Cu}_{27,5}$-Filmen bei $150 \mathrm{~Hz}$ Resonanzfrequenz haben Kempen et al. für den wie hergestellten Zustand E' zu 75 \pm 10 GPa bestimmt [134]. Über die Poisson-Zahl v steht der Schermodul G mit dem Elastizitätsmodul E gemäß Gleichung (7.4) in Beziehung. Da der Schermodul für die ,wie hergestellte“ Schicht bei Raumtemperatur etwa 30 GPa beträgt (Abbildung 7.6), lässt sich die Poisson-Zahl mit den vergleichbaren Kempen-Daten berechnen. Sie liegt für den amorphen Film bei etwa 0,25 . Der Wert von $E^{\prime}$ für die kristalline Legierung beträgt bei Kempen $140 \mathrm{GPa}$ und ist damit doppelt so groß wie für die ,wie hergestellte“ Probe. Die Veränderung der elastischen Anteile bei der Schicht auf DPO 19 lässt sich aus Abbildung 7.2 bestimmen. Für die amorphe Schicht beträgt der elastische Anteil zur Frequenzverschiebung bei Raumtemperatur etwa 3,7 Hz, für die kristalline Schicht liegt er etwa 1,5 Hz darüber, was einem Faktor von 1,4 entspricht. Demnach beträgt der Schermodul G' für die kristalline Schicht bei Raumtemperatur $42 \mathrm{GPa}$. Aus diesen Daten ergibt sich eine Poisson-Zahl von 0,6. Für die reinen Metalle Zirkon, Kupfer und Aluminium ist $v$ jeweils 0,37, 0,35 und 0,34 [140]. Im Rahmen dieser groben Abschätzung sind die Ergebnisse aus DMA, Vibrating Reed und DPO-Messungen plausibel und vergleichbar. Diskrepanzen haben ihre Ursache neben dem statistischen Messfehler in der ungenauen Bestimmung der exakten Probengeometrie im Fall von Vibrating Reed- und DMA-Messungen und natürlich den verschiedenen Anregungsfrequenzen.

\subsubsection{Viskoses Fließen und $\alpha$-Relaxation}

In diesem Abschnitt wird davon ausgegangen, dass der gesamte Relaxationsvorgang ausschließlich durch viskoses Fließen der einzelnen Legierungsatome verursacht wird. Wie in Kapitel 2.2.6 gezeigt ist, kann diese primäre Relaxation im mechanischen Verlust mit einer Funktion nach Havriliak und Negami (Gleichung 2.18) beschrieben werden. Die AS2-Resonanzfrequenz beträgt bei DPO 19 etwa $5440 \mathrm{~Hz}$ und wird als konstant angenommen. Die charakteristische Relaxationszeit wird oberhalb des Glasübergangs durch eine Vogel-FulcherTammann-Abhängigkeit (Gleichung 2.7) beschrieben. Aus Viskositätsmessungen in der unterkühlten Schmelze [21] hat M. Weiß die VFT-Parameter $T_{\text {VFT }}$ zu $367 \mathrm{~K}$ und D zu 26,3 für $\mathrm{Zr}_{65} \mathrm{Al}_{7,5} \mathrm{Cu}_{27,5}$ bestimmt. In Abbildung 7.6 ist ersichtlich, dass der enorme Anstieg des Verlustmoduls bei etwa $625 \mathrm{~K}$ einsetzt, weshalb hier der Glasübergang angenommen wird. Für 
die folgende Kurvenanpassung werden daher nur die Messpunkte verwendet, die bei höheren Temperaturen liegen. Mit Hilfe eines in das Auswerteprogramm OriginPro7.0 implementierten Levenberg-Marquardt-Algorithmus wird die Kurvenanpassung mit den fest vorgegebenen VFT-Parametern $\mathrm{T}_{\mathrm{VFT}}$ und D sowie dem Breiteparameter $\alpha=1$ (der Einfachheit halber wird eine Cole-Davidson-Funktion angenommen) durchgeführt. Als Resultat ergeben sich die in Tabelle 7.1 zusammengefassten Fitparameter. Die berechnete Havriliak-Negami-Funktion ist in Abbildung 7.8 zusammen mit den Messdaten in linearer (linkes Bild) und logarithmischer Auftragung (rechtes Bild) dargestellt.

\begin{tabular}{|c|c|c|c|}
\hline Parameter & Bezeichnung & Wert & fest/frei \\
\hline $\mathrm{T}_{\mathrm{VFT}}$ & VFT-Temperatur & $367 \mathrm{~K}$ & fest \\
\hline $\mathrm{D}$ & VFT-Stärkeparameter & 26,3 & fest \\
\hline$\tau_{0}$ & VFT-Anklopfzeit & $2,8 \times 10^{-12} \mathrm{~s}$ & frei \\
\hline$\alpha$ & Breiteparameter & 1 & fest \\
\hline$\gamma$ & Asymmetrieparameter & 0,32 & frei \\
\hline $\mathrm{G}_{\mathrm{S}}$ & Schermodul statische Frequenz & $34,75 \mathrm{GPa}$ & frei \\
\hline $\mathrm{G}_{\infty}$ & Schermodul unendliche Frequenz & $9,25 \mathrm{GPa}$ & frei \\
\hline
\end{tabular}

Tabelle 7.1: Parameter für Kurvenanpassung mit einer Havriliak-Negami-Funktion. In der letzten Spalte ist angegeben, ob der Parameter bei der Kurvenanpassung fest vorgegeben oder frei angepasst wurden.

Erstaunlicherweise entsprechen $\mathrm{G}_{\mathrm{S}}$ und $\mathrm{G}_{\infty}$ relativ gut den Werten von $\mathrm{G}^{\prime}$ bei Raumtemperatur und am Glasübergang. Die Differenz aus $G_{S}$ und $G_{\infty}$ ist die Relaxationsstärke. $\tau_{0}$ legt bei festgehaltenen $T_{V F T}$ und D die horizontale Position der Kurve fest. Sind Relaxationsstärke und horizontale Position ermittelt, so bleibt $\gamma$ als unabhängiger Asymmetrieparameter übrig, der sich letztlich aus der Steigung der Messwerte ergibt.

Die Position des Maximums befindet sich bei etwa 950 K. Da das Verhältnis aus thermodynamischer Schmelztemperatur $T_{m}$ und Glasübergangstemperatur $T_{g}$ für viele metallische Gläser zwischen 0,6 und 0,7 liegt [3,4], befindet sich das Maximum etwa am thermodynamischen Schmelzpunkt der kristallinen $\mathrm{Zr}_{65} \mathrm{Al}_{7,5} \mathrm{Cu}_{7,5}$-Legierung. Die erhaltene Havriliak-Negami-Kurve entspricht daher dem in Abbildung 2.1 gelb eingezeichneten $\alpha$-Relaxationspeak, der durch das viskose Fließen verursacht wird. 

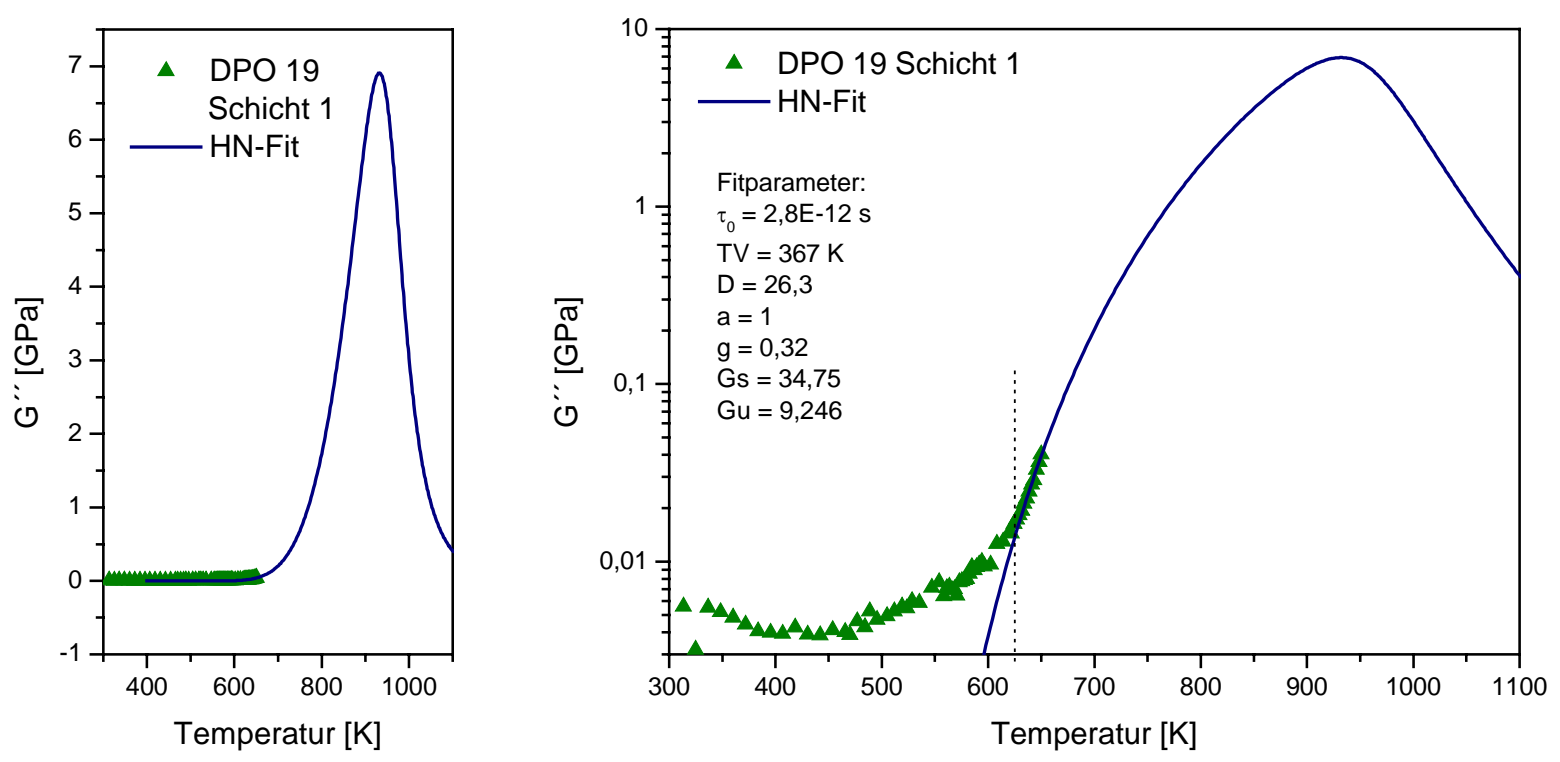

Abbildung 7.8: Darstellung der gemessenen Daten an DPO 19 Schicht 1 mit Havriliak-Negami Fitfunktion in linearer (links) und logarithmischer Auftragung (rechts).

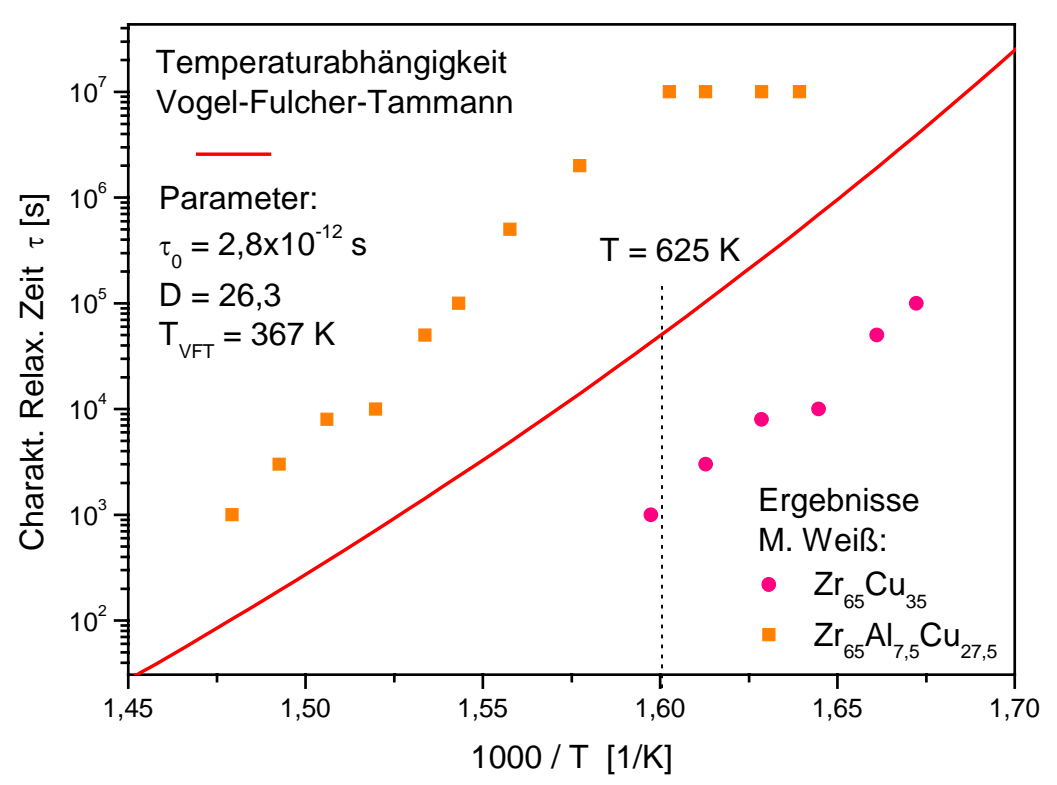

Abbildung 7.9:Vergleich der berechneten Relaxationszeiten nach Vogel-Fulcher Tammann (rote Linie) mit den an zwei metallischen Gläsern unterschiedlicher Zusammensetzung von M. Weiß gemessenen.

In Abbildung 7.9 sind die mit den gegebenen Fitparametern berechneten Relaxationszeiten im Temperaturintervall zwischen $690 \mathrm{~K}$ und $588 \mathrm{~K}$ in Arrheniusauftragung über $1000 \mathrm{~K} / \mathrm{T}$ dargestellt. Sie stimmen gut mit ebenfalls eingezeichneten Messergebnisse von M. Weiß [21] an zwei ZrAlCu-Gläser mit unterschiedlicher chemischer Zusammensetzung überein. 


\subsubsection{Zusätzliche Relaxationsbeiträge bei tiefen Temperaturen}

Ganz offensichtlich genügt die in Abbildung 7.8 gezeichnete einzelne Havriliak-NegamiFunktion nicht, um den Imaginärteil des Schermoduls bei tieferen Temperaturen hinreichend gut zu beschreiben. Es muss daher im Bereich des Glasübergangs und darunter zusätzliche Beiträge zur Relaxation in $\mathrm{Zr}_{65} \mathrm{Al}_{7,5} \mathrm{Cu}_{27,5}$ geben. Anders als in organischen Glasbildnern gibt es in metallischen Legierungen keine intramolekularen Freiheitsgrade, deren Schwingungsmoden Dämpfung verursachen könnten. Sekundäre, als Peak oder Schulter auftretende $\beta$-Prozesse werden daher ausgeschlossen. Abweichungen vom $\alpha$-Peak können nur durch den „excess-wing“ verursacht sein, dessen mikroskopische Ursache bislang unklar ist. Wenn es sich bei dem „wing“ aber um ein universelles Verhalten von glasbildenden Systemen handelt, dann müsste sein Einfluss auch in metallischen Gläsern zu finden sein. Für den Nachweis hierfür werden alternative Wege verfolgt.

\subsubsection{1 „wing“ als Niedertemperaturflanke einer JG- $\beta$-Relaxation}

Eine Ursache für den „wing“ besteht in der Annahme, dass es einen universellen, sekundären Relaxationsprozess gibt, der breiter ist als das viskose Fließen und bei hohen Temperaturen bzw. niedrigen Frequenzen unter dem dominanten $\alpha$-Peak verborgen liegt [118]. In diesem Fall ist der „wing“ nichts anderes als die Niedertemperatur- bzw. Hochfrequenzflanke eines Johari-Goldstein- $\beta$-Prozesses. Wie die primäre, so sollte sich auch die sekundäre Relaxation mit einer HN-Funktion beschreiben lassen. Die Messwerte ergeben sich dann aus der mathematischen Überlagerung der primären (HN1) und der sekundären (HN2) Havriliak-NegamiFunktion. In Abschnitt 2.3 ist auf die Problematik der Unabhängigkeit beider Prozesse eingegangen. Bei der Diskussion im Rahmen dieser Arbeit wird aber ausschließlich die additive Superposition angewandt. Das Ergebnis einer Kurvenanpassung mit der Summe zweier HNFunktionen ist in Abbildung 7.11 rechts dargestellt und beschreibt die Messdaten von $550 \mathrm{~K}$ bis $625 \mathrm{~K}$ relativ gut. Allerdings bleibt die mikroskopische Begründung für den sekundären Relaxationsprozess unklar und das mathematische Verfahren wegen der vielen freien Fitparameter fragwürdig. 


\subsubsection{2 Überlagerung vieler unabhängiger Relaxationsprozesse}

Wie in Abschnitt 2.2.3 dargestellt, gibt es viele prominente Theorien zu dynamischen Eigenschaften unterkühlter Flüssigkeiten, die auf dynamische Heterogenitäten, Dichtefluktuationen und der kollektiven Bewegung von Atomgruppen („Cluster“) aufbauen. In der Freien-Volumen-Theorie nennen Turnbull, Cohen und Grest diese Regionen ,solid-like“ und „liquidlike“. Analog dazu gibt es „,cooperative rearranging regions“ im molekularkinetischen Ansatz von Adam und Gibbs. Johari und Goldstein nennen ihre Cluster ,,islands of mobility“. Denkbar für die Interpretation wäre daher eine Vielzahl sich überlagernder unabhängiger Relaxationsfunktionen, die sich mit der Clustertheorie begründen lassen [174]. Verschiedene Szenarien für eine solche Überlagerung von Relaxationsfunktionen unabhängiger Cluster, die sich bei isothermen Messungen in einer Verteilung von Relaxationszeiten wiederspiegelt und sich in einem KWW- $\beta$ kleiner als 1 zeigen, sind in [175] diskutiert und für den imaginären Schermodul von $\mathrm{Zr}_{65} \mathrm{Al}_{7,5} \mathrm{Cu}_{27,5}$ in Abbildung 7.10 schematisch gezeigt. Die dargestellten Relaxationskurven sind dabei als nicht statisch anzusehen sondern visualisieren das spontane Bilden und die Bewegung von Atomgruppen. Im tiefen Glaszustand ist die Wahrscheinlichkeit für diese Ereignisse gering. Die Größe und Lebensdauer der Heterogenitäten hängt stark von der Temperatur ab. Für $\mathrm{Zr}_{65} \mathrm{Al}_{7,5} \mathrm{Cu}_{27,5}$ wurde die mittlere Clustergröße zu 2,1 nm am Glasübergang bestimmt [21]. Steigt die Temperatur, verändern sich auch die Heterogenitäten. Die Relaxationspeaks nehmen an Stärke zu und ihr Beitrag zur Gesamtrelaxation steigt. In der unterkühlten Schmelze schließlich, bleibt der dominierende primäre Relaxationsprozess übrig. Die Mobilität der einzelnen Atome ist nun so groß, dass sie sich unabhängig voneinander bewegen können.

Diese Beschreibung deckt sich mit Erkenntnissen aus Molekulardynamik Simulationen von Teichler [176] an $\mathrm{Ni}_{0,5} \mathrm{Zr}_{0,5}$, der sich kooperativ bewegende Atome als die Dynamik des Systems bestimmenden Prozess identifiziert. In Simulationsstudien von Vollmayr-Lee et al. [177] an tief unterkühlten Lennard-Jones Flüssigkeiten werden die dort ebenfalls gefundenen Heterogenitäten auf Dichteschwankungen zurückgeführt. Dass die Größe der Heterogenitäten mit abnehmender Temperatur zunimmt, wurde von Kob et al. [178] nachgewiesen. 


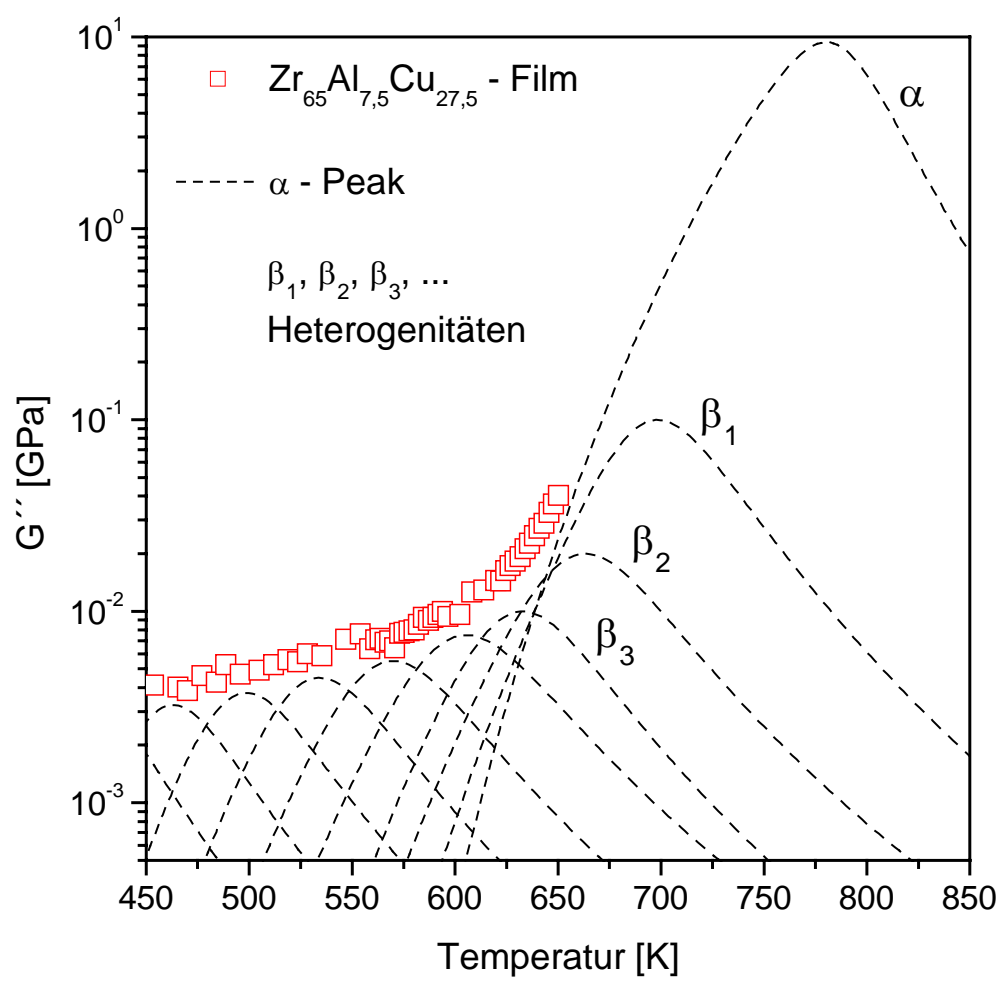

Abbildung 7.10: Schematische Relaxationsbeiträge verschiedener Einzelprozesse durch dynamische Heterogenitäten. Der $\alpha$-Peak entspricht dem viskosen Fließen einzelner Atome.

Der Abfall des Verlustmoduls mit abnehmender Temperatur erklärt sich nun mit der Überlagerung dieser Einzelprozesse. Mathematisch lässt sich für den Beitrag aller Heterogenitäten im einfachsten Fall ein Potenzgesetz annehmen:

$$
G_{\text {Cluster }}^{\prime \prime}=A \cdot\left(T-T_{c}\right)^{p}
$$

In Abbildung 7.11 links ist deshalb die Überlagerung der primären HN1-Funktion mit einer solchen Funktion gezeigt. Auch für diesen Fall werden die Messdaten relativ gut beschrieben.

\subsubsection{Vergleich des JG- $\beta$ - und des „Cluster“-Ansatzes}

Um die in den Abschnitten 7.4.5.1 und 7.4.5.2 vorgestellten mathematischen Verfahren vergleichen zu können, wird das viskose Fließen im $\alpha$-Prozess in beiden Fällen durch die exakt gleiche Havriliak-Negami-Funktion (HN1) beschrieben. Allerdings unterscheiden sich die Parameter von denen der HN-Funktion, falls sich die Betrachtung auf einen einzigen Relaxationsprozess beschränkt (Abschnitt 7.4.4). Insbesondere die charakteristische Relaxationszeit 
$\tau_{0}$ und der Asymmetrieparameter $\gamma$ ändern sich signifikant. Die Fitparameter für die verschiedenen Funktionen sind in Tabelle 7.2 zusammengefasst.
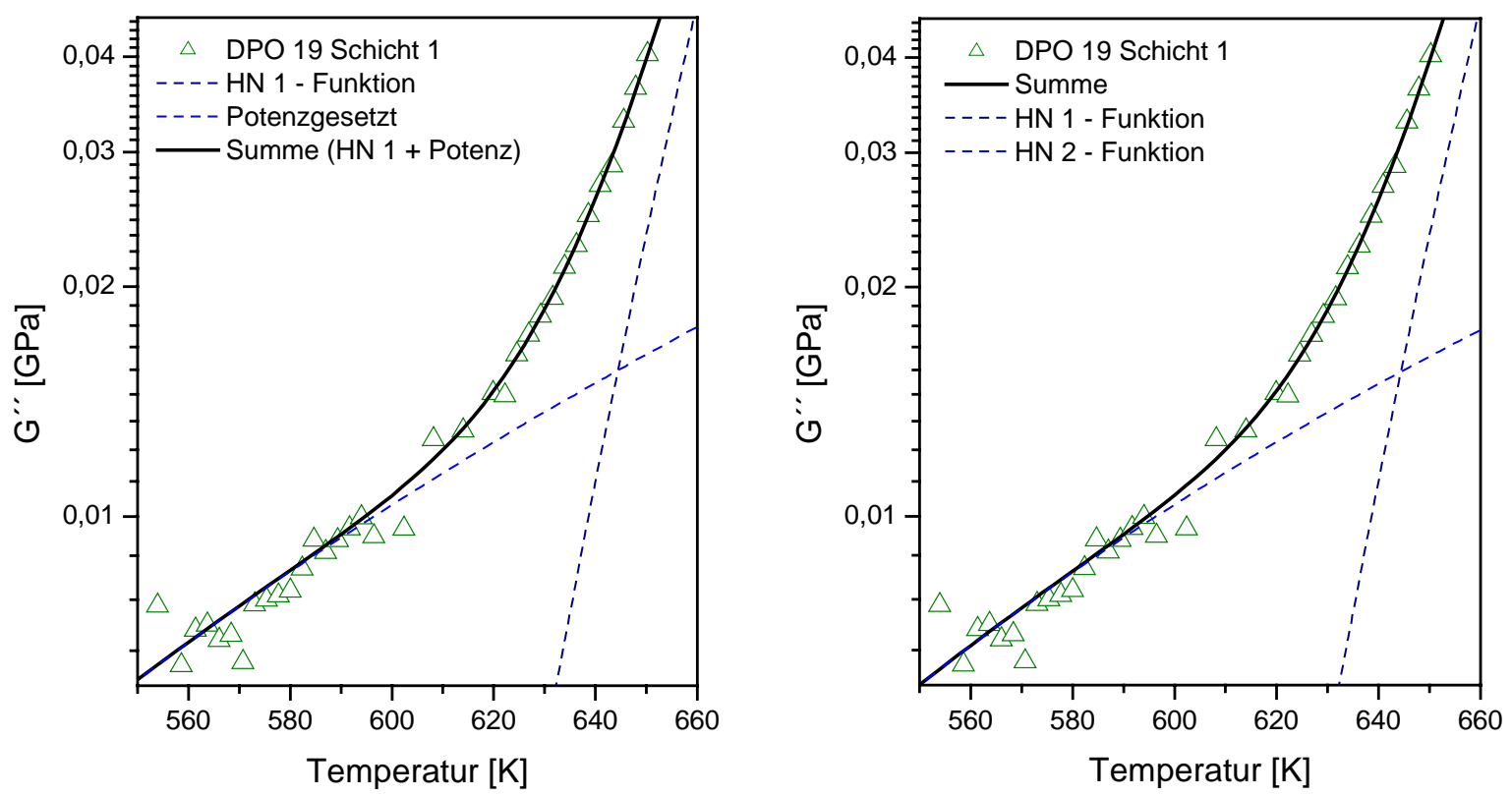

Abbildung 7.11: Alternative Fitfunktionen für die Anpassung an die Messwerte von DPO 19 Schicht 1. Der aProzess wird in beiden Fällen durch die HN1-Funktion beschrieben (gestrichelte Linie), die Erweiterung zu tiefen Temperaturen links durch ein Potenzgesetz und rechts durch eine weitere Havriliak-Negami-Funktion.

\begin{tabular}{|c|c|c|c|c|}
\cline { 2 - 5 } \multicolumn{1}{c|}{} & $\begin{array}{c}\text { Nur } \alpha \text {-Relaxation } \\
\text { (Abschnitt 7.4.4) }\end{array}$ & \multicolumn{2}{|c|}{$\begin{array}{c}\alpha \text {-Relaxation (HN1) und sekundäre Prozesse (HN2) } \\
\text { Abschnitt 7.4.5.1 bzw. Potenzgesetz Abschnitt 7.4.5.2) }\end{array}$} \\
\hline Parameter & HN-Funktion & HN1 & HN2 & Potenzgesetz \\
\hline $\mathrm{T}_{\mathrm{VFT}}$ & $367 \mathrm{~K}$ & $366 \mathrm{~K}$ & $85,8 \mathrm{~K}$ & \\
\hline $\mathrm{D}$ & 26,3 & 26,0 & 44,0 & \\
\hline$\tau_{0}$ & $2,8 \times 10^{-} 12 \mathrm{~s}$ & $5,16 \times 10^{-} 15 \mathrm{~s}$ & $6,38 \times 10^{-6} \mathrm{~s}$ & \\
\hline$\alpha$ & 1 & 1 & 1 & \\
\hline$\gamma$ & 0,32 & 0,61 & 0,69 & \\
\hline $\mathrm{G}_{\mathrm{S}}$ & $34,75 \mathrm{GPa}$ & $34,1 \mathrm{GPa}$ & $9,61 \mathrm{GPa}$ & \\
\hline $\mathrm{G}_{\infty}$ & $9,25 \mathrm{GPa}$ & $9,9 \mathrm{GPa}$ & $8,96 \mathrm{GPa}$ & \\
\hline $\mathrm{T}_{\mathrm{c}}$ & & & & $6,5 \times 10^{-11} \mathrm{GPa}$ \\
\hline $\mathrm{A}$ & & & & 3,25 \\
\hline $\mathrm{p}$ & & & & \\
\hline
\end{tabular}

Tabelle 7.2: Überblick über die Fitparameter zur Kurvenanpassung der Messdaten 
Bei der Gegenüberstellung von Havriliak-Negami- und Kohlrausch-Williams-Watts-Parametern in [64] wird folgender Zusammenhang angegeben:

$$
\alpha \gamma=\beta_{K W W}^{1,23}
$$

Mit den HN1-Parametern für das viskose Fließen errechnet sich damit ein $\beta_{\mathrm{Kww}}$ von 0,67. Dieses Ergebnis deckt sich mit Beobachtungen von M. Weiß für Temperaturen unterhalb des Glasübergangs [21] und ist ein Hinweis darauf, dass die einzelne HN-Funktion in Abschnitt 7.4.4 zwar die Hochtemperaturdaten gut beschreibt, aber für die Gesamtbetrachtung nicht ausreichend ist.

Die in Abbildung 7.11 gezeigten Summenfunktionen unterscheiden sich für beide Fällen nur geringfügig, weshalb eine Folgerung, welches der beiden Szenarien zutreffend ist, daraus nicht gezogen werden kann.

\subsubsection{Identifikation des „wing“ in $\mathrm{Zr}_{65} \mathrm{Al}_{7,5} \mathrm{Cu}_{27,5}$}

Obwohl die gezeigten Summenfunktionen die Messwerte in beiden Fällen gut beschreiben ist diese Vorgehensweise umstritten. Insbesondere ist fraglich, ob der Knick bei etwa $625 \mathrm{~K}$ in $\mathrm{G}^{\prime \prime}$ überhaupt etwas mit dem „wing“ zu tun hat. Dass die Messwerte in der logarithmischen Darstellung unterhalb $\mathrm{T}_{\mathrm{g}}$ deutlich langsamer abfallen, als für höhere Temperaturen, kann auch mit dem Verlassen des Gleichgewichtszustands und dem dazugehörigen Einfrieren der Dynamik am Glasübergang erklärt werden. In der Tat haben Johari und Goldstein [87] dieses Abknicken auch an Propylencarbonat gefunden, und unabhängig davon einen „excess-wing“ in diesem organischen Glasbildner ohne ausgeprägte $\beta$-Relaxation, nachgewiesen. Demnach gibt es keine mikroskopische Begründung, die Beiträge bei Temperaturen unterhalb von $\mathrm{T}_{\mathrm{g}}$ (sub$\mathrm{T}_{\mathrm{g}}$-Beiträge) und die Abweichungen vom $\alpha$-Prozess durch den „,wing“ oberhalb von $\mathrm{T}_{\mathrm{g}}$ in einer geschlossenen Funktion darzustellen. Beide Beiträge werden daher wie in Abbildung 7.12 schematisch als Geraden in der logarithmischen Darstellung angedeutet. Hier wird besonders deutlich, dass der lineare Anstieg der Dämpfung im Glaszustand durch sub- $\mathrm{T}_{\mathrm{g}}$-Beiträge (blau) ab etwa $625 \mathrm{~K}$ durch den ,wing“ (hellgrün) dramatisch zunimmt, aber immer noch klar von dem mit der HN1-Funktion gelb angedeuteten $\alpha$-Peak abweicht. Dieses Abweichen ist ein Nachweis dafür, dass es den ,wing“ auch in metallischen Gläsern gibt. Da hier weder kovalente Netzwerke wie in Silikatgläsern, noch intramolekulare Eigenmoden wie in 
selbst einfachen organischen Molekülen existieren, ist die hier zu ziehende Folgerung, dass der „wing“ eine universelle Eigenschaft von glasbildenden Systemen sein muss.

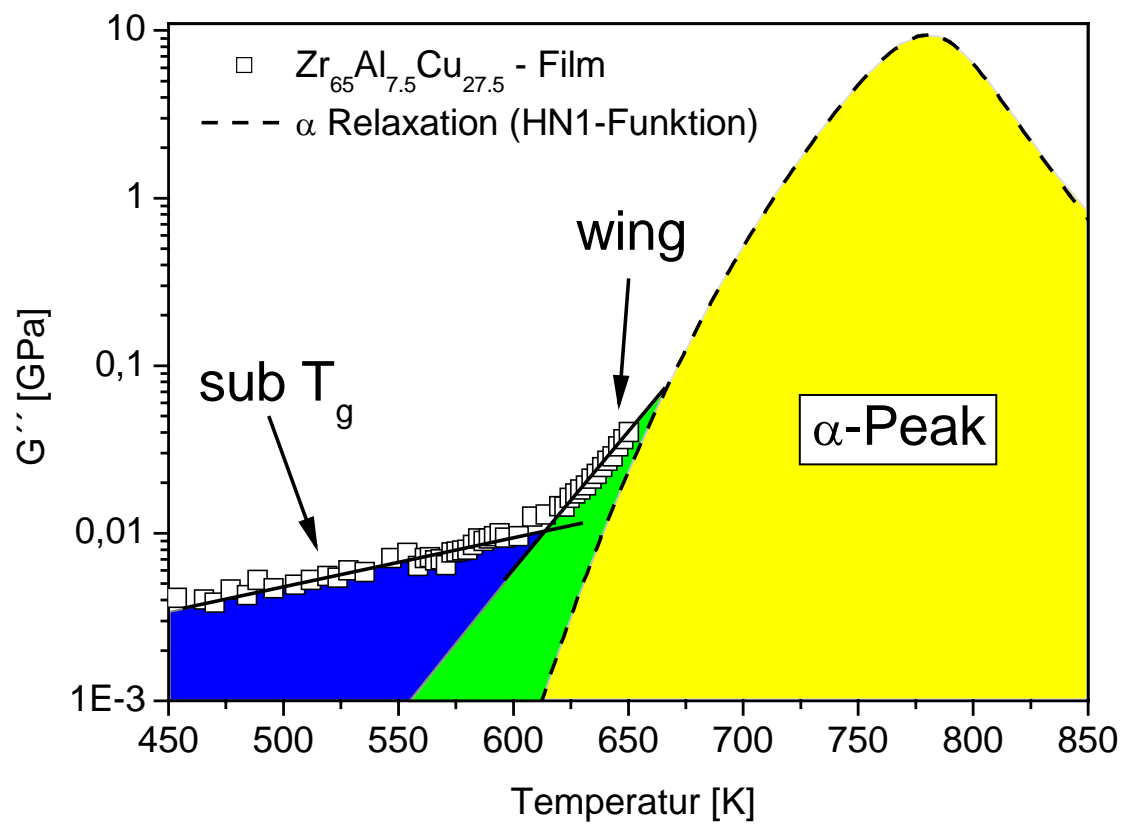

Abbildung 7.12: Trennung der zusätzlichen dynamischen Beiträge zum primären $\alpha$-Peak (gelb) bei tiefen Tem-

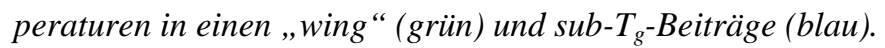

\subsection{Modul oder Nachgiebigkeit}

In der Literatur ist es umstritten, ob der Schermodul oder die Schernachgiebigkeit mechanisch dynamische Eigenschaften besser beschreiben [134, 179]. Zwischen dem komplexen Modul $\mathrm{G}^{*}$ und der komplexen Nachgiebigkeit $\mathrm{C}^{*}$ besteht der folgende einfache Zusammenhang:

$$
C^{*}=\frac{1}{G^{*}}=\frac{1}{G^{\prime}+i G^{\prime \prime}}=\frac{1}{G^{\prime 2}+G^{\prime 2}}\left(G^{\prime}+i G^{\prime \prime}\right)
$$

Da in der dielektrischen Spektroskopie meist die Permittivität, also eine Nachgiebigkeit, betrachtet wird, ist es auch aus Gründen der Vergleichbarkeit von mechanischen und dielektrischen Messungen interessant, die komplexe Schernachgiebigkeit zu bestimmen.

In Abbildung 7.13 ist der so berechnete Imaginärteil der Nachgiebigkeit (rote Kreise) und der Imaginärteil des Schermoduls vergleichend dargestellt. In beiden Größen ist der Knick am Glasübergang zu erkennen, angedeutet durch die jeweils eingezeichneten Geraden. Dabei scheint das Abknicken für die Nachgiebigkeit bei $625 \mathrm{~K}$ um etwa $10 \mathrm{~K}$ höher zu liegen als für den Modul. Betrachtet man die Skala, so steigt die Nachgiebigkeit über beinahe zwei Größen- 
ordnungen mit der Temperatur an, während der Modul nur um etwa eine Größenordnung zunimmt. Qualitativ ähneln sich beide Kurvenverläufe stark, weshalb alle bislang gemachten Aussagen über den Schermodul für die Nachgiebigkeit entsprechend gelten.

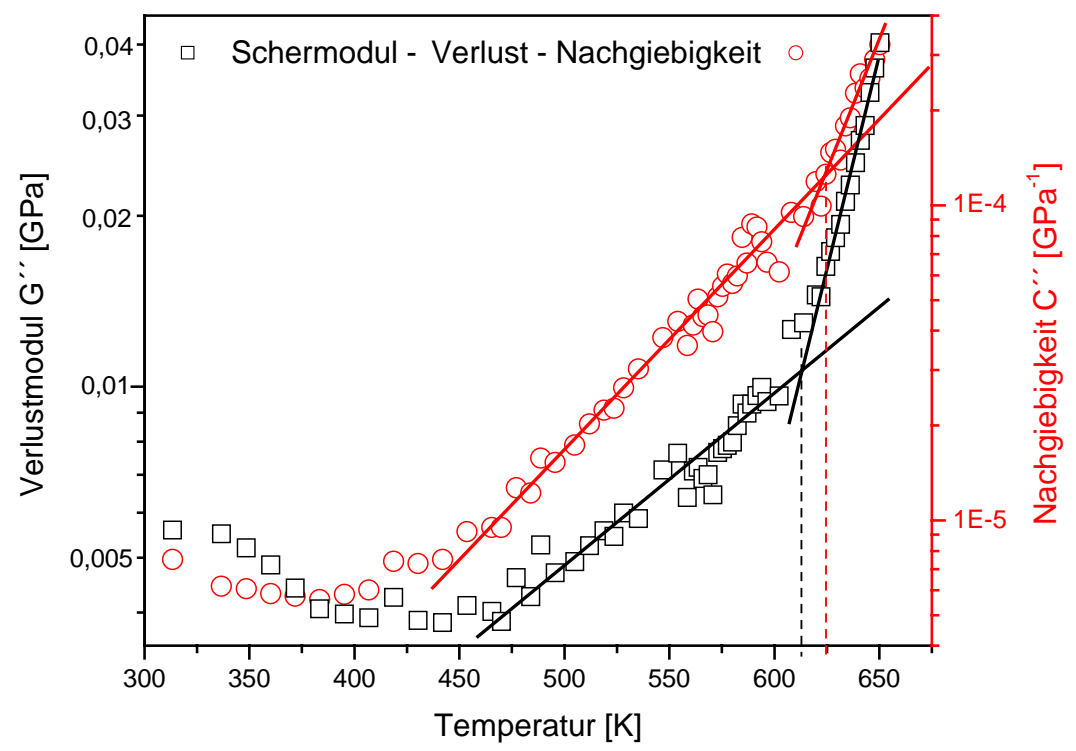

Abbildung 7.13: Vergleich des Imaginärteils der komplexen Nachgiebigkeit mit dem Imaginärteil des komplexen Schermoduls DPO 19 Schicht 1

\subsection{Kristallisationskinetik in $\mathrm{ZrAlCu}$}

Die ursprüngliche Idee, $\mathrm{Zr}_{65} \mathrm{Al}_{7,5} \mathrm{Cu}_{27,5}$-Schichten bis knapp über die Glastemperatur zu erwärmen, um einen relaxierten, metastabilen Gleichgewichtszustand herzustellen und die Schichten danach so langsam abzukühlen, dass das System im Gleichgewicht bleibt, konnte nicht realisiert werden. Aus umfangreichen Untersuchungen zum Kristallisationsverhalten am Zirkon-Kupfer-System $[180,181,182]$ ist bekannt, dass bereits $20 \mathrm{~K}$ unterhalb von $\mathrm{T}_{\mathrm{g}}$ kristalline $\mathrm{Zr}_{2} \mathrm{Cu}$-Keime entstehen, deren Bildung und Wachstum bei weiterer Auslagerung irreversibel ist. In Abschnitt 5.2.1.3 sind isotherme Messungen der AS2-Resonanzfrequenz und der Dämpfung von Schicht 1 auf DPO 12 bei $623 \mathrm{~K}$ gezeigt, in Abschnitt 5.2.3.3 entsprechende Daten für Schicht 1 auf DPO 19 bei $654 \mathrm{~K}$.

Ordnungsumwandlungen - in diesem Fall die primäre Kristallisation der amorphen Schicht lassen sich nach der Theorie von Kolmogorov, Johnson, Mehl und Avrami (KJMA) [183, 184, 185] formal in drei Einzelschritte zerlegen [186, 187] 
- Zunächst bilden sich Kristallisationskeime. Die Potentialbarriere ist gegeben durch die Konkurrenz von aufzubringender Grenzflächenenergie und freiwerdender Volumenenergie.

- Haben sich solche Keime gebildet, so wachsen sie in die amorphe Matrix hinein. Die Kinetik dieses Wachstumsprozesses kann mit dem Modell von Johnson und Mehl beschrieben werden. Bezeichnet N die Zahl der vorhandenen Keime pro Volumen und v die Geschwindigkeit der sich verschiebenden Grenzfläche, dann wächst der Anteil des geordneten Volumens X mit der Zeit t gemäß

$$
X=1-\exp \left(-\frac{4}{3} \pi v^{3} N t^{3}\right)
$$

Die Geschwindigkeit der Grenzfläche v hängt dabei zweifach von der Temperatur ab. Zum einen nimmt die Sprungrate der Atome bzw. der Diffusionskoeffizient mit der Temperatur zu. Im Bereich des Glasübergangs und in der unterkühlten Schmelze ist die Diffusionskonstante mit der Viskosität gekoppelt. Die Sprungrate und die Viskosität hängen nach Gleichung (2.7) sehr empfindlich von der Temperatur ab, weshalb dies auch für v gilt. Zum anderen wächst die treibende Kraft durch die bei der Kristallisation freiwerdende Energie mit sinkender Temperatur.

- Stoßen die einzelnen geordneten Domänen aneinander, so wird ihr radiales Wachstum eingeschränkt. Selbst wenn schließlich die ganze amorphe Matrix kristallisiert ist, enthält der Kristall eine große Zahl von Antiphasengrenzen. Wenn große Domänen auf Kosten von kleinen Domänen wachsen, kann die freie Energie durch Auflösen dieser Phasengrenzen weiter vermindert werden. Dieser Vorgang wird als Ostwaldreifung bezeichnet. Die mittlere Domänengröße verändert sich dabei proportional zur Quadratwurzel der Zeit.

Bei isothermer Auslagerung treten während der Kristallisation alle drei Prozesse gleichzeitig auf. Für die exakte Beschreibung ist auch zu berücksichtigen, dass sich die Keimbildungsrate oder die Wachstumsgeschwindigkeit der Grenzfläche mit zunehmendem Anteil an kristallisiertem Volumen verändern können. Ein Maß für gesamte Kristallisationskinetik ist der Avrami-Exponent n. Er verknüpft den Anteil des bereits kristallisierten Volumens X mit dem neu hinzukommenden, gerade kristallisierenden Anteil y [187]. Hängt y von der Zeit ab, so ist auch $n$ zeitabhängig und wie folgt definiert: 


$$
n(t)=\frac{\partial[\ln (y(t))]}{\partial[\ln (t)]}
$$

$\mathrm{y}$ und $\mathrm{X}$ stehen miteinander in Beziehung:

$$
\frac{\partial X(t)}{\partial y(t)}=1-X(t)
$$

Integriert und umgeformt ergibt sich daraus

$$
y(t)=-\ln [1-X(t)]
$$

Die Betrachtung zur Kristallisationskinetik beschränkt sich im Folgenden auf DPO 19, da hier auf eine Tantal-Diffusionsbarriere aufgedampft wurde. Fehlt diese Zwischenschicht, findet die Kristallisation schon bei deutlich niedrigeren Temperaturen statt, was auf die erleichterte Keimbildung an der Silizium - $\mathrm{Zr}_{65} \mathrm{Al}_{7,5} \mathrm{Cu}_{27,5}$ Grenzschicht zurückgeführt wird. Davon abgesehen führt die nun folgende Auswertung aber zu ähnlichen Resultaten.

Abbildung 7.14 zeigt noch einmal den isothermen Kuvenverlauf für den Realteil (oben) und den Imaginärteil des Schermoduls (unten).

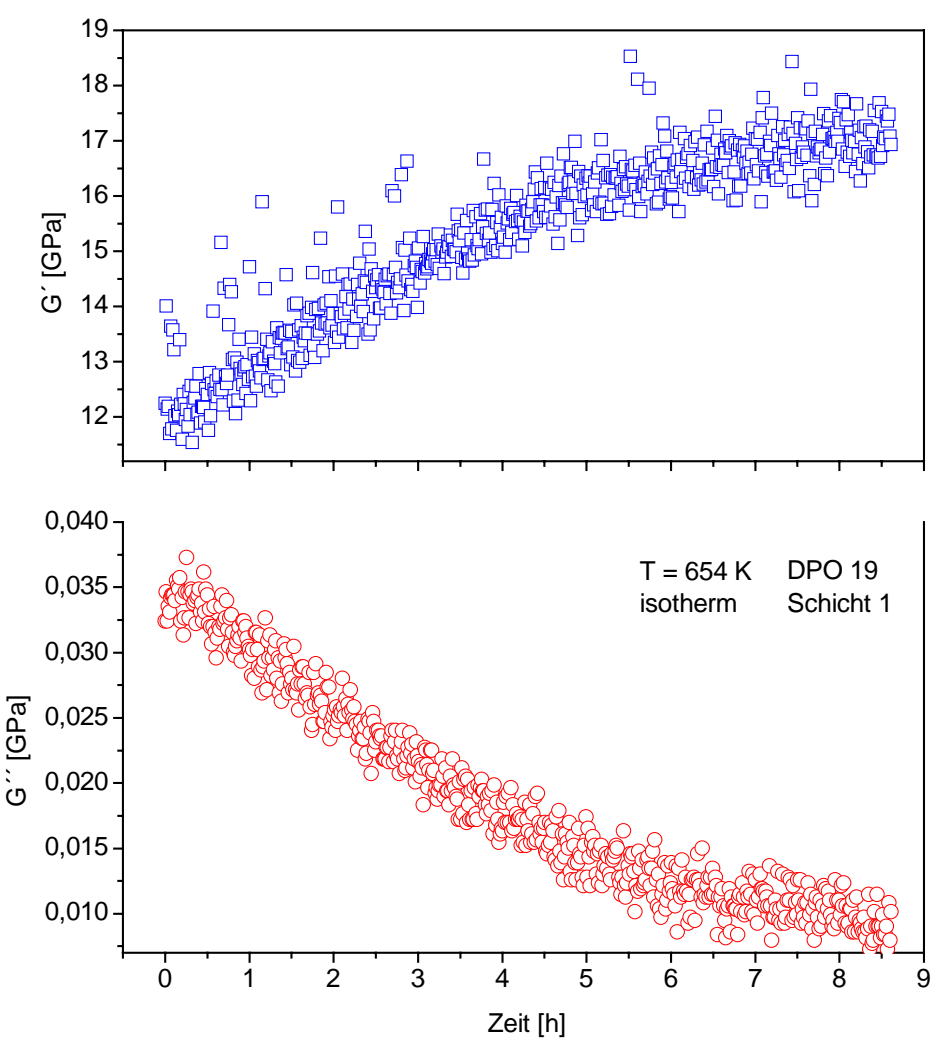

Abbildung 7.14: Isotherme Messung des Realteils (oben) und des Imaginärteils (unten) des Schermoduls bei 654 K über 8,5 Stunden bei DPO 19 Schicht 1. 
Aus beiden Kurven kann nun der bereits kristallisierte Volumenbruchteil X(t) berechnet werden. Dabei wird davon ausgegangen, dass $G^{\prime}$ für die vollständig kristallisierte Schicht bei $17,3 \mathrm{GPa}$ liegt, während für die völlig amorphe Schicht $\mathrm{G}^{\prime}$ nur 11,8 GPa beträgt. Für den Imaginärteil $\mathrm{G}^{\prime \prime}$ wird analog angenommen, dass er für die kristallisierte Schicht bei 0,01 GPa liegt und für die amorphe Probe 0,035 GPa beträgt. Damit wird der kristalline Volumenanteil jeweils aus dem Speichermodul G'(t) bzw. dem Verlustmodul $G^{\prime \prime}(t)$ berechnet:

$$
X(t)=\frac{G^{\prime}(t)-11,8 G P a}{17,3 G P a-11,8 G P a} \text { bzw. } X(t)=\frac{0,035 G P a-G^{\prime \prime}(t)}{0,035 G P a-0,01 G P a}
$$

Beide so berechneten Anteile sind in Abbildung 7.15 dargestellt. Die Werte liegen nahezu aufeinander, was zeigt, dass sich der Real- und der Imaginärteil des Schermoduls gleichermaßen aufgrund der zunehmenden Kristallisation verändern.

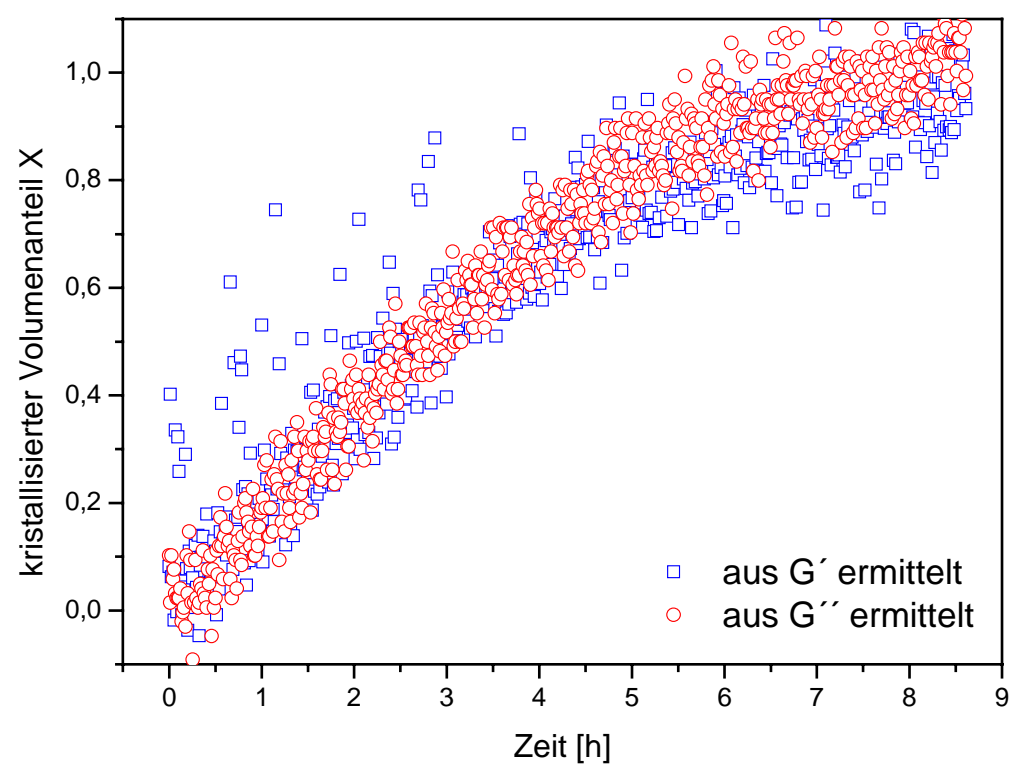

Abbildung 7.15: Zeitliche Entwicklung des kristallisierten Volumenanteils $X$ bei isothermer Auslagerung von Schicht 1 auf DPO 19 bei $654 \mathrm{~K}$.

Mit der Gleichung (7.10) ergibt sich daraus y(t) und mit Gleichung (7.8) der Avrami-Exponent $\mathrm{n}$ aus der Steigung der Messdaten in Abbildung 7.16. Der Avrami-Exponent beträgt etwa 1,5 und ist über das betrachtete Zeitintervall konstant. Er liegt damit in dem für die Kristallisation metallischer Gläser erwarteten Intervall für den Avrami-Exponenten zwischen 1,3 und 2,5 [187]. Die Konstanz ist ein Indiz dafür, dass sich die Keimbildungsrate im Verlauf der Kristallisation nicht ändert. Dass Inoue et al. [12] für schmelzgesponnene $\mathrm{Zr}_{65} \mathrm{Al}_{7,5} \mathrm{Cu}_{27,5^{-}}$ Bänder aus differentialkalorimetrischen Experimenten einen Avrami-Exponenten von etwa 3 
berechnen, steht im Widerspruch zu den hier gezeigten Daten. Allerdings ist zu berücksichtigen, dass nicht klar ist, wie groß der Anteil des Volumens ist, der schon während des Aufheizens auf $654 \mathrm{~K}$ kristallisiert ist, und ob die Prozesse nach 9 Stunden tatsächlich vollständig abgeschlossen sind. Es könnte auch sein, dass dünne Schichten und bulk-Proben ein unterschiedliches Kristallisationsverhalten zeigen, worauf der Avrami-Exponent hindeutet.

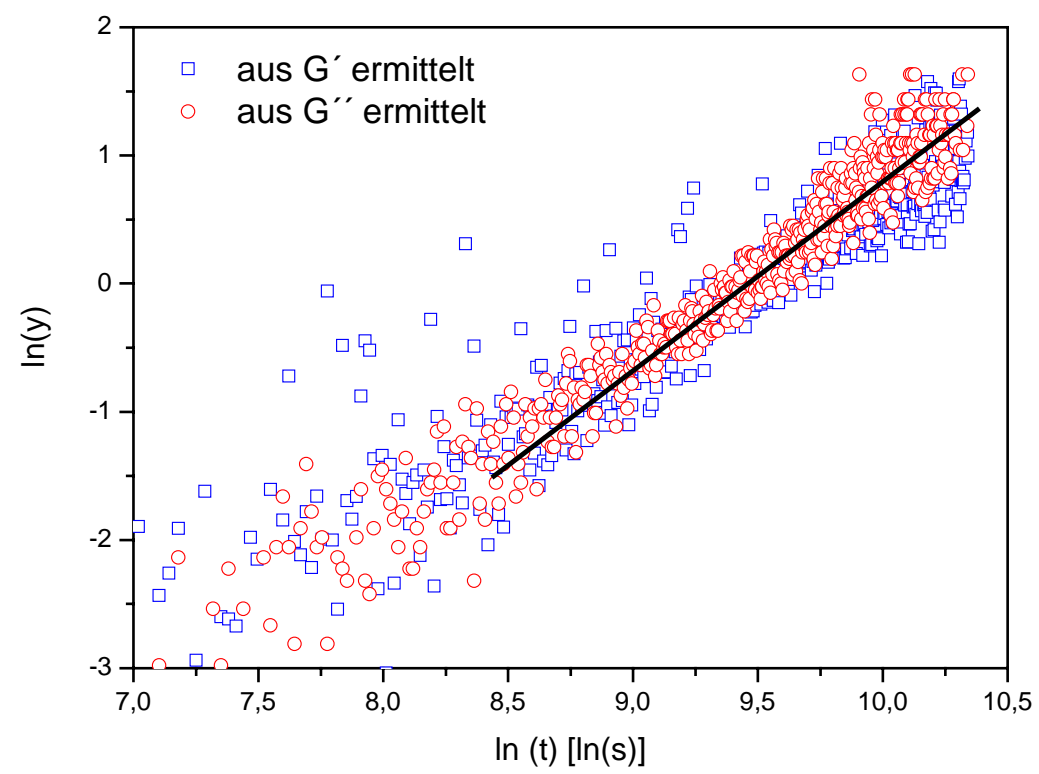

Abbildung 7.16: Der Avrami-Exponent von Schicht 1 auf DPO 19 bei 654 K ergibt sich aus der Steigung der eingezeichneten Gerade zu etwa 1,5.

\subsection{Charakterisierung der PMMA-Schichten}

In Abschnitt 6.1.2 wurde die Herstellung dünner PMMA-Schichten nach dem PLD-Verfahren beschrieben. Der exakte Ablationsprozess bei der Bestrahlung von PMMA ist unklar. Die Energie von Lichtquanten der Wellenlänge $248 \mathrm{~nm}$ liegt mit $5 \mathrm{eV}$ im Bereich der kovalenten Bindungsenergie der Makromoleküle. In der Literatur wird daher ein photochemischer Prozess vorgeschlagen [188]. Die Energie der Strahlung wird auf Atombindungen übertragen, die auf energetisch höhere Niveaus angeregt werden [189]. Zerfallen diese angeregten Zustände in einem einzigen Schritt, so können Bindungen brechen, was zur Dissoziation von Kettenbruchteilen führt.

Die Zusammensetzung der Plasmakeule und des deponierten Materials deutet aber auch auf einen thermischen Prozess hin [190, 191]. Angeregte Atombindungen zerfallen dabei über mehrere Zwischenschritte. Die eingestrahlte Energie wird in Form von Schwingungen als 
Wärme auf das PMMA-Target übertragen. Die Oberfläche heizt sich innerhalb weniger Nanosekunden so stark auf, dass thermische Zersetzung einsetzt. Diese Vermutung wird durch die Beobachtung gestützt, dass als Hauptbestandteil in der Plasmakeule Methylmethacrylat-Moleküle identifiziert wurden, die beim Auftreffen auf das Substrat neu polymerisieren [192]. Die Frage, ob es mit dem PLD-Verfahren gelingt, die Polymerstruktur auf die Doppel-Paddel Oszillatoren zu übertragen, ohne die Moleküle dabei fundamental zu verändern, erscheint vor diesem Hintergrund berechtigt. Daher werden in den folgenden Abschnitten die strukturellen Eigenschaften der Schichten mittels FTIR, die Abhängigkeit des Glasübergangs von der Kettenlänge und die thermischen Eigenschaften der so hergestellten Schichten diskutiert.

\subsubsection{Strukturelle Eigenschaften von PMMA (FTIR)}

Mit der in Abschnitt 3.3 beschriebenen FTIR-Spektroskopie lassen sich Informationen über die chemische Struktur von Polymeren gewinnen. Ist die Energie des durchstrahlenden Lichtes gerade so groß, dass eine intramolekulare Eigenschwingung angeregt wird, so beobachtet man bei dieser Wellenlänge in Transmission eine deutlich verminderte Intensität.

\begin{tabular}{|c|c|}
\hline Wellenzahl & Zuordnung / Absorptionskante \\
\hline $2993 \mathrm{~cm}^{-1}$ & $\left(v_{\mathrm{a}}\right) \mathrm{CH}_{3}-\mathrm{O}$ und $\left(v_{\mathrm{a}}\right) \mathrm{CH}_{2}$ \\
\hline $2950 \mathrm{~cm}^{-1}$ & $\left(v_{\mathrm{s}}\right) \mathrm{CH}_{3}-\mathrm{O},\left(v_{\mathrm{a}}\right) \alpha-\mathrm{CH}_{3},\left(v_{\mathrm{s}}\right) \alpha-\mathrm{CH}_{3}$ und $\left(v_{\mathrm{s}}\right) \mathrm{CH}_{2}$ \\
\hline $1729 \mathrm{~cm}^{-1}$ & $(v) \mathrm{C}=\mathrm{O}$ \\
\hline $1452 \mathrm{~cm}^{-1}$ & $(\delta) \mathrm{CH}_{2}$ \\
\hline $1438 \mathrm{~cm}^{-1}$ & $\left(\delta_{\mathrm{s}}\right) \mathrm{CH}_{3}-\mathrm{O}$ \\
\hline $1386 \mathrm{~cm}^{-1}$ & $\left(\delta_{\mathrm{s}}\right) \alpha-\mathrm{CH}_{3}$ \\
\hline $1268 \mathrm{~cm}^{-1}$ & $\left(v_{\mathrm{a}}\right) \mathrm{C}-\mathrm{C}-\mathrm{O}$ gekoppelt mit $\left(v_{\mathrm{a}}\right) \mathrm{C}-\mathrm{O}$ \\
bis $1238 \mathrm{~cm}^{-1}$ & \begin{tabular}{c} 
Deformationsschwingungen \\
\hline $1191 \mathrm{~cm}^{-1}$ \\
bis $650 \mathrm{~cm}^{-1}$
\end{tabular} \\
\hline $987 \mathrm{~cm}^{-1}$ & $\left(\gamma_{\mathrm{r}}\right) \mathrm{CH}_{3}-\mathrm{O}$ gekoppelt mit Skelett-Valenzschwingungen \\
\hline $752 \mathrm{~cm}^{-1}$ & $\left(\gamma_{\mathrm{r}}\right) \mathrm{CH}_{2}$ gekoppelt mit Skelett-Valenzschwingungen \\
\hline
\end{tabular}

Tabelle 7.3: Schwingungsbanden in ataktischem PMMA nach Dechant [193]. Die Bezeichnungen der Schwingungen bedeuten: $v_{s / a}$-symmetrische / antisymmetrische Streckschwingung, $\delta$ - Deformationsschwingung (Änderung des Valenzwinkels) und $\gamma$ - Deformationsschwingung (aus der Molekülebene heraus). 
In Tabelle 7.3 ist die Position der wichtigsten Absorptionsbanden von PMMA, bezüglich der in der Literatur üblichen Wellenzahl, dargestellt [193]. Die Wellenzahl ist dabei der Reziprokwert der Wellenlänge. Abbildung 7.17 zeigt IR-Spektren von drei verschiedenen PMMA-Proben. Die Daten werden zur übersichtlicheren Darstellung normiert und abzüglich einer Untergrund-Basislinie dargestellt.

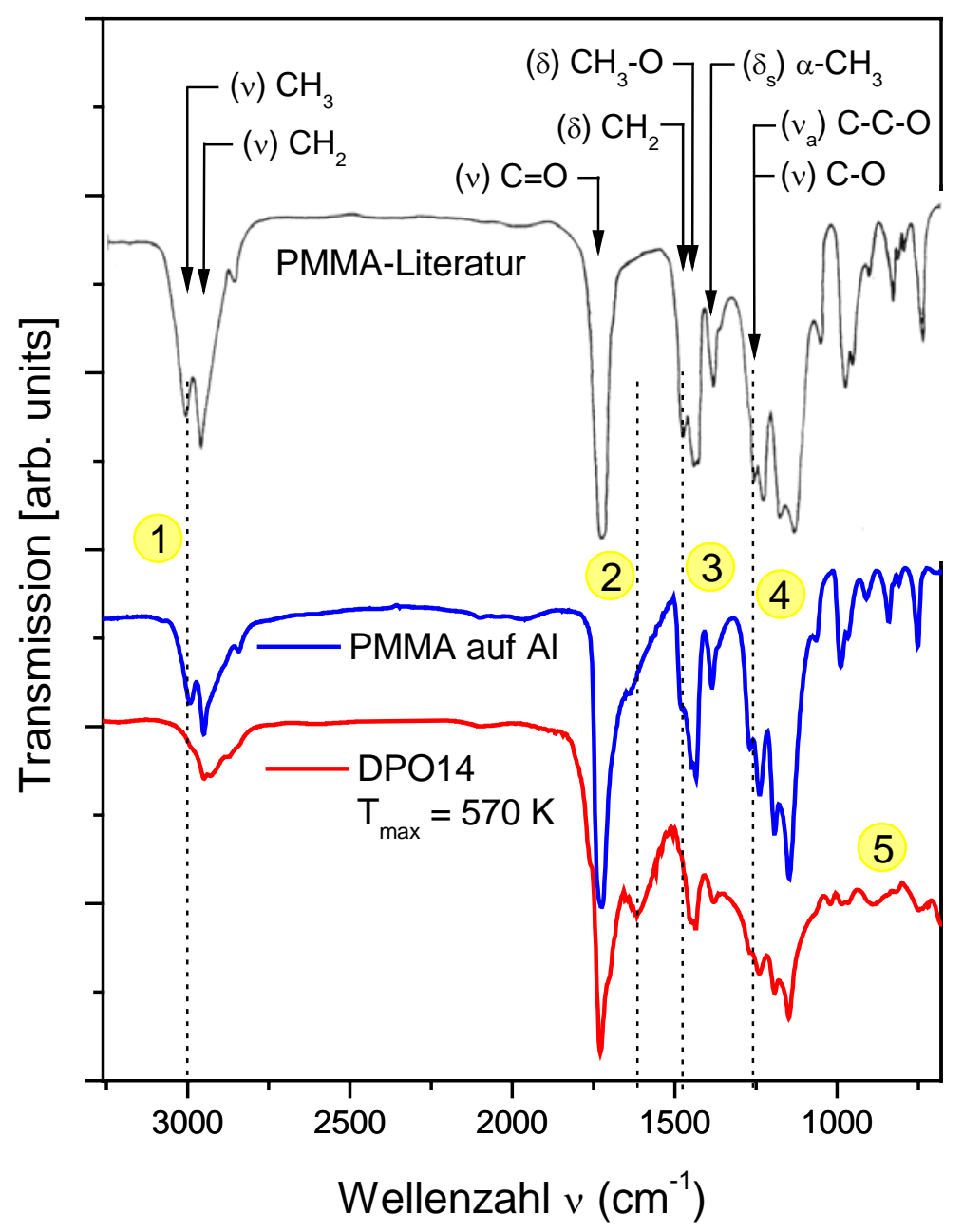

Abbildung 7.17: IR-Spektrum des PMMA Targets (schwarz) mit Bezeichnung der Absorptionsbanden nach Dechant [193], eines laserdeponierten PMMA-Films direkt nach der Herstellung (blau) und des PMMA-Films auf DPO 14 nach Beendigung der mechanischen Messungen und einer maximal erreichten Temperatur von 570 K (rot). Signifikante Unterschiede zwischen den Spektren treten bei den eingezeichneten gestrichelten Hilfslininen (1) bis (4), sowie im Bereich kleiner Wellenzahlen (5) auf.

In schwarz eingezeichnet ist das Spektrum von ataktischem PMMA, wie es als Target verwendet wird. Wegen experimentellen Schwierigkeiten bei der Messung des Massivmaterials wird dieses Spektrum der Literatur entnommen [193]. Blau eingetragen ist das Spektrum eines auf Aluminiumfolie laserdeponierten Films direkt nach der Herstellung. Offensichtlich 
gibt es eine eins-zu-eins Korrespondenz der Banden, was auf eine chemisch intakte Schicht hinweist. Geringe Unterschiede in der Absorption einzelner Banden können mit dem Verlust oder der Modifikation von Seitengruppen, beispielsweise durch Vernetzung, erklärt werden.

Die rote Kurve zeigt das Spektrum der auf DPO 14 deponierten Schicht nach Beendigung aller mechanischen Messungen. Diese Schicht hat ein umfangreiches Temperaturprogramm durchlaufen und wurde bis auf $570 \mathrm{~K}$ aufgeheizt (Tabelle 6.1). Bei dieser roten Kurve sind signifikante Änderungen zu beobachten, auf die in Abbildung 7.17 durch die von (1) bis (5) nummerierten Markierungen hingewiesen wird. Der Absorptionsverlust der $\mathrm{CH}_{3}-$ und $\mathrm{CH}_{2}$ Streckschwingung (1) könnte auf eine Abspaltung von Seitengruppen hinweisen. Eine andere Interpretation ist, dass es Vernetzungen an diesen Seitengruppen gibt. Eine solche chemische Reaktion könnte auch zur Bildung einer neuen Absorptionslinie bei $1600 \mathrm{~cm}^{-1}$ führen (2), die sich in der blauen Kurve andeutet, im Spektrum des Targets aber überhaupt nicht zu sehen ist. Auch an der größeren, in Abbildung 6.1 blau markierten Seitengruppe gibt es Veränderungen. Die Intensität der Absorptionsbanden von Atomschwingungen, an denen Sauerstoff beteiligt ist (3) und (4) geht deutlich zurück. Von fundamentaler Bedeutung sind auch die Veränderungen im Bereich kleiner Wellenzahlen (5). Da die hier liegenden Resonanzen vornehmlich durch Skelett-Valenzschwingungen des Polymer-Backbones verursacht werden, lässt die Verringerung der Intensität dieser Linien darauf schließen, dass der Backbone wenn nicht zerstört, so doch deutlich beschädigt, oder aber durch entstandene Vernetzungen in seinen Schwingungen stark eingeschränkt ist. Dafür spricht auch der Verlust der $\mathrm{CH}_{2}$-Streckschwingung bei der Wellenzahl $1452 \mathrm{~cm}^{-1}$.

\subsubsection{Abhängigkeit der Glastemperatur von der Kettenlänge}

Die Glasübergangstemperatur $\mathrm{T}_{\mathrm{g}}$ eines Polymers hängt von seiner Kettenlänge $a b$. Hinter dieser lange bekannten Beobachtung steht die Annahme, dass der Beitrag von Endstücken von Molekülketten zum gesamten freien Volumen deutlich größer ist, als der von Kettensegmenten [194]. Ausgehend von einer empirischen Beziehung von Fox und Flory [194] haben O’Driscoll und Sanayei [195] für PMMA folgenden Zusammenhang gefunden: 


$$
T_{g}=T_{g, \infty}-\frac{K}{X_{n}^{2 / 3}}
$$

Dabei ist $\mathrm{X}_{\mathrm{n}}$ die durchschnittliche Anzahl an Monomeren in einer Molekülkette und $\mathrm{T}_{\mathrm{g}, \infty}$ der asymptotische Wert für die Glasübergangstemperatur bei sehr langkettigen Molekülen. Zur Bestimmung der Parameter in Gleichung (7.14) haben die Autoren PMMA durch katalytische Polymerisation mit unterschiedlicher Kettenlänge hergestellt. Der Glasübergang wurde differentialkalorimetrisch bei einer Heizrate von $25 \mathrm{~K} / \mathrm{min}$ bestimmt. Aus den Messwerten ergeben sich mit Kurvenanpassung an Gleichung (7.14) die Parameter $\mathrm{T}_{\mathrm{g}, \infty}=386 \mathrm{~K}$ und $\mathrm{K}=353$. Die durchgezeichnete Kurve in Abbildung 7.18 zeigt den Zusammenhang nach Gleichung (7.14) mit den angegebenen Parametern.

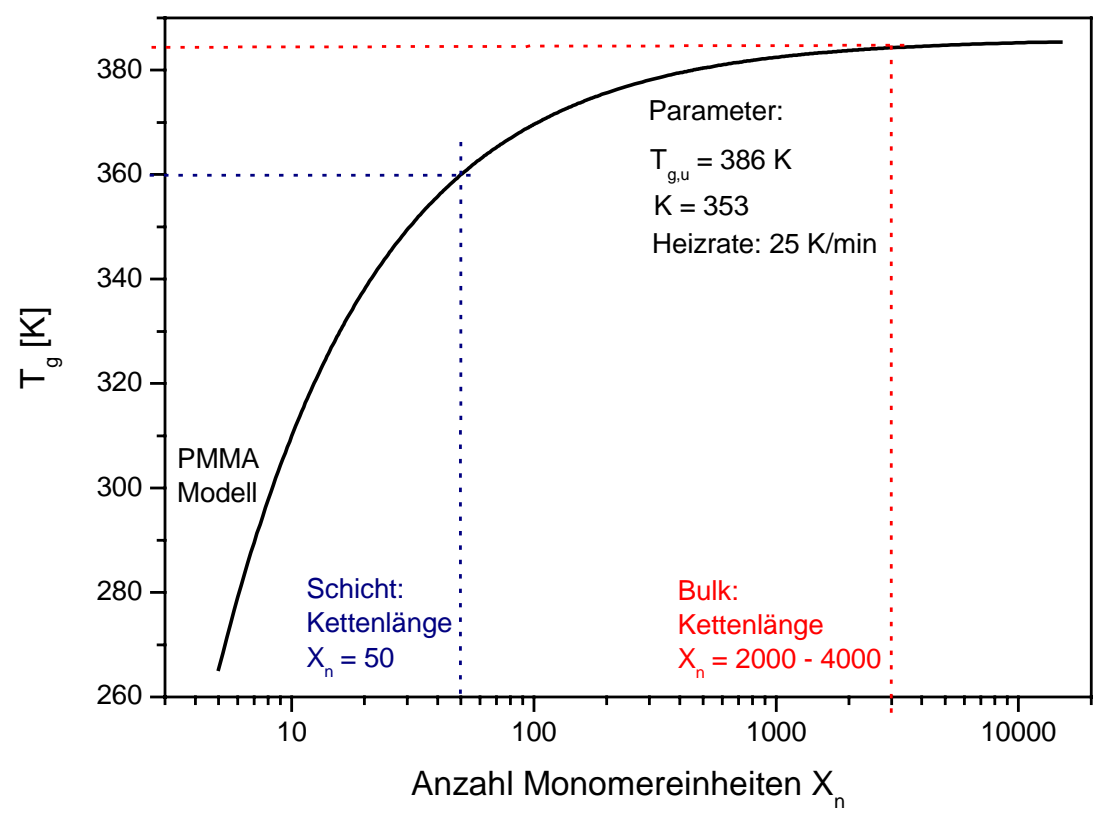

Abbildung 7.18: Abhängigkeit der Glasübergangstemperatur von der PMMA-Kettenlänge.

Geht man davon aus, dass das ataktische PMMA-Ausgangsmaterial für die im Rahmen dieser Arbeit hergestellten Filme eine Kettenlänge $X_{n}$ zwischen etwa 2000 und 4000 aufweist, so müsste der Glasübergang nach Gleichung (7.14) bei 385 K (rot gepunktete Linien) liegen. In den laserdeponierten Filmen ist die Kettenlänge auf etwa 50 Monomereinheiten verkürzt, was durch Messungen mittels Größenausschlusschromatographie (SEC) [196] nachgewiesen wurde. $\mathrm{T}_{\mathrm{g}}$ wird dadurch um rund $25 \mathrm{~K}$ zu niedrigeren Temperaturen verschoben (blau gepunktete Linien). 


\subsubsection{Thermische Eigenschaften von PMMA}

Bei einer Heizrate von $20 \mathrm{~K} / \mathrm{min}$ wurde der Glasübergang an PMMA-Ausgangsmaterial differentialkalorimetrisch (DSC) mit einem Perkin Elmer TGA 7 Gerät zu 387 K bestimmt [159, 196]. Wegen der geringen Menge an zur Verfügung stehendem Schichtmaterial ist eine vergleichende Messung nicht möglich. Allerdings steht mit Gleichung (7.14) eine Möglichkeit zur Verfügung, $T_{g}$ für PMMA zu berechnen, das in der Schicht eine Kettenlänge von $X_{n}=50$ aufweist, während eine Kette im Ausgangsmaterial aus 2000 bis 4000 Monomereinheiten besteht. Wie in Abbildung 7.18 skizziert, ist $\mathrm{T}_{\mathrm{g}}$ für die Schicht um rund $25 \mathrm{~K}$ kleiner als im Ausgangsmaterial und müsste daher bei etwa $362 \mathrm{~K}$ liegen.

Erhitzt man Polymere stark, beginnen sie sich zu zersetzen. Der damit verbundene Masseverlust lässt sich thermogravimetrisch (TGA) bestimmen. Dabei wird die Probe unter Inertgas mit einer Heizrate von $20 \mathrm{~K} / \mathrm{min}$ erhitzt und mit einer empfindlichen Waage fortwährend ihre Masse bestimmt. Im PMMA Ausgangsmaterial beginnt der Masseverlust bei etwa $513 \mathrm{~K}$. Er ist bei $723 \mathrm{~K}$ abgeschlossen. Im Schichtmaterial dagegen beginnt die Gewichtsabnahme schon bei $473 \mathrm{~K}$. Bei $673 \mathrm{~K}$ sind noch etwa $20 \%$ des Materials vorhanden, allerdings verlangsamt sich der Gewichtsverlust hier stark. Erst bei Temperaturen größer als $853 \mathrm{~K}$ kann kein PMMA Film mehr nachgewiesen werden $[159,196]$.

\subsubsection{Veränderungen in der Schicht bei hohen Temperaturen}

Resultate aus unterschiedlichen Experimenten deuten darauf hin, dass sich die Schichten bei isothermer Auslagerung bei hohen Temperaturen irreversibel verändern. So hat Süske [159] eine sukzessive Zunahme der Vickershärte bei Raumtemperatur festgestellt, nachdem die Proben vorher bei zunehmend höheren Temperaturen ausgelagert worden sind. Bei den DPO-Experimenten verschiebt sich die für DPO 14 und DPO 16 in Abbildung 6.5 gezeigte Resonanzfrequenz in Abhängigkeit von der erreichten Maximaltemperatur zu kleineren negativen Werten, was einer Zunahme des Schermoduls entspricht (siehe auch Abschnitt 7.8.1).

In Kapitel 6.3.3 sind isotherme Messungen sowohl der Frequenz, als auch der Dämpfung bei der Schicht auf DPO 14 für drei verschiedenen Temperaturen vorgestellt. Qualitativ ist in bei allen Temperaturen eine Zunahme des Schermoduls der Schicht und der Dämpfung zu beobachten. Im Verlauf der jeweils etwa 30 Stunden dauernden Experimente ist für beide Größen eine Sättigung zu erkennen. 
Die Veränderungen sind signifikant, und wurden auch in PMMA-Kompaktmaterial beobachtet. Sane und Knauss [197] weisen auf starke Effekte durch physikalische Alterungsprozesse hin. Die mikroskopischen Ursachen dafür sind allerdings unklar und konnten auch im Rahmen dieser Arbeit nicht abschließend geklärt werden. Allerdings gibt es zwei Interpretationsmöglichkeiten Es könnte sein, dass in Abhängigkeit von der Auslagerungstemperatur verschiedene Relaxationsprozesse in der Schicht angeregt werden. Dabei könnte es sich um Bildung von Vernetzungen, Reorientierung der Molekülketten, um weitergehende Polymerisation oder auch um Verdampfen kurzkettiger Moleküle oder Fragmente handeln. Ebenso denkbar ist, dass all diese Prozesse bei den drei Auslagerungstemperaturen parallel stattfinden (alle drei Temperaturen sind oberhalb von $\mathrm{T}_{\mathrm{g}}$ gewählt), allerdings nicht vollständig ablaufen. Vielmehr steigt die erforderliche thermische Energie mit zunehmendem Fortschreiten dieser Vorgänge.

\subsection{Mechanische Eigenschaften von PMMA}

\subsubsection{Komplexer Schermodul in PMMA}

Mit dem gleichen Verfahren wie für die metallischen Schichten wird nun der komplexe Schermodul von PMMA berechnet. Exemplarisch werden die Heizkurven von DPO 14 run 2, run 6 und run 10 ausgewertet. Mit den Folgerungen aus Abschnitt 7.2, dass die Schichtdicke auf diesem Oszillator nicht wie angenommen $1000 \mathrm{~nm}$, sondern $1800 \mathrm{~nm}$ beträgt, bestimmt sich der Masseanteil der Frequenzverschiebung nach Gleichung (4.43) zu -8,83 Hz. Die AS2Resonanzfrequenz von DPO 14 beträgt $5720 \mathrm{~Hz}$. Die Dichte von Silizium ist $2332 \mathrm{~kg} / \mathrm{m}^{3}$ und von PMMA $1200 \mathrm{~kg} / \mathrm{m}^{3}$. Der mit den Gleichungen (4.47), (4.55) und (4.59) bestimmte komplexe Schermodul ist in Abbildung 7.19 dargestellt.

Der Realteil zeigt für alle drei Heizdurchläufe einen qualitativ ähnlichen Kurvenverlauf. Allerdings ist $\mathrm{G}^{\prime}$ im Verlauf des Temperaturprogramms zu höheren Werten verschoben. Der Widerstand gegenüber Scherspannungen nimmt deutlich zu. Schon nach dem ersten Aufheizen über $T_{g}$ beträgt $G^{\prime}$ bei Raumtemperatur $6 \mathrm{GPa}$. Dieser Wert ist deutlich größer als der des Targetmaterials mit etwa 1 bis 2 GPa (nach Angabe des Herstellers). In Torsionsexperimenten an PMMA Massivmaterial wurde $\mathrm{G}^{\prime}$ von Muzeau et al. [198] zu etwa 1 GPa bestimmt. Dass $\mathrm{G}^{\prime}$ für die Schichten höher liegt, erklärt sich durch die ausführlich diskutierten Vorgänge bei der Deposition und Repolymerisation der Schicht auf dem DPO-Substrat. Die Verschiebung 
der Kurven zu größeren $\mathrm{G}^{\prime}$-Werten im Lauf des Temperaturprogramms wurde bereits in Abschnitt 7.7.4 diskutiert.

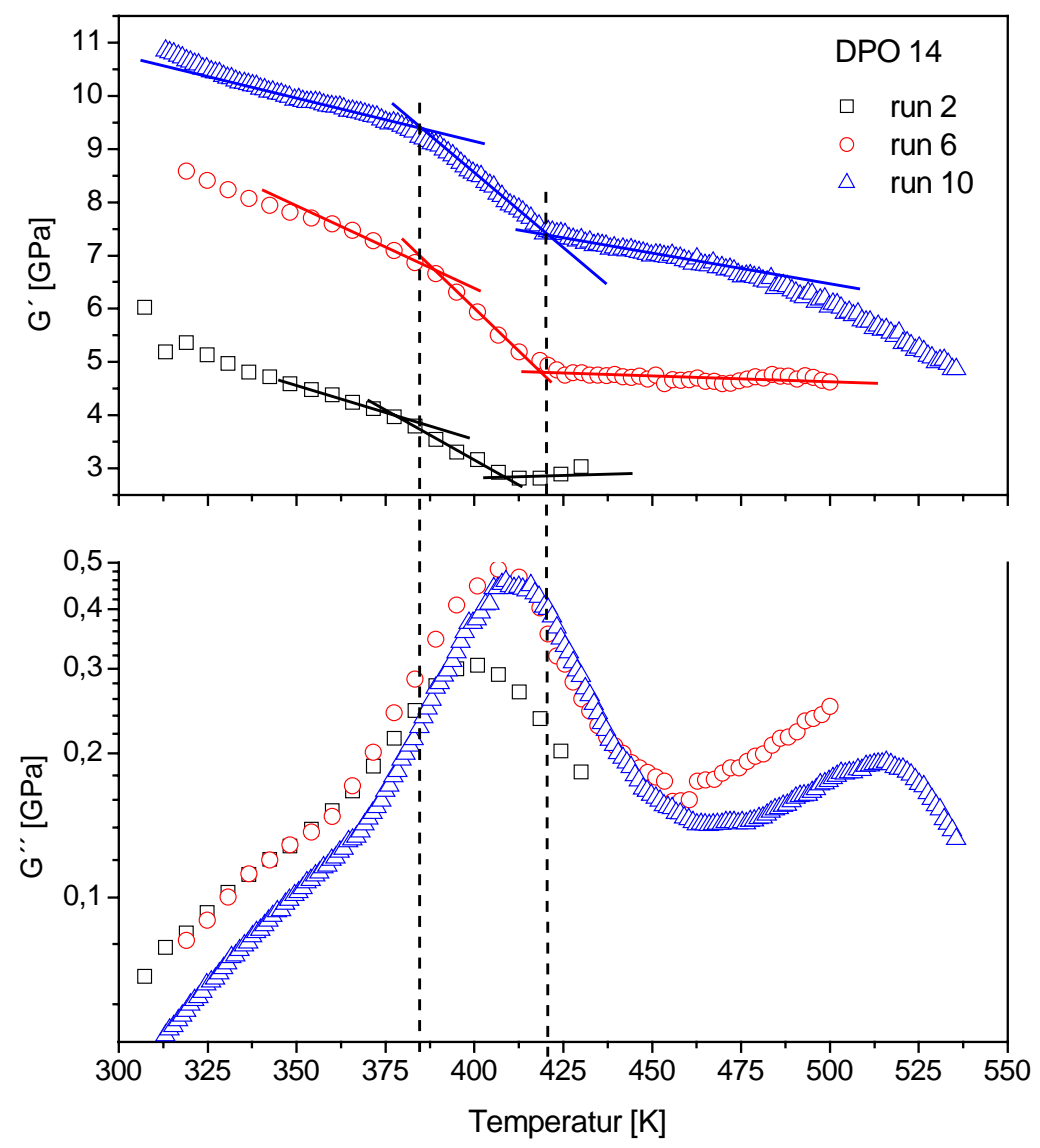

Abbildung 7.19: Komplexer Schermodul von PMMA auf DPO 14, berechnet an drei exemplarisch herausgegriffenen Heizzyklen. Die beiden senkrechten Linien deuten die charakteristischen Wechsel der Steigung in $G^{\prime}$ bei run 10 an und dienen zur Orientierung.

Bei allen drei Kurven finden sich zwei charakteristische Änderungen in der Steigung von G'. Die erste liegt bei etwa $385 \mathrm{~K}$ und könnte mit dem kalorischen Glasübergang korreliert sein, der zu $387 \mathrm{~K}$ bestimmt wurde (Abschnitt 7.7.3). Die Ursache für den zweiten Knick bei $420 \mathrm{~K}$ ist offen. $G^{\prime}$ mündet nun sowohl in run 6 , als auch in run 10 in ein Plateau ein, was zweierlei bedeuten kann. Entweder die Polymerschmelze erfährt in diesem Stadium keine Änderung der elastischen Eigenschaften mehr, was auch bedeutet, dass sich die Viskosität nun nicht mehr ändert, oder das weitere Absinken der Viskosität und des Speichermoduls G' wird durch parallel ablaufende Prozesse in der Schicht gerade kompensiert. Solche Prozesse könnten weitere Vernetzung bedeuten, Abdampfen von kurzkettigen Molekülen oder Fragmenten und einer einher gehenden Verdichtung des Materials. Dafür spricht auch die Zunahme der mechani- 
schen Härte nach Auslagern bei hohen Temperaturen [159]. Der Schermodul des auf Höhe des Plateaus verdichteten Materials würde nun beim Abkühlen auf Raumtemperatur größer sein, als beim Start der Heizkurve, was in der Tat ja auch beobachtet wird. Dass für run 10 die Messdaten ab etwa $500 \mathrm{~K}$ von diesem Niveau abfallen, wird mit der einsetzenden thermischen Zersetzung der Schichten erklärt.

Die Interpretation des Verlustmoduls $\mathrm{G}^{\prime \prime}$ beschränkt sich auf run 10, da bei diesen Durchlauf Messpunkte im Abstand von 0,15 K mit hoher Genauigkeit aufgenommen wurden. Für die Interpretation des Kurvenverlaufs ist der Vergleich mit dielektischen Messungen und die Betrachtung der unterschiedlichen Wirkung der anregenden Kräfte in beiden Methoden hilfreich.

\subsubsection{Vergleich des dielektrischen und des mechanischen Verlustes}

In Abbildung 7.20 ist sowohl der mechanische Verlust in der Schicht auf DPO 14 bei run 10 als auch der dielektrisch gemessenen Verlust einer $1 \mu \mathrm{m}$ dicken Schicht, bei gleicher Anregungsfrequenz $\mathrm{f}=5,4 \mathrm{kHz}$, gegenüber der Temperatur aufgetragen.

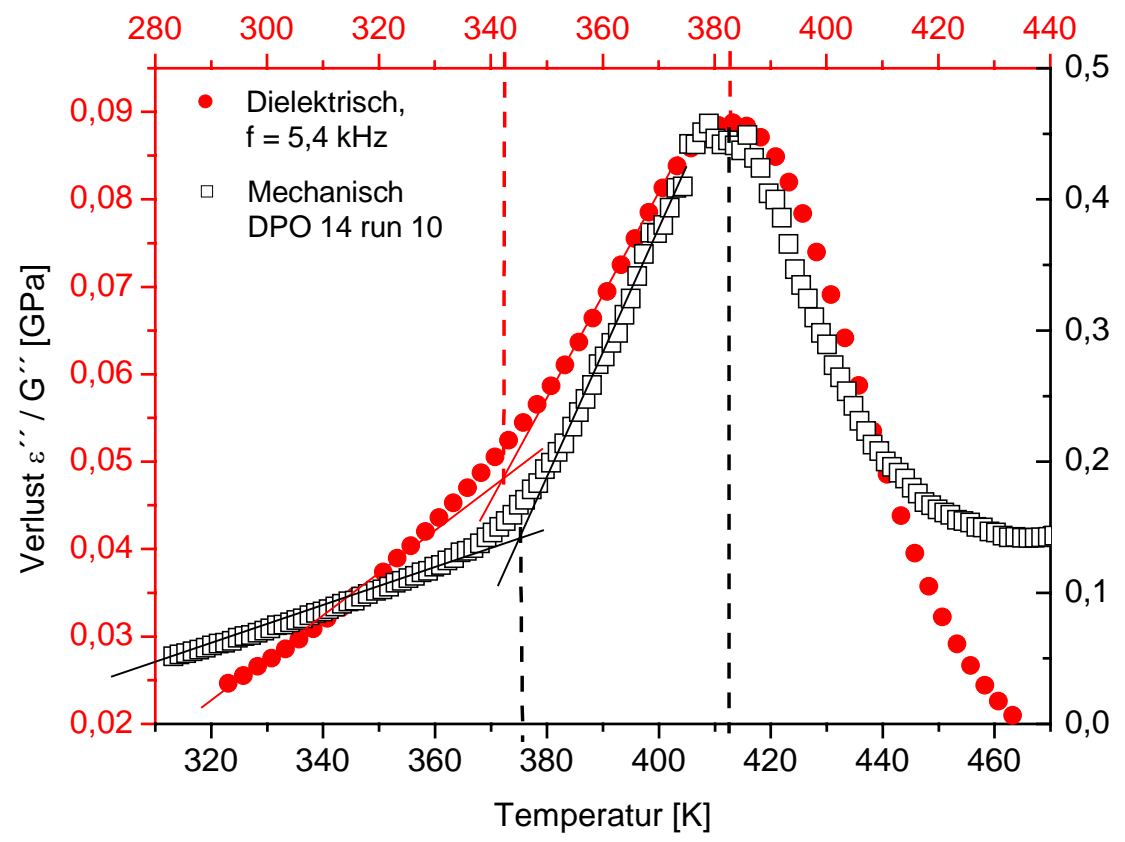

Abbildung 7.20: Vergleich des dielektrischen und des mechanischen Verlustes an PMMA-Schichten bei 5,4 kHz Anregungsfrequenz. 
Obwohl sich beide Spektren auf den ersten Blick ähneln, gibt es signifikante Unterschiede. Besonders auffällig ist die unterschiedliche Position und Form des dominierenden Peaks. Er ist für die mechanische Messung (schwarze Quadrate, untere Temperaturachse) schmaler als für die dielektrische und nimmt sein Maximum bei $412 \mathrm{~K}$ an. Der breitere Peak bei den dielektrischen Daten (rote Kreise, obere Temperaturskala) ist bei $383 \mathrm{~K}$ positioniert. Auch die Form der Niedertemperaturflanke ist für beide Methoden verschieden. Der durch die jeweils eingezeichneten Linien angedeutete Knick im Temperaturverlauf ist im mechanischen Verlust deutlich stärker ausgeprägt als im dielektrischen Fall.

Einen Erklärungsansatz liefert die Betrachtung der Kopplungsmechanismen und der Wirkung der anregenden Kräfte auf die PMMA-Moleküle.

Im dielektischen Fall koppelt das elektrische Wechselfeld am Hauptdipolmoment des Kettenmoleküls an den Seitengruppen an. Diese werden zur Rotation angeregt ( $\beta$-Prozess). Über diese Rotation und das „merging“ von primärer und sekundärer Relaxation wird die Anregung schließlich auch das viskose Fließen der Kettenmoleküle übertragen. Im dielektrischen Verlust ist daher die Relaxationsstärke für den $\beta$-Peak deutlich größer [161, 162]. Da bei $5400 \mathrm{~Hz}$ beide Prozesse ihr Verlustmaximum im gleichen Temperaturbereich haben, lassen sie sich bei den rot gezeichneten Punkten nicht eindeutig voneinander separieren.

Bei der mechanischen Anregung bewirkt die anliegende Scherspannung eine makroskopische Bewegung der Moleküle. Die Schicht kann der äußeren Kraft nur folgen, wenn sich die einzelnen Moleküle gegeneinander verschieben, was der primären Relaxation entspricht. Dass gleichzeitig durch die makroskopische Bewegung auch die Seitengruppen wegen des „merging“ gegeneinander rotieren können, erscheint zwar wahrscheinlich, allerdings kann dieser Prozess bei der mechanischen Spektroskopie nicht der dominierende Vorgang sein.

Vor diesem Hintergrund müssen die resultierenden Verlustspektra, die sich als Überlagerung beider Prozesse zeigen, unterschiedlich sein. Im dielektrischen Verlust zeigt sich hauptsächlich der $\beta$-Prozess, der viskoses Fließen überhaupt erst stimuliert, während im mechanischen Verlust im Wesentlichen der $\alpha$-Prozess angeregt wird und die Rotation der Seitengruppen nur eine untergeordnete Rolle spielt. Zur weiteren Aufklärung der Unterschiede beider Methoden müssten Experimente bei verschiedenen Frequenzen durchgeführt werden. 


\subsubsection{Interpretation des mechanischen Verlustes}

Nun wird davon ausgegangen, dass der dominierende Verlustpeak in Abbildung 7.19 durch viskoses Fließen während der primären Relaxation verursacht ist. Aus dielektrischen Messungen ist bekannt, dass die $\alpha$-Relaxationszeit $\tau_{\mathrm{HN}}$ einem Vogel-Fulcher-Gesetz mit $\mathrm{D}=2,3$ gehorcht. PMMA gehört damit zu den fragilen Gläsern. Entsprechend wurde KWW- $\beta$ bei $\mathrm{T}_{\mathrm{g}} \mathrm{zu}$ 0,2 bestimmt [72]. Der HN-Parameter $\gamma$ ist im Temperaturintervall zwischen $250 \mathrm{~K}$ und $500 \mathrm{~K}$ konstant bei 0,5 , während $\alpha$ linear mit der Temperatur ansteigt und sich näherungsweise nach der Gleichung $\alpha=0,002 \mathrm{~T}-0,21$ berechnet. Mit diesen Parametern wurde eine Kurvenanpassung mit der HN-Funktion (2.18) durchgeführt. Das Ergebnis ist in Abbildung 7.21 gelb dargestellt. Als Fitparameter für die VFT-Funktion (2.7) ergeben sich $\mathrm{T}_{\mathrm{VFT}}=334,5 \mathrm{~K}$ und die charakteristische Relaxationszeit $\tau_{0}=2,5 \times 10^{-9} \mathrm{~s}$. Die noch freien Parameter der HN-Funktion sind $\mathrm{G}_{\mathrm{S}}=10,25 \mathrm{GPa}$ und $\mathrm{G}_{\infty}=8 \mathrm{GPa}$. Die Relaxationsstärke beträgt daher etwa 2,25 GPa, was in Abbildung 7.19 (oben) gerade dem Verlust in $\mathrm{G}^{\prime}$ zwischen den beiden senkrechten Linien entspricht. Dass der dominierende Peak mit seinem Maximum bei $410 \mathrm{~K}$ auf diese Weise gut angepasst werden kann, spricht stark dafür, dass es sich dabei tatsächlich um die durch viskoses Fließen dominierte $\alpha$-Relaxation handelt.

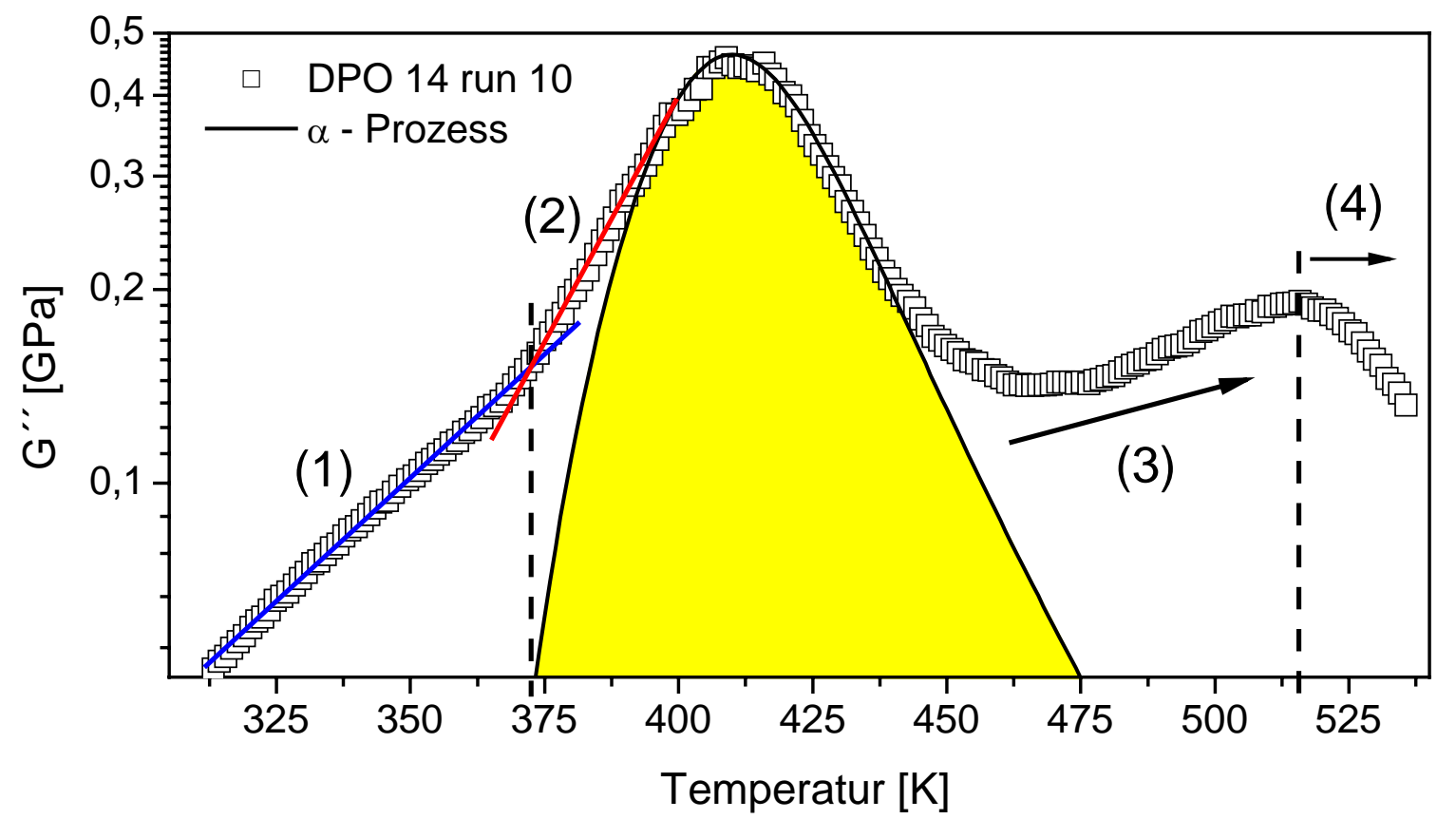

Abbildung 7.21: Mechanischer Verlust G" in PMMA auf DPO 14 während run 10. Gelb dargestellt ist der $\alpha$ Prozess, die charakteristischen Bereiche (1) bis (4) werden im Text erklärt. 
Um die Abweichungen auf der Niedertemperaturflanke (1) und (2) mikroskopischen Prozessen zweifelsfrei zuordnen zu können, wären vergleichende Messungen bei verschiedenen Frequenzen nötig. Es gibt allerdings verschiedene Ansätze, wie sich die Abweichungen verstehen lassen:

- Das in dielektrischen Messungen beobachtet „,merging“ der $\alpha$-Relaxation mit der Rotation von Seitengruppen könnte dazu führen, dass auch mechanisch der $\beta$-Prozess beobachtet wird und in der Niedertemperaturflanke erscheint [72]. In der Tat haben Muzeau et al. [198] in Torsionsexperimenten an PMMA-Stäben das Maximum im Verlust durch den $\beta$ Prozess bei einer Anregungsfrequenz von $1 \mathrm{~Hz}$ bei $275 \mathrm{~K}$ gefunden.

- Auch bei PMMA muss das Einfrieren der Dynamik bei $T_{g}$ zu einem Knick im mechanischen Verlust führen. Ein solcher Knick wird bei $373 \mathrm{~K}$ beobachtet (gestrichelte Linie zwischen (1) und (2) und liegt damit etwas tiefer als der kalorische Glasübergang.

- Da in dielektrischen Messungen die Seitengruppenrotation das Spektrum dominiert gibt es bislang keine Untersuchungen zum Auftreten eines „wing“ in PMMA. In mechanischen Messungen, die den $\alpha$-Prozess wiederspiegeln, könnte der „wing“ aber im Bereich (2) zu erkennen sein.

- Die Verteilung der Kettenlängen in den mit PLD hergestellten PMMA-Schichten ist unklar. Darüber hinaus wurden in GPC-Messungen auch Dimere, Monomere und Fragmente von Polymerketten gefunden. Diese sind deutlich kleiner als die durchschnittlichen PMMA-Moleküle. Ihr Glasübergang liegt daher bei tieferen Temperaturen. Im Bereich (1) könnten sich die Messwerte durch Überlagerung von $\alpha$-Peaks der Bestandteile mit niedriger Masse ergeben.

Der Bereich (3), in dem die Messwerte auf der Hochtemperaturflanke vom $\alpha$-Prozess abweichen entspricht etwa dem Plateau in Abbildung 7.19 (oben). Abdampfen von leicht flüchtigen Bestandteilen und unter Umständen weitergehende Repolymerisation oder Vernetzungsbildung führt insgesamt zu einer Verdichtung des Materials. Der mechanische Verlust G" durch dissipierte Reibungsenergie wird größer. Ab etwa $515 \mathrm{~K}$ in Bereich (4) fällt $\mathrm{G}^{\prime \prime} \mathrm{ab}$. Wird höher geheizt, als bis zu dieser Temperatur, so lässt sich bei späteren Temperaturzyklen eine Abnahme der Intensität $\mathrm{G}^{\prime \prime}$ über den gesamten Messbereich beobachten. Ab $515 \mathrm{~K}$ zersetzt sich PMMA thermisch. 


\section{Zusammenfassung und Ausblick}

In dieser Arbeit wurden dynamische Eigenschaften von $\mathrm{Zr}_{65} \mathrm{Al}_{7,5} \mathrm{Cu}_{27,5^{-}}$und PMMA-Schichten im Bereich des Glasübergangs untersucht. Insbesondere dem ,wing“-Phänomen als $\mathrm{Zu}$ satzbeitrag zur Niedertemperaturflanke des primären Relaxationspeaks galt dabei das Interesse. Als Methode wurde die mechanische Torsions-Spektroskopie mit dem Doppel-Paddel Oszillator gewählt. Eine entsprechende Apparatur zum Betrieb des DPO bei hohen Temperaturen wurde zu diesem Zweck gebaut. Dieses Kapitel fasst die wesentlichen Ergebnisse zusammen, stellt kritisch offen gebliebene Fragen und gibt Anregungen für weitere Experimente.

\section{Betrieb des DPO bei hohen Temperaturen}

Erstmals wurde der DPO oberhalb von Raumtemperatur als Substrat eingesetzt, um den Schermodul dünner Schichten zu messen. Die dafür entwickelte und gebaute Apparatur hat sich als wirkungsvolles und empfindliches Experiment erwiesen. Insbesondere konnte gezeigt werden, dass die Dämpfung des unbedampften Oszillators bis zu $400{ }^{\circ} \mathrm{C}$ durch den thermoelastischen Effekt dominiert wird. Die Güte bleibt groß genug, um selbst kleine Effekte in dünnen Schichten nachzuweisen. Für die Zukunft steht daher eine Methode zur Verfügung, nahezu beliebige Materialien auf ihre dynamischen Eigenschaften oder Phasenübergänge wie beispielsweise die Kristallisation hin temperaturabhängig zu untersuchen. Die einzige Bedingung ist, dass sie sich als dünne Schicht definiert auf den Oszillator aufbringen lassen. Der begrenzende Faktor für Geschwindigkeit und Empfindlichkeit der Experimente ist die Temperaturmessung und -regelung. Für weitere Experimente wäre auch zu klären, warum lasergeschnittene Oszillatoren stets eine geringere Güte haben als durch Photolithografie hergestellte und ob möglicherweise zu Silizium alternative Materialien als Substrat eingesetzt werden können.

\section{Der „wing“ in $\mathrm{Zr}_{65} \mathrm{Al}_{7,5} \mathrm{Cu}_{27,5}$}

Die gezeigten Daten des Verlustmoduls in diesem metallischen Glas lassen keinen Zweifel daran, dass es den „wing“ auch in metallischen Gläsern gibt. Da Beiträge aufgrund intramolekularer Freiheitsgrade ausgeschlossen sind, könnte es sich dabei um eine universelle Eigenschaft glasbildender Systeme handeln. Werden dynamische Heterogenitäten und kooperative Prozesse im Glas und in der unterkühlten Schmelze postuliert, lässt sich der „wing“ als Überlagerung verschiedener Relaxationsprozesse interpretieren. Wegen der bereits nahe am Glasübergang einsetzenden Kristallisation wäre es wünschenswert, amorphe Metalle mit größerer 
thermischer Stabilität zu untersuchen. Auch der Vergleich des hier untersuchten relativ starken Zr-basierten Systems mit einem fragilen Glas könnte weiteren Aufschluss über die zusätzlichen Relaxationsbeiträge geben. Das Zeit-Temperatur-Prinzip sollte durch entsprechende Experimente bei kleineren und größeren Frequenzen überprüft werden. So würde sich insbesondere klären, ob die Havriliak-Negami-Fitparameter temperaturabhängig sind.

\section{Mechanische und dielektrische Spektroskopie an PMMA}

Obwohl die mechanischen Messungen an PMMA-Schichten zunächst nur dazu gedacht waren, die Apparatur zu kalibrieren und die Empfindlichkeit des DPO zu testen, haben sich bei der Untersuchung interessante Aspekte ergeben. Es wurde gezeigt, dass sich die aus dielektrischer und mechanischer Spektroskopie gewonnenen Daten signifikant unterscheiden. Um die These zu manifestieren, dass dies durch die unterschiedliche Wirkung der elektrischen und mechanischen anregenden Kraft verursacht ist, wäre es hilfreich, ein System zu untersuchen, das möglichst wenig intramolekulare Freiheitsgrade besitzt und daher keinen ausgeprägten $\beta$ Peak zeigt. Nicht klar geworden ist auch, wie sich die PMMA Moleküle durch das Herstellungsverfahren mit PLD verändern. Interessant wäre daher auch die Untersuchung von alternativ hergestellten Schichten.

Abschließend sei angemerkt, dass die Dynamik von Gläsern und unterkühlten Schmelzen ein spannendes Forschungsgebiet ist - und wohl auch noch eine Weile bleiben wird. Auch nach drei Jahren intensiver Bemühungen und lebhafter Debatten bleibt der Glasübergang ein ,unsolved problem“, wie es Anderson ausdrückte. Allerdings erscheinen die Experimente an den metallischen Gläsern in Kombination mit Molekulardynamik Simulationen durchaus geeignet, der Natur weitere Einzelheiten zu diesem Phänomen zu entlocken. 


\section{Danke}

Der Erfolg, nach dreieinhalb Jahren Promotion am I. Physikalischen Institut der GeorgAugust Universität Göttingen über amorphe Materialien, Glasphysik und Doppel-Paddel Technik die Resultate meiner Arbeit in Form dieses Buches in der Hand zu halten, hat viele Mütter und Väter. Mein aufrichtiger Dank gilt daher:

- Prof. Dr. Konrad Samwer, an dessen Lehrstuhl in Augsburg ich zum ersten Mal echte Laborluft schnuppern durfte. Das hat nicht nur zur Zulassungsarbeit für das LehramtsStaatsexamen geführt, sondern mir so viel Freude bereitet, dass ich dann auch noch Diplomphysiker geworden bin. Der Aussicht, weiterhin sein Schüler zu sein, hatte nicht einmal meine starke Bindung zur bayerischen Heimat etwas entgegenzusetzen. Herzlichen Dank, Herr Samwer, dass ich bei Ihnen in Göttingen promovieren durfte, dass Sie mich gefördert und gefordert haben und mir den fachlichen Austausch national und mit dem Cornell-Aufenthalt und der Kreta-Tagung auch international ermöglichten. Danke auch für Ihr stetes und motivierendes Interesse am Fortschritt meiner Arbeit („Paddelt's noch“?) und für die vielen fachlichen und persönlichen Gespräche.

- Prof. Dr. Hilmar Teichler für sein Interesse und die Erstellung des Zweitgutachtens.

- Prof. Dr. Robert Otto Pohl, Xiao Liu, Tom Metcalf und Robert Merithew für Ihre Gastfreundschaft an der Cornell-University in Ithaca, die mir mit viel Geduld die experimentelle Technik rund um den Doppel-Paddel Oszillator beigebracht haben und deren Rat mir in der Aufbauphase sehr geholfen hat. Danke, Bobby, für die intensiven Diskussionen, danke Xiao für die Versorgung mit Oszillatoren.

- PD. Dr. Susanne Schneider für Ihre Betreuung als Teilprojektleiterin im SFB 602.

- PD. Dr. Michael Seibt und Dr. Yue-Lin Huang für die TEM-Untersuchungen.

- Prof. Dr. Hans-Ulrich Krebs, Erik Süske, Thorsten Scharf und Jörg Faupel für die PMMA-PLD-Schichten und die intensiven Diskussionsrunden.

- Dr. Yuansu Luo für das Sputtern der Tantal-Diffusionsbarieren.

- Prof. Dr. Alois Loidl, PD. Dr. Peter Lunkenheimer und Robert Wehn (Universität Augsburg) für die dielektrischen Messungen an PMMA und die wertvollen Tips zur Interpretation der Daten. 
- Luca Haiberger (Universität Düsserldorf) und Dr. Matthias Sperl (TU München) für den fachlichen Austausch zu Experimenten und Glastheorie.

- Dr. Manfred Weiß für das Überlassen seiner umfangreichen Paper-Sammlung.

- Christian Wald für seine Hilfe bei der Mathematik.

- Andreas Dohrn vom Fraunhofer Institut für Lasertechnik in Aachen für das Laserschneiden der Silizium-Wafer.

- Ludwig Beyland, Hartmut Eichenberg und den Mitarbeitern der feinmechanischen Werkstatt für die Konstruktion und den Bau des DPO-Einsatzes.

- Carsten Mahn, Uta Bete und Katrin Gehrke für all ihre Unterstützung mit Computern, Elektronik, UHV-Technik und im Labor.

- Den Mitgliedern des ersten Physikalischen Instituts, insbesondere meinen Kollegen Thomas Becker, Alberto Bracchi, Jörg Hachenberg, Dr. Henning Harms, Dr. Andrea Käufler, Frank Leuenberger, Björn Sass, Janina Schmidt und Christoph Streng für das angenehme Arbeitsklima und ihre große Hilfsbereitschaft, viele Grillabende und Kaffeerunden. Immerhin habe ich gelernt, dass Kaffee und Bier stets pur zu genießen sind, Hamburg keinen Nordsee-Strand hat und Göttingen strenggenommen nicht in Preußen liegt.

- Der Deutschen Forschungsgemeinschaft für die umfangreiche finanzielle Förderung meiner Arbeit im Rahmen des Schwerpunktprogramms Unterkühlte Metallschmelzen, des SFB 602 und des Graduiertenkollegs 782.

- Meiner Freundin Iris für die moralische Unterstützung, wenn es manchmal eben doch nicht so geklappt hat, wie ich es gerne gehabt hätte.

- Meiner Familie für ihr Vertrauen, ihre jahrelange Unterstützung, Geduld und Förderung, ohne die weder mein Studium, noch diese Arbeit möglich gewesen wäre. 


\section{Anhang 1 - Dokumentation der LabView Software}

Im Rahmen dieser Arbeit wurden LabView Programme und Unterprogramme entwickelt. Sie übernehmen wichtige Aufgaben zur Steuerung der Elektronik und zur Auswertung der Daten und werden hier vorgestellt und beschrieben. Die Programme wurden mit LabView Version 5.1 erstellt.

Die Unterprogrammen SENLesen.vi, MAGLesen.vi, PHALesen.vi, LockinlesenV.vi und LockinlesenIII.vi dienen zur Datenerfassung mit dem EG\&G 5210 Lock-In-Verstärkers, der auf GPIB Adresse 2 gesetzt ist.

Frequlesen.vi liest den Frequenzzähler FLUKE PM 6669 auf GPIB Adresse 4 aus, während Frequysnthschreiben.vi den HP 3325 B Frequenzgenerator (GPIB Adresse 1) ansteuert. frequscan.vi nimmt ein Bode-Diagramm auf (Amplitude und Phasenlage in Abhängigkeit von der extern vorgegebenen Frequenz). scandauer.vi ist ein Unterprogramm, das von frequscan.vi aufgerufen wird. RingdownAUTO.vi und ringdownseveral.vi sind Programme, mit denen Ring-Down-Experimente durchgeführt und ausgewertet werden. Dabei dient LinRegQ.vi zur Berechnung des Dämpfungsfaktors. Die Kommunikation mit dem Omega-Temperaturregler übernehmen OmegaTlesen.vi und OmegaTwrite.vi.

Hauptprogramm für die temperaturabhängige Aufnahme von Frequenz- und Dämpfungsdaten ist das Programm MastermitTProg.vi. Omegarampeinit.vi initialisiert darin die Temperatursteuerung, fTtschreiben.vi übernimmt die Datensicherung.

Das Umschalten zwischen Antrieb mit dem Frequenzgenerator und selbstangeregtem Phase-Lock-Loop-Modus wird durch die Programme PLLaktivieren.vi, PLLretten.vi, driveFGPLL.vi und driveONOFF.vi kontrolliert und gesteuert.

Im Folgenden werden für alle 21 Programme die Anschlussfelder mit den zugehörigen Daten Ein- und Ausgängen dargestellt. Auf dem Frontpanel sind die Bedien- und Anzeigenelemente des Programms sichtbar, während das Blockdiagramm den tatsächlichen Quellcode darstellt. Bei Fallunterscheidungen oder Case-Anweisungen ist jeweils ein Fall im Diagramm dargestellt, die anderen Fälle finden sich nebenstehend. Wegen der Übersichtlichkeit wurde bei allen Programmen auf die Verwendung von übereinander liegenden Strukturen verzichtet. Alle Programme besitzen einen Fehlereingang und einen Fehlerausgang. Verfolgt man diesen, so läuft man genau auf dem linear sequenziellen Weg, den die Programme abarbeiten. 


\section{SENLesen.vi}
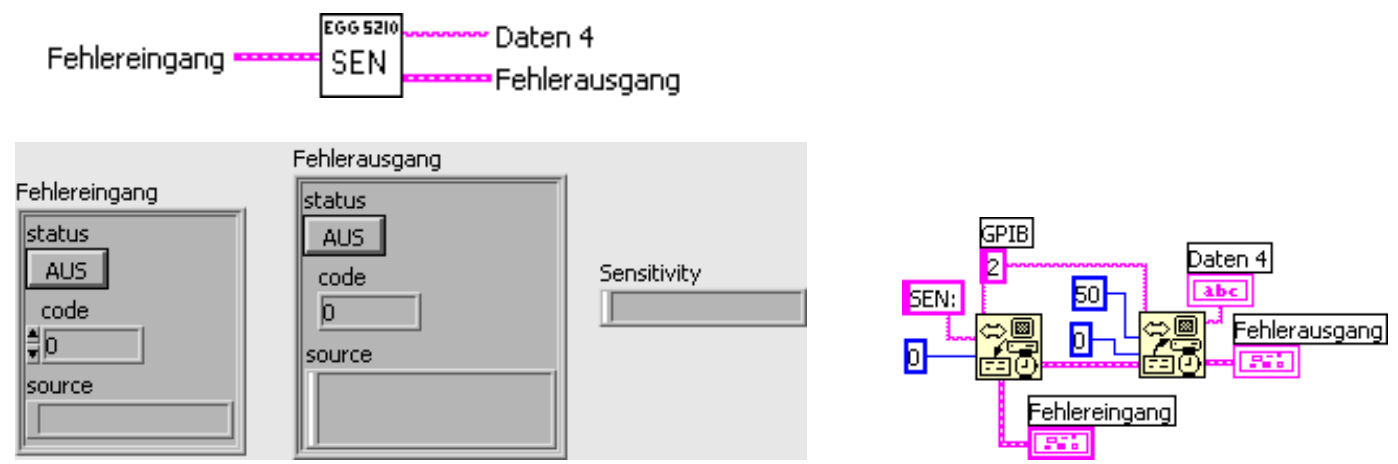

SENlesen.vi ist ein Basis-VI auf der untersten Ebene. Auf die GPIB Adresse 2 wird der String „SEN:“ geschrieben. Die am Lock-In-Verstärker eingestellte Empfindlichkeit des Eingangsverstärkers wird ausgegeben (maximal 50 Zeichen). Das VI wird als Unterprogramm von LockinlesenIII.vi aufgerufen und bei Frequenzscans benötigt, in denen die Amplitude mit Hilfe des SEN-Signals in mV umgerechnet wird.

\section{MAGLesen.vi}
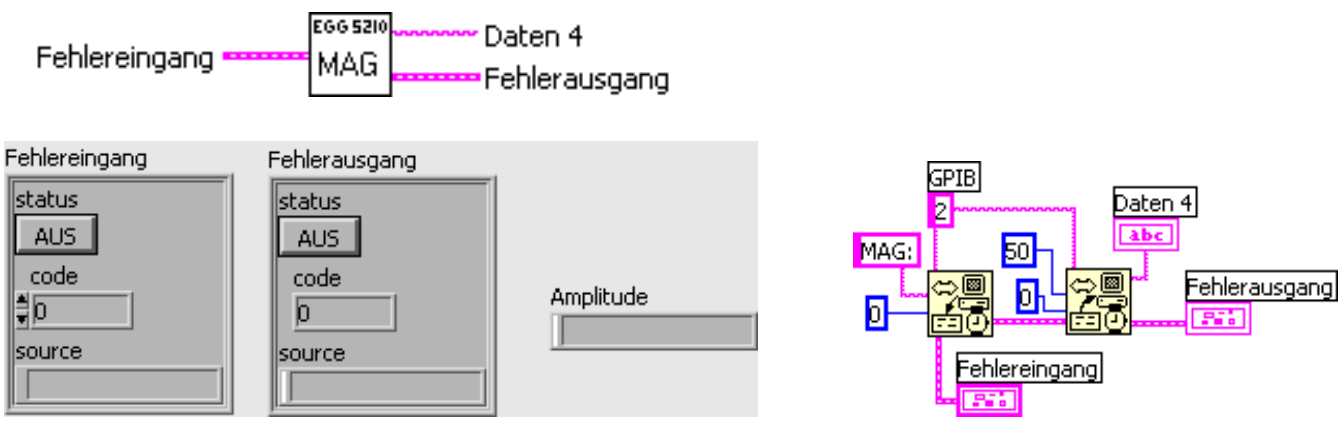

Auf den EG\&G 5210 Lock-In Verstärker, der auf GPIB Adresse 2 liegt, wird der String „MAG:“" geschrieben, worauf als Antwort die Amplitude geliefert wird.

\section{PHALesen.vi}
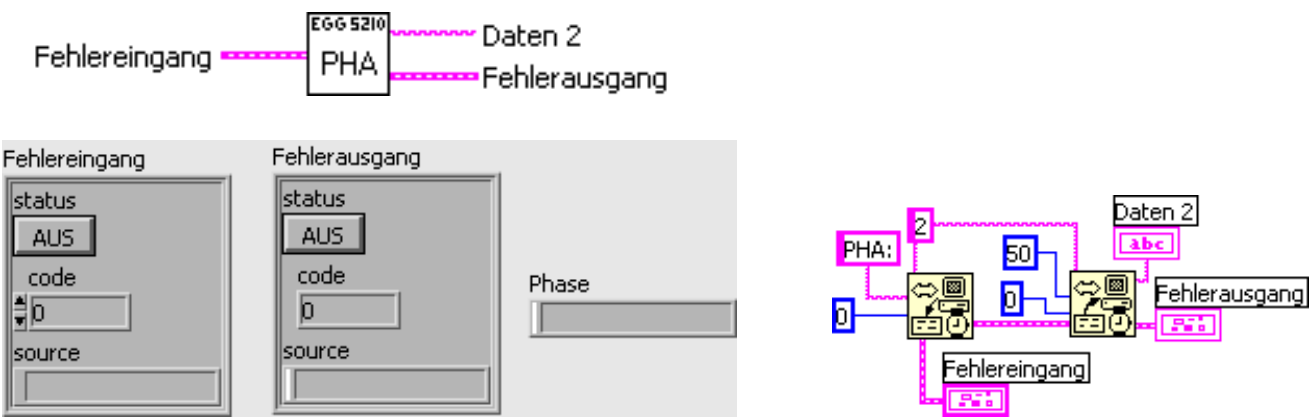
Auch PHAlesen.vi ist ein Basis-VI auf der untersten Ebene. Bei Betrieb des EG\&G 5210 im R- $\Theta-$ Modus liest dieses VI die Phasenverschiebung $\Theta$ zwischen Signal und Referenz aus. Auf GPIB Adresse 2 wird der String „PHA:“ geschrieben, worauf den Phasenwinkel als String ausgegeben wird. Das VI wird als Unterprogramm von LockinlesenIII.vi aufgerufen und bei Frequenzscans benötigt, in denen die Amplitude und die Phasenlage aufgezeichnet werden.

\section{LockinlesenV.vi}
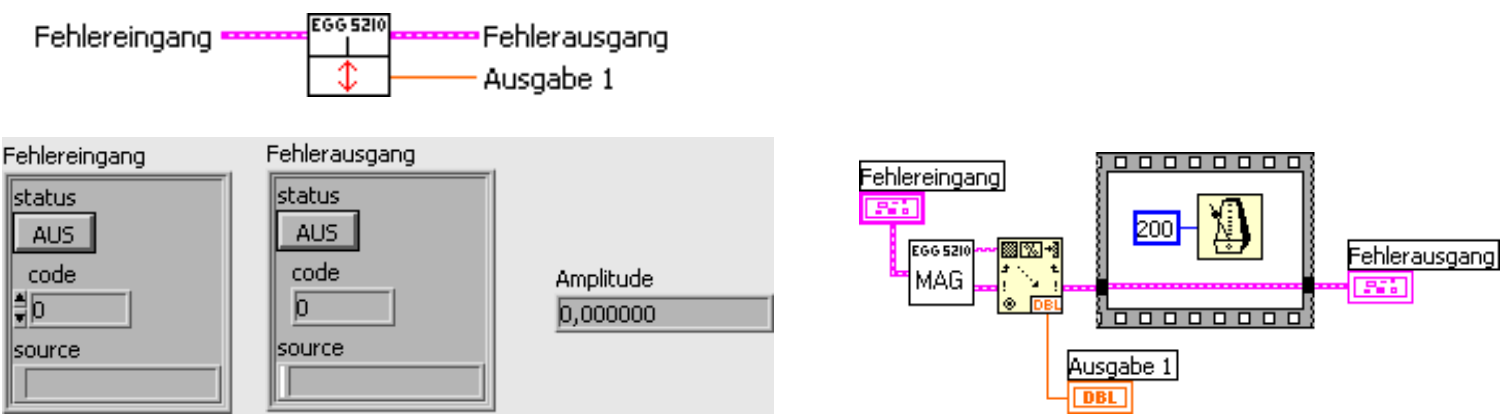

LockinlesenV.vi liest die über das Sub-VI Maglesen.vi die Amplitude des EG\&G 5210 Lock-In-Verstärkers einmalig aus. Hier, wie auch bei allen anderen Programmen, die den EG\&G 5210 ansprechen, wird eine 200 ms lange Warteschleife eingebaut, die ein sofortiges nochmaliges Ansprechen des Verstärkers verhindert, was zu einem GPIB Fehler führen würde. Dieses VI wird eingesetzt, um die als Exponentialfunktion abfallenden Amplituden während des Ring-Down-Modus zu messen. Ausgegeben wird die Amplitude als Zahl in der Einheit, die am Lock-In Verstärker als Messbereich eingestellt ist.

\section{LockinlesenllI.vi}
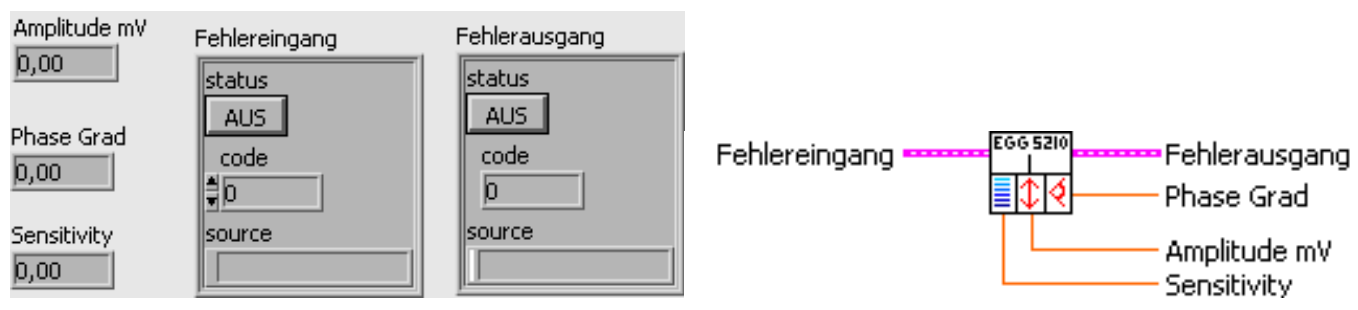


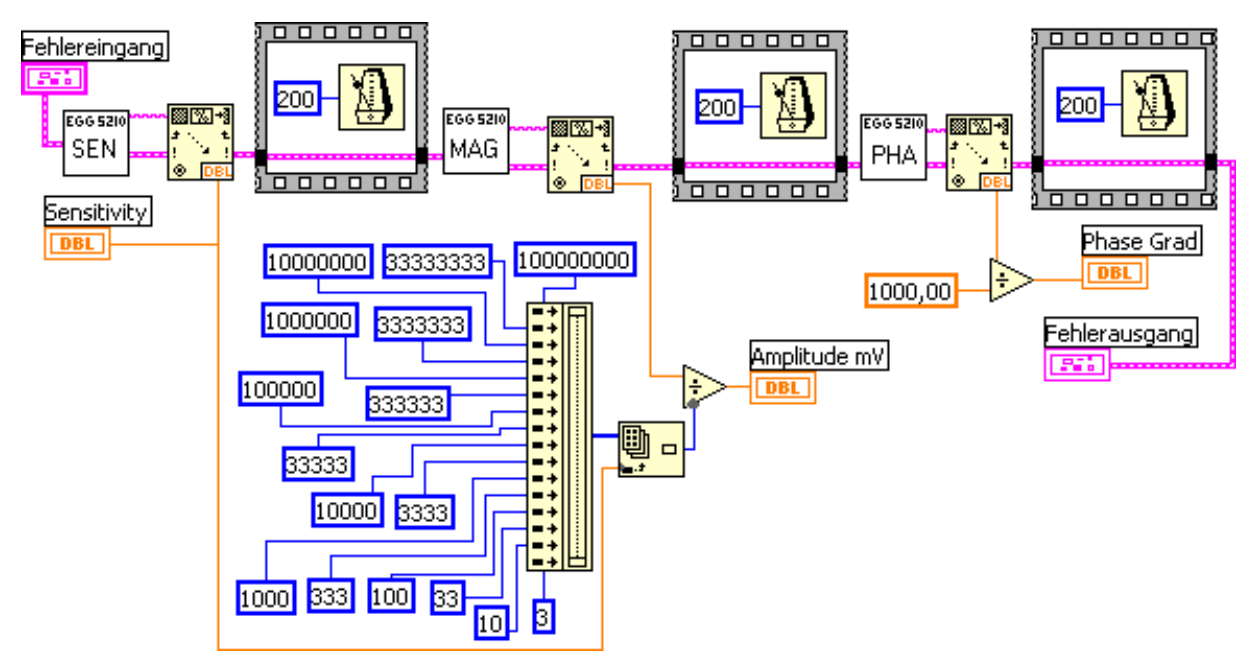

Im Gegensatz zu LockinlesenV.vi produziert LockinlesenIII.vi detailliertere und mehr Daten des EG\&G 5210 Lock-In-Verstärkers. Dabei liefern SENlesen.vi, MAGlesen.vi und PHAlesen.vi die eingestellte Empfindlichkeit des Eingangsverstärkers, die Amplitude und die Phasenbeziehung zwischen Referenz und Signal. Die von den Unterprogrammen gelieferten Strings werden zunächst in DBL-Zahlwerte umgewandelt. Die Sensitivity nimmt dabei Werte zwischen 1 und 16 an. Nun wird mit einem als konstant angegebenen 1-dimensionalen Array der passende Umrechnungsfaktor bestimmt, mit Hilfe dessen die Amplitude in $\mathrm{mV}$ umgerechnet wird. So werden verschiedene Messungen vergleichbar. Der Sensitivity-Zahlwert wird ebenso ausgegeben, wie die korrigierte Amplitude. In Programmen höherer Entwicklungsstufe wird jedoch auf den Sensitivity-Wert meist verzichtet. Schließlich wird die Phase ausgelesen und ebenfalls als DBL weitergegeben.

Das VI LockinlesenIII.vi wird bei den Frequenzscans aufgerufen, weshalb die Wartezeit zwischen den einzelnen Befehlen an den Lock-In-Verstärker 200ms beträgt. GPIB-Fehler werden so vermieden. Die Gesamtdauer von LockinlesenV.vi beträgt $600 \mathrm{~ms}$ und ist damit dreimal so lang wie bei LockinlesenIII.vi. Bei der Erfassung der Amplitude im RingDown-Modus, wobei möglichst viele Werte in kurzer Zeit eingelesen werden sollen, wird daher LockinlesenIII.vi verwendet.

\section{Frequlesen.vi}

Mit diesem Sub-VI wird die Frequenz des Fluke PM 6669 Frequenzzähler ausgelesen. Dieser hat die GPIB-Adresse 4. Zunächst wird der Befehl „EOI ON;MTIME 1“ zum Frequenzzähler geschickt. MTIME 1 fordert eine Integrationszeit von einer Sekunde, wodurch die Frequenzgenauigkeit auf $\pm 0,5 \mathrm{mHz}$ festgelegt ist. Das Resultat wird eingelesen und 
der zurück gelieferte String so zerlegt und in DBL-Format umgewandelt, dass schließlich die Frequenz als Zahl ausgegeben wird.
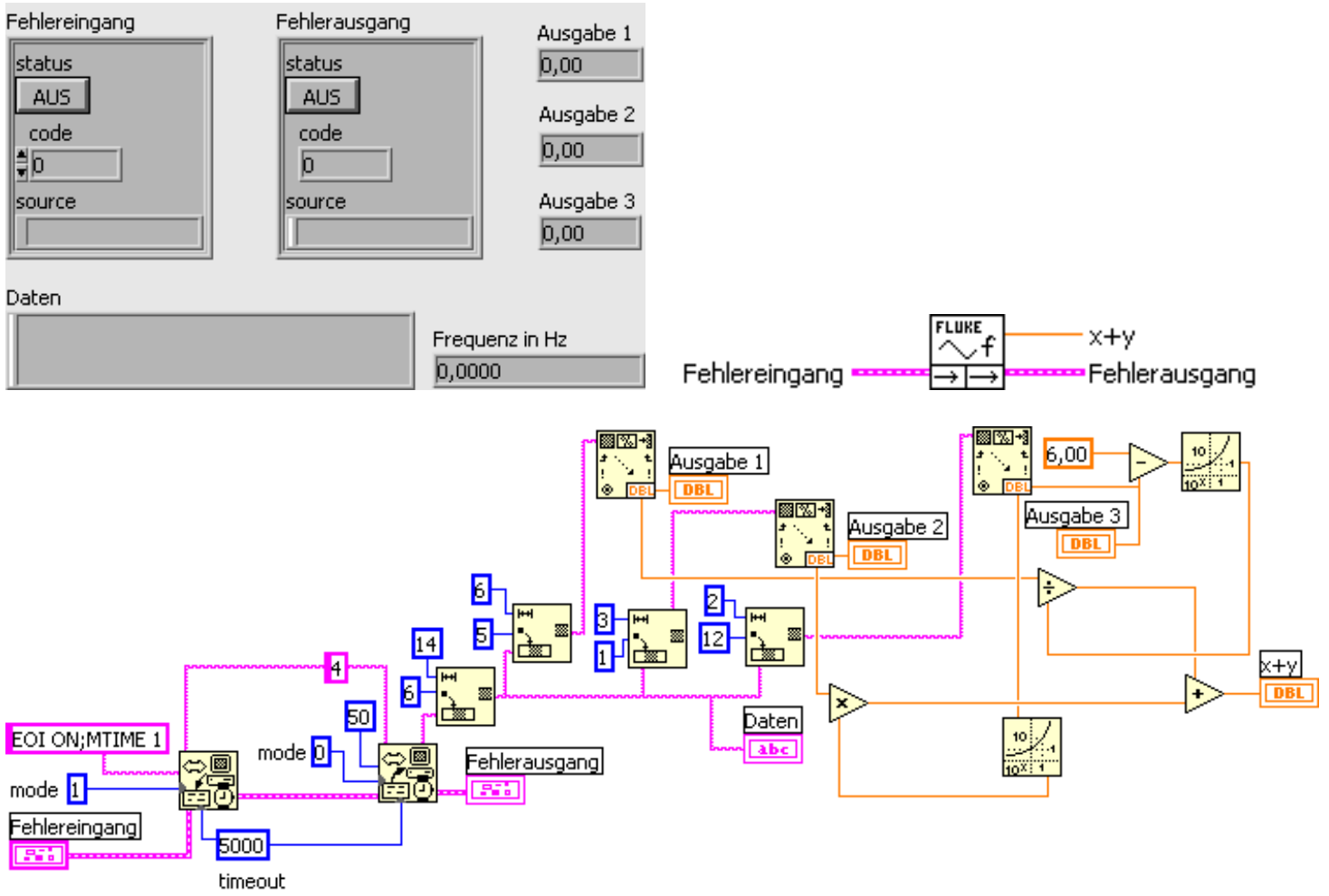

\section{frequsynthschreiben.vi}
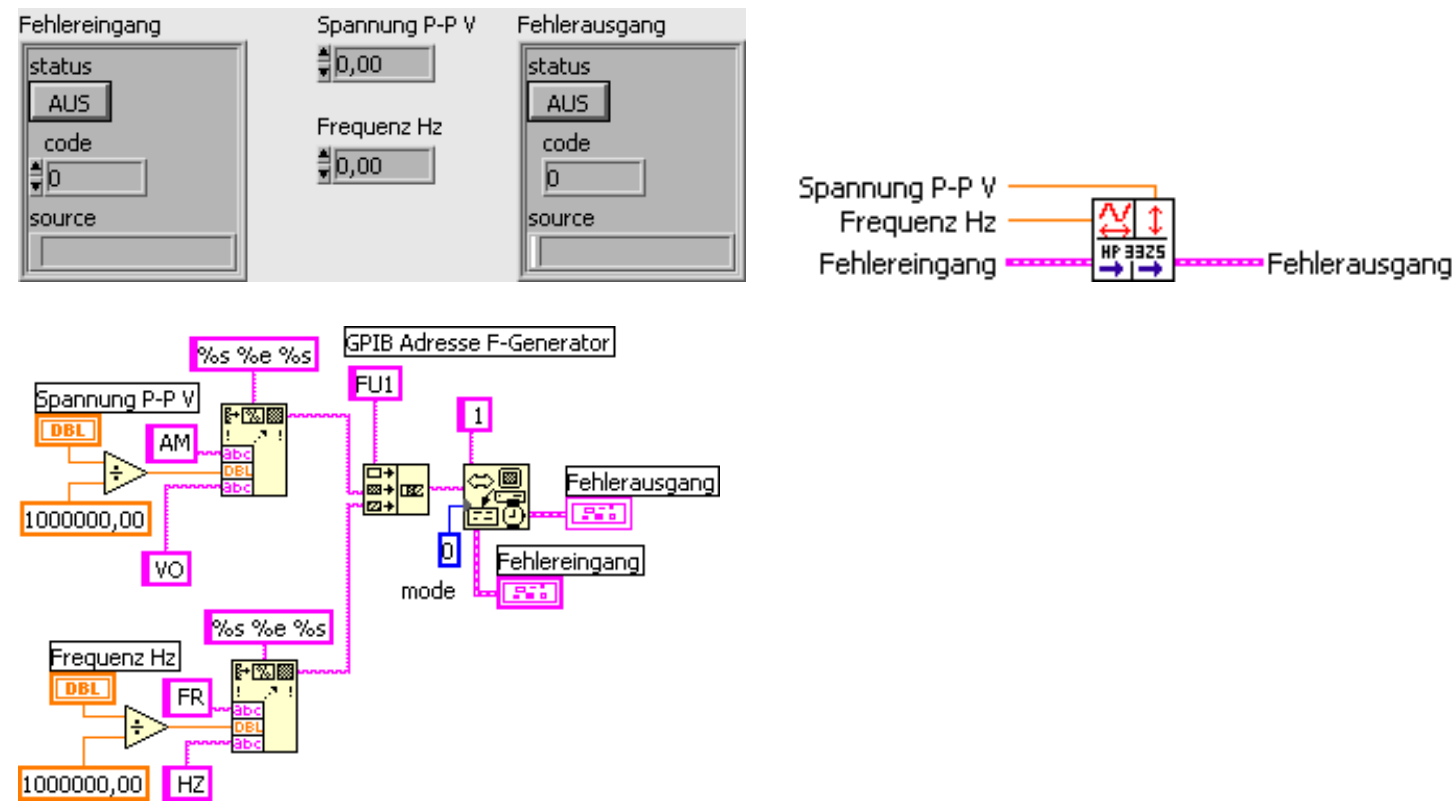

Fehlereingang

$$
\text { Filerausgang }
$$

Mit diesem Unterprogramm wird der HP 3325 B Frequenzgenerator für die externe Anregung der Paddelschwingung angesteuert. Die Peak-to-Peak Spannung wird in Volt, die Frequenz in $\mathrm{Hz}$ eingegeben. Beide Größen werden entsprechend umgerechnet, zu einem 
gemeinsamen String verknüpft und in einem gemeinsamen Vorgang auf den Frequenzgenerator, GPIB Adresse 1, geschrieben.

\section{frequscan.vi}

Eingabeparameter in dieses Programm, das in der Hierarchie auf höchster Stufe steht und nirgends sonst als Unterprogramm verwendet wird, ist die Startfrequenz, die Stopfrequenz, die Schrittweite, die Amplitude und die Einschwingzeit. Schrittweise werden dem HP Frequenzgenerator die Frequenzen übergeben, die mit der eingegebenen Amplitude den DPO antreiben. Ist die Frequenz eingestellt, wird für die Dauer der Einschwingzeit gewartet, mittels LockinlesenIII.vi Amplitude und Phasenlage der tatsächlichen DPO-Schwingung ermittelt und somit das Bode-Diagramm aufgezeichnet. Zur besseren Abschätzung ermittelt das Sub-VI scandauer.vi die Dauer des gesamten Frequenzscans.

Das Programm eignet sich sowohl für sehr grobe Scans (Schrittweite 0,2 bis $1 \mathrm{~Hz}$ ) als auch für sehr feine und langsame Scans. Die kleinste mögliche Schrittweite ist dabei $1 \mathrm{mHz}$. Hierbei ist zu beachten, dass bei der Untersuchung von Resonanzpeaks, beispielsweise um die Dämpfung aus der FWHM zu bestimmen, zwischen den einzelnen Frequenzschritten eine gewisse Einschwingzeit abgewartet werden muss, um Schwingungsüberlagerungen zu vermeiden und Einschwingvorgänge auszublenden. Weitere Ausgabeparameter sind die aktuelle Frequenz, die Amplitude und die Phasenlage. Die beiden letzteren werden in den Diagrammen auch grafisch dargestellt.

Mit den eingegebenen Parametern wird zunächst die Dauer des Scans berechnet und ausgegeben. Dominierend ist dann die große While-Schleife, die endet, falls die aktuelle Frequenz über der Stopfrequenz liegt. Jeder einzelne Schleifendurchgang beginnt mit der Messung von Signalamplitude und Phasenverschiebung mit LockinlesenIII.vi. Beide Werte werden im Diagramm dargestellt und zusammen mit der Frequenz in einer Datei gespeichert. Nun wird zu dieser Frequenz einmal die Schrittweite hinzuaddiert und die neue Frequenz auf den Frequenzgenerator geschrieben. Für das Einhalten der nötigen Einschwingzeit sorgt die sich anschließende Warte-Sequenz.

In der Datei werden die Daten in drei Spalten, getrennt durch TAB, in der Reihenfolge Frequenz (in $\mathrm{Hz}$ ), Phasenlage (in ${ }^{\circ}$ ) und Amplitude (in $\mathrm{mV}$ ) gespeichert. 


\section{Frequenz-Scan}

\begin{tabular}{|c|c|c|c|c|}
\hline Startfrequenz & * 0,00000 & $\mathrm{~Hz}$ & Scandauer & 0,00 \\
\hline Stopfrequenz & * 0,00000 & $\mathrm{~Hz}$ & & \\
\hline Schrittweite & $\neq 0,00000$ & $\mathrm{~Hz}$ & Ist-Frequenz & 0,0000 \\
\hline Amplitude & $=0,00$ & $V p-p$ & Ist-Amplitude & 0,000000 \\
\hline Einschwingzeit & $\approx 0,00$ & $s$ & Ist-Phase & 0,00 \\
\hline
\end{tabular}

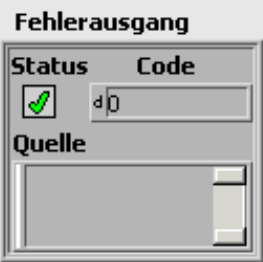

Amplitudenverlauf

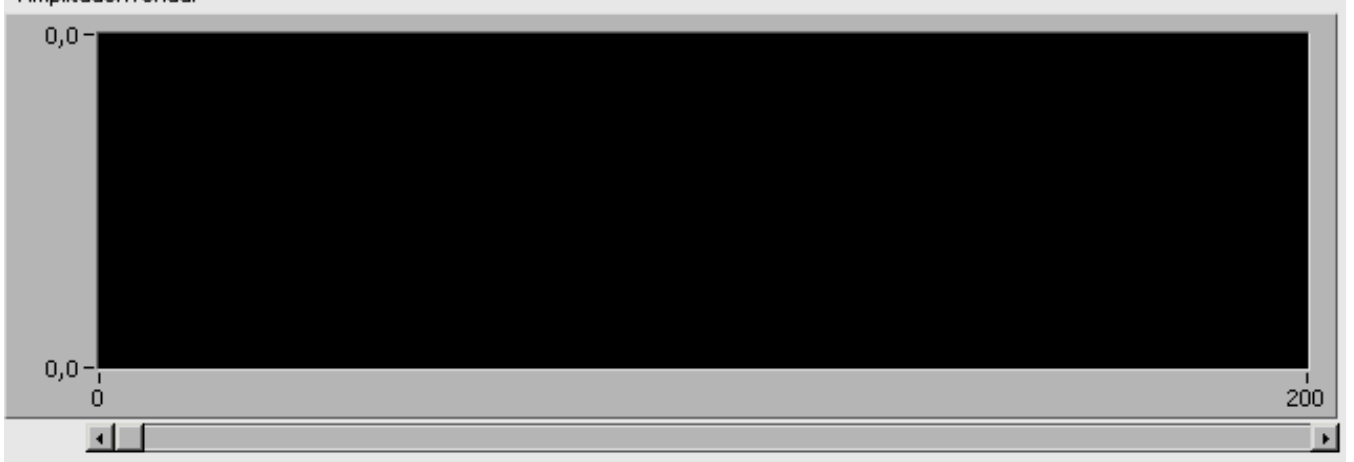

Phasenverlauf
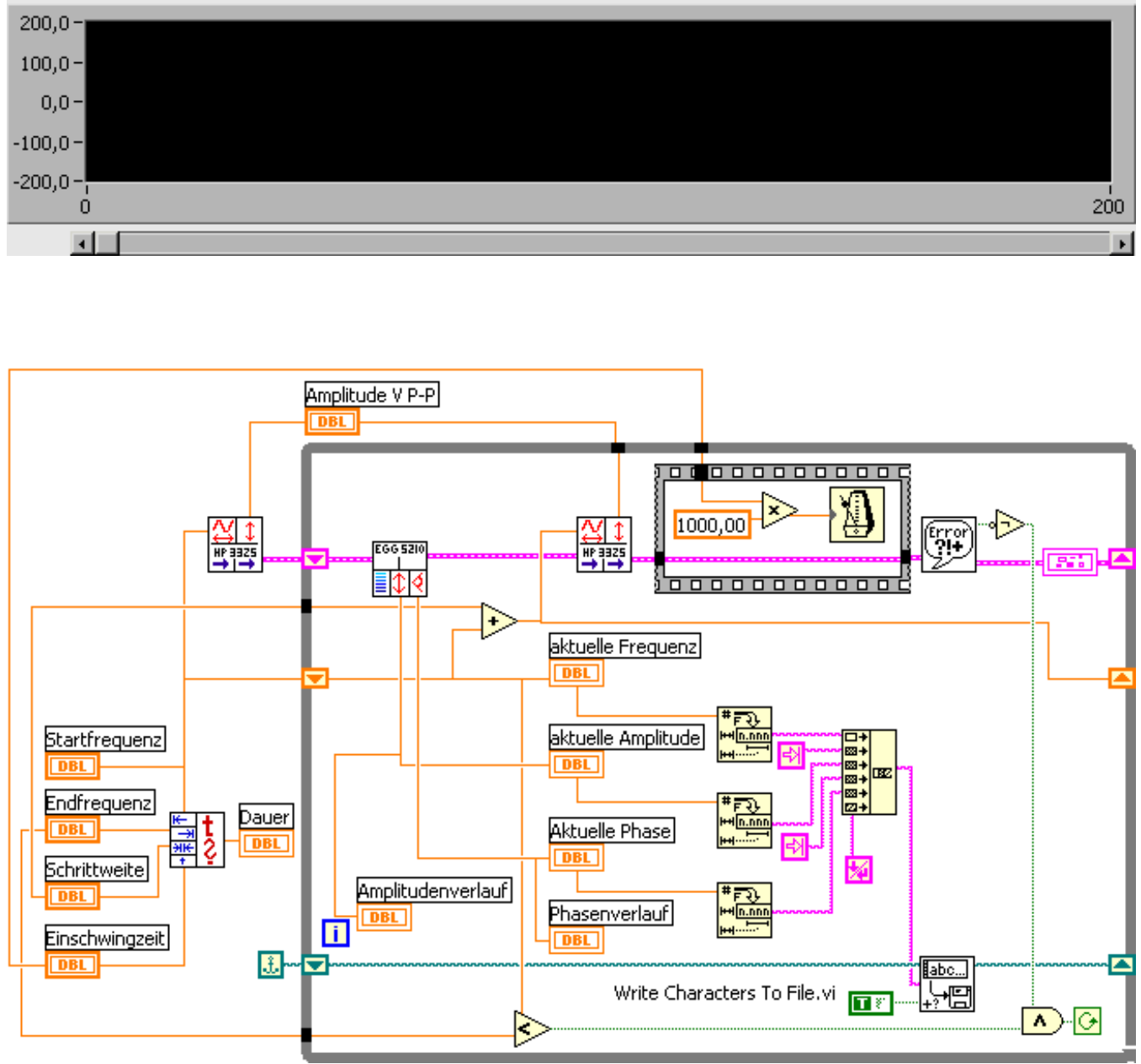


\section{scandauer.vi}
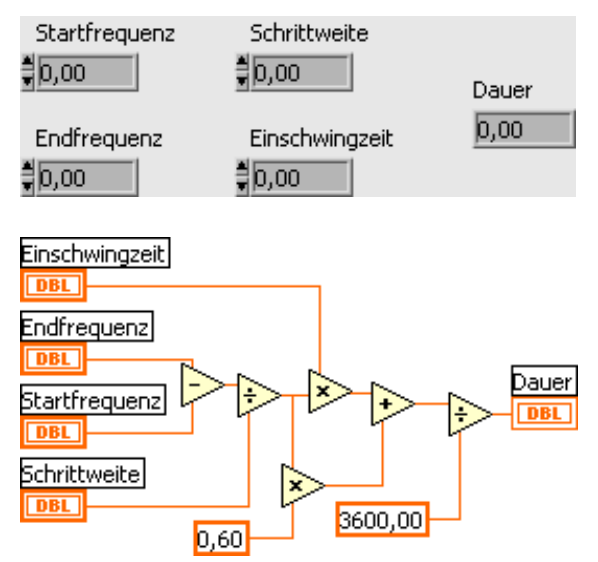

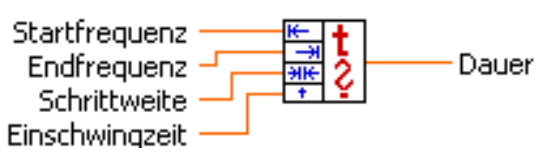

Dieses Sub-VI steht in der Hierarchie ganz unten und wird als Basis-VI nur von dem Programm frequscan.vi aufgerufen. Aus den eingehenden Daten Einschwingzeit, Startfrequenz, Endfrequenz und Schrittweite ermittelt es die tatsächliche Dauer des gesamten Frequenzscans in Stunden. Dabei wird als zeitlimitierender Vorgang das Auslesen des LockIn-Verstärkers angesehen, das pro Frequenzschritt 0,6 s dauert. Multipliziert mit der Anzahl der Messpunkte und der Einschwingzeit ergibt sich die Gesamtdauer des Scans.

\section{ringdownAUTO.vi}

Das Programm steht in der Hierarchie auf höchster Stufe und wird nirgends als Unterprogramm verwendet. Beim Programmstart wird die Anregung (PLL) ausgeschaltet und der Ring-Down-Modus gestartet. Die Daten werden grafisch im Diagramm dargestellt und in der angegebenen Datei gespeichert (Zeit und Amplitude). Der Dämpfungskoeffizient wird berechnet. Als Zusatzinformation wird die Frequenz angezeigt. Dieses Programm wird immer dann verwendet, wenn zwischendurch relativ schnell aus einem einzelnen RingDown-Experiment der Dämpfungsfaktor bestimmt werden soll.

Zunächst wird die Frequenz und die Amplitude bei angeregtem PLL gemessen. Nach Abschalten des Drive-Signals ist eine Wartezeit von 400 ms eingebaut. Dadurch werden Einschwingvorgänge abgewartet. Nach Messung der aktuellen Systemzeit, die dem Startsignal für das eigentliche Ring-Down-Experiment entspricht, beginnt die While-Schleife. Sie endet, wenn die Amplitude unter den zehnten Teil der Startamplitude abgefallen ist. In jedem einzelnen Durchlauf wird zunächst die Systemzeit ermittelt. Davon wird die Startzeit subtrahiert. Das Ergebnis dividiert durch 1000 ergibt die bislang vergangene Zeit des Experiments in Sekunden. Diese wird ausgegeben und in die erste Spalte der Datei gespeichert. 


\section{DPO-Abklingkurven}

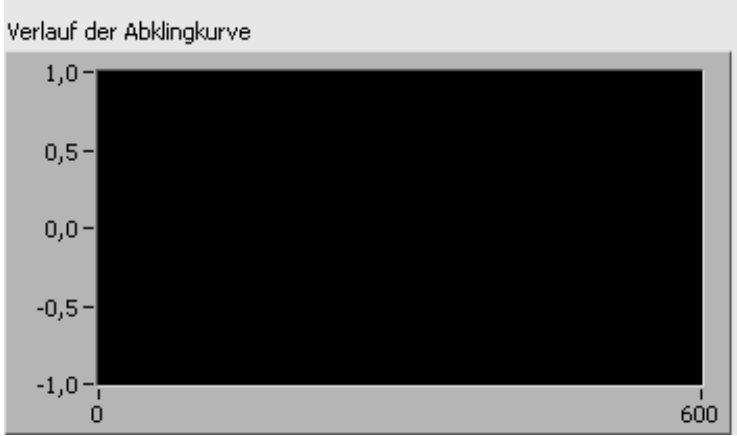

Dateiname für

die Abklingkurve

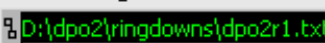

Frequenz

0,0000

Amplitude

0,000000

$\begin{array}{lll}\text { Zeit } & \text { Dämpfung Q-1 } \\ 0,00 & 0,00\end{array}$

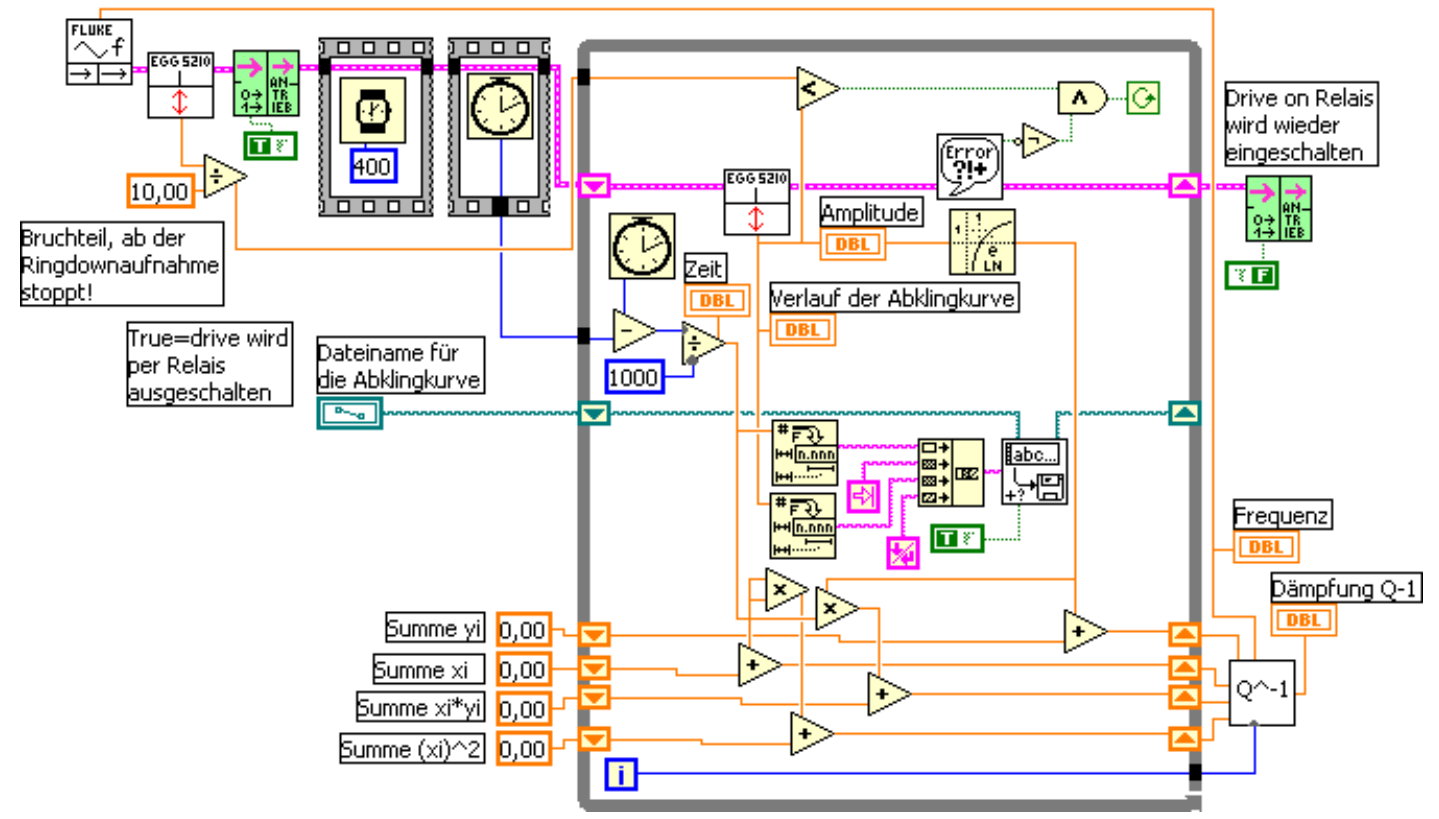

Nun wird die Amplitude gemessen, ebenfalls ausgegeben und in der zweiten Spalte der Datei gespeichert. Aus Zeit und Amplitude werden nach der Methode der kleinsten Quadrate die Dämpfungskoeffizienten ermittelt.

\section{ringdownsseveral.vi}

Das Programm ringdownseveral.vi steht auf der höchsten Hierarchie-Ebene und wird nirgends als Unterprogramm verwendet. Es dient dazu, bei einer festen Frequenz und Temperatur den Dämpfungsfaktor aus mehreren Ring-Down-Experimenten zu bestimmen. Manuell werden die am PAR-Lock-In-Verstärker eingestellte Filterfrequenz, und die tatsächliche Drive-Amplitude (Ausgangssignal des PAR-Lock-In-Verstärkers) eingegeben. Diese Werte werden im Programm nicht verwendet, sie werden jedoch in der Datei mit abgelegt. Im Feld „Anzahl Wiederholungen“ wird die Anzahl der hintereinander ausgeführten Ring- 
Down-Experimente festgelegt. Die Daten werden in zwei unterschiedlichen Dateien gespeichert. In die erste Datei (innerhalb der while-Schleife) werden die Ring-Down-Daten in die Spalten Zeit und Amplitude geschrieben. Die zweite Datei hinterlegt für jeden Programmdurchlauf nur eine Zeile, bestehend aus den Daten Temperatur, Filterfrequenz, Drive-Amplitude, Startamplitude des Signals, Phasenverschiebung zwischen Anregung und Signal, Resonanzfrequenz, Mittelwert des Dämpfungsfaktors, Standardabweichung des Dämpfungsfaktors.

\section{Ring-Down Aufnahme}

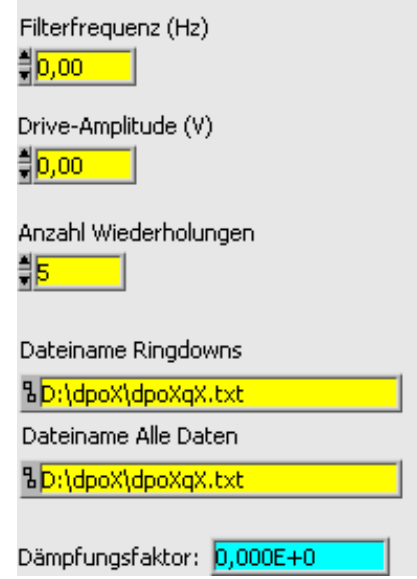
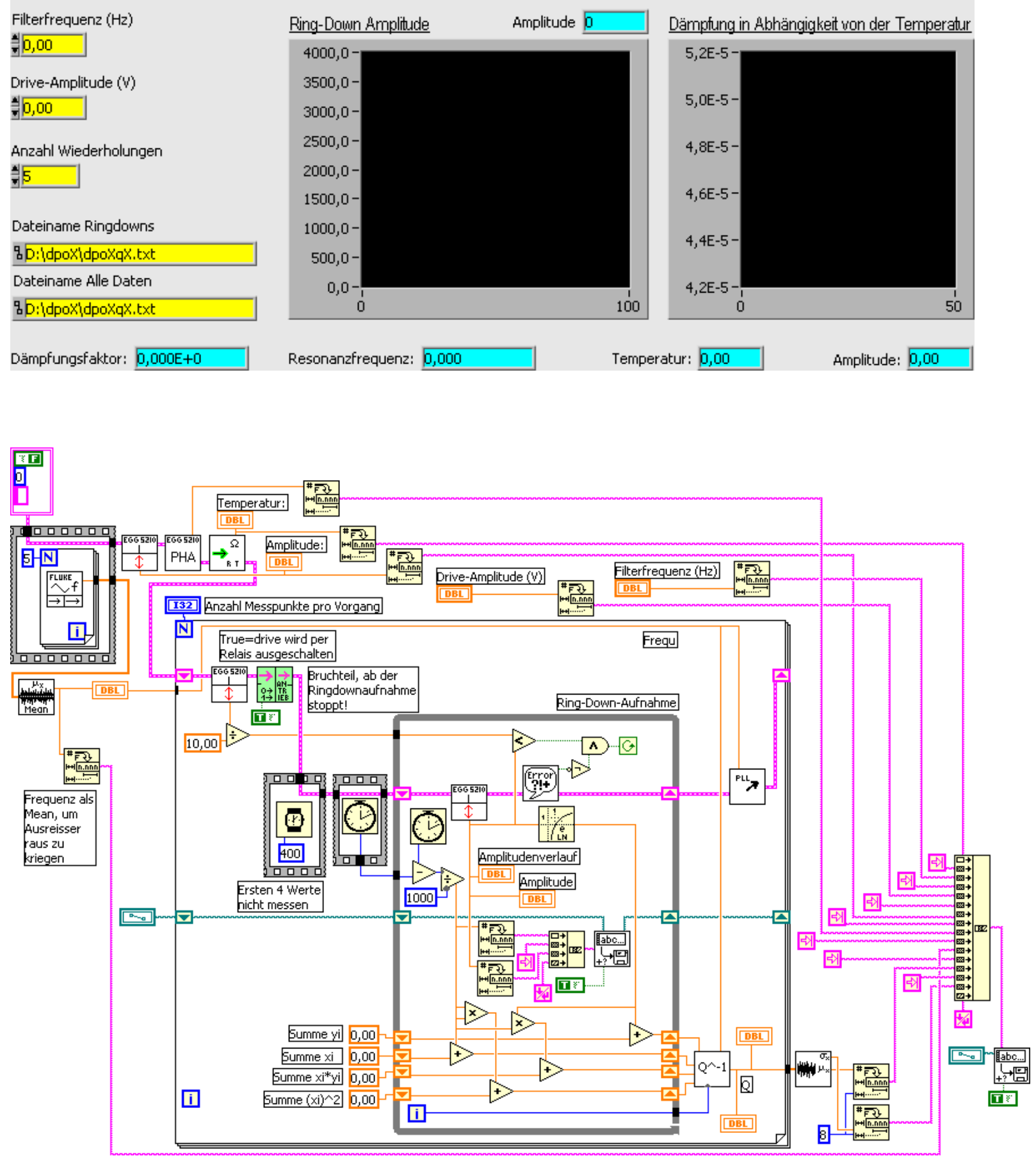
Je besser die Statistik für den Dämpfungsfaktor sein soll, desto mehr Messungen müssen durchgeführt werden. Das Programm eignet sich besonders für die Untersuchung des Dämpfungsfaktors, oder der Signalamplitude in Abhängigkeit von der Drive-Amplitude, der eingestellten Filterfrequenz oder zum Verfolgen der zeitlichen Stabilität.

Bevor die Ring-Down-Experimente starten wird fünf mal die Frequenz gemessen. Es hat sich gezeigt, dass in unregelmäßigen Abständen der Frequenzzähler Abweichungen von etwa einem Hertz liefert. Solche Fehler werden durch die Verwendung des Mittelwertes aus fünf Messungen reduziert. Der Mittelwert wird gespeichert und dient im weiteren Programmverlauf zum selbständigen Wiederanregen des PLL.

Die große For-Next-Schleife wird so oft durchlaufen, wie Ring-Down-Experimente durchgeführt werden sollen. In jedem Durchlauf wird der Dämpfungskoeffizient ermittelt. Ist die Schleife beendet, so wird von den als Array an der Schleifengrenze zur Verfügung gestellten Dämpfungsfaktoren Mittelwert und Standardabweichung ausgerechnet. Diese werden mit jeweils achtstelliger Genauigkeit gespeichert.

Ein einzelner Durchlauf der For-Next-Schleife lässt sich anhand der Fehlerlinie in die folgenden Elemente gliedern:

- Messung der aktuellen Signalamplitude bei eingeschwungenem und laufendem PLL. Fällt später im Ring-Down-Experiment die Amplitude auf den zehnten Teil dieses Ausgangswertes, so stoppt die Messung.

- Abschalten des Drive-Signals. Der DPO befindet sich nun in freier Schwingung.

- Nach der Wartezeit von 400 ms sind Einschwingvorgänge abgeschlossen.

- Ermitteln der aktuellen Systemzeit. Alle späteren Zeiten während der Aufnahme der Abklingkurve werden von dieser Startzeit subtrahiert.

- Aufnahme der Ring-Down-Daten in einer While-Schleife mit anschließender Berechnung des Dämpfungskoeffizienten nach der statistischen Methode der kleinsten quadratischen Abweichung.

- Wieder anregen des PLL. 


\section{LinRegQ.vi}
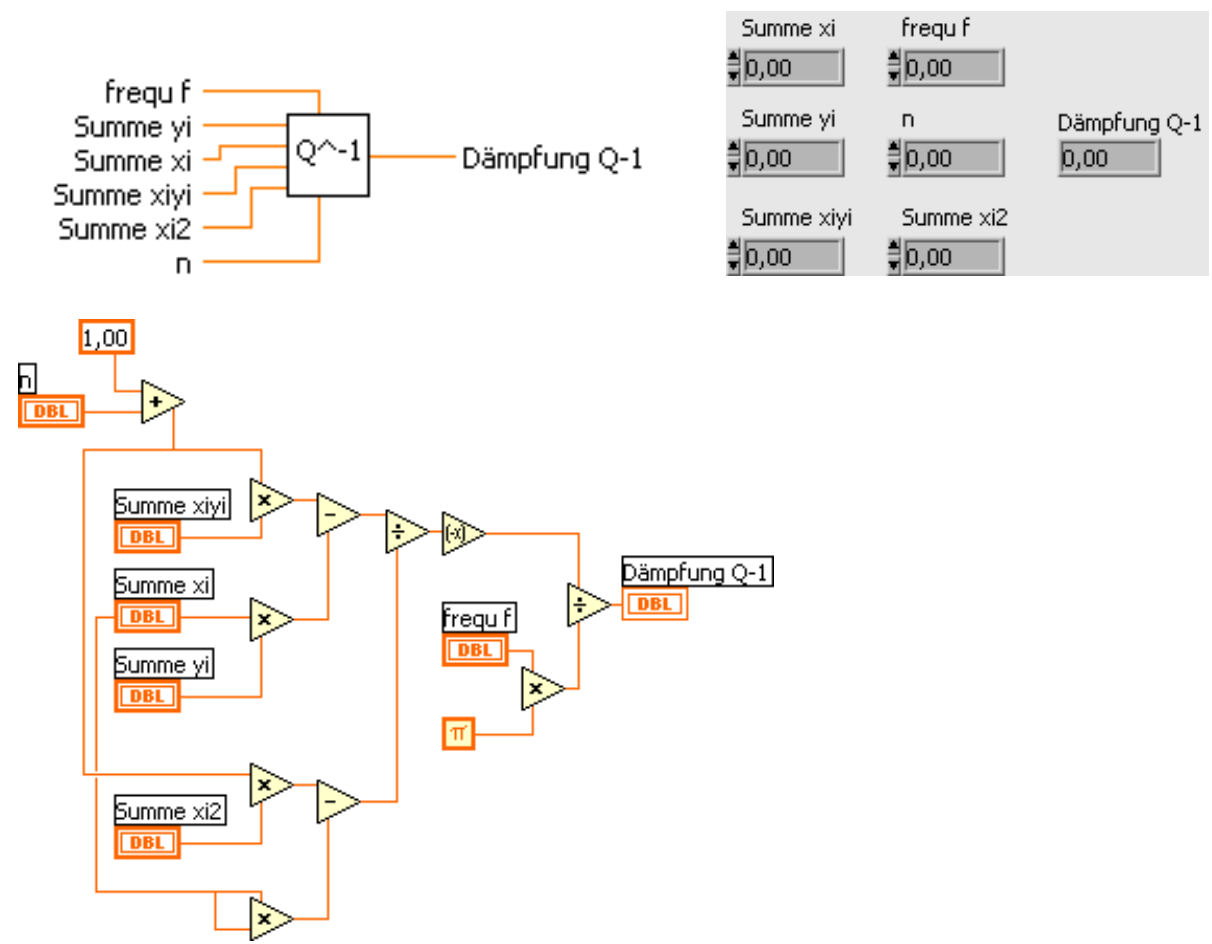

\section{Mathematische Beschreibung der Ring-Down-Experimente}

Der während den Ring-Down-Experimenten gemessene Verlauf der Schwingungsamplituden folgt mathematisch einer abklingenden Exponentialfunktion, die sich durch

$$
U(t)=U_{0} \cdot e^{-t / \tau}
$$

beschreiben lässt. Dabei ist $\mathrm{U}(\mathrm{t})$ die Signalamplitude, $\mathrm{U}_{0}$ die Amplitude zum Zeitnullpunkt, $\tau$ die Abklingkonstante und $\mathrm{t}$ die aktuelle Zeit. Aus dem Abklingkoeffizienten $\tau$ lässt sich der Gütefaktor $\mathrm{Q}^{-1}$ wie folgt berechnen:

$$
Q^{-1}=\frac{1}{\tau \cdot f_{0} \cdot \pi}
$$

$\mathrm{f}_{0}$ ist dabei die Resonanzfrequenz der Schwingung. In den Experimenten werden $\mathrm{U}(\mathrm{t})$ und $\mathrm{t}$ gemessen. Logarithmiert Gleichung (A1.1) so ergibt sich mathematisch eine Gerade mit negativer Steigung:

$$
\ln U(t)=\ln U_{0}-\ln \frac{1}{\tau} \cdot t
$$

Substituiert man $y \equiv \ln U(t), A \equiv \ln U_{0}, B \equiv \ln \frac{1}{\tau}$ und $x \equiv t$ so erhält man die Form 


$$
y=A+B x
$$

\section{Bestimmung der Parameter nach der Methode der kleinsten Quadrate}

Das Ziel ist nun, genau diejenige Gerade $y=A+B x$ zu finden, die am Besten zu einer Menge von zusammengehörigen Messwerten $\left(\mathrm{x}_{\mathrm{i}}, \mathrm{y}_{\mathrm{i}}\right)$ passt. Vereinfachend sei angenommen, dass zwar die Messwerte für y (Signalamplitude) fehlerbehaftet sind, aber die Unsicherheit der gemessenen $\mathrm{x}$-Werte (Zeit) vernachlässigbar klein ist. Das ist nicht nur einfacher, sondern auch zulässig, da die Unsicherheiten in der Amplitudenmessung viel größer sind, als die der Zeitmessung. Ferner sei angenommen, dass die statistischen Fehler für alle Messwerte y gleich groß sind.

Wenn A und B bekannt wären, dann ließe sich zu jedem Wert $x_{i}$ den wahren Wert von $y_{i}$ mit Gleichung (A1.4) berechnen. Würde $y_{i}$ sehr oft gemessen, so wären die Ergebnisse normalverteilt mit dem Bestwert beim ,wahren Wert" und dem Breiteparameter $\delta y$. Die Wahrscheinlichkeit dafür, den beobachteten Wert $y_{i}$ tatsächlich $z u$ erhalten folgt dann aus der Normalverteilung (in Abhängigkeit der als bekannt vorausgesetzten A und B):

$$
P_{A, B}\left(y_{i}\right) \propto \frac{1}{\delta y} \exp \left[\frac{-\left(y_{i}-A-B x_{i}\right)^{2}}{2(\delta y)^{2}}\right]
$$

Die Wahrscheinlichkeit, nicht nur einen Wert $\mathrm{y}_{\mathrm{i}}$ so $\mathrm{zu}$ erhalten, sondern tatsächlich alle beobachteten Werte in Abhängigkeit von ihrem jeweiligen $\mathrm{x}_{\mathrm{i}}$-Wert, ergibt das Produkt der Einzelwahrscheinlichkeiten, das in der Exponentialfunktion dann zu einer Summe wird:

$$
P_{A, B}\left(y_{1} \ldots y_{n}\right)=P_{A, B}\left(y_{1}\right) \cdots P_{A, B}\left(y_{n}\right) \propto \frac{1}{(\delta y)^{n}} \exp \left[-\frac{\chi^{2}}{2}\right]
$$

dabei ist

$$
\chi^{2}=\sum_{i=1}^{n} \frac{\left(y_{i}-A-B x_{i}\right)^{2}}{(\delta y)^{2}}
$$

Nun liegt es auf der Hand, dass die Bestwerte für die unbekannten Parameter A und B diejenigen sind, für die die Wahrscheinlichkeit, alle y-Werte wie beobachtet zu erhalten, maximal ist. Soll die Gesamtwahrscheinlichkeit maximal sein, muss $\chi^{2}$ minimal werden, die partiellen Ableitungen nach den Parametern A und B also gleichzeitig null sein. 


$$
\begin{gathered}
\frac{\partial \chi^{2}}{\partial A}=\left(-\frac{2}{(\delta y)^{2}}\right) \sum_{i=1}^{n}\left(y_{i}-A-B x_{i}\right)=0 \\
\frac{\partial \chi^{2}}{\partial B}=\left(-\frac{2}{(\delta y)^{2}}\right) \sum_{i=1}^{n} x_{i}\left(y_{i}-A-B x_{i}\right)=0
\end{gathered}
$$

Zur Vereinfachung der Schreibweise werden die Summenindizes im Folgenden weggelassen. Es ergeben sich Gleichungssystem für A und B:

$$
\begin{gathered}
A n+B \sum x_{i}=\sum y_{i} \\
A \sum x_{i}+B \sum x_{i}^{2}=\sum x_{i} y_{i}
\end{gathered}
$$

Die Lösung dieses Gleichungssystems liefert die Schätzwerte für die Parameter A und B nach der Methode der kleinsten Quadrate:

$$
\begin{gathered}
A=\frac{\left(\sum x_{i}^{2}\right)\left(\sum y_{i}\right)-\left(\sum x_{i}\right)\left(\sum x_{i} y_{i}\right)}{n\left(\sum x_{i}^{2}\right)-\left(\sum x_{i}\right)^{2}} \\
B=\frac{n\left(\sum x_{i} y_{i}\right)-\left(\sum x_{i}\right)\left(\sum y_{i}\right)}{n\left(\sum x_{i}^{2}\right)-\left(\sum x_{i}\right)^{2}}
\end{gathered}
$$

\section{Bestimmung von $\mathbf{Q}^{-1}$}

Im vorliegenden speziellen Fall ist die Konstante A nicht von Interesse, sehr wohl jedoch die Konstante B. Deshalb werden während der Aufnahme der Ring-Down-Parameter die in B eingehenden Summen direkt berechnet. Nach Beendigung des Experiments werden sie dem Unterprogramm LinRegQ.vi übergeben, das daraus nach dem angegebenen Algorithmus den Dämpfungsfaktor $\mathrm{Q}^{-1}$ bestimmt und ausgibt. $\mathrm{Zu}$ Beachten ist, dass die WhileSchleife beim Zählen mit der ,,null“ beginnt. Um dem Unterprogramm für die Berechnung die richtige Anzahl an Wertepaaren zu übergeben, wird zu n noch die Zahl 1 hinzuaddiert.

\section{OmegaTlesen.vi}

Dieses Unterprogramm liest die aktuelle Prozesstemperatur vom Omega Temperaturregler ein und gibt sie an übergeordnete Programme weiter. Die Grammatik für den seriellen Code ist relativ kompliziert. Der auf den Regler geschriebene Befehl „\$0101R05C1“ bewirkt das Bereitstellen der Ist-Temperatur am seriellen Ausgang. Allerdings dauert es eini- 
ge Millisekunden, bis tatsächlich der volle Ausgabestring bereitsteht. Eine Wartezeit von $100 \mathrm{~ms}$ bis zum Lesevorgang hat sich in allen Fällen als ausreichend erwiesen. Mit Hilfe einiger stringverarbeitenden Module wird schließlich der Zahlwert für die Temperatur extrahiert und als DBL ausgegeben. Nach einem Neustart des Computers müssen unbedingt die Parameter der seriellen Schnittstelle überprüft werden: baud rate $=9600$, data bits $=8$, parity $=$ none, stop-bits $=1$, flow control $=$ none. Nach Verstellen dieser Parameter ist ein Neustart ebenso nötig, wie ein anschließendes Ein- und Ausschalten des Reglers.
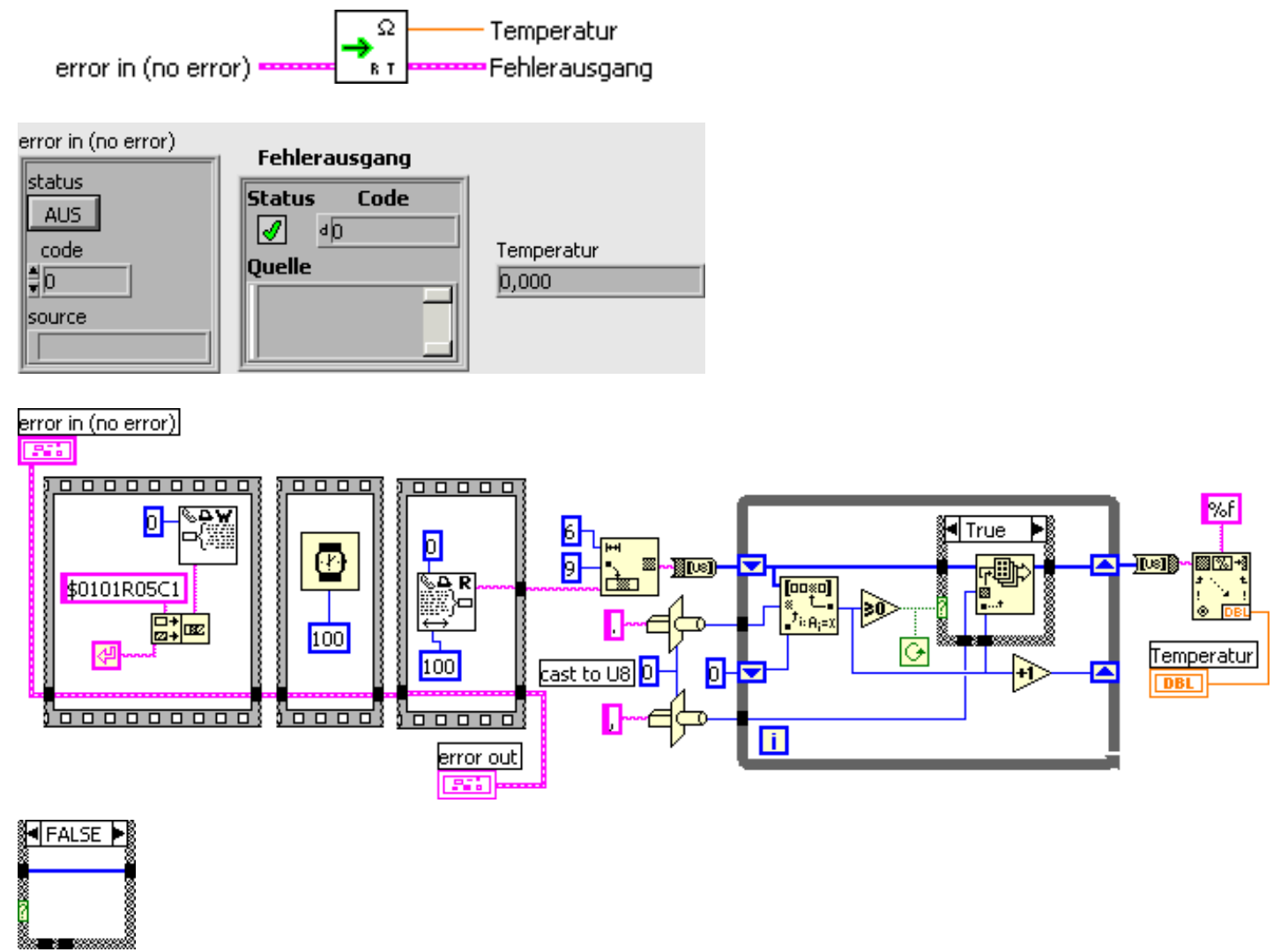

\section{OmegaTwrite.vi}
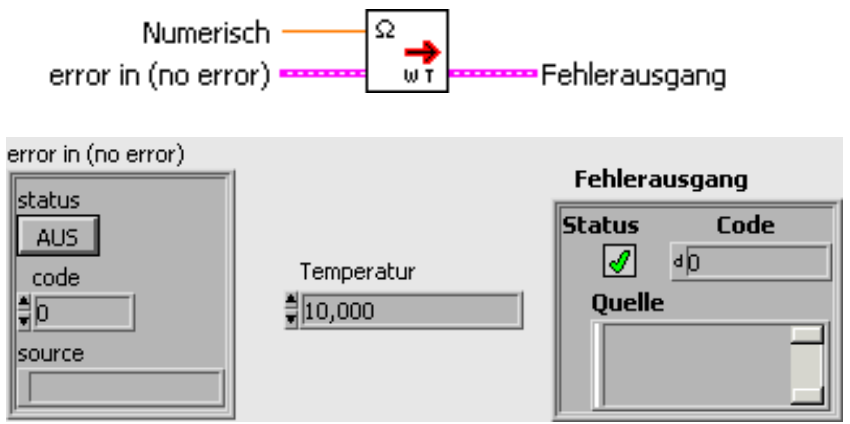

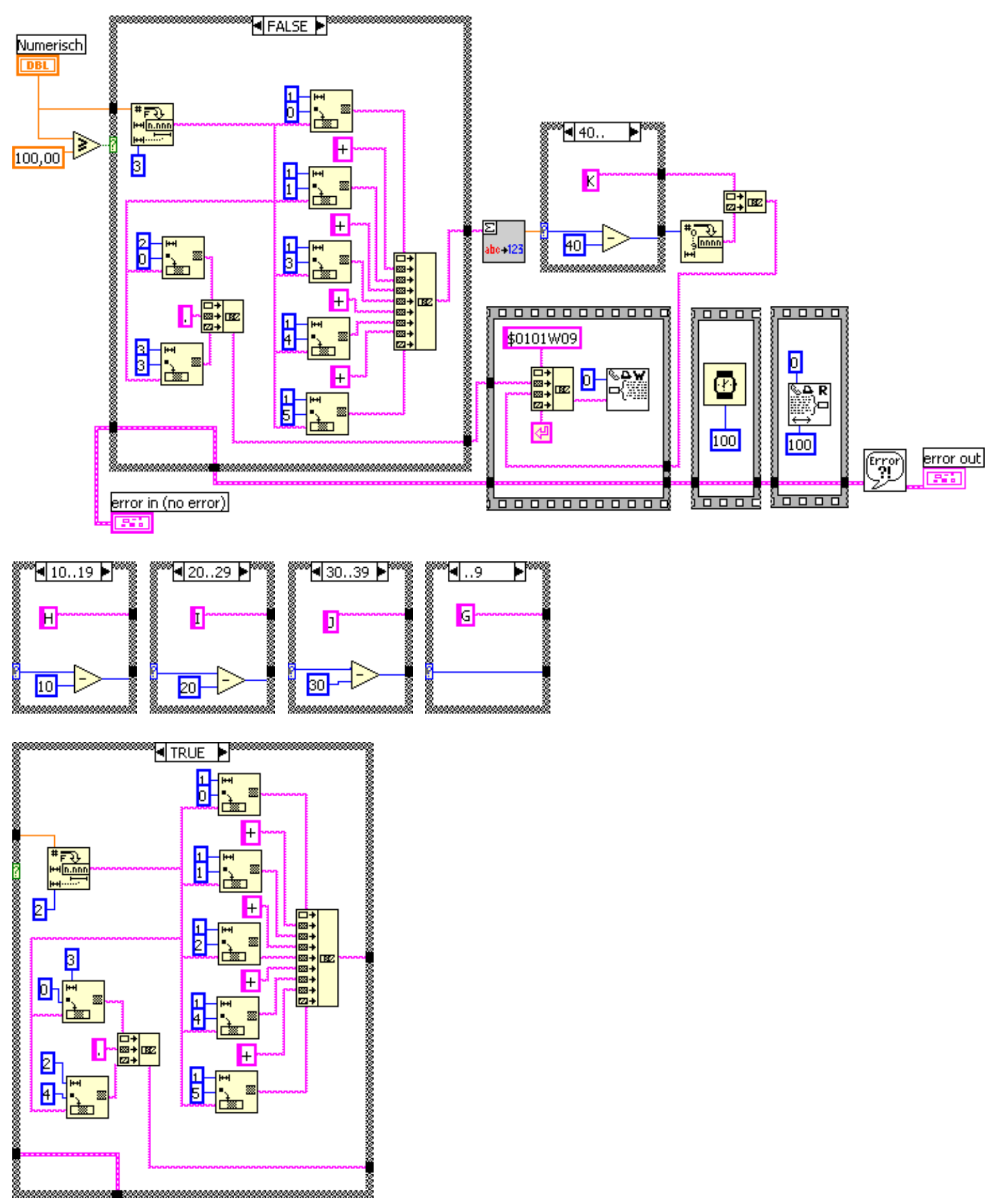

Wieder stellt sich die Aufgabe als vermeintlich leicht dar, nämlich dem Omega-Temperaturregler eine neue Soll-Temperatur mitzuteilen. Allerdings erfordert die Programmierung einige Tricks. Der Kern des Programms besteht aus den drei letzten Sequenzanweisungen, wenn man dem Fehlerstrang folgt. In der drittletzten Anweisung wird der eigentliche Befehl „\$0101W09“ mit dem String kombiniert, der die Temperatur und die Kontrollsumme enthält und an die serielle Schnittstelle übergeben. Nach $100 \mathrm{~ms}$ Wartezeit findet man das Ergebnis im Puffer der Schnittstelle und liest es aus. Das Auslesen ist sehr wichtig, um den Puffer der Schnittstelle wieder zu leeren. Würde der Puffer nicht geleert und versucht, die 
Temperatur wieder auszulesen, so würde der neue Temperaturstring an den nicht gelöschten angehängt. Ein Verarbeiten dieses Doppelstrings ist mit den zur Verfügung stehenden Programmen nicht möglich.

Die eigentliche Kunst besteht nun darin, die als DBL-Variable vorliegende Temperatur in einen String mit dem zugehörigen korrekten Kontrollparameter umzuwandeln. Zunächst wird unterschieden, ob die Temperatur zwei- oder drei Stellen vor dem Komma hat. Ist sie kleiner $100{ }^{\circ} \mathrm{C}$, so finden sich zwei Ziffern vor und drei hinter dem Komma. Andernfalls sind drei Stellen vor und zwei hinter dem Komma. Der Kontrollparameter errechnet sich aus der Quersumme des ASCII-Codes der im Befehl vorkommenden Ziffern und setzt sich aus einem Buchstaben und einer Ziffer zusammen. Die Quersumme wird berechnet und mit der Case-Anweisung in diesen Code umgesetzt.

\section{MastermitTProg.vi}

Dieses Programm erfasst automatisch die Resonanzfrequenz und Dämpfung in Abhängigkeit von der Temperatur. Die Temperatur wird nach einem vorgegebenen Programm geregelt, das aus bis zu acht Einzelschritten bestehen kann. Für jeden einzelnen run - im Frontpanel durch einen 1x6 Array festgelegt - sind dabei folgende Variablen festzulegen:

- T Start $\left[{ }^{\circ} \mathrm{C}\right]$ ist die Starttemperatur, die von der aktuellen Prozesstemperatur ausgehend in einer ersten Rampe angefahren wird. Findet sich hier der Eintrag „,0“, so wird dieser run nicht mehr ausgeführt und das Programm endet. Die zuletzt eingestellte Temperatur wird gehalten.

- Start Rate $\left[{ }^{\circ} \mathrm{C} / \mathrm{min}\right]$ ist die Rate, mit der die Starttemperatur ausgehend von der aktuellen Prozesstemperatur angefahren wird.

- Wartezeit [min] ist die Zeitspanne zwischen Erreichen der Zieltemperatur und der Messung der Dämpfung. Diese Wartezeit ist nötig, um ein Gleichgewicht zwischen der Temperatur am Paddelhals, messbar durch die Eigenfrequenz und am Paddelblock, wo sich das Thermoelement zur Messung der Prozesstemperatur befindet, einzustellen. Diese Zeit gilt sowohl für die Wartezeit nach der ersten Rampe, als auch für die Wartezeiten jedes einzelnen Temperaturschritts innerhalb des runs. Folgende Zeiten können als Faustregel gelten: zwischen Raumtemperatur und $100{ }^{\circ} \mathrm{C}$ : 15 Minuten, zwischen $100{ }^{\circ} \mathrm{C}$ und $200{ }^{\circ} \mathrm{C}: 10$ Minuten, größer $200^{\circ} \mathrm{C}: 5$ Minuten. 
- Delta $\mathrm{T}\left[{ }^{\circ} \mathrm{C}\right]$ ist der Abstand zwischen zwei Temperaturpunkten, bei denen die Dämpfung gemessen werden soll. Z. B. alle $5{ }^{\circ} \mathrm{C}$ oder alle $2{ }^{\circ} \mathrm{C}$. Bei Aufheizkurven ist die Temperatur positiv, bei Abkühlkurven negativ zu wählen.

- Rate $\left[{ }^{\circ} \mathrm{C} / \mathrm{min}\right]$ ist die Heiz- bzw. Kühlrate zwischen den einzelnen Temperatursteps. Sowohl beim Heizen, als auch beim Kühlen muss die Rate positiv gewählt werden. Sollen große Temperaturdifferenzen zwischen Paddel und Einspannung vermieden werden, so sollte die Rate nicht größer als $0,5^{\circ} \mathrm{C} / \mathrm{min}$ sein. Bei Temperaturen oberhalb von $300{ }^{\circ} \mathrm{C}$ sollte die Rate kleiner $0,3{ }^{\circ} \mathrm{C} / \mathrm{min}$ gewählt werden.

- $\mathrm{T}$ Ende $\left[{ }^{\circ} \mathrm{C}\right]$ ist die Temperatur, bei der die letzte Messung stattfindet.

\section{Automatisches Erfassen von Resonanzfrequenz und Dämpfung in Abhängigkeit der Temperatur} Temperaturprogramm

\begin{tabular}{|c|c|c|c|c|c|c|}
\hline \multirow[b]{2}{*}{ 1. run } & \multirow{2}{*}{$\begin{array}{l}\text { T Start } \\
{\left[{ }^{\circ} \mathrm{C}\right]} \\
\sqrt[40,00]{40}\end{array}$} & $\begin{array}{l}\text { Start Rate } \\
{\left[{ }^{\circ} \mathrm{C} / \mathrm{min}\right]}\end{array}$ & $\begin{array}{l}\text { Wartezeit } \\
\text { [ min ] }\end{array}$ & \multirow{2}{*}{$\begin{array}{l}\text { Delta T } \\
{\left[{ }^{\circ} \mathrm{C}\right]}\end{array}$} & \multicolumn{2}{|c|}{$\begin{array}{ll}\text { Rate } & \text { TEnde } \\
{\left[{ }^{\circ} \mathrm{C} / \text { min }\right]} & {\left[{ }^{\circ} \mathrm{C}\right]}\end{array}$} \\
\hline & & $\sqrt[4]{1,00}$ & $\sqrt[*]{15,00}$ & & $\sqrt[0,50]{0,0}$ & $\sqrt{100,00}$ \\
\hline 2. run & $\sqrt[*]{105,00}$ & $* 0,50$ & $|*| \frac{10,00}{10,0}$ & : 5,00 & 10,50 & 200,00 \\
\hline 3. run & $\leqslant 205,00$ & $\approx 0,50$ & 8,00 & : 5,00 & $\leqslant 0$ & * 300,00 \\
\hline & $\leqslant 302,00$ & $* 0,30$ & $|*| \sqrt{5,00}$ & $\leqslant 2,00$ & $\because 0,30$ & 340,00 \\
\hline 'un & 338,00 & $\approx 0,30$ & $\leqslant 5,00$ & $\sqrt{-2,00}$ & $\leqslant 0,30$ & 300,00 \\
\hline & 295,00 & 0,50 & $\approx 8,00$ & 粘 $-5,00$ & 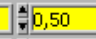 & 200,00 \\
\hline & $\sqrt[4]{195,00}$ & $\approx 0,50$ & $=10,00$ & : $-5,00$ & $\div 0,50$ & $\sqrt[*]{100,00}$ \\
\hline & $\approx 95,00$ & 0,50 & $\sqrt[*]{15,00}$ & : $-5,00$ & 50 & 40,00 \\
\hline
\end{tabular}
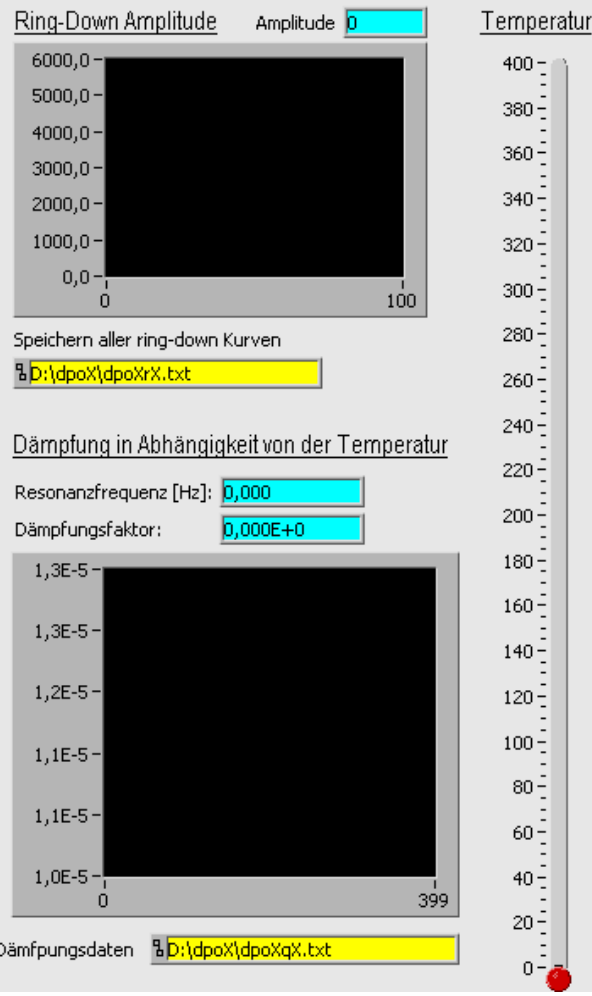

Bei allen einzugebenden Temperaturen ist darauf $\mathrm{zu}$ achten, dass dies Temperaturen sind, die am Paddelblock gemessen werden. Die tatsächliche Temperatur am Paddelkopf ist deutlich größer und kann nach Gleichung (4.1) berechnet werden.

Gemeinsam sind allen runs folgende Parameter:

- Wartezeit pro Messung [s]: Werden mehrere ring-down Kurven hintereinander aufgenommen um Mittelwert und Standardabweichung ermitteln zu können, so muss jedes Mal der Phase-Lock-Loop (PLL) wieder angeregt werden. Je nachdem, wie groß der 
Dämpfungsfaktor ist, dauert es eine gewisse Zeit, bis die Schwingung wieder vollständig angeregt ist. So sollte bei $\mathrm{Q}^{-1}$ von $10^{-5}$ die Wartezeit etwa $30 \mathrm{~s}$ betragen.

- Anzahl Messungen pro T-Step: Um gesichertere Messwerte zu bekommen, werden pro eingeregelter Temperatur mehrere ring-down-Kurven aufgenommen.

Die Daten werden in drei verschiedenen .txt Dateien gesichert:

- dpoXfTtX.txt: Der Verlauf der Frequenz und der Temperatur mit der Zeit wird sowohl während der Rampen, als auch während der Einregelzeit protokolliert. Die Speicherung übernimmt das SubVI fTtschreiben.vi. In den drei Spalten der Tabelle steht zuerst die Zeit in Sekunden vom Programmstart an gerechnet, dann die gemessene Resonanzfrequenz $(\mathrm{Hz})$ gefolgt von der Temperatur $\left({ }^{\circ} \mathrm{C}\right.$ am Block).

- dpoXrX.txt: Zur nachträglichen Auswertung und Überprüfung werden alle ring-downKurven in ihren Originaldaten aufgezeichnet. Die Tabelle hat zwei Spalten, Zeit (s) und Amplitude.

- dpoXqX.txt: Die eigentlichen Dämpfungsmessungen werden statistisch zusammengefasst und in eine Tabelle mit sechs Spalten geschrieben: Temperatur $\left({ }^{\circ} \mathrm{C}\right.$ am Block), Resonanzfrequenz (Hz), größter Messwert, kleinster Messwert, Mittelwert und Standardabweichung der gemessenen Dämpfungskoeffizienten.

Um das Blockdiagramm zu verstehen, folgt man am Einfachsten dem Weg, den die roten Fehlerverbindungen vorgeben. Sie führen zu einer Linearisierung der Programmschritte, da der jeweils nächste erst begonnen wird, wenn der vorhergehende abgeschlossen ist und „kein Fehler“" weitergibt.

Das Blockdiagramm ist von einer umfassenden For-Next-Schleife dominiert, in denen die einzelnen runs ausgeführt werden. Die Anzahl der Schleifendruchläufe bestimmt sich aus der Anzahl der T-Start-Variablen im Temperatur-Programm Block, die nicht gleich null sind. Sollen beispielsweise drei runs nacheinander ausgeführt werden, so würde die große Schleife dreimal durchlaufen. Um die Anzahl der Durchgänge zu bestimmen, überprüft eine kleine While-Schleife den Temperatur-Programm-Array auf den ersten Eintrag von TStart der gleich null ist. Die Anzahl i, wie oft diese Schleife durchlaufen wurde ist mit N der großen For-Next-Schleife verbunden. Zu beachten ist, dass das Zählen bei 0 beginnt. Soll die große Schleife also dreimal durchlaufen werden, so wäre $\mathrm{N}=2$. 

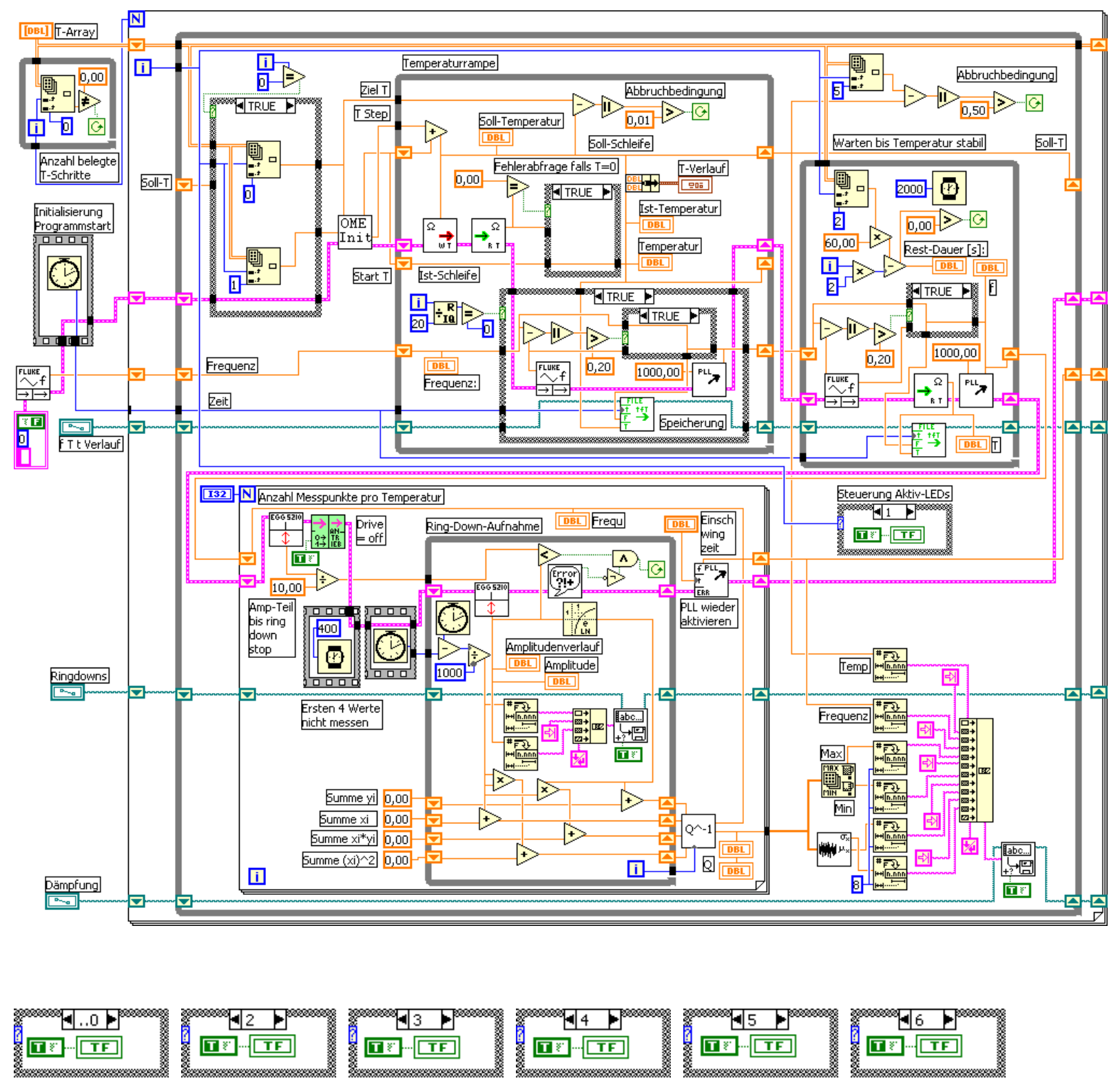

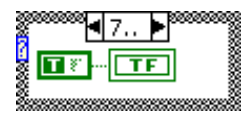

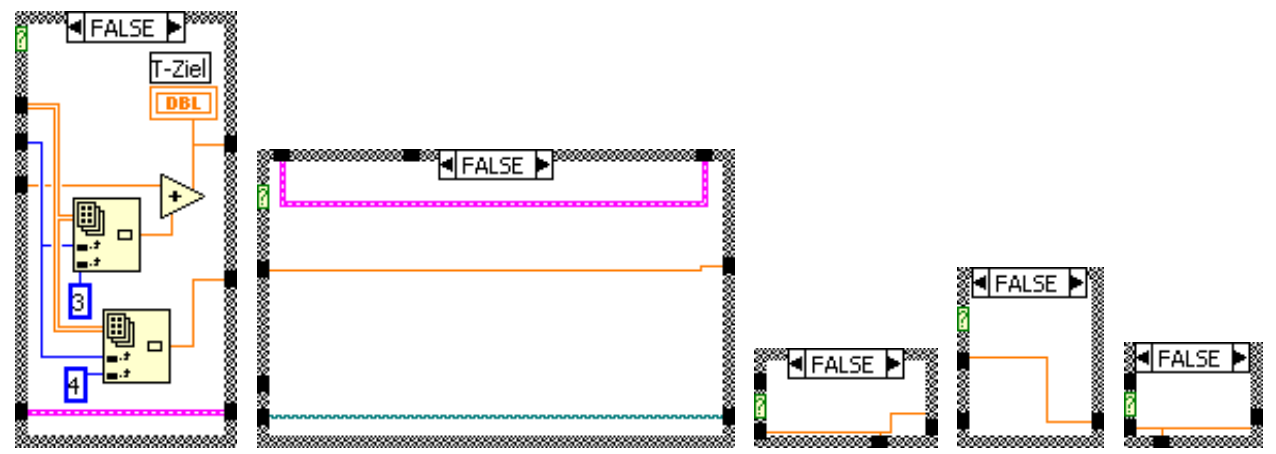


Außerhalb der Schleife befinden sich eine erstmaligen Frequenzmessung, die Dateinamen für die Datensicherung und der Start-Schalter der Stoppuhr des Programms. Von dem hier in die Schleife eingeführten Zeitwert werden alle späteren subtrahiert, so dass man ein fortlaufendes Zeitsignal erhält.

Innerhalb der großen For-Next-Schleife befindet sich sofort eine große While-Schleife. In dieser Schleife wird ein einzelner run abgearbeitet. Die wichtigsten Eingänge sind die aktuelle Zahl i der großen For-Next-Schleife (sie enthält den run, der gerade behandelt wird) und natürlich der Array für die Temperatursteuerung mit den eingegebenen Daten. Die erste Case-Abfrage überprüft, ob die Schleife zum ersten Mal angesprochen wird. In diesem Fall ist i der While-Schleife null und als erste Zieltemperatur und erste Rate werden aus dem Temperatur-Programm-Array „T Start“ und „Start Rate“ des entsprechenden runs ge-

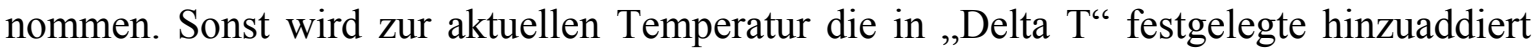
und die Rate ausgegeben, die bei „Rate“ für den run festgelegt wurde. Die große WhileSchleife endet, falls die Temperatur bis auf $0,5^{\circ} \mathrm{C}$ an die festgelegte Endtemperatur herangekommen ist.

Das Innere der großen While-Schleife besteht aus drei Blöcke mit folgenden Aufgaben:

- Zunächst stellt der Temperaturregler in Form der programmierten Rampe die Zieltemperatur ein. Grafisch dargestellt wird die Soll- und die Ist-Temperatur.

- Ist die Zieltemperatur erreicht, wartet die nächste Schleife, bis die Temperatur stabil ist. Zur Kontrolle wird die gemessene Frequenz alle zwei Sekunden aufgezeichnet.

- Schließlich werden die ring-down-Kurven aufgenommen. Dabei wird nach der oben dargestellten Methode der kleinsten Quadrate eine Anpassung an eine abfallende Exponentialkurve vorgenommen. Statistisch ausgewertet werden sie schließlich in die Datei geschrieben.

\section{Beschreibung der einzelnen Programmteile}

\section{Temperaturrampe}

Eingänge für die While-Schleife sind die Zieltemperatur, die eingestellt werden soll, die Temperaturschritte, in denen gesteigert werden soll und die Systemzeit. Mit drei Schieberegistern werden die jeweils aktuellen Prozessparameter verfolgt: Die Soll-Temperatur, die Ist-Temperatur und die Frequenz. 
Ein einzelner Schleifen-Durchgang besteht aus folgenden Schritten:

Zunächst wird zur bisherigen Soll-Temperatur ein T-Step addiert, was die neue Soll-Temperatur ergibt. Diese wird dem Omega-Regler übergeben. Danach wird die aktuelle IstTemperatur vom Omega-Regler eingelesen und beide Werte grafisch im Diagramm dargestellt. Aus ungeklärten Gründen liefert der Omega-Regler für die Ist-Temperatur in seltenen Fällen den Wert null. In diesem Fall (obere Case-Anweisung True) wird der vorhergehende Ist-Wert übernommen. Falls ein realistischer Temperatur-Ist-Wert kommt (Case-Anweisung False) wird dieser Wert als neuer Ist-Wert dem Schieberegister übergeben.

Alle 20 Temperatur-Steigerungsschritte (untere Case-Anweisung True) wird die Frequenz gemessen und die Prozessdaten in die fTt-Datei geschrieben. In dieser Case-Anweisung wird auch überprüft, ob die Amplitude des PLL noch groß genug ist. Wird die Amplitude zu klein, so meldet der PAR-Lock-In einen ,unlocked“ Status und der PLL fällt aus. Also wird ein Unterprogramm zwischengeschalten, das die Schwingung noch einmal neu per Frequenzgenerator anregt. Auch der Frequenzmesser liefert in unregelmäßigen Abständen „falsche“ Werte, die mehrere Hertz daneben liegen können. (Interessanterweise gibt es keine Fehlerwerte, die nur ganz knapp daneben liegen). Deshalb wird der gerade gemessene Frequenzwert mit dem vorherigen verglichen. Liegt er mehr als 0,2 Hz daneben, so ist er per definitionem falsch und der bisherige Wert wird nochmals dem Schieberegister übergeben.

\section{Warten bis die Temperatur stabil ist}

Während des Heizens und Kühlens treten Temperaturabweichungen zwischen dem DPO und dem Mini-Schraubstock auf, in dem das Paddel eingespannt ist. Findet keine Temperaturänderung mehr statt, so dauert es noch eine gewisse Zeit, bis sich ein stationäres Gleichgewicht eingestellt hat und sich die Paddelfrequenz nicht mehr ändert (was ein gutes Indiz für eine konstante Temperatur am Paddel ist). Alle zwei Sekunden wird die Temperatur vom Omega-Regler eingelesen und die Frequenz gemessen, die dann grafisch dargestellt wird. Die Werte werden in die gleiche fTt-Datei geschrieben, wie die im Programmteil Temperaturrampe ermittelten. Eingang in die While-Schleife ist die Spalte Wartezeit aus der Programm-Steuerung und die Frequenz, die auch hier einem Schieberegister übergeben wird. Auch in diesem Programmblock wird jeweils die Amplitude überprüft und ggf. das Unterprogramm PLLretten.vi aufgerufen. Die Frequenzkontrolle funktioniert wie oben. 


\section{Dämpfungsmessung}

Die For-Next-Schleife wird so oft durchlaufen wie in „Anzahl Messungen pro T-Step“ im Kontrollbereich festgelegt wurde. Die Frequenz wird im Schieberegister weitergegeben. Die Ring-down-Messung erfolgt in mehreren Schritten:

Zunächst wird die Amplitude gemessen. Hat sich später die Amplitude auf den 10ten Teil reduziert, so ist die Messung beendet. Nun wird der Antrieb ausgeschalten. Das bedeutet, die Schwingung wird sich selbst überlassen und die Amplitude sollte in Form einer Exponentialfunktion abklingen. Um elektrische Einschwingvorgänge zu kappen ist eine Wartezeit von 400 ms eingebaut, bevor die eigentliche Messung beginnt. Zum Start der Messung wird die aktuelle Systemzeit genommen, von der alle späteren Zeiten subtrahiert werden. Die nun folgende While-Schleife misst die Amplitude und die Zeit und schreibt beide in die Datei, in der alle Ring-down-Kurven gespeichert werden.

\section{Omegarampeinit.vi}

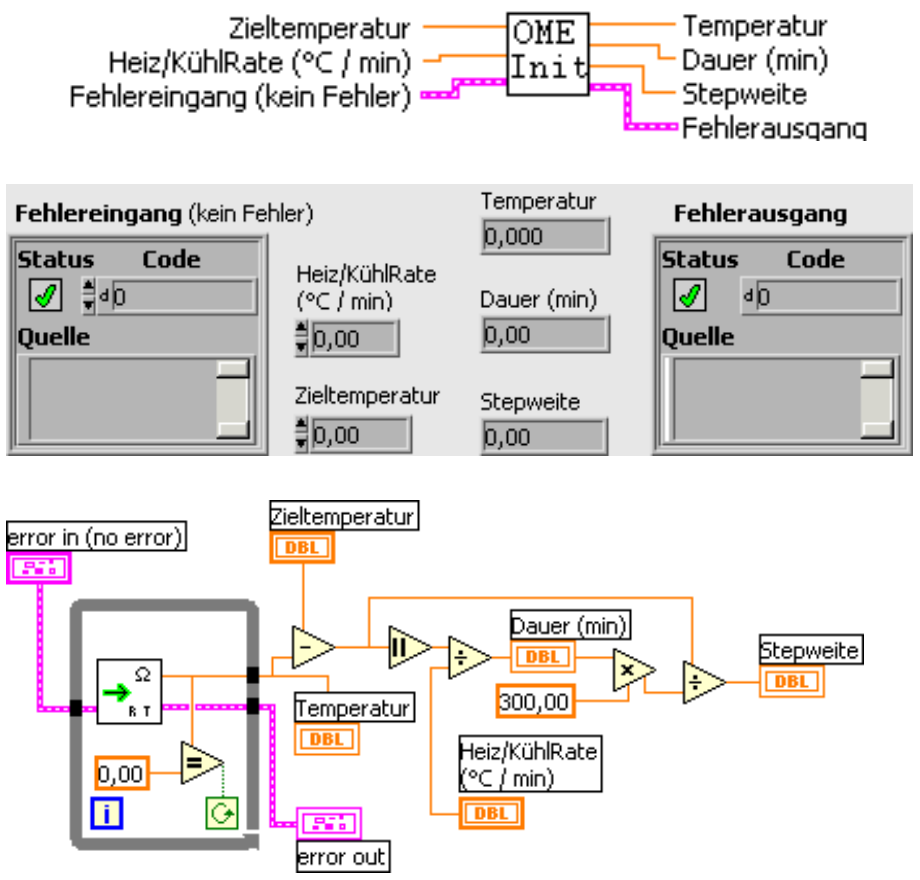

Das Unterprogramm bestimmt aus der vorgegebenen Endtemperatur und der Rate, mit der diese erreicht werden soll, die nötigen Steuerdaten für die programmierte Rampe. Zunächst wird daher die aktuelle Temperatur gemessen. Dies findet in Form einer While-Schleife statt, um fehlerhaft vorkommende Null-Ergebnisse ausschalten zu können. Aus der Diffe- 
renz zwischen Start- und Zieltemperatur und der Heiz-/Kühlrate wird die Dauer der Rampe in Minuten berechnet. Da während der späteren Rampe sowohl das Lesen der aktuellen Temperatur als auch das Einschreiben einer neuen Temperatur als Soll-Wert für den Regler jeweils $100 \mathrm{~ms}$ dauert, können pro Sekunde fünf Temperaturschritte erfolgen - pro Minute also 300. Der Rampe wird die Dauer und die Stepweite als Ausgabeparameter übergeben.

\section{fTtschreiben.vi}
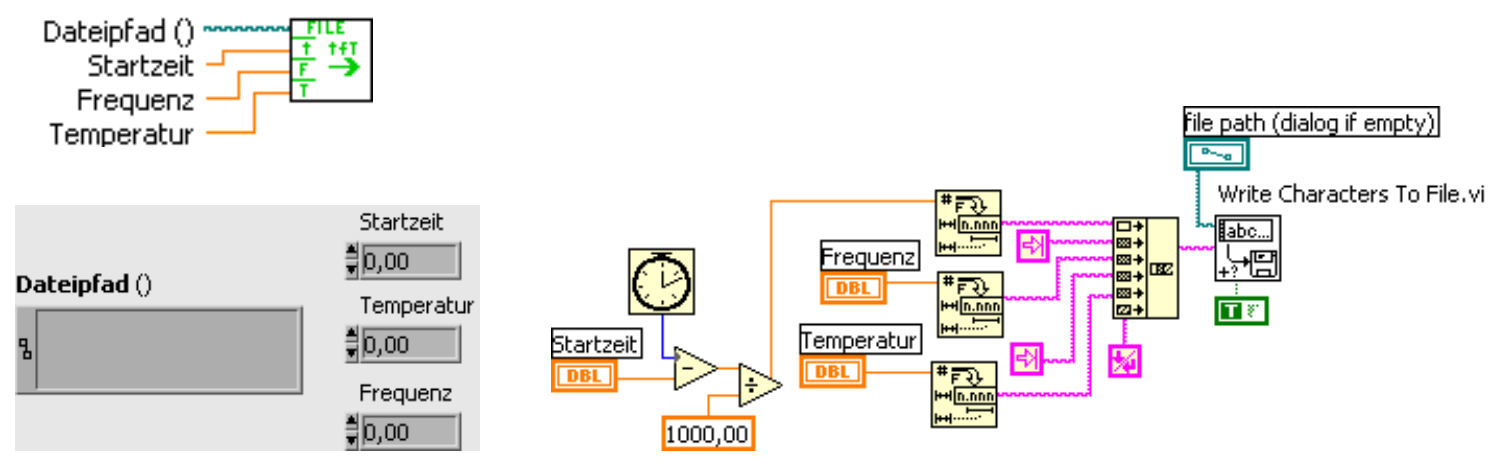

Dieses Sub-VI wird in MastermitTProg.vi eingesetzt und dokumentiert die Prozessparameter während der Temperaturrampe und der anschließenden Wartezeit. Eingabeparameter sind die Temperatur, die Frequenz und die Startzeit. Diese wird von der aktuellen Zeit subtrahiert, so dass man die vergangene Zeit seit Programmstart erhält. Diese drei Parameter werden in die entsprechende Datei geschrieben.

\section{PLLaktivieren.vi}
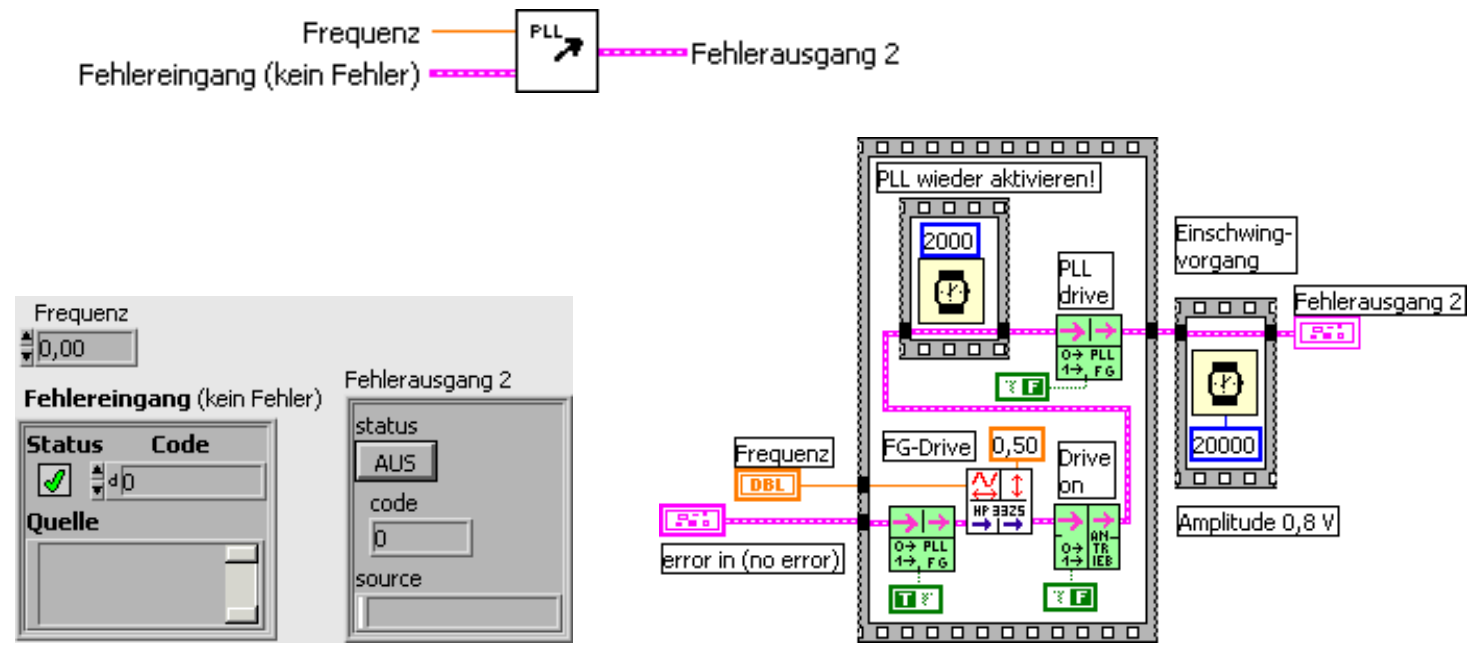
Einschwingvorgänge sorgen bei sich schnell ändernden Frequenzen (z.B. wegen schneller Temperaturänderungen) manchmal für einen rapiden Abfall der Schwingungsamplitude, so dass Gefahr besteht, den PLL zu verlieren. Fällt die Amplitude daher unter einen kritischen Wert, so wird das Programm PLLaktivieren.vi gestartet. Der Eingabeparameter Frequenz wird dem HP Frequenzgenerator einbeschrieben und die Schwingung extern neu angeregt.

Dementsprechend wird zunächst der Antrieb vom PLL auf den Funktionsgenerator umgeschalten, dann der Funktionsgenerator mit 0,5V P-P Drive-Amplitude (was bewusst hoch gewählt ist) angesprochen, der Antrieb noch einmal auf „on“ gestellt, zwei Sekunden gewartet und der PLL wieder aktiviert. Während der nächsten 20 Sekunden Wartezeit sollte sich die Schwingung komplett wieder aufgeschaukelt haben.

Dieses Programm wird auch von allen anderen Programmen verwendet, um den PLL nach erfolgtem Ring-Down-Experiment wieder neu anzuregen.

\section{PLLretten.vi}

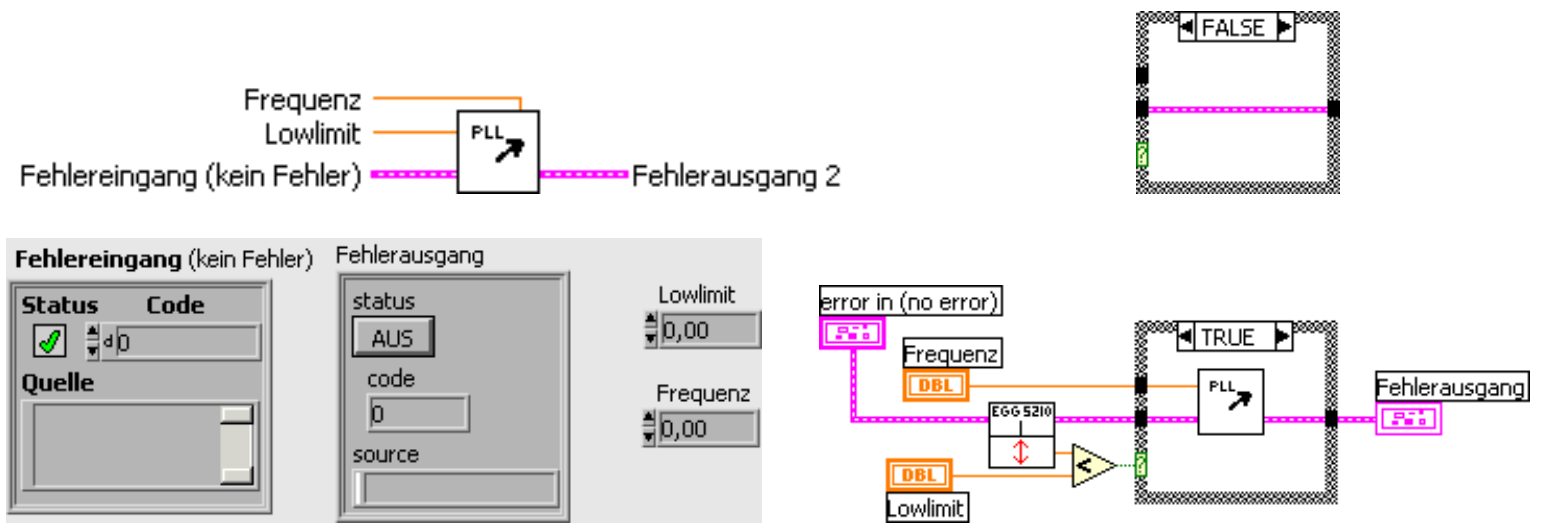

In diesem VI wird getestet, ob die Signalamplitude unter einen kritischen Wert gefallen ist (lowlimit). Falls dem so ist, wird das Sub-VI PLLaktivieren.vi aufgerufen, falls nicht, passiert nichts und das aufrufende VI läuft ganz normal weiter.

\section{driveFGPLL.vi}

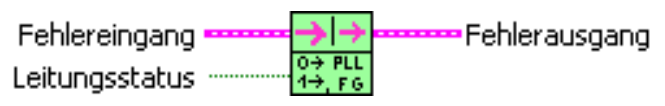



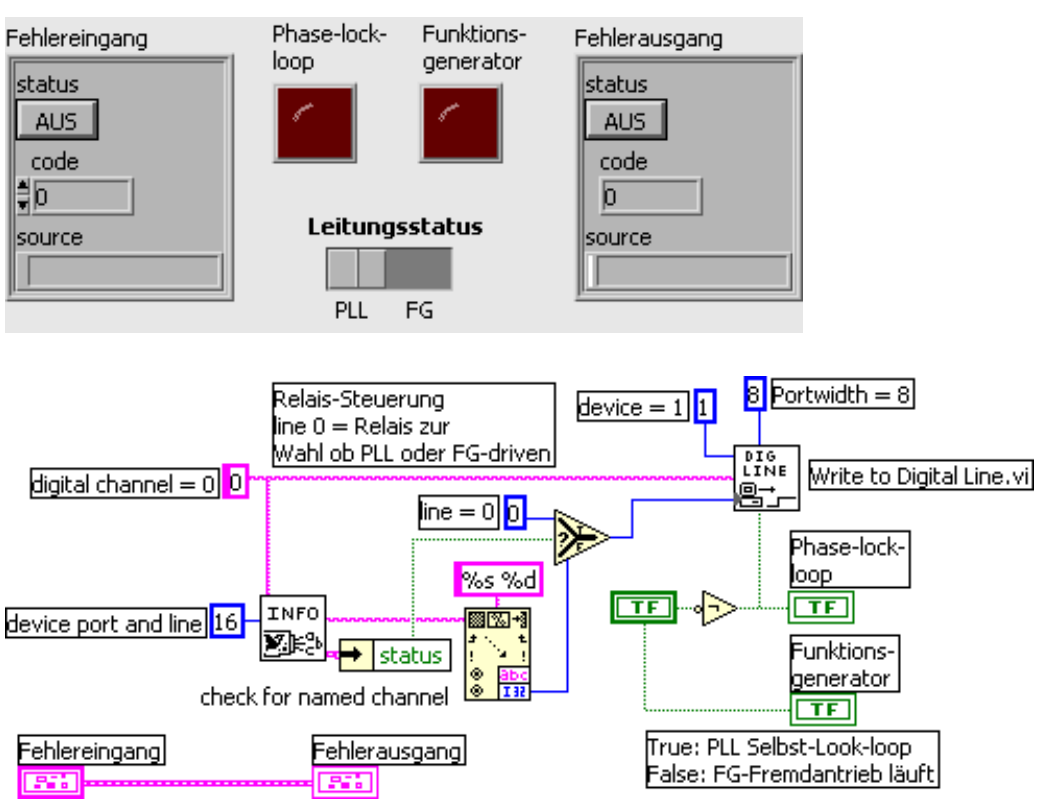

Über den Analogausgang OI $0($ line $=0)$ der seriellen Schnittstelle wird ein Relais angesprochen, das die Quelle für den Antrieb des DPOs regelt. Bei der Schalterstellung True ist der PLL aktiv, sonst der Frequenzgenerator.

\section{driveONOFF.vi}
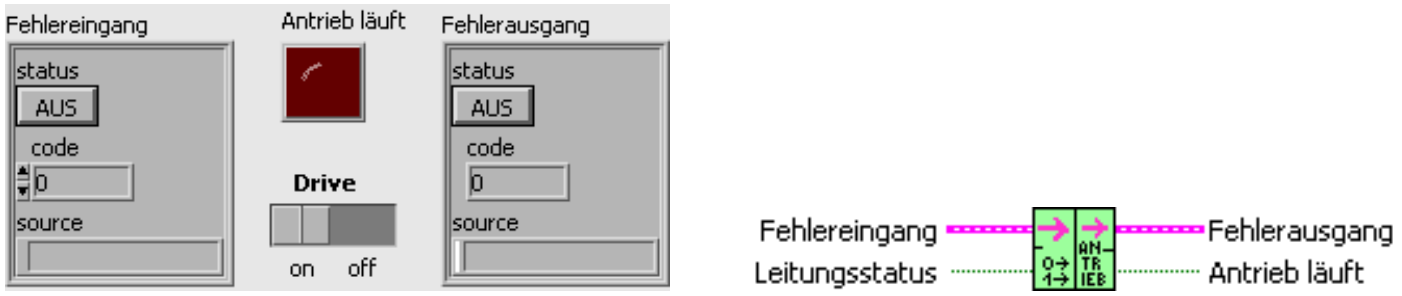

Relais-Steuerung

line 1 = Relais zur wahl ob

Freier ring down oder nicht

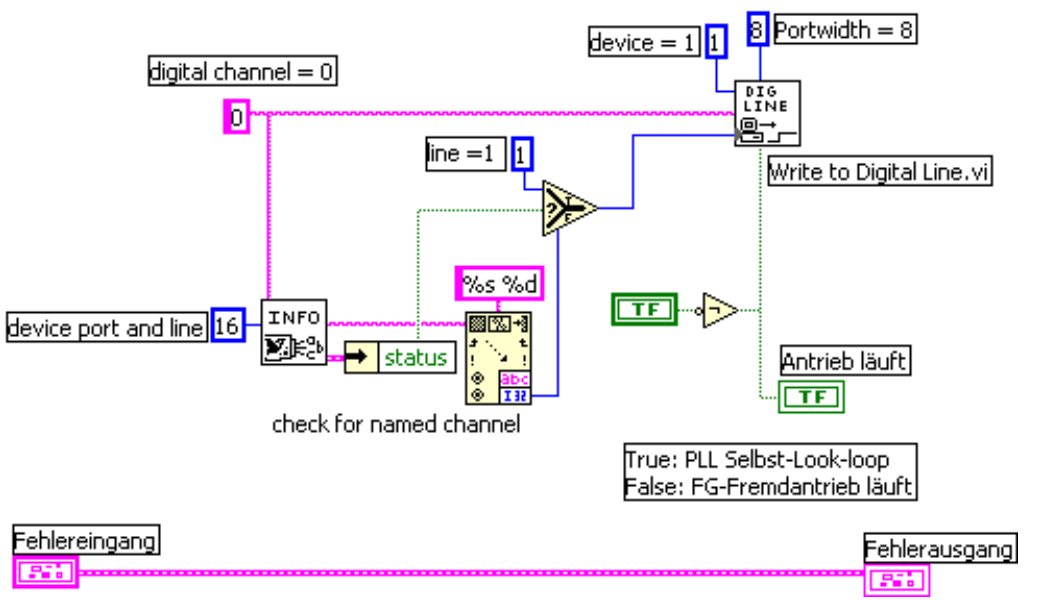


Über den Analogausgang OI $1($ line $=1)$ der seriellen Schnittstelle wird ein Relais angesprochen, das bei der Schalterstellung true den Antrieb ein-, bei false abschaltet. 


\section{Anhang 2 - Real- und Imaginärteil der HN-Funktion}

In Gleichung (2.16) wurde die komplexe Havriliak-Negami Funktion eingeführt und ihr Real- und Imaginärteil in (2.17) und (2.18) angegeben. Da deren Berechnung nicht ganz trivial ist, wird sie hier im Detail durchgeführt. Dazu wird von der HN-Funktion

$$
G^{*}=G_{\infty}+\frac{\left(G_{s}-G_{\infty}\right)}{\left[1+\left(i \omega \tau_{H N}\right)^{\alpha}\right]^{\gamma}}
$$

ausgegangen und zunächst die komplexe Zahl z wie folgt definiert:

$$
\frac{1}{Z}=\frac{1}{1+\left(i \omega \tau_{H N}\right)^{\alpha}}
$$

Eine beliebige komplexe Zahl z lässt sich in der Euler-Schreibweise immer auch als

$$
z=r \exp (i \varphi)
$$

darstellen. Für ihren Kehrbruch gilt:

$$
\frac{1}{Z}=\frac{\exp (-i \varphi)}{r}
$$

Mit der komplexen Identität $i=\exp \left(i \frac{\pi}{2}\right)$ und $\exp (i \varphi)=\cos (\varphi)+i \sin (\varphi)$ folgt:

$$
\begin{aligned}
z & =1+\left(i \omega \tau_{H N}\right)^{\alpha} \\
& =1+\left(\omega \tau_{H N}\right)^{\alpha} i^{\alpha} \\
& =1+\left(\omega \tau_{H N}\right)^{\alpha} \exp \left(i \frac{\pi}{2}\right)^{\alpha} \\
& =1+\left(\omega \tau_{H N}\right)^{\alpha} \exp \left(i \alpha \frac{\pi}{2}\right)= \\
& =1+\left(\omega \tau_{H N}\right)^{\alpha} \cdot \cos \left(\alpha \frac{\pi}{2}\right)+i \cdot\left(\omega \tau_{H N}\right)^{\alpha} \cdot \sin \left(\alpha \frac{\pi}{2}\right)
\end{aligned}
$$

Aus dem Real- und dem Imaginärteil dieser komplexen Zahl lassen sich nun umgekehrt $r$ und $\varphi$ bestimmen:

$$
\varphi=\arctan \left[\frac{\left(\omega \tau_{H N}\right)^{\alpha} \cdot \sin \left(\alpha \frac{\pi}{2}\right)}{1+\left(\omega \tau_{H N}\right)^{\alpha} \cdot \cos \left(\alpha \frac{\pi}{2}\right)}\right]
$$




$$
\begin{aligned}
& r=|z|=\sqrt{\left[1+\left(\omega \tau_{H N}\right)^{\alpha} \cdot \cos \left(\alpha \frac{\pi}{2}\right)\right]^{2}+\left[\left(\omega \tau_{H N}\right)^{\alpha} \cdot \sin \left(\alpha \frac{\pi}{2}\right)\right]^{2}}= \\
& =\sqrt{1+2 \cdot\left(\omega \tau_{H N}\right)^{\alpha} \cdot \cos \left(\alpha \frac{\pi}{2}\right)+\left[\left(\omega \tau_{H N}\right)^{\alpha} \cdot \cos \left(\alpha \frac{\pi}{2}\right)\right]^{2}+\left[\left(\omega \tau_{H N}\right)^{\alpha} \cdot \sin \left(\alpha \frac{\pi}{2}\right)\right]^{2}}= \\
& =\sqrt{1+2 \cdot\left(\omega \tau_{H N}\right)^{\alpha} \cdot \cos \left(\alpha \frac{\pi}{2}\right)+\left(\omega \tau_{H N}\right)^{2 \alpha}\left\{\left[\cos \left(\alpha \frac{\pi}{2}\right)\right]^{2}+\left[\sin \left(\alpha \frac{\pi}{2}\right)\right]^{2}\right\}}= \\
& =\sqrt{1+2 \cdot\left(\omega \tau_{H N}\right)^{\alpha} \cdot \cos \left(\alpha \frac{\pi}{2}\right)+\left(\omega \tau_{H N}\right)^{2 \alpha}}
\end{aligned}
$$

Eingesetzt in Gleichung (A2.4) gilt damit:

$$
\frac{1}{Z}=\frac{1}{1+\left(i \omega \tau_{H N}\right)^{\alpha}}=\frac{\exp (-i \varphi)}{\sqrt{1+2 \cdot\left(\omega \tau_{H N}\right)^{\alpha} \cdot \cos \left(\alpha \frac{\pi}{2}\right)+\left(\omega \tau_{H N}\right)^{2 \alpha}}}
$$

Nun wird betrachtet:

$$
\begin{aligned}
& \left(\frac{1}{z}\right)^{\gamma}=\frac{1}{\left(1+\left(i \omega \tau_{H N}\right)^{\alpha}\right)^{\gamma}}=\frac{\exp (-i \gamma \varphi)}{\left(1+2 \cdot\left(\omega \tau_{H N}\right)^{\alpha} \cdot \cos \left(\alpha \frac{\pi}{2}\right)+\left(\omega \tau_{H N}\right)^{2 \alpha}\right)^{\frac{\gamma}{2}}}= \\
& =\frac{\cos (\gamma \varphi)-i \sin (\gamma \varphi)}{\left(1+2 \cdot\left(\omega \tau_{H N}\right)^{\alpha} \cdot \cos \left(\alpha \frac{\pi}{2}\right)+\left(\omega \tau_{H N}\right)^{2 \alpha}\right)^{\frac{\gamma}{2}}}
\end{aligned}
$$

Mit diesen Vorarbeiten berechnet sich der Realteil $G^{\prime}$ und der Imaginärteil $G^{\prime \prime}$ der komplexen Havriliak-Negami Funktion wie in (2.18) und (2.19) dargestellt zu:

$$
\begin{aligned}
& G^{\prime}=G_{\infty}+\left(G_{s}-G_{\infty}\right) \cdot \frac{\cos (\gamma \varphi)}{\left(1+2 \cdot\left(\omega \tau_{H N}\right)^{\alpha} \cdot \cos \left(\alpha \frac{\pi}{2}\right)+\left(\omega \tau_{H N}\right)^{2 \alpha}\right)^{\frac{\gamma}{2}}} \\
& G^{\prime \prime}=\left(G_{\infty}-G_{s}\right) \cdot \frac{\sin (\gamma \varphi)}{\left(1+2 \cdot\left(\omega \tau_{H N}\right)^{\alpha} \cdot \cos \left(\alpha \frac{\pi}{2}\right)+\left(\omega \tau_{H N}\right)^{2 \alpha}\right)^{\frac{\gamma}{2}}}
\end{aligned}
$$

Dabei ist zu beachten, dass $G_{\infty}$ und $G_{s}$ reelle Zahlen sind. 


\section{Literaturverzeichnis}

[1] P. Lunkenheimer, U. Schneider, R. Brand und A. Loidl, Contemp. Phys. 41, 1, 15 (2000).

[2] W. Schirmacher, G. Diezemann und C. Ganter, Physica B 263-264, 160 (1999).

[3] J. Zarzycki, „Glasses and the vitreous state“, Cambridge University Press, Cambridge, UK (1991).

[4] S. R. Elliott, "Physics of amorphous materials, $2^{\text {nd }}$ edition", Longman Scientific \& Technical (1990).

[5] W. Kauzmann, Chem. Rev. 43, 219 (1948).

[6] K. Binder, J. Non-Cryst. Sol. 307-310, 1 (2002).

[7] W. Götze und L. Sjögren, Rep. Prog. Phys. 55, 241 (1992).

[8] T. Kirkpatrick und D. Thirumalai, Phys. Rev. B 37, 4439 (1988).

[9] H. Schumacher, „Untersuchungen mittels Röntgenabsorption von Glasübergang und Kristallisation metallischer Gläser“, Mensch und Buch Verlag, Augsburg (2000).

[10] W. Zhou, J. Non-Cryst. Sol. 201, 256 (1996).

[11] T. Zhang, A. Inoue, T. Masumoto, Mat. Trans. JIM 32, 1005 (1991).

[12] A. Inoue et al., Mat. Sci. Eng. A 178, 255 (1996).

[13] A. Peker und W. L. Johnson, Appl. Phys. Lett. 63:17, 2342 (1993).

[14] E. Bakke, R. Busch und W. L. Johnson, Appl. Phys. Lett. 67:22, 3260 (1995).

[15] A. Meyer, J. Wuttke, W. Petry, A. Peker, R. Bormann, G. Coddens, L. Kranich, O. G. Randl, H. Schober, Phys. Rev. B 53:18, 12107 (1996).

[16] I.-R. Lu, G. Wilde, G. P. Görler, R. Willnecker, J. Non-Cryst. Solids 250-252, 577 (1999).

[17] R. Böhmer, K. L. Ngai, C. A. Angell und D. J. Plazek, J. Chem. Phys. 99, 4201 (1993).

[18] C. A. Angell, Science 267, 1924 (1995).

[19] C. A. Angell, J. Non-Cryst. Sol. 102, 205 (1988). 
[20] D. J. Plazek und K. L. Ngai, Macromolecules 24, 1222 (1991).

[21] M. Weiß, „Mechanische Eigenschaften und thermische Stabilität tief unterkühlter Metallschmelzen im Bereich der Glastemperatur“, Verlag Neue Wissenschaft, Frankfurt am Main (2000).

[22] R. Kohlrausch, Ann. Phys. 91, 56 (1954) und Ann. Phys. 91, 179 (1854).

[23] G. Williams und D. C. Watts, Trans. Faraday Soc. 66, 80 (1970).

[24] A. Kursmovic und B. Cantor, Scripta Mater. 34, 1655 (1996).

[25] M. H. Cohen und D. Turnbull, J. Chem. Phys. 31, 1164 (1959).

[26] M. H. Cohen und G. S. Grest, Phys. Rev. B 20, 1077 (1979).

[27] G. S. Grest und M. H. Cohen, Advances in chemical physics 48, 455 (1981).

[28] G. Adam und J. H. Gibbs, J. Chem. Phys 43, 139 (1965).

[29] R. V. Chamberlin, R. Böhmer, E. Sanchez und C. A. Angell, Phys. Rev. B, 46, 5787 (1992).

[30] E. A. DiMarzio und I. C. Sanchez, in "Transport and Relaxation in Random Materials", edited by J. Klafter, R. J. Rubin and M. Shlesinger, World Scientific, London (1986).

[31] E. A. DiMarzio und A. J. M. Yang, J. Res. Natl. Inst. Stand. Technol. 102, 135 (1997).

[32] C. T. Moynihan und J. Schroeder, J. Non-Cryst. Sol. 160, 52 (1993).

[33] P. Rösner, M. Weiß, S. Schneider und K. Samwer, J. Non-Cryst. Sol. 307-310, 848 (2002).

[34] H. Teichler, J. Non-Cryst. Solids 312 - 314, 533 (2002).

[35] R. Böhmer, K. L. Ngai, C. A. Angell und D. J. Plazek, J. Chem. Phys. 99, 4201 (1993).

[36] U. Bengtzelius, W. Götze und A. Sjölander, J. Phys C 17, 5915 (1984).

[37] S. Arrhenius, Z. Phys. Chem. 4, 226 (1889).

[38] R. Brand, „Breitbandige dielektrische Spektroskopie zur Untersuchung der Glasdynamik plastischer Kristalle“, Dissertation, Augsburg (2000).

[39] H. Vogel, Phys. Z. 22, 645 (1921).

[40] G. S. Fulcher, J. Am. Ceram. Soc. 8, 229 (1923). 
[41] G. Tammann und W. Hesse, Z. Anorg. Allg. Chem. 156, 245 (1926).

[42] T. Odagaki, Phys. Rev. Lett. 75, 3701 (1995).

[43] C. A. Angell, in K. L. Ngai und G. B. Wright, Hg., „Relaxations in complex systems“, National Technical Information Service, US Dept. of Commerce, Springfield (1985).

[44] R. Böhmer und C. A. Angell, „Disorder effects on relaxational processes“, Springer Verlag, Berlin (1994).

[45] A. K. Doolittle, J. Appl. Phys. 22, 1471 (1951).

[46] M. L. Williams, R. F. Landel und J. D. Ferry, J. Am. Chem. Soc. 77, 3701 (1955).

[47] D. Kivelson, S. A. Kivelson, X.-L. Zhao, Z. Nussinov und G. Tarjus, Physica A 219, 27 (1995).

[48] G. Tarjus, D. Kivelson und S. A. Kivelson, in J. T. Fourkas, D. Kivelson, U. Mohanty und K. A. Nelson, Hg., "Supercooled Liquids: Advances and Novel Applications", ACS Publications, Washington (1997).

[49] A. Meyer, H. Franz, B. Sepiol, J. Wuttke und W. Petry, Europhys. Lett. 36, 379 (1996).

[50] A. Meyer, J. Wuttke und W. Petry, J. Non-Cryst. Sol. 250-252, 116 (1999).

[51] V. Zollmer, K. Ratzke, F. Faupel und A. Meyer, Phys. Rev. Lett. 90, 195502 (2003).

[52] P. Debye, Phys. Z. 3, 97 (1912).

[53] P. Debye, Ber. D. Phys. Ges. 16, 777 (1913).

[54] H. A. Kramers, Phys. Z. 30, 52 (1929).

[55] R. de Kronig, J. Opt. Soc. Am. 12, 547 (1926).

[56] R. Brand, P. Lunkenheimer und A. Loidl, J. Chem. Phys. 116, 10386 (2002).

[57] K. Schröter und E. Donth, J. Non-Cryst. Sol. 307-310, 270 (2002).

[58] J. Colmenero, J. Non-Cryst. Sol. 131-133, 860 (1991).

[59] J. Colmenero, A. Alegria, J. M. Alberdi und B. Frick, J. Non-Cryst. Sol. 131-133, 949 (1991).

[60] S. Havriliak und S. Negami, Polymer 8, 161 (1967).

[61] D. W. Davidson und R. H. Cole, J. Chem. Phys. 18, 1417 (1950). 
[62] D. W. Davidson und R. H. Cole, J. Chem. Phys. 19, 1484 (1951).

[63] K. S. Cole und R. H. Cole, J. Chem. Phys. 9, 341 (1941).

[64] F. Alvarez, A. Alegria und J. Colmenero, Phys. Rev. B 44, 7306 (1991).

[65] F. Alvarez, A. Alegria und J. Colmenero, Phys. Rev. B 47, 125 (1993).

[66] C. R. Snyder und F. I. Mopsik, Phys. Rev. B 60, 984 (1999).

[67] A. Meyer, R. Busch und H. Schober, Phys. Rev. Lett. 83, 5027 (1999).

[68] P. K. Dixon, L. Wu, S. R. Nagel, B. D. Williams und J. P. Carini, Phys. Rev. Lett. 65, 1108 (1990).

[69] N. Menon, K. P. O’Brian, P. K. Dixon, L. Wu, S. R. Nagel, B. D. Williams und J. P. Carini, J. Non-Cryst. Sol. 141, 61 (1992).

[70] N. Menon und S. R. Nagel, Phys. Rev. Lett. 74, 1230 (1995).

[71] Z. Dendzik, M. Paluch, Z. Gburski und J. Ziolo, J. Phys.: Cond. Matter 9, L339 (1997).

[72] R. Bergman, F. Alvarez, A. Alegria und J. Colmenero, J. Chem. Phys. 109, 7546 (1998).

[73] L. Onsager, J. Amer. Chem. Soc. 58, 1486 (1936).

[74] J. G. Kirkwood, J. Chem. Phys. 7, 911 (1939).

[75] H. Fröhlich, „Theory of Dielectrics, Second Edition”, Oxford University Press, Oxford (1958).

[76] C. J. F. Böttcher und P. Bordewijk, „Theory of electric polarization I and II, second Edition“, Elsevier, Amsterdam (1992).

[77] N. G. McCrum, B. E. Read und G. Williams, „Anelastic and Dielectric Effects in Polymeric Solids", John Wiley\&Sons, New York (1967).

[78] N. O. Birge, J. H. Jeong, S. R. Nagel, S. Bhattacharya und S. Susman, Phys. Rev. B 30, 2306 (1984).

[79] L. Wu und S. R. Nagel, Phys. Rev. B 46, R11198 (1992).

[80] A. Arbe, D. Richter, J. Colmenero und B. Farago, Phys. Rev. E 54, 3853 (1996). 
[81] A. Kudlik, C. Tschirwitz, S. Benkhof, T. Blochowicz und E. Rössler, Europhys. Lett. 40, 649 (1997).

[82] G. P. Johari, Ann. New York Acad. Sci. 279, 117 (1976).

[83] A. Arbe, U. Buchenau, L. Willner, D. Richter, B. Farago und J. Colmenero, Phys. Rev. Lett. 76, 1872 (1996).

[84] S. Corezzi, S. Capaccioli, G. Gallone, A. Livi und P. A. Rolla, J. Phys.: Condens. Matter 9, 6199 (1997).

[85] E. Donth, S. Kahle, J. Korus und M. Beiner, J. Phys. I (France) 7, 581 (1997).

[86] S. Yagihara, M. Yamada, M. Asano, Y. Kanai, N. Shinyashiki, S. Mahimo und K. L. Ngai, J. Non-Cryst. Sol. 235-237, 412 (1998).

[87] G. P. Johari und M. Goldstein, J. Chem. Phys. 53, 2372 (1970).

[88] F. Garwe, A. Schönhals, H. Lockwenz, M. Beiner, K. Schröter und E. Donth, Macromolecules 29, 247 (1997).

[89] F. Garwe, A. Schönhals, M. Beiner, K. Schröter und E. Donth, J. Phys.: Condens. Matter 6, 6941 (1994).

[90] E. Donth, K. Schröder und S. Kahle, Phys. Rev. E 60, 1099 (1999).

[91] A. Arbe, J. Colmenero, D. Gómez, D. Richter und B. Farago, Phys. Rev. E 60, 1103 (1999).

[92] P. Lunkenheimer, R. Wehn, Th. Riegger und A. Loidl, J. Non-Cryst. Sol. 307-310, 336 (2002).

[93] G. Williams und D. C. Watts, Trans. Farad. Soc. 67, 1971 (1971).

[94] G. Williams und D. C. Watts, in P. Diehl, E. Fluck und R. Kosfeld, Hrsg., „NRM:

Basic Principles and Progress“, Springer Verlag, Berlin, S. 271 (1971).

[95] G. P. Johari und M. Goldstein, J. Chem. Phys. 53, 2372 (1970).

[96] S. S. N. Murthy, J. Chem. Soc. Faraday Trans. 85, 581 (1989).

[97] A. Kudlik, C. Tschirwitz, T. Blochowicz. S. Benkhof und E. Rössler, J. Non-Cryst. Sol. 235-237, 406 (1998). 
[98] G. P. Johari und M. Goldstein, J. Phys. Chem. 74, 2034 (1970).

[99] G. P. Johari und M. Goldstein, J. Chem. Phys. 55, 4245 (1971).

[100] M. Goldstein, J. Chem. Phys. 51, 3728 (1969).

[101] K. L. Ngai, Comments Solid State Phys. 9, 127 (1979).

[102] H. Wagner und R. Richert, J. Non-Cryst. Sol. 242, 19 (1998).

[103] M. Vogel und E. Rössler, J. Phys. Chem. B 104, 4285 (2000).

[104] R. L. Leheny und S. R. Nagel, Europhys. Lett. 39, 447 (1997).

[105] P. Lunkenheimer und A. Loidl, Chem. Phys. 284, 205 (2002).

[106] P. Lunkenheimer, A. Pimenov, B. Schiener, R. Böhmer und A. Loidl, Europhys. Lett. 33, 611 (1996).

[107] A. Kudlik, S. Benkhof, T. Blochowicz, C. Tschirwitz und E. Rössler, J. Mol. Struct. 479, 201 (1999).

[108] S. Hensel-Bielowka und M. Paluch, Phys. Rev. Lett. 89, 025704 (2002).

[109] L. Wu, Phys. Rev. B 43, 9906 (1991).

[110] R. V. Chamberlin, Phys. Rev. B 48, 15638 (1993).

[111] R. V. Chamberlin, Phys. Rev. Lett. 82, 2520 (1999).

[112] A. Hofmann, F. Kremer, E. W. Fischer und A. Schönhals in R. Richert und A. Blumen, Hrsg., „Disorder effects on relaxational processes“, Springer Verlag, Berlin, S. 309 (1994).

[113] N. B. Olsen, J. Non-Cryst. Sol. 235-237, 399 (1998).

[114] M. Jiménez-Ruiz, M. A. Gonzáles, F. J. Bermejo, M. A. Miller, N. O. Birge, I. Cendoya und A. Alegriá, Phys. Rev. B 59, 9155 (1999).

[115] C. León und K. L. Ngai, J. Phys. Chem. B 103, 4045 (1999).

[116] C. León, K. L. Ngai und C. M. Roland, J. Chem. Phys. 110, 11585 (1999).

[117] H. Wagner und R. Richert, J. Chem. Phys. 110, 11660 (1999).

[118] U. Schneider, R. Brand, P. Lunkenheimer und A. Loidl, Phys. Rev. Lett. 84, 5560 (2000). 
[119] A. Segmüller, Thin Solid Films 18, 287 (1973).

[120] W. Hink, W. Petzold, Z. Angew. Phys. 10, 553 (1958).

[121] Reimer, „Transmission electron microscopy”, Springer Verlag, Berlin (1993).

[122] D. B. Williams, C. B. Butler, "Transmission Electron Microscopy: A Textbook for Materials Science", Plenum Press, New York und London (1996).

[123] H. Günzler und H. M. Heise, IR-Spektroskopie: eine Einführung, VCH, 3. Auflage (1996).

[124] R. N. Kleiman, G. K. Kaminsky, J. D. Reppy, R. Pindak and D .J. Bishop, Rev. Sci. Instr. 56, 2088 (1985).

[125] R. N. Kleiman, G. Agnolet and D. J. Bishop, Phys. Rev. Lett. 59, 2079 (1987).

[126] B. E. White, Jr and R. O. Pohl, Phys. Rev. Lett. 75, 4437 (1995).

[127] X. Liu and R. O. Pohl, Phys. Rev. B 58, 9067 (1998).

[128] M. Layer, M. Heitz, J. Classen, C. Enss and S. Hunklinger, J. Low. Temp. Phys. 124, 419 (2001).

[129] J. Classen, V. Keppens and S. Hunklinger, Physica B 284-288, 377 (2000).

[130] T. H. Metcalf and R. O. Pohl, J. Low Temp. Phys. 122, 545 (2001).

[131] C. L. Spiel, R. O. Pohl and A. T. Zehnder, Rev. Sci. Instr. 72, 1482 (2001).

[132] X. Liu, S. F. Morse, J. F. Vignola, D. M. Photiadis, A. Sarkissian, M. H. Marcus and B. H. Houston, Appl. Phys. Lett. 78, 1346 (2001).

[133] B. H. Houston, D. M. Photiadis, M. H. Marcus, J. A. Bucaro, X. Liu and J. F. Vignola, Appl. Phys. Lett. 80, 1 (2002).

[134] L. Kempen, U. Harms, H. Neuhäuser, D. Scholz, E. Peiner and A. Schlachetzki, J. de Physique IV, Colloque C8, 6, 643 (1996).

[135] R. A. Wind und M. A. Hines, Science 460, 21 (2000).

[136] T. H. Metcalf, Ph.D. thesis, Cornell University (2002).

[137] R. Merithew, Private Mitteilungen (2001). 
[138] Christian Gehrtsen, Helmut Vogel, Gehrtsen Physik, 19. Auflage, Springer Verlag, Berlin, S. 306 (1997).

[139] J. E. Van Cleve, Ph.D. thesis, Cornell University (1991).

[140] Horst Kuchling, Taschenbuch der Physik, Fachbuchverlag Leipzig (1991).

[141] S. P. Timoshenko und J. N. Goodier, in „Theory of Elasticity“, 3. Auflage, McGrawHill, New York (1970).

[142] V. B. Braginski, V. P. Mitrofanov, V. I. Panov, Systems with Small Dissipation, The University of Chicago Press, Chicago and London (1985).

[143] Xiao Liu, S. F. Morse, J. F. Vignola, D. M. Photiadis, A. Sarkissian, M. H. Marcus und B. H. Houston, Appl. Phys. Lett. 78, 1346 (2001).

[144] C. L. Spiel, R. O. Pohl and A. T. Zehnder, Rev. Sci. Instr. 72, 1482 (2001).

[145] G. Simmons and H. Wang, Single Crystal Elastic Constants and calculated Aggregate Properties: A Handbook, The M.I.T. Press, Cambridge, Massachussetts and London, England (1971).

[146] C. Zener, Elasticity and Anelasticity in Metal, The University of Chicago Press, Chicago (1994).

[147] EMIS Datareviews Series No. 4 - Properties of Silicon, edited by T. K. Ning, INSPEC, New York (1988).

[148] P. Rösner, K. Samwer, R. O. Pohl und S. Schneider, Rev. Sci. Instr. 74, 3395 (2003).

[149] T. Klitsner, Ph.D. thesis, Cornell University (1987).

[150] B. E. White, Ph.D. thesis, Cornell University (1996).

[151] A. Sarkissian, Private Mitteilung, Naval Research Laboratory, Washington (2003).

[152] M. Dopfer, „Spezifische Wärmemessungen von neuen metallischen Gläsern in der Nähe des Glasübergangs“, Diplomarbeit, Augsburg (1995).

[153] O. Müller, „Mechanische Spannungen und elektrische Transporteigenschaften von amorphen $\mathrm{Zr}_{65} \mathrm{Al}_{7.5} \mathrm{Cu}_{27.5}$ Schichten“, Diplomarbeit, Augsburg (1996).

[154] R. Rambousky, M. Moske und K. Samwer, Z. Phys. B. 99, 387 (1996). 
[155] B. M. Reinker, ,STM-Untersuchungen an amorphen ZrCo und ZrAlCuAufdampfschichten“, Dissertation, Wißner-Verlag, Augsburg (1997).

[156] S. G. Mayr, Dissertation, „Wachstum amorpher Schichten: Experiment und Simulation im Bereich Oberflächenrauhigkeit und mechanische Spannungen“, Göttingen (2001).

[157] A. Regenbrecht, G. von-Minnigerode und K. Samwer, Z. Phys. B: Condens. Matter 79, 25 (1990).

[158] A. R. Käufler, „Amorphe weichmagnetische CoFeNiSiB-Detektionsschichten in Spinventilen“, Dissertation, Göttingen (2002).

[159] E. Süske, „Laserdeposition von Polymer-Metall-Schichten“, Diplomarbeit, Göttingen (2001).

[160] H.-U. Krebs, M. Weisheit, J. Faupel, E. Süske, T. Scharf, C. Fuhse, M. Störmer, K. Sturm, M. Seibt, H. Kijewski, D. Nelke, E. Panchenko und M. Bubak, Phys. Chem. Chem. Phys. 5, 1365 (2003).

[161] K. Fukao, S. Uno, Y. Miyamoto, A. Hoshino und H. Miyaji, Phys. Rev. E 64, 051807 (2001).

[162] R. Bergman, F. Alvarez, A. Alegria und J. Colmenero, J. Chem. Phys. 109, 19, 7546 (1998).

[163] R. Wehn, Universität Augsburg, private Mitteilungen (2003).

[164] D. Landau und E. M. Lifschitz, „Lehrbuch der Theoretischen Physik VII, Elastizitätstheorie“, Akademie-Verlag, Berlin (1991).

[165] N. W. Ashcroft und N. D. Mermin, „Festkörperphysik, Kapitel 22“, OldenbourgVerlag, München, Wien (2001).

[166] W. A. Brantley, J. Appl. Phys. 44, 534 (1973).

[167] Taechung Yi, Lu Li und Chang-Jin Kim, Sens. Actuators A 83, 172 (2000).

[168] S. A. Chambers und J. H. Weaver, J. Vac. Sci. Technol. A 3, 1929 (1985).

[169] I. Stolt und F. m. d'Heurle, Thin Solid Films 189, 269 (1990).

[170] A. Cros, M. O. Aboelfotoh und K. N. Tu, J. Appl. Phys. 67, 3328 (1990).

[171] K. Holloway und P. M. Fryer, Appl. Phys. Lett. 57, 1736 (1990). 
[172] K. M. Latt, Y. K. Lee, T. Osipowicz und H. S. Park, Mat. Sci. and Eng. B 94, 111 (2002).

[173] R. Rambousky, „Dynamisch-mechanische Eigenschaften neuer ternärer und mehrkomponentiger Gläser“, Diplomarbeit, Universität Augsburg (1994).

[174] P. Rösner, K. Samwer und P. Lunkenheimer, Phys. Rev. Lett., submitted (2003).

[175] B. Jérôme und J. Commandeur, Nature 386, 589 (1997).

[176] H. Teichler, J. Non-Cryst. Sol. 293-295, 339 (2001).

[177] K. Vollmayr-Lee, W. Kob, K. Binder und A. Zippelius, J. Chem. Phys. 116, 5158 (2002).

[178] W. Kob, C. Donati, S. J. Plimpton, P. H. Poole und S. C. Glotzer, Phys. Rev. Lett. 79, 2827 (1997).

[179] K. Schröter, G. Wilde, R. Willnecker, M. Weiss, K. Samwer und E. Donth, Eur. Phys. J. B 5, 1 (1998).

[180] K. H. J. Buschow, J. Appl. Phys. 52, 3319 (1980).

[181] Z. Altounian, T. Gou-hua und J. O. Strom-Olsen, J. Appl. Phys. 53, 4755 (1982).

[182] E. Kneller, Y. Khan und U. Gorres, Z. Metallkunde 77, 152 (1986).

[183] W. A. Johnson und P. A. Mehl, Trans. Am. Inst. Mining Metall. Eng 135, 416 (1939).

[184] A. N. Kolmogorov, Bull. Acad. Sci. USSR, Phys. Ser. 1, 355 (1937).

[185] M. Avrami, J. Chem. Phys. 7, 1103 (1939), 8, 212 (1940) und 9, 177 (1941).

[186] P. Haasen, „Physikalische Metallkunde“, 3. Auflage, Springer-Verlag, (1994) S. 148.

[187] T. Prandell, D. Crespo, N. Clavaguera und M. T. Clavaguera-Mora, J. Phys.: Condens. Matter 10, 3833 (1998).

[188] R. Larciprete und M. Stuke, Appl. Phys. B 42, 181 (1987).

[189] B. Garrison und R. Srinivasan, J. Appl. Phys. 57, 2909 (1985).

[190] M. Tsunekawa, S. Nishio und H. Sato, J. Appl. Phys. 76, 5598 (1994).

[191] G. B. Blanchet, P. Cotts und C. R. Fincher Jr, J. Appl. Phys. 88, 2975 (2000).

[192] G. B. Blanchet und C. R. Fincher, Jr., Appl. Phys. Lett. 65, 1311 (1994). 
[193] J. Dechant, „Ultrarotspektroskopische Untersuchungen an Polymeren“, AkademieVerlag, Berlin (1972).

[194] T. G. Fox und P. J. Flory, J. Appl. Phys. 21, 581 (1950).

[195] K. O’Driscoll und R. A. Sanayei, Macromolecules 24, 4479 (1991).

[196] E. Süske, T. Scharf, P. Schaaf, E. Panchenko, D. Nelke, M. Buback, H. Kijewski und H. U. Krebs, Appl. Phys. A, eingereicht (2003).

[197] S. B. Sane und W. G. Knauss, Mech. Time-Dep. Mat. 5, 293 (2001).

[198] E. Muzeau, J. Perez und G. P. Johari, Macromolecules 24, 4713 (1991). 


\section{Lebenslauf}

Name:

Geburtsdatum:

Geburtsort:

Familienstand:

Staatsangehörigkeit:

Sept. 1978 bis Juli 1982:

Sept. 1982 bis Juli 1992:

Nov. 1992 bis Aug. 1999:

Sept. 1997 bis Nov. 1998:

Diplomarbeit bei Prof. Dr. F. Haider, Thema: „Bestimmung der

Freien Enthalpie bei der Martensitumwandlung in $\mathrm{NiTi}^{*}$

Sept. 1999 bis Juli 2000: Zivlidienst bei der ökum. Sozialstation Meitingen

Sept. 2000 bis März 2004: Promotionsstudium Physik an der Universität Göttingen

Wissenschaftlicher Mitarbeiter im SFB 602, B8

Assoziierter Kollegiat im GRK 782

Anfertigen der vorliegenden Dissertation am I. Physikalischen

Institut der Universität Göttingen bei Prof. Dr. Konrad Samwer

Göttingen, 22.1.04

(Peter Christian Rösner) 